\title{
Regesten Kaiser Friedrichs III.
}

$$
\text { (1440-1493) }
$$

nach Archiven und Bibliotheken geordnet

begründet von Heinrich Koller

herausgegeben von

Paul-Joachim Heinig, Christian Lackner und Alois Niederstätter

Heft 34

Die Urkunden und Briefe des Österreichischen Staatsarchivs in Wien, Abt. Haus-, Hof- und Staatsarchiv: Allgemeine Urkundenreihe, Familienurkunden und Abschriftensammlungen (1476-1479)

\author{
bearbeitet von \\ Kornelia Holzner-Tobisch \\ nach Vorarbeiten von Anne-Katrin Kunde
}


Regesten Kaiser Friedrichs III. (1440-1493)

Heft 34 


\section{Regesten Kaiser Friedrichs III.}

(1440-1493)

nach Archiven und Bibliotheken geordnet

begründet von Heinrich Koller

herausgegeben von

Paul-Joachim Heinig, Christian Lackner und Alois Niederstätter

Heft 34

Die Urkunden und Briefe des Österreichischen Staatsarchivs in Wien, Abt. Haus-, Hof- und Staatsarchiv: Allgemeine Urkundenreihe, Familienurkunden und Abschriftensammlungen (1476-1479)

bearbeitet von

Kornelia Holzner-Tobisch

nach Vorarbeiten von Anne-Katrin Kunde

BÖHLAU VERLAG WIEN KÖLN WEIMAR 


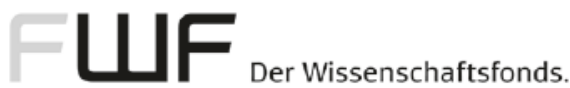 \\ Veröffentlicht mit Unterstützung des Austrian Science Fund (FWF): PUB 597-G28}

Open Access: Wo nicht anders festgehalten, ist diese Publikation lizenziert unter der Creative-Commons-

Lizenz Namensnennung 4.0; siehe http://creativecommons.org/licenses/by/4.0/

Die Publikation wurde einem anonymen, internationalen Peer-Review-Verfahren unterzogen.

Bibliografische Information der Deutschen Nationalbibliothek:

Die Deutsche Nationalbibliothek verzeichnet diese Publikation in der

Deutschen Nationalbibliografie; detaillierte bibliografische Daten sind im Internet über http://dnb.d-nb.de abrufbar.

(C) 2019 by Böhlau Verlag Ges.m.b.H \& Co. KG, Wien, Kölblgasse 8-10, A-1030 Wien

Korrektorat: Jörg Eipper-Kaiser, Graz

Satz: Michael Rauscher, Wien

Druck und Bindung: Generaldruckerei, Szeged

Gedruckt auf chlor- und säurefrei gebleichtem Papier

Printed in the EU

Vandenhoeck \& Ruprecht Verlage | www.vandenhoeck-ruprecht-verlage.com

ISBN 978-3-205-23217-9 


\section{Inhalt}

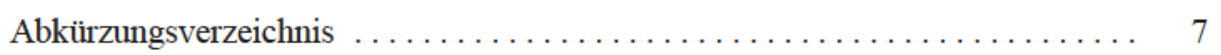

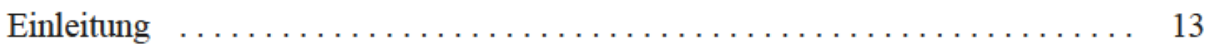

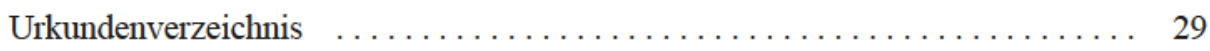

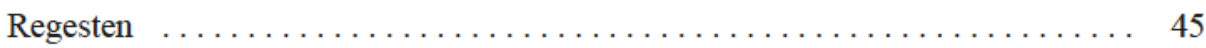

Quellen- und Literaturverzeichnis $\ldots \ldots \ldots \ldots \ldots \ldots \ldots \ldots \ldots \ldots \ldots \ldots \ldots \ldots \ldots$

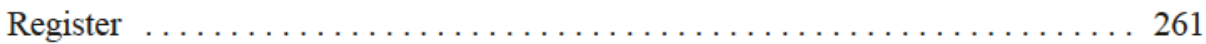




\section{Abkürzungsverzeichnis}

$\begin{array}{ll}\text { Abb. } & \text { Abbildung(en) } \\ \text { abgeg. } & \text { abgegangen } \\ \text { Abt. } & \text { Abteilung } \\ \text { a.d. } & \text { an der } \\ \text { A.m.d.i. } & \text { Ad mandatum domini imperatoris } \\ \text { A.m.d.i.i.c. } & \text { Ad mandatum domini imperatoris in consilio } \\ \text { A.m.d.i.p. } & \text { Ad mandatum domini imperatoris proprium } \\ \text { A.m.p.d.i. } & \text { Ad mandatum proprium domini imperatoris } \\ \text { Anh. } & \text { Anhang } \\ \text { anh. } & \text { anhängend } \\ \text { Anm. } & \text { Anmerkung } \\ \text { AO } & \text { Ausstellungsort (der von K.F. ausgestellten Urkunden) } \\ \text { AÖG } & \text { Archiv für österreichische Geschichte (Bde. 1-33: Archiv für } \\ & \text { Kunde österreichischer Geschichts-Quellen) } \\ \text { ARS } & \text { Arhiv Republike Slovenije } \\ \text { Art. } & \text { Artikel } \\ \text { aufgedr. } & \text { aufgedrückt } \\ \text { Aufl. } & \text { Auflage } \\ \text { Aug. } & \text { August } \\ \text { AUR } & \text { Allgemeine Urkundenreihe } \\ \text { Ausst. } & \text { Aussteller/Ausstellerin } \\ \text { Bd./Bde. } & \text { Band/Bände } \\ \text { Bearb. } & \text { Bearbeiter(-in) } \\ \text { bearb. } & \text { bearbeitet } \\ \text { Beil. } & \text { Beilage } \\ \text { bes. } & \text { besonders } \\ \text { Best. } & \text { Bestand } \\ \text { betr. } & \text { betreffend } \\ \text { Bf./Bff. } & \text { Bischof/Bischöfe } \\ \text { Bggf./Bggff. } & \text { Burggraf/Burggrafen } \\ \text { böhm. } & \text { böhmisch } \\ \text { bzgl. } & \text { bezüglich } \\ \text { bzw. } & \text { beziehungsweise } \\ \text { ca. } & \text { circa } \\ \text { Chmel } & \text { Regesta chronologico-diplomatica, hg. v. J. Chmel } \\ \text { C.d.i.i.c. } & \text { Commissio domini imperatoris in consilio } \\ \text { C.d.i.p. } & \text { Commissio domini imperatoris propria } \\ & \end{array}$


collat.

d.

d. Ä.

dems.

Dep.

Dép.

ders.

desgl.

Dez.

Diöz.

Diss.

d.J.

$d n$

DO

Dr.

Dr. iur.

Dr. iur. utr.

Dr. med.

DRW

dt.

e.

Eb.

ebd.

Ehz./Ehzn.

eingedr.

erg.

Erg.-Bd.

erw.

etc.

evtl.

f./ff.

Fasz.

Febr.

fl.

fl. rh.

fl. ung.

fol.

Fotokop.

Friedr. collationiert

das, dem, den, der, des, die

der Ältere

demselben

Deperditum

Département

derselbe

desgleichen

Dezember

Diözese

Dissertation

der Jüngere

denarii

Datierungsort (= Ausstellungsort der nicht von K.F. ausgestellten Urkunden)

Doctor

Doctor iuris

Doctor iuris utriusque

Doctor medicinae

Deutsches Rechtswörterbuch

deutsch

ein(-e, -em, -en, -er, -es)

Erzbischof

ebenda

Erzherzog/Erzherzogin

eingedrückt

ergänzt

Ergänzungsband

erwähnt

et cetera

eventuell

folgend/folgende

Faszikel

Februar

Gulden

rheinische(r) Gulden

ungarische(r) Gulden/Dukaten

Folio

Fotokopie

Friedrich 
Ftm.

$\mathrm{FU}$

gen.

Gf./Gff./Gfn./Gft.

gg.

$\mathrm{H}$.

Habil.-Schr.

HAUrk

$\mathrm{Hg}$.

hg.

HHStA

Hs.

HStA
Fürstentum

Familienurkunden

genannt

Graf/Grafen/Gräfin/Grafschaft

gegen

Heft

Habilitationsschrift

Hauptarchiv-Urkunden

Herausgeber(-in, -innen)

herausgegeben

Haus-, Hof- und Staatsarchiv

Handschrift

Hauptstaatsarchiv

Hz./Hzm./Hzn./Hzz. Herzog/Herzogtum/Herzogin/Herzöge

ital.

Jan.

Jg.

Jh.

$\mathrm{K}$.

K.F.

Kf./Kff.

$\mathrm{KG}$

$\mathrm{Kg} . / \mathrm{Kgn} . / \mathrm{Kgr}$.

$\mathrm{kgl}$.

KLA

Kler.

Konv.

Konz.

Kop.

ksl.

Kt.

$\mathrm{KVr}$

$\mathrm{KVv}$

lat.

Ldgf./Ldgft.

Lfg.

Lic. decr.

Lic. iur. can.

Lic. iur. utr.

Lit. italienisch

Januar

Jahrgang

Jahrhundert

Kaiser

Kaiser Friedrich

Kurfürst/Kurfürsten

Kammergericht

König/Königin/Königreich

königlich

Kärntner Landesarchiv

Kleriker

Konvolut

Konzept

Kopie

kaiserlich

Karton

Kanzleivermerk auf der Vorderseite der Urkunde

Kanzleivermerk auf der Rückseite der Urkunde

lateinisch

Landgraf/Landgrafschaft

Lieferung

Licentiatus decretorum

Licentiatus iuris canonici

Licentiatus iuris utriusque

Literatur 
MC

Mgf./Mgft.

$\mathrm{MGH}$

mglw.

MHVSt

MIÖG

MÖSTA

Mon. Habsb.

n.

n./nn.

ND

NDB

NF

niederösterr.

NÖ

nö.

Nov.

Nr.

nw.

oberösterr.

ö.

öff.

österr.

Okt.

o.O.

OÖLA

Org./Orgg.

P.

p.

päpstl.

Pap.

Perg.

Pf.

Pfd.
Monumenta historica ducatus Carinthiae

Markgraf/Markgrafschaft

Monumenta Germaniae Historica

möglicherweise

Mitteilungen des Historischen Vereines für Steiermark

Mitteilungen des Instituts für Österreichische

Geschichtsforschung

Mitteilungen des Österreichischen Staatsarchivs

Monumenta Habsburgica

nördlich

Nummer/Nummern

Nachdruck

Neue Deutsche Biographie

Neue Folge

niederösterreichisch

Niederösterreich

nordöstlich

November

Nummer

nordwestlich

oberösterreichisch

östlich

öffentlich

österreichisch

Oktober

ohne Ort

Oberösterreichisches Landesarchiv

Original/Originale

Papst

Pagina

päpstlich

Papier

Pergament

Pfennig

Pfund

Pfgf./Pfgfn./Pfgft. Pfalzgraf/Pfalzgräfin/Pfalzgrafschaft

phil.-hist.

philosophisch-historisch

Privil.

Privileg

Ps

Pergamentstreifen

purpurf.

purpurfarben 


\begin{tabular}{ll} 
QGStW & Quellen zur Geschichte der Stadt Wien \\
QIÖG & Quelleneditionen des Instituts für Österreichische Geschichtsfor- \\
& schung \\
r & recto \\
RA & Reichs-Archiv \\
REC & Regesta episcoporum Constantiensium \\
red. & redigiert \\
Reg./Regg. & Regest/Regesten \\
Regg.F.III. & Regesten Kaiser Friedrichs III. \\
RI & Regesta Imperii \\
röm. & römisch \\
röm.-dt. & römisch-deutsch \\
Rta & Registrata \\
rücks. & rückseitig \\
S & Siegel \\
S. & Seite \\
s. & siehe \\
$\beta$ & solidi \\
Sch. & Schilling \\
Sept. & September \\
Sign. & Signatur \\
sö. & südöstlich \\
sog. & sogenannt \\
Sp. & Spalte \\
SS & Siegel (Plural) \\
Ss & Seidenschnur \\
St. & Sankt \\
StA & Staatsarchiv \\
StadtA & Stadtarchiv \\
StLA & Steiermärkisches Landesarchiv \\
STUF & Studien und Forschungen aus dem Niederösterreichischen \\
& Institut für Landeskunde \\
sub dat. & sub dato \\
sü. & südlich \\
sw. & südwestlich \\
Tab. & Tabelle \\
Tf. & Tafel \\
Tl./Tle. & Teil/Teile \\
tlw. & teilweise \\
u. & und \\
& \\
\hline
\end{tabular}




$\begin{array}{ll}\text { u.a. } & \text { und andere/unter anderem } \\ \text { UB } & \text { Urkundenbuch } \\ \text { Urk. } & \text { Urkunde } \\ \text { UStA } & \text { Ungarisches Staatsarchiv } \\ \text { v } & \text { verso } \\ \text { v. } & \text { von/vom } \\ \text { Veröff. } & \text { Veröffentlichungen } \\ \text { VIÖG } & \text { Veröffentlichungen des Instituts für Österreichische } \\ & \text { Geschichtsforschung } \\ \text { vmtl. } & \text { vermutlich } \\ \text { vorders. } & \text { vorderseitig } \\ \text { w. } & \text { westlich } \\ \text { wachsf. } & \text { wachsfarben } \\ \text { Wr. Neust. } & \text { Wiener Neustadt } \\ \text { WStLA } & \text { Wiener Stadt- und Landesarchiv } \\ \text { Z.B. } & \text { zum Beispiel } \\ \text { ZGO } & \text { Zeitschrift für die Geschichte des Oberrheins } \\ \text { ZHF } & \text { Zeitschrift für Historische Forschung } \\ \text { ZHVStmk } & \text { Zeitschrift des Historischen Vereines für Steiermark } \\ \text { ZRG } & \text { Zeitschrift der Savigny-Stiftung für Rechtsgeschichte } \\ \text { Zw. } & \text { Zwischen }\end{array}$




\section{Einleitung}

Der vorliegende Regestenband ist das siebente Heft aus der Wiener Reihe der Regesten von Urkunden und Briefen Kaiser Friedrichs III. aus dem Haus-, Hof- und Staatsarchiv (HHStA) in Wien. Er schließt mit 332 Regesten zu den Jahren 1476 bis 1479 chronologisch an das Heft 27 an, das Regesten zu den Jahren 1470 bis 1475 bietet. Die durch das zwischenzeitliche Erscheinen des Heftes 30 mit Regesten zu den Jahren 1483 bis 1488 bedingte chronologische Lücke in der Wiener Reihe, die sich durch den Bearbeiterwechsel im Rahmen der vom österreichischen Wissenschaftsfonds FWF finanzierten, befristeten Projekte ergab, kann somit ein Stück weit geschlossen werden. Regesten zu den drei „fehlenden“ Jahren 1480 bis 1482 werden von Petra Heinicker vorgelegt (Heft 35). Der die Bearbeitung des HHStA abschließende neunte Band der Wiener Reihe zu den letzten Regierungsjahren Friedrichs III., 1489 bis 1493, wird von Daniel Luger erarbeitet.

Wie in den Vorgängerbänden wurden aus den Beständen Allgemeine Urkundenreihe (AUR), Familienurkunden und Urkundenabschriften des 18./19. Jahrhunderts des HHStA Wien original und kopial überlieferte Urkunden und Briefe Friedrichs III. aufgenommen. ${ }^{1}$ Ebenfalls berücksichtigt wurden die in der AUR vereinzelt überlieferten Konzepte. Als „Deperdita“ im Sinne der Richtlinien der Gesamtreihe der Regesten Kaiser Friedrichs III. wurden jene Stücke aufgenommen, die zum einen im bearbeiteten Bestand nicht überliefert sind, sich aber sicher erschließen lassen, etwa durch Erwähnung eines kaiserlichen Briefes, und zum anderen in den bisher erschienenen Regestenbänden der Gesamtreihe noch nicht erfasst wurden. Diese „Deperdita“ müssen somit nicht unbedingt unbekannt sein und können im Rahmen der künftigen Bearbeitung weiterer Archive bzw. Archivlandschaften noch regestiert werden. Auch Urkunden, die nur als Regest bei Joseph Chmel bekannt sind und sich im HHStA befunden haben oder befinden, bislang aber nicht gefunden werden konnten, sind aufgenommen worden. Eine Sondergruppe bilden die um 1980 an das ehemalige Jugoslawien abgegebenen Stücke (heute im Arhiv Republike Slovenje in Ljubljana), die in Form von Fotokopien in der AUR eingereiht blieben und daher als Kopialüberlieferung berücksichtigt wurden. Regesten auf der Grundlage der Originale bietet zwar das 2014 erschienene Heft 29 der Friedrich III.-Regesten, allerdings nur in knapper Form, weshalb in diesem Band ausführliche Regesten anhand der Fotokopien erstellt wurden. Aufgenommen wurde auch eine bei Chmel gedruckte Urkunde des in den 1950er-Jahren an das Oberösterreichische Landesarchiv abgegebenen Bestands Spital am Pyhrn sowie ein nach einer Überlieferung im HHStA Wien bei Chmel genanntes Privileg für Klosterneuburg, das sich im dortigen Stadtarchiv befindet.

1 Zur Geschichte des Archivs und der Urkundenbestände s. den Überblick in den Regg.F.III. H. 12 S. If. 


\section{1. Überlieferung}

\begin{tabular}{|l|c|c|c|c|c|}
\hline Jahr & gesamt & Original & kopial & $\begin{array}{c}\text { nach } \\
\text { Konzept }\end{array}$ & Deperditum \\
\hline $\mathbf{1 4 7 6}$ & 45 & $\begin{array}{c}18 \\
(40 \%)\end{array}$ & $\begin{array}{c}3 \\
(6,7 \%)\end{array}$ & $\begin{array}{c}24 \\
(53,3 \%)\end{array}$ \\
\hline $\mathbf{1 4 7 7}$ & 64 & $\begin{array}{c}16 \\
(25 \%)\end{array}$ & $\begin{array}{c}10 \\
(15,6 \%)\end{array}$ & $\begin{array}{c}38 \\
(59,4 \%)\end{array}$ \\
\hline $\mathbf{1 4 7 8}$ & 113 & $\begin{array}{c}28 \\
(24,8 \%)\end{array}$ & $\begin{array}{c}11 \\
(9,7 \%)\end{array}$ & $\begin{array}{c}20 \\
(17,7 \%)\end{array}$ & $\begin{array}{c}54 \\
(47,8 \%)\end{array}$ \\
\hline $\mathbf{1 4 7 9}$ & 110 & $\begin{array}{c}26 \\
(23,6 \%)\end{array}$ & $\begin{array}{c}7 \\
(6,4 \%)\end{array}$ & $\begin{array}{c}21 \\
(19,1 \%)\end{array}$ & $\begin{array}{c}56 \\
(50,9 \%)\end{array}$ \\
\hline Gesamt & 332 & $\begin{array}{c}88 \\
(26,6 \%)\end{array}$ & $\begin{array}{c}31 \\
(9,3 \%)\end{array}$ & $\begin{array}{c}41 \\
(12,3 \%)\end{array}$ & $\begin{array}{c}172 \\
(51,8 \%)\end{array}$ \\
\hline
\end{tabular}

Tab. 1: Urkundenüberlieferung 1476-1479

Der Jahresausstoß an Urkunden ist mit durchschnittlich 83 Stücken im Vergleich zu den Jahren 1470 bis 1475 mit 47 Stücken deutlich gestiegen. ${ }^{2}$ Insbesondere in den Jahren 1478 und 1479 ist ein sprunghafter, über dem Jahresschnitt liegender Anstieg zu verzeichnen, der sich zum einen aus der Überlieferung von Konzepten mit umfangreichen Empfängerlisten ${ }^{3}$ ergibt und zum anderen aus der ab Januar 1478 einsetzenden dichten Überlieferung an erbländischen Pfleg-, Amt-, Pfand- und Lehensreversen, die möglicherweise auf den Aufenthalt Friedrichs III. in Graz zurückzuführen ist. Nach dem Friedensschluss mit König Matthias von Ungarn im Dezember 1477 weilte er von Januar 1478 bis Dezember 1479 durchgehend in Graz. Denkbar ist, dass die Kontinuität von Kaiser und Kanzlei vor Ort auch die Überlieferungschancen der im Ausstellerarchiv verwahrten Stücke durch eine verbesserte Archivierung und Registratur erhöhte. ${ }^{4}$

Nur etwa ein Viertel der regestierten Stücke - nämlich 88 - sind als Originale überliefert, darunter allerdings als besonders wertvolle Stücke ein verloren geglaubtes, im Jahr 2015 wiedergefundenes eigenhändiges Schreiben Friedrichs III., das in den 1930erJahren versehentlich in den Bestand der „Fridericiana“ eingereiht worden war, sowie eine mit einem eigenhändigen Vermerk des Kaisers versehene Urkunde. ${ }^{5}$ Zwei weitere eigenhändige Schreiben des Kaisers werden zwar im bearbeiteten Zeitraum als Beilage

2 Zu den Jahren 1470-1475 s. Regg.F.III. H. 27 S. 14.

3 Siehe nn. 171-187 und 244-264.

4 Zur Schriftlichkeit in der Verwaltung s. ausführlich unten.

5 Siehe nn. 157, 324. 
zu einer kaiserlichen Urkunde aus dem Jahr 1479 verwahrt (Sign. AUR 1479 X 14), datieren jedoch in die Jahre 1480 und 1481 und sind daher im Folgeband von Petra Heinicker regestiert. ${ }^{6}$ Teil der Originalüberlieferung sind zudem zwei Noteln, eine zwischen Konzept und Ausfertigung stehende Urkundenform mit rechtsverbindlichem Charakter, der bei beiden Stïcken durch die Besiegelung gegeben ist. ${ }^{7}$ Während der Kaiser bei dem einen Stück - dem von Matthias Corvinus für Böhmen zu leistenden Lehenseid - mit Ausnahme des Siegels überhaupt nicht als Aussteller auftritt, wird er bei dem zweiten, als abrede bezeichneten Stück zunächst - wie bei vielen vergleichbaren beredmusszedeln - in der 3. Person als Vertragspartei genannt, jedoch erfolgt, da es sich um eine von ihm beschlossene abrede handelt, die Siegelankündigung in der 1. Person.

Kopial überliefert sind im bearbeiteten Zeitraum 31 Stücke in Form von fünf Vidimus, vier Notariatsinstrumenten, zehn Abschriften aus dem 15. Jahrhundert und vier Abschriften aus dem 18./19. Jahrhundert sowie acht Fotokopien (der an das heutige Slowenien abgetretenen Urkunden).

Fünf Konzepte, zwei davon mit beigefügtem Empfängerverzeichnis, sind die Grundlage für 41 Urkunden. ${ }^{8}$

Mit mehr als der Hälfte der Überlieferung ( $51,8 \%$ ) bilden Deperdita die weitaus größte Gruppe der Regesten; unter Berücksichtigung der auf Grundlage der Konzepte erschlossenen Urkunden würde sich der Anteil sogar auf über 60 \% erhöhen. Gegenüber den in den Wiener Vorgängerbänden bearbeiteten Regierungsjahren 1440 bis 1475 ist somit ein weiterer prozentueller Anstieg der Deperdita zu verzeichnen, deren Anteil an der Überlieferung sich in den 1480er-Jahren dann auf etwa $60 \%$ stabilisiert. ${ }^{9}$ Die Mehrheit der Deperdita, nämlich 129 Stück (75\%), wurde aus Reversen erschlossen, deren große Zahl im bearbeiteten Bestand - immerhin $39 \%$ der Gesamtüberlieferung - eine Besonderheit des HHStA Wien als Ausstellerarchiv und somit Empfängerarchiv für Reverse ist. ${ }^{10}$ Die in den 1480er-Jahren etwa gleichbleibende, ebenfalls überwiegend auf Reversen basierende Überlieferung von Deperdita würde die Annahme stützen, dass hier eine in den 70er-Jahren einsetzende Verdichtung der Schriftlichkeit in der Verwaltung und nachhaltige Verbesserung der Archivierung greifbar werden könnte. ${ }^{11}$

6 Siehe Regg.F.III. H. $35 \mathrm{nn} .1$ und 159 sowie den Kommentar zu unserer n. 320.

7 Siehe nn. 108, 326 und dazu ausführlich ErBL, Entwurf.

8 Siehe nn. 121, 171 (+ Empfängerverzeichnis), 190, 212, 244 (+ Empfängerverzeichnis).

9 Zur Entwicklung der Anteils der Deperdita an der Überlieferung des HHStA Wien für die Jahre 14401475 mit einem Anstieg von $28,5 \%$ auf $40,3 \%$ s. ausführlich Regg.F.II. H. 22 S. 14, wobei in den Jahren 1464-1469 nahezu eine prozentuelle Verdoppelung zu verzeichnen ist; für die Jahre 1470-1475 bildete der prozentuelle Anteil $46 \%$, s. Regg.F.III. H. 27 S. 14. - Zur Entwicklung in den Folgejahren 1480-1482 (60\%) und 1483-1488 (59,9\%) s. Regg.F.II. H. 35 S. 11 und H. 30 S. 14.

10 Zur Überlieferung der Reverse s. ausführlich Regg.F.III. H. 22 S. 16-18.

11 Für die Jahre 1483-1488 wurden 55 \% der Deperdita aus Reversen erschlossen, s. Regg.F.III. H. 30 S. 14. 
Die Grundlage der restlichen Deperdita bildet sehr vielfältiges Material, von Friedensverträgen, habsburgischer Korrespondenz und päpstlichen Schreiben über Prozessund Aufsendungsurkunden bis zu Quittungen und Urfehdebriefen, wobei insbesondere auf die im Rahmen von Friedensverträgen und Waffenstillständen - u.a. mit Matthias Corvinus - im Ausstellerarchiv überlieferten Gegenurkunden verwiesen werden soll. ${ }^{12}$

\section{2. Äußere Merkmale der Urkunden}

Von den regestierten 88 Originalurkunden wurde der Großteil, nämlich 72, auf Pergament ausgefertigt, 16 auf Papier, womit, wie in den Vorheften, ein mit der Eigenheit des Wiener Archivs begründbares Übergewicht der Pergamenturkunde ( $82 \%$ ) vorliegt. ${ }^{13}$ In der Regel handelt es sich bei den Ausfertigungen auf Pergament um Belehnungen, Privilegien, Urkundenbestätigungen, Verpfändungen, Bestandgaben, Verträge, Gerichtsurteile und Schutzbriefe. Papier wurde hingegen für litterae clausae, Anweisungen an Amtleute sowie Mandate, gerichtliche Ladungen und Noteln verwendet; auch das eigenhändige Schreiben Friedrichs III. ist auf Papier geschrieben. Beide Beschreibstoffe fanden bei Schuldverschreibungen und Bevollmächtigungen Verwendung, eine systematisch-inhaltliche Abgrenzung lässt sich daher nicht vornehmen und wäre, wie schon früher festgestellt wurde, in Anbetracht der im bearbeiteten Bestand überaus begrenzt überlieferten Ausfertigungen auf Papier auch gar nicht aussagekräftig. ${ }^{14}$

In lateinischer Sprache sind nur zwei Originalurkunden verfasst, beide aus dem kirchlichen Umfeld, betreffend die Präsentation eines Geistlichen und den Konstanzer Bistumsstreit. ${ }^{15}$ Weitere sieben lateinische Stïcke sind als Abschrift, Konzept oder Deperditum überliefert und haben bis auf eine Ausnahme (n. 212) kirchliche, ungarische oder französische Adressaten und Betreffe. ${ }^{16}$

Bis auf drei Urkunden waren alle Originale besiegelt, ${ }^{17}$ überwiegend mit dem roten S 21: 48-mal in einer wachsfarbenen Schüssel an Pergamentstreifen mit dem auf der Rückseite der Wachsschüssel eingedrückten wachsfarbenen Sekretsiegel S 16, 14-mal rückseitig aufgedrückt, davon zweimal als Verschluss und einmal - bei einer der beiden Noteln - vorderseitig aufgedrückt. Bei zwei Urkunden hat sich das Siegelbild in der

12 Siehe n. 69 (Waffenstillstand mit böhmischen Adeligen) und die nn. 105, 106 (Friedensvertrag mit Matthias Corvinus).

13 Im Unterschied zu den Vorheften wurden für die Auswertung die an das ARS Ljubljana abgegebenen Urkunden nicht berücksichtigt, die in Regg.FIII. H. 29 bereits ausgewertet wurden; zum Beschreibstoff Pergament s. Regg.F.III. H. 13 S. 6.

14 Siehe dazu Regg.F.II. H. 27 S. 17.

15 Siehe nn. 73, 326.

16 Siehe nn. 105 (Dep.), 106 (Dep.)., 121 (Konz.), 144 (Kop.), 171 (Konz.), 190 (Konz.), 212 (Konz.).

17 Die Nummerierung der Siegel erfolgt nach der von Otto PossE erstellten Systematik. 
Wachsschüssel nicht erhalten, doch ist S 21 wahrscheinlich, desgleichen im Falle eines Siegelverlustes. Bemerkenswert ist die seltene Kombination des rückseitig aufgedrückten S 21 mit dem vorderseitig aufgedrückten S 16. Diese Art der Besiegelung findet sich auf fünf Stücken, zwei davon Ausfertigungen auf Pergament, und verweist auf ein besonderes Interesse des Kaisers, wobei die Betreffe unterschiedlich sind: zwei kaiserliche Schuldbriefe, eine Zahlungsanweisung zugunsten Maximilians, ein Freiheitsbrief für einen Kärntner Juden und die Kraftloserklärung eines Reverses für Kaspar von Rogendorf. ${ }^{18}$ Die alleinige Verwendung des Sekretsiegels S 16 findet sich nur auf dem erwähnten eigenhändigen Schreiben Friedrichs III.

Aufgrund der Dominanz der erbländischen Materien im bearbeiteten Bestand fand das als „Dienstsiegel“ der römischen Kanzlei ${ }^{19}$ gebrauchte rote S 18 nur in acht Fällen Verwendung, viermal in wachsfarbener Schüssel mit rückseitig eingedrücktem S 16 an Pergamentstreifen, dreimal rückseitig und einmal - auf einer Notel - vorderseitig aufgedrückt. In zwei Fällen mit Siegelverlust ist S 18 anzunehmen.

Zwei Urkunden für Erzherzog Sigmund von Österreich wurden mit dem wachsfarbenen Majestätssiegel S 15 (Münzsiegel) an purpurfarbener Seidenschnur beglaubigt, wobei in einem Fall das vorderseitig eingedrückte wachsfarbene S 16 möglicherweise aufgrund der (schwachen) roten Spuren ursprünglich rot war. ${ }^{20}$ Bei den drei, Burgund betreffenden Lehensurkunden für Erzherzog Maximilian und Herzogin Maria von Burgund sind die Siegel nicht erhalten, doch ist ebenfalls das repräsentative S 15 anzunehmen. ${ }^{21}$ Die Beglaubigung mit dem österreichischen Münzsiegel S 24 an purpurfarbener Seidenschnur erfolgte bei drei Urkunden, jeweils mit vorderseitig eingedrücktem S 16.22

Drei Originale blieben unbesiegelt, zweimal fehlt das Siegel, einmal der Siegelabdruck in der Wachsschale. ${ }^{23}$ Da bei diesen drei Stücken auch der Kanzleivermerk fehlt, ist davon auszugehen, dass sie nicht ausgefertigt wurden.

Bei den Kanzleivermerken dominieren - wenig überraschend - jene aus der österreichischen Kanzlei. Von den 84 Originalen mit vorderseitig angebrachten Auftragsvermerken tragen 49 den Commissio-Vermerk der erbländischen Kanzlei und 14 den Ad mandatum-Vermerk der römischen Kanzlei. Der Commissio-Vermerk tritt in den Varianten Commissio domini imperatoris in consilio (31-mal) und Commissio domini imperatoris propria (18-mal) auf, stets ohne Nennung von Referenten. Der Ad manda-

18 Siehe nn. 35 (Pap.), 56 (Pap.), 57 (Pap.), 133 (Perg.), 205 (Perg.) und dazu auch die nn. 270 und 287 (nach

Fotokop.): Zahlungsanweisungen mit vorderseitig aufgedrücktem $\mathrm{S} 16$.

19 Zum „Dienstsiegel" s. Regg.F.III. H. 23 S. 27.

20 Siehe nn. 158 (Libell), 293.

21 Siehe nn. 146-148.

22 Siehe nn 12, 30, 42; zum Münzsiegel S 24 s. auch unten Anm. 49

23 Siehe nn. 97, 206, 304. 
tum-Vermerk tritt zweimal als Ad mandatum domini imperatoris auf, davon einmal mit Nennung des Protonotars Johannes Waldner, dreimal als Ad mandatum domini imperatoris in consilio - bemerkenswerterweise auch auf dem Privileg für die Stadt Klosterneuburg ${ }^{24}$ - und neunmal als proprium-Vermerk in Form von Ad mandatum domini imperatoris proprium bzw. Ad mandatum proprium domini imperatoris, wobei in zwei Fällen wieder Johann Waldner und einmal der Kämmerer Sigmund Prüschenk als Referenten genannt werden. Der auf einen persönlichen Einfluss Friedrichs III. verweisende proprium-Vermerk der römischen Kanzlei findet sich im vorliegenden Bestand nur auf Urkunden, die mit Erzherzog Maximilian, Erzherzog Sigmund und - bezieht man auch die kopial überlieferten Stücke ein - mit den Königen Ludwig XI. von Frankreich und Wladislaw II. von Böhmen in Zusammenhang stehen. ${ }^{25}$ Der wesentlich häufigere propria-Vermerk der österreichischen Kanzlei findet sich in Urkunden verschiedenen Inhalts wie Belehnungen, Ämterübertragungen und wohl prioritär zu behandelnden Zahlungsanweisungen, sodass auch hier die Verwendung vermutlich mit dem Empfängerkreis in Zusammenhang steht. So weisen beispielsweise die Urkunden für Kaspar von Rogendorf und die Brüder Prüschenk, die seit den ausgehenden 1470er-Jahren zum engsten Kreis Friedrichs III. gehörten, den proprium-Vermerk auf. ${ }^{26}$

21 Urkunden tragen keinen vorderseitigen Kanzleivermerk, jedoch ist nur bei drei Stücken aufgrund der fehlenden Besiegelung auf eine Nicht-Ausfertigung zu schließen.

Registratur-Vermerke finden sich auf 41 Originalen, wie üblich in der Form Rta mittig auf der Urkundenrückseite. Bei drei Stücken, die Finanzangelegenheiten betreffen und eine vorderseitige Besiegelung mit S 16 aufweisen, wurde der Rta-Vermerk auf der Vorderseite im rechten unteren Eck angebracht. ${ }^{27}$ Als Registrator ist im bearbeiteten Bestand nur Lukas Snitzer belegt, der auf vier Urkunden der römischen Kanzlei genannt wird. Vermerke auf der Rückseite der Siegelwachsschüsseln, deren gehäuftes Auftreten ab 1470 mit Veränderungen im Registraturwesen der österreichischen Kanzlei in Zusammenhang gebracht wird, treten regelmäßig in allen Jahren des bearbeiteten Zeitraums auf. ${ }^{28}$ Diese in der Regel den Namen der Empfänger nennenden Vermerke weisen 23 Urkunden mit erbländischen Betreffen auf, davon 12-mal zugleich mit einem Rta-Vermerk auf der Urkundenrückseite und in 15 Fällen mit einem Commissio-Vermerk. Bei acht Stücken fehlt der Auftragsvermerk, wobei in zwei Fällen allerdings der Registratur-Vermerk vorhanden ist. ${ }^{29}$ Mit Ausnahme der eindeutigen Zuweisung dieser

\footnotetext{
24 Siehe n. 15.

25 Siehe die Ad mandatum proprium-Vermerke nach Org in den nn. 146-148, 158, 207, 208, 293, 311, 312 sowie nach Kop. in den nn. 82, 87, 144, 195, 196; zum proprium-Vermerk ausführlich HEING, Kanzleipraxis S. 432-437.

26 Siehe nn. 31, 204, 222, 313, 320 .

27 Siehe $\mathrm{nn} .35,56,57$; ein vorderseitiger Rta-Vermerk auch in den nn. 270 und 287 (nach Fotokop.).

$28 \mathrm{Zu}$ diesen Vermerken s. Regg.F.III. H. 27 S. 19 f.

29 Siehe nn. 62, 303.
} 
Form der Registratur in die österreichische Kanzlei ist daher anhand des bearbeiteten Materials (noch) keine systematische Verwendung erkennbar. Bei zwei Urkunden wurden auf dem Pergamentstreifen Taxvermerke angebracht. ${ }^{30}$

Eigenhändige Vermerke des Kaisers sind zweimal überliefert, einmal die sogenannte „kleine“ Form der Unterfertigung per mamum propriam auf dem erwähnten autographen Schreiben und einmal eine Notiz auf der Plica als inhaltliche Ergänzung zur Urkunde, diese wieder für Sigmund Prüschenk. ${ }^{31}$

\section{Inhaltliche Besonderheiten}

Aufgrund des inhaltlich sehr disparaten Materials beschränken sich die folgenden Ausführungen auf ausgewählte Aspekte des bearbeiteten Bestands. Es soll aber kurz verwiesen werden auf die als Notel überlieferte Entscheidung des Kaisers im Konstanzer Bistumsstreit im Jahr 1479, ${ }^{32}$ auf die Stücke zum Salzburger Bistumsstreit, ${ }^{33}$ der einen thematischen Schwerpunkt des Folgebandes für die Jahre 1480 bis 1482 von Petra Heinicker bildet und daher dort besprochen wird, weiters auf ein reichs- und kirchenpolitisch überaus interessantes, undatiertes Konzept (samt Empfängerverzeichnis) mit einer inserierten päpstlichen Bulle von 1478 Juli 1, das eine beträchtliche Einschränkung des Wahlrechts der Domkapitel zugunsten Kaiser und Papst beinhaltet, ${ }^{34}$ und schließlich auf den Herrschaftsausbau Kaspars von Rogendorf und der Brüder Prüschenk. ${ }^{35}$

Im bearbeiteten Zeitraum ist besonders 1477 ein Jahr, dessen Ereignisse das folgende Jahrzehnt nachhaltig bestimmen sollten. Im Westen des Reiches begannen nach dem Tod Karls des Kühnen am 5. Januar 1477 und der Eheschließung Maximilians und Marias von Burgund am 19. August 1477 (per procuram am 21. April) die kriegerischen Auseinandersetzungen um die burgundischen Länder. Im Osten führten die jahrelangen Spannungen zwischen dem Kaiser und Matthias Corvinus mit der ungarischen Offensive gegen die österreichischen Erblande im Juni 1477 - als Reaktion auf die Belehnung König Wladislaws ${ }^{36}$ mit Böhmen wenige Tage zuvor - zum offenen Krieg.

30 Siehe nn. 6,10 .

31 Siehe nn. 157, 324; ausführlich dazu WAGENDORFER, Eigenhändige Unterfertigungen. - Zu den autographen Schreiben und eigenhändig unterfertigten Urkunden Friedrichs III. für die Brüder Prüschenk S. auch Regg.F.III. H. 35 nn. 1, 131, 150, 151, 159 (Jahre 1480-1482), H. 30 nn. 143, 309, 310 (Jahre 1483-1488).

32 Siehe n. 326.

33 Siehe nn. 225, 244-265, 272, 273; zum Konflikt ausführlich Regg.F.III. H. 35 S. 14 f.

34 Siehe nn. 171-187.

$35 \mathrm{Zu}$ Kaspar von Rogendorf s. nn. 13, 31, 205, 222, 313, zu den Brüdern Sigmund und Heinrich Prüschenk S. nn. $156,204,320,324$.

36 Siehe n. 82 (1477 Juni 10), auch n. 87 (Privilegienbestätigung von 1477 Juni 24). 
Sowohl der burgundische Erbfolgekrieg als auch der Krieg mit Ungarn sollten erst in den 1490er-Jahren beendet werden.

Einblick in die bereits jahrelang schwelenden, mehr oder weniger offen auf „,Nebenschauplätzen" geführten Konflikte mit dem ungarischen König gibt ein Schreiben Friedrichs an die opponierenden, unter dem Schutz des Corvinen stehenden österreichischen Adeligen von November $1476 .{ }^{37}$ Das Schreiben ist in einer Abschriftensammlung überliefert, die auch die zeitgleich von September bis November laufenden und von einem sich zunehmend verschärfenden Ton geprägten Unterhandlungen mit Matthias Corvinus enthält, auch wenn von ungarischer Seite der offene Bruch noch vermieden wurde. ${ }^{38} \mathrm{Im}$ Dezember 1476 erfolgte der Abschluss eines ausdrücklich gegen Matthias gerichteten Bündnisses Friedrichs III. mit Wladislaw von Böhmen, ${ }^{39}$ der gemäß seiner Zusage zwar im Juni 1477 persönlich mit seinem Heer in Wien erschien, sich aber nach der Belehnung mit Böhmen und der Absage des ungarischen Königs sehr schnell wieder zurückzog. Das ungarische Heer drang rasch und ohne nennenswerten Widerstand in Niederösterreich vor. Bereits im August begannen die Friedensverhandlungen, deren Ergebnis der mit 1. Dezember 1477 datierte, von Matthias erst am 18. Dezember ratifizierte Friedensvertrag sowie ein nur in der Überlieferung des HHStA Wien bekanntes und letztlich nur ,auf dem Papier“ gebliebenes Geheimabkommen war. Die Urkunden zum Frieden von Gmunden - Vertrag, Geheimabkommen, Lehenseid des Matthias für Böhmen, Huldigungsrevers, Mandate beider Herrscher zur Einhaltung des Friedens - bilden zweifellos einen der wichtigsten Urkundenkomplexe des bearbeiteten Bestands, der zudem ergänzt wird durch eine zur Aktenüberlieferung gehörende - und daher hier nicht regestierte - Materialsammlung im HHStA Wien zur Belehnung des Ungarnkönigs mit Böhmen. ${ }^{40}$ Neben dem Konzept eines Mandats Friedrichs III. an die böhmischen Untertanen (samt Empfängerverzeichnis), Matthias als König von Böhmen Gehorsam zu leisten, ist auch ein mit 2. Dezember 1477 datiertes Konzept der bislang nicht bekannten und daher sogar als gar nicht existent vermuteten Lehensurkunde überliefert, weshalb eine Ausfertigung entgegen den bisherigen Vermutungen wohl anzunehmen ist. ${ }^{41}$

37 Siehe n. 38 und auch die nn. 55, 69: Vergleich mit den österreichischen Adeligen (Ulrich von Grafenegg u.a.) von 1477 März 2 und Waffenstillstand mit den ebenfalls von Matthias unterstützten böhmischen Adeligen (Bohuslav von Schwanberg u.a.) von 1477 April 20.

38 Siehe zur Abschriftensammlung n. 38, Anm. 14. - Da die Verhandlungsprotokolle zwar im Namen des Kaisers geführt, von ihm aber weder ausgestellt noch besiegelt wurden, erfolgte gemäß den Richtlinien der Gesamtreihe keine Aufnahme im vorliegenden Band; sie sind gedruckt bei CHMEL, Mon. Habsb. I/2 S. $79-90$ nn. 5-8.

39 Siehe $\mathrm{n} .42$.

40 Zum Frieden von Gmunden s. nn. 105-108 und 110, zur Materialsammlung im HHStA Wien n. 107, Anm 7.

41 Siehen. 107. 
So erleichternd der Abzug der ungarischen Truppen für Österreich unter der Enns zweifellos war, so belastend erwies sich die vertragliche Zahlungsverpflichtung des Kaisers und der österreichischen Stände gegenüber Matthias Corvinus in Höhe von 100.000 Gulden. ${ }^{42} \mathrm{Im}$ vorliegenden Bestand sind allerdings nur wenige Belege für die Bemühungen um Einbringung der Geldsumme überliefert. ${ }^{43}$ Die Nichtbezahlung und damit der Bruch des Friedensvertrags war schließlich neben der zunehmenden Polarisierung des habsburgisch-ungarischen Gegensatzes, unter anderem im Salzburger Bistumsstreit, einer der vielen Gründe für die erneute Kriegserklärung des ungarischen Königs im Jahr 1482.

Während der Urkundenkomplex zum Gmundner Friedensvertrag durch den vor allem von Chmel gebotenen Druck gut erschlossen ist und in der Literatur daher breit rezipiert wurde, gilt dies für den im vorliegenden Bestand zweiten großen, überaus bedeutsamen Urkundenkomplex zur Belehnung Erzherzog Maximilians mit Burgund am 19. April 1478 nicht in gleicher Weise. Die bislang nur unzulänglich erschlossenen Burgund-Urkunden hielten daher Überraschendes bereit. Überliefert sind drei Diplome für Maximilian und Maria von Burgund: Eine Urkunde beinhaltet die Belehnung mit Geldern und Zutphen, ${ }^{44}$ die beiden anderen die Belehnung mit den burgundischen Ländern, die nach dem Druck bei Chmel, der nur ein Stück im Volltext und das zweite auszugsweise bietet, als nahezu wortgleich galten. ${ }^{45}$ Tatsächlich weisen beide Stücke in der Dispositio grundlegende Unterschiede auf. In der einen Urkunde erfolgt, wie zu erwarten, die Belehnung mit den burgundischen Reichslehen (n. 148), in der anderen hingegen bemerkenswerterweise die Belehnung mit allen von Karl dem Kühnen hinterlassenen Ländern und Herrschaften (n. 147), also auch mit den von Frankreich lehenrührigen Teilen. Maximilians Nachfolgerecht wird hier in der Tradition Karls des Kühnen, der gegenüber der französischen Krone die - von König Ludwig XI. nie akzeptierte - Souveränität der burgundischen Länder behauptete, mit dem Geburtsrecht Marias als „geborene" Herzogin von Burgund begründet, wie auch ihr Titel in der Urkunde lautet. Was dem Diplom allerdings besondere Brisanz verleiht, ist die durch die Belehnung gegebene, scheinbare Eingliederung aller burgundischen Länder in den Reichslehensverband. Die Urkunde entbehrt zwar jeglicher Rechtsgrundlage, doch ist sie ein großartiges Beispiel politischer Strategie, indem sie einen als „Faktum“ formulierten und im Falle eines Sieges Maximilians im Erbfolgekrieg vielleicht durchsetz-

42 Siehe n. 106, Artikel 7.

43 Siehe nn. 210, 279.

44 Siehe n. 146.

45 Siehe nn. 147, 148; Chmel, Mon. Habsb. I/2 S. 398-400 nn. 88/2, 88/3 (Druck nach einem Vidimus von 1505); s. dazu auch n. 148, Anm 8. 
baren Anspruch dokumentiert. ${ }^{46}$ Durch die unzureichende Quellenerschließung wurde dieser Urkundenkomplex bislang kaum wahrgenommen.

Die Belehnung mit Burgund berührt auch die Frage der Titulatur Maximilians, der in allen drei Lehensurkunden mit dem Titel eines ,Erzherzogs von Österreich und Burgund" geführt wird. ${ }^{47}$ Bekanntermaßen hatte Friedrich III. die Erzherzogswürde 1453 mit der Bestätigung der sogenannten ,österreichischen Freiheitsbriefe“48 jenen habsburgischen Fürsten vorbehalten, die die Herzogtümer Steiermark, Kärnten und Krain regierten, also der steirischen Linie, wobei in der Folgezeit diese Würde nur von Albrecht VI. in der Intitulatio geführt wurde, nicht hingegen von Friedrich und Maximilian. ${ }^{49} \mathrm{Im}$ Dezember 1477 gestattete der Kaiser auch Sigmund von Tirol die Führung des Titels. ${ }^{50}$ Dieser bezog sich gemäß Intitulatio stets auf die Gesamtheit der habsburgischen Herzogtümer (Österreich, Steiermark, Kärnten und Krain), weshalb es möglich war, dass Maximilian den Erzherzogstitel durch Voranstellung zunächst undifferenziert auf die burgundischen Länder ausdehnen konnte; eine Differenzierung der Titel für Österreich und Burgund in der Intitulatio sollte erst später erfolgen..$^{51}$

Bislang nicht eindeutig geklärt werden konnte der Zeitpunkt sowie der - in der Regel vage mit dem Erwerb Burgunds in Verbindung gebrachte - Hintergrund der Annahme des Titels „Erzherzog von Österreich und Burgund“.52 Wesentliche Hinweise finden sich dazu im Material des bearbeiteten Zeitraums, allerdings nicht in den zu spärlich überlieferten kaiserlichen Urkunden und Briefen, die Maximilian zum Empfänger haben oder ihn nennen, sondern in den relativ dicht überlieferten Pflegreversen der Amtleute. Da es sich bei diesen Reversen in der Regel um Kanzleiausfertigungen handelt, die von den Ausstellern nur besiegelt wurden, und ihr Formular das Versprechen des Gehorsams gegenüber dem Kaiser, seinem Sohn Maximilian (samt Titel) und ihren

46 Siehe dazu auch die Kommentare zu den nn. 147 und 148.

47 Zum vollen Titel Maximilians in den drei Urkunden s. n. 146, Anm. 2, und n. 147, Anm. 1. - Aufgrund der markanten Veränderung der Titulatur Maximilians im Jahr 1478 wurde diese in den Regesten immer aufgenommen (Ehz. von Österreich und Burgund).

48 Siehe Regg.F.III. H. 13 n. 258.

49 Friedrich III. führte allerdings den Erzherzogshut auf Darstellungen (Wiener Neustädter Wappenwand, Vorauer Porträt) sowie in Wappen und Siegeln (Münzsiegel S 24), s. dazu BENNA, Hut oder Krone S. $97-$ 99.

50 Bekannt ist nur ein knapper Revers Sigmunds von 1477 Dezember 8 im HHStA Wien, s. n. 109, Anm. 1, und dazu Luger, Bestätigung S. 257.

51 Nach LACKNER, Herzogtum S. 297, wurde nach der Wahl Maximilians zum röm. König 1486 vor der Nennung der burgundischen Herzogtümer der Herzogstitel gesetzt (,Erzherzog von Österreich, Herzog von Burgund ${ }^{\text {c) }}$. - In den burgundischen Urkunden erfolgte die Führung des Erzherzogstitel für Österreich zusätzlich zum burgundischen Herzogstitel bereits fallweise ab 1482 nach dem Tod Marias von Burgund und ab 1484 häufiger, s. Regg.F.III. Supplemente 1 S. 28.

52 Koller, Probleme S. 151, nennt für die Annahme das Jahr 1477, hingegen LACKNER, Herzogtum S. 297, das Jahr 1479 . 
Erben beinhaltet, können sie als Beleg für den Gebrauch der Titulatur Maximilians in der erbländischen Kanzlei herangezogen werden. ${ }^{53}$

Während Maximilian in den burgundischen Urkunden 1477 als „Herzog von Burgund" urkundete, wird er in der kaiserlichen Kanzlei noch bis Ende des Jahres 1477 durchwegs als „Herzog zu Österreich etc. “ tituliert. ${ }^{54}$ Eine Änderung erfolgte im Januar 1478, bislang erstmals bekannt in einem kaiserlichen Schreiben an die Stadt Frankfurt von 7. Januar 1478 und dann in einem Pflegrevers von 14. Januar. ${ }^{55}$ Nun wurden die burgundischen Länder in die Titulatur aufgenommen und mit dem Erzherzogstitel verbunden, meist in der Form „Erzherzog zu Österreich, Burgund, Brabant, Graf zu Flandern und Tirol“" oder nur „Erzherzog zu Österreich und Burgund“. Wurde in den ersten Wochen des Jahres fallweise statt dem Erzherzogstitel noch der Herzogstitel (Herzog zu Österreich, Burgund, Brabant, Graf zu Flandern und Tirol) verwendet, ${ }^{56}$ ist danach zumindest in den Reversen die konsequente Verwendung des Erzherzogstitels festzustellen. In den kaiserlichen Urkunden gibt es - mit Ausnahme des erwähnten Stücks von 7. Januar - nach derzeitigem Stand der Erschließung dafür erst gehäufte Belege ab April 1478, zuvor ist der Titelgebrauch schwankend. ${ }^{57}$

Die Annahme des Erzherzogstitels seitens Maximilian selbst im Januar 1478 belegt ein ebenfalls im HHStA Wien überliefertes Diplom Maximilians und Marias von 24. Januar, in dem sie als „Erzherzog und Erzherzogin zu Österreich, Burgund und Brabant etc., Grafen zu Flandern und Tirol“ urkunden, was insofern bemerkenswert ist, da das burgundische Herzogspaar als gemeinsame Aussteller üblicherweise den Herzogstitel gebrauchte. ${ }^{58}$ Auch der Kanzleivermerk auf der Plica lautet $d$. archidux

53 Durchgesehen wurden alle in der AUR überlieferten Reverse, das heißt auch jene, die im vorliegenden Band nicht als Grundlage eines Deperditums berücksichtigt wurden. Das Gehorsamsversprechen in den Reversen entspricht den ksl. Pflegbriefen, s. nn. 93, 204, 240.

54 Zur Titulatur des burgundischen Herzogspaares s. Regg.F.III. Supplemente 1 S. 28; in der kaiserlichen Kanzlei zuletzt als „Herzog von Österreich" tituliert im Revers des Achaz von Ernau von 1477 Dezember 29 im HHStA Wien (Sign. AUR 1477 XII 29).

55 Zum Schreiben von 1478 Januar 7 s. Regg.F.III. H. 4 n. 789; hingegen im Schreiben an Köln vom selben Tag und in derselben Sache „Herzog von Österreich und Burgund“", s. Regg.F.III. H. 7 n. 621; zum Pflegrevers Niklas Raubers von 1478 Januar 14 s. n. 111.

56 So im Schreiben an die Stadt Köln von Januar 7 (s. Anm. 55) sowie in den Reversen Friedrich Dürrers und Leonhard Harrachers von 1478 Januar 16 und Februar 13, s. nn. 113 und 125.

57 Zum Gebrauch des Erzherzogstitels ab April 1478 s. nn. 139, 140, 144, 146-149, 153; weiters Regg. F.III. H. 4 nn. 792, 796, H. 7 n. 623; ChмeL, Mon. Habsb. I/2 S. 352f. n. 46 (Urkunde von 1478 April 5). Zum schwankenden Gebrauch s. auch CHMEL, Conzeptensammlung 2 S. 188 nn. 414, 415 zu Februar 10 (Erzherzog), aber Herzog in n. 430 (S. 320) zu Februar 8 (Herzog); in Briefen des Kaisers von Januar bis März 1478 wird Maximilian mit „Herzog" angesprochen, s. CHMEL, Mon. Habsb. I/2 S. 392-394 nn. 82, 83,85 .

58 Urkunde von 1478 Januar 24 im HHStA Wien (Sign. AUR 1478 I 24); Druck: Chmel, Mon Habsb. I/2 S. 391f. n. 81. - Zur Führung des Erzherzogstitels in der Intitulatio Maximilians s. auch unsere n. 
in consilio. Diese Urkunde könnte einen Anhaltspunkt liefern hinsichtlich des Hintergrunds der Änderung der Titulatur in genau diesem Zeitraum, da sich unmittelbar anlassbedingte Bezüge zu Burgund nicht zwingend herstellen lassen. Die Eheschließung lag vier Monate zurück (19. August) und die Belehnung erfolgte vier Monate später (19. April). Empfänger dieser Urkunde ist allerdings kein burgundischer Gefolgsmann, sondern Sigmund von Österreich, zu dessen Gunsten Maximilian und Maria auf die ihnen als Erben Karls des Kühnen verpfändete Grafschaft Pfirt verzichten. Sigmund wird selbstverständlich mit dem ihm wenige Wochen zuvor kraft kaiserlicher Erlaubnis zugestandenen Erzherzogstitel („Erzherzog zu Österreich etc.“) genannt. Die Urkunde lässt daher den Rückschluss zu, dass Maximilian mit der Führung des Erzherzogstitels gegenüber Sigmund dieselbe ihm zustehende Würde bekundete, sehr anschaulich formuliert nicht nur in der Intitulatio, sondern auch in der Dispositio, wo beide Fürsten nebeneinander genannt werden: beid fürsten ertzhertzog Sigmund und ertzhertzog Maximilian von Österreich etc.

Es scheint somit sehr wahrscheinlich, dass die Annahme des Erzherzogstitels mit der zeitnah erfolgten diesbezüglichen kaiserlichen Erlaubnis für Sigmund in Zusammenhang stand und wohl auch auf Betreiben Maximilians erfolgt sein dürfte. Offen muss bleiben, ob die Erweiterung auf Burgund bzw. die burgundischen Herzogtümer ohne Differenzierung zwischen dem Erzherzogstitel für Österreich und dem burgundischen Herzogstitel, wie sie später erfolgte, ${ }^{59}$ eine Vorrangstellung gegenüber dem Tiroler Vetter zum Ausdruck bringen sollte.

In Anbetracht der großen Zahl an Reversen im bearbeiteten Bestand sollen abschlieBend damit im Zusammenhang stehende Fragen zur Schriftlichkeit in der erbländischen Verwaltung kurz thematisiert werden. Von den in der AUR des HHStA Wien für den Zeitraum 1476 bis 1479 überlieferten über 200 Reversen wurden - wie auch in allen anderen Friedrich III.-Bänden der Wiener Reihe - nur jene berücksichtigt, die einen „kaiserlichen Brief“ erwähnen und damit gesichert auf eine Urkunde Friedrichs III. rückschließen lassen ${ }^{60}$ Reverse ohne diesen Hinweis wurden nicht aufgenommen, auch wenn die Ausstellung einer entsprechenden Urkunde des Kaisers zu vermuten ist. Deren Aufnahme bzw. Nichtaufnahme als Deperditum wurde daher immer wieder von den Bearbeiterinnen und Bearbeitern diskutiert.

146, Anm. 6 (Versicherung bzgl. Geldern von 1479 März 1) und n. 292 (Schreiben an Friedrich III. von 1479 Juni 5, unterzeichnet mit ,Erzherzog von Österreich und Burgund"), weiters CHмrL, ebd. S. 417-421 n. 109; zur burgundischen Titulatur s. Anm. 54.

59 Siehe dazu Anm. 51.

60 Nicht berücksichtigt wurden allerdings jene Reverse mit Erwähnung einer kaiserlichen Urkunde, die bereits in einem Band der Friedrich III-Regesten nach dem Original oder einer Kopialüberlieferung regestiert wurde. 
So sind in dem für diesen Band bearbeiteten Archivbestand noch weitere 88 Reverse ohne Erwähnung eines zuvor ausgestellten kaiserlichen Briefs überliefert, bei denen es sich überwiegend um in der kaiserlichen Kanzlei ausgefertigte Pfleg- und Amtreverse handelt. ${ }^{61}$ Ein Vergleich beider Gruppen von Reversen - mit Erwähnung (A) und ohne Erwähnung (B) einer kaiserlichen Urkunde - lässt vermuten, dass zwei verschiedene Urkundenformulare verwendet worden sein könnten. Eine kaiserliche Urkunde wird in jenen Reversen genannt - laut seiner kaiserlichen gnaden brief darumb ausganngen -, die eine Verpfändung oder eine Ämterverpachtung oder die Übertragung einer Pflege in Verbindung mit der Bestandgabe der Erträge oder sonstigen regelmäßigen Geld- und Sachleistungen zum Inhalt haben (A). Hingegen erfolgt keine Erwähnung in Pfleg- und Amtreversen, die eine treuhändische Verwaltung mit Pflicht zur Abrechnung beinhalten (B), wobei bei der Übertragung von Burgen üblicherweise auch die Kosten für die Burghut geregelt werden.

Sollte es sich bei den Reversen der beiden Gruppen tatsächlich um Urkundenformulare für unterschiedliche Rechtsformen der Ämterübertragung handeln, ist aus der Nicht-Erwähnung einer kaiserlichen Urkunde nicht zwingend auf deren Nicht-Vorhandensein zu schließen, sondern vielmehr ebenfalls von einer schriftlichen Übertragung zu treuen Händen durch den Kaiser auszugehen. ${ }^{62}$

Die Systematik hinsichtlich eines (angenommenen) Formulars trifft auf nahezu alle im bearbeiteten Bestand überlieferten Reverse zu, allerdings gibt es auch Ausnahmen. Vereinzelt wird auch bei der Übertragung zu treuhändischer Verwaltung ein kaiserlicher Brief genannt. ${ }^{63}$ Umgekehrt kann dieser wiederum fehlen, obwohl mit der Übertragung der Burg die Bestandgabe der Erträge oder eine sonstige regelmäßige finanzielle Leistung verbunden ist. ${ }^{64}$ Auch bei Verlängerungen von Bestandgaben wird in den Reversen mitunter nur die Urkunde über die frühere Verpachtung genannt, nicht aber jene über die Verlängerung. ${ }^{65}$ Zwei Pflegreverse mit der gleichlautenden Auflage für die Pfleger, sich die Burghut und einen ihnen zustehenden Sold aus den zugehörigen Erträgen selbst zu bezahlen - der eine mit Erwähnung eines kaiserlichen Briefs, der andere ohne - lassen sich überhaupt nicht einordnen. ${ }^{66}$

61 Eine große Zahl dieser Reverse regestiert bei ChMmL, Mon. Habsb. I/3 S. 638-657.

62 Ein Beispiel bei ChMrL, Mon. Habsb. I/2 S. 841 n. 1025: Urkunde für Hans Wolkensteiner von 1478 Februar 19

63 Siehe nn. 19, 70 (beide Male das Amt Stockenboi betreffend), n. 74 (Landgericht Graz), n. 237 (Ungeld zu Herzogenburg), n. 239 (Pflege Schloss Waxenegg).

64 Pflegrevers Christoph Sachsenlanders von 1478 August 19 mit Pacht der Erträge, s. n. 18, Anm. 3; Pflegrevers Sigmund Seldners von 1478 September 5 mit jährlicher Zahlungsverpflichtung im HHStA Wien (Sign. AUR 1478 IX 5).

65 Pflegrevers Leonhard Helds von 1479 Juni 11, s. Kommentar zu n. 39; Amtrevers Valentins von Lamberg von 1479 Juli 6, s. Kommentar zu n. 115.

66 Revers des Hofmarschalls Jörg Fuchs von Fuchsberg, s. n. 18; Revers Bajezid Ottmans von 1476 No- 
Die angeführten Stücke sind zwar Einzelfälle, doch folgt daraus, dass entweder das Urkundenformular in der Kanzleipraxis nicht unbedingt streng gehandhabt wurde oder aber dass tatsächlich nur dann eine Urkunde Friedrichs III. genannt wurde, wenn sie auch ausgestellt worden war. In diesem Zusammenhang ist auf einen Revers zu verweisen, wonach die Pflege der Burg ab dem Datum der Verschreibung des Pflegers - und nicht des kaiserlichen Befehls - beginnen sollte. ${ }^{67}$ Demnach wäre zumindest denkbar, dass die von der Kanzlei ausgefertigten Verschreibungen bzw. Reverse der Amtleute, die diese nur zu besiegeln hatten, einer Übertragung mit Brief und Siegel ohne Vorliegen einer Kaiserurkunde gleichkamen.

Dann ist allerdings immer noch zu fragen, wie die Übertragung der Burgen und Ämter - die Übermittlung der entsprechenden Befehle - praktisch erfolgt sein soll. Dass dies prinzipiell auch mündlich geschehen konnte, belegt eine Urkunde des Kaisers für Kaspar von Rogendorf, der zufolge er diesem das Ungeld zu Waidhofen/Ybbs und zu Ybbs für zwei Jahre in bestanndweis mundlich gelassen habe. ${ }^{68}$ Demnach wurden auch Bestandgaben - in diesem Fall mit der jährlich hohen Bestandsumme von 1.200 Pfd. Pf. - nur mündlich übertragen. Eine weitere Form konnten kurze eigenhändige Schreiben des Kaisers (,Zetteln“) sein, wie sie für Heinrich Prüschenk zweimal für die Verlängerung der Bestandgabe der Maut zum Sarmingstein überliefert sind. ${ }^{69}$ In allen drei Fällen sind, gleichsam als „Gegenprobe“, keine Reverse überliefert. Es ist allerdings fraglich, ob sich solche vom Kaiser persönlich veranlassten - und selbstverständlich kaum überlieferten - Amtseinsetzungen auf den großen Kreis der Amtleute übertragen lassen und nicht vielmehr nur auf einen kleinen Kreis Vertrauter beschränkt waren.

Es ist wohl letztlich von der Übermittlung eines schriftlichen Befehls auszugehen, möglicherweise in Form eines geschlossenen oder offenen Briefs auf Papier, auch wenn bislang kein Beleg - das heißt eine „Doppelüberlieferung" von kaiserlichem Schreiben und entsprechendem Revers der Gruppe B - gefunden werden konnte. Die Überlieferung im HHStA Wien als Ausstellerarchiv bietet dafür kaum die geeignete Quellenbasis, die Urkunden finden sich, wenn überhaupt, in den Empfängerarchiven. Durchgesehen wurde allerdings ein überaus umfangreiches Kanzleibuch ${ }^{70}$ mit hunder-

vember 16 im HHStA Wien (Sign. AUR 1476 XI 16), gedruckt bei ChMEL, Mon. Habsb. I/2 S. $259 f$. n. 87.

67 Revers des Hans vom Turm von 1477 Februar 12 im HHStA Wien (Sign. AUR sub. dat. 1477 II 3).

68 Siehe n. 31.

69 Siehe dazu Anm. 6.

$70 \mathrm{Zu}$ diesem Kanzleibuch (Konzeptbuch) im HHStA Wien (Sign. Hs. B 53, olim Ms. 163) s. CHmel, Conzeptensammlung 1 und Conzeptensammlung 2, die restlichen Urkunden von ihm geboten vor allem in den Mon. Habsb. I/2, inhaltlich nach den Kapiteln verteilt; s. auch seine Einleitung ebd. S. XII; einige Stücke auch in den Mon. Habsb. I/1 und I/3; zur Beschreibung der Handschrift s. BöHM, Handschriften S. 59 n. 129. 
ten Urkunden Friedrichs III. mit erbländischen Betreffen, vornehmlich für das Jahr 1478, jedoch ohne Ergebnis. ${ }^{71}$

Diese Handschrift überliefert eine überaus dichte Verwaltungsschriftlichkeit, insbesondere in Form von kaiserlichen Schreiben an die Pfleger und Amtleute. Erst deren Erschließung durch die Bearbeitung der Empfängerarchive oder der Kopial- und Registerbücher der kaiserlichen Kanzlei im HHStA Wien kann letztlich darüber Aufschluss geben, wie die Organisation von Herrschaft und Raum im 15. Jahrhundert funktionierte. Wenn man bedenkt, dass allein jede Amtsübertragung mit Befehlen an die früheren Amtleute zur Abtretung und an die zugehörigen Untertanen zur Gehorsamsleistung begleitet war, ${ }^{72}$ zudem viele Reverse nicht mehr vorhanden sind, da sie nach der Abtretung des Amtes zurückgegeben oder, wenn nicht mehr auffindbar, für ungültig erklärt wurden, ${ }^{73}$ sind in Anbetracht des tatsächlichen Urkundenausstoßes der Kanzlei die im vorliegenden Band erschlossenen Urkunden Friedrichs III. nur die „Spitze eines Eisberges", dessen Größe trotz jahrzehntelanger Bearbeitung der Urkunden dieses Herrschers kaum abschätzbar ist.

\section{Danksagung}

Allen voran ist an dieser Stelle den drei Herausgebern der Reihe, den Universitätsprofessoren Dr. Paul-Joachim Heinig, Dr. Alois Niederstätter und Dr. Christian Lackner, zu danken, die das Manuskript nicht nur durchgesehen und korrigiert haben, sondern für Fragen und Diskussionen immer zur Verfügung standen. Bedanken möchte ich mich auch bei Dr. Paul Herold, dem Bibliothekar des Instituts für Österreichische Geschichtsforschung, für die Unterstützung bei Forschungsarbeiten in der Institutsbibliothek sowie bei Mag. Thomas Just, Direktor des HHStA Wien, und MMag. Kathrin Kininger für ihre unbürokratische Hilfe bei den letzten Archivrecherchen.

Besonderen Dank gilt meinen Kolleginnen und Kollegen der Abteilung Editionsunternehmen und Quellenforschung des Instituts für Mittelalterforschung sowie dem Abteilungsleiter Doz. Dr. Andreas Zajic für die von Fachkompetenz, Kollegialität und Teamgeist geprägte Atmosphäre, in der die Arbeit mit mittelalterlichen Quellen ganz besondere Freude macht. Zu ihnen gehörte lange Zeit auch Dr. Petra Heinicker, die

71 Hingegen gibt es zahlreiche Belege für eine „Doppelüberlieferung “c der Gruppe A(Revers mit Erwähnung eines kaiserlichen Briefs), bislang zwar selten in Form einer Originalüberlieferung der Kaiserurkunde (s. nn. 3, 55 [Anm. 10], 61, 240, 320), aber hāufig belegt nach dem Kanzleibuch bzw. kopialer Überlieferung: s. etwa die nn. $29,47,61,92,93,111-114,116,118,119,122,124,136,138,143,145,152,155$, $156,162,165,170$.

72 Siehe z.B. nn. 124, 130, 138, 145, 155, 156, 170, 191; auch CHMEL, Mon. Habsb. I/2 S. 741 zu n. 744.

73 Siehe z.B. n. 205; auch CHмrL, ebd. 
zeitgleich ein Regestenmanuskript (Heft 35) bearbeitete und inzwischen an der Berliner Arbeitsstelle der Friedrich-III.-Regesten tätig ist. Der Abschied fiel schwer, denn wir haben viele Stunden mit Diskussionen über die Regesten und „unseren Friedrich“ verbracht.

An dem vorliegenden Band waren zwei Mitarbeiterinnen des Instituts für Mittelalterforschung beteiligt: Dr. Anne-Katrin Kunde hat zunächst den Archivbestand gesichtet, die Aufnahme der Schriftstücke vorgenommen und ein erstes Manuskript vorgelegt. Nach ihrem Ausscheiden aus dem Projekt wurde dieses Manuskript auf Bitte der Herausgeber Kornelia Holzner-Tobisch zur Überarbeitung übergeben. Sie hat im Sinne einer weitgehenden inhaltlichen und sprachlichen Neufassung den nun vorliegenden Band erarbeitet. Zudem erfolgten weitere Literatur- und Archivrecherchen, die Ergänzung von Literaturangaben und Kommentaren, die Erstellung des Apparates, die Formulierung der Einleitung und die Vorbereitung für die Drucklegung.

Kornelia Holzner-Tobisch 


\section{Urkundenverzeichnis}

1 [Vor 1476 Jan. 13, - ]

2 [Vor 1476 Jan. 23, - ]

31476 Jan. 31, Wr. Neust.

4 [Vor 1476 Febr. 15, -]

51476 Febr. 16, Wr. Neust.

61476 März 8, Wr. Neust.

7 [Vor 1476 März 10, - ]

81476 März 11, Wr. Neust.

91476 März 20, Wr. Neust.

101476 März 20, Wr. Neust.

111476 März 29, Wr. Neust.

121476 April 5, Wr. Neust.

13 [1476 April 10, - ]

141476 April 19, Wr. Neust.

151476 April 24, Wr. Neust.

16 [Vor 1476 April 29, -]

17 [Vor 1476 Mai 6, - ]

18 [Um 1476 Mai 10, - ]

19 [Vor 1476 Mai 29, - ]

20 [Vor 1476 Mai 31, - ]

21 [Vor 1476 Juni 7, -] überträgt R. Ennser d. Donaubrückenmeisteramt zu Wien bestandweise.

verschreibt $\mathrm{H}$. Holobersy d. Teich zu Biedermannsdorf.

überträgt A. Wagen d. Grundlsee bei Aussee bestandweise.

überträgt A. Giebinger Schloss Katsch pfleg- u. bestandweise.

nimmt $\mathrm{H}$. Winkler als Hofkaplan auf.

belehnt Ch. v. Rappach mit Gütern in Österreich u.

Steier.

belehnt J. Eschelweck mit Gütern in Österreich.

belehnt Sigmund auf dem perg mit Hube in Lesach.

beauftragt Gf. L. von Görz mit Anhörung betr.

Wippach.

belehnt B. v. Ferndorf mit Stücken ebd.

erklärt Schuldbrief d. L. v. Herberstein für ungültig. bestätigt Privilegien d. Klosters Vornbach u. d. Propstei Gloggnitz.

befiehlt K. v. Rogendorf Schuldenrückzahlung.

belehnt A. v. Uttweiler mit Reichslehen.

verleiht d. Stadt Klosterneuburg zweiten Jahrmarkt. überträgt d. Stadt Leoben Viehaufschlag u. Maut bestandweise.

überträgt B. Stettner Maut, Geleit u. Taverne zu Lieserhofen bestandweise.

überträgt J. Fuchs v. Fuchsberg Schloss Starhemberg pflegweise.

überträgt P. Feistritzer Amt u. Maut Stockenboi treuhändisch.

überträgt S. Krell u. J. Peurbeck Fron u. Wechsel in Ortenburg u. genannten Gerichten bestandweise. überträgt K. Rösler Wiese bei Judenburg bestandweise. 
22 [Vor 1476 Juni 8, - ]

23 [Vor 1476 Juni 8, - ]

241476 Juni 25, Wr. Neust.

251476 Juli 9, Wr. Neust.

26 [1476 Juli 9, Wr. Neust.]

27 [1476 Juli 9, Wr. Neust.]

281476 Juli 10, Wr. Neust.

29 [1476 Juli 13, Wr. Neust.]

301476 Juli 29, Wr. Neust.

311476 Aug. 1, Wr. Neust.

32 [Vor 1476 Aug. 20, - ]

33 [Vor 1476 Aug. 20, - ]

34 [Vor 1476 Sept. 4, - ]

351476 Sept. 30, Wr. Neust.

361476 Okt. 2, Wr. Neust.

371476 Okt. 11, Wr. Neust.

381476 Nov. 13, Wr. Neust.

39 [Vor 1476 Nov. 21, -]

40 [Vor 1476 Nov. 23, - ]

41 [Vor 1476 Dez. 2, -]

421476 Dez. 8, Wr. Neust.

43 [Vor 1476 Dez. 12,-] überträgt S. Krell u. J. Peurbeck Silberkauf bestandweise.

überträgt J. Peurbeck Amt u. Landgericht Großkirchheim bestandweise.

belehnt K. Melz mit Mannsburger Lehen.

beurkundet KG-Urteil im Prozess Hz. S. von

Österreich gg. Ehzn. M. v. Österreich u. Gf. E. v.

Württemberg.

lädt Ehzn. M. v. Österreich vor d. KG.

lädt Gf. E. v. Württemberg vor d. KG.

bevollmächtigt Gesandte zu Friedensverhandlungen mit Mähren.

belehnt B. Rietmair mit e. Hof bei Feldkirchen.

bestätigt A. Rindscheit Altarstiftung in d. Grazer Pfarrkirche.

verlängert K. v. Rogendorf Bestand d. Ungelds zu Waidhofen/Ybbs u. Ybbs.

überträgt W. Lesch d. Salzamt Gmunden bestandweise.

überträgt W. Lesch d. Ungeld zu Gmunden bestandweise.

überträgt R. Steiner Schloss Pittersberg u. d. Amt Lesach pfleg- u. bestandweise.

befiehlt Ch. v. Mörsberg Zahlung an Hz. M. v. Österreich.

überträgt d. Stadt Wels diverse Ämter bestandweise. bestätigt d. Wiener Minoritenkloster e. Schenkung. antwortet H. v. Liechtenstein, U. v. Grafenegg u.a. überträgt L. Held Schloss Schönau u. d. Maut zu Neudorf u. Sollenau pfleg- u. bestandweise. überträgt F. Schottl Gericht, Maut u. Ungeld zu Neunkirchen bestandweise.

überträgt G. Elssenberger d. Amt Greifenburg bestandweise.

schließt mit Kg. W. v. Böhmen Bündnis gg. Kg.

Matthias v. Ungarn.

überträgt K. Mores d. Maut zu Neumarkt bestandweise. 
441476 Dez. 14, Wr. Neust.

45 [Vor 1476 Dez. 17, -]

461477 Jan. 4, Wr. Neust.

47 [1477 Jan. 4, Wr. Neust.]

48 [Vor 1477 Febr. 3, - ]

491477 Febr. 21, Wien

50 [1477 Febr. 25, -]

51 [1477 Febr. 25, Wien]

52 [Vor 1477 Febr. 26, -]

531477 Febr. 26, Wien

54 [Vor 1477 Febr. 28, -]

551477 März 2, Wien

561477 März 14, Wien

571477 März 14, Wien

581477 März 14, Wien

591477 März 14, Wien

60 [Vor 1477 März 16, -]

61 [1477 März 17, Wien]

621477 März 17, Wien

63 [Vor 1477 März 21, -]

64 [Vor 1477 März 28, -]

65 [Vor 1477 März 28, -]

661477 März 28, Wien

67 [Vor 1477 März 31, -]

68 [Vor 1477 April 8, - ] belehnt Ch. v. Rojach mit e. Gut zu Feicht. verpfändet $\mathrm{H}$. Blay Schloss Grafenwart. gestattet M. Melz Fleischbank in Laibach. überträgt S. Skodel Schloss Finkenstein pfleg- u. bestandweise.

überträgt J. Schrott Schloss Klamm pfleg- u. bestandweise.

belehnt N. Dürrer mit Huben in Unterbärental. schreibt Hz. A. v. Bayern(-München) betr. Hilfe für Burgund.

fordert von d. Stadt Köln e. Darlehen.

lädt S. Pirckheimer rechtlich vor sich betr. R. Kärgl. lädt S. Pirckheimer erneut rechtlich vor sich betr. $R$. Kärgl.

überträgt W. v. Auersperg Ungeld u. Landgericht zu Weikersdorf bestandweise.

beurkundet Vergleich mit U. v. Grafenegg u.a.

gibt N. Groß d.Ä. Schuldverschreibung.

gibt Brüdern Hörlin Schuldverschreibung.

bestätigt D. Moyker Verschreibung von Widerlage

u. Morgengabe.

bestätigt S. Reichenauer Verpfändung v. Gütern.

überträgt $\mathrm{Ch}$. Stecher d. Ungeld zu Neulengbach bestandweise.

überträgt $d$. Stadt Wien halbe Donaubrückenmaut bestandweise.

belehnt M. Grasser mit Feste Hornsburg.

überträgt d. Stadt Klosterneuburg Gericht $u$. Maut ebd. bestandweise.

befiehlt d. Stadt Neuenburg, Bf. v. Basel nicht zu behindern.

hebt Befehl an d. Stadt Neuenburg auf.

befiehlt Bf. J. v. Basel, d. Stadt Neuenburg nicht zu behindern.

überträgt S. Gruber d. Ungeld zu Wr. Neustadt bestandweise.

überträgt J. Rehwein d. Teich bei Stockern bestandweise. 
69 [1477 April 20, -]

70 [Vor 1477 April 23, -]

71 [Vor 1477 April 29, - ]

72 [Vor 1477 Mai 6, - ]

731477 Mai 14, Wien

74 [Vor 1477 Mai 16,-]

75 [1477 Mai 20, Wien]

76 [1477 vor Mai 25, - ]

77 [1477 vor Mai 25, - ]

78 [1477 vor Mai 25, - ]

79 [1477 vor Mai 25, - ]

801477 Mai 25, Wien

811477 Juni 3, Wien

821477 Juni 10, Wien

83 [Vor 1477 Juni 13, -]

84 [Vor 1477 Juni 15, -]

851477 Juni 17, Wien

861477 Juni 20, Wien

$87 \quad 1477$ Juni 24, Wien

88 [Vor 1477 Juli 4, -]

89 [Vor 1477 Juli 4, -]

90 [Vor 1477 Juli 11, -]

911477 Juli 17, Wien

92 [1477 Aug. 8,-]

93 [1477 Aug. 9, -] schließt Waffenstillstand mit B. v. Schwanberg u. böhm. Landherren.

überträgt W. Paulseer Amt u. Maut Stockenboi treuhändisch.

überträgt d. Stadt Laa Gericht, Maut u. Ungeld ebd. bestandweise.

schreibt Hz. S. v. Österreich betr. Burgund.

präsentiert J. Grumel für d. Pfarrkirche Grafenwarth.

überträgt $\mathrm{H}$. Weidinger d. Landgericht zu Graz treuhändisch.

gibt Hz. M. v. Österreich Geleitbrief für d. Zug nach Burgund.

antwortet Hz. S. v. Österreich betr. Burgund.

schreibt Hzn. Maria v. Burgund.

schreibt Hzn. Margarete v. Burgund.

schreibt an d. Untertanen der burgundischen Länder. übermittelt Hz. M. v. Österreich Briefe.

belehnt P. u. B. Langauer mit d. Turm zu Winklern.

belehnt Kg. W. v. Böhmen mit Böhmen.

verschreibt G. Peuscher Schloss Falkenstein satz- u. pflegweise.

überträgt H. Murstetter d. Wiener Stadtgericht bestandweise.

gebietet d. Mainzer Domkapitel Befolgung e. KGUrteils.

beurkundet Entscheidung im Erbstreit zw. J. Rehwein u. U. Lembacher.

bestätigt Kg. W. v. Böhmen d. Privilegien d. böhmischen Krone.

verpfändet d. Stift Spital am Pyhrn Schloss Klaus. verpfändet d. Stift Spital am Pyhrn d. Amt Molln. verschreibt W. Perler Güter d. H. Judel. gibt W. Galler Schuldverschreibung. verpfändet $C h$. Jörger Schloss Roith. verpfändet Eb. J. v. Gran Schloss u. Stadt Steyr u. Ämter u. Erträge zu Korneuburg. 
94 [Vor 1477 Aug. 12, -]

95 [Vor 1477 Aug. 12, -]

961477 Aug. 23, Krems

971477 Aug. 29, Krems

98 [Vor 1477 Sept. 3, - ]

99 [Vor 1477 Sept. 9, -]

1001477 Sept. 9, Krems

1011477 Okt. 19, Steyr

102 [Vor 1477 Okt. 22, -]

1031477 Okt. 23, Steyr

1041477 Nov. 8, Gmunden

105 [Um 1477 Nov. 30, Gmunden]

106 [1477 Dez. 1, Gmunden]

107 [1477 Dez. 2, Gmunden]

108 [1477 Dez. 2, Gmunden]

1091477 Dez. 2, Gmunden

1101478 Jan. 9, Graz

111 [1478 Jan. 9, Graz]

112 [1478 Jan. 9, Graz]

113 [1478 Jan. 15, Graz]

114 [1478 Jan. 15, Graz]

115 [Vor 1478 Jan. 17, -] überträgt W. v. Lusthal Schloss Altenburg pflegweise.

überlässt d. Ehepaar Rorbeck e. Wohnung in Wien. bestätigt W. v. Prem Erbrecht an e. Hof. privilegiert L. Meuting d.Ä. von Augsburg. gestattet J. Waitzinger alleinige Nutzung e. Fischweide bei Lavamünd.

überträgt U. Harnstein Schloss Lavamünd pfleg- u. bestandweise.

belehnt J. Schandacher mit Gütern in d. Pfarre Ybbs.

belehnt d. Ehepaar Dorfner mit e. Hube in d. Pfarre Kematen (a.d. Krems).

überträgt H. v. Sinzendorf d. Ungeld zu Kremsmünster bestandweise.

verleiht $H$. Vischmeister Sege u. Fischweide.

gewährt d. Stift Spital am Pyhrn Steuerfreiheiten. schließt mit Kg. M. v. Ungarn e. Geheimabkommen.

beurkundet d. Friedensvertrag mit Kg. M. v. Ungarn.

belehnt Kg. M. v. Ungarn mit Böhmen. beglaubigt Lehenseid v. Kg. M. v. Ungarn.

befiehlt Pfandinhabern Eidesleistung gegenüber $\mathrm{Hz}$. S. v. Österreich.

befiehlt Einhaltung d. Friedens mit Kg. M. v. Ungarn.

überträgt N. Rauber d. Hauptmannschaft Mitterburg pfleg- u. bestandweise.

überträgt N. Rauber Kastell u. Ämter zu Kastav, Veprinac u. Moschenitz bestandweise.

überträgt F. Dürrer d. Hauptmannschaft Portenau pfleg- u. bestandweise.

überträgt d. Stadt Gmunden Schloss Wildenstein sowie Salzamt u. Ungeld zu Gmunden pfleg- und bestandweise.

überträgt V. v. Lamberg Amt, Maut u. Landgericht zu Stein in Krain bestandweise. 
116 [1478 Jan. 18, Graz]

1171478 Jan. 26, Graz

118 [1478 Jan. 28, Graz]

119 [1478 Jan. 30, Graz]

120 [Vor 1478 Jan. 31, -]

121 [1478 etwa Febr., -]

122 [1478 Febr. 4, Graz]

123 [Vor 1478 Febr. 6, -]

124 [1478 Febr. 7, Graz]

125 [Vor 1478 Febr. 10,-]

126 [Vor 1478 Febr. 13, -]

1271478 Febr. 13, Graz

128 [Vor 1478 Febr. 17, -]

1291478 Febr. 21, Graz

1301478 März 1, Graz

131 [Vor 1478 März 2, -]

132 [Vor 1478 März 2, -]

1331478 März 4, Graz

1341478 März 8, Graz

1351478 März 23, Graz

136 [1478 März 30, Graz]

1371478 März 30, Graz

138 [1478 April 1, Graz] überträgt H. Glanhofer Maut bei Prem bestandweise.

bestätigt d. Spital zu Gurkfeld Schenkungen. überträgt M. Moser Fron u. Wechsel zu Schladming bestandweise.

überträgt $d$. Stift Vorau Fischweide zu Reinberg bestandweise.

überträgt S. Zmollner Schloss St. Peter ob Leoben pfleg- u. bestandweise.

bezeugt für Gf. S. v. St. Georgen u. Bösing.

überträgt $H$. Reisacher Fischweide in d. Mürz bei Kindberg bestandweise.

überträgt L. v. Herberstein d. Turm zu Wippach u. diverse Güter pfleg- und bestandweise.

überträgt L. Saldorfer Fischweide zu Döbriach bestandweise.

überträgt L. Harracher Schloss Weitersfeld pfleg- u. bestandweise.

befiehlt Burgleuten v. Hallstatt Schätzung der Saline.

befiehlt A. Wagen u. A. Fuchsberger Schätzung der Saline Hallstatt.

überträgt d. Stadt Möttling Gericht u. Maut ebd. bestandweise.

belehnt B. Span mit Hof zu Hausmannstätten. verkauft H. Pickl e. Haus in Voitsberg. überträgt J. Katzendorfer Schloss Montpreis pflegu. bestandweise.

erlaubt B. Heustadl Aufenthalt u. Handel. gewährt d. Juden Kifel Gerichtsfreiheiten. befiehlt Beachtung d. Rechte d. Klosters Viktring. belehnt K. v. Spangstein mit e. Hube zu UnterUrscha.

überträgt S. Piers Landgericht in d. Gottschee bestandweise.

verleiht C. Parilo e. Hube zu Kaufrecht. überträgt J. v. Obritschan Schloss Schärffenberg pfleg- u. bestandweise. 
1391478 April 4, Graz

1401478 April 4, Graz

141 [Vor 1478 April 6, -]

1421478 April 15, Graz

143 [Vor 1478 April 16,-]

1441478 April 16, Graz

145 [1478 April 18, Graz]

1461478 April 19, Graz

1471478 April 19, Graz

1481478 April 19, Graz

149 [1478 zw. April 19 u. Aug. 20,-]

150 [Vor 1478 April 20,-]

151 [Vor 1478 April 21, -]

152 [1478 April 21, Graz]

1531478 April 23, Graz

154 [Um 1478 April 24, -]

155 [1478 April 27, Graz]

156 [1478 April 30, Graz]

1571478 Mai 3,- bevollmächtigt Kardinal G. Hessler u. Ehz. M. v. Österreich zu Verhandlungen mit Ldgf. H. v. Hessen.

bevollmächtigt Kardinal G. Hessler u. Ehz. M. v. Österreich zu Verhandlungen mit Pfgf. Ph. bei Rhein.

belehnt M. Erdinger mit e. Hof zu Thalheim. belehnt d. Brüder Lueger mit Schloss Rudenegg. überträgt d. Stadt Völkermarkt Jahrmarkt u. Maut ebd. bestandweise.

ermahnt Kg. L. v. Frankreich zur Herausgabe von Cambrai u. Freilassung d. Bf. v. Verdun.

überträgt H. Gall Schloss Hohenmauthen pfleg- u. bestandweise.

belehnt Ehz. M. v. Österreich u. Hzn. M. v. Burgund mit Geldern u. Zutphen.

belehnt Ehz. M. v. Österreich u. Hzn. M. v. Burgund mit d. burgundischen Ländern.

belehnt Ehz. M. v. Österreich u. Hzn. M. v. Burgund mit d. burgundischen Reichslehen.

befiehlt Kardinal G. Hessler Entgegennahme d.

Lehenseids v. Ehz. M. v. Österreich u. Hzn. M. v. Burgund.

überträgt G. Prantner d. Maut zu Cilli u. Freudenthal bestandweise.

überträgt W. Praun Schloss Kammer im Attersee pfleg- u. bestandweise.

überträgt St. Widmer d. Amt Hochenegg bestandweise.

ersucht Ehz. M. v. Österreich, nicht nach Metz zu reisen.

überträgt S. Krell Fron u. Wechsel in Ortenburg u. genannten Gerichten bestandweise.

überträgt $\mathrm{H}$. Gabelhuber d. Ungeld im Mürztal bestandweise.

überträgt S. Prüschenk Schloss Bernstein pfleg- u. bestandweise.

quittiert d. Juden Isaak Entrichtung d. Steuer. 
1581478 Mai 5, Graz

1591478 Mai 14, Graz

160 [Vor 1478 Juni 3, -]

1611478 Juni 5, Graz

162 [1478 Juni 9, Graz]

163 [Vor 1478 Juni 10, -]

164 [Vor 1478 Juni 11, -]

165 [Vor 1478 Juni 15, -]

1661478 Juni 18, Graz

167 [1478 Juni 18, Graz]

1681478 Juni 20, Graz

169 [Vor 1478 Juni 25, -]

170 [1478 Juni 27, Graz]

171 [Nach 1478 Juli $1,-]$

172 [Nach 1478 Juli 1, -]

173 [Nach 1478 Juli 1, - ]

174 [Nach 1478 Juli 1, -]

175 [Nach 1478 Juli 1, -]

176 [Nach 1478 Juli 1, -]

177 [Nach 1478 Juli 1, -]

178 [Nach 1478 Juli 1, -]

179 [Nach 1478 Juli 1, -]

180 [Nach 1478 Juli 1, -]

181 [Nach 1478 Juli 1, -]

182 [Nach 1478 Juli 1, -]

183 [Nach 1478 Juli 1, -]

184 [Nach 1478 Juli 1, - ] bestätigt Ehz. S. v. Österreich Urkunden betr. Trient. belehnt J. Glojacher mit Gütern zu Hausmannstätten, Afram, Stocking u. Stiefing.

überträgt H. v. Trautmannsdorf Schloss Kammern pfleg- u. bestandweise.

gibt W. Pandorfer Schuldverschreibung.

überträgt A. v. Teufenbach Schloss Eppenstein pfleg- u. bestandweise.

überträgt K. Zirkendorfer Schloss Weitra u. d. Teich ebd. pfleg- u. bestandweise.

überträgt N. Haller Schloss Landskron pfleg- u. bestandweise.

überträgt A. Kamrer Ungeld u. Weinaufschlag zu Judenburg bestandweise.

lädt K. Seus rechtlich vor sich.

lädt d. Ehepaar Holfelder rechtlich vor sich.

belehnt E. Lueger mit Gütern d. Bggf. Haug v.

Lienz.

verschreibt A. v. Ernau Schloss Falkenstein satz- u. pflegweise.

überträgt $\mathrm{H}$. Stubich d. Ungeld zu Leoben u.

St. Peter ob Leoben bestandweise.

befiehlt d. Domkapitel v. Utrecht Einhaltung d.

Bulle P. Sixtus IV. betr. Bischofswahl.

desgl. an d. Domkapitel v. Lüttich.

desgl. an d. Domkapitel v. Cambrai.

desgl. an d. Domkapitel v. Trier.

desgl. an d. Domkapitel v. Köln.

desgl. an d. Domkapitel v. Mainz.

desgl. an d. Domkapitel v. Würzburg.

desgl. an d. Domkapitel v. Bamberg.

desgl. an d. Domkapitel v. Eichstätt.

desgl. an d. Domkapitel v. Salzburg.

desgl. an d. Domkapitel v. Straßburg.

desgl. an d. Domkapitel v. Passau.

desgl. an d. Domkapitel v. Augsburg.

desgl. an d. Domkapitel v. Freising. 
185 [Nach 1478 Juli 1, -]

186 [Nach 1478 Juli 1, -]

187 [Nach 1478 Juli 1, -]

188 [Vor 1478 Juli 4, -]

189 [Vor 1478 Juli 5, -]

190 [Nach 1478 Juli 6, -]

191 [Vor 1478 Juli 8, -]

192 [Vor 1478 Aug. 13, -]

193 [Vor 1478 Aug. 18, -]

1941478 Aug. 19, Graz

1951478 Aug. 26, Graz

1961478 Aug. 26, Graz

197 [1478 Aug. 26, Graz]

198 [1478 Aug. 26, Graz]

199 [1478 Aug. 26, Graz]

200 [Vor 1478 Aug. 28, -]

2011478 Sept. 14, Graz

202 [1478 Sept. 14, Graz]

203 [1478 Sept. 14, Graz]

2041478 Sept. 24, Graz

2051478 Sept. 24, Graz

2061478 Sept. 25, Graz

2071478 Sept. 27, Graz

2081478 Sept. 27, Graz

209 [Vor 1478 Okt. 5,-]

210 [Vor 1478 Okt. 11, -]

2111478 Okt. 12, Graz desgl. an d. Domkapitel v. Münster.

desgl. an d. Domkapitel v. Regensburg.

desgl. an d. Domkapitel v. Besançon.

überträgt H. Laak Schloss Haasberg mit d. Amt Seeberg pfleg- $u$. bestandweise.

überträgt S. Piers d. Amt in der Rieg bestandweise. schreibt P. Sixtus IV. betr. Erzbistum Köln.

überträgt W. Lesch Schloss Mödling sowie Ungeld, Bergrecht u. Zehent ebd. pfleg- u. bestandweise.

überträgt P. Ebenauer Hals- u. Landgericht Glei-

chenberg bestandweise.

empfiehlt Eb. J. v. Gran bei P. Sixtus IV.

bestätigt Privilegien d. Stadt Eggenburg.

befiehlt Churwalden, Lenz, Schanfigg u. Langwies, Ehz. S. v. Österreich zu huldigen.

untersagt Schwyz Unterstiutzung v. Churwalden, Lenz, Schanfigg zu St. Peter u. Langwies.

desgl. an Unterwalden.

desgl. an Uri.

desgl. an d. Stadt Zürich.

verpfändet J. Hauser Schloss Asparn.

lädt Ehz. S. v. Österreich rechtlich vor sich.

lädt Ehzn. M. v. Österreich rechtlich vor sich.

lädt Gf. E. v. Württemberg rechtlich vor sich.

verpfändet S. Prüschenk Schloss u. Herrschaft Weitenegg.

erklärt Revers d. K. v. Rogendorf betr. Weitenegg

für ungültig.

bestätigt d. Leuten zu Tinjan Kauf e. Berges.

belehnt Ehz. S. v. Österreich mit d. Bergwerken in

d. Herrschaft Schelklingen.

befiehlt Bf. G. v. Brixen Entgegennahme d. Lehenseids v. Ehz. S. v. Österreich.

verkauft $H$. Weinich e. Haus in Graz.

beauftragt $H$. Vilshover u. J. Burckhart mit d. Ein-

hebung e. Aufschlags in d. Steiermark.

bestätigt d. Privilegien d. Stadt Friedberg. 
2121478 Okt. 13, Graz

2131478 Okt. 16, Graz

2141478 Okt. 25, Graz

215 [Vor 1478 Okt. 28, - ]

216 [1478 Nov. $5,-]$

217 [Vor 1478 Nov. 23, -]

218 [Vor 1478 Nov. 23, -]

2191478 Nov. 23, Graz

2201478 Nov. 23, Graz

2211478 Dez. 5, Graz

2221478 Dez. 7, Graz

223 [Vor 1479 Jan. 2, -]

224 [Vor 1479 Jan. 9, -]

225 [Vor 1479 Jan. 10, -]

226 [Vor 1479 Jan. 11, -]

227 [Vor 1479 Jan. 14, -]

228 [Vor 1479 Jan. 15, -]

229 [Vor 1479 Jan. 16, -]

230 [Vor 1479 Jan. 16, - ]

231 [Vor 1479 Jan. 18, -]

2321479 Jan. 19, Graz

233 [Vor 1479 Jan. 21, -]

234 [1479 Jan. 27, -] nimmt d. Brüder Nikolitsch in seinen Schutz. gewährt H. Apfaltrer Weinschankrecht im Dorf zu St. Martin in der Littai.

gewährt K. Harder Markt- u. Weinschankrecht für dessen Leute in Gleisdorf.

überträgt A. Hertenfelder Schloss Neuburg a.d.

Kanker u. d. Amt Primskau pfleg- u. bestandweise. verbietet d. Abt v. Allerheiligen u. d. Stadt Schaffhausen Gebrauch d. Wildbanns.

lädt $\mathrm{H}$. Oberhaimer rechtlich vor sich.

lädt $\mathrm{H}$. Oberhaimer rechtlich vor sich.

befiehlt $\mathrm{H}$. Oberhaimer, d. Forderungen H. Oberhaimers v. Bernau zu befriedigen.

befiehlt H. Oberhaimer, d. Forderungen d. Brüder J. u. W. Oberhaimer zu befriedigen.

gewährt d. Heilig-Geist-Spital zu Konstanz Herrschaftsrechte im Dorf Sipplingen.

belehnt K. v. Rogendorf mit Schloss Pöggstall. überträgt d. Stadt Graz Ungeld u. Landgericht ebd. belehnt B. Stadler u. M. Reiffenberger mit Schloss Kirchstetten.

ermahnt Eb. B. von Salzburg zu resignieren.

überträgt J. Fleischhacker d. Amt Schwarzenbach bestandweise.

überträgt W. Kransdorfer d. zu Schloss Dürnstein gehörige Ungeld bestandweise.

überträgt M. Reiffenberger Schloss Hernstein pflegweise.

überträgt P. Tenter d. Ungeld zu Klosterneuburg bestandweise.

überträgt B. Krannagel d. Amt (Windisch-)Feistritz mit Gericht und Stadtsteuer bestandweise.

überträgt H. Raschberger Schloss Wolkenstein pflegweise.

belehnt J. Hauser mit Ortenburger u. Kärntner Lehen. tauscht mit A. Rindscheit Güter u. Gülten. verkauft W. Schandel e. Haus in Wr. Neustadt. 
235 [Vor 1479 Jan. 30, -]

236 [Vor 1479 Febr. 2, -]

237 [Vor 1479 Febr. 2, -]

2381479 Febr. 10, Graz

239 [Vor 1479 Febr. 15, -]

2401479 Febr. 22, Graz

2411479 Febr. 24, Graz

242 [Vor 1479 Febr. 27, -]

243 [Vor 1479 März 6, -]

2441479 März 9, Graz

2451479 März 9, Graz

2461479 März 9, Graz

2471479 März 9, Graz

2481479 März 9, Graz

2491479 März 9, Graz

2501479 März 9, Graz

2511479 März 9, Graz

2521479 März 9, Graz

2531479 März 9, Graz

2541479 März 9, Graz

2551479 März 9, Graz

2561479 März 9, Graz

2571479 März 9, Graz

2581479 März 9, Graz

2591479 März 9, Graz

2601479 März 9, Graz

2611479 März 9, Graz

2621479 März 9, Graz

2631479 März 9, Graz überträgt d. Stadt Leoben Viehaufschlag u. Maut. überträgt J. Metnitzer Schloss Arnfels pfleg- u. bestandweise.

überträgt B. Murstetter d. Ungeld zu Herzogenburg treuhändisch.

belehnt R. Pretzner mit Wiesen bei Klagenfurt.

überträgt W. Kundorfer Schloss Waxenegg pflegweise.

überträgt G. Slaher Schloss Laxenburg u. d. Ungeld zu Himberg pflegweise.

überträgt $B$. Eggenberger Ungeld $u$. Weinaufschlag zu Judenburg.

überträgt W. Kundorfer Amt u. Landgericht Waxenegg bestandweise.

überträgt J. Metnitzer d. Ämter zum Entrich u. zu

Tunau bestandweise.

verbietet Bf. Ch. v. Seckau Unterstützung d. Eb. B.

v. Salzburg.

desgl. an Bf. G. v. Chiemsee.

desgl. an Bf. J. v. Lavant.

desgl. an d. Stift Berchtesgaden.

desgl. an d. Stift Baumburg.

desgl. an d. Stift Gars.

desgl. an d. Stift (Herren-)Chiemsee.

desgl. an d. Stift Au am Inn.

desgl. an d. Stift St. Peter zu Salzburg.

desgl. an d. Stift Seeon.

desgl. an d. Stadt Salzburg.

desgl. an d. Stadt Radstadt.

desgl. an d. Stadt Hallein.

desgl. an d. Stadt Laufen.

desgl. an d. Stadt Tittmoning.

desgl. an d. Stadt Friesach.

desgl. an d. Stadt Gmünd (in Kärnten).

desgl. an d. Stadt Pettau.

desgl. an d. Stadt Rann.

desgl. an d. Stadt St. Andrä im Lavanttal. 
2641479 März 9, Graz

2651479 März 9, Graz

266 [Vor 1479 März 10, -]

2671479 März 10, Graz

268 [Vor 1479 März 11, -]

269 [Vor 1479 März 24, -]

2701479 März 30, Graz

271 [Vor 1479 April 7, -]

272 [Vor 1479 April 7, -]

2731479 April 13, Graz

2741479 April 15, Graz

2751479 April 20, Graz

276 [1479 April 20, Graz]

277 [Vor 1479 April 24, -]

278 [1479 Mai 1, Graz]

2791479 Mai 2, Graz

2801479 Mai 4, Graz

281 [Vor 1479 Mai 5, - ]

282 [Vor 1479 Mai 19, -]

283 [Vor 1479 Mai 20, - ]

284 [Vor 1479 Mai 21, -]

2851479 Mai 21, Graz

2861479 Mai 24, Graz

2871479 Mai 24, Graz desgl. an Prälaten, Ritter, Städte u. d. Landschaft d. Erzstifts Salzburg (Generalmandat).

desgl. an d. Stadt Mühldorf (am Inn).

erlässt Ordnung für d. Wochenpfennig in Kärnten.

befiehlt Einhebung d. Wochenpfennigs in Kärnten.

verpfändet Eb. J. v. Gran Schloss u. Herrschaft

Kreuzenstein u. weitere Erträge.

belehnt W. Kadauer mit Buchberg, Plank u.a.

befiehlt A. v. Kraig Zahlung an A. Dietprant.

verlängert A. Spangstein Bestand v. Schloss, Amt u.

Landgericht Eibiswald.

schreibt P. Sixtus IV. betr. Resignation d. Eb. B. v.

Salzburg.

schreibt Eb. J. v. Gran.

beurkundet Vergleich zw. M. Scharffwindt u. H.

Gall v. Buchenstein.

lädt d. Ehepaar Holfelder rechtlich vor sich.

lädt K. Seus rechtlich vor sich.

belehnt Th. Praitenwiser mit Gütern zu Press.

überträgt d. Kloster Ossiach Fischweide in d.

Feistritz bestandweise.

gestattet den österr. Ständen Aufnahme e. Darlehens zur Bezahlung d. Kg. M. v. Ungarn.

gewährt Gf. J. L. v. Nassau-Saarbrücken Aufschub der Belehnung.

überträgt J. Leininger Schloss und Landgericht Landskron pfleg- u. bestandweise.

überträgt d. Stadt Leoben d. Ungeld ebd. u. zu

St. Peter ob Leoben bestandweise.

überträgt J. Dürrer Schloss Pemont pfleg- u. bestandweise.

verpfändet N. Prothowicz Schloss Asparn/Zaya.

belehnt K. Zebinger mit Pettauer Gütern.

überlässt A. am Greith e. Grund im Amt Gschaid zinsweise.

befiehlt A. v. Kraig Zahlung an S. v. Kraig als Heiratssteuer. 
288 [Vor 1479 Mai 25, -]

289 [Vor 1479 Mai 25, -]

290 [Vor 1479 Mai 28, -]

291 [Vor 1479 Mai 28,-]

292 [Vor 1479 Mai 30, -]

2931479 Juni 5, Graz

294 [Vor 1479 Juni 11, -]

295 [Vor 1479 Juni 18, -]

2961479 Juni 19, Graz

297 [Vor 1479 Juni 25, -]

298 [Vor 1479 Juli 1, -]

2991479 Juli 5, Graz

3001479 Juli 13, Graz

301 [Vor 1479 Juli 16, -]

302 [Vor 1479 Juli 18, -]

3031479 Juli 18, Graz

3041479 Juli 20, Graz

3051479 Juli 21, Graz

306 [1479 Aug. 6, Graz]

3071479 Aug. 11, Graz

3081479 Aug. 15, Graz

309 [Vor 1479 Aug. 19, -] überträgt S. Tellitzer Schloss Wald pfleg- u. bestandweise.

überträgt S. Tellitzer d. Ungeld zu St. Pölten bestandweise.

überträgt W. Lembacher Schloss Lankowitz pflegu. bestandweise.

überträgt K. Auer Amt u. Herrschaft Hornstein bestandweise.

schreibt Ehz. M. v. Österreich betr. Tag zu Nürnberg.

bestätigt Gerichtshoheit d. Landgerichts Rankweil über d. Appenzeller.

überträgt H. Stubich Schloss St. Peter ob Leoben pfleg- u. bestandweise.

nimmt D. Heller mit Familie in seinen Schutz.

belehnt H. Dolacher mit Huben zu Treßdorf.

überträgt J. v. Lamberg Schloss Adelsberg samt Maut pfleg- u. bestandweise.

überträgt $\mathrm{K}$. Wenger $\mathrm{d}$. Teich zu Waidhofen/Thaya. belehnt L. v. Wehingen mit d. Feste Sitzenberg, d. österr. Türhüteramt u.a.

nimmt J. Wartenauer als Diener auf.

überträgt H. Vogt v. Summerau Schloss Fürstenfeld u. d. Amt Feldbach pfleg- u. bestandweise.

überträgt H. Aichelperger Schloss Klamm pfleg- u. bestandweise.

belehnt A. Stermoler u. Geschwister mit Cillier u. Rohitscher Lehen.

gestattet Ehz. S. v. Österreich Einlösung d. Landvogtei Schwaben.

belehnt d. Brüder Rosegger mit e. Alm bei Hohenburg.

verkauft B. Neupaur e. Haus in Wien.

bestätigt Ordnung $\mathrm{d}$. Wiener Leinwaterzeche.

befiehlt Anhörung im Streit zw. Bf. Ph. v. Bamberg u. Ch. Ungnad.

verpfändet B. Ottman Schloss Rauhenstein. 
310 [Vor 1479 Aug. 26, - ]

3111479 Sept. 4, Graz

3121479 Sept. 5, Graz

3131479 Sept. 6, Graz

314 [Vor 1479 Sept. 7, -]

315 [Vor 1479 Sept. 11, -]

316 [Vor 1479 Sept. 16, -]

317 [Vor 1479 Sept. 18, - ]

318 [Vor 1479 Sept. 22, -]

319 [Vor 1479 Okt. 2,-]

3201479 Okt. 14, Graz

321 [Vor 1479 Okt. 17, -]

322 [Vor 1479 Nov. 1, - ]

323 [Vor 1479 Nov. 8,-]

3241479 Nov. 10, Graz

325 [Vor 1479 Nov. 12, - ]

3261479 Nov. 24, Graz

327 [Vor 1479 Nov. 26, -]

3281479 Nov. 28 , Graz überträgt H. Heidenreich d. Forstamt im Eisenerz u.a. bestandweise.

belehnt Ehz. S. v. Österreich mit Lehen d. P. v. Raron.

befiehlt d. Reichsuntertanen Beachtung d. Belehnung d. Ehz. S. v. Österreich.

belehnt K. v. Rogendorf mit Schloss Ottenschlag. überträgt J. Kätzler e. Hof im Winkl zu Leoben bestandweise.

belehnt M. Fritz mit Zehenten in d. Pfarren Vöcklamarkt, Pöndorf u. Gampern.

überträgt d. Markt Aussee d. Ungeld ebd. bestandweise.

überträgt $P$. Engelhart d. Gericht zu Radmannsdorf bestandweise.

überträgt $\mathrm{H}$. Lenghaimer d. Land- u. Marktmaut zu Weixelburg bestandweise.

überträgt J. Kranperger d. Ungeld zu Nussdorf u. Purkersdorf zur Schuldenbegleichung.

überträgt H. Prüschenk Schloss Sarmingstein u. d. Maut ebd. pfleg- und bestandweise.

überträgt $\mathrm{W}$. v. Auersperg Fischweide in d. Mürz bei Kindberg bestandweise.

überträgt J. Dachauer d. Kaltmaut zu Korneuburg u. Wien bestandweise.

überträgt S. Kötzler d. Ungeld zu Leibnitz bestandweise.

überträgt S. Prüschenk Ämter in d. Herrschaften Gutenstein, Windischgraz u. Holenstein bestandweise.

überträgt H. Gressl d. Hansgrafenamt im Ftm.

Österreich bestandweise.

besiegelt Einigung im Konstanzer Bistumsstreit.

belehnt H. Halder u. G. Stadler mit d. Sitz Tandlesbach.

bevollmächtigt B. v. Schärffenberg mit d. Einsetzung eines Lehensgerichts betr. Tandlesbach. 
3291479 Nov. 28, Graz

330 [Vor 1479 Dez. 16, -]

331 1479 Dez. 16, Graz

332 [Vor 1479 Dez. 29, -] befiehlt d. Lehensleuten im Ftm. Österreich Gehorsam gegenüber B. v. Schärffenberg.

überträgt E. Lueger Schloss Stein in Krain u. Amt, Maut u. Landgericht ebd. pfleg- und bestandweise. belehnt G. Scheyrer mit Zehent u. Bergrechten im Ftm. Krain.

überträgt K. v. Stein d. Amt Primskau bestandweise. 
Regesten 
K.F. überträgt Ruprecht Ennser und dessen Erben das Brückenmeisteramt der Wiener Donaubrücken bestandweise für zwei Jahre ab Datum dieses Briefs gegen Zahlung von jährlich 1.400 Pfd. Pf., die je zur Hälfte dem K. und der Stadt Wien zu entrichten sind.

Org. oder Kop. im bearbeiteten Bestand nicht überliefert. - Dep.: Erw. im Revers Ruprecht Ennsers von 1476 Januar 13 im HHStA Wien (Sign. AUR 1476 I 13), Perg., grünes S des Wiener Bürgers Hans Ponhaimer in wachsf. Schüssel an Ps und $\mathrm{S}$ des Wiener Bürgers Jörg Talhaimer ${ }^{1}$ samt Ps ab und verloren, mit Rta-Vermerk auf der Rückseite. ${ }^{2}$

Lit.: PERGER, Wiener Ratsbürger S. 191f.; zur Donaubrückenmaut s. BRUNNER, Finanzen S. $117 f$.

1 Siehe zu ihm Perger, Wiener Ratsbürger S. 183.

2 Reg.: Lichnowsky(-BiRk) 7 n. 1913; CHMeL, Mon. Habsb. I/3 S. 647 n. 42; der Revers auch inseriert im Bürgschaftsbrief Hans Holobersys von 1476 Januar 17 im HHStA Wien (Sign. AUR 1476 I 17); Reg.: LichNowsKy(-BrRK) 7 n. 1914; CHMEL, ebd. n. 43.

\section{[Vor 1476 Januar 23, -]}

K.F. verschreibt dem Ritter Hans Holobersy auf Lebenszeit den kleinen Teich zu Biedermannsdorf.

Org. oder Kop. im bearbeiteten Bestand nicht überliefert. - Dep.: Erw. im Revers Hans Holobersys von 1476 Januar 23, Wien, im HHStA Wien (Sign. AUR 1476 I 23), Perg., 2 grüne SS des Ausst. und Jörg Plessers in wachsf. Schüsseln an Ps, mit Rta-Vermerk auf der Rückseite. ${ }^{1}$

Lit.: Zu Hans Holobersy (wohl ident mit Jan Holub von Štokov) s. HeINIG, Friedrich III./1 S. 429; TRESP, Söldner aus Böhmen S. 118f.

1 Reg.: Lichnowsky(-BIRK) 7 n. 1918; ChMEL, Mon. Habsb. I/3 S. 647 n. 44.

\section{Januar 31, Wiener Neustadt}

K.F. überlässt Andreas Wagen, seinem Verweser der Saline Aussee, und dessen Erben wissenntlich mit dem brief seinen See bei Aussee, genannt der Grundlsee (Krumelsee), samt Rechten und Zubehör, wie ihn Wolfgang Praun innehatte, bestandweise für zwei Jahre ab weichnachten yetzverganngen 1476 (1475 Dezember 25) bis weichnachten schiristkomenden 1478 (1477 Dezember 25) mit der Verpflichtung, jährlich 32 Pfd. Pf. 
in guter landläufiger Münze und 1.000 Saiblinge zuhanden des K. zu entrichten und den See samt Zubehör nach Ablauf der zwei Jahre abzutreten. An mittichen vor unser lieben Frawntag der liechtmess. ${ }^{1}$

KVr: C.d.i.i.c. $-\mathrm{KVv}$ : Rta.

Org. im HHStA Wien (Sign. AUR 1476 I 31), Perg., rotes S 21 in wachsf. Schüssel mit wachsf. S 16 rücks. eingedr. an Ps.

Reg.: ChMel n. 7033; DeRs., Mon. Habsb. I/3 S. 714 n. 242.

Lit.: Muchar, Geschichte 8 S. 90; HollwöGER, Ausseer Land S. 62; zum ksl. Türhüter Andreas Wagen s. HeINIG, Türhüter S. 364.

1 Siehe dazu den Revers Andreas Wagens von 1476 Februar 5 im HHStA Wien (Sign. AUR 1476 II 5); Reg.: GöTH, Urkunden-Regesten 9 n. 630.

\section{[Vor 1476 Februar 15, -]}

K.F. überträgt Andreas Giebinger bis auf Widerruf die Pflege von Schloss Katsch samt Zubehör und überlässt ihm die zugehörigen Nutzen und Renten bestandweise gegen Zahlung von jährlich 400 Pfd. Pf.

Org. oder Kop. im bearbeiteten Bestand nicht überliefert. - Dep.: Erw. im Revers Andreas Giebingers von 1476 Februar 15 im HHStA Wien (Sign. AUR sub dat. 1476 II 14), Perg., 2 grüne SS des Ausst. und des Hofmarschalls Jörg Fuchs von Fuchsberg ${ }^{1}$ in wachsf. Schüsseln an Ps, mit Rta-Vermerk auf der Rückseite. ${ }^{2}$

Lit:: Muchar, Geschichte 8 S. 90; Baravalle, Burgen S. 484; EBNer, Burgen Steiermark 1 S. 74; zu Katsch s. auch DERS., Herrschaft Katsch.

1 Siehe zu ihm HeIng, Friedrich III./1 S. 70-73, 299f.

2 Reg.: Muchar, Urkunden-Regesten n. 238; GötH, Urkunden-Regesten 9 n. 631.

\section{Februar 16, Wiener Neustadt}

K.F. nimmt Hans Winkler, Pfarrer von Wieselburg, wissenlich mit dem briefe wegen dessen erberkait und guter siten als seinen Kaplan auf und zusammen mit den zu dessen Pfarrkirche gehörigen Leuten und Gütern in seinen Schutz. Er verfügt, dass sie alle Ehren, Freiheiten, Rechte und gute Gewohnheiten, die den anderen Hofkaplänen und den unter seinem Schutz stehenden Personen zustehen, nach Recht und Gewohnheit 
ungehindert gebrauchen sollen, und gebietet allen Hauptleuten, Landmarschällen, Gff., Freiherren etc., Verwesern, Vizedomen, Pflegern, Bggff., Bürgermeistern, Landrichtern, Richtern, Räten, Bürgern, Gemeinden und allen anderen Amtleuten und Untertanen die Beachtung dieses Privilegs. Am freytag nach sannd Valentins tag.

KVr: fehlt.

Org. im HHStA Wien (Sign. AUR 1476 II 16), Perg., rotes S 21 in wachsf. Schüssel mit wachsf. S 16 rücks. eingedr. an Ps.

Druck: ChMEL, Mon. Habsb. I/2 S. 238f. n. 70.

Reg.: CHMel n. 7034.

Lit.: Zu den Hofkaplänen s. HeINIG, Friedrich III./1 S. 801-804; auch LACKNER, Hof und Herrschaft S. 153-158.

\section{März 8, Wiener Neustadt}

K.F. belehnt seinen Diener Christoph von Rappach wissenntlich mit dem brief, was wir im zu recht daran verleihen sullen oder mugen, mit nachfolgend genannten ererbten Festen, Gülten, Gütern und Zehenten samt Zubehör aus der Lehenschaft der Fürstentïmer Österreich und Steier und verfügt, dass Christoph und dessen Erben die Lehen innehaben und nutzen sowie mit den Festen ihm als Lehensherrn gehorsam zu Diensten sein sollen gemäß Lehens- und Landrecht.

Lehen des Ftm. Österreich: ein Viertel des Getreide- und Viehzehents sowie der Weinzehent in der Eben zu Simmering (Symaning); zu Schwechat ein Viertel des Getreidezehents in Unserr Frawnveld; zu Kledering (Gletarn) ein Viertel des Getreidezehents und der Viehzehent; zu Lanzendorf der halbe Getreidezehent auf vier Lehen; zu Atzgersdorf (Etzkestorff) ein Viertel des Getreide- und Weinzehents in der Eben und der Viehzehent; zu Altmannsdorf ein Viertel des Getreide-, Wein- und Viehzehents; zu Wien ein Viertel des Weinzehents von den Weingärten in den beiden Ämtern Ottakring und Krautgeb sowie ein Viertel des Getreidezehents; ein halber Krautzehent zu Erdberg bei der Donau, genannt im Gussen; die halbe Fischweide zu Achau (Aychaw) und fünf Metzen Vogthafer; im Markt Schottwien 6 Sch. 29 Pfd. Geld; in der Pfarre Klamm 3 Pfd. 6 Sch. 21 Pf. Geld auf drei Höfen und ein Getreidezehent auf vier Höfen mit einem Dienst von 80 Pf. sowie zu Pielachperg 1 Pfd. 80 Pf. Geld auf behaustem Gut.

Lehen des Ftm. Steier: die Feste Brunn bei Wiener Neustadt in der Pfarre Fischau; in der Prein 2 Pfd. 3 Sch. Pf. Geld, gelegen in der Pfarre Payerbach (Pierbach); in der Pfarre Haus 7 Pfd. 70 Pf. Geld; in der Pfarre Trofaiach (Trafey) 3 Pfd. 4 Pf. Geld; zu Liezen (Lutzen) im Ennstal 23 Pfd. 28 Pf. Geld; ein Gut zu Au bei der Gaysgassen; zwei Güter im Liechtmoss; ein die Gnoppen genanntes Gut in der Pfarre Aussee und ein 
Getreidezehent zu Kalwang (Chechelwanng). An freytag vor dem suntag Reminiscere in der vassten.

KVr: C.d.i.i.c. - Rückseite der Wachsschüssel: Cristoff von Rappach. - Ps: xvifl.

Org. im HHStA Wien (Sign. AUR 1476 III 8), Perg., rotes S 21 in wachsf. Schüssel mit wachsf. S 16 rücks. eingedr. an Ps.

Druck: ChMEL, Mon. Habsb. I/2 S. 241f. n. 72.

Reg.: LICHNOWSKY(-BIRK) 7 n. 1930.

Lit.: MUCHAR, Geschichte 8 S. 88.

\section{[Vor 1476 März 10, -]}

K.F. belehnt Jörg Eschelweck mit Stücken und Gütern im Ftm. Österreich.

Org. oder Kop. im bearbeiteten Bestand nicht überliefert. - Dep.: Erw. in einem Schreiben Afras von Rottau, der Witwe Jörg Eschelwecks, an K.F. mit der Bitte um Verleihung ihrer Erbgüter in Österreich an ihren Ehemann Wilhelm von Rottau zu Madau, Pfleger von Vilshofen, als ihren Lehensträger von 1476 März 10 im HHStA Wien (AUR 1476 III 10), Pap., 2 SS des Vilshofner Kastners Stephan Planck und des Vilshofner Bürgers Simon Maurer vorders. aufgedr. unter Papieroblate. ${ }^{1}$

1 Reg.: Lichnowsky(-BIRK) 7n. 1932; ChMeL, Mon. Habsb. I/3 S. 709 n. 223. - Siehe auch das Bittschreiben Wilhelms von Rottau an K.F. von 1477 Januar 20 im HHStA Wien (Sign. AUR 1477 I 20); Reg.: LichNowsKy(-BIRK) 7 n. 2003; CHMEL, ebd. n. 225 (wohl ident mit n. 224 zu 1476 Januar 20).

\section{März 11, Wiener Neustadt}

K.F. belehnt Sigmund auf dem perg auf Bitte von dessen Vetter Jakob Pucher in Tilliach wissenlich mit dem briefe, was wir im zu recht daran verleihen sollen oder mugen, mit einer Hube samt Zubehör in Lesach, gelegen auf dem perg ob der wisen, genant am Ort ${ }^{1}$ aus der in den vergangenen Kriegen von den Gff. von Görz an den K. gekommenen Lehenschaft, die Jakob Pucher ${ }^{2}$ diesem übergeben und mit einem offenen besiegelten Brief aufgesandt hat, und verfügt, dass Sigmund und dessen Erben das Gut gemäß Lehens- und Landrecht innehaben und nutzen sollen. Am montag nach dem sunntag Reminiscere.

KVr: fehlt. - Rückseite der Wachsschüssel: Vermerk nicht lesbar (wohl Name des Empfängers). 
Org. im HHStA Wien (Sign. AUR 1476 III 11), Perg., rotes S 21 in wachsf. Schüssel mit wachsf. S 16 rücks. eingedr. an Ps.

Reg.: ToMASCHEK, Regesten Kärnten 3 n. 475.

1 Mit hoher Wahrscheinlichkeit handelt es sich um das Orthner (Orther)-Gut in Xaveriberg bei St. Lorenzen im Lesachtal, s. NeUManN, Kärntner Lesachtal S. 113.

2 Zur Belehnung Jakob Puchers von 1461 Juli 16 s. Regg.F.III. H. 26 n. 592.

\section{März 20, Wiener Neustadt}

K.F. erinnert Gf. Leonhard von Görz, dass Leonhard von Herberstein, sein Pfleger von Wippach, ${ }^{1}$ widerrechtliche Eingriffe in die Herrschaft Wippach durch dessen (des Gf.) Verweser zu Görz und dessen Pfleger zu Reifenberg, nämlich den Entzug u.a. von Zehenten sowie Gewalttätigkeiten gegen Leute und Holden, ihm (K.F.) angezeigt habe, und bekundet sein Befremden, dass trotz des Erbietens Leonhards, die Beteiligten anzuhören und den Schaden zu beseitigen, bisher nichts geschehen sei. Vielmehr sei der Görzer Verweser mit etlichem Volk zu Pferd und zu Fuß auf die ksl. Gründe gezogen und schädige nach wie vor die Leute und entwende gewaltsam ihr Gut. K.F. fordert daher von Gf. Leonhard, für eine Anhörung der Parteien und nötigenfalls eine Besichtigung vor Ort zu sorgen und anzuordnen, dass dem Herbersteiner, den ksl. Leuten und auch ihm (K.F.) für das ihm gehörige Gut Schadenersatz geleistet werde. Leonhard würde ihm damit zusambt der pillichait einen gut gevallen erweisen, wofür er verspricht, sich gnädig zeigen zu wollen. An mittichen vor dem suntag Letare in der vasten

KVr: C.d.i.i.c. - KVv: Dem wolgebornen Lienharten graven zu Görtz, unserm lieben oheim und fürsten (Adresse, Blattmitte). - L Herbersteiner (linker Blattrand).

Org. im HHStA Wien (Sign. AUR 1476 III 20), ${ }^{2}$ Pap., rotes S 21 als Verschluss rücks. aufgedr. (aufgebrochen).

1 Zur Übertragung der Pflege im Jahr 1470 s. Regg.F.III. H. 27 n. 34; zu Leonhard von Herberstein s. HEINGG, Friedrich III./1 S. 188, zu Wippach (Vipava) Kos, Burg und Stadt S. 294-297.

2 Dem ksl. Schreiben beiliegend das Begleitschreiben Leonhards von Herberstein an Leonhard von Görz von 1476 Juni 23 und zwei Konzepte für Antwortschreiben des Görzers an K.F. und den Herbersteiner.

K.F. belehnt Barbara, Tochter des verstorbenen Walters von Ferndorf(Vedendorff), wissenntlich mit dem brief, was wir ir zu recht daran verleihen sullen oder mugen, mit folgenden drei Stücken aus der Lehenschaft der Gft. Ortenburg, die jährlich eine Mark und 
20 Agleier in das Amt Ferndorf dienen und die sie und ihre Erben vorbehaltlich dieser Zinse gemäß Lehens- und Landrecht innehaben und nutzen sollen: zwei Mahd Gras bei der Lakhen oberhalb Ferndorfs und dazu eine Taverne in ihrem Haus zu Ferndorf; vier Mahd Gras in der Awen und eine Mühle an der Drau oberhalb Ferndorfs. An mittichen nach dem suntag Oculi in der vassten.

KVr: C.d.i.i.c. $-\mathrm{KVv}$ : Rta. - Ps: $x$ gulden und ein weiterer nicht lesbarer Vermerk.

Org. im HHStA Wien (Sign. AUR 1476 III 20), Perg., rotes S 21 in wachsf. Schüssel (beschädigt) mit wachsf. S 16 rücks. eingedr. an Ps.

Reg.: ToMASCHEK, Regesten Kärnten 3 n. 476.

\section{März 29, Wiener Neustadt}

K.F. erklärt einen Schuldbrief Leonhards von Herberstein, seines Pflegers von Wippach, ${ }^{1}$ über $300 \mathrm{fl}$. ung. für das Dorf Schönberg, dessen sich Gf. Ivan von Veglia bemächtigt hatte, das er (K.F.) aber zurückgewonnen und Leonhard wiedergegeben hat, wissenntlich mit dem brief für ganntz tod und krafftlos, da Leonhard die Summe zwar bezahlt hat, der gelltbrief aber nicht verfügbar ist. Aus dessen Vorlage soll daher weder dem K. und seinen Erben ein Nutzen noch dem Herbersteiner und dessen Erben ein Schaden entstehen. K.F. verspricht, den Schuldbrief, sollte er zuhanden sein, gegen diesen tottbrief auszuhändigen. An freitag vor dem suntag Judica in der vasten.

$\mathrm{KVr}$ : C.d.i.i.c. $-\mathrm{KVv}$ : Rta (Blattmitte), - Quit(ung) umb die iii ${ }^{C}$ ducaten von wegen Schonberg, der schuldbrief ligt noch in kaiserlicher m(ajesta)t schatzbriefn, aber das ain sigill ist davon (zeitnaher Vermerk, Blattmitte).

Org. im HHStA Wien (Sign. AUR 1476 III 29), Perg., rotes S 21 in wachsf. Schüssel mit wachsf. S 16 rücks. eingedr. an Ps.

Reg.: ChMel n. 7038; Ders., Mon. Habsb. I/3 S. 725 n. 270.

1 Zur Übertragung der Pflege im Jahr 1470 s. Regg.F.II. H. 27 n. 34; zu Leonhard von Herberstein s. HeING, Friedrich III./1 S. 188, zu Wippach (Vipava) Kos, Burg und Stadt S. 294-297.

\section{April 5, Wiener Neustadt}

K.F. bestätigt und erneuert aus ksl. Macht und als Herr und Landesfürst von Österreich Abt Leonhard ${ }^{1}$ und dem Konvent des Benediktinerklosters Vornbach in Ansehung ihres geistlichen Lebens und ihrer täglichen Gottesdienste, derer er tailhefftig zu werden hofft, 
von sundern gnaden, mit rechter wissen und wissenntlich mit dem brief alle ihnen und ihrer (Propstei-)Kirche zu Gloggnitz ${ }^{2}$ von seinen Vorfahren verliehenen Privilegien und Rechte in allen stukhen, punkhten und artikeln, als ob sie wortwörtlich inseriert wären. Er gebietet allen geistlichen und weltlichen Fürsten, Gff., Freiherren etc., Verwesern, Vizedomen, Pflegern, Bggff., Bürgermeistern, Schultheißen, Richtern, Räten, Bürgern, Gemeinden und allen anderen Amtleuten und Untertanen des Reichs und seiner Fürstentümer bei seiner und des Reichs schweren Ungnade und einer Pön von 100 Mark Gold, zahlbar je zur Hälfte an die ksl. Kammer und das Kloster, die Beachtung der Freiheiten und Rechte der Klosterleute. Am freitag vor dem heiligen Palmtag. ${ }^{3}$

KVr: fehlt.

Org. im HHStA Wien (Sign. AUR sub dat. 1476 IV 6), Perg., wachsf. S 24 mit wachsf. S 16 vorders. eingedr. an purpurf. Ss. - Kop.: Abschrift des 18. Jh. ebd. (Sign. Urkundenabschriften Österr. Urkunden, Kt. 45 sub dat. 1476 April 6), Pap.

Druck: ChMel, Mon. Habsb. I/2 S. 246f. n. 75 (zu April 6).

Reg.: CHMel n. 7040 (zu April 6).

1 Abt Leonhard I. Strasser (1474-1501), s. Germania Benedictina II/3 S. 2450 (Äbteliste).

2 Zur Propstei Gloggnitz s. Mochry-Weutin u.a., Wehrbauten 3 S. 41-80.

3 Siehe dazu die Privilegienbestätigungen von 1440 Dezember 3 (Regg.F.II. H. 2 n. 1) und 1485 Mai 13 (CHMel n. 7735).

\section{[1476 April 10, - $]^{1}$}

K.F. befiehlt Kaspar von Rogendorf, ihm die wegen der Gefangenen schuldigen 500 Pfd. Pf. zu geben; desgleichen die 150 Gulden von der Remanenz des Ungelds ${ }^{2}$ zu Waidhofen a.d. Ybbs und zu Ybbs, die der Krabath ${ }^{3}$ dem Rogendorfer übergeben hat (nach Registereintrag).

KVr: C.d.i. per dominum Sigismundum Prueschinckh camerarium (nach Registereintrag).

Org. oder Kop. im bearbeiteten Bestand nicht überliefert. - Dep.: Auszugsweise wiedergegeben in zwei von verschiedenen Händen verfassten Abschriften (A, B) eines Registereintrags (im „blauen Register“) im HHStA Wien (Sign. AUR 1476 IV 10), beide Pap., Anfang 16. Jh. ${ }^{4}$

Lit.: BeRgMANN, Rogendorf S. 524 [S. 8] (zu April 11); zur Person des Rogendorfers s. ZAJI, Kaspar von Roggendorf, bes. S. 14; NeIDHART, Geschichte Pöggstalls S. 41-58; auch HeINIG, Friedrich III./1 S. 195f.; zur Familie s. ZAлC, Rog[g]endorf S. 1207-1214. 
Der Registereintrag hält das Rechtsgeschäft auszugsweise in Regestenform in objektiver Form fest, wonach Kaspar von Rogendorf dem Kaiser die schuldigen Geldsummen geben soll. K.F. wird nicht als Aussteller genannt, auf eine ksl. Urkunde weist nur der Kanzleivermerk, der - wie auch das Datum - rechts unterhalb des Textes vermerkt ist.

Das als „blaues Register“ bezeichnete Kanzleiregister (s. Anm. 4) befand sich unter dem Nachlass Johann Waldners, ab 1475 Leiter der röm. Kanzlei und unter Maximilian I. Kanzler des niederösterreichischen Regiments (bis 1501), der 1502 durch Suizid starb. ${ }^{5}$

1 Datierung nach Abschrift des Registereintrags: Actum feria quarta post Palmarum.

2 Zur Bestandgabe des Ungelds im Jahr 1474 s. n. 31.

3 Andreas von Lappitz, s. HeINIG, Friedrich III./1 S. 294.

4 Vermerk unterhalb des Textes: (A) Disz aufzaichen ist gezogen aus ainem canntzleyregister mit ainem plaben coopert und ligt neben anndern des Waldners briefen; (B): Disz aufzaichen oder auszug steet geschriben in dem plaben register so unnder anndern des Waldners briefen liget und ist ein canntzleyregister. - Rücks. Vermerke: (A) Rogendorff schuldhalben; (B) Rogendorff schuldhalben den raitreten zuzuschickhen. - Druck (Abschrift B): CHMEL, Mon. Habsb. I/3 S. 655 n. 80; Reg.: Lichnowsky(-BIRK) 7 n. 1939.

5 Zu Johann Waldner s. Herng, Friedrich III./1 S. 721-731; SchwARZ, Salzburger Kürschnersohn, und DERS., Johann Waldner.

\section{April 19, Wiener Neustadt}

K.F. belehnt Adam von Uttweiler für sich und als Lehensträger seiner Brüder und Vettern Heinrich, Friedrich, Michael, Eberhard und Melchior, mit den von Adolf von Uttweiler ererbten Burglehen zu Hagenau und Wilshausen sowie mit den Stücken und Gütern, die Georg von Uttweiler von ihm und dem Reich zu Lehen hatte. Am fritag nach dem heiligen Ostertag (nach Kop.).

Org. im bearbeiteten Bestand nicht überliefert, der Kop. zufolge Perg. mit anh. S. - Kop.: Vidimus Konrads von Wilsberg und Hans' von Mittelhausen von 1494 Januar 2 im HHStA Wien (Sign. AUR sub dat. 1476 III 29), Pap., 2 SS der Ausst. vorders. aufgedr. unter Papieroblate; ${ }^{1}$ davon Abschrift des 18. Jh. ebd. (Sign. AUR 1476 IV 19), Pap.

Reg.: Ein ausführliches Regest bieten die Regg.F.III. H. 17 n. 290 (nach Kop.).

1 Das Vidimus weist von anderer Hand vorgenommene Streichungen (u.a. der Begünstigten Friedrich und Melchior) und Textverweise mit mehrzeiligen Anmerkungen am linken Rand auf. Diese den Urkundentext „aktualisierenden“ Korrekturen stammen mit hoher Wahrscheinlichkeit aus der Kanzlei Maximilians I., vermutlich diente das Vidimus als Vorlage einer Bestätigung. 
K.F. verleiht aus ksl. Macht und als Herr und Landesfürst von Österreich dem Richter, Rat und allen Bürgern von Klosterneuburg zur Förderung der Stadt von sundern gnaden wissentlich mit dem brief einen zweiten Jahrmarkt ${ }^{1}$ am Montag nach Fronleichnam mit fürstlicher freyung vierzehn Tage davor und danach und verfügt, dass sie den Jahrmarkt auf ewig nach den für andere Jahrmärkte im Ftm. Österreich geltenden Freiheiten, Rechten und Gewohnheiten abhalten sollen, jedoch unbeschadet der Rechte anderer Jahrmärkte. Er gebietet allen Hauptleuten, Landmarschällen, Gff., Freiherren etc., Verwesern, Hubmeistern, Pflegern, Bggff., Bürgermeistern, Richtern, Räten, Bürgern, Gemeinden und allen anderen Amtleuten und Untertanen bei seiner schweren Ungnade, dieses Privileg zu beachten und niemand am Besuch des Jahrmarkts zu hindern. Geben zu der Newnstat an mittichen sand Jorgen tag (nach Org.).

KVr: A.m.d.i.i.c. (nach Org.).

[Org. im StadtA Klosterneuburg. $]^{2}$ - Genannt bei CHMEL nach einer Überlieferung im HHStA Wien.

Druck: ZeIBIG, UB Stadt Klosterneuburg S. 339f. n. 29 (nach Kop.). ${ }^{3}$

Reg.: CHMEL n. 7043 (zu April 22); JÄGER-SunstenAU, Archiv Klosterneuburg S. 29 n. 54.

Lit.: StARZer, Klosterneuburg S. 224; ${ }^{4}$ Wellner, Klosterneuburg S. 122; Perger, Klosterneuburg S. 194.

1 Zur Verleihung des ersten Jahrmarkts von 1453 Juli 7 s. ZeIBIG, UB Stadt Klosterneuburg S. 337 n. 26 (zu Juni 30).

2 StadtA Klosterneuburg (Sign. AStK1 Urk. Nr. 54)

3 Nach einem Kopialbuch des 16. Th. in der Stiftsbibliothek Klosterneuburg; dem Druck zufolge Bitte der Stadt um einen Jahrmarkt auf Sonntag (!) nach Fronleichnam, die Verleihung erfolgte aber wie im Org. auf Montag nach Fronleichnam.

4 Bei STARZER irrtümlich Jahrmarkt auf Sonntag nach Fronleichnam (wohl nach ZeIBIG).

\section{[Vor 1476 April 29, -]}

K.F. überlässt dem Richter, Rat und der Stadt Leoben den Viehaufschlag sowie die zur Instandhaltung der Brücken und Wege eingehobene Maut ${ }^{1}$ zu Leoben samt Zwiespil bestandweise für drei Jahre ab St. Georgstag 1476 (April 24) ${ }^{2}$ gegen Zahlung von jährlich 1.300 Pfd. Pf. 
Org. oder Kop. im bearbeiteten Bestand nicht überliefert. - Dep.: Ergibt sich aus dem Bürgschaftsbrief der Leobener Bürger Gregor Leubner, Jörg Kätzler und Wolfgang Steyrer von 1476 April 29 im HHStA Wien (Sign. AUR 1476 IV 29), Perg., 5 grüne SS der Ausst. und der Leobener Ratsherren Hans Haindorfer und Hans Pringsauf in wachsf. Schüsseln an Ps, mit Rta-Vermerk auf der Rückseite. ${ }^{3}$

1 Zur Leobener Maut s. SchILLINGER-PRASSL, Rechtsquellen Leoben S. 71f.

2 Zur Datierung des St. Georgstags auf April 24 s. Regg.F.III. H. 12 n. 6, Anm. 2.

3 Die Aussteller verbürgten sich, im Falle von Zahlungsrückständen seitens der Stadt die Bezahlung des Bestandgeldes zu übernehmen (wie auch in n. 235). - Reg.: MuchAR, Urkunden-Regesten n. 240 (irrtümlich als Bestandgabe an die drei Leobener Bürger); GöTH, Urkunden-Regesten 9 n. 634; erw. bei MucHAR, Geschichte 8 S. 90. Von den als Bürgen oder Siegler beteiligten Leobener Bürgern hatten Leubner, Steyrer und Pringsauf in den 1470er- und 1480er-Jahren auch das Stadtrichteramt inne, s. SchILLINGER-PRAssL, Rechtsquellen Leoben, Anh. S. 314.

[Vor 1476 Mai 6, -]

K.F. überlässt Berthold Stettner bis auf Widerruf die Maut, das Geleitgeld und die Taverne zu Lieserhofen samt Zubehör bestandweise ab Sonntag vor dem St. Urbanstag 1476 (Mai 19) gegen Zahlung von jährlich 800 Pfd. Pf.

Org. oder Kop. im bearbeiteten Bestand nicht überliefert. - Dep.: Erw. im Revers Berthold Stettners von 1476 Mai 6 im HHStA Wien (Sign. AUR 1476 V 6), Perg., 2 grüne SS des Ausst. und des Ritters Reichart Kärgl in wachsf. Schüsseln an Ps, mit Rta-Vermerk auf der Rückseite. ${ }^{1}$

1 Reg.: TomaschEK, Regesten Kärnten 3 n. 478.

[Um 1476 Mai 10, -]

K.F. überträgt seinem Hofmarschall Jörg Fuchs von Fuchsberg bis auf Widerruf die Pflege von Schloss Starhemberg ${ }^{1}$ unter der Bedingung, dass dieser sich von den Nutzen und Renten die Burghut und den Sold für das Hofmarschallamt ${ }^{2}$ selbst bezahlt und der $\mathrm{K}$. ihm darüber hinaus nichts zu geben verpflichtet ist. ${ }^{3}$

Org. oder Kop. im bearbeiteten Bestand nicht überliefert. - Dep.: Erw. im Revers des Hofmarschalls Jörg Fuchs von Fuchsberg von 1476 Mai 10 im HHStA Wien (Sign. AUR 1476 V 10), Perg., 2 grüne SS des Ausst. und Christophs von Rappach in wachsf. Schüsseln an Ps, mit Rta-Vermerk auf der Rückseite. ${ }^{4}$ 
Lit.: ZAHN, Hernstein S. 81; zu Jörg (Georg) Fuchs von Fuchsberg s. HeINIG, Friedrich III./1 S. $70-73$, bes. 73 , und 299 f.

1 Starhemberg in Niederösterreich (bei Wiener Neustadt) und nicht, wie der Rückvermerk des 16. Jh. $o b$ der Enns auf dem Revers nahelegt, Starhemberg in Oberösterreich.

2 Der Sold betrug jährlich 400 Pfd. Pf., s. HeINIG, Friedrich III/1 S. 72

3 Wohl in engem Zusammenhang mit der Übertragung Starhembergs an Jörg Fuchs von Fuchsberg steht die Einigung mit dem früheren Pfleger und ksl. Rat Hans von Spaur, der am 9. Mai 1476 dem K. die Befriedigung aller seiner Forderungen bestätigte; s. dazu den Quittbrief Hans' von Spaur von 1476 Mai 9 und dessen Urfehdebrief von 1476 Mai 13 im HHStA Wien (beide Sign. AUR sub dat. 1476 V 9); Druck: ChMel, Mon. Habsb. I/2 S. 249f. nn. 78, 79; s. auch ChMel nn. 7049, 7051. - Jörg Fuchs von Fuchsberg hatte die Pflege nur kurzzeitig inne, da er 1477/78 verstarb; ihm folgte als Pfleger Christoph Sachsenlander, s. dessen Pflegrevers von 1478 August 19 ebd. (Sign. AUR sub dat. 1478 VIII 13); Reg.: CHMEL n. 7220; DERS., Mon. Habsb. I/3 S. 640 n. 16.

4 Reg. (Revers): ChMEL n. 7050; DERS., Mon. Habsb. I/3 S. 638 n. 7; LichNowsKY(-BrRK) 7 n. 1947.

\section{[Vor 1476 Mai 29, -]}

K.F. überträgt Peter Feistritzer bis auf Widerruf das in der Gft. Ortenburg gelegene Amt Stockenboi und die dortige Brückenmaut zu treuen Händen. ${ }^{1}$

Org. oder Kop. im bearbeiteten Bestand nicht überliefert. - Dep.: Erw. im Revers Peter Feistritzers von 1476 Mai 29, Wiener Neustadt, im HHStA Wien (Sign. AUR 1476 V 29), Perg., 2 grüne SS des Ausst. und des Hofmarschalls Jörg Fuchs von Fuchsberg ${ }^{2}$ in wachsf. Schüsseln an Ps, mit Rta-Vermerk auf der Rückseite. ${ }^{3}$

1 Siehe dazu auch n. 70.

2 Siehe zu ihm HeInIG, Friedrich III./1 S. 70-73, 299f.

3 Reg.: TOMASCHEK, Regesten Kärnten 3 n. 480; MC 11 n. 457.

\section{[Vor 1476 Mai 31, -]}

K.F. überlässt Simon Krell und Jörg Peurbeck Fron und Wechsel des Bergbaus in der Herrschaft Ortenburg und in den Gerichten Vellach, Rottenstein und Greifenburg bestandweise für zwei Jahre ab St. Georgstag 1476 (April 24) ${ }^{1}$ gegen Zahlung von jährlich 400 Mark Silber, vorbehaltlich seines (Vor-)Kaufrechts auf das gewonnene Silber zum Preis von $6 \mathrm{fl}$. ung. für eine Mark Silber.

Org. oder Kop. im bearbeiteten Bestand nicht überliefert. - Dep.: Erw. im Revers Simon Krells und Jörg Peurbecks von 1476 Mai 31, Wiener Neustadt, im HHStA Wien (Sign. AUR sub dat. 1476 VI 1), Perg., 4 grüne SS der Ausst., des Wiener Neustädter Bürgermeisters Kaspar 
Seefelder und des Ratsherrn Prokop Zinner ${ }^{2}$ in wachsf. Schüsseln an Ps, mit Rta-Vermerk auf der Rückseite. ${ }^{3}$

Lit.: WIESSNER, Kärntner Bergbau 1 S. 41f.; FRÄss-EHRFELD, Geschichte Kärntens 1 S. 566, 671.

Laut Revers Simon Krells von 1480 Januar 26 (s. n. 154) hatten er und Jörg Peurbeck bis 1478 auch die Anteile des 1472 verstorbenen Hans von Kainach ${ }^{4}$ samt den Hütten in Bestand. Im vorliegenden Revers werden diese zwar nicht als Inhalt der ksl. Urkunde genannt, aber im Zusammenhang mit den Verpflichtungen der Bestandnehmer erwähnt. Möglicherweise hatten Krell und Peurbeck die Kainacher-Anteile bereits früher bestandweise erhalten; nicht auszuschließen ist, dass sie ebenfalls Gegenstand der ksl. Urkunde waren.

Siehe nn. 22, 154.

1 Zur Datierung des St. Georgstags auf April 24 s. Regg.F.III. H. 12 n. 6, Anm. 2.

2 Zu Kaspar Seefelder (Bürgermeister 1475-1478) und Prokop Zinner s. MAYER, Wiener Neustadt I/2 S. $133,491 f$.

3 Druck: ChMel, Mon. Habsb. I/3 S. 717-719 n. 254.

4 Siehe zu ihm HärTEL, Kainacher S. 13.

[Vor 1476 Juni 7, -]

K.F. überlässt Katharina, Witwe des Judenburger Bürgers Wolfgang Rösler, auf Lebenszeit eine Wiese bei Judenburg bestandweise gegen Zahlung von jährlich $6 \mathrm{Pfd}$. Pf. ${ }^{1}$

Org. oder Kop. im bearbeiteten Bestand nicht überliefert. - Dep.: Erw. im Revers Katharina Röslers von 1476 Juni 7, Wiener Neustadt, im HHStA Wien (Sign. AUR 1476 VI 7), Perg., 2 grüne SS des Wiener Neustädter Bürgermeisters Kaspar Seefelder ${ }^{2}$ und des Stadtschreibers Wolfgang Spitzweg ${ }^{3}$ in wachsf. Schüsseln an Ps. ${ }^{4}$

Lit.: FeLSER, Herkunft S. C.

1 Laut Revers hatte Katharina Rösler die an der zu den Kellchen führenden Straße gelegene Wiese, ehemals im Besitz des Scheller - wohl der Judenburger Stadtrichter Stephan Scheller - urkundlich K.F. zur freien Verfügung überlassen; zu Stephan Scheller s. FeLSER, Herkunft S. CVIf.

2 Bürgermeister 1475-1478, s. MAYER, Wiener Neustadt I/2 S. 133.

3 Wolfgang Spitzweg war ksl. Diener, Sekretär und Notar in der röm. Kanzlei und ab 1473 Stadtschreiber von Wiener Neustadt, s. zu ihm MAYER, ebd. S. 106 und 135; HenNG, Friedrich III./1 S. $749 f$.

4 Reg.: GöTH, Urkunden-Regesten 9 n. 636. 
K.F. überlässt Simon Krell und Jörg Peurbeck zusätzlich zu Fron und Wechsel des Bergbaus $^{1}$ in der Herrschaft Ortenburg und in den Gerichten Vellach, Rottenstein und Greifenburg den Silberkauf bestandweise für zwei Jahre ab St. Veitstag 1476 (Juni 15) gegen Zahlung von jährlich 200 Mark Silber.

Org. oder Kop. im bearbeiteten Bestand nicht überliefert. - Dep.: Erw. im Revers Simon Krells und Jörg Peurbecks von 1476 Juni 8, Wiener Neustadt, im HHStA Wien (Sign. AUR 1476 VI 8), Perg., 4 grüne SS der Ausst., des Wiener Neustädter Bürgermeisters Kaspar Seefelder und des Ratsherrn Prokop Zinner ${ }^{2}$ in wachsf. Schüsseln an Ps, mit Rta-Vermerk auf der Rückseite. ${ }^{3}$

Lit.: WIESSNER, Kärntner Bergbau 1 S. 41f.; FRäss-EHRFELD, Geschichte Kärntens 1 S. 566, 671.

1 Siehe n. 20.

2 Zu Kaspar Seefelder (Bürgermeister 1475-1478) und Prokop Zinner s. MAYER, Wiener Neustadt I/2 S. $133,491 f$.

3 Druck: MC 11 n. 458 (Teildruck); Reg.: GöTH, Urkunden-Regesten 9 n. 637.

\section{[Vor 1476 Juni 8, - ]}

K.F. überlässt Jörg Peurbeck Amt und Landgericht zu Großkirchheim bestandweise für fünf Jahre ab St. Georgstag 1476 (April 24) ${ }^{1}$ gegen Zahlung von jährlich 190 Pfd. Pf. ${ }^{2}$

Org. oder Kop. im bearbeiteten Bestand nicht überliefert. - Dep.: Erw. im Revers Jörg Peurbecks von 1476 Juni 8, Wiener Neustadt, im HHStA Wien (Sign. AUR 1476 VI 8), Perg., 3 grüne SS des Ausst., des Wiener Neustädter Bürgermeisters Kaspar Seefelder und des Ratsherrn Prokop Zinner ${ }^{3}$ in wachsf. Schüsseln an Ps, mit Rta-Vermerk auf der Rückseite. ${ }^{4}$

1 Zur Datierung des St. Georgstags auf April 24 s. Regg.F.III. H. 12 n. 6, Anm. 2.

2 Im Jahr 1481 übertrug K.F. Amt und Landgericht zu Großkirchheim Kaspar Pischofer zu treuhändischer Verwaltung, s. dessen Revers von 1481 Mai 20 im HHStA Wien (Sign. AUR sub dat. 1478 V 20).

3 Zu Kaspar Seefelder (Bürgermeister 1475-1478) und Prokop Zinner s. MAYER, Wiener Neustadt I/2 S. $133,491 f$.

4 Reg.: TomascheK, Regesten Kärnten 3 n. 481. 
K.F. belehnt Kaspar Melz, Bürger von Laibach, wissenntlich mit dem brief, was wir im $z$ recht daran verleihen sullen oder mugen, mit nachfolgend genannten Äckern und Zehenten samt Zubehör aus der Lehenschaft der verstorbenen Mannsburger, nachdem der ksl. Diener Jörg Schrott die Äcker und Peter Kremser, Bürger von Stein (in Krain), die Zehenten nach deren Verkauf an Melz mit offenen besiegelten Briefen aufgesandt haben: mehrere Äcker in der Pfarre Laibach bei St. Christoph hinter der hergruben, die mit einer Seite an die Äcker des Laibacher Bürgers Primos Kolman und mit den anderen beiden Seiten an die dortige Gemeinde stoßen, und der halbe Zehent ${ }^{1}$ von zehn Huben in Pfaffendorf, Maierhof, Aich und Worst (under dem Varst) sowie von einem Hof zu Prunn bei Mannsburg (Mannigspurg). Er verfügt, dass Melz und dessen Erben die Güter und Gülten gemäß Lehens- und Landrecht innehaben und nutzen sollen. An eritag nach sannd Johanns tag zu sunewennden (nach Kop.).

KVr: C.d.i.i.c. $-\mathrm{KVv}$ Rta (nach Kop.).

[Org. im ARS Ljubljana, zuvor im HHStA Wien.] - Fotokop. vom Org. im HHStA Wien (Sign. AUR 1476 VI 25).

Reg.: Regg.F.III. H. 29 n. 188 (nach Org., mit Druckbelegen); weiters OTOREPEC, Gradivo VI/34 (Druck); Göтн, Urkunden-Regesten 9 n. 638.

1 Zur Verleihung von je einem Viertel des Zehents an zwei Steiner Bürger von 1473 April 10 s. Regg.F.II. H. 27 nn. 201, 202, H. 29 nn. 178, 179.

\section{Juli 9, Wiener Neustadt}

K.F. beurkundet das auf heut unter seinem persönlichen Vorsitz ergangene Urteil seines Kammergerichts, dem zufolge der Klage ${ }^{1}$ seines Vetters Hz. Sigmund von Österreich gegen die verwitwete Ehzn. Mechthild von Österreich, geborene Pfgfn. bei Rhein, seine swester, und Gf. Eberhard (V.) d.Ä. von Württemberg, seinen swager, ${ }^{2}$ stattgegeben und dem Hz. das Recht zugesprochen wird, die Burgställe Hohenberg und Wehingen gegen Entrichtung der Pfandsumme von $2.000 \mathrm{fl}$. rh. aus dem Pfandbesitz Mechthilds einzulösen sowie Schadenersatz ${ }^{3} z u$ fordern. Dem Antrag der Anwälte ${ }^{4}$ des Klägers auf Ausstellung eines Urteilsbriefs sowie von Gebotsbriefen und Ladungen ${ }^{5}$ wegen der Kosten und Schäden wird stattgegeben.

Beisitzer: die ksl. Räte Bf. Anton von Triest, Bf. Sigmund von Laibach, Abt (Johann) von St. Lambrecht, die Gff. Haug von Werdenberg und Wilhelm von Thierstein, ${ }^{6}$ der Konstanzer Dompropst Thomas (Prelokar) von Cilli, ${ }^{7}$ der ksl. Hofmarschall Georg 
Fuchs (von Fuchsberg), ${ }^{8}$ Dr. Hartung von Cappel,,${ }^{9}$ Hofmeister Bartholomäus von Lichtenstein ${ }^{10}$ und der ksl Protonotar Meister Johannes Rehwein ${ }^{11}$. Geben mit urteil [...] am newndten tag des monets julii.

KVr: A.m.d.i. Jo(hann) Waldner prothonotarius etc. - KVv: Urteilbrief herczog Sigmunden (rechter Blattrand). - Urteilbrief zwischen meinem g. heren und meiner frawen von Osterrich 1476 (Empfängervermerk, Blattmitte).

Org. im HHStA Wien (Sign. AUR 1476 VII 9), ${ }^{12}$ Perg., rotes S 18 in wachsf. Schüssel mit wachsf. S 16 rücks. eingedr. an Ps. - Kop.: Abschrift des 18. Jh. ebd. (Sign. Urkundenabschriften Österr. Urkunden, Kt. 45 sub dat.), Pap.

Druck: ChMEL, Mon. Habsb. I/2 S. 181-187 n. 43.

Reg.: Ein ausführliches Regest auf der Grundlage einer Abschrift des 15. Jh. bieten die Regg.F.III. H. 23 n. 643; s. auch LichNowsKy(-BIRK) 7 n. 1957.

Lit. siehe Regg.F.III. H. 23 n. 643; weiters ERnst, Eberhard im Bart S. 163; MiLBRADT, Kammergericht S. 38; BAUM, Sigmund der Münzreiche S. 416, 419f.; DERS., Habsburger S. 700f.; PRESS, Eberhard im Bart S. 19; zum Prozessverlauf s. Protokoll- und Urteilsbücher 3 S. 13291331 Verfahrensnr. 619.

Siehe nn. 26, 27, 201-203.

1 Zur Streitsache s. Regg.F.III. H. 23 nn. 98, 605, 627-631, 634, 644, 645, 664, 669; auch Regg.F.III. H. 27 nn. $60,62,85,113$.

2 Eberhard d.A. „im Bart" war Mechthilds zweitgeborener Sohn aus ihrer ersten Ehe mit Gf. Ludwig I. von Württemberg. Thre zweite Ehe mit Ehz. Albrecht VI. von Österreich begründete die Verschwägerung zwischen Habsburg und Württemberg.

3 Der Schadenersatz wurde ebenfalls auf 2.000 fl. rh. beziffert (s. Regg.F.III. H. 23 nn. 644, 645), wodurch Hz. Sigmund die Möglichkeit erhielt, die verpfändeten Herrschaften kostenneutral zurückzuerhalten. Mechthild appellierte am 10. September 1476 an K.F. persönlich gegen das Urteil und eine erneute Vorladung, s. Regg.F.III. H. 23 n. 644 (Kommentar).

4 Johann (Hans) Keller, zugleich ksl. Fiskalprokurator, und Sigmunds Diener Jörg Schetzer; zu ihrer Bevollmächtigung von 1476 Mai 28 s. Anm. 13, zu Johann Keller s. HenNIG, Friedrich III/1 S. 123-134, bes. 128 .

5 Siehe nn. 26, 27 und Regg.F.III. H. 23 nn. 644, 645.

6 Zu Haug von Werdenberg und Wilhelm von Thierstein s. HeINIG, Friedrich III./1 S. 336-347 und $365 f$

7 Zu Dr. iur. utr. Thomas Prelokar (Prelager, Berlower) von Cilli, Protonotar der österr. Kanzlei, s. ebd. S. 612-618; LUGER, Humanismus S. 110-119.

$8 \mathrm{Zu}$ Georg (Jörg) Fuchs von Fuchsberg s. HenNIG, ebd. S. 70-73 und 299f.

$9 \mathrm{Zu}$ Hartung Molitoris von Cappel s. ebd. S. 111-118.

10 Hofmeister Hz. Maximilians von Österreich, s. Regg.F.III. H. 23 n. 643, Anm. 4.

$11 \mathrm{Zu}$ Lic. iur. utr. Johannes Rehwein (Rechwein, Reben), 1470-1481 Leiter der österr. Kanzlei, s. HeINIG, Friedrich III./1 S. 592-595; LUGER, Humanismus S. 97-110. 
12 Dem Urteil beiliegend drei Vollmachten für die Anwälte beider Parteien: je eine Vollmacht Ehzn. Mechthilds und Gf. Eberhards für Hermann von Sachsenheim von 1476 April 27 sowie eine Vollmacht Hz. Sigmunds von 1476 Mai 28 für Johann Keller und Jörg Schetzer; die Vollmacht Sigmunds auch als Abschrift des 18. Jh. überliefert im HHStA Wien (Sign. AUR 1476 V 28); s. dazu auch die erneute Bevollmächtigung Kellers und Schetzers von 1477 Januar 24 ebd. (Sign. AUR 1477 I 24 [Org.]; Urkundenabschriften Österr. Urkunden, Kt. 45 sub dat. [Kop., 18. Jh.]).

\section{[1476 Juli 9, Wiener Neustadt $]^{1}$}

K.F. lädt die verwitwete Ehzn. Mechthild von Österreich, geborene Pfgfn. bei Rhein, in ihrem Streit mit Hz. Sigmund von Österreich wegen der Burgställe Hohenberg und Wehingen vor sein Kammergericht, um gemäß ergangenem Urteil² die Schadenersatzleistung und die Gerichtskosten bemessen zu lassen.

Org. oder Kop. im bearbeiteten Bestand nicht überliefert. - Dep.: Erw. in der Prozessvollmacht Ehzn. Mechthilds von Österreich, Pfgfn. bei Rhein, von 1476 Oktober 4, Rottenburg, im HHStA Wien (Sign. AUR 1476 X 4), Perg., rotes S der Ausst. in wachsf. Schüssel an Ps. ${ }^{3}$

Lit. siehe bei $n .25$.

In der Vollmacht wird auch der ksl. Befehl an Ehzn. Mechthild erwähnt, gemäß Urteil von 1476 Juli 9 Hz. Sigmund die verpfändeten Burgställe Hohenberg und Wehingen zur Einlösung zu geben; zu diesem Mandat s. Regg.F.III. H. 23 n. 644.

1 Datierung nach den Mandaten an Ehzn. Mechthild und Gf. Eberhard von Württemberg von 1476 Juli 9 , S. Regg.F.III. H. $23 \mathrm{nn} .644,645$.

2 Siehe n. 25.

3 Bevollmächtigung der Doktoren Bernhard Schöferlin und Martin Kellner unter Widerrufung der Hermann von Sachsenheim erteilten Vollmacht von 1476 April 27 (s. n. 25, Anm. 12); Druck: CHMEL, Mon. Habsb. I/2 S. 194-196 n. 45A; Reg.: LichNowsKY(-BIRK) 7 n. 1981.

[1476 Juli 9, Wiener Neustadt] ${ }^{1}$

K.F. lädt desgleichen Gf. Eberhard (V.) d.Ä. von Württemberg vor sein Kammergericht.

Org. oder Kop. im bearbeiteten Bestand nicht überliefert. - Dep.: Erw. in der Prozessvollmacht Gf. Eberhards von Württemberg von 1476 Oktober 4 im HHStA Wien (Sign. AUR 1476 $\mathrm{X} 4)$, Perg., rotes S des Ausst. in wachsf. Schüssel an Ps. ${ }^{2}$

Lit. siehe bei n. 25 .

In der Vollmacht wird auch der ksl. Befehl an Gf. Eberhard erwähnt, gemäß Urteil von 1479 Juli 9 (s. n. 25) Hz. Sigmund nicht an der Einlösung der Burgställe Hohenberg und Wehingen zu 
hindern; der Befehl ist wohl ident mit dem Mandat von 1476 Juli 9, s. Regg.F.III. H. 23 n. 645 (nach Kop.).

1 Datierung wie n. 26.

2 Bevollmächtigung der Doktoren Bernhard Schöferlin und Martin Kellner unter Widerrufung der Hermann von Sachsenheim erteilten Vollmacht von 1476 April 27 (s. n. 25, Anm. 12); Druck: CHMEL, Mon. Habsb. I/2 S. 196f. n. 45B; Reg.: LichNowsKy(-BIRK) 7 n. 1982.

\section{Juli 10, Wiener Neustadt}

K.F. erteilt seinen Räten Rüdiger von Starhemberg, ${ }^{1}$ Wilhelm von Puchheim ${ }^{2}$ und Sigmund von Eitzing ${ }^{3}$ sowie Raphael Lischinsky ${ }^{4}$, Leopold Wultzendorfer und Virgil Schrutauer, seinem Anwalt im Wiener Rat, ${ }^{5}$ wissenlich mit dem brief die Vollmacht, auf dem auf freitag vor sannd Maria Magdalentag nechstkomenden (Juli 19) anberaumten Tag über die Beilegung der nach dem Frieden von Breslau ${ }^{6}$ erfolgten Friedensbrüche zwischen dem Ftm. Österreich und der Mgft. Mähren ${ }^{7}$ in seinem Namen zu verhandeln, und verspricht, die von ihnen ausgehandelten Beschlüsse einzuhalten. Am mittichen vor sanndt Margreten tag.

\section{KVr: C.d.i.i.c.}

Org. im HHStA Wien (Sign. AUR 1476 VII 10), Perg., rotes S 21 in wachsf. Schüssel mit wachsf. S 16 rücks. eingedr. an Ps. - Kop.: 2 Abschriften des 18. Jh. ebd. (Sign. Urkundenabschriften Österr. Urkunden, Kt. 45 sub dat.; Urkundenabschriften Collat. Urkunden, Kt. 73 sub dat.), Pap.

Druck: ChMEL, Mon. Habsb. I/1 S. 497f. n. 183.

Reg.: ChMel n. 7057; Lichnowsky(-BIRK) 7 n. 1960 (zu Juli 17).

Lit.: BACHMANN, Reichsgeschichte 2 S. 546; VANCSA, Geschichte 2 S. 493.

1 Zu Rüdiger von Starhemberg s. Zernatto, Herrenstand S. 162f;; HenNig, Friedrich III./1 S. 252.

2 Zu Wilhelm von Puchheim s. Zernatto, ebd. S. 135; TePPerberg, Herren von Puchheim S. 84; Heinig, ebd. S. 256.

3 Zu Sigmund von Eitzing s. HeINIG, ebd. S. $264 f$.

4 Zu Raphael Lischinsky s. ebd. S. 424.

5 Zum langjährigen Wiener Stadtanwalt Virgil Schrutauer (1468-1479, 1485-1490) S. PeRger, Wiener Ratsbürger S. 244; HEING, ebd. S. $618 \mathrm{f}$.

6 Breslauer Frieden von 1474 Dezember 8 zwischen $\mathrm{Kg}$. Matthias von Ungarn und $\mathrm{Kg}$. Wladislaw von Böhmen, in dem ein zweijähriger Waffenstillstand ab Pfingsten 1475 (Mai 14) vereinbart wurde und dem K.F. und Kg. Kasimir IV. von Polen beitraten; Druck: Scriptores rerum Silesiacarum 13 S. 166-175 n. 217; BachmanN, Urkundliche Nachträge n. 326; Reg.: Weise, Staatsverträge 3 S. 5153 n. 446; zum 
Beitritt des K. s. das Schreiben an die Untertanen des Ftm. Österreich von 1475 Februar 9; Druck: CFMEL, Mon. Habsb. I/3 S. 680f. n. 157; s. dazu VANCSA, Geschichte 2 S. 493; NeHRING, Matthias Corvinus S. 72.

7 Am 12. Mai 1476 war mit dem Hauptmann von Mähren, Ctibor von Zinnburg und Tobitschau, ein Waffenstillstand geschlossen worden; Druck: CHMEL, Materialien 2 S. 332 n. 264.

\section{[1476 Juli 13, Wiener Neustadt] ${ }^{1}$}

K.F. belehnt Bartholomäus Rietmair, Hofschreiber zu Hallstatt, mit dem durch den Tod Meinhard Kellerbergers ledig gewordenen Hof beim Burgstall am Gradisch bei Feldkirchen aus der Lehenschaft des Ftm. Kärnten.

Org. oder Kop. im bearbeiteten Bestand nicht überliefert. - Dep.: Erw. im Revers Bartholomäus Rietmairs von 1476 Juli 17 im HHStA Wien (Sign. AUR 1476 VII 17), Perg., grünes S des Hofmarschalls Jörg Fuchs von Fuchsberg ${ }^{2}$ in wachsf. Schüssel an Ps und S des Ritters Reichart $\mathrm{Kärgl}^{3}$ an Ps ab und verloren, mit Rta-Vermerk auf der Rückseite. ${ }^{4}$

Reg.: ChMel, Mon. Habsb. I/3 S. 719 n. 255.

\footnotetext{
Datierung und Ausstellungsort nach Reg. bei CHMEL.

Siehe zu ihm HeInIG, Friedrich III./1 S. 70-73, $299 \mathrm{f}$.

Reichart Kärgl starb in den Folgemonaten, s. n. 53, Anm 4.

4 Reg.: Chmel, Mon. Habsb. I/3 S. 719 n. 256.
}

\section{Juli 29, Wiener Neustadt}

K.F. entspricht der Bitte Andreas Rindscheits in der Hoffnung, des dadurch geförderten Gottesdienstes tailhefftig zu werden, und nimmt sich von sunder gnaden der von dessen verstorbenem Vater Pankraz ${ }^{1}$ gestifteten Ewigmesse am Margaretenaltar der Grazer (Dom-)Pfarrkirche St. Ägidius (Gilgen) als obrister stiffter an, bestätigt als Herr und Landesfürst die Stiftung wissentleich mit dem brief, was wir daran verwilligen, bestetten und nemen sullen und mugen, und nimmt sie samt dem Kaplan, den zugehörigen Leuten, Holden, Gütern und dem anderen Zubehör in seinen besonderen Schutz. Er verfügt, dass die stifft, gab und ordnung in allen stucken und puncten, als ob sie wortwörtlich inseriert wären, in Kraft bleiben sollen und einzuhalten sind und dass niemand dagegen handeln oder den Kaplan, die Leute und die Güter beschweren darf. Sollten ihnen gegenüber Ansprüche geltend gemacht oder Klage erhoben werden, sind diese vor ihn zu bringen. K.F. gebietet allen Hauptleuten, Gff., Freiherren etc., Verwesern, Landschreibern, Vizedomen, Pflegern, Bggff., Bürgermeistern, Richtern, Räten, Bürgern, Gemeinden und allen anderen Amtleuten und Untertanen bei seiner schweren Ungnade und einer Pön von 50 Mark Gold, zahlbar an die ksl. Kammer, die Beachtung dieses Privilegs. Am montag nach sannt Jacobs tag im snit des heiligen zwelfbotten. 
KVr: fehlt.

Org. im HHStA Wien (Sign. AUR 1476 VII 29), Perg., wachsf. S 24 mit wachsf. S 16 vorders. eingedr. an purpurf. Ss.

Reg.: CHMEL n. 7063.

1 Zu Pankraz Rindscheit, ksl. Kämmerer und Rat sowie Landschreiber in der Steiermark, s. HenNIG, Friedrich III/1 S. $191 \mathrm{f}$.

\section{August 1, Wiener Neustadt}

K.F. verlängert seinem Truchsess Kaspar von Rogendorf, Pfleger von Weitenegg, ${ }^{1}$ und dessen Erben wissenntlich mit dem brief den Bestand des Ungelds ${ }^{2}$ zu Waidhofen a.d. Ybbs und zu Ybbs samt Zubehör, das er ihm zuvor in bestanndweis mundlich für zwei Jahre ab sannd Kathrein tag 1474 (November 25) gegen Zahlung von jährlich 1.200 Pfd. Pf. überlassen hat, erneut für zwei Jahre bis zum sannd Kathrein tag 1478 (November 25) gegen Entrichtung derselben Summe pro Jahr. Er verpflichtet sie, das Ungeld treulich und aufrichtigklich innezuhaben und zu verwalten, das Bestandgeld wie gewöhnlich zu den Quatembern zu entrichten, die zugehörigen Bürger und Leute nicht ungebührlich gegen altes Herkommen zu belasten oder dies zu gestatten und das Ungeld nach Ablauf der zwei Jahre abzutreten. An phintztag sand Peters tag ad vincula.

KVr: C.d.i.p. - KVv: Rta. - Rückseite der Wachsschüssel: Vermerk nicht lesbar.

Org. im HHStA Wien (Sign. AUR 1476 VIII 1), Perg., rotes S 21 in wachsf. Schüssel mit wachsf. S 16 rücks. eingedr. an Ps.

Reg.: LichNowsky(-BIRK) 7 n. 1965; ChMEL, Mon. Habsb. I/3 S. 647 n. 45.

Lit:: BeRGMANN, Rogendorf S. 525 [S. 9]; ZAлIC, Kaspar von Rogendorf S. 14; zur Person des Rogendorfers s. auch NeIDHART, Geschichte Pöggstalls S. 41-58; HeINIG, Friedrich III./1 S. 195f., zur Familie s. ZАлC, Rog[g]endorf S. 1207-1214.

1 Zur Verpfändung von Weitenegg an die Rogendorfer im Jahr 1470 s. Regg.F.III. H. 27 n. 35, zur Abtretung Weiteneggs im Jahr 1478 s. unten n. 205.

2 Siehe dazu n. 13. 
K.F. überlässt Wilhelm Lesch das Amt Gmunden bestandweise für ein Jahr bis zum St. Michaelstag 1477 (September 29) gegen Zahlung von 3.000 fl. rh.

Org. oder Kop. im bearbeiteten Bestand nicht überliefert. - Dep.: Erw. im Revers Wilhelm Leschs von 1476 August 20 im HHStA Wien (Sign. AUR 1476 VIII 20), ${ }^{1}$ Perg., 2 grüne SS des Ausst. und des Hofmarschalls Jörg Fuchs von Fuchsberg ${ }^{2}$ in wachsf. Schüsseln an Ps, mit Rta-Vermerk auf der Rückseite. ${ }^{3}$

Lit.: Zur Verpachtung des Salzamts Gmunden s. KRACKowizer, Gmunden 2 S. 294f.; HuFNAGL, Maut Zu Gmunden S. 146f.

Laut Revers war Wilhelm Lesch - wie später auch die Stadt Gmunden (s. n. 114) - verpflichtet, aus den Erträgen des Salzamts Gmunden die Saline Hallstatt zu unterhalten und das Gotteszeilensalz $z^{4}$ an etliche Kirchen und Klöster zu entrichten. Hinsichtlich der Abtretung des Amts enthält der Revers vom üblichen Formular abweichende Bestimmungen: Lesch soll dem Kaiser den Schätzwert für den übernommenen Vorrat an Salz, Holz und Material (Blech, Seile, Unschlitt etc.) bezahlen, während ihm der Wert für bei der Abtretung hinterlassenes Salz, Holz etc. entgolten oder vom Bestandgeld abgezogen wird; zudem verspricht er, innerhalb eines halben Jahres für eine Bürgschaft für die $3.000 \mathrm{fl}$. rh. zu sorgen.

Siehe nn. 32, 114.

1 Unter dieser Sign. gemeinsam verwahrt mit n. 33 (Ungeld Gmunden) und dem Pflegrevers Wilhelm Leschs für Schloss und Landgericht Wildenstein von 1476 Oktober 17, s. dazu CHMEL n. 7075 und DERS., Mon. Habsb. I/3 S. 696 n. 187.

2 Siehe zu ihm HeInIG, Friedrich III./1 S. 70-73, $299 \mathrm{f}$.

3 Druck: Chмет, Mon. Habsb. I/2 S. 252-254 n. 81; Reg.: ChмеL n. 7065.

4 Bezeichnung für als Almosen geltende Salzschenkungen an Kirchen und Klöster, die auf eine oder mehrere „Zeilen“ Fuder lauteten (eine Zeile $=30$ Fuder), wovon sich die Bezeichnung ableitete, s. HuFNAGL, Maut zu Gmunden S. 212.

[Vor 1476 August 20, -]

K.F. überlässt Wilhelm Lesch das Ungeld zu Gmunden bestandweise für ein Jahr ab St. Michaelstag 1476 (September 29) gegen Zahlung von 230 Pfd. Pf.

Org. oder Kop. im bearbeiteten Bestand nicht überliefert. - Dep.: Erw. im Revers Wilhelm Leschs von 1476 August 20 im HHStA Wien (Sign. AUR 1476 VIII 20), ${ }^{1}$ Perg., 2 grüne SS 
des Ausst. und des Hofmarschalls Jörg Fuchs von Fuchsberg ${ }^{2}$ in wachsf. Schüsseln an Ps, mit $R t a$-Vermerk auf der Rückseite. ${ }^{3}$

Lit.: WALTER, Ungeld S. 65.

Siehe nn. 32, 114.

1 Unter dieser Sign. gemeinsam verwahrt mit n. 32 und dem Pflegrevers Wilhelm Leschs für Schloss Wildenstein (s. ebd., Anm. 1).

2 Siehe zu ihm HeInIG, Friedrich III./1 S. 70-73, 299f.

3 Reg.: ChMel n. 7066; DERS., Mon. Habsb. I/3 S. 699 n. 198.

\section{[Vor 1476 September 4, -]}

K.F. überträgt Ruprecht Steiner bis auf Widerruf Schloss Pittersberg und das Amt Lesach(-tal) samt Nutzen, Renten und allem Zubehör pfleg- und bestandweise gegen Zahlung von jährlich $100 \mathrm{Pfd}$. Pf. und der Verpflichtung, vom Rest der Erträge das Schloss zu behüten und zu erhalten.

Org. oder Kop. im bearbeiteten Bestand nicht überliefert. - Dep.: Erw. im Revers Ruprecht Steiners von 1476 September 4 im HHStA Wien (Sign. AUR 1476 IX 4), Perg., 2 grüne SS des Ausst. und Berthold Magers, Verweser (der Hauptmannschaft) von Kärnten, ${ }^{1}$ in wachsf. Schüsseln an Ps, mit Rta-Vermerk auf der Rückseite. ${ }^{2}$

Lit.: WiesSNER/VYORAL-TsCHAPKA, Burgen Kärnten 3 S. 35; s. auch KorAK, Burggrafen S. LXf.

1 1476-1493 Verweser der Hauptmannschaft von Kärnten, s. FRÄss-EHRFELD, Geschichte Kärntens 1 S. 575.

2 Reg.: ToMAscher, Regesten Kärnten 3 n. 482; MC 11 n. 459.

\section{September 30, Wiener Neustadt}

K.F. weist seinen Rat Christoph von Mörsberg, Bggf. zu Graz, an, von den Nutzen und Renten, die dieser für ihn einnimmt, seinem Sohn Hz. Maximilian von Österreich unverzüglich $100 \mathrm{fl}$. ung. zu geben, und verspricht, gegen Vorlage dieses Briefs die Summe bei Rechnungslegung in Abzug zu bringen. An montag nach sand Michels tag.

KVr: C.d.i.p. - Rta (unterer Blattrand rechts). $-\mathrm{KVv}$ : - . - Item der gulden hat die czeit goltten $x \beta x d n$ (wohl Empfängervermerk, rechte untere Blattseite). 
Org. im HHStA Wien (Sign. AUR 1476 IX 30), Pap., rotes S 21 rücks. aufgedr. (großteils abgefallen) und rotes S 16 vorders. aufgedr. - Kop.: Abschrift des 18. Jh. ebd. (Sign. Urkundenabschriften Österr. Urkunden, Kt. 45 sub dat.), Pap.

Druck: Chmel, Mon. Habsb. I/2 S. 255f. n. 83.

Reg.: CHMEL n. 7071.

Lit: $\mathrm{Zu}$ Christoph von Mörsberg s. Popelka, Geschichte Graz 1 S. 315; HeInIG, Friedrich III./1 S. 204-206 mit weiterführender Lit.

\section{Oktober 2, Wiener Neustadt}

K.F. überlässt Richter, Rat und allen Bürgern von Wels sowie ihren Nachkommen wissenntlich mit dem brief das Ungeld zu Wels und auf dem zugehörigen Land samt dem Ungeld in der Klaus und der dortigen Salz- und Straßenmaut, weiters Maut und Zoll zu Ebelsberg (Ebersperg) und Gmunden sowie das Gericht zu Wels bestandweise für zwei Jahre ab weichnachten nagstkunftigen 1477 (1476 Dezember 25) bis weichnachten 1479 (1478 Dezember 25) gegen Zahlung von jährlich 970 Pfd. Pf. in guter landläufiger Münze. Er verpflichtet sie, die Ämter trewlich innezuhaben und zu verwalten, das Bestandgeld wie gewöhnlich zu den Quatembern zu entrichten, die zu Ungeld und Gericht gehörigen Leute sowie jene, die mit ihren Waren und Gütern zu den Maut- und Zollstellen kommen, nicht ungebührlich gegen altes Herkommen zu belasten und die Ämter nach Ablauf der zwei Jahre abzutreten. An mittichen nach sand Michels tag.

KVr: fehlt.

Org. im HHStA Wien (Sign. AUR 1476 X 2), Perg., rotes S 21 in wachsf. Schüssel mit wachsf. $\mathrm{S} 16$ rücks. eingedr. an Ps.

Druck: ChMEL, Mon. Habsb. I/2 S. 256f. n. 84.

Reg.: ChMEL n. 7072; PrItZ, Geschichte 2 S. 721 n. 467.

Lit:: MeINDL, Geschichte Wels 1 S. 64; WALTER, Ungeld S. 67.

\section{Oktober 11, Wiener Neustadt}

K.F. bestätigt dem Guardian und Konvent des Minoritenklosters zu Wien die Schenkung Barbaras, Witwe des Wiener Bürgers Philipp Sailer, ${ }^{1}$ wissenlich mit dem briefe, was wir zurecht daran bestetten sullen oder mugen, die dem Kloster zum Lob Gottes und für ihr Seelenheil etliche im Burgfried von Wien gelegene Stücke und Weingärten sowie eine Kapelle laut dem darüber ausgestellten Brief übertragen hat, und verfügt, 
dass das Kloster die Güter und die Kapelle samt Zubehör gemäß den Schenkungsurkunden innehaben und nutzen sollen, als solher gab und lannds recht ist. Am freytag vor sannd Colmans tag.

KVr: C.d.i.i.c. - Rückseite der Wachsschüssel: Sailerin.

Org. im HHStA Wien (Sign. AUR 1476 X 11), Perg., rotes S 21 in wachsf. Schüssel mit wachsf. S 16 rücks. eingedr. an Ps. - Kop.: Abschrift des 18. Jh. ebd. (Sign. Urkundenabschriften Österr. Urkunden, Kt. 45 sub dat.), Pap.

Druck: CHMEL, Mon. Habsb. I/2 S. 257f. n. 85.

Reg.: CHMEL n. 7074.

1 Verstorben vor 1458 April 14, s. QGStW II/3 n. 3780 (Nennung Barbara Sailers als Witwe).

\section{November 13, Wiener Neustadt}

K.F. antwortet Heinrich von Liechtenstein zu Nikolsburg, ${ }^{1}$ Ulrich von Grafenegg, ${ }^{2}$ Jörg von Pottendorf, ${ }^{3}$ Heinrich von Puchheim, ${ }^{4}$ Veit von Ebersdorf, Friedrich von Pottendorf ${ }^{5}$ und Heinrich Dachauer auf deren Schreiben, dem zufolge sie von ihm ungenediclich behandelt und durch seine Leute geschädigt worden seien.

Der K. erklärt, das ihm, Land und Leuten von ihnen seit langer Zeit zugefügte unrecht nicht länger dulden zu wollen. ${ }^{6}$ Auch wenn sie sich damit rechtfertigen würden, dass er sie gegen altes Herkommen und ihre Freiheit belastet hätte, so finndet sich doch das im grunt der warhait nicht, da er immer fürstlich rechtlich und frumbklich regiert und weder sie noch andere unbillig beschwert habe. Sie hingegen haben das lanndsrecht $[\ldots]$ zestört und den von ihm eingesetzten lanndesman dazu gebracht, daz er das hat auf sagen mussen, da sie auch dem lanndsrechten nicht gehorsam sein wollten. Namentlich dem Grafenegger wirft er vor, die von ihm (K.F.) geschlagene gute aufrichtige bewerte Münze gegen dessen Ehre und Gelübde, mit denen er dem K. für seine (Münz-) Freiheit ${ }^{7}$ verpflichtet sei, gefelscht zu haben und noch zu fälschen. Da die unermesslich hohen Aufschläge das Land zugrunde richten, verstehe jeder, dass ihm, Land und Leuten Unrecht geschehe und sie nicht aus Recht, sondern aus Eigennutz handeln würden.

Weiters wirft ihnen der K. vor, ihn beraubt, die Feinde ins Land geführt, beherbergt und tail mit ihnen gehabt zu haben sowie die Seinen auf ihren Schlössern schetzn und peiken zu lassen. Mehrmals sei er wegen ihres Ungehorsams um Verhandlungen ${ }^{8}$ ersucht worden, die aber an ihnen gescheitert seien, und ab dem Zeitpunkt, als sie sich einer fremden Herrschaft unterstellten, ${ }^{9}$ hätten sie in ihrem Ungehorsam verachtleich verharrt, keine Vereinbarung gehalten und ihm als ihren rechten und naturlichem Lan- 
desfürsten keinerlei Gehorsam erwiesen. Wenn sie, wie sie schreiben, zur Versöhnung wirklich bereit gewesen wären, hiet es nicht not tan, sie seitens des ungarischen $\mathrm{Kg}$. als seine (K.F.) ihm feindlich gesinnten Landleute in den Breslauer Anstand ${ }^{10}$ einzubeziehen, zumal sie diesen nicht eingehalten hätten, sondern wiederum neue Aufschläge eingehoben und die alten Aufschläge nicht aufgehoben haben. Namentlich der Grafenegger habe über dessen berechtigten Forderungen hinaus mehr als $80.000 \mathrm{Pfd}$. Pf. eingenommen, ihm (K.F.) aber die Schlösser wider [...] brief und sigel und alle pillikait vorenthalten. ${ }^{11}$ Die Einhebung der Aufschläge sei widerrechtlich, da der K. keinem von ihnen nach Recht etwas schuldig sei.

Abschließend erklärt K.F., dass er ihnen für ihren Ungehorsam nie einen Grund gegeben, sondern sich vielmehr ihnen gegenüber gnädiger als seine Vorgänger erwiesen habe. Sollten sie von ihrem Ungehorsam und der Bedrückung des Landes ablassen und sich ihm gegenüber treu und gehorsam zeigen, verspricht er, keinerlei Ungnade walten zu lassen und ihr genediger herr und lannsfurst zu sein, woran jeder erkenne möge, dass es bei ihm nicht an pillikait fehle. An mitichen nach sannd Merten tag (nach Kop.). ${ }^{12}$

KVr: -. - KVv: Unsern lieben getrewn Hainreichen von Liechtenstain von Nicolspurg, Ulreichen von Gravenegkh, Jörgen von Potendorff, Hainreichen von Puchaim, Veiten von Eberstorff, Fridreichen von Potendorff und Hainreichen von Dachaw (Adresse, nach Kop.). ${ }^{13}$

Kop.: Zeitnahe Abschrift im HHStA Wien (Sign. AUR 1472-1476), Pap.-Libell, fol. 6v-7v. ${ }^{14}$

Druck: Lichnowsky(-Birk) 7, Beil. C S. 493f. n. 8; ChMel, Mon. Habsb. I/2 S. 91 f. n. 9 (ohne Adresse).

Reg.: LichNowsKy(-BIRK) 7 n. 1991.

Lit:: Bachmann, Reichsgeschichte 2 S. 590; VANCSA, Geschichte 2 S. 495; BuzzI, Pottendorf S. 125 .

Zeitgleich zum vorliegenden Schreiben intensivierten sich die Vorbereitungen zu einem Feldzug gegen den von $\mathrm{Kg}$. Matthias von Ungarn unterstïtzten österreichischen Adelsbund. K.F. sicherte sich die Unterstïtzung Kg. Wladislaws von Böhmen, mit dem er am 8. Dezember 1476 ein Bündnis schloss (s. n. 42). Am 2. März 1477 kam es schließlich zu einem Vergleich mit Ulrich von Grafenegg, den Pottendorfern und den Puchheimern (s. n. 55).

$1 \mathrm{Zu}$ Heinrich von Liechtenstein s. FALKE, Geschichte Liechtenstein 1 S. 486-490.

2 Zu Ulrich von Grafenegg (Grafeneck) s. HALLER-REIFFENSTEIN, Ulrich von Grafeneck; HeINIG, Friedrich III//1 S. 269-272.

3 Zu Jörg (Georg) von Pottendorf (Linie Ebenfurt) s. ausführlich BuzzI, Pottendorf S. 122-130; weiters ZERNATTO, Herrenstand S. 124f.; HeINIG, ebd. S. 255 f. 
$4 \mathrm{Zu}$ Heinrich von Puchheim s. HeInig, ebd. S. 258; weiters Zernatto, ebd. S. 137; TePPERBerg, Herren von Puchheim S. 85f.

5 Zu Friedrich von Pottendorf (Linie Kirchschlag) s. BuzzI, Pottendorf S. 141f.

$6 \mathrm{Zu}$ den Konflikten mit einigen österreichischen Adeligen, die während der Reise des $\mathrm{K}$. in das Binnenreich 1471 die Abgaben auf Hintersassen und Durchreisende deutlich erhöht hatten und bei $\mathrm{Kg}$. Matthias von Ungarn Schutz fanden (s. Anm. 10) s. VANCSA, Geschichte 2 S. 486-489; HaLleR-ReIFFENSTEIN, UIrich von Grafeneck S. 139f.; HoENSCH, Matthias Corvinus S. 130f.; s. auch Regg.F.III. H. 27 nn. 166-168 und 180 .

7 Ein Münzprivileg für Ulrich von Grafenegg ist bislang zwar nicht bekannt, jedoch ist belegt, dass er Münzen prägen ließ, s. HaLleR-REIFFENSTEIN, ebd. S. 130.

$8 \mathrm{Zu}$ den Verhandlungen und Vertragsentwürfen von 1475 s. CrmeL, Materialien 2 S. 326-332 nn. 260263; HALlER-REIFFENSTEn, ebd. S. 140.

$9 \mathrm{Kg}$. Matthias von Ungarn hatte am 13. Juni 1472 den österreichischen Adelsbund unter seinen Schutz genommen, s. ChMel, Mon. Habsb. I/2 S. 3-5 n. 1 (nach Abschrift im HHStA Wien, Sign. AUR 1472-1476, Pap., fol. 13rv); s. dazu auch dessen Schreiben an K.F. von 1472 September 6 bei LichNowsKy(-Birk) 7, Anh. S. 477-479 n. 3.

10 Gemäß dem Breslauer Frieden von 1474 Dezember 8 (s. n. 28, Anm. 6) behielt sich Kg. Matthias das Recht vor, die österreichischen Adeligen zu schützen; s. dazu NeHrnng, Matthias Corvinus S. 72.

$11 \mathrm{Zu}$ den Schlössern Ulrichs von Grafenegg s. n. 55.

$12 \mathrm{Zu}$ den Reaktionen auf dieses Schreiben s. VANCSA, Geschichte 2 S. 495f;; auch CHMEL, Materialien 2 S. 333f. n. 267.

13 Die Adresse ist in der Abschrift am Ende des Schreibens angegeben (fol. 7v).

14 Das Libell (19 fol.) ist eine zeitnahe Abschriftensammlung, die (mit dem vorliegenden Stück) insgesamt 14 Schriftstücke aus den Jahren 1472 und $1476 \mathrm{zu}$ den Auseinandersetzungen zwischen K.F. und $\mathrm{Kg}$. Matthias enthält. Bis auf eine Urkunde Kg. Matthias' von 1472 (fol. 17r-19r) liegen alle Stücke als Druck vor: zum Notenwechsel zwischen K.F. und Matthias Corvinus im Herbst 1476 s. CHMEL, Mon. Habsb. I/2 S. 79-90 nn. 5-8 (Stellungnahme des K. von 1476 September 3 [fol. 1r], Antwort Kg. Matthias' von Oktober 1476 [fol. 2r-3r], ksl. Antwort von 1476 November 10 [fol. 4r-6v], Antwort Kg. Matthias' von November (?) 1476 [fol. 8r]); s. auch LichnowsKy(-BIRK) 7, Beil. C nn. 6, 7. - Zu den weiteren Stücken aus dem Jahr 1476 s. ChMEL, ebd. S. 93 n. 10 (fol. 15v), S. 93-96 n. 11 (fol. 9r-10r), und DERS., Materialien 2 S. 333f. nn. 267a (fol. 15rv), 267b (fol. 15r). - Zu den Stücken aus dem Jahr 1472 s. Regg.F.II. H. 27 nn. 166, 168 und ChMeL, Mon. Habsb. I/2 S. 3-5 n. 1 (fol. 13rv), S. 16-18 n. 10 (fol. 14rv), S. 19f. n. 12 (fol. 12rv), S. 23-25 n. 15 (fol. 11rv).

\section{[Vor 1476 November 21, -]}

K.F. überträgt Leonhard Held die Pflege von Schloss Schönau und überlässt ihm dazu die Maut zu (Wiener-)Neudorf und Sollenau bestandweise für zwei Jahre ab St. Martinstag 1476 (November 11) gegen Zahlung von jährlich 150 Pfd. Pf.

Org. oder Kop. im bearbeiteten Bestand nicht überliefert. - Dep.: Erw. im Revers Leonhard Helds von 1476 November 21 im HHStA Wien (Sign. AUR 1476 XI 21), Perg., 3 grüne SS des Ausst., seines Bruders Jörg Held sowie Wolfgang Voits in wachsf. Schüsseln an Ps, mit Rta-Vermerk auf der Rückseite. ${ }^{1}$ - Ebenfalls erw. im Revers Leonhard Helds von 1479 Juni 11 
ebd. (Sign. AUR 1479 VI 11), ${ }^{2}$ Perg., Petschaft des Ausst. und 2 grüne SS Kaspar Harders und Wolfgang Lembachers in wachsf. Schüsseln an Ps, mit Rta-Vermerk auf der Rückseite. ${ }^{3}$

Laut Revers Leonhard Helds von 1479 Juni 11 überließ ihm K.F. nach Ablauf der zwei Jahre erneut Schönau sowie die Maut zu Neudorf und Sollenau pfleg- bzw. bestandweise, nun aber bis auf Widerruf gegen jährlich 170 Pfd. Pf., allerdings wird nur die ksl. Urkunde von 1476 ausdrücklich erwähnt, nicht aber eine Urkunde zur Verlängerung aus dem Jahr 1479.

1 Reg. (Revers von 1476): ChMel n. 7080; Ders., Mon. Habsb. I/3 S. 639 n. 10.

2 Unter dieser Sign. gemeinsam verwahrt mit den nn. 298, 302.

3 Reg. (Revers von 1479): ChMEL n. 7294; Ders., Mon. Habsb. I/3 S. 642 n. 24.

[Vor 1476 November 23, -]

K.F. überlässt Franz Schottl, Bürger von Neunkirchen, Gericht, Maut und Ungeld zu Neunkirchen bestandweise für drei Jahre ab St. Georgstag 1477 (April 24) ${ }^{1}$ gegen Zahlung von jährlich $400 \mathrm{Pfd}$. Pf.

Org. oder Kop. im bearbeiteten Bestand nicht überliefert. - Dep.: Erw. im Revers Franz Schottls von 1476 November 23 im HHStA Wien (Sign. AUR 1476 XI 23), Perg., 2 grüne SS der Wiener Neustädter Bürger Gotthard Vindorfer und Wolfgang Rorbeck, Ratsherr, in wachsf. Schüsseln (davon eine beschädigt) an Ps, mit Rta-Vermerk auf der Rückseite. ${ }^{2}$

Lit.: WALTER, Ungeld S. 143.

1 Zur Datierung des St. Georgstags auf April 24 s. Regg.F.III. H. 12 n. 6, Anm. 2

2 Reg.: ChMel n. 7081; Ders., Mon. Habsb. I/3 S. 648 n. 46.

\section{[Vor 1476 Dezember 2, -]}

K.F. überlässt Gilg Elssenberger bis auf Widerruf das Amt Greifenburg bestandweise gegen Zahlung von jährlich $220 \mathrm{Pfd}$. Pf.

Org. oder Kop. im bearbeiteten Bestand nicht überliefert. - Dep.: Erw. im Revers Gilg Elssenbergers von 1476 Dezember 2 im HHStA Wien (Sign. AUR sub dat. 1476 X 2), Perg., 2 grüne SS des Ritters und Hofmarschalls Jörg Fuchs von Fuchsberg ${ }^{1}$ und Gotthard Vindorfers in wachsf. Schüsseln an Ps, mit Rta-Vermerk auf der Rückseite. ${ }^{2}$ 
Lit.: Zum Söldnerführer Gilg von Greifenburg, ksl. Pfleger auf Greifenburg, s. Dopsch, Geschichte Salzburgs I/1 S. 550; zu Greifenburg WIESSNER/VYORAL-TsCHAPKA, Burgen Kärnten 3 S. $62-67$, bes. 66 .

1 Siehe zu ihm HennI, Friedrich III/ 1 S. 70-73, 299f.

2 Reg.: TOMASChEK, Regesten Kärnten 3 n. 483.

\section{Dezember 8, Wiener Neustadt}

K.F. und Kg. Wladislaw (II.) von Böhmen schließen aufgrund ihrer anngesiptenn unnde angebornnen liebe unnde freuntschafft zum Schutz ihrer Länder und Leute sowie zum Kampf gegen die Türken und gegen ihre widersetzlichen Untertanen und Landleute nach guter vorbetrachtung, zeittigenn rate unnde rechter wissen und wissenntlich incraft des briefs folgendes Bündnis ${ }^{1}$ gegen $\mathrm{Kg}$. Matthias von Ungarn und dessen Verbündeten, weil Matthias ihren Ländem und Leuten an all redlich ursach wider recht mutwilliglich unnde on unnderlos lange Zeit durch Brand und Verwüstung schwerste Schäden zugefügt und die Türken zum Schaden von Land, Leuten und der ganzen Christenheit ohne jeglichen Widerstand durch Ungarn in die Länder des K. ziehen lassen habe sowie die unngehorsamen und widerwertigenn Landleute in Österreich gegen den $\mathrm{K}$. unterstütze und nach crone, stanndt, eren, wirde, lanndt unnde lewten $\mathrm{Kg}$. Wladislaws trachte.

$(1)^{2} \mathrm{Kg}$. Wladislaw verpflichtet sich, auf mittvassten schiristkunftig (1477 März 16) oder, falls erforderlich, ehebaldigst aus dem Kgr. Böhmen mit ayner wagennpurgk, zewg und in annder wege zugericht unnde geschickt, als in veld unnd herczug gehort, und einer Streitmacht von mindestens 10.000 Mann zu Pferd und zu Fuß nach Österreich an den von K.F. gewünschten Ort persönlich gegen dessen ungehorsame Landleute zu ziehen, diese zu strafen und zum Gehorsam zu bringen. Er wird so lange in Österreich bleiben, wie es der $\mathrm{K}$. benötigt, und erst dann abziehen, wenn es ihrer beider Wille ist, sowie keinerlei bericht, fride, teyding, unnderrede, tractat noch anders mit den Ungehorsamen oder sonst jemandem in Österreich ohne Zustimmung des K. schließen und dessen gehorsame Landleute weder zur Huldigung zwingen noch schädigen.

(2) K.F. verpflichtet sich, auf dieselbe zeit ebenfalls persönlich mit $10.000 \mathrm{Mann}$ zu Pferd und zu Fuß dort, wo es erforderlich ist oder ihm am besten erscheint, gegen seine ungehorsamen Landleute ins Feld zu ziehen und für die Eroberung der Städte und Schlösser hawbtpuchssenn, stain unnde pulfer, terres unnde pleidenn und sonstiges Zubehör zur Verfügung zu stellen, welches sie, wenn sie gemeinsam im Feld sind, auch gemeinsam hüten und bewahren.

(3) Sollten sie nicht gemeinsam im Feld sein, wird vereinbart, dass K.F. den Kg. mit den Waffen samt Zubehör versorgt, während dieser sie zu bewahren und auf Erfordern des K. ohne Verlust zurückzugeben hat. 
(4) Eroberte Städte, Schlösser samt Besatzung und Güter in den ksl. Ländern stehen K.F. zu, Eroberungen in Böhmen und Mähren dem Kg. und der Krone von Böhmen.

(5) $\mathrm{Da} \mathrm{Kg}$. Matthias den mit ihnen geschlossenen Breslauer Anstand ${ }^{3}$ nicht eingehalten hat, will $\mathrm{Kg}$. Wladislaw auf Erfordern des $\mathrm{K}$. an dessen Seite persönlich gegen Matthias ziehen oder, sollte K.F. nicht persönlich ins Feld ziehen, seinen Hauptmann an der Seite des ksl. Hauptmanns mit der genannten Streitmacht nach Ungarn ins Feld schicken.

(6) Beide verpflichten sich, ohne Einverständnis des anderen weder das Feld zu verlassen noch mit Kg. Matthias oder dessen Verbündeten Frieden zu schließen oder darüber zu verhandeln, sondern einander Beistand zu leisten und den Krieg gemeinsam zu führen. Für den Fall, dass Matthias gegen einen von ihnen oder einen ihrer Verbündeten ziehen sollte, sei es in Ungarn oder in ihren Erblanden, verpflichten sie sich zu Hilfe und Zuzug, um ihn aufzuhalten.

(7) Dieses Bündnis soll unbeschadet sämtlicher zuvor mit Dritten geschlossener Bündnisse und getätigter Verschreibungen sein. An suntag unnser liebenn Frawenn tag concepcionis.

Der Corroboratio zufolge zwei gleichlautende Ausfertigungen, davon ein Org. im HHStA Wien (Sign. AUR 1476 XII 8), Perg., wachsf. S 24 mit wachsf. S 16 vorders. eingedr. an purpurf. Ss und rotes S Kg. Wladislaws von Böhmen in wachsf. Schüssel an rot-weißer Ss. - Kop.: Inseriert im Revers Beneschs von Weitenmühl, Bggf. von Karlstein und Münzmeister des Kgr. Böhmen, von 1476 Dezember 9, Wiener Neustadt, ebd. (Sign. AUR sub dat. 1476 XII 8), Perg., 2 SS des Ausst. (rot) und des Hofmarschalls Jörg Fuchs von Fuchsberg ${ }^{4}$ (grün) in wachsf. Schüsseln an Ps. -2 Abschriften (Org.) des 18. Jh. ebd. (Sign. Urkundenabschriften Österr. Urkunden, Kt. 45 sub dat.; Urkundenabschriften Collat. Urkunden, Kt. 73 sub dat.), Pap.; Abschrift (Revers) des 18. Jh. ebd. (Sign. Urkundenabschriften Österr. Urkunden, Kt. 45 sub dat.), Pap.

Abb.: Kaiserurkunden in Abb., Lfg. XI, Tf. 18.

Druck: KuRz, Oesterreich 2 S. 249-252, Beil. n. 40; ChMel, Mon. Habsb. I/1 S. 500-503 n. 187 (Org.) und S. 503f. n. 188 (Revers Beneschs von Weitenmühl).

Reg.: ChMel nn. 7083f.; LichNowsky(-BIRK) 7 nn. 1996-1998; Kaiserurkunden in Abb., Textbd. S. 500 .

Lit.: PalackÝ, Geschichte Böhmen 5/1 S. 147; BachmanN, Reichsgeschichte 2 S. 592; VANCSA, Geschichte 2 S. 495f.; NeHrIng, Matthias Corvinus S. 80f.; HoENsCH, Matthias Corvinus S. 154; s. auch Čornej/Bartlová, Velké dějiny 6 S. 432f.; Kalous, Matyáš Korvín S. 168; HoLtz, Länder der böhmischen Krone S. 33.

Im Revers bestätigt Benesch von Weitenmühl die Übernahme der ksl. Vertragsurkunde mit Majestätssiegel, um sie Kg. Wladislaw zu überbringen, und verspricht, dem Kaiser bis spätestens 
2. Februar 1477 eine gleichlautende Urkunde mit dem kgl. Siegel zukommen zu lassen. Das vorliegende Stück ist daher das in der böhmischen Kanzlei ausgestellte Vertragsexemplar. ${ }^{5}$

Im Vorfeld des Bündnisabschlusses hatte $\mathrm{Kg}$. Wladislaw laut einem ksl. Schreiben ${ }^{6}$ von 5. Dezember 1476 zugesagt, innerhalb von drei bis vier Wochen nach der Rückkehr Beneschs von Weitenmühl 3.000 bis 4.000 Mann nach Österreich zu schicken und bis 16. März 1477 persönlich mit einer Streitmacht zu erscheinen. K.F. hatte seinerseits dem Kg. versprochen, ihn innerhalb von acht bis zehn Tagen nach Grenzübertritt mit den Regalien des Kgr. Böhmen zu belehnen. Die Belehnung erfolgte am 10. Juni 1477 (s. n. 82).

Siehe nn. 38, 55, 82, 87 .

1 Siehe dazu das Bündnis zwischen K.F. und Kg. Wladislaw (II.) von 1474 März 11, das sich allerdings nicht, wie der vorliegende Vertrag, ausdrücklich gegen Dritte richtete: Regg.F.II. H. 26 n. 670 (ausführliches Reg.), auch H. 11 n. 436 und H. 27 n. 226 (beide nach unzulänglicher Überlieferung).

2 Die Gliederung in einzelne Vertragspunkte wurde von der Bearbeiterin vorgenommen.

3 Zum Breslauer Frieden von 1474 Dezember 8 s. n. 28, Anm. 6. - Ein Absage seitens Kg. Matthias an $\mathrm{Kg}$. Wladislaw war bereits im Oktober 1476 erfolgt, s. dazu den kurzen Bericht von 1476 Oktober 21 über den Prager Landtag bei Bachmann, Urkundliche Nachträge n. 392, und NeHRING, Matthias Corvinus S. 80 (zu Oktober 1).

4 Siehe zu ihm HeInIG, Friedrich III./1 S. 70-73, 299f.

5 Auch aus der Orthographie (Vokalismus) ist auf eine Ausstellung in der böhmischen Kanzlei zu schließen, s. Kaiserurkunden in Abb., Textbd. S. 500.

6 Druck: ChMeL, Mon. Habsb. I/1 S. 499f. n. 186; auch Ders., Materialien 2 S. 334 n. 268 (mit geringfügigen Abweichungen); Reg.: LICHNOwsKY(-BIRK) 7n. 1995.

\section{[Vor 1476 Dezember 12, -]}

K.F. überlässt Klaus Mores bis auf Widerruf die Maut zu Neumarkt (in Steiermark) bestandweise $a b$ Weihnachten (1476 Dezember 25) gegen Zahlung von jährlich 100 Pfd. Pf.

Org. oder Kop. im bearbeiteten Bestand nicht überliefert. - Dep.: Erw. im Revers des Klaus Mores von 1476 Dezember 12 im HHStA Wien (Sign. AUR 1476 XII 12), Perg., 2 grüne SS des Ausst. und des Wiener Neustädter Bürgers und Ratsherrn Simon Gruber in wachsf. Schüsseln an Ps, mit Rta-Vermerk auf der Rückseite. ${ }^{1}$

Lit.: Muchar, Geschichte 8 S. 90.

1 Reg.: Muchar, Urkunden-Regesten n. 242. 


\section{Dezember 14, Wiener Neustadt}

K.F. belehnt Christian von Rojach als Sohn des verstorbenen Niklas von Rojach und Lehensträger seiner Geschwister Clemens, Afra und Barbara wissenntlich mit dem brief, was wir im zu recht daran verleihen sullen oder mugen, mit einem von ihrem Vater ererbten Gut samt Zubehör zu Feicht (Fewcht) aus der Lehenschaft der Herrschaft Ortenburg, das sie und ihre Erben gemäß Lehens- und Landrecht innehaben und nutzen sollen. An sambstag nach sannd Lucien tag.

KVr: C.d.i.i.c. $-\mathrm{KVv}$ : Rta.

Org. im HHStA Wien (Sign. AUR sub dat. 1476 XII 13), Perg., rotes S 21 in wachsf. Schüssel mit wachsf. S 16 rücks. eingedr. an Ps.

\section{[Vor 1476 Dezember 17, -]}

K.F. verschreibt Hans Blay vertragsgemäß Schloss Grafenwarth, genannt Kostel, satzund pflegweise für $700 \mathrm{fl}$. ung. ${ }^{1}$

Org. oder Kop. im bearbeiteten Bestand nicht überliefert. - Dep.: Erw. im Revers Hans Blays von 1476 Dezember 17, Wiener Neustadt, im HHStA Wien (Sign. AUR 1476 XII 17), Perg., 2 grüne SS des Ausst. und des Ritters Wilhelm von Auersperg ${ }^{2}$ in wachsf. Schüsseln an Ps, mit Rta-Vermerk auf der Rückseite. ${ }^{3}$

Lit.: Zu Grafenwarth s. Kos, Burg und Stadt S. 219f.

1 Laut Revers erfolgte die Verpfändung gemäß einem das Schloss betreffenden Vertrag (teiding und verttrag) mit K.F.

2 Ksl. Kämmerer und Rat, s. zu ihm HeInIG, Friedrich III./1 S. 231f.

3 Reg.: TomascheK, Urkunden-Regesten Krain 2n. 42.

\section{Januar 4, Wiener Neustadt}

K.F. gestattet Michse Melz, Bürger von Laibach, von sundern gnaden wissenntlich mit dem brief die Errichtung einer Fleischbank in der Stadt Laibach auf der oberen Brücke, wo sich die anderen Fleischbänke befinden, und erlaubt ihm und dessen Erben, dort das Fleischhackerhandwerk persönlich oder durch Knechte mit allen den anderen Inhabern des Fleischhackerrechts zustehenden Rechten, Gewohnheiten und Freiheiten gegen einen jährlich in das Vizedomamt Laibach zu leistenden Zins von 2 Pfd. Pf. in guter landläufiger Münze auszuüben. Er gebietet Richter, Rat und allen Bürgern von Laibach, insbesondere den Inhabern des Fleischhackerrechts und allen Fleischhackerknechten, 
dieses Privileg zu beachten und ihm (K.F.) den jährlichen Zins von der Fleischbank vorzubehalten. An sambstag vor der heiligen dreyr Kunig tag (nach Kop.).

KVr: C.d.i.i.c. $-\mathrm{KVv}$ : Rta (nach Kop.).

[Org. im ARS Ljubljana, zuvor im HHStA Wien.] - Fotokop. vom Org. im HHStA Wien (Sign. AUR 1477 I 4).

Reg.: Regg.F.III. H. 29 n. 191 (nach Org., mit Druckbelegen); s. weiters OTOREPEC, Gradivo VI/36 (Druck); TomascheK, Urkunden-Regesten Krain 2 n. 43.

\section{[1477 Januar 4, Wiener Neustadt] ${ }^{1}$}

K.F. überträgt [seinem Silberkämmerer] ${ }^{2}$ Sigmund Skodel ${ }^{3}$ bis auf Widerruf die Pflege von Schloss Finkenstein und überlässt ihm die zugehörigen Nutzen, Renten und Zinse bestandweise gegen Zahlung von jährlich $100 \mathrm{Pfd}$. Pf.

KVr: C.d.i.p. (nach CHMEL).

Org. oder Kop. im bearbeiteten Bestand nicht überliefert. - Dep.: Erw. im Revers Sigmund Skodels von 1477 Januar 6 im HHStA Wien (Sign. AUR 1477 I 6), Perg., 2 grüne SS des Ausst. und des Hofmarschalls Jörg Fuchs von Fuchsberg ${ }^{4}$ in wachsf. Schüsseln an Ps, mit Rta-Vermerk auf der Rückseite. ${ }^{5}$

Reg.: ChMel, Mon. Habsb. I/3 S. 719 n. 258.

Lit.: Zu (Alt-)Finkenstein s. METNITZ, Kärntner Burgenkunde 2 S. 33f.; WIESSNER/VYORALTschAPKA, Burgen Kärnten 3 S. 135-137.

1 Datierung und Ausstellungsort nach Reg. bei CHMEL.

2 Nach ChMеL; im Revers wird das Amt nicht genannt.

3 Bei ChMEL „Schödl".

4 Siehe zu ihm HeInIG, Friedrich III./1 S. 70-73, 299 f.

5 Reg.: ToMAsCHEK, Regesten Kärnten 3 n. 484 (zu Januar 8); MC 11 n. 464 (zu Januar 13).

\section{[Vor 1477 Februar 3, -]}

K.F. überträgt Jörg Schrott bis auf Widerruf die Pflege von Schloss Klamm und überlässt ihm die zugehörigen Nutzen und Renten bestandweise gegen Zahlung von jährlich 160 Pfd. Pf. ${ }^{1}$ 
Org. oder Kop. im bearbeiteten Bestand nicht überliefert. - Dep.: Erw. im Revers Jörg Schrotts von 1477 Februar 3 im HHStA Wien (Sign. AUR 1477 II 3), Perg., 2 grüne SS des Ausst. und Dr. iur. utr. Martin Haidens in wachsf. Schüsseln an Ps, mit Rta-Vermerk auf der Rückseite.

Reg.: ChMel n. 7086.

Lit.: Zu Klamm s. KüHTREIBER u.a., Wehrbauten $1 \mathrm{~S} .124-136$, bes. 134.

1 Siehe dazu auch n. 302.

\section{Februar 21, Wien}

K.F. belehnt Niklas Dürrer auf Bitte Ostermans von Auersperg wissenntlich mit dem brieve, was wir im zu recht daran verleihen sullen oder mugen, mit fünf Huben samt Zubehör im Dorf Unterbärenthal (Nidersperntal) in der Treffener Pfarre aus der Lehenschaft des Ftm. Krain, die Auersperger diesem verkauft und mit einem offenen besiegelten Brief ${ }^{1}$ aufgesandt hat, und verfügt, dass Dürrer und dessen Erben die Stücke gemäß Lehens- und Landrecht innehaben und nutzen sollen. An freytag vor dem suntag Invocavit in der vassten.

KVr: fehlt. - Rückseite der Wachsschüssel: Durrer lehenbr(ief).

Org. im HHStA Wien (Sign. AUR sub dat. 1477 II 26), Perg., rotes S 21 in wachsf. Schüssel mit wachsf. S 16 rücks. eingedr. an Ps.

1 Aufsandbrief Ostermans von Auersperg von 1476 November 7 im HHStA Wien (Sign. AUR 1476 XI 7).

[1477 Februar 25, - $]^{1}$

K.F. schreibt Hz. Albrecht (IV.) von Bayern(-München), sich mit Berittenen und Fußvolk zu rüsten und auf Erfordern ihm zu helfen, die Reichszugehörigkeit der von Hz. Karl von Burgund hinterlassenen Fürstentümer und Länder zu sichern.

Org. oder Kop. im bearbeiteten Bestand nicht überliefert. - Dep.: Erw. im Schreiben der Hzz. Ludwig (IX.) von Bayern(-Landshut) und Albrecht (IV.) von Bayern(-München) an K.F. von 1477 März 30 im HHStA Wien (Sign. AUR 1477 III 30), Pap., zwei rote SS der Ausst. als Verschluss rücks. aufgedr. unter Papieroblate (aufgebrochen); ${ }^{2}$ davon Abschrift des 18 . Jh. ebd. (Sign. Urkundenabschriften Österr. Urkunden, Kt. 45 sub dat.), Pap.

Lit.: Rausch, Burgundische Heirat S. 168; BachmanN, Reichsgeschichte 2 S. 579; PrIEBATSCH, Correspondenz 2 S. 283 zu n. 271 (Anm. 3); HollweG, Georg Heßler S. 55. 
In dem Schreiben der bayerischen Herzöge an K.F. wird ausdrücklich nur ein ksl. Schreiben an Hz. Albrecht erwähnt, während Hz. Ludwig durch die ksl. Gesandten Bf. Georg von Metz und Dr. Georg Heßler ${ }^{3}$,ersucht“ wurde, vermutlich mündlich, den Zug Hz. Maximilians in die burgundischen Länder mit Berittenen zu unterstützen. Ein entsprechendes ksl. Schreiben an Hz. Ludwig ist aus der Vorlage daher nicht zwingend zu erschließen.

1 Datierung nach PrieBATSCH (nach Org. im HStA München).

2 Druck: Chmel, Mon. Habsb. I/1 S. 463f. n. 171; Reg.: ChMel n. 7106; Lichnowsky(-BIRK) 7 n. 2029.

3 Druck ihres Geleitbriefs von 1477 Februar 15 bei CHMEL, Mon. Habsb. I/1 S. 139 n. 36; s. auch PRIEBATSCH, Correspondenz 2 S. 283f. n. 271.

\section{[1477 Februar 25, Wien] ${ }^{1}$}

K.F. fordert von Bürgermeister und Rat der Stadt Köln, seinen Räten Bf. Georg von Metz und Georg Heßler, Lehrer der Rechte und sein Protonotar, für den Kauf von Kleinodien ${ }^{2} 1.000$ fl. rh. auf den Kölner Zoll zu leihen, die er (K.F.) bei der nächstfälligen Zollzahlung in Abzug bringen wird (nach Druck).

Org. oder Kop. im bearbeiteten Bestand nicht überliefert. - Dep.: Erw. in der Quittung Bf. Georgs von Metz und Dr. iur. utr. Georg Heßlers, ksl. Protonotar, über den Erhalt der Geldsumme von 1477 April 14, Mecheln, im HHStA Wien (Sign. FU n. 732), Perg., zwei SS Bf. Georgs von Metz (rot) und Georg Heßlers (grün in wachsf. Schüssel) an Ps; ${ }^{3}$ davon Abschrift des 18. Jh. ebd. (Sign. Urkundenabschriften Österr. Urkunden, Kt. 45 sub dat.), Pap.

Druck: ChMel, Mon. Habsb. I/3 S. 593f. n. 103.

Lit.: Rausch, Burgundische Heirat S. 168; HollweG, Georg Heßler S. 54f.; zu Georg Heßler S. auch HeINIG, Friedrich III./1 S. 709-720.

Vom gleichen Tag datiert ein Konzept einer an Köln, Frankfurt und Nürnberg auszufertigenden ksl. Beglaubigung für Bf. Georg von Metz und Georg Heßler, in der angekündigt wird, dass Hz. Maximilian in das Reich zu kommen plant, um mercklich sachen zu hanndeln, und die ksl. Gesandten den Auftrag haben, wegen des dafür benötigten Geldes mit ihnen zu verhandeln. Die ksl. Geldforderungen dienten der Finanzierung des Zugs Maximilians nach Burgund. ${ }^{4}$

1 Datierung und Ausstellungsort nach Druck bei CHMEL.

2 Laut Quittung waren die Kleinodien für Hzn. Maria von Burgund bestimmt.

3 Druck: ChmeL, Mon. Habsb. I/1 S. 465f. n. 173; Reg.: Lichnowsky(-BIRK) 7 n. 2032.

4 Druck des Konzepts: Chmel, Mon. Habsb. I/3 S. 595 n. 106; zu den Zahlungsbefehlen an Frankfurt und Nürnberg s. ebd. S. 593 nn. 101, 102 und Regg.F.III. H. 4 n. 774; zum Zug nach Burgund s. unten n. 80. 
K.F. stellt Sigmund Pirckheimer einen Ladungs- und Inhibitionsbrief in der Reichart Kärgl betreffenden Streitsache aus.

Org. oder Kop. im bearbeiteten Bestand nicht überliefert. - Dep.: Erw. in n. 53.

Möglicherweise erfolgte die Ausstellung zweier ksl. Urkunden, einer Ladung Sigmund Pirckheimers sowie einer Inhibition. Reichart Kärgl hatte in dem seit den 1460er-Jahren laufenden Prozess um Wolfersdorf 1474 zunächst an das herzogliche Hofgericht zu Landshut appelliert. ${ }^{1}$

1 Zur Streitsache s. EtTelt-SchöNwaLd, Ludwig der Reiche 2 S. 470f.

\section{Februar 26, Wien}

K.F. setzt Sigmund Pirckheimer von der Klage des ksl. Fiskalprokurators (Johann Keller) ${ }^{1}$ in Kenntnis, der zufolge er wegen schwerer Verfehlungen gegen $\mathrm{K}$. und Reich der Pön seiner kgl. „Reformatio“2 verfallen soll, nachdem Pirckheimer den ksl. Notar Nikolaus Lehner wegen Verkündigung der Appellation und ladung und inhibicion brieff ${ }^{3}$, den verstorbenen ksl. Diener und hoffgesind Reichart Kärgl ${ }^{4}$ betreffend, gefangen gesetzt und zu Urfehde und Eiden gezwungen hat, uns und dem heyligen Reich zu smahe und verachtung unnser kayserlicher oberkeit und gerichtzwangk. K.F. lädt ihn auf den 45. Tag nach Erhalt dieser Ladung bzw. den ersten darauf folgenden Gerichtstag peremptorisch zu rechtlicher Verantwortung vor sich oder seinen Bevollmächtigten. Er fordert ihn auf, rechtlich anzuerkennen, der genannten Pön verfallen zu sein und ihm (K.F.) und Nikolaus Lehner abtrag, kerung und wandel zu schulden, oder dagegen gute Gründe vorzubringen, und weist darauf hin, auch in dessen Abwesenheit auf Erfordern des Fiskals rechtlich zu verfahren, wie es sich gebührt. Am sechsundzwanczigsten tag des monats februarii (nach Kop.).

Kop.: Notariatsinstrument des öff. Notars Wolfgang Staud, Kler. des Bistums Eichstätt, von 1477 September 25, Ingolstadt, im HHStA Wien (Sign. AUR sub dat. 1477 II 26), Perg., Notarssignet. $^{5}$

Druck: ChMEL, Mon. Habsb. I/3 S. 596f. n. 108.

Lit.: KNOLLE, Reichsfiskalat S. 108; zur Streitsache zwischen Kärgl und Pirckheimer um Wolfersdorf s. ETTELT-SCHÖNWALD, Ludwig der Reiche 2 S. 470f.

1 Lic. (oder Dr.) iur. utr. Johann Keller, ksl. Rat und seit 1474 alleiniger Fiskal, s. zu ihm HeINIG, Friedrich III./1 S. 123-134. 
2 Zur sog. Frankfurter „Reformatio Friderici“ von 1442 August 14 s. Regg.F.III. H. 4 n. 41 mit Druckbelegen und Lit.

3 Siehe n. 52.

4 Reichart Kärgl zu Wolfersdorf, Rat Hz. Ludwigs IX. von Bayern(-Landshut), verstorben nach 1476 Juli 17, da Kärgl an diesem Tag noch einen Revers bezeugte (s. n. 29); s. zu ihm ETTELT-ScHÖNwALD, Ludwig der Reiche 1 S. 209, 213, 216 und 2 S. 470 f.

5 Das Notariatsinstrument wurde anlässlich der Übergabe der ksl. Ladung an Sigmund Pirckheimer vor den Zeugen Ulrich Mair von Gaimersheim, Kler. des Bistums Eichstätt, und Konrad Koppel aus dem Würzburger Bistum (wohl Kler.) ausgestellt.

\section{[Vor 1477 Februar 28, -]}

K.F. überlässt Wilhelm von Auersperg Ungeld und Landgericht zu (Groß-)Weikersdorf bestandweise für ein Jahr ab Sonntag Oculi 1477 (März 9) gegen Zahlung von 300 Pfd. Pf.

Org. oder Kop. im bearbeiteten Bestand nicht überliefert. - Dep.: Erw. im Revers Wilhelms von Auersperg von 1477 Februar 28, Wien, im HHStA Wien (Sign. AUR 1477 П 28), Pap., S des Ausst. rücks. aufgedr. unter Papieroblate, mit Rta-Vermerk auf der Rückseite.

Lit.: Zu Wilhelm von Auersperg, ksl. Kämmerer und Rat, s. HeINIG, Friedrich III./1 S. $231 \mathrm{f}$.

\section{März 2, Wien}

K.F. beurkundet den von Eb. Johann von Gran ${ }^{1}$ vermittelten Vergleich ${ }^{2}$ zwischen ihm (K.F.) einerseits und den Freiherren Ulrich ${ }^{3}$ und dessen Sohn Wolf von Grafenegg, Jörg und Friedrich von Pottendorf $f^{4}$ sowie Heinrich und Georg von Puchheim ${ }^{5}$ andererseits zur Beendigung ihrer Feindseligkeiten:

(1) ${ }^{6}$ Ulrich von Grafenegg und sein Sohn sollen K.F. alle ihre nachstehend genannten Städte, Schlösser, Märkte, Dörfer, Zehenten, Bergrechte, Gülten, Häuser und liegenden Güter übergeben und $\mathrm{Eb}$. Johann von Gran zuhanden des $\mathrm{K}$. überantworten, namentlich Trautmannsdorf (a.d. Leitha), ${ }^{7}$ Stadt und Schloss Litschau, Grafenegg (New Wolfenrewt $)^{8}$ samt Gülten, Leuten, Gütern und allem, was einst zu Schloss Winkl im Besitz Georg Schecks (von Wald) gehört hat, ${ }^{9}$ Wald mit Zubehör, ${ }^{10}$ Schauenstein,,${ }^{11}$ Götzendorf, die besatzung zu Rackenhof (Regkenhof) mit der Gült und dem zu Ebenthal gehörigen Zubehör, das Scheckenamt bei Steyr, die Bergrechte zu Währing und Klosterneuburg (Newnburg) sowie die einst im Besitz Georg Schecks gewesenen Bergrechte, die zwei Häuser in Wien und die Häuser in Wiener Neustadt und Steyr mit Zubehör sowie alle ihre anderen liegenden Güter im Ftm. Österreich samt dem Dorf Reisenberg, das Ulrich von Grafenegg von den Wallseern satzweise innehat. Die Grafenegger und ihre Erben haben künftig keinerlei Ansprüche oder Rechte auf die genannten Güter; des- 
gleichen haben K.F. und seine Erben keine Ansprüche auf deren Schlösser und Güter außerhalb des Ftm. Österreich.

(2) Gemeinsam mit den vorgenannten Schlössern und Gütern hat Ulrich von Grafenegg unverziiglich auch Stadt und Schloss Bruck a.d. Leitha samt den Pfandbriefen, ${ }^{12}$ Schloss Sarmingstein samt den Befestigungen, ${ }^{13}$ die er zusätzlich innehat, und alle anderen Tabore und Besatzungen im Ftm. Österreich in der genannten Weise dem K. abzutreten, was desgleichen für die Tabore zu Ebersdorf (Heberstorf) und gegenüber gilt, welche die Pottendorfer und Puchheimer innehaben.

(3) Eb. Johann soll die genannten Besitzungen so lange innehaben, bis er Ulrich von Grafenegg die vom K. zu zahlende Summe von $50.000 \mathrm{fl}$. ung. gegen Quittung entrichtet hat, wobei $32.000 \mathrm{fl}$. ung. am Tag der Abtretung und $18.000 \mathrm{fl}$. ung. zu sand Johanns tag zu sunnewennden schiristkunfftig (Juni 24) fällig sind. ${ }^{14}$

(4) Ulrich von Grafenegg soll den Pfandbrief für Reisenberg herausgeben, wofür ihm K.F. einen Willebrief Reinprechts von Wallsee ${ }^{15}$ ausstellen lassen wird.

(5) Über die gegenseitigen Forderungen des K., Jörgs (Georgs) von Pottendorf und Heinrichs von Puchheim bezüglich der Schlösser Waltersdorf und Pottschach und anderes soll durch je zwei Vertreter beider Parteien und Eb. Johann als obman eine gütliche Einigung erzielt und bis zum nagsten suntag nach Ostern nagstkomenden (April 13) ein Vertrag geschlossen werden. ${ }^{16}$

(6) Nach Entrichtung der $50.000 \mathrm{fl}$. ung. soll Bf. Johann die genannten Schlösser, Städte, Märkte, Dörfer und Güter dem K. abtreten.

(7) Die Fahrhabe des Grafeneggers in den Städten, Schlössern und besatzungen jenseits (nördlich) der Donau wird von ksl. Leuten sicher nach Gratzen gebracht, seine Fahrhabe diesseits (südlich) der Donau nach Landsee. Auch soll K.F. darauf achten, dass die Pottendorfer und Puchheimer ihre Besitztümer in den Taboren sicher zu ihren Schlössern bringen können.

(8) Es wird vereinbart, dass alle im Krieg erzwungenen Huldigungen von Märkten, Dörfern, Höfen, Leuten und Gütern aufgehoben, die Gefangenen freigelassen und alle gegenseitigen Absagen zurückgegeben werden sowie ungnad, veindtschafft und unwillen nichtig sind.

(9) Alle von K.F. oder Ulrich von Grafenegg ausgestellten Briefe und Verschreibungen über die Aufschläge und Schloss Sarmingstein oder aber Kraftloserklärungen, falls die haubtbrief nicht verfïgbar waren, sollen zusammen mit dem von K.F. dem Grafenegger ausgestellten munssbrief $E$ Eb. Johann überantwortet werden. ${ }^{17}$

(10) K.F. verspricht, sich beim Papst (Sixtus IV.) für die Lösung vom Kirchenbann ${ }^{18}$ und die Restituierung der sich im Bann befindlichen Priesterschaft einzusetzen. Alle Priester, die aus Gehorsam zum Papst ihre Kirchen verlassen haben, sollen wieder zurückkehren können. An suntag Reminiscere in der vassten (nach Kop.). 
Org. im bearbeiteten Bestand nicht überliefert, der Kop. zufolge (Perg.) mit anh. S. - Kop.: Abschrift des 15. Jh. im HHStA Wien (Sign. AUR 1477 III 2), Pap. ${ }^{19}$

Druck: ChMEL, Mon. Habsb. I/2 S. 267-269 n. 95; DERS., Materialien 2 S. 339-341 n. 274 (nach anderer kopialer Überlieferung) ${ }^{20}$.

Reg.: ChMel n. 7092; LichNowsKy(-BIRK) 7 nn. 2016, 2017.

Lit.: Keiblinger, Aggstein S. 58f.; MAYer, Abdankung S. 180; BACHMANn, Reichsgeschichte 2 S. 594f.; VANCSA, Geschichte 2 S. 496; NeHRING, Matthias Corvinus S. 83; DoPsCH, Geschichte Salzburgs S. 546; BuzzI, Pottendorf S. 125f.; HalleR-ReiffensteIn, Ulrich von Grafeneck S. 141f.; HeINIG, Friedrich III./1 S. 271f.

In der Abschrift wird im Anschluss an den Vertrag die Ausstellung folgender Urkunden vermerkt: eine gleichlautende Gegenurkunde der Grafenegger, Pottendorfer und Puchheimer, ${ }^{21}$ ein Pfandbrief für Eb. Johann von Gran über Steyr und Korneuburg für die geliehenen $37.000 \mathrm{fl}$. sowie dessen Revers (zu beiden Stïcken s. n. 93). ${ }^{22}$

Siehe nn. 38, 93.

1 Johann Beckenschlager (Beckensloer) (Eb. von Gran 1472-1487), s. zu ihm ZAISBERGER, Rohr, bes. S. 58-62; GATZ, Bischöfe 2 S. 36f.; HeING, Friedrich III./1 S. 449-452 mit weiterführender Lit.

2 K.F. hatte den Friedensschluss bereits in einem Schreiben von 1477 Februar 27 seinen Untertanen bekanntgegeben und die Einstellung der Feindseligkeiten befohlen, s. FucHs, Urkunden Göttweig 3 S. $101 \mathrm{f}$ n. 1870 .

$3 \mathrm{Zu}$ Ulrich von Grafenegg s. bes. HALLER-ReIFFENSTEIN, Ulrich von Grafeneck; weiters HeINIG, Friedrich III/1 S. 269-272.

4 Zu Jörg (in der Urkunde auch Georg) von Pottendorf (Linie Ebenfurt), Erbschenk in Österreich, dessen Schwester Katharina mit Ulrich von Graffenegg verheiratet war, s. BuzzI, Pottendorf S. 122-130; weiters ZernatTo, Herrenstand S. 124f.; HeInIG, ebd. S. 255f.; zu Friedrich (Linie Kirchschlag), dem letzten Pottendorfer, s. Buzzi, ebd. S. 141f.

$5 \mathrm{Zu}$ Heinrich von Puchheim, Erbtruchsess in Österreich und ksl. Rat, und dessen Sohn Georg (Jörg) s. ZernatTo, ebd. S. 137f.; HeInIG, ebd. S. 258; erw. bei TePPERBERg, Herren von Puchheim S. 85.

6 Die Gliederung in einzelne Vertragspunkte wurde von der Bearbeiterin vorgenommen.

7 Zur Übertragung von Trautmannsdorf s. Regg.F.III. H. 18 n. 149 (1459 September 12); auch BIRK, Urkunden-Auszïge (AÖG 10) n. 459; s. dazu HallER-REIFFENSTEIN, Ulrich von Grafeneck S. 125; zur Herrschaft s. Feigl, Trautmannsdorf, bes. S. 44.

8 Das nach den Grafeneggern benannte Schloss Grafenegg hatte im 15. Jh. aufgrund von Besitzerwechsel verschiedene Namen (Aspersdorf, Tahenstein, Wolfenreut); Ulrich erwarb das Schloss 1470 unter dem Namen „Neuwolfenreut" ${ }^{\prime \prime}$, s. HALLER-REIFFENSTEIN, ebd. S. 136.

9 Nach dem Aussterben der Herren von Winkl um 1425 kamen Schloss Winkl und der Markt (Groß-)Weikersdorf zunächst an die mit ihnen verschwägerten Scheck von Wald und danach an den Grafenegger, s. Marian, Adel im Tullnerfeld S. 114. 
10 Zur Verpfändung von Schloss Wald an Sigmund Schlick von 1477 April 27 s. Regg.F.III. H. 26 n. 685; der Revers Schlicks von 1477 April 28 überliefert im HHStA Wien (Sign. AUR 1477 IV 28). - Zur Übertragung an Sigmund Tellitzer im Jahr 1479 s. unten n. 288.

11 Zur Übertragung Schauensteins an Kaspar Ringkl s. dessen Pflegrevers von 1477 April 29 im HHStA Wien (Sign. AUR sub dat. 1477 III 17); Reg.: ChMEL n. 7120; DERS., Mon. Habsb. I/3 S. 639 f. n. 13.

12 Zur Verpfändung von Bruck a.d. Leitha von 1462 Oktober 4 s. Regg.F.II. H. 18 n. 288. - Zur Übertragung von Schloss Bruck an Leopold von Wulzendorf s. dessen Pflegrevers von 1477 Mai 9 im HHStA Wien (Sign. AUR sub dat. 1477 V 2); Reg.: CHMeL n. 7125; DERS., Mon. Habsb. I/3 S. 640 n. 15.

13 Zur Verpfändung Sarmingsteins s. Anm. 17.

1437.000 fl. ung. lieh sich K.F. von Eb. Johann von Gran, s. n. 93; ksl. Schuldbriefe für je $1.000 \mathrm{fl}$ ung. ergingen zudem an die beiden ksl. Räte Christoph Ungnad und Balthasar von Weißpriach, an Wilhelm von Auersperg und Gebhard Peuscher sowie für $500 \mathrm{fl}$. ung. an Ulrich von Graben, s. CHMEL, Conzeptensammlung 1 S. $80 \mathrm{n} .1$.

$15 \mathrm{Zu}$ Reinprecht von Wallsee, ksl. Rat, s. Doblinger, Walsee, bes. S. 248-259 [482-493]; HeInIG, Friedrich III./1 S. 251f.

$16 \mathrm{Zu}$ Pottschach s. MochTY-Weutin u.a., Wehrbauten 3 S. 163-180, bes. 172. - Zur weiteren Überlieferung der Sache im HHStA Wien s. unten Anm. 22.

17 Zur Verpfändung der Salz- und Weinaufschläge sowie Sarmingsteins von 1470 März 21 s. Regg.F.III. H. 27 n. 25. - Ein Münzprivileg für Ulrich von Grafenegg ist zwar nicht überliefert, jedoch ist belegt, dass er Münzen prägen ließ, s. HALLER-ReIFFenstern, Ulrich von Grafeneck S. 130; s. dazu auch die Erwähnung einer ksl. Verschreibung wegen der münss bei CнмеL, Materialien 2 S. 327 n. 261.

18 Papst Sixtus IV. bestätigte 1476 den Bannbrief des Kardinallegaten Marcus Barbo von 1474 Mai 27 über Ulrich von Grafenegg, Wolfgang von Schaunberg, Heinrich von Liechtenstein, Johann von Starhemberg, Zdenko von Sternberg, Jörg von Pottendorf u.a.; zum Bann von 1474 s. CHMEL, Materialien 2 S. 315-318 n. 257 (1474), zum Bann Sixtus' IV. ebd. S. 335f. n. 270 (1476), auch S. 319f. zu n. 257 (nach Kop.); s. in der Sache auch das Schreiben Heinrichs von Liechtenstein, Ulrichs von Grafenegg und Jörgs von Pottendorf an Bf. Ulrich von Passau von 1477 April 23 ebd. S. 341f. n. 275.

19 Die Abschrift des Vertrags ist Beilage eines ebenfalls abschriftlich überlieferten Schreibens Peter Altenhausers, des Stallmeisters Maximilians I., aus den Jahren 1508/09 an den Statthalter mit der Bitte, die Übersendung des Vertrags an das Kammergericht in Wiener Neustadt zu veranlassen, um seine Rechte auf das ihm von Maximilian übertragene Dorf Wagram (RI XIV 4,1 n. 18085), das aus diesem Vertrag herrühren soll, geltend zu machen. Aus dem Konzept des Antwortschreibens geht hervor, dass die Suche nach Belegen zu diesem Dorf ergebnislos blieb.

20 Druck nach einer nicht im bearbeiteten Bestand überlieferten Handschrift im HHStA Wien, wobei Schloss Wald bei den Besitzungen der Grafenegger nicht genannt wird.

21 Gegenurkunde von 1477 März 17, ausgestellt von Ulrich und Wolf von Grafenegg, Jörg und Friedrich von Pottendorf und Heinrich und Jörg von Puchheim in Trautmannsdorf, überliefert als Org. und Kop. (18. Jh.) im HHStA Wien (Sign. AUR 1477 III 17; Urkundenabschriften Österr. Urkunden, Kt. 45 sub. dat.); Druck: Kurz, Oesterreich 2 S. 253-256, Beil. n. 41; ChMEL, Mon. Habsb. I/2 S. 270-273 n. 96; Reg.: ChMel n. 7101; LichNowsKy(-BIRK) 7 n. 2023.

22 Siehe in der Sache auch folgende Stücke im HHStA Wien: Schriftstücke betreffend die gegenseitigen Forderungen des K., Jörgs von Pottendorf und Heinrichs von Puchheim (Sign. AUR sub dat. 1477 V 24); Druck: ChMEL, Mon. Habsb. I/2 S. 286-308 nn. 107-117; Reg.: LichNowsky(-BIRK) 7 n. 2052. - Urfehdebriefe Pankraz Tiemingers von 1477 März 3 (Sign. AUR 1477 III 3) und Wolfgang Ruckendorfers von 1477 April 27 (Sign. AUR 1477 IV 27); Druck: CHMEL, Mon. Habsb. I/2 S. 279f. n. 100 (Ruckendorfer); Reg.: ChMel nn. 7093, 7117; LichNowsKY(-Birk) 7 nn. 2018, 2042. 
K.F. bestätigt dem Nürnberger Bürger Niklas Groß d.Ä. und dessen Erben, ihnen 600 fl. ung. für Tuch, das dieser ihm zur Bezahlung seiner Söldner verkauft hat, zu schulden und das Geld bis sannd Michels tag schierestkomend (September 29) zu bezahlen. An freitag vor dem suntag Letare zu mittervasten.

KVr: C.d.i.i.c. - Rta (unterer Blattrand rechts). - KVv: Das gepurt graf Hawgen und herr Jobsten Hawser zuverraitten. Item herr Jobst $\mathrm{m}^{C}$ rh. gulden auch vom Grossen (oberer Blattrand).

Org. im HHStA Wien (Sign. AUR 1477 III 14), ${ }^{1}$ Pap., rotes S 21 rücks. aufgedr. und rotes S 16 vorders. aufgedr. unter Papieroblate.

Reg.: ChMel n. 7094; LichNowsky(-BIRK) 7 n. 2022.

1 Unter dieser Sign. gemeinsam verwahrt mit $\mathrm{n} .57$ sowie einem Schuldbrief des Obersten Hauptmanns Gf. Haug von Werdenberg für Niklas Groß von 1477 Dezember 18, worin er statt K.F. eine von ihm namens des K. übernommene Schuld von $80 \mathrm{fl}$. ung. für Tuch zur Bezahlung der ksl. Dienstleute sowie deren Begleichung durch den K. bis 1478 Juni 24 bestätigt.

\section{März 14, Wien}

K.F. bestätigt den Brüdern Matthias und Lukas Hörlin (Herly) und ihren Erben, ihnen $100 \mathrm{fl}$. ung. für Tuch, das sie ihm zur Bezahlung seiner Söldner verkauft haben, zu schulden und das Geld bis sannd Michels tag schierstkomend (September 29) zu bezahlen. An freitag vor dem suntag Letare zu mittervasten.

KVr: C.d.i.i.c. - Rta (unterer Blattrand rechts).

Org. im HHStA Wien (Sign. AUR 1477 III 14), ${ }^{1}$ Pap., rotes S 21 rücks. aufgedr. und rotes S 16 vorders. aufgedr. unter (abgelöster) Papieroblate.

Reg.: ChMel n. 7097; LichNowsKy(-BIRK) 7 n. 2021.

1 Unter dieser Sign. gemeinsam verwahrt mit $\mathrm{n}$. 56 sowie einem Schuldbrief Gf. Haugs von Werdenberg von 1477 Dezember 18 (s. ebd., Anm. 1).

K.F. bestätigt als Herr und Landesfürst der Dorothea, Ehefrau des Hans Moyker, von sundern gnaden wissenntlich mit dem brief die ihm vorgelegte Verschreibung und gemecht ihres Ehemanns, der ihr als Widerlage und Morgengabe seinen Hof zu Hunds- 
heim und einen dort gelegenen, Rainfal genannten Weingarten aus der Lehenschaft der Herrschaft Orth verschrieben hat, und verfügt, dass sie die Güter samt Zubehör gemäß der Verschreibung innehaben und nutzen soll, als solher verschreibung heiratgut widerlegung morgengab und lannds recht ist, jedoch unbeschadet seiner und seiner Erben Lehenschaft und Rechte. An freitag vor dem suntag Letare zu mittervasten.

KVr: fehlt. - Rückseite der Wachsschüssel: nur Moykerin lesbar.

Org. im HHStA Wien (Sign. AUR 1477 III 14), ${ }^{1}$ Perg., rotes S 21 in wachsf. Schüssel mit wachsf. S 16 rücks. eingedr. an Ps.

Reg.: ChMel n. 7095.

1 Unter dieser Sign. gemeinsam verwahrt mit n. 59.

\section{März 14, Wien}

K.F. bestätigt als Herr und Landesfürst dem Sebald Reichenauer von sundern gnaden wissenntlich mit dem brief den satzbrief, den Matthias Grasser, Pfleger am Kahlenberg, diesem über die Verpfändung etlicher Güter und Gülten zu Oberfellabrunn aus der Lehenschaft des Ftm. Österreich ausgestellt hat, und verfügt, dass Reichenauer die Güter und Gülten gemäß dem Pfandbrief innehaben und nutzen soll, als solhs satz verschreibung und lannds recht ist, jedoch unbeschadet seiner und seiner Erben Lehenschaft und Rechte. An freitag vor dem suntag Letare zu mittervasten.

KVr: fehlt. - Rückseite der Wachsschüssel: nur Reihenawer lesbar.

Org. im HHStA Wien (Sign. AUR 1477 III 14), ${ }^{1}$ Perg., rotes S 21 in wachsf. Schüssel mit wachsf. S 16 rücks. eingedr. an Ps.

Reg.: ChMeL n. 7096; DeRs., Mon. Habsb. I/3 S. 658 n. 91.

1 Unter dieser Sign. gemeinsam verwahrt mit n. 58.

[Vor 1477 März 16, -]

K.F. überlässt Christoph Stecher bis auf Widerruf das Ungeld zu (Neu-)Lengbach bestandweise gegen Zahlung von jährlich $250 \mathrm{Pfd}$. Pf. 
Org. oder Kop. im bearbeiteten Bestand nicht überliefert. - Dep.: Erw. im Revers Christoph Stechers von 1477 März 16 im HHStA Wien (Sign. AUR 1477 III 16), Pap., 2 grüne SS des Ausst. und Wolfgang Ludmansdorfers rücks. aufgedr. unter (abgelöster) Papieroblate, mit Rta-Vermerk auf der Papieroblate. ${ }^{1}$

Lit.: WALTER, Ungeld S. 117.

1 Reg.: ChMel n. 7098; Ders., Mon. Habsb. I/3 S. 648 n. 47.

\section{[1477 März 17, Wien] ${ }^{1}$}

K.F. überlässt Bürgermeister, Rat, Genannten und der Gemein der Stadt Wien auf ewig den halben Teil der Brückenmaut auf den drei Donaubrücken zu Wien bestandweise gegen Zahlung von jährlich $1.000 \mathrm{Pfd}$. Pf. ${ }^{2}$

[Org. im WStLA.] $]^{3}$ - Erw. im Revers von Bürgermeister, Rat, Genannten und der Gemein von Wien von 1477 März 17, Wien, im HHStA Wien (Sign. AUR 1477 III 17), ${ }^{4}$ Perg., rotes S der Stadt Wien in wachsf. Schüssel an Ps, mit Rta-Vermerk auf der Rückseite. ${ }^{5}$

Reg.: QGStW II/3 n. 4637.

Lit.: Zur Wiener Donaubrückenmaut s. BRUNNER, Finanzen S. 117f.

1 Datierung und Ausstellungsort nach Org.: Geben zu Wenn am montag nach sand Gregorien tag.

2 Zum Dank für ihren Widerstand gegen Kg. Matthias von Ungarn erließ K.F. der Stadt Wien am 3. Februar 1479 die Zahlung des Bestandgelds für die Brückenmaut sowie die Stadtsteuer für das Jahr 1479; Org. im WStLA (Sign. HAUrk 4720), s. monasterium.net (AT-WStLA, Bestand Hauptarchiv-Urkunden, sub dat.); Druck: CHMEL, Mon. Habsb. I/3 S. 691 n. 177; Reg.: QGStW II/3 n. 4720.

3 WStLA (Sign. HAUrk 4637 [= Privil. Nr. 49]), s. monasterium.net (AT-WStLA, Bestand HauptarchivUrkunden, sub dat.).

4 Unter dieser Sign. gemeinsam verwahrt mit den nn. 67, 68, 71.

5 Druck: ChMeL, Mon. Habsb. I/2 S. 274f. n. 97; Reg.: ChMEL n. 7099.

\section{März 17, Wien}

K.F. belehnt Matthias Grasser, seinen Pfleger am Kahlenberg, auf Bitte Sebald Reichenauers wissenntlich mit dem brief, was wir im zu recht daran verleihen sullen oder mugen, mit der Feste Hornsburg (Horensperg) samt dem Dorf Pyrha (Pirchach) und allem Zubehör aus der Lehenschaft des Ftm. Österreich, die Reichenauer diesem verkauft und mit einem offenen besiegelten Brief aufgesandt hat, und verfügt, dass Grasser und 
dessen Erben die Lehen innehaben und nutzen und mit der Feste ihm als Lehensherrn treu zu Diensten sein sollen gemäß Lehens- und Landrecht. An montag nach dem suntag Letare.

KVr: fehlt. - KVv: Rta. - Rückseite der Wachsschüssel: Grasser lehenbr(ief).

Org. im HHStA Wien (Sign. AUR 1477 III 17), Perg., rotes S 21 in wachsf. Schüssel mit wachsf. S 16 rücks. eingedr. an Ps.

Reg.: ChMEL n. 7100; DERS., Mon. Habsb. I/3 S. 658 n. 92; QGStW I/5 n. 5038.

Lit.: ReICHHALTER/KüHTREIBER, Burgen Weinviertel S. 237.

\section{[Vor 1477 März 21, -]}

K.F. überlässt dem Richter und Rat der Stadt Klosterneuburg Gericht und Maut zu Klosterneuburg bestandweise für zwei Jahre ab Donnerstag vor dem Sonntag Judica 1477 (März 20) gegen Zahlung von jährlich 150 Pfd. Pf.

Org. oder Kop. im bearbeiteten Bestand nicht überliefert. - Dep.: Erw. im Revers von Richter und Rat von Klosterneuburg von 1477 März 21 im HHStA Wien (Sign. AUR 1477 III 21), Pap., grünes S der Stadt Klosterneuburg rücks. aufgedr. ${ }^{1}$

1 Reg.: ChMel n. 7103.

\section{[Vor 1477 März 28, -]}

K.F. gebietet der Stadt Neuenburg (am Rhein) unter Androhung schwerer Strafen, den Bf. von Basel bei der Nutzung der zu den hochstiftlichen Dörfern Schliengen und Steinenstadt gehörigen Güter nicht zu behindern sowie den Ihren zu verbieten, im Rhein zwischen den Dörfern nach Gold zu suchen.

Org. oder Kop. im bearbeiteten Bestand nicht überliefert. - Dep.: Erw. in n. 66.

\section{[1477 März 28, Wien] ${ }^{1}$}

K.F. hebt den in n. 64 ausgesprochenen Befehl an die Stadt Neuenburg (am Rhein) auf und bestätigt die althergebrachten Rechte der Stadt an den Rheinauen zwischen Bellingen und Grißheim gemäß ihren Freiheiten und Privilegien. Er gebietet allen Reichs- 
untertanen, insbesondere aber den Bff. von Basel, bei seiner und des Reichs schweren Ungnade die Beachtung dieses Privilegs (nach Druck).

[Org. im StadtA Neuenburg am Rhein.] - Erw. in n. 66.

Druck: MERK, Oberrheinische Stadtrechte II/3 S. 72-74 n. 49 (nach Org.).

Reg.: SieverT, Archivalien Müllheim S. m19 n. 88 (zu März 26); HugGle, Neuenburg 2 S. 170 (zu März 26).

1 Datierung und Ausstellungsort nach Druck bei MERK.

\section{März 28, Wien}

K.F. gebietet aus ksl. Machtvollkommenheit Bf. Johann von Basel ${ }^{1}$, von seinem auf dessen Vorbringen ergangenen Befehl ${ }^{2}$ an die Stadt Neuenburg (am Rhein) keinen Gebrauch zu machen, dem zufolge er (K.F.) der Stadt untersagt hat, den Bf. an der Nutzung der Hölzer, Felder, Weiden, zewnen, werden, Äcker und matten, die zu den Dörfern Schliengen und Steinenstadt gehören, welche der Bf. als Teil der Regalien von $\mathrm{K}$. und Reich zu Lehen trägt, zu behindern sowie im Rhein zwischen den genannten Dörfern Gold zu suchen. Der Befehl wurde auf Bitte der Stadt aufgehoben, ${ }^{3}$ da die Neuenburger ihm durch Vorlage ihrer Freiheiten und Privilegien glaubhaft darlegen konnten, dass sie diese Güter schon lange, bevor die beiden Dörfer an das Hochstift Basel gekommen seien, durch seine Vorfahren im Reich zum Schutz vor dem Rhein ${ }^{4}$ besessen hätten und darin auch von ihm (K.F.) in seiner Königszeit bestätigt worden seien. Er gebietet Bf. Johann daher, die Neuenburger in der Nutzung der genannten Güter gemäß ihren Freiheiten und ihrem alten Besitzrecht nicht zu behindern, sollte dieser aber darauf Rechte geltend machen, sei er (K.F.) gegenüber den Neuenburgern zu recht mechtig und willig, ihm diese auf dessen gepurlich Erfordern furderlich einzuräumen. Am achtundczwanczigisten tag des monats marcii (nach Kop.).

Org. im bearbeiteten Bestand nicht überliefert, der Kop. zufolge mit rücks. aufgedr. S. - Kop.: Inseriert im Notariatsinstrument des öff. Notars Johannes Lingk von München, Kler. des Bistums Freising, über die Appellation Bf. Johanns von Basel an K.F. von 1477 Mai 17 im HHStA Wien (Sign. AUR sub dat. 1477 III 28), Perg., Notarssignet. ${ }^{5}$

1 Johann von Venningen (Bf. 1458-1478), s. zu ihm Helvetia Sacra I/1 S. 197f.; GATz, Bischöfe 2 S. 723; HIRsCH, Hof, bes. S. $36-41$.

2 Siehe n. 64.

3 Siehe n. 65. 
4 Die Stadt erlitt immer wieder schwere Hochwasserschäden, s. MERK, Oberrheinische Stadtrechte II/3 S. XVII; SPECK/TrefFeISEN, Neuenburg am Rhein S. 33; ausführlich Huggle, Neuenburg 2 S. 211-214.

5 Das Notariatsinstrument wurde im Schloss Pruntrut, Diöz. Besançon, vor den Zeugen Antonius von Regisheim, Domherr zu Basel, und Antonius Retzinger, Prokurator des Erzpriesterhofs zu Basel, ausgestellt.

[Vor 1477 März 31, -]

K.F. überlässt Simon Gruber, Bürger von Wiener Neustadt, das Ungeld zu Wiener Neustadt bestandweise für ein Jahr ab Palmsamstag 1477 (März 29) gegen Zahlung von 950 Pfd. Pf.

Org. oder Kop. im bearbeiteten Bestand nicht überliefert. - Dep.: Erw. im Revers Simon Grubers von 1477 März 31 im HHStA Wien (Sign. AUR sub dat. 1477 III 17), ${ }^{1}$ Perg., 2 grüne SS des Ausst. und Gotthard Vindorfers in wachsf. Schüsseln an Ps. ${ }^{2}$

Lit.: WALTER, Ungeld S. 141; zum Wiener Neustädter Ratsherrn Simon Gruber S. MAYER, Wiener Neustadt I/2 S. 149 und 509.

1 Unter dieser Sign. gemeinsam verwahrt mit den nn. 61, 68, 71.

2 Reg.: ChMel n. 7127 (zu Mai 31); Ders., Mon. Habsb. I/3 S. 648 n. 48.

\section{[Vor 1477 April 8, -]}

K.F. überlässt Lic. iur. utr. Johannes (Hans) Rehwein, Pfarrer von Gars, seinen Teich bei Stockern samt den zugehörigen kleinen Teichen und dem Fischbesatz, wie er von dem Stockhorner ${ }^{1}$ an ihn (K.F.) gekommen ist, bestandweise für fünf Jahre ab Datum dieses Briefs gegen Zahlung von jährlich $10 \mathrm{fl}$. ung.

Org. oder Kop. im bearbeiteten Bestand nicht überliefert. - Dep.: Erw. im Revers Johannes Rehweins von 1477 April 8 im HHStA Wien (Sign. AUR sub dat. 1477 III 17), ${ }^{2}$ Perg., 2 SS des Ausst. (rot) und seines Bruders, des Wiener Bürgers Jakob Rehwein (grün), ${ }^{3}$ in wachsf. Schüsseln an Ps. ${ }^{4}$

Lit.: BRUNNER, Eggenburg 1 S. 216; zu Johannes Rehwein (Rechwein), Pfarrer von Gars (am Kamp) und Eggenburg, ksl. Rat und Leiter der erbländischen Kanzlei (1470-1481), s. bes. HEINIG, Friedrich III./1 S. 592-595; LUGER, Humanismus S. 97-110.

1 Das im Besitz der Stockhorner (Stok[h]arner, Stockerner) befindliche Schloss Stockern wurde 1474 auf ksl. Befehl von Stephan von Eitzing gebrochen, nachdem sich die Stockhorner der Adelsopposition an- 
geschlossen hatten; s. BRunNER, Eggenburg 1 S. 215; DAIM/KüHTreIBER, Burgen Waldviertel-Wachau S. 329; zu den Stockhorner auch TurBa, Ritterstand S. 124-128.

2 Unter dieser Sign. gemeinsam verwahrt mit den nn. 61, 67, 71.

3 Siehe zu ihm Perger, Wiener Ratsbürger S. 233 (Jakob d.J. Rechwein).

4 Reg.: ChMel n. 7110; Ders., Mon. Habsb. I/3 S. 648 n. 49.

\section{[1477 April 20, - $]^{1}$}

K.F. schließt mit Bohuslav von Schwanberg, dem Obersten Hauptmann und Obersthofmeister des Kgr. Böhmen, sowie Wok von Rosenberg, Jan von Schwanberg zu Strakonitz, Diepold von Lobkowitz, Peter Kapler von Winterberg und Peter Stupenský einen Waffenstillstand bis zum Sonnenuntergang des kommenden St. Michaelstag (September 29). Beide Parteien vereinbaren, in dieser Zeit Frieden zu halten und einander weder anzugreifen noch zu schädigen. Der Frieden soll in drei Tagen beginnen und Bohuslav von Schwanberg durch Reinprecht von Wallsee ${ }^{2}$ verkündet werden. In der Zeit des Waffenstillstands setzen Gf. Haug von Werdenberg ${ }^{3}$ und der Wallseer einen Tag zur gütlichen Einigung fest, zu dem jede Partei die andere mit notwendigem Geleit versehen soll. Der dort gefällte Spruch Haugs von Werdenberg und Reinprechts von Wallsee ist von beiden Parteien einzuhalten. Der Waffenstillstand kann unter Einhaltung einer achtwöchigen Kündigungsfrist jederzeit von beiden Seiten beendet werden. ${ }^{4}$

Org. oder Kop. im bearbeiteten Bestand nicht überliefert. - Dep.: Erw. in dem von Bohuslav von Schwanberg und Wok von Rosenberg besiegelten Vertragsexemplar der böhmischen Landherren von 1477 April 20 im HHStA Wien (Sign. AUR 1477 IV 20), Pap., 2 rote SS Bohuslavs von Schwanberg und Woks von Rosenberg vorders. aufgedr. unter Papieroblate; 5 davon Abschrift des 18. Jh. ebd. (Sign. Urkundenabschriften Österr. Urkunden, Kt. 46 sub dat.), Pap.

Lit.: PALACKX́, Geschichte Böhmen 5/1 S. 157; CoRI, Grenzfehden S. 29.

Das in der Corroboratio als bered zedel bezeichnete Vertragsexemplar der böhmischen Landherren beginnt mit Vermerkht und nennt nach der Siegelankündigung ein von K.F. besiegeltes, gleichlautendes Stïck.

1 Datierung nach dem Datum der Gegenurkunde: Beschehen an suntag vor sand Jorgen tag.

2 Ksl. Rat, s. zu ihm DoBlinger, Herren von Walsee S. 248-259 [482-493]; HenNG, Friedrich III./1 S. 251 .

3 Ksl. Rat und seit 1476 Oberster Hauptmann im Kampf gegen die von Matthias Corvinus unterstützten böhmischen Adeligen, s. Henng, Friedrich III./1 S. 341; auch CHMEL n. 7073.

4 Der Waffenstillstand wurde am 24. September 1477 zunächst bis zum Neujahrstag 1478 und am 31. Dezember 1477 bis 24 . April 1478 verlängert, s. CнмеL, Mon. Habsb. I/2 S. 516-518 n. 143.

5 Druck (böhm. Vertragsexemplar): ChMEL, Mon. Habsb. I/1 S. 505f.n. 189; Reg.: ChMrL n. 7113. 
K.F. überträgt Wilhelm Paulseer bis auf Widerruf das in der Gft. Ortenburg gelegene Amt Stockenboi und die dortige Brückenmaut zu treuen Händen. ${ }^{1}$

Org. oder Kop. im bearbeiteten Bestand nicht überliefert. - Dep.: Erw. im Revers Wilhelm Paulseers von 1477 April 23 im HHStA Wien (Sign. AUR 1477 IV 23), Perg., 2 grüne SS des Ausst. und Leonhard Vetters von (Donau-)Wörth in wachsf. Schüsseln an Ps, mit Rta-Vermerk auf der Rückseite. ${ }^{2}$

1 Siehe dazu auch n. 19.

2 Reg.: ToMAschEK, Regesten Kärnten 3 n. 486.

[Vor 1477 April 29, -]

K.F. überlässt dem Richter und Rat der Stadt Laa Gericht, Maut und Ungeld zu Laa bestandweise für drei Jahre ab Mariä Verkündigung 1477 (März 25) gegen Zahlung von jährlich 500 Pfd. Pf.

Org. oder Kop. im bearbeiteten Bestand nicht überliefert. - Dep.: Erw. im Revers von Richter und Rat von Laa von 1477 April 29, Laa, im HHStA Wien (Sign. AUR sub dat. 1477 III 17), ${ }^{1}$ Perg., grünes S der Stadt Laa in wachsf. Schüssel an Ps, mit Rta-Vermerk auf der Rückseite. ${ }^{2}$

Lit.: WALTER, Ungeld S. 100.

1 Unter dieser Sign. gemeinsam verwahrt mit den nn. 61, 67, 68 .

2 Reg.: Chmel n. 7121.

\section{[Vor 1477 Mai 6, -]}

K.F. schreibt an Hz. Sigmund von Österreich betreffend Burgund.

Org. oder Kop. im bearbeiteten Bestand nicht überliefert. - Dep.: Erw. in einem Schreiben Hz. Sigmunds von Österreich an K.F. von 1477 Mai 6, Innsbruck, im HHStA Wien (Sign. AUR 1477 V 6), Pap., rotes S des Ausst. als Verschluss rücks. aufgedr. (aufgebrochen); davon Abschrift des 18. Jh. ebd. (Sign. Urkundenabschriften Österr. Urkunden, Kt. 46 sub dat.), Pap. ${ }^{1}$

Lit.: BaChManN, Reichsgeschichte 2 S. 580; Baum, Sigmund der Münzreiche S. 370.

Siehe nn. 76, 80 . 
1 In dem Schreiben ersucht $\mathrm{Hz}$. Sigmund den $\mathrm{K}$. erneut um Belehnung mit Burgund und Mailand; Druck: Chmel, Mon. Habsb. I/2 S. 207f. n. 51; Reg.: Chmel n. 7123; Lichnowsky(-BrRK) 7 n. 2045. - Siehe dazu das Schreiben Hz. Maximilians an Sigmund von 1477 April 21, worin er Sigmund ersucht, von seinen Ambitionen auf Burgund abzulassen; Druck: ChMEL, Mon. Habsb. I/1 S. 153f. n. 45; Reg.: LICHNOWSKY(-BIRK) 7 n. 2038.

\section{Mai 14, Wien}

K.F. präsentiert als Hz. von Krain und Inhaber des Patronatsrechts dem Hofkaplan und Rektor der Pfarrkirche in Reiffnitz, Thomas Freinperger, den Priester Johannes Grumel aus der Diöz. Speyer für die Pfarrkirche der seligen Jungfrau Maria in Grafenwarth, Diöz. Aquileia, die durch den Tod ihres früheren Rektors Vitus Frostl vakant wurde, und fordert ihn auf, Grumel canonice instituere und $\mathrm{zu}$ investieren, ut moris est. Die quartadecima mensis maii.

KVr: C.d.i.i.c. - Rückseite der Wachsschüssel: Grumel.

Org. (lat.) im HHStA Wien (Sign. AUR 1477 V 14), Perg., rotes S 21 (tlw. abgefallen) in wachsf. Schüssel mit wachsf. S 16 rücks. eingedr. an Ps.

Druck: CHMEL, Mon. Habsb. I/2 S. 281f. n. 102.

Reg.: LichNOwsKY(-Birk) 7 n. 2050.

Lit.: $\mathrm{Zu}$ den Hofkaplänen s. HeINIG, Friedrich III./1 S. 801-804; auch LACKNER, Hof und Herrschaft 153-158.

\section{[Vor 1477 Mai 16, -]}

K.F. überträgt Hans Weidinger, Weisbote in Steier, bis auf Widerruf das Landgericht zu Graz zu treuen Händen mit der Verpflichtung, ab 1478 jährlich zum St. Johannestag zur Sonnwende (Juni 24) abzurechnen und ihm oder seinen Erben ein Drittel der Gefälle zu überantworten.

Org. oder Kop. im bearbeiteten Bestand nicht überliefert. - Dep.: Erw. im Revers Hans Weidingers von 1477 Mai 16 im HHStA Wien (Sign. AUR 1477 V 16), Perg., 2 grüne SS des Ausst. und des Wiener Bürgers Sigmund Gwaltshofer ${ }^{1}$ in wachsf. Schüsseln an Ps, mit Rta-Vermerk auf der Rückseite. ${ }^{2}$

Lit:: Muchar, Geschichte 8 S. 90 (zu 1476); PolPelKa, Geschichte Graz 1 S. 396.

1 Siehe zu ihm Perger, Wiener Ratsbürger S. 207.

2 Reg.: Muchar, Urkunden-Regesten n. 247; GöTH, Urkunden-Regesten 9 n. 644. 
K.F. gibt aus ksl. Macht seinem Sohn Hz. Maximilian von Österreich und dessen Gefolge Sicherheit und Geleit für den $\mathrm{Zug}^{2}$ zu dessen Gemahlin Hzn. Maria von Burgund ${ }^{3}$ und gebietet den Kff., geistlichen und weltlichen Fürsten und allen anderen Reichsuntertanen bei seiner und des Reichs schweren Ungnade, sie frei, sicher und ungehindert durch seine und ihre Länder ziehen zu lassen und ihnen auf Erfordern Geleit zu geben (nach Druck).

Org. oder Kop. im bearbeiteten Bestand nicht überliefert. - Dep.: Mit hoher Wahrscheinlichkeit handelt es sich bei dem in $n .80$ erwähnten ksl. Schreiben an die Reichsuntertanen um diesen Geleitbrief für Maximilian.

Druck: ChMel, Mon. Habsb. I/1 S. 468f. n. 177 (nach Konz.).

Lit. siehe bei $n .80$.

1 Datierung und Ausstellungsort nach Druck bei CHMEL.

2 Zur Reise Maximilians nach Burgund von Mai bis August 1477 s. den Kommentar zu n. 80.

3 Die Eheschließung war am 21. April 1477 in Brügge per procuram erfolgt, s. WIESFLECKER, Kaiser Maximilian I/1 S. 126.

\section{[1477 vor Mai 25, -]}

K.F. antwortet auf ein Schreiben Hz. Sigmunds von Österreich ${ }^{1}$ betreffend Burgund.

Org. oder Kop. im bearbeiteten Bestand nicht überliefert. - Dep.: Erw. in n. 80 .

1 Mit hoher Wahrscheinlichkeit das Schreiben Sigmunds von 1477 Mai 6, s. n. 72.

\section{[1477 vor Mai 25, -]}

K.F. schreibt an Hzn. Maria von Burgund.

Org. oder Kop. im bearbeiteten Bestand nicht überliefert. - Dep.: Erw. in n. 80 . 
K.F. schreibt an Hzn. Margarete von Burgund, Witwe Hz. Karls von Burgund.

Org. oder Kop. im bearbeiteten Bestand nicht überliefert. - Dep.: Erw. in n. 80.

[1477 vor Mai 25, -]

K.F. schreibt allen Untertanen der von Hz. Karl von Burgund hinterlassenen Länder.

Org. oder Kop. im bearbeiteten Bestand nicht überliefert. - Dep.: Erw. in n. 80.

\section{Mai 25, Wien}

K.F. übermittelt seinem Sohn Hz. Maximilian von Österreich folgende offen briefe ${ }^{1}$ und abgeschriffen ${ }^{2}$ mit der Empfehlung, die Briefe wol zugebrauchen: seine Antwort ${ }^{3}$ auf das diesem bereits bekannte Schreiben Hz. Sigmunds von Österreich ${ }^{4}$ sowie seine (K.F.) Schreiben an Maximilians Gemahlin Hzn. Maria von Burgund ${ }^{5}$, an Hzn. Margarete von Burgund, ${ }^{6}$ Witwe Hz. Karls von Burgund, an die Untertanen der von Hz. Karl hinterlassenen Länder ${ }^{7}$ und an die Untertanen des Reichs ${ }^{8}$. An suntag dem heiligen Pfingsttag (nach Kop.).

KVr: C.d.i.i.c. (nach Kop.). - KVv: Dem hochgebornnen Maximilian hertzogen zu Osterreich etc., unserm lieben sun und fursten (Adresse, nach Kop.).

Org. ehemals im HHStA Wien, dem Druck zufolge Pap. - Kop.: Zwei Abschriften des 18. Jh. ebd. (Sign. Urkundenabschriften Österr. Urkunden, Kt. 46 sub dat.), Pap.

Druck: ChMEL, Mon. Habsb. I/1 S. 155f. n. 46 (nach Org.)

Reg.: LICHNOwsKY(-BIRK) 7 n. 2053 (nach Org.).

Lit.: RausCH, Burgundische Heirat S. 173; zur Reise nach Burgund s. ebd. 174-177; WIESFLECKER, Kaiser Maximilian I./1 S. 129-131; ausführlich Bock, Maximilian S. 26-29; auch HoLLwEG, Georg Heßler S. 59.

Hz. Maximilian brach am 21. Mai 1477 auf und reiste über Graz, Salzburg, Frankfurt und Köln in die burgundischen Länder. Am 18. August 1477 zog er in Gent ein, am folgenden Tag fand die Hochzeit mit Maria von Burgund statt. ${ }^{9}$

1 Wohl der Geleitbrief für Maximilian (n. 75) und das Schreiben an die Untertanen der burgundischen Länder (n. 79). 
2 Wohl Abschriften der nn. 76, 77, 78 (Schreiben an Hz. Sigmund und an die Herzoginnen Maria und Margarete von Burgund).

3 Siehe n. 76.

4 Mit hoher Wahrscheinlichkeit das Schreiben Hz. Sigmunds von 1477 Mai 6, s. dazu n. 72.

5 Siehe n. 77; zur Eheschließung per procuram am 21. April 1477 in Brügge s. WIEsFLECKER, Kaiser Maximilian I/1 S. 126.

6 Siehe n. 78.

7 Siehe $\mathbf{n} .79$.

8 Wohl der Geleitbrief von 1477 Mai 20 für die Reise nach Burgund, s. n. 75.

9 Zur Finanzierung der Reise s. n. 51; zur Aufforderung an die Reichsuntertanen von 1477 April 2 bzgl. Entsendung von Berittenen als Geleit Maximilians s. CHMEL, Mon. Habsb. I/1 S. 142-144 n. 39 (mit Empfängerliste); zum Itinerar ebd. S. 163f. zu n. 49. -Eine Kop. (19. Jh.) des Ehevertrags von $1477 \mathrm{Au}-$ gust 18 im HHStA Wien (Sign. FU n. 734).

\section{Juni 3, Wien}

K.F. belehnt Peter Langauer und dessen Ehefrau Barbara auf Bitte von Gabriel und Margarete Waching, den Eltern Barbaras, wissenlich mit dem brief, was wir in zurecht daran verleihen sullen oder mugen, mit dem Turm samt Zubehör zu Winklern im Großkirchheimer Gericht aus der Lehenschaft des Ftm. Kärnten, den das Ehepaar Waching ihnen verkauft und mit einem offenen besiegelten Brief aufgesandt hat, und verfügt, dass sie und ihre Erben den Turm gemäß Lehens- und Landrecht innehaben und nutzen sollen. Am eritag vor Gotzleichnambstag.

KVr: C.d.i.i.c. $-\mathrm{KVv}$ : Lanngenawer (Blattmitte unten). - Rückseite der Wachsschüssel: Vermerk nicht lesbar (wohl Name der Empfänger).

Org. im HHStA Wien (Sign. AUR 1477 VI 3), Perg., rotes S 21 in wachsf. Schüssel mit wachsf. S 16 rücks. eingedr. an Ps.

Reg.: GöTH, Urkunden-Regesten 9 n. 646; TомASCHEK, Regesten Kärnten 3 n. 487; MC 11 n. 467.

Lit.: WIISSNER/VYORAL-TsCHAPKA, Burgen Kärnten 3 S. 126; zum Turm zu Winklern s. auch KoHLA, Kärntner Burgenkunde 1 S. $370 \mathrm{f}$.

\section{Juni 10, Wien}

K.F. belehnt den vor ihm erschienenen Kg. Wladislaw (II.) von Böhmen mit den Regalien und Lehen, dem Kurfürstentum und dem Erzschenkenamt des Reichs samt den Markgrafschaften und allen Fürstentümern, Ländern, Herrschaften etc. sowie allen anderen zur Krone Böhmens gehörigen Rechten. Am zehennden tag des monadts junii (nach Kop.). 
KVr: A.m.d.i.p. Jo(hann) Waldner prothonotarius etc. - KVv: Rta Lucas Snitzer (nach Kop.).

Kop.: Abschrift des 18. Jh. im HHStA Wien (Sign. Urkundenabschriften Böhm. Urkunden, Kt. 85 sub dat.), Pap.; beiliegend zwei Abschriften (18. Jh.) eines Vidimus ${ }^{1}$ Ernsts von Schleinitz, Administrator des Erzbistums Prag, von 1541 Mai 14, Pap.

Druck: BEGERT, Böhmen S. 603f. n. 3B.

Reg.: Ein ausführliches Regest bieten die Regg.F.III. H. 26 n. 688 (nach Org.) und H. 10 n. 432 (nach Kop.) mit weiteren Druckbelegen und Lit.; s. auch H. 9 n. 337 und H. 23 n. 650 (jeweils nach unzulänglichen Überlieferungen).

Lit.: BEGERT, Böhmen S. 217-229; HoLTz, Länder der böhmischen Krone S. 33.

Das Ringen um den Urkundentext in der Frage der lehensrechtlichen Abhängigkeit Böhmens vom Reich, die seitens Wladislaws und der böhmischen Stände möglichst gering gehalten werden sollte, dokumentiert ein im HHStA Wien überliefertes Konzept, dem zufolge K.F. Wladislaw ursprünglich mit dem Kgr. Böhmen und der Mgft. Mähren samt Kurfürstentum und Erzschenkenamt belehnen sollte, aber - wie die Korrekturen und Streichungen sowie die Letztfassung der Urkunde zeigen - eine eindeutige Formulierung der Lehensoberhoheit nicht durchsetzen konnte. ${ }^{2}$

Als Reaktion auf die Belehnung Wladislaws erfolgte am 12. Juni 1477 die Absage $\mathrm{Kg}$. Matthias' von Ungarn an K.F. mit einer ausführlichen Begründung. ${ }^{3}$ Am 24. Juni 1477 nahm der Kaiser in einem Schreiben an die Einwohner Ungarns dazu Stellung. ${ }^{4}$

Siehe nn. 42, 87, 106, 107.

1 Vidimiert wurden die vorliegende Urkunde und eine Urkunde Karls V. von 1531 Dezember 27 (Belehnung Ferdinands I. mit Böhmen).

2 Siehe ausführlich BEGERT mit Abb. (S. 220f.) und Druck des Konzepts (S. 601f. n. 3A).

3 Org. im HHStA Wien (Sign. AUR 1477 VI 12); Druck: CHMEL, Mon. Habsb. I/2 S. 96-98 n. 12; BACHMANN, Urkundliche Nachträge S. 422-424 n. 416 (nach Kop. in lat. Übersetzung); s. dazu NeHRING, Matthias Corvinus S. 85f.; HoENsCH, Matthias Corvinus S. $156 f$.

4 Siehe Regg.F.III. H. 15 n. 330; s. auch den Druck bei CHмEL, Mon. Habsb. I/2 S. 98-108 nn. 13a (dt., 1477 Juni 24), 13b (lat., 1477 Juni 26) nach einem Konzept im HHStA Wien, sowie den Druck bei PrAY, Annales Hungariae 4 S. 109-112. - Weitere Böhmen und den Krieg mit Matthias Corvinus betreffende Stücke im HHStA Wien: Fehdebriefe (tschechisch) an K.F. (Sign. AUR 1477 VII 21), s. ChMEL, Mon. Habsb. I/2 S. 108f. n. 14; die Schreiben P. Sixtus' IV., der im Interesse des Türkenkriegs Matthias unterstützte, an K.F. von 1477 September 16 (Org., Sign. AUR 1477 IX 16) und September 19 sowie an die böhmischen Stände von September 24 (beide Kop., Sign. AUR sub dat. 1477 IX 24), s. CHMEL n. 7145 und DERs., Mon. Habsb. I/1 S. 379f. n. 133; LICHNOwSKY(-BIRK) 7 n. 2089; Beschwerdebrief Kg. Matthias' an Nürnberg von 1477 Oktober 26 (Kop., Sign. AUR 1477 X 26), diesem beigelegt in dt. Abschrift das päpstliche Schreiben an K.F. von 1477 September 16 und die Bannbulle Sixtus' IV. gegen die Gegner des Corvinen vom selben Tag, s. LICHNOwSKY(-BIRK) 7 n. 2106; s. weiters auch die nicht im bearbeiteten Bestand überlieferten Stücke bei CнMEL, Mon. Habsb. I/2 S. 110-119 nn. 15, 16. 
K.F. verschreibt Gebhard Peuscher und dessen Erben Schloss Falkenstein im Ftm. Kärnten satz- und pflegweise gegen ein Darlehen von 2.000 fl. ung. ${ }^{1}$

Org. oder Kop. im bearbeiteten Bestand nicht überliefert. - Dep.: Erw. im Revers Gebhard Peuschers von 1477 Juni 13 im HHStA Wien (Sign. AUR 1477 VI 13), Perg., 2 grüne SS des Ausst. und des Ritters Hans von Aichberg, Hofmeister des Eb. (Bernhard II.) von Salzburg, in wachsf. Schüsseln an Ps, mit Rta-Vermerk auf der Rückseite. ${ }^{2}$

Lit.: ZAISBERGER, Briefe S. 158; MetNITZ, Kärntner Burgenkunde 2 S. 29; zu Gebhard Peuscher s. ReINLE, Peuscher S. 934-941, bes. 939; zu Falkenstein s. auch WIESSNER/VyoraLTsCHAPKA, Burgen Kärnten 3 S. 49-52.

Niederschlag der Türkeneinfälle in Kärnten, zuletzt im Oktober 1476, ist die laut Revers getroffene Vereinbarung, dass Peuscher die Pfandschaft unter Einhaltung einer Frist von einer Quatember kündigen kann, sollten die Erträge durch die Türken vernichtet werden. ${ }^{3}$

1 Zur Ablösung bereits im Jahr 1478 s. n. 169.

2 Reg.: GöTH, Urkunden-Regesten 9 n. 647; ToMASCHEK, Regesten Kärnten 3 n. 489; MC 11 n. 468.

$3 \mathrm{Zu}$ den Türkeneinfällen in Kärnten ab 1473 s. Fräss-EHRFELD, Geschichte Kärntens 1 S. 598-602, zum Einfall im Oktober 1476 auch MC $11 \mathrm{nn} .465,466$; ausführlich zu den Einfällen in Innerösterreich KLEIN, Geschichte.

\section{[Vor 1477 Juni 15, -]}

K.F. überlässt Hans Murstetter, Bürger von Wien, das Stadtgericht zu Wien bestandweise für zwei Jahre ab Mittwoch nach dem St. Veitstag 1477 (Juni 18) gegen Zahlung von jährlich $350 \mathrm{Pfd}$. Pf.

Org. oder Kop. im bearbeiteten Bestand nicht überliefert. - Dep.: Erw. im Revers Hans Murstetters von 1477 Juni 15, Wien, im HHStA Wien (Sign. AUR sub dat. 1477 V 2), ${ }^{1}$ Perg., 2 grüne SS des Ausst. und des Wiener Bürgers Hans Lichtenperger ${ }^{2}$ in wachsf. Schüsseln an Ps, mit Rta-Vermerk auf der Rückseite. ${ }^{3}$

Lit:: Zu Hans Murstetter, Wiener Stadtrichter 1477-1479, s. PERGER, Wiener Ratsbürger S. 277.

1 Unter dieser Sign. gemeinsam verwahrt mit n. 102.

2 Siehe zu ihm PERGER, Wiener Ratsbürger S. 220.

3 Reg.: ChMel n. 7130; Ders., Mon. Habsb. I/3 S. 648 n. 50. 
K.F. gebietet dem Dekan und Kapitel der Mainzer Domkirche aus ksl. Macht, dem auf Klage des Fiskalprokurators (Johann Keller) ${ }^{1}$ gegen sie ergangenen Kammergerichtsurteil $^{2}$ wegen Missachtung seines Gebots, ein Floß zur Rettung der belagerten Stadt Neuss auf dem Rhein passieren zu lassen, ${ }^{3}$ Folge zu leisten, und lädt sie im Falle des Ungehorsams peremptorisch vor sich. Am siebenziehenden tag des monadtz junii (nach Kop.).

KVr: A.m.d.i. (nach Kop.).

Kop.: Zeitnahes Vidimus des öff. Notars Wilhelm ten Putte aus Büderich, Kler. der Diöz. Köln, im HHStA Wien (Sign. AUR 1477 VI 17), Perg., 15. Jh. ${ }^{4}$

Druck: ChMel, Mon. Habsb. I/3 S. 602f. n. 119.

Reg.: Ein ausführliches Regest bieten die Regg.F.III. H. 4 n. 782 (nach Kop.).

1 Lic. (oder Dr.) iur. utr. Johann Keller, ksl. Rat und seit 1474 alleiniger Fiskal, s. zu ihm HenNG, Friedrich $\mathrm{III} / 1$ S. 123-134.

2 Zum bislang noch nicht aufgetauchten Urteil s. Regg.F.III. H. 4 n. 781.

3 Mandat von 1475 Mai 24, s. Regg.F.III. H. 4 n. 720.

4 Rücks. Vermerk: Anno Domini $1482 x_{x x t^{t a}}$ mensis marcii ist dechannt und capitel des thumstiffts Menntz gegen der ksl. camer procurator fiscal nach lautt der zuruck geschriben copei einer ksl. ladung in gericht verlesen gerufft worden. Johannes Laventaller imperialis cancelarie notarius manu propria scripsit (Blattmitte) - - Zu Johann Laventaler, einem der wichtigsten Kanzlisten in der Spätzeit Friedrichs III., s. HEINIG, Friedrich III./1 S. $786 f$.

\section{Juni 20, Wien}

K.F. beurkundet die Entscheidung seiner Räte im Erbstreit um ein Haus gegenüber dem Propsthof in Wien aus dem Besitz des verstorbenen Grazer Bürgers Christoph Epishauser ${ }^{1}$ zwischen dem Wiener Bürger Jakob Rehwein ${ }^{2}$ anstatt seiner verstorbenen Ehefrau Elisabeth und seiner beiden Schwäger, des Laibacher Chorherrn Lic. iur. can. Ulrich Huber und des Radkersburger Bürgers Pankraz Huber ${ }^{3}$, einerseits und dem (inzwischen) verstorbenen Pankraz Peddersdorfer als Bevollmächtigten Ursulas, Ehefrau Erhard Lembachers und Witwe Christoph Epishausers, andererseits. Nachdem die Streitparteien vor Bürgermeister, Richter und Rat von Wien in recht gestanden sind und das Urteil ${ }^{4}$ zuerklern durch Peddersdorfer an ihn (K.F.) gedingt worden war, haben seine Räte dieses Urteil in der Weise erledigt und erklert, dass die von Erhard Lembacher vorgelegten ksl. brieve $e^{5}$ in Kraft bleiben sollen. Sollten die Wiener oder 
Jakob Rehwein und seine Miterben etwas dagegen vorzubringen haben, es sey irer statt freyhait, geschefftbrieve, gerichtsbrieve oder annders, worauf sie ihren Rechtsspruch gründeten, verspricht er, dass sie gehört werden und dann geschehe, was recht ist. An freytag nach sannd Veits tag.

KVr: C.d.i.i.c. - KVv: Rta (Blattmitte). - erklerung (unterer Blattrand).

Org. im HHStA Wien (Sign. AUR 1477 VI 20), Perg., rotes S 21 in wachsf. Schüssel mit wachsf. S 16 rücks. eingedr. an Ps.

Druck: CHMEL, Mon. Habsb. I/2 S. 282f. n. 103.

Reg.: CHMEL n. 7132; QGStW I/5 n. 5047.

Der Grazer Bürger Christoph Epishauser hatte seiner Ehefrau Ursula mit einem von K.F. als Landesfürsten und obersten Grundherrn der Stadt Wien bestätigten Gemächtbrief sein gesamtes Vermögen, also auch sein Haus in Wien, vermacht. Das Gemächt wurde allerdings nicht unter Einhaltung der rechtlich vorgeschriebenen Frist vor dem Wiener Rat eingebracht. Nach Epishausers Tod (vor 1466 Dezember 6, s. Anm. 1) erhoben daher seine Verwandten Elisabeth Rehwein und deren Brüder Erbansprüche auf das Haus, während die Witwe Ursula auf ihrem Besitzrecht gemäß dem Gemächt bestand. Im Prozess vor dem Wiener Rat 1472 legte Peddersdorfer als Bevollmächtigter der inzwischen mit Erhard Lembacher wiederverheirateten Witwe die ksl. Bestätigung des Gemächtbriefs sowie das ksl. Mandat, sie in das Grundbuch einzutragen, vor (s. Anm. 5). Seitens der Kläger wurde gegen die von der beklagten Partei vorgebrachte, oberste grundherrliche Autorität der ksl. Bestätigungsurkunde mit der Fristversäumnis und dem damit verbundenen Verlust der Gewere gemäß Stadtrecht argumentiert, dem sich der Wiener Rat anschloss. Mit Ratsurteil von 1472 April 28 (s. Anm. 4) wurde das Besitzrecht Ursula Lembachers auf das Haus aufgrund Nichteinbringung des Gemächts binnen Jahresfrist für kraftlos erklärt und das Erbrecht der Kläger mit Eintrag in das Grundbuch bestätigt. Durch dieses Urteil wurden die ksl. Urkunden außer Kraft gesetzt, worauf Peddersdorfer an K.F. appellierte.

1 Zu Christoph Epishausers, Grazer Ratsbürger und Bürgermeister (1456), s. DiENEs, Bürger von Graz S. LXIVf.; auch PopelKA, Geschichte Graz 1 S. 484 (Bürgermeisterliste); im ksl. Mandat von 1466 Dezember 6 (QGStW II/3 n. 4138) wird er als verstorben erwähnt.

2 Zu Jakob Rehwein, Bruder des damaligen Leiters der österr. Kanzlei Johannes Rehwein, s. PERGER, Wiener Ratsbürger S. 233 (Jakob d.J. Rechwein).

3 Ulrich und Pankraz Huber waren Brüder Elisabeth Rehweins, Christoph Epishauser deren Vetter, s. Regg.F.III. H. 27 n. 153.

4 Rechtsspruch des Wiener Rats von 1472 April 28 zugunsten Elisabeth Rehweins und ihrer Miterben (Org. im HHStA Wien, Sign. AUR 1472 IV 28); s. dazu die ksl. Befehle an den Wiener Rat auf Vorbringen Jakob Rehweins von 1471 Oktober 31 und 1472 April 24, Elisabeth Rehwein im Erbstreit gegen Ursula Lembacher Recht ergehen zu lassen (QGStW II/3 nn. 4401, 4428; s. auch Regg.FIII. H. 27 n. 153, hier allerdings irrtümlich Urteil zugunsten Ursula Lembachers). Während diese ksl. Mandate die Rehwein- 
Partei begünstigten, unterstützten die früher ausgestellten Mandate (s. Anm. 5) sowie die darauf basierende Erklärung der ksl. Räte von 1477 die Ansprüche der Gegenpartei.

5 Ksl. Bestätigung eines Gemächtbriefs Christoph Epishausers zugunsten seiner Frau Ursula von 1466 Dezember 7 (Regg.F.III. H. 22 n. 149); am Vortag erging der ksl. Befehl an den Wiener Rat, Epishausers Witwe Ursula in das Grundbuch einzutragen (QGStW II/3 n. 4138), dem bis Juli 1467 fünf weitere Mandate gleichen Inhalts folgten (ebd. nn. 4147, 4160, 4161, 4175, 4182); s. in der Sache weiters ebd. nn. 4847, 5183.

\section{Juni 24, Wien}

K.F. bestätigt Kg. Wladislaw (II.) von Böhmen alle Privilegien und Rechte der Krone zu Böhmen, des Kurfürstentums und des Erzschenkenamts des Reichs und aller zur Krone und dem Kgr. Böhmen gehörigen Länder und gebietet allen Untertanen des Reichs sowie der Krone und des Kgr. Böhmen bei seiner und des Reichs schweren Ungnade und einer Pön von 1.000 Mark Gold, zahlbar je zur Hälfte an die ksl. Kammer und Kg. Wladislaw, die Beachtung dessen Privilegien. Am vierundzwanczigsten tag des monadts junii (nach Kop.).

KVr: A.m.d.i.p. Jo(hann) Waldner prothonotarius etc. (nach Kop.).

Kop.: Abschrift des 18. Jh. im HHStA Wien (Sign. Urkundenabschriften Böhm. Urkunden, Kt. 85 sub dat.), Pap.

Reg.: Ein ausführliches Regest bieten die Regg.F.III. H. 26 n. 695 (nach Org.) und H. 10 n. 432 (nach Kop.) mit Druckbelegen.

Lit.: BaChMANN, Reichsgeschichte 2 S. 597; HoLtz, Länder der böhmischen Krone S. 33.

Siehe n. 82 .

\section{[Vor 1477 Juli 4, -]}

K.F. verpfändet Dekan und Kapitel des Kollegiatstifts Spital am Pyhrn Schloss Klaus (am Pyhrn) samt Nutzen, Renten und allem Zubehör für $1.600 \mathrm{fl}$. ung., um die sie das Schloss auf seinen Befehl von Heinrich von Kainach abgelöst haben, und verpflichtet sie, ihm von den Nutzen und Renten des Schlosses jährlich 20 Pfd. Pf. zu geben oder aber diesen Betrag bei Bezahlung der Pfandsumme abzuziehen.

Org. oder Kop. im bearbeiteten Bestand nicht überliefert. - Dep.: Erw. im Revers Dekan Urbans (von Weix) und des Kapitels von Spital am Pyhrn von 1477 Juli 4 im HHStA Wien (Sign. AUR 1477 VII 4), Perg., 2 wachsf. SS des Dekans (spitzoval) und des Kapitels (rund) an Ps. ${ }^{1}$

Lit.: PrITZ, Geschichte Spital am Pyrn S. 276; HäRTEL, Kainacher S. 14; ${ }^{2}$ zu Schloss Klaus S. BAUMERT/GRÜLL, Burgen Oberösterreich 3 S. 71-74. 
1 Reg.: GöTH, Urkunden-Regesten 9 n. 649.

2 Bei HäRTEL irrtümliche Angabe der Ablösesumme von 1.000 fl. ung. (Summe für das Amt Molln, s. n. 89) statt $1.600 \mathrm{fl}$. ung.

[Vor 1477 Juli 4, -]

K.F. verpfändet Dekan und Kapitel des Kollegiatstifts Spital am Pyhrn das zur Herrschaft Steyr gehörende Amt Molln für 1.000 fl. ung., um die sie das Amt auf seinen Befehl von Heinrich von Kainach, dem es von Ehz. Albrecht (VI.) von Österreich für dieselbe Summe verpfändet worden war, abgelöst haben, und verpflichtet sie, ihm von den Nutzen und Renten jährlich 80 Pfd. Pf. zu geben oder aber diesen Betrag bei Bezahlung der Pfandsumme abzuziehen

Org. oder Kop. im bearbeiteten Bestand nicht überliefert. - Dep.: Erw. im Revers Dekan Urbans (von Weix) und des Kapitels von Spital am Pyhrn von 1477 Juli 4 im HHStA Wien (Sign. AUR 1477 VII 4), Perg., wachsf. S des Dekans (spitzoval) an Ps, S des Kapitels (rund) samt Ps ab und verloren. ${ }^{1}$

Lit.: HÄRTEL, Kainacher S. $15 .^{2}$

1 Reg.: GöTH, Urkunden-Regesten 9 n. 648.

2 Bei HärTEL irrtümliche Angabe der Ablösesumme von 1600 fl. ung. (Summe für Schloss Klaus, s. n. 88) statt $1.000 \mathrm{fll}$. ung.

[Vor 1477 Juli 11, -]

K.F. verschreibt Wolfgang Perler ${ }^{1}$ seine Güter bei Judenburg, Obdach, Schöder und Ranten, die der verstorbene Voitsberger Bürger Hans Judel innehatte, gegen ein Darlehen von $100 \mathrm{fl}$. ung. und gestattet ihm, sich die Summe aus den Nutzen und Renten selbst zu bezahlen.

Org. oder Kop. im bearbeiteten Bestand nicht überliefert. - Dep.: Erw. im Revers Wolfgang Perlers von 1477 Juli 11 im HHStA Wien (Sign. AUR 1477 VII 11), Perg., 2 grüne SS Virgil Schrutauers, ksl. Stadtanwalt in Wien, ${ }^{2}$ und Sigmund Gwaltshofers, Münzmeister in Österreich, ${ }^{3}$ in wachsf. Schüsseln an Ps., mit Rta-Vermerk auf der Rückseite. ${ }^{4}$

Lit.: EBNER, Bürgerfamilie Judel S. 12.

1 Laut Revers gesessen auf der Sperlmul.

2 Langjähriger Wiener Stadtanwalt (1468-1479, 1485-1490), s. zu ihm PERGER, Wiener Ratsbürger S. 244; HEINIG, Friedrich III./1 S. $618 \mathrm{f}$. 
3 Siehe zu ihm Perger, ebd. S. 207, der ihn erst ab 1478 als Münzmeister nennt.

4 Reg.: Muchar, Urkunden-Regesten n. 248; GöTH, Urkunden-Regesten 9 n. 650; s. dazu auch CHMEL, Conzeptensammlung 2 S. 189 n. 418.

\section{Juli 17, Wien}

K.F. bestätigt Wolfgang Galler (Geler) und dessen Erben, ihnen 2.000 fl. ung., die dieser ihm yecz zu unsern notdurften berait geliehen hat, zu schulden und innerhalb von zwei Monaten ab Datum dieses briefs zurückzuzahlen. An phincztag vor sand Marien Magdalenen tag.

$\mathrm{KVr}$ C.d.i.i.c. $-\mathrm{KVv}$ : Rta - Quitantia Friderici imperatoris pro 2000 aureorum, quos debuit Wollfgango Geler (Blattmitte).

Org. im HHStA Wien (Sign. AUR 1477 VII 17), Perg., rotes S 21 in wachsf. Schüssel (beschädigt) mit wachsf. S 16 rücks. eingedr. an Ps.

Reg.: LICHNOWSKY(-BIRK) n. 2070.

Lit.: MUCHAR, Geschichte 8 S. 94.

\section{[1477 August 8, - $]^{1}$}

K.F. verschreibt Christoph Jörger und dessen Erben Schloss Roith satz- und pflegweise auf vier Jahre gegen ein Darlehen von $400 \mathrm{fl}$. rh.

Org. oder Kop. im bearbeiteten Bestand nicht überliefert. - Dep.: Erw. im Revers Christoph Jörgers von 1477 September 10 im HHStA Wien (Sign. AUR 1477 IX 10), Perg., 2 grüne SS des Ausst. und seines Vetters Hans Sinzendorfer in wachsf. Schüsseln an Ps, mit Rta-Vermerk auf der Rückseite. ${ }^{2}$

Reg.: ChMel, Mon. Habsb. I/3 S. 697 n. 190.

Lit.: Zu Christoph Jörger s. ausführlich WuRM, Jörger von Tollet S. 24-32, zu Roith bes. 26f.; ${ }^{3}$ HeINIG, Friedrich III./1 S. 293.

1 Datierung nach Reg. bei CHMEL.

2 Reg.: CHMEL n. 7144; DeRs., Mon. Habsb. I/3 S. 698 n. 194.

3 WURM nennt eine satz- und pflegweise Übertragung an Christoph Jörger auf zehn Jahre von 1477 Mai 24 gegen ein Darlehen von $400 \mathrm{fl}$., was allerdings in dem drei Tage später ausgestellten Pflegrevers des Jörgers von 1477 Mai 27 keine Entsprechung findet (nur pflegweise Übertragung bis auf Widerruf); der Revers überliefert im HHStA Wien (Sign. AUR sub dat 1477 V 2); Reg.: CHMEL n. 7126 (zu Mai 22); DERS., Mon. Habsb. I/3 S. 697 n. 189. 
K.F. verpfändet Eb. Johann von Gran ${ }^{2}$ Schloss und Stadt Steyr mit dem dortigen Ungeld und allen anderen zugehörigen Nutzen, Renten und Gülten zusammen mit seinen Ämtern, Nutzen und Renten zu Korneuburg, die nicht dem Schloss Kreuzenstein ${ }^{3}$ verschrieben sind, gegen ein Darlehen von $37.000 \mathrm{fl}$. ung. zur Bezahlung der $50.000 \mathrm{fl}$. ung. an Ulrich von Grafenegg ${ }^{4}$ für dessen in Österreich gelegenen Schlösser und Güter. Er verfügt, dass Eb. Johann und dessen Erben Schloss und Stadt Steyr samt Zubehör sowie die Ämter und Erträge zu Korneuburg pfandweise innehaben und alle Erträge ohne Abschlag auf die $37.000 \mathrm{fl}$. ung. einnehmen sollen, und bevollmächtigt sie, die Korneuburger Erträge nach Gutdünken durch die dortigen Bürger oder ihre eigenen Amtleute und Diener einheben zu lassen, unbehindert durch ihn (K.F.), seine Erben und Nachkommen. ${ }^{5}$ Er verpflichtet sie, ihm, nach seinem Tod seinem Sohn Hz. Maximilian von Österreich und nach dessen Tod ihren Erben mit Stadt und Schloss Steyr treu und gehorsam zu sein, diese auf seine (K.F.) Kosten und unbeschadet dieser Verschreibung offen zu halten und die zugehörigen Bürger und Leute über die gewöhnlichen Zinse, Steuern und Robotleistungen hinaus nicht zu belasten, sondern sie gegen Gewalt und Unrecht zu schützen. K.F. untersagt ihnen, ohne seine Erlaubnis und sein Wissen von Steyr aus Krieg zu führen und den Entzug seiner Rechte an Schloss und Stadt Steyr und den Ämtern zu Korneuburg zuzulassen. Auf Erfordern sind die genannten Güter und Erträge ihm oder seinem Bevollmächtigten gegen Bezahlung der $37.000 \mathrm{fl}$. ung. abzutreten und samt Zeug, Urbarregistern und anderem Zubehör zu überantworten. Die Pfandlösung hat unter Einhaltung einer Kündigungsfrist von einem Quatember für die seinerseitige Einlösung wie auch die ihrerseitige Aufkündigung der Pfandschaft ohne Widerspruch zu erfolgen gemäß der Verschreibung Eb. Johanns (nach Druck).

Org. oder Kop. im bearbeiteten Bestand nicht überliefert. - Dep.: Erw. im Revers Eb. Johanns von Gran von 1477 August 9, Wien, im HHStA Wien (Sign. AUR 1477 VIII 9), Perg., rotes S des Ausst. in wachsf. Schüssel an Ps, mit Rta-Vermerk auf der Rückseite. ${ }^{6}$

Druck: Chmel, Mon. Habsb. I/2 S. 264-266 n. 93 (nach Kop.).

Lit: Pritz, Geschichte Steyr S. 160; MAYER, Abdankung S. 180; STARZER, Korneuburg S. 125; BRANDL, Steyr S. 290; DopsCH, Geschichte Salzburgs S. 546.

Die bei CHMEL nach einer undatierten kopialen Überlieferung im HHStA Wien gedruckte ksl. Urkunde ist mit Sicherheit der im Revers Eb. Johanns von Gran erwähnte Pfandbrief, dessen Inhalt mit dem Revers weitgehend übereinstimmt. Eine Ausnahme bildet die im Revers nicht genannte Vollmacht zur freien Wahl der Einnehmer in Korneuburg. Da die Verschreibung bzw. der Revers Eb. Johanns in der ksl. Urkunde erwähnt wird, wurde sie mit hoher Wahrscheinlichkeit am selben Tag ausgestellt. 
1 Zur Datierung s. den Kommentar.

2 Johann Beckenschlager (Beckensloer) (Eb. von Gran 1472-1487), s. zu ihm ZAISBERGER, Rohr, bes. S. 58-62; GATZ, Bischöfe 2 S. 36f.; HEINIG, Friedrich III./1 S. 449-452 mit weiterführender Lit.

3 Zur Verpfändung Kreuzensteins an Johann Beckenschlager im Jahr 1479 s. n. 268.

4 Zum Vertrag mit Ulrich von Grafenegg von 1477 März 2 s. n. 55.

5 Siehe dazu den Revers der Stadt Korneuburg für Eb. Johann von Gran von 1478 Dezember 23 im HHStA Wien (Sign. AUR 1478 XII 23) über die Verpachtung von Ungeld und Ämtern an die Stadt für drei Jahre gegen Zahlung von jährlich 1.000 Pfd. Pfd.; Druck: CHMEL, Mon. Habsb. I/2 S. 632 n. 463.

6 Reg.: ChMel, Mon. Habsb. I/3 S. 700 n. 200; auch ChMel n. 7139 und LichNowsKr(-BIRK) 7 n. 2078 (beide nach Erwähnung bei KuRZ, Oesterreich 2 S. 129, Anm. c).

[Vor 1477 August 12, - ] $]^{1}$

K.F. überträgt Wenko von Lusthal bis auf Widerruf die Pflege von Schloss Altenburg mit der Verpflichtung, von den Nutzen und Renten das Schloss zu behüten und ihm davon jährlich 50 Pfd. Pf. zu entrichten.

Org. oder Kop. im bearbeiteten Bestand nicht überliefert. - Dep.: Erw. im Revers Wenkos von Lusthal von 1477 August 12 im HHStA Wien (Sign. AUR 1477 VIII 12), Perg., 2 grüne SS des Ausst. und des Ritters Laurenz Haiden ${ }^{2}$ in wachsf. Schüsseln an Ps, mit Rta-Vermerk auf der Rückseite. $^{3}$

1 Der Revers ist datiert mit Dienstag nach St. Tiburtius (an eritag nach sannd Tiburtien tag), dessen Festtag am 14. April und 11. August begangen wird; laut rücks. Vermerk des 15. Jh. erfolgte die Ausstellung am 12. August.

2 Wohl der gleichnamige Wiener Ratsbürger, s. zu ihm PERGER, Wiener Ratsbürger S. 207f

3 Reg.: Muchar, Urkunden-Regesten n. 245; Götr, Urkunden-Regesten 9 n. 642; erw. bei Muchar, Geschichte 8 S. 95 (jeweils zu April 15).

\section{[Vor 1477 August 12, -]}

K.F. überlässt dem Wiener Bürger Thomas Rorbeck ${ }^{1}$ und dessen Ehefrau Anna bis auf Widerruf die untere Wohnung in seinem, ehemals dem Wallseer ${ }^{2}$ gehörenden Haus an der Ecke bei St. Michael in Wien samt den Gemächern, die der verstorbene Hans Welser $^{3}$ von ihm (K.F.) innehatte, gegen einen jährlichen zu St. Michaelstag (September 29) fälligen Zins von 5 Pfd. Pf. 
Org. oder Kop. im bearbeiteten Bestand nicht überliefert. - Dep.: Erw. im Revers des Ehepaars Thomas und Anna Rorbeck von 1477 August 12 im HHStA Wien (Sign. FU n. 733), Perg., 2 grüne SS des Wiener Stadtrichters Thomas Tenk und des Ratsherrn Ernreich Köppl ${ }^{4}$ in wachsf. Schüsseln an Ps. ${ }^{5}$

1 Laut Rückvermerk (15. Jh.) auf dem Revers war Thomas Rorbeck Schuster.

2 Reinprecht (V.) von Wallsee hatte K.F. das Haus im Jahr 1460 verkauft (CHMEL n. 3827), s. Doblnnger, Walsee S. 247 [481]; zur Person s. ebd. S. 248-259 [482-493]; HeINIG, Friedrich III/1 S. 251f.

3 Hans Welser starb um 1457/59, s. Perger, Wiener Ratsbürger S. 257.

$4 \mathrm{Zu}$ Thomas Tenk und Ernreich Köppl s. ebd. S. 185 und 180, Köppl allerdings in der Ratsherrenfunktion nicht für 1477 genannt, sondern für die Jahre davor und danach.

5 Reg.: ChMEL n. 7140; DERS., Mon. Habsb. I/3 S. 666 n. 113.

\section{August 23, Krems}

K.F. bestätigt Wolfgang von Prem das Erbrecht an dem Striell genannten Hof gegenüber Schloss Prem wissenntlich mit dem briefe, was wir zu recht daran bestetten sullen oder mugen, nachdem im Jahr 1468 der ksl. Pfleger von Montpreis, Reinprecht Reichenburger, ${ }^{1}$ den Hof samt Äckern, Wiesen und allem Zubehör mit Vollmacht Reinprechts von Wallsee ${ }^{2}$ dem verstorbenen Achaz von Prem und dessen Erben laut dem darüber ausgestellten Brief zu Erbrecht gegeben hatte und in der Folgezeit das Erbrecht auf dessen Sohn Wolfgang übergegangen und die Herrschaft sowie das Urbar zu Prem, wohin der Hof dienstpflichtig ist, an den K. gefallen ist. K.F. verfügt, dass Wolfgang und dessen Erben den Hof samt Zubehör erbrechtsweise innehaben und nutzen sollen, und verpflichtet sie, jährlich zu sannd Mertten tag (November 11) $148 \mathrm{Sch}$. Agleier, je einen halben Metzen Weizen und Hirse sowie einen Metzen Hafer in sein Urbaramt zu Prem zu dienen, den Hof stifftlich und pewlich zu erhalten und alles zu tun, wozu alle anderen zu Prem gehörigen Leute und Holden verpflichtet sind und was früher von dem Hof geleistet wurde. An sambstag sannd Bartholomees abennt des heiligen zwelfboten (nach Kop.).

KVr: fehlt.

[Org. im ARS Ljubljana, zuvor im HHStA Wien.] - Fotokop. vom Org. im HHStA Wien (Sign. AUR sub dat. 1477 VIII 24).

Reg.: Regg.F.III H. 29 n. 196 (nach Org., zu August 24); s. auch MucHAR, Urkunden-Regesten n. 472; Gӧтн, Urkunden-Regesten 9 n. 652.

1 Pfleger von Montpreis 1461-1478, s. SCHÄFFER, Reinprecht von Reichenburg S. 120.

2 Ksl. Rat, s. zu ihm DoblnNger, Walsee S. 248-259 [482-493]; HenNG, Friedrich III./1 S. 251 f. 
K.F. gibt, aus redlichen ursachen veranlasst, seinem Diener Ludwig Meuting d.Ä. von Augsburg aus ksl. Macht wissentlich in crafft diss briefs Sicherheit und Geleit, um in Augsburg zu wohnen und überall im Reich seinen Geschäften nachzugehen, da dieser von Bürgermeister und Rat der Stadt Augsburg in seinen ihm auf zehn Jahre gewährten ksl. Freiheiten ${ }^{1}$ behindert wird. Er gebietet allen geistlichen und weltlichen Fürsten, Gff., Freiherren etc., Hauptleuten, Amtleuten, Vögten, Pflegern, Verwesern, SchultheiBen, Bürgermeistern, Richtern, Räten, Bürgern und Gemeinden, insbesondere aber Bürgermeister und Rat von Augsburg, sowie allen anderen Reichsuntertanen bei seiner und des Reichs schweren Ungnade, seine und des Reichs Sicherheit für Meutings Leib, Hab und Gut zu gewährleisten, ihn durch seine und ihre Länder, Herrschaften, Städte, Märkte, Dörfer und Gebiete zu Wasser und zu Land frei und sicher reisen und bei ihnen wohnen zu lassen, ihm bei Bedarf Geleit zu geben und weder dagegen zu handeln noch dies zu gestatten. Am newnundundzweinczigisten tag des monets augusti.

KVr: fehlt. - KVv: Gleit Meuting (oberer Blattrand).

Org. im HHStA Wien (Sign. AUR 1477 VIII 29), Perg., der Siegelankündigung zufolge mit unserm keiserlichen aufgedrucktem innsigel, aber unbesiegelt.

Druck: ChmeL, Mon. Habsb. I/3 S. 609 n. 125 (Teildruck).

Reg.: CHMel n. 7142.

Lit.: EHRENBERG, Zeitalter der Fugger 1 S. 188; Heinig, Friedrich III./1 S. 807 und 2 S. 989; zur Augsburger Kaufmanns- und Patrizierfamilie Meuting s. STEINER, Meuting, bes. S. 43-46; weiters RIEBARTSCH, Augsburger Handelsgesellschaften S. 123-125, 308f. (Anh. 3.1.), 329-335 (Anh. 3.2.1.); Blendinger, Art. Meuting S. 275-277; GeFFCKen/SChMÖLZ-HäBERLein, Art. Meuting (Stadtlexikon Augsburg online).

Aufgrund des Fehlens von Kanzleivermerk und Besiegelung sowie der Überlieferung im Ausstellerarchiv ist anzunehmen, dass die Urkunde nicht ausgefertigt wurde.

1 Blendinger, Art. Meuting S. 276, erwähnt einen ksl. Freiheits- und Wappenbrief von 1474 für Ludwig Meuting; s. auch den ksl. Dienst- und Schirmbrief von 1465 Dezember 11 für ihn und seine Familie bei ChMel n. 4308.

\section{[Vor 1477 September 3, -]}

K.F. gestattet Jörg Waitzinger, Bürger von Lavamünd, dass niemand auf der Fischweide oberhalb von dessen Mühle zu Lavamünd Fischwehren errichten darf sowie bestehende 
Wehren abgebaut werden müssen, gegen die Verpflichtung, zusätzlich zu den 4 Pfd. Pf. jährlichen Dienst 12 Sch. Pf. jährlich von der Mühle und der Fischweide in sein Amt zu Lavamünd zu entrichten.

Org. oder Kop. im bearbeiteten Bestand nicht überliefert. - Dep.: Erw. im Revers Jörg Waitzingers von 1477 September 3, Krems, im HHStA Wien (Sign. AUR 1477 IX 3), Perg., 2 grüne SS Wolfgang Aichpergers, Bürgermeister von Krems und Stein, und des Stadtschreibers Hans Schurger in wachsf. Schüsseln an Ps, mit Rta-Vermerk auf der Rückseite. ${ }^{1}$

1 Reg.: ToMAscheK, Regesten Kärnten 3 n. 491.

\section{[Vor 1477 September 9, -]}

K.F. überträgt Ulrich Harnstein bis auf Widerruf die Pflege von Schloss Lavamünd und überlässt ihm die zugehörigen Nutzen und Renten bestandweise ab St. Michaelstag 1477 (September 29) gegen Zahlung von jährlich 150 Pfd. Pf.

Org. oder Kop. im bearbeiteten Bestand nicht überliefert. - Dep.: Erw. im Revers Ulrich Harnsteins von 1477 September 9 im HHStA Wien (Sign. AUR 1477 IX 9), Perg., 2 grüne SS des Ausst. und Bernhard Karlingers, Mautner zu Stein, in wachsf. Schüsseln an Ps, mit Rta-Vermerk auf der Rückseite. ${ }^{1}$

1 Reg.: Tomascher, Regesten Kärnten 3 n. 492; MC 11 n. 470.

\section{September 9, Krems}

K.F. belehnt Jörg Schandacher auf Bitte des Hans Sachs zu Almegg und des Wolfgang Sachs wissenntlich mit dem brieve, was wir im zurecht daran verleyhen sullen oder mugen, mit folgenden in der Pfarre Ybbs gelegenen Lehen des Ftm. Österreich, die diese dem Schandacher verkauft haben: zwei von Hans Sachs mit einem offenen besiegelten Brief aufgesandte Güter zu Donaudorf; ein halbes Lehen zu Donaudorf, das Wolfgang Sachs als Lehensträger für sich und seine Brüder Jesse und Pilgrim aufgesandt hat; drei halbe Lehen zu Donaudorf und sechs halbe Lehen zu Reitering (Riettarn), die Wolfgang Sachs als Lehensträger der Kinder seines obgenannten verstorbenen Bruders Jesse Sachs, nämlich Jörg, Jesse, Christoph, Leonhard und Hans, zusammen mit dem genannten halben Lehen zu Donaudorf mit einem offenen besiegelten Brief aufgesandt hat. K.F. verfügt, dass Schandacher und dessen Erben die Güter gemäß Lehens- und Landrecht innehaben und nutzen sollen. An eritag nach unser lieben Frawntag nativitatis. 
KVr: fehlt. - Rückseite der Wachsschüssel: Schandacher.

Org. im HHStA Wien (Sign. AUR 1477 IX 9), Perg., rotes S 21 in wachsf. Schüssel mit wachsf. S 16 rücks. eingedr. an Ps.

Reg.: CHMEL n. 7143; DERS., Mon. Habsb. I/3 S. 658 n. 94; auch DERS., Conzeptensammlung 1 S. 83 n. 8.

\section{Oktober 19, Steyr}

K.F. belehnt Andreas Dorfner, Bürger von Steyr, und dessen Ehefrau Dorothea auf Bitte des Wiener Bürgers Hans Leschenprant wissenntlich mit dem brieve, was wir in zurecht daran verleihen sullen oder mugen, mit einer in der Pfarre Kematen (a.d. Krems) gelegenen Hube, genannt Greilhueb, aus der Lehenschaft des Ftm. Österreich ob der Enns, die Leschenprant ihnen verkauft und mit einem offenen besiegelten Brief aufgesandt hat, ${ }^{1}$ und verfügt, dass sie und ihre Erben das Gut gemäß Lehens- und Landrecht innehaben und nutzen sollen. An suntag nach sannd Gallntag.

KVr: C.d.i.i.c. - Rückseite der Wachsschüssel: Dorffer.

Org. im HHStA Wien (Sign. AUR 1477 X 19), Perg., rotes S 21 in wachsf. Schüssel mit wachsf. S 16 rücks. eingedr. an Ps.

Reg.: ChMel n. 7157; DeRs., Mon. Habsb. I/3 S. 709 n. 226.

1 Zu Hans Leschenprant s. Aspernig, Wiener Bürgermeister S. 55f. (mit Erwähnung des Verkaufs); PerGER, Wiener Ratsbürger S. 220.

\section{[Vor 1477 Oktober 22, -]}

K.F. überlässt Hans von Sinzendorf bis auf Widerruf das Ungeld zu Kremsmünster, wie es der verstorbene Bernhard Jörger ${ }^{1}$ innehatte, bestandweise gegen Zahlung von jährlich 32 Pfd. Pf.

Org. oder Kop. im bearbeiteten Bestand nicht überliefert. - Dep.: Erw. im Revers des Hans von Sinzendorf von 1477 Oktober 22 im HHStA Wien (Sign. AUR sub dat. 1477 V 2), ${ }^{2}$ Perg., 2 grüne SS des Ausst. und seines Vetters Christoph Jörger ${ }^{3}$ in wachsf. Schüsseln an Ps, mit Rta-Vermerk auf der Rückseite. ${ }^{4}$

Lit.: WALTER, Ungeld S. 68; HeINIG, Friedrich III./1 S. 276. 
1 Verstorben um 1477, s. zu ihm Wurm, Jörger von Tollet S. 41 f.

2 Unter dieser Sign. gemeinsam verwahrt mit n. 84.

3 Siehe zu ihm ausführlich WuRM, Jörger von Tollet S. 24-32; auch HeINIG, Friedrich III./1 S. 293.

4 Reg.: ChMEL n. 7159; DERS., Mon. Habsb. I/3 S. 700 n. 201.

\section{Oktober 23, Steyr}

K.F. verleiht Hieronymus Vischmeister zu Neu-Attersee ${ }^{1}$ von sundern gnaden wissenntlich mit dem brief, was wir im zurecht daran verleihen sullen oder mugen, eine halbe $\mathrm{Sege}^{2} \mathrm{zu}$ Unterach im Attersee und die halbe Fischweide in der Ach, welche dieser geerbt hat. Er verfügt, dass Vischmeister und dessen Erben die Stïcke innehaben und nutzen sowie jährlich von der halben Sege $10 \mathrm{Sch}$. Pf. und von der Fischweide $9 \mathrm{Sch}$. Pf. in guter landläufiger Münze in sein Schloss Kogl dienen sollen gemäß altem Herkommen und Lehens- und Landrecht. An pfintztag nach sannd Ursulen tag.

KVr: C.d.i.i.c. $-\mathrm{KVv}$ : Rta.

Org. im HHStA Wien (Sign. AUR 1477 X 23), Perg., rotes S 21 in wachsf. Schüssel mit rücks. eingedr. S (Siegelbild abgefallen, wohl S 16) an Ps.

Reg.: ChMel n. 7160; DERS., Mon. Habsb. I/3 S. 709 n. 227.

1 Zu Neu-Attersee (Kogl) s. BAUMERT/GrüLL, Burgen Oberösterreich 3 S. 16-18.

2 Sege, Säge, Segen: sehr großes Schleppnetz für den Fischfang, s. Grmм, Deutsches Wörterbuch 14 Sp. 1649,16 Sp. 81.

\section{November 8, Gmunden}

K.F. erklärt, den Dekan und das Kapitel des Kollegiatstifts Spital am Pyhrn aufgrund ihres Vorbringens, dem zufolge sie durch die dem Prälatenstand des Ftm. Österreich ob und unter der Enns auferlegten Steuern im Gegensatz zu den anderen Prälaten erheblich belastet werden, weshalb das Stift vasst in abnemen keme und wieder zu einer Pfarrkirche gedeyhen werde, sowie in Ansehung ihrer täglichen Gottesdienste, derer er tailhefftig zu werden hofft, von sunndern gnaden von den österreichischen Prälaten ganncz gesunndert zu haben, und gewährt ihnen die sunnder gnad und freyhait wissenlich in krafft des brieffs, bei Steuern auf den Prälatenstand Österreichs ob und unter der Enns nur $10 \mathrm{fl}$. oder Pfd. Pf., bei Steuern allein auf die obderennsischen Prälaten $20 \mathrm{fl}$. oder Pfd. Pf. je veranschlagte $1.000 \mathrm{fl}$. oder Pfd. Pf. zu leisten. Er verfügt, dass die österreichischen Prälaten künftig keinerlei Gewalt haben, von ihnen Steuern zu fordern, sowie diese nur ihm (K.F.) oder dem jeweiligen Einnehmer in genannter Höhe zu entrichten 
sind, und gebietet allen Prälaten, Adeligen und Städten sowie Amtleuten, Anwälten und Steuereinnehmern des Ftm. Österreich die Beachtung dieses Privilegs. Am sambstag nach sannd Lienhartstag (nach Druck).

KVr.: C.d.i.i.c. (nach Druck).

[Org. im OÖLA Linz, zuvor im HHStA Wien.] ${ }^{1}$ - Gedruckt bei ChMEL nach Org. im HHStA Wien.

Druck: ChMEL, Mon. Habsb. I/2 S. 285f. n. 106.

Reg.: CHMel n. 7165.

1 OÖLA (Sign. Urkunden Spital am Pyhrn [1190-1798], 1477 XI 08), s. monasterium.net (AT-OOeLA, Bestand Urkunden Spital am Pyhrn, sub dat.). - Der Bestand Spital am Pyhrn kam in den 1950er-Jahren vom HHStA Wien in das OÖLA.

\section{[Um 1477 November 30, Gmunden] $]^{1}$}

K.F.schließt mit Kg. Matthias von Ungarn folgende Übereinkunft: $(1)^{2}$ K.F. wird Gian Galeazzo (Sforza) und dessen Mutter Bona (von Savoyen), die unrechtmäßig als Hzz. von Mailand auftreten, ${ }^{3}$ innerhalb eines Jahres gerichtlich vorladen, zu gewaltsamen Okkupatoren und Inhabern fremden Guts erklären und entsprechende Urkunden ausstellen. (2) Nach dem Urteilsspruch wird er Friedrich, Sohn Kg. Ferdinands (I.) von (Neapel und) Sizilien, mit der Herrschaft Mailand samt allen Gebieten und Orten, derer sich Gian Galeazzo und Bona bemächtigt haben, als Vikariat gemäß Gewohnheit des Reichs erblich belehnen. Über die ihm (K.F.) und seinen Nachfolgern zu leistende jährliche Steuer soll auf dem in der Sache am kommenden St. Georgstag (1478 April 24) ${ }^{4}$ anberaumten Tag in Wien oder dort, wo sich der K. in seinen Erblanden aufhalten wird, durch seine, $\mathrm{Kg}$. Ferdinands und Kg. Matthias' Gesandten eine Übereinkunft erzielt werden. (3) Zur Erlangung Mailands wird er Friedrich mit allen nur möglichen Gnaden selbst versehen wie auch durch andere versehen lassen. (4) Sobald dieser in den Besitz der Herrschaft und vor allem der Stadt Mailand gelangt, wird ihm K.F. innerhalb von drei Jahren seine Tochter Kunigunde zur Gemahlin geben laut den auf dem genannten Tag ausgehandelten Bedingungen. (5) Stimmt Kg. Ferdinand den Vertragsartikeln zu, sollen jene $100.000 \mathrm{fl}$., die gemäß anderen Verträgen ${ }^{5}$ von Österreich an $\mathrm{Kg}$. Matthias zu zahlen sind, dem $\mathrm{K}$. zufallen; verweigert $\mathrm{Kg}$. Ferdinand aber seine Zustimmung, fallen die $100.000 \mathrm{fl}$. vertragsgemäß an $\mathrm{Kg}$. Matthias. (6) Abschließend wird vereinbart, dass die Gesandten für den genannten Tag mit allen Vollmachten, auch hinsichtlich erforderlicher Vorkehrungen gegen die Türkeneinfälle, ausgestattet sowie der $\mathrm{Kg}$. von Kastilien und der Hz. von Burgund ebenfalls zur Entsendung von Bevollmächtigten aufgefordert werden sollen. 
Org. oder Kop. im bearbeiteten Bestand nicht überliefert. - Dep.: Ein gleichlautender ,Zettel“ (cedula) erw. im Vertragsexemplar (lat.) Kg. Matthias' von Ungarn von 1477 November 30, Korneuburg, im HHStA Wien (Sign. AUR 1477 XI 30), Pap., rotes S des Ausst., rücks. aufgedr. (großteils abgefallen). ${ }^{6}$

Lit.: Fraknó, Mathias Corvinus S. 196; MAYER, Abdankung S. 192-196; BachmanN, Reichsgeschichte 2 S. 602f.; StARZER, Klosterneuburg S. 121; RÁszó, Feldzüge S. 6; NEHRING, Matthias Corvinus S. 92; Isenmann, Kaiserliche Obrigkeit S. 319; HoENsch, Matthias Corvinus S. 158.

Das in der Corroboratio als cedula bezeichnete Vertragsexemplar Kg. Matthias' von Ungarn beginnt mit den Worten Memorie comendatur quod serenissimi principes [...] und nennt beide Vertragsparteien in objektiver Form in der dritten Person. Nur die Siegelankündigung erfolgt durch Kg. Matthias in der ersten Person.

Die nur in dieser Überlieferung bekannte und daher immer unter dem Datum 1477 November 30 zitierte Übereinkunft ist ein Geheimabkommen ${ }^{7}$ zum Friedensvertrag von 1477 Dezember 1 (s. n. 106), der allerdings seitens Matthias tatsächlich erst am 18. Dezember angenommen und rückdatiert wurde. Nach MAYER (S. 192) und NEHRING (S. 92) waren vermutlich die Geheimartikel der Grund für die Verzögerung. Auch wenn die Ausstellung des ksl. „Vertragszettels“ erst im Laufe dieses Zeitraums erfolgt sein dürfte, ist eine (Rück-)Datierung auf den 30. November entsprechend dem ungarischen Vertragsexemplar oder auf den 1. Dezember, dem Datum des Friedensvertrags, mit hoher Wahrscheinlichkeit anzunehmen.

Das Geheimabkommen begünstigte zwar vorrangig die Italienpolitik Kg. Matthias' - Prinz Friedrich war ein Bruder der Kgn. Beatrix -, eröffnete aber dem Kaiser die Option auf die laut Hauptvertrag an Matthias zu leistende Entschädigung von $100.000 \mathrm{fl} .{ }^{8}$ Hinsichtlich der vereinbarten Verurteilung Hzn. Bonas und Gian Galeazzos wurde seitens K.F. nichts unternommen; auch fand keine Zusammenkunft in der Angelegenheit im April 1478 statt. Im Januar 1479 kündigte Matthias Corvinus das Abkommen, entband K.F. von dem Heiratsversprechen und forderte die Zahlung des Geldes ein. ${ }^{9}$

1 Zur Datierung s. den Kommentar, zum Ausstellungsort CHMrL, Mon. Habsb. I/3 S. 633 f. n. 4 (Kredenzbrief Kg. Matthias' von 1479 Januar 12 für Propst Georg von Pressburg), wo es in Bezug auf das vorliegende Abkommen heißt: super secretis illis articulis apud Gmunden conceptis.

2 Die Nummerierung der Artikel folgt der Gliederung des Vertragstextes, in dem jeder Artikel mit conventum et conclusum est eingeleitet wird.

3 Eine reichsrechtliche Legitimierung der Herrschaft der Sforza als Hzz. von Mailand (seit 1450) durch K.F., der auf eine Resignation Mailands an das Reich bestand, war nie erfolgt, s. REINLE, Ulrich Riederer S. $258 \mathrm{f}$.

4 Zur Datierung des St. Georgstags auf April 24 s. Regg.F.III. H. 12 n. 6, Anm. 2.

5 Siehen 106.

6 Druck (ungarisches Vertragsexemplar): CHMEL, Mon. Habsb. I/2 S. 117-119 n. 17; Reg.: Kollar, Ursinus Velius S. 320f. n. 2; CHMEL n. 7169; LichNowsKY(-BIRK) 7 n. 2115. - Rücks. Ausstellervermerk: 
Concordie et conventiones inter illustrissimos dominos Fredericum Romanorum imperatorem et Mathiam Hungarie regem super quibusdam articulis contra Joannem Galiatz et eius genitricem occasione violente occupationis et iniuste detentionis ducatus Mediolani etc. concluse Closterneuburg [!] et sigillate per predictum regem Hungarie. Anno Domini mccccloxvii.

7 Zur Bezeichnung als, ,geheime Artikel"s. Anm. 1.

8 Siehe n. 106, Artikel 7.

9 Siehe dazu Chmel (wie Anm. 1); MaYer, Abdankung S. 196f.; NeHring, Matthias Corvinus S. 109

\section{[1477 Dezember 1, Gmunden] ${ }^{1}$}

K.F. beurkundet den durch Vermittlung des päpstlichen Legaten Bf. Gabriel von Erlau, des Elekten Johann von Großwardein, des Zipser Grafen Emmerich Zapolya, des Pressburger Gespans Nikolaus Bánfi und des Propstes Georg von Pressburg, päpstlicher Protonotar, geschlossenen Frieden mit $\mathrm{Kg}$. Matthias von Ungarn:

$(1)^{2}$ Zwischen dem K. und seinen Nachfolgern als Hzz. von Österreich sowie ihren Erblanden einerseits und $\mathrm{Kg}$. Matthias und seinen Nachfolgern als Könige von Ungarn sowie dem Kgr. Ungarn andererseits soll ein aufrichtiger und beständiger Frieden bestehen. Die beiden Fürsten sollen sich wie Vater und Sohn zugetan sein und einander wie wahre Freunde und engste Verbündete fördern und vor Übel bewahren. Es sollen keinerlei Feindseligkeiten gegen die Herrschaften des anderen gerichtet werden und alle diesem Bündnis entgegenstehenden Bündnisse und Verträge aufgehoben sein, mit Ausnahme jener mit dem Papst und Kg. Ferdinand (I.) von (Neapel und) Sizilien.

(2) Zur Wahrung des Friedens verpflichten sich beide Parteien, in ihren Ländern und Herrschaften keinen Gefolgsmann zum Schaden des anderen zu unterhalten.

(3) Weiters sollen sie nichts mehr von den Untertanen des anderen ohne dessen ausdrückliche Zustimmung einfordern, sondern alle Leute zum vollen Gehorsam gegenüber ihrem Herrn entlassen. Entgegenstehende Verpflichtungen werden hiermit aufgehoben; ksl. Briefe, die dem Kg. zugunsten der Verbündeten gegeben wurden, sollen K.F. zurückgestellt werden.

(4) Sofort nach Übergabe der in gleichlautender Form wie dem Erstgeborenen (Wladislaw II. von Böhmen) ${ }^{3}$ des Kg. (Kasimir IV.) von Polen ausgestellten Urkunden über die Belehnung Kg. Matthias' mit dem Kgr. Böhmen und dem Kurfürstentum des Reichs soll dieser sein Heer aus den Ländern des K. abziehen und die den Lehenseid enthaltenden Reverse ausstellen. ${ }^{4}$ Die persönliche Belehnung des Kg. durch K.F. wird bei ihrer ersten Zusammenkunft erfolgen.

(5) Sollten der Erstgeborene des Kg. von Polen oder der Kg. von Polen selbst wegen der Belehnung einen Krieg gegen K.F. beginnen, verpflichtet sich Kg. Matthias zur Hilfeleistung.

(6) Vereinbart wird die Einberufung eines Landtags der Stände Österreichs ob und unter Enns auf den kommenden Tag der Erscheinung des Herrn (1478 Januar 6) in 
Krems mit freiem Geleit für jeden Teilnehmer für die Hin- und Rückreise und den Aufenthalt. ${ }^{5}$

(7) Die vier Stände Österreichs ob und unter der Enns sollen sich dort entsprechend den darüber ausgestellten ksl. Briefen zur Zahlung von insgesamt $100.000 \mathrm{fl}$. an Kg. Matthias verschreiben, zahlbar zu je $50.000 \mathrm{fl}$. am St. Martinstag (November 11) 1478 und 1479 in Hainburg zuhanden des Kg. oder seines Bevollmächtigten. Im Falle nicht termingerechter Zahlung ist Kg. Matthias berechtigt, die Summe mit allen Mitteln einzufordern ohne den Frieden zu verletzen, der in allen anderen Punkten in Kraft bleibt. $^{6}$

(8) Die Schuldbriefe des K. und der Stände und die kgl. Ledigsag- und Quittbriefe aller Burgen und Städte werden gleichzeitig zu treuen Händen hinterlegt. Danach erfolgt unverzüglich die Restituierung aller von $\mathrm{Kg}$. Matthias im Krieg eingenommenen Burgen, Städte und Orte.

(9) Deren Einwohnern soll keinerlei Gewalt geschehen und der Abzug der Leute des Kgr. Ungarn unbehelligt von beiden Seiten erfolgen.

(10) Die mit Kg. Matthias verbündeten Untertanen des K. sollen für ihre Person und ihre Güter denselben ksl. Schutz genießen wie die Anhänger des K., auf Wunsch ksl. Gnadenbriefe erhalten, sich umgekehrt zu Treue und Gehorsam verschreiben, alle ihnen entzogenen Güter zurückerhalten, die zu Kriegszeiten errichteten Befestigungen über der Donau und anderswo zerstören, von der Einhebung aller Zölle zu Land und zu Wasser und anderer unrechtmäßigen Aufschläge unverzüglich ablassen und dem K. den schuldigen Gehorsam erweisen; die Exkommunizierten sollen absolviert werden.

(11) Gegenseitige Schuldbriefe des Kg. und seiner Verbündeten sind zurückzustellen und werden zudem hiermit annulliert.

(12) Alle Adeligen und Nichtadeligen, alle geistlichen und weltlichen Personen, die sich im Krieg Kg. Matthias unterworfen haben oder auf seiner Seite gestanden sind, sollen Sicherheit und Schutz genießen.

(13) Über die gegenüber K.F. erhobenen Soldforderungen der zu Kg. Matthias übergelaufenen Söldner soll auf dem Tag zu Krems eine Einigung erzielt werden.

Org. oder Kop. im bearbeiteten Bestand nicht überliefert, dem Druck zufolge (Perg.) mit anh. ksl. S. - Dep.: Eine gleichlautende Urkunde mit anh. ksl. S erw. in der Corroboratio des Vertragsexemplars (lat.) Kg. Matthias' von Ungarn von 1477 Dezember 1, Korneuburg, im HHStA Wien (Sign. AUR 1477 XII 1), Perg., rotes S des Ausst. in wachsf. Schüssel an Ps, mit rücks. Kanzleivermerk (Blattmitte): Verainigung kunig Mathias zu Hungern fur sich und sein nachkomen des kunigreichs zu Hungern mit kayser Friderichen und seinen nachkomen hertzogen zu Osterreich des datum laut anno Domini etc. 1477 ?

Druck: TelEkI, Hunyadiak kora 12 S. $37-42$ n. 610 (nach Kop.). ${ }^{8}$

Reg.: LICHNOwsKY(-BIRK) 7 n. 2117; Regg.F.III. H. 11 n. 491 (Auszug in dt. Übersetzung). 
Lit.: Fraknó, Mathias Corvinus S. 196f.; MAYeR, Abdankung S. 192-196; BachmanN, Reichsgeschichte 2 S. 603f.; STARZER, Korneuburg S. 126f.; VANCSA, Geschichte 2 S. 501f;; Nehring, Matthias Corvinus S. 91-95; IsenManN, Kaiserliche Obrigkeit S. 318f.; Hoensch, Matthias Corvinus S. 158; BEGERT, Böhmen S. 226; knapp NIEDERSTäTTER, Jahrhundert der Mitte S. 355; Kovács, Ungarn im Spätmittelalter S. 178; Kalous, Matyáš Korvín S. 168; Holtz, Länder der böhmischen Krone S. 33; s. auch die Quellensammlung von NEHRING, Quellen S. $96 f$. nn. 55-61.

Der Friedensvertrag wurde von K.F. am 1. Dezember 1477 ausgestellt, von Kg. Matthias aber erst am 18. Dezember angenommen und rückdatiert; Grund der Verzögerung waren vermutlich die Verhandlungen über das geheime Zusatzabkommen (s. n. 105). Trotz noch nicht erfolgter Ratifizierung seitens Matthias wurde aber von beiden Seiten bereits mit der Umsetzung der Vertragsbestimmungen begonnen (s. nn. 107, 108). Am 18. Dezember 1477 gebot Matthias den unter seinem Schutz stehenden österreichischen Adeligen die Einhaltung des Friedens, am 9. Januar 1478 folgte das ksl. Friedensgebot (s. zu beiden Mandaten n. 110). Der feierliche Austausch der Vertragsurkunden erfolgte zu Weihnachten 1477 in Korneuburg. ${ }^{9}$

1 Datierung und Ausstellungsort nach Druck bei TeLEKr: Datum in oppido nostro Gmundensi die prima mensis decembris.

2 Die Nummerierung der Artikel folgt der Gliederung des Vertragstextes, in dem jeder Artikel mit conclusum est eingeleitet wird.

3 Wladislaw II. von Böhmen wird in der Urkunde nur als primogenitus regis Polonie bezeichnet.

4 Zur Lehensurkunde für Wladislaw von Böhmen von 1477 Juni 10 s. n. 82; zur Lehensurkunde für Kg. Matthias und dessen Huldigungsrevers s. nn. 107, 108.

5 Der Landtag trat schließlich am 19. Februar 1478 in Krems zusammen und dann erneut am 20. April in Wien; s. zu den Landtagen ChMEL, Materialien 2 S. 345-348 n. 281; DERS., Mon. Habsb. I/2 S. 524f. n. 160 , S. $549-555$ n. 248 und S. 127 f. n. 22 (Geleitbrief Kg. Matthias' fur die Landtagsbesucher von 1477 Dezember 19); s. auch DERs., Conzeptensammlung 2 S. 319 n. 427, S. 340 f. n. 433, S. 345 n. 449, S. 384 n. 533.

6 Auf dem Wiener Landtag von 1478 April 20 (s. Anm. 5) wurde die Unterfertigung des Schuldbriefs und die Modalitäten der Aufbringung der Entschädigung beschlossen; zum Schuldbrief des K. und der Stände s. CHMEL, Mon. Habsb. I/2 S. 314 f. n. 4 (zu Februar 1478), weiters dazu DERs., Mon. Habsb. I/3 S. 670 f nn. 127, 128, S. 687 n. 169. - Die Bezahlung der Entschädigung sowie die Befriedigung der Forderungen der Söldner gemäß Artikel 13 des Vertrags und die im Gegenzug erhobenen Forderungen der Stände an K.F. waren eine in der Folgezeit zahlreiche Landtage bestimmende Thematik (zur Bezahlung s. auch unten die nn. 210 und 279); laut einem ksl. Schreiben von 1480 März 20 (Regg.F.II. H. 11 n. 580) war zu diesem Zeitpunkt erst die Hälfte der Summe bezahlt worden; s. dazu ScновеR, Eroberung S. 17-23; VANCSA, Geschichte 2 S. 502-505 (mit tlw. irrigen Quellenangaben); IsENMANN, Kaiserliche Obrigkeit S. 322-332; NeHring, Matthias Corvinus S. $119 \mathrm{f}$.

7 Druck (ungarisches Vertragsexemplar): PRAY, Annales Hungariae IV S. 114-116; KuRZ, Oesterreich 2 S. 258-262, Beil. n. 43; ChMmL, Mon. Habsb. I/2 S. 119-122 n. 18; Reg.: Kollar, Ursinus Velius S. 321 n. 3; CHMEL n. 7170.

8 Bestätigung Kg. Wladislaws II. für Kardinal Thomas (Tamás) Bakócz, Eb. von Gran, von 1504 Juli 16 im UStA (Sign. DL 18137); die Edition von Telekr nach NeHRING, Matthias Corvinus S. 91, allerdings fehlerhaft. 
9 Zur Rückdatierung s. MAYER, Abdankung S. 192; ihm folgt NeHRnNG, ebd.; die Annahme des Vertrags am 18. Dezember durch Matthias Corvinus auch erw. in einem ksl. Schreiben von 1477 Dezember 29 (ChMEL, Conzeptensammlung 1 S. 98 n. 51) und im Mandat von 1478 Januar 9 (s. n. 110); zum Austausch der Vertragsurkunden s. CHMEL, Materialien 2 S. 344f. n. 280.

\section{[1477 Dezember 2, Gmunden] ${ }^{1}$}

K.F. belehnt Kg. Matthias von Ungarn als Kg. von Böhmen mit dem Erzschenkenamt und Kurfürstentum des Reichs und der Mgft. Mähren samt allen anderen zur Krone Böhmens gehörigen Ländern, Herrschaften, Burgen, Städten etc. ${ }^{2}$

Org. oder Kop. im bearbeiteten Bestand nicht überliefert. - Dep.: Erw. im Huldigungsrevers (lat.) Kg. Matthias' von 1477 Dezember 13, Korneuburg, im HHStA Wien (Sign. AUR 1477 XII 13), ${ }^{3}$ Perg., rotes S des Ausst. in wachsf. Schüssel an grüner $\mathrm{Ss} ;{ }^{4}$ davon sechs zeitgleiche $\mathrm{Ab}-$ schriften ebd. (Sign. AUR 1477 XII 13 [3], AUR sub dat. 1480 III 23 [1], Urkundenabschriften Österr. Urkunden, Kt. 46 sub dat. [zeitgleiche Abschrift mit Korrekturen des 18. Jh.], Urkundenabschriften Böhm. Urkunden, Kt. 85 sub dat. [1]), alle Pap.; eine Abschrift des 18. Jh. ebd. (Sign. Urkundenabschriften Böhm. Urkunden, Kt. 85 sub dat.), Pap. ${ }^{5}$

Reg.: Regg.F.III. H. 23 n. 653.

Lit. siehe bei $\mathrm{n} .106$.

Bislang ist keine Lehensurkunde für $\mathrm{Kg}$. Matthias bekannt, weshalb vermutet wurde, dass sich darauf Bezug nehmende Formulierungen auf den Friedensvertrag von 1477 Dezember 1 (s. n. 106) beziehen. ${ }^{6}$ In seinem Huldigungsrevers erwähnt $\mathrm{Kg}$. Matthias allerdings einen ksl. Brief (iuxta contenta litterarum suarum imperialium nobis desuper datarum). Zudem ist im HHStA Wien in einer die Belehnung betreffenden Materialsammlung ${ }^{7}$ der ksl. Kanzlei das unvollständige Konzept eines Lehensbriefs überliefert, dieser datiert mit 1477 Dezember 2, Gmunden, und der Siegelankündigung zufolge besiegelt mit dem Majestätssiegel, weshalb eine Urkundenausfertigung anzunehmen ist.

1 Die Datierung ergibt sich aus dem im HHStA Wien überlieferten, mit 1477 Dezember 2 datierten Konzept und aus den am selben Tag ausgefertigten ksl. Mandaten, Matthias als $\mathrm{Kg}$. von Böhmen Gehorsam zu leisten (s. Anm. 7 und den Kommentar).

2 Die Lehensurkunde für $\mathrm{Kg}$. Matthias hatte gemäß Artikel 4 des Friedensvertrags von Gmunden (s. n. 106) den gleichen Wortlaut wie jene für Wladislaw II. (s. n. 82) zu haben. - Zum Inhalt der Belehnung laut dem Huldigungsrevers, der analog zur Lehensurkunde Wladislaws II. die Lehensnahme des Kgr. Böhmen nicht ausdrücklich erwähnt (s. dazu den Kommentar zu n. 82), und dem damit verbundenen Diskurs über die lehensrechtliche Abhängigkeit Böhmens vom Reich s. BEGERT, Böhmen S. 226 unter Heranziehung der im HHStA Wien überlieferten Konzepte des Huldigungsreverses (s. unten Anm. 7); auch der Dorsualvermerk der ksl. Kanzlei (s. Anm. 4) nennt nur die Belehnung mit der Mgft. Mähren. Da der Lehenseid 
(s. n. 108) allerdings dem „Standard" der Reichsfürstenbelehnungen entspricht, schließt BEGERT, ebd. S. 227, dass K.F. seine Rechtsposition hinsichtlich der Lehensabhängigkeit des Kgr. Böhmen wahren konnte.

3 Dem Revers beiliegend dessen Konzept (lat., Pap.) und die von K.F. besiegelte deutsche Eidesformel (s. n. 108).

4 Rücks. zeitgleicher Kanzleivermerk (Blattmitte): Kunig Mathias zu Hungern und Behaim revers der lehen halben die marggrafschafft Merhern von kayser Friderichen emphangen des datum laut anno Domini etc. 1477 .

5 Druck (Revers): ChмеL, Mon. Habsb. I/2 S. 124-126 n. 20 und I/3 S. 278f. n. 115 b (jeweils nach zeitgleicher Kop.); Reg.: LichNowsKY(-BIRK) 7 n. 2126.

6 Siehe Regg.F.III. H. 10 n. 437, H. 11 n. 492 , H. 23 n. 653 , H. 26 n. 700.

7 Die nicht im bearbeiteten Bestand überlieferte Materialsammlung mit Konzepten und zeitgleichen Abschriften zur Belehnung ist verwahrt unter der Sign. Österr. Akten, Böhmen, Kt. 1, Fasz. 1 sub dat. 1477, 1-2 Dec., die unvollständige Lehensurkunde auf fol. 32rv (fehlend Intitulatio, Publicatio und Narratio). Teil des Materials ist neben drei Konzepten des Huldigungsreverses Kg. Matthias' und einem Auszug des Friedensschlusses (s. n. 106) auch das Konzept eines ksl. Mandats (lat.) von 1477 Dezember 2 an die Einwohner Böhmens, Matthias den schuldigen Gehorsam zu leisten, samt einem Empfängerverzeichnis; Mandat und Verzeichnis gedruckt bei ChMEL, Mon. Habsb. I/2 S. 123f. n. 19; Reg.: ChMEL n. 7172; LichNowsKY(-BRRK) 7 n. 2119. - Das Empfängerverzeichnis umfasst Adelige, Städte, die schlesischen Herzöge und die böhmischen Kronvasallen; einige dieser Mandate, alle datiert mit 1477 Dezember 2, sind in den Regg.F.III. bereits erfasst: s. H. 10 n. 437, H. 11 nn. 492, 493, H. 20 n. 249, H. 23 n. 654, H. $26 \mathrm{nn}$. 700, 701, 702; s. auch LichNowsKy(-BrRK) 7 n. 2120. - Siehe dazu ein weiteres ksl. Mandat zur Gehorsamsleistung an die Untertanen des Kgr. Böhmen von 1477 Dezember 4 bei Crmel n. 7174 und LichNowSKY(-BIRK) 7 n. 2121 (nach Kop.).

\section{[1477 Dezember 2, Gmunden] ${ }^{1}$}

K.F. beglaubigt durch Besiegelung den von Kg. Matthias von Ungarn und Böhmen zu leistenden Lehenseid. Dieser gelobt und schwört auf das Evangelium, dem K. und dessen Nachfolgern im Reich, röm. Kaisern und Königen, sowie dem Reich treu und gehorsam zu sein, gegen dessen Person, Ehre, Würde oder stand in keiner Weise zu handeln, sondern Ehre und Nutzen des K. und des Reichs nach ganzem Vermögen zu fördern, Vorhaben oder Handlungen gegen dessen Person oder die ksl. Majestät zu verhüten und den $\mathrm{K}$. unverzüglich davor zu warnen sowie alles zu tun, wie es einem $\mathrm{Kg}$. von Böhmen, Mgf. von Mähren, Kurfürsten und getrewen lehenman des Reichs nach Recht und Gewohnheit zu tun gebührt. ${ }^{2}$

Von K.F. besiegelte, undatierte Notel (dt.), beiliegend dem Huldigungsrevers Kg. Matthias' von 1477 Dezember 13, Korneuburg, im HHStA Wien (Sign. 1477 XII 13), Pap., rotes S 18 vorders. aufgedr. (großteils abgefallen) unter (abgelöster) Papieroblate.

Druck: BEGERT, Böhmen S. 605 n. 3C.

Reg.: LichNowsKy(-BIRK) 7 n. 2127 (zu Dezember 13). 
Lit.: BEGERT, Böhmen S. 227f.; s. auch die in n. 106 genannte Lit.

Der Lehenseid im Huldigungsrevers des Matthias (s. n. 107) ist die lateinische Übersetzung der von K.F. an Matthias übersandten Eidesformel. Die Notel enthält nur die Eidesformel, beginnend mit Ich, Matthias, kunig zu Hungern und zu Beheim etc., und ist unterhalb des Textes von K.F. besiegelt.

1 Datierung und Ausstellungsort nach Druck bei BEGERT, der den 2. Dezember annimmt, allerdings als Ausstellungsort irrtümlich Gmünd statt Gmunden angibt. Mit hoher Wahrscheinlichkeit erfolgte die Ausstellung der Notel am selben Tag wie alle anderen die Belehnung betreffenden Stücke.

2 Zur Eidesformel s. auch n. 107, Anm. 2.

\section{Dezember 2, Gmunden}

K.F. setzt alle Reichsuntertanen, die von ihm und dem hauss Österreich Pfandschaften innehaben und mit diesem Brief oder einer glaubwürdigen Abschrift in der Sache ersucht werden, von der Beschwerde seines Vetters Hz. Sigmund von Österreich in Kenntnis, der zufolge sie die Untertanen und Einwohner an den schuldigen Eiden diesem gegenüber hindern würden, obgleich jedem Fürsten und dem Haus Österreich die oberkait vorbehalten sei. Er gebietet ihnen daher unter Androhung des Verlustes der Pfandschaft sowie seiner Strafe und Ungnade, ihre Untertanen und Einwohner zur Eidesleistung gegenüber $\mathrm{Hz}$. Sigmund als regierundem fursten, irem rechten herrnn, und dem Haus Österreich zu veranlassen, unbeschadet ihrer Pfandschaften und Verschreibungen, um nicht zum Handeln gegen sie gezwungen zu werden. Am anderen tag des monads december (nach Kop.).

KVr: A.m.p.d.i. (nach Kop.).

Org. im bearbeiteten Bestand nicht überliefert, der Kop. zufolge mit rücks. aufgedr. S. - Kop.: Vidimus Abt Alexius' von Wilten von 1477 Dezember 13, Wilten, im HHStA Wien (Sign. AUR 1477 XII 13), Perg., rotes (spitzovales) S des Ausst. in wachsf. Schüssel an Ps; davon Abschrift des 18. Jh. ebd. (Sign. Urkundenabschriften Österr. Urkunden, Kt. 46 sub dat.), Pap.

Druck: ChMel, Mon. Habsb. I/2 S. 213f. n. 53.

Reg.: ChMel n. 7171; LichNowsKy(-BIRK) 7 n. 2118.

Das Vidimus wurde auf Wunsch „Erzherzog“ Sigmunds von Österreich ausgestellt, dem wenige Tage nach der Ausstellung der vorliegenden ksl. Urkunde - in dieser noch als Herzog tituliert - von K.F. vermutlich ad personam die Führung des Erzherzogstitel gestattet worden war. ${ }^{1}$

1 Die Erlaubnis zur Führung des Erzherzogstitels nur überliefert im Revers Ehz. Sigmunds von 1477 De- 
zember 8 im HHStA Wien (Org. und Kop., Sign. FU 736/1,2); Druck: ChMel, Mon. Habsb. I/2 S. $214 f$. n. 54; Reg.: LICHNOwSKY(-BIRK) 7 n. 2125; s. dazu LUGER, Bestätigung S. 257; zum Erzherzogstitel s. auch oben die Einleitung S. 22-24.

\section{Januar 9, Graz}

K.F. befiehlt allen Hauptleuten, Landmarschällen, Adeligen, Städten und Märkten, Amtleuten, Rottmeistern, Dienstleuten und allen anderen Untertanen seiner Erbländer, den ewigen cristenliche (n) Frieden ${ }^{1}$ zwischen ihm und $\mathrm{Kg}$. Matthias von Ungarn und Böhmen, seinem Sohn, sowie ihren Ländern, Leuten und Untertanen, der am phintztag vor sannd Thomas tag des heyligen zwelifboten nagstverganngen (1477 Dezember 18) enntlich beschlossen und angenommen wurde, stet und vesticklich zu halten. Da vertragsgemäß $\beta^{2}$ alle Gefangenen, geschetzt oder ungeschetzt sowie auch jene, für die noch kein Lösegeld bezahlt wurde, freigelassen werden sollen, befiehlt er ihnen zudem, alle noch gefangenen Diener, Bürger, Leute, Dienstleute und Holden des ungarischen $\mathrm{Kg}$. unverzüglich und unentgeltlich freizulassen, sie und ihre Bürgen bzw. schriftliche Bürgschaften freizusprechen und künftig nicht mehr zu belangen. An freitag nach der heyligen dreyr Kunig tag. ${ }^{3}$

$\mathrm{KVr}$ : C.d.i.i.c.

Org. im HHStA Wien (Sign. AUR 1478 I 9), Pap., rotes S 21 rücks. aufgedr. - Kop.: Abschrift des 18. Jh. ebd. (Sign. Urkundenabschriften Collat. Urkunden, Kt. 73 sub dat.), Pap.

Druck: ChMel, Mon. Habsb. I/2 S. 518f. n. 144 (zu Januar 7).

Reg.: Kollar, Ursinus Velius S. 321 n. 5; ChMel n. 7177; LichNowsky(-BIRK) 8 n. 8; s. auch CHMEL, Conzeptensammlung 1 S. 103 n. 68 (zu Januar 2).

Lit.: KURZ, Oesterreich 2 S. 138; s. auch die in n. 106 genannte Lit.

1 Siehe n. 106.

2 Ein entsprechender Passus zur Freilassung der Gefangenen ist allerdings im Vertrag nicht enthalten.

$3 \mathrm{Kg}$. Matthias hatte am 18. Dezember 1477 allen unter seinem Schutz stehenden österreichischen Adeligen die Einhaltung des Friedens befohlen und entband am 28. Februar 1478 alle Österreicher von den ihm geleisteten Eiden; Orgg. im HHStA Wien (Sign. AUR 1477 XII 18, AUR 1478 II 28); Druck: KuRZ, Oesterreich 2 S. 262-264, Beil. nn. 44, 45; CHMEL, Mon. Habsb. I/2 S. 126f. n. 21, S. 313 n. 3.

\section{[1478 Januar 9, Graz ${ }^{1}$}

K.F. überträgt Niklas Rauber bis auf Widerruf die Pflege der Hauptmannschaft Mitterburg samt Schloss und allem Zubehör und überlässt ihm die zugehörigen Nutzen 
und Renten, wie sie die verstorbenen ksl. Hauptleute Thomas Elacher und Jakob Raunacher ${ }^{2}$ innehatten, bestandweise ab Weihnachten 1478 (1477 Dezember 25) gegen Zahlung von jährlich 1.200 fl. ung., inklusive jener Nutzen und Renten, die der Elacher und der Raunacher treuhändisch gegen Verrechnung innehatten.

Org. oder Kop. im bearbeiteten Bestand nicht überliefert. - Dep.: Erw. im Revers Niklas Raubers, Hauptmann zu Triest und Mitterburg, von 1478 Januar 14 im HHStA Wien (Sign. AUR sub dat. 1478 VI 10), ${ }^{3}$ Perg., 7 grüne SS des Ausst., seiner vier Bürgen Wilhelm und dessen Bruder Jörg von Auersperg, Leonhard von Herberstein und Christoph Mindorfer ${ }^{4}$ sowie des Zobelberger Pflegers Hermann Rauber und des Premer Pflegers Niklas Obernburger in wachsf. Schüsseln an Ps, mit Rta-Vermerk auf der Rückseite. ${ }^{5}$

Reg.: Chmel, Conzeptensammlung 1 S. 111 n. 93.

Siehe n. 112.

1 Datierung und Ausstellungsort nach Reg. bei CнмеL.

2 Zu Jakob Raunacher, ksl. Rat, und Thomas Elacher s. HeInIG, Friedrich III./1 S. $234 f$.

3 Der Revers wurde am Freitag vor dem St. Antoniustag 1478 ausgestellt, hier der Festtag am 17. Januar (Antonius der Große, der Einsiedler). Die Datierung (und daher Sign.) im HHStA Wien erfolgte nach dem Antoniustag am 13. Juni (Antonius von Padua).

$4 \mathrm{Zu}$ den ksl. Räten Christoph Mindorfer und Wilhelm von Auersperg s. Heıng, Friedrich III./1 S. $208 \mathrm{f}$. und 231f., zu Leonhard von Herberstein ebd. S. 188.

5 Reg.: СнмгL, Conzeptensammlung 1 S. 119 n. 121; zur Übergabe der Hauptmannschaft s. ebd. S. $112 f$. nn. 96, 102, und DERS., Conzeptensammlung 2 S. 120 n. 309.

\section{[1478 Januar 9, Graz $]^{1}$}

K.F. überlässt Niklas Rauber, Hauptmann zu Triest und Mitterburg, bis auf Widerruf die Kastelle und Ämter zu Kastav, Veprinitz und Moschenitz samt Nutzen, Renten und Zubehör, wie sie der verstorbene Jakob Raunacher ${ }^{2}$ innehatte, bestandweise ab Weihnachten 1478 (1477 Dezember 25) gegen Zahlung von jährlich 250 fl. ung.

Org. oder Kop. im bearbeiteten Bestand nicht überliefert. - Dep.: Erw. im Revers Niklas Raubers von 1478 Januar 9, Graz, im HHStA Wien (Sign. AUR 1478 I 9), Perg., 2 grüne SS des Ausst. und des Ritters Wilhelm von Auersperg ${ }^{3}$ in wachsf. Schüsseln an Ps, mit Rta-Vermerk auf der Rückseite. ${ }^{4}$

Reg.: ChMEL, Conzeptensammlung $1 \mathrm{~S} .111 \mathrm{n} .95 .^{5}$

Siehe n. 111 .

1 Datierung und Ausstellungsort nach Reg. bei CнмmEL. 
2 Ksl. Rat, s. zu ihm HenNig, Friedrich III./1 S. $234 \mathrm{f}$.

3 Ksl. Kämmerer und Rat, s. zu ihm ebd. S. $231 \mathrm{f}$.

4 Reg.: TOMAScheK, Urkunden-Regesten Krain 2 n. 46.

5 Zur Übergabe der Kastelle s. ChMEL, Conzeptensammlung $1 \mathrm{~S} .112$ f. nn. 96, 102 und Conzeptensammlung 2 S. 120 n. 309.

\section{[1478 Januar 15, Graz $]^{1}$}

K.F. überträgt Friedrich Dürrer bis auf Widerruf die Hauptmannschaft von Schloss und Stadt Portenau pfleg- und bestandweise ab Mariä Lichtmess 1478 (Februar 2) gegen Zahlung von jährlich $400 \mathrm{fl}$. ung.

Org. oder Kop. im bearbeiteten Bestand nicht überliefert. - Dep.: Erw. im Revers Friedrich Dürrers von 1478 Januar 16 im HHStA Wien (Sign. AUR sub. dat. 1478 VI 12), ${ }^{2}$ Perg., 2 grüne SS des Ausst. und Erhard Lembachers in wachsf. Schüsseln an Ps, mit Rta-Vermerk auf der Rückseite.

Reg.: CHMEL, Mon. Habsb. I/2 S. 922 n. $1277 .^{3}$

1 Datierung und Ausstellungsort nach Reg. bei CHMEL.

2 Der Revers wurde am Freitag vor dem St. Antoniustag 1478 ausgestellt, hier der Festtag am 17. Januar (Antonius der Große, der Einsiedler). Die Datierung (und daher Sign.) im HHStA Wien erfolgte nach dem Antoniustag am 13. Juni (Antonius von Padua).

3 Mit Erwähnung des Reverses Friedrich Dürrers.

\section{[1478 Januar 15, Graz $]^{1}$}

K.F. überträgt Richter, Rat und allen Bürgern der Stadt Gmunden bis auf Widerruf die Pflege von Schloss Wildenstein samt dem Landgericht und überlässt ihnen bestandweise das Amt Gmunden mit der Saline Hallstatt und das Ungeld zu Gmunden mit allem Zubehör, wie von Wilhelm Lesch innegehabt, ${ }^{2}$ für ein Jahr ab Mittwoch vor dem Sonntag Reminiscere 1478 (Februar 11) gegen Zahlung von 3.000 fl. rh. für Saline und Amt und 230 Pfd. Pf. für das Ungeld. ${ }^{3}$

Org. oder Kop. im bearbeiteten Bestand nicht überliefert. - Dep.: Erw. im Revers von Richter, Rat und den Bürgern von Gmunden von 1478 Januar 29 im HHStA Wien (Sign. AUR 1478 I 29), ${ }^{4}$ Perg., 5 grüne SS der Stadt Gmunden, des Gmundner Stadtrichters Hans Pinter und der Gmundner Ratsherren Kaspar Tainsteter, Kolman Studnitzer und Wolfgang Traint in wachsf. Schüsseln an Ps, mit Rta-Vermerk auf der Rückseite. ${ }^{5}$ - Ebenfalls erw. in n. 127.

Reg.: CHMEL, Conzeptensammlung 1 S. 120 n. 123. 
Lit.: Krackowizer, Gmunden 2 S. 294; Walter, Ungeld S. 65; HufNaGel, Maut zu Gmunden S. 146; s. auch BrandL, Gmunden S. 158, 161.

Laut Revers war die Stadt Gmunden - wie zuvor auch Wilhelm Lesch (s. n. 32) - verpflichtet, aus den Erträgen des Salzamts Gmunden die Saline zu unterhalten und das Gotteszeilensalz $z^{6}$ an etliche Kirchen und Klöster zu entrichten.

1 Datierung und Ausstellungsort nach Reg. bei CHмEL.

2 Zur Bestandgabe an Wilhelm Lesch im Jahr $1476 \mathrm{~s}$. nn. 32, 33. - Aufgrund der Zugehörigkeit Hallstatts zur Herrschaft Wildenstein war das Salzamt Gmunden meist mit der Pflege von Schloss Wildenstein verbunden, s. HurNAGEL, Maut zu Gmunden S. 153; auch Wilhelm Lesch war Wildenstein samt Landgericht von K.F. bis auf Widerruf übertragen worden, s. n. 32, Anm. 1. - Zum Amt Gmunden s. auch die Urkunde Wolfgang Prauns von 1478 April 27 im HHStA Wien (Sign. AUR 1478 IV 27) über dessen Verzicht auf alle das Amt Gmunden und Schloss Kammer betreffenden Forderungen gegenüber K.F.; Reg.: CHMEL n. 7202; DERS., Mon. Habsb. I/2 S. 656 n. 517.

3 Zur Übergabe an Gmunden s. nn. 126, 127 sowie die ksl. Mandate von Januar und Februar 1478 bei CHMEL, Mon. Habsb. I/2 S. 635 nn. 466, 467, und DeMs., Conzeptensammlung 1 S. 121 n. 124 und Conzeptensammlung 2 S. 144 n. 354 , S. 378 n. 506.

4 Unter dieser Sign. gemeinsam verwahrt mit den nn. 119, 151, 152.

5 Reg.: CHMEL n. 7181; DERS., Mon. Habsb. I/2 S. 636 n. 470.

6 Bezeichnung für als Almosen geltende Salzschenkungen an kirchliche Institutionen, die auf eine oder mehrere „Zeilen“ Fuder lauteten (eine Zeile $=30$ Fuder), wovon sich die Bezeichnung ableitete, s. HuFNAGL, Maut zu Gmunden S. 212.

\section{[Vor 1478 Januar 17, -] ${ }^{1}$}

K.F. überlässt Valentin von Lamberg Amt, Maut und Landgericht zu Stein in Krain bestandweise für weitere zwei Jahre ${ }^{2}$ ab dem Dreikönigstag 1478 (Januar 6) gegen Zahlung von jährlich 750 Pfd. Pf.

Org. oder Kop. im bearbeiteten Bestand nicht überliefert. - Dep.: Erw. im Revers Valentins von Lamberg von 1478 Januar 17 im HHStA Wien (Sign. AUR sub dat. 1478 VI 13), Perg., 2 grüne SS des Ausst. und seines Vetters Kaspar von Lamberg in wachsf. Schüsseln an Ps, mit Rta-Vermerk auf der Rückseite; auch erw. im Revers Valentin Lambergers von 1479 Juli 6 über die erneute bestandweise Überlassung auf zwei Jahre ebd. (Sign. AUR 1479 VII 6), Perg., 2 grüne SS des Ausst. und Jörg Schrotts in wachsf. Schüsseln an Ps, mit Rta-Vermerk auf der Rückseite. ${ }^{3}$

1 Zur Datierung: Der Revers wurde am St. Antoniustag 1478 ausgestellt; aufgrund der Bestandgabe ab 1478 Januar 6 handelt es sich mit hoher Wahrscheinlichkeit um den Festtag am 17. Januar (Antonius der Große, der Einsiedler) und nicht um jenen am 13. Juni (Antonius von Padua). Die Datierung (und daher Sign.) im HHStA Wien erfolgte nach dem Antoniustag im Juni. 
2 Laut Revers hatte Valentin von Lamberg die Ämter zu Stein in Krain in den vergangenen zwei Jahren bis 1478 Januar 6 gegen Zahlung von 700 Pfd. Pf. in Bestand.

3 Der zweite Revers Valentins von Lamberg von 1479 Juli 6 über die weitere Bestandgabe bis 1482 Januar 6 erwähnt nur die hier erschlossene ksl. Urkunde, nicht aber eine Urkunde über die erneute Verlängerung um zwei Jahre; er verstarb allerdings noch im Jahr 1479 (s. n. 330).

\section{[1478 Januar 18, Graz $]^{1}$}

K.F. überlässt Hans Glanhofer, Bürger von Laibach, bis auf Widerruf die Maut zu Sannd Vyor bei Prem, wie sie der verstorbene Veit Perl innehatte, bestandweise ab St. Erasmustag 1478 (Juni 3) gegen Zahlung von jährlich 40 Mark Venediger Schilling.

Org. oder Kop. im bearbeiteten Bestand nicht überliefert. - Dep.: Erw. im Revers Hans Glanhofers von 1478 Januar 25 im HHStA Wien (Sign. AUR 1478 I 25), Perg., 2 grüne SS Niklas Raubers, Hauptmann von Triest und Mitterburg, und Kaspar Melz', Verweser (der Hauptmannschaft) in Krain, in wachsf. Schüsseln an Ps, mit Rta-Vermerk auf der Rückseite. ${ }^{2}$

Reg.: CHMEL, Conzeptensammlung $1 \mathrm{~S} .130 \mathrm{n} .148 .^{3}$

1 Datierung und Ausstellungsort nach Reg. bei CHMEL.

2 Druck: OTOREPEC, Gradivo VI/42; Reg.: ChMEL, Conzeptensammlung 2 S. 120 n. 310.

3 Siehe dazu auch Crmer, Mon. Habsb. I/2 S. 906 n. 1233 (zu 1478 Mai).

\section{Januar 26, Graz}

K.F. bestätigt als Herr und Landesfürst dem (Heilig-Geist-)Spital in der Stadt Gurkfeld von sonndern gnaden wissenntlich mit dem brief folgende Schenkungen des verstorbenen Gf. Friedrich von Cilli, der Gfn. Katharina, Witwe Gf. Ulrichs von Cilli, und des Pfarrers Martin von Gurkfeld: von Gf. Friedrich von Cilli eine Hofstatt neben dem Gurkfelder Friedhof aus dessen Lehenschaft, die einst dem Pfarrer Martin für das Spital zu Eigen übertragen wurde, sowie ein sechs Huben umfassendes Dorf am Rain samt Zubehör, gelegen in seinem (K.F.) Urbar zu Gurkfeld und vormals dorthin zinspflichtig; von Gfn. Katharina ein ödes Dorf zu St. Ulrich, auch in seinem Urbar gelegen; von Pfarrer Martin etliche Äcker mit zwei Teilen des Zehents, eine Wiesmahd und einen Teil des Zehents im Dorf zu Obretz im Ranner Urbar. K.F. verfügt, dass das Spital und die dortigen Armen die Dörfer, Zehenten und anderen Schenkungen gemäß dem Inhalt ihrer brief innehaben und nutzen sollen und damit nach ihrem Bedarf ungehindert verfahren können, und gebietet allen Hauptleuten, Gff., Herren etc., Verwesern, Vizedomen, Pflegern, Bggff., Bürgermeistern, Richtern, Räten, Bürgern, Gemeinden und allen 
anderen Amtleuten und Untertanen, insbesondere aber seinem Rat Andreas von Kraig, ${ }^{1}$ Pfleger von Gurkfeld, die Beachtung dieser Bestätigung. An montag nach sannd Pauls tag der bekerung.

[Org. im ARS Ljubljana, zuvor im HHStA Wien.] - Fotokop. vom Org. im HHStA Wien (Sign. AUR 1478 I 26).

Druck: ChMEL, Mon. Habsb. I/2 S. 889f. n. 1170.

Reg.: Regg.F.III. H. 29 n. 200 (nach Org.); s. weiters ChMEL n. 7180; DERS., Conzeptensammlung 1 S. 147f. n. 192.

Lit.: Muchar, Geschichte 8 S. 97; Dimitz, Geschichte Krains S. 312; Gubo, Geschichte Cilli S. 183 .

1 Ksl. Rat und seit 1471 Erbkämmerer in Kärnten, s. zu ihm HeINIG, Friedrich III./1 S. $212 f$.

\section{[1478 Januar 28, Graz $]^{1}$}

K.F. überlässt Matthias Moser, Bergrichter zu Schladming, Fron und Wechsel vom Silbererz zu Schladming, soweit das Pfarrgebiet reicht, bestandweise für zwei Jahre ab Mariä Lichtmess 1478 (Februar 2) gegen Entrichtung von jährlich 20 Mark Feinsilber.

Org. oder Kop. im bearbeiteten Bestand nicht überliefert. - Dep.: Erw. im Revers Matthias Mosers von 1478 Januar 31, Graz, im HHStA Wien (Sign. AUR 1478 I 31), Perg., 2 grüne SS des Grazer Stadtrichters Christoph Patriarch und des Grazer Ratsherrn Hans Payer mit dem Bären in wachsf. Schüsseln an Ps, mit Rta-Vermerk auf der Rückseite. ${ }^{2}$

Reg.: CHMrL, Conzeptensammlung 1 S. 152f. n. 203 (mit Reg. des Reverses).

Lit.: KunNeRT, Beiträge S. 24; DERS., Geschichte S. 48.

1 Datierung und Ausstellungsort nach Reg. bei CHMEL.

2 Zu den Sieglern Christoph Patriarch und Hans Payer s. PopelKa, Geschichte Graz 1 S. 484, 486; Dienes, Bürger von Graz S. IXf., diesem zufolge war Patriarch auch ksl. Diener. - Zeugen der Siegelbitte sind die Grazer Ratsbürger Tibold Brunner und Hans (Krafft) Büchsenmeister, s. zu ihnen DIENES, ebd. S. XXXIV und XLIXf.; zu Brunner auch PopelKa, ebd. S. 486f. 
K.F. überlässt Propst und Konvent des Augustiner-Chorherrenstifts Vorau bis auf Widerruf die Fischweide zu Reinberg auf der Lafnitz und dem Voraubach, wie sie vormals der Kellermeister innehatte, bestandweise ab Mariä Lichtmess 1478 (Februar 2) gegen Lieferung von jährlich 300 lebenden oder frisch geräucherten Forellen an den ksl. Hof.

KVr: C.d.i.i.c. (nach CHMEL).

Org. oder Kop. im bearbeiteten Bestand nicht überliefert. - Dep.: Erw. im Revers Propst Leonhards und des Konvents des Stifts Vorau von 1478 Januar 30 im HHStA Wien (Sign. AUR sub dat. 1478 I 29), ${ }^{2}$ Perg., 2 SS des Propstes (rot) und Konvents (grün) in wachsf. Schüsseln an Ps, mit Rta-Vermerk auf der Rückseite. ${ }^{3}$

Reg.: CHMEL, Conzeptensammlung 1 S. 156f. n. 217 (mit Nennung des Reverses).

Lit.: Muchar, Geschichte 8 S. 102.

1 Datierung und Ausstellungsort nach Reg. bei CHMEL.

2 Unter dieser Sign. gemeinsam verwahrt mit den $\mathrm{nn} .114,151,152$.

3 Reg.: ChMEL n. 7182.

\section{[Vor 1478 Januar 31, -]}

K.F. überträgt Stephan Zmollner bis auf Widerruf erneut die Pflege von Schloss St. Peter ob Leoben samt dem Landgericht und überlässt ihm die zugehörigen Nutzen und Renten bestandweise gegen Zahlung von jährlich $100 \mathrm{Pfd}$. Pf.

Org. oder Kop. im bearbeiteten Bestand nicht überliefert. - Dep.: Erw. im Revers Stephan Zmollners von 1478 Januar 31 im HHStA Wien (Sign. AUR 1478 I 31), Perg., 2 grüne SS des Ausst. und Andreas Prämers, Pfleger von Gösting, ${ }^{1}$ in wachsf. Schüsseln an Ps, mit Rta-Vermerk auf der Rückseite. ${ }^{2}$

Lit.: Muchar, Geschichte 8 S. 102 (zu Februar 1); zum Schloss St. Peter (St. Peter-Freienstein) S. BARAVALLE, Burgen S. 373-375; EBNER, Burgen Steiermark 2 S. 124.

Siehe n. 294.

1 Siehe zu ihm HenNig, Friedrich III./1 S. 208f.

2 Reg.: Muchar, Urkunden-Regesten n. 252; Göth, Urkunden-Regesten 9 n. 655 (jeweils mit Bestandgeld von jährlich 500 Pfd. Pf., so auch bei Muchar, Geschichte 8 S. 102). 
K.F. bezeugt gegenüber den zu Ofen (Buda) versammelten Prälaten, Baronen, Rittern und Städten des Kgr. Ungarn auf Bitte Gf. Sigmunds von St. Georgen und Bösing, dass sich dieser auf seinen Wunsch zum festum Penthecosten 1477 (Mai 25) in Wien eingefunden hätte, er mit ihm ausschließlich über die Einlösung der von Bggf. Michael von Maidburg an Sigmund verpfändeten Burg Hardegg und anderer Güter, die zwischen beiden strittig sei, verhandelt und, nachdem keine Einigung erzielt werden konnte, ihn dann entlassen habe. Zwischen ihnen sei kein einziges Wort gegen Kg. Matthias von Ungarn und Böhmen, Kurfürst und filium meum carissimum, gewechselt worden. K.F. weist damit ausdrücklich den bei $\mathrm{Kg}$. Matthias gegen den Grafen vorgebrachten Verdacht zurück, dieser hätte ihm im Vorjahr in Wien gegen Schenkung der genannten Pfandschaften Beistand gegen Kg. Matthias versprochen.

Org. im bearbeiteten Bestand nicht überliefert. - Konz. (lat., undatiert) mit Korrekturen im HHStA Wien (Sign. AUR sub dat. 1478), Pap. (fol. 1r). ${ }^{2}$

1 Die Datierung in das Jahr 1478 ergibt sich aus der Bezeichnung des Jahres 1477 als superiori anno; auf Februar dieses Jahres verweist die Erwähnung der zu Ofen versammelten ungarischen Stände, da Kg. Matthias für Februar 1478 einen Tag zu Ofen einberufen hatte, s. NeHRING, Matthias Corvinus S. 95.

2 Gemeinsam überliefert mit zwei weiteren Konzepten, s. nn. 190, 212.

\section{[1478 Februar 4, Graz $]^{1}$}

K.F. überlässt Hans Reisacher bis auf Widerruf seine Fischweide im Mürztal auf der Mürz ober- und unterhalb von Kindberg bestandweise ab St. Martinstag 1477 (November 11) gegen Zahlung von jährlich $40 \mathrm{fl}$. ung. und der Verpflichtung, bei Aufenthalten des K. in Kindberg oder bei der Durchreise Fische für den ksl. Hof zu liefern. ${ }^{2}$

Org. oder Kop. im bearbeiteten Bestand nicht überliefert. - Dep.: Erw. im Revers Hans Reisachers von 1478 Februar 5 im HHStA Wien (Sign. AUR 1478 II 5), Perg., 2 grüne SS des Ausst. und Wulfings von Fladnitz in wachsf. Schüsseln an Ps, mit Rta-Vermerk auf der Rückseite. ${ }^{3}$

Reg.: ChmeL, Conzeptensammlung 2 S. 173 n. 374 (mit Nennung des Reverses).

Lit.: Muchar, Geschichte 8 S. 102 (zu Februar 1).

1 Datierung und Ausstellungsort nach Reg. bei CHMEL.

2 Siehe dazu auch n. 321.

3 Reg.: GöTH, Urkunden-Regesten 9 n. 656. 
K.F. überträgt Leonhard von Herberstein bis auf Widerruf die Pflege des Turms zu Wippach aus dem Besitz des verstorbenen Andreas Baumkircher ${ }^{1}$ und überlässt ihm die zugehörigen Nutzen und Renten sowie das einst dem Mordaxer gehörende Gut mit 6 Mark Sch. jährlichem Dienst und das von Hans Gall an ihn (K.F.) gekommene Gut mit eineinhalb Gulden jährlichem Dienst bestandweise ab Mariä Lichtmess 1478 (Februar 2) gegen Zahlung von jährlich $154 \mathrm{fl}$. ung. und $4 \mathrm{Pfd}$. Venediger Münze.

Org. oder Kop. im bearbeiteten Bestand nicht überliefert. - Dep.: Erw. im Revers Leonhards von Herberstein von 1478 Februar 6 im HHStA Wien (Sign. AUR 1478 II 6), Perg., 2 grüne SS des Ausst. und des Grazer Ratsherrn Thomas Peheim² in wachsf. Schüsseln an Ps, mit Rta-Vermerk auf der Rückseite. ${ }^{3}$

Lit.: VIIfFAN, Andreas Baumkircher S. 267f., 270f., 288f. (mit Karte); DERS., Baumkircherturm; zu Leonhard von Herbstein, seit 1470 Pfleger von Wippach, s. HeINIG, Friedrich III./1 S. 188.

1 Hingerichtet am 23. April 1471, s. ScHÄFFER, Baumkircherfehde S. 166f.; auch DERS., Untreue und Verrat; zur Person bes. Haller-ReIFFensteIn, Friedrich III. und Andreas Baumkircher; s. auch HeInig, Friedrich III// S. 229-231.

2 Siehe zu ihm DienEs, Bürger von Graz S. XVIIIf.

3 Reg.: TomascheK, Urkunden-Regesten Krain 2 n. 48.

\section{[1478 Februar 7, Graz $]^{1}$}

K.F. überlässt Leonhard Saldorfer, Vizedom zu Ortenburg, bis auf Widerruf die Fischweide zu Döbriach am Millstätter See bestandweise gegen eine jährliche Lieferung von 400 Seeforellen ${ }^{2}$ am 1. Mai.

Org. oder Kop. im bearbeiteten Bestand nicht überliefert. - Dep.: Erw. im Revers Leonhard Saldorfers von 1478 Februar 10 im HHStA Wien (Sign. AUR 1478 II 10), Perg., 2 grüne SS des Ausst. und des Melchior Posch zu Spittal (a.d. Drau) in wachsf. Schüsseln an Ps, mit Rta-Vermerk auf der Rückseite. ${ }^{3}$

KVr: C.d.i. per d. Sig. Pruschinckh (nach ChMeL).

Reg.: ChMEL, Conzeptensammlung 1 S. 109 n. 88.

1 Datierung und Ausstellungsort nach Reg. bei CHMEL. 
2 Im Revers zalvorichen.

3 Reg.: ToмAschEK, Regesten Kärnten 3 n. 495; der Revers sowie ein ksl. Befehl an den früheren Inhaber, die Fischweide abzutreten, auch erw. bei CHMEL zu n. 88 (zu Februar 7).

[Vor 1478 Februar 10, -]

K.F. überträgt Leonhard Harracher bis auf Widerruf die Pflege von Schloss Weitersfeld und überlässt ihm die zugehörigen Nutzen und Renten bestandweise gegen Zahlung von jährlich 150 Pfd. Pf.

Org. oder Kop. im bearbeiteten Bestand nicht überliefert. - Dep.: Erw. im Revers Leonhard Harrachers von 1478 Februar 13 im HHStA Wien (Sign. AUR 1478 II 13), Perg., 2 grüne SS des Ausst. und Friedrichs von Hollenegg in wachsf. Schüsseln an Ps, mit Rta-Vermerk auf der Rückseite; ergibt sich auch aus dem Revers Leonhard Harrachers von 1478 Februar 10 ebd. (Sign. AUR 1478 II 10), Pap., grünes S (Petschaft) des Ausst. vorders. aufgedr. unter Papieroblate. ${ }^{1}$

Lit.: Muchar, Geschichte 8 S. 102 (zu Februar 1); Baravalle, Burgen S. 541; zu Leonhard Harracher, ksl. Diener, s. HeINIG, Friedrich III./1 S. $214 f$.

Der erste Revers Leonhard Harrachers von 1478 Februar 10 ist im Unterschied zu jenem von Februar 13 keine Kanzleiausfertigung, sondern ein eigenhändiges Schreiben Harrachers, worin dieser - wie im späteren Revers - die Übertragung der Pflege von Weitersfeld und die Bestandgabe bestätigt (ohne Nennung einer ksl. Urkunde) und verspricht, jährlich 3 Pfd. Pf. Amtsgeld in die ksl. Kanzlei zu entrichten, was der zweite, dem üblichen Formular folgende Revers mit den Pflichten des Bestandnehmers nicht enthält.

1 Reg.: Muchar, Urkunden-Regesten nn. 253, 254; GöTH, Urkunden-Regesten 9 n. 657.

\section{[Vor 1478 Februar 13, -]}

K.F. befiehlt den Burgleuten zu Hallstatt, eine Schätzung der Saline Hallstatt für die Übergabe an die Stadt Gmunden vorzunehmen.

Org. oder Kop. im bearbeiteten Bestand nicht überliefert. - Dep.: Erw. in n. 127.

\section{Februar 13, Graz}

K.F. befiehlt Andreas Wagen und Andreas Fuchsberger, seinen Verwesern der Saline Aussee, sich wegen der Abtretung der Saline Hallstatt und des Amts Gmunden durch Wilhelm Lesch ${ }^{1}$ nach Hallstatt und Gmunden zu begeben, nachdem er Saline und Amt 
dem Richter und Rat von Gmunden übertragen hat. ${ }^{2}$ Sie sollen gemeinsam mit den ebenfalls schriftlich informierten Hallstätter Burgleuten ${ }^{3}$ Holz, Eisen, Seile und insteund Salz zu Gmunden sowie alles andere zur Saline und dem Amt Gehörige in der Weise verzeichnen, schätzen und ihm schriftlich übermitteln, wie sie es vormals bei der Übergabe von Amt und Saline an Wilhem Lesch gemacht haben. An freitag vor dem suntag Reminiscere in der vasten (nach Kop.).

KVr: C.d.i.p. - KVv: Unnseren getrewn Andreen Wagen und Andreen Fuchsperger, unnseren verweseren unsers Halls zu Aussee (Adresse) (nach Kop.). ${ }^{4}$

Kop.: Zeitgleiche Abschrift als Beilage des von dem Ausseer Verweser Andreas Fuchsberger und den Hallstätter Burgleuten erstellten Übergabeverzeichnisses über die Saline Hallstatt und das Amt Gmunden von 1478 Februar 11 im HHStA Wien (Sign. AUR sub dat. 1478 II 17), Pap. (1 fol.). ${ }^{5}$

Druck: ChMel, Mon. Habsb. I/2 S. 642 n. 473 (= Conzeptensammlung 2 S. 343 n. 441).

Lit.: Krackowizer, Gmunden 2 S. 294; HufNaGel, Maut zu Gmunden S. 146; zum ksl. Türhüter Andreas Wagen s. HeINIG, Türhüter S. 364.

1 Zur Übertragung an Wilhelm Lesch im Jahr 1476 s. n. 32 .

2 Zur Übertragung an Gmunden s. n. 114.

3 Siehe n. 126; die Burgleute zu Hallstatt hatten die Pfannstätten als Burglehen inne, s. HurNagel, Maut zu Gmunden S. 156f.

4 Der Kanzleivermerk ist unterhalb des Textes auf fol. 1r, die Adresse auf fol. 1v angegeben.

5 Druck des Übergabeverzeichnisses: CHMEL, Mon. Habsb. I/2 S. 637-642 n. 472; im Übergabeverzeichnis wird erwähnt, dass die ksl. Schreiben an Andreas Fuchsberger und die Hallstätter Burgleute abschriftlich beiliegen (CHмkL S. 637).

\section{[Vor 1478 Februar 17, -]}

K.F. überlässt Richter, Rat und allen Bürgern der Stadt Möttling bis auf Widerruf das dortige Gericht und die Maut bestandweise ab St. Michaelstag 1477 (September 29) gegen Zahlung von jährlich 132 Pfd. Pf.

Org. oder Kop. im bearbeiteten Bestand nicht überliefert. - Dep.: Erw. im Revers von Richter, Rat und den Bürgern von Möttling von 1478 Februar 17 im HHStA Wien (Sign. AUR 1478 II 17), Perg., grünes S der Stadt Möttling an Ps, mit Rta-Vermerk auf der Rückseite. ${ }^{1}$

1 Reg.: Tomaschex, Urkunden-Regesten Krain 2 n. 49. 


\section{Februar 21, Graz}

K.F. belehnt Barbara Span, Tochter des verstorbenen Leonhard Span (Gspan), wissenntlich mit dem brief, was wir ir zu recht daran verleihen sullen oder mugen, mit dem von ihr ererbten Hof zu Hausmannstätten ${ }^{1}$ samt Zubehör aus der Lehenschaft des Ftm. Steier, den sie und ihre Erben gemäß Lehens- und Landrecht innehaben und nutzen sollen, und bestimmt als Lehensträger ihren Vetter Andreas Span zur Leistung der schuldigen Dienste. An sambstag vor dem suntag Oculi in der vasten.

KVr: C.d.i.p. - KVv: Rta. - Rückseite der Wachsschüssel: Vermerk nicht lesbar (tlw. nicht mehr vorhanden).

Org. im HHStA Wien (Sign. AUR 1478 II 21), Perg., rotes S 21 in wachsf. Schüssel (beschädigt) mit wachsf. S 16 rücks. eingedr. an Ps.

Reg.: Muchar, Urkunden-Regesten n. 257; GöTH, Urkunden-Regesten 9 n. 659; STARZER, Lehen S. 319 n. $298 / 2$.

Lit:: MUCHAR, Geschichte 8 S. 100.

1 Zur Übertragung des Hofs in Hausmannstätten an Leonhard (Lienhard) Span im Jahr 1472 s. Regg.F.III. H. 27 n. 178.

\section{März 1, Graz}

K.F. verkauft Hans Pickl, seinem Amtmann zu Voitsberg, und dessen Erben wissenntlich mit dem brief das einst dem Andreas Greisenegger ${ }^{1}$ gehörende Haus in Voitsberg am Hauptplatz (am Platz), genannt Dornhaws, das mit einer Seite an das Haus des Vogel und mit der anderen an die Kirchengasse grenzt, für eine bereits gänzlich bezahlte Geldsumme. Er verfügt, dass sie das Haus samt Zubehör und allen von alters her zugehörigen Rechten innehaben und nutzen sollen, es verkaufen oder in anderer Weise damit wie mit ihrem anderen Eigengut verfahren können, und erklärt, im Klagsfall ihr recht gaber, gewer und scherm zu sein, als solhs verkauffs und lannds recht ist. An suntag Letare zu mittervassten. ${ }^{2}$

KVr: fehlt. ${ }^{3}$

Org. im HHStA Wien (Sign. AUR 1478 III 1), Perg., S (Siegelbild abgefallen, wohl rotes S 21) in wachsf. Schüssel mit rücks. eingedr. S (Siegelbild abgefallen, wohl wachsf. S 16) an Ps.

Reg.: ChMel n. 7190; DeRs., Mon. Habsb. I/2 S. 707 n. 615, I/3 S. 716 n. 251. 
1 Hingerichtet gemeinsam mit Andreas Baumkircher am 23. April 1471, s. SCHÄFrER, Baumkircherfehde S. 166f.; zur Person s. Hennig, Friedrich III./1 S. 217f.

2 Zur Abtretung des Hauses an Pickl durch Wilhelm von Saurau, Verweser der Hauptmannschaft in Steier, S. CHMEL, Mon. Habsb. I/2 S. 707 zu n. 615 (Befehl von 1478 Februar 28).

3 Nach CHMEL, ebd.: C.d.i. per Prueschinkh (nach Konz.).

\section{[Vor 1478 März 2, -]}

K.F. überträgt Jörg Katzendorfer bis auf Widerruf die Pflege von Schloss Montpreis und überlässt ihm die zugehörigen Nutzen und Renten gegen Zahlung von jährlich 250 fl. ung.

Org. oder Kop. im bearbeiteten Bestand nicht überliefert. - Dep.: Erw. im Revers Jörg Katzendorfers von 1478 März 2 im HHStA Wien (Sign. AUR 1478 III 2), Perg., 2 grüne SS des Ausst. und Wulfings d.Ä. von Fladnitz in wachsf. Schüsseln an Ps, mit Rta-Vermerk auf der Rückseite.

Lit.: Zu Schloss Montpreis s. PIRCHEGGER, Untersteiermark S. 243f.

\section{[Vor 1478 März 2, -]}

K.F. erlaubt Balthasar Heustadl auf Bitte Ehz. Sigmunds von Österreich, sich wieder in seinen Ländern und Herrschaften aufzuhalten und Handel zu treiben. ${ }^{1}$

Org. oder Kop. im bearbeiteten Bestand nicht überliefert. - Dep.: Erw. im Urfehdebrief Balthasar Heustadls von 1478 März 2, Innsbruck, im HHStA Wien (Sign. AUR 1478 III 2), ${ }^{2}$ Perg., 2 grüne SS des Ausst. und des Ritters Burkhard von Knöringen in wachsf. Schüsseln an Ps, mit Rta-Vermerk auf der Rückseite. ${ }^{3}$

1 Dem Urfehdebrief Heustadls zufolge hatte dieser nach der Freilassung aus längerer ksl. Gefangenschaft geschworen, sich nicht mehr in den Ländern des K. aufzuhalten; s. zu ihm auch CHMEL n. 3933 (Urfehdebrief von 1462).

2 Unter dieser Sign. gemeinsam verwahrt mit dem Urfehdebrief Bartholomäus Brunpecks von 1478 April 24, s. ChMel n. 7201 und Ders., Mon. Habsb. I/2 S. 748 n. 767 (Druck)

3 Zeugen der Siegelbitte sind Wolfhard Kobinger und Jörg Costentzer. - Druck: CHMEL, Mon. Habsb. I/2 S. 471f. n. 118; Reg.: CHMEL n. 7191.

\section{März 4, Graz}

K.F. gewährt dem Juden Kifel zu St. Veit in Kärnten wissenlich mit dem brief bis auf Widerruf die sunnder gnad, sich nur vor Jakob von Ernau, seinem Pfleger von Karls- 
berg und Vizedom in Kärnten, ${ }^{1}$ oder jenem, dem er das in sunderhait befiehlt, verantworten zu müssen, und gebietet allen Hauptleuten, Landmarschällen, Gff., Freiherren etc., Verwesern, Landschreibern, Vizedomen, Pflegern, Bggff., Bürgermeistern, Richtern, Räten, Judenrichtern, Bürgern, Gemeinden und allen anderen Amtleuten und Untertanen, den Kifel bei diesen Freiheiten zu belassen, jedoch nur bis auf Widerruf. Am mittichen nach dem sunntag Letare zu mittervassten.

KVr: C.d.i.p.

Org. im HHStA Wien (Sign. AUR 1478 III 4), Perg., rotes S 21 rücks. aufgedr. und rotes S 16 vorders. aufgedr. (beide SS großteils abgefallen).

Druck: MC 11 n. 485 (Teildruck, mit dem Namen Kisel).

Reg.: CHMEL n. 7192; DeRs., Mon. Habsb. I/3 S. 720 n. 260; WIENER, Regesten 1 S. 96 n. 111. ${ }^{2}$

Lit:: WADL, Geschichte der Juden S. 137.

1 1452-1479 Pfleger von Karlsberg und 1462-1479 Vizedom in Kärnten, s. zu ihm LerTNER, Herren von Ernau S. 193-195.

2 Auch erw. bei CHMrL, Mon. Habsb. I/2 S. 842 zu n. 1030.

\section{März 8, Graz}

K.F. befiehlt allen Hauptleuten, Gff., Freiherren, Rittern, Knechten, Verwesern, Landschreibern, Vizedomen, Pflegern, Bggff., Bürgermeistern, Richtern, Räten, Bürgern, Gemeinden und allen anderen Amtleuten und Untertanen, insbesondere Konrad Lochner, seinem Pfleger zu Hollenburg, ${ }^{1}$ wie auch den künftigen Pflegern, auf Beschwerde des Abtes des Zisterzienserklosters Viktring über Eingriffe in das von verstorbenen Priestern und seeltrager( $n)$ der inkorporierten Pfarrkirchen hinterlassene Gut sowie in die zugehörigen Pfarrhöfe, die Hinterlassenschaften und Pfarrhöfe der Priester und Seelsorger nicht anzutasten und den Abt von Viktring damit nach Bedarf ungehindert verfahren zu lassen. Am sunntag Judica in der vassten. ${ }^{2}$

KVr: C.d.i.i.c.

Org. im HHStA Wien (Sign. AUR 1478 III 8), Perg., rotes S 21 rücks. aufgedr. (abgefallen).

Druck: ChMEL, Mon. Habsb. I/2 S. 845f. nn. 1040, 1042 (Teildruck); MC 11 n. 486 (Teildruck).

Reg.: LichNowsKY(-BIRK) 8 n. 33.

Lit.: RoschER, Geschichte Viktring S. 137. 
1 Pfleger 1472-1482, s. zu Hollenburg Metnitz, Kärntner Burgenkunde 2 S. 68-70; WiessNer/SEEBACH, Burgen Kärnten 2 S. 67-69.

2 Siehe in der Sache auch CHMғL, Mon. Habsb. I/2 S. 845f. nn. 1041, 1043: vom selben Tag entsprechender Befehl an Konrad Lochner sowie ein Privileg für das Kloster Viktring, über die Hinterlassenschaft der Priester verfugen zu können.

\section{März 23, Graz}

K.F. belehnt Kristina, Tochter des verstorbenen Hermann Hafner und Ehefrau des Andreas von Spangstein, wissenlich mit dem brief, was wir ir zu recht daran verleihen sullen oder mugen, mit einer von ihrer Mutter Elisabeth ererbten Hube samt Zubehör zu Unterurscha (Nidern Urfar) ${ }^{1}$ in der Eggersdorfer Pfarre aus der Lehenschaft des Ftm. Steier, die sie und ihre Erben gemäß Lehens- und Landrecht innehaben und nutzen sollen, und bestimmt als Lehensträger ihren Ehemann Andreas zur Leistung der schuldigen Dienste. Am montag in den heiligen Osterveirtagen.

KVr: C.d.i.p.

Org. im HHStA Wien (Sign. AUR sub dat. 1478 III 16), Perg., rotes S 21 in wachsf. Schüssel mit wachsf. S 16 rücks. eingedr. an Ps.

Reg.: Muchar, Urkunden-Regesten n. 260; GöTH, Urkunden-Regesten 9 n. 662; StARZER, Lehen S. 320 n. $299 / 5 .^{2}$

Lit.: MUchaR, Geschichte der Steiermark 8 S. 100 (,Niedern Urfar“ in der „Eberstorfer“ Pfarre).

1 Wohl Verschreibung statt „Nidern Ursar" oder „Nidern Ursa“; ;. auch StarZER, Lehen S. 268 n. 193/1 zu 1479 Dezember 20: Verkauf der Hube „zu Nidern Ursa“ durch Kristina von Spangstein.

2 Bei StARZER irtümlich Belehnung Andreas Spangsteiners als Lehensträger seiner Frau Elisabeth mit der von ihrem Vater Hermann ${ }_{\text {,Hoffner }}{ }^{c c}$ ererbten Hube.

\section{[1478 März 30, Graz] ${ }^{1}$}

K.F. überlässt Sigmund Piers (Pfleger von Friedrichstein) ${ }^{2}$ bis auf Widerruf das zum Schloss Friedrichstein gehörige Landgericht in der Gottschee bestandweise gegen Zahlung von jährlich $32 \mathrm{fl}$. ung.

Org. oder Kop. im bearbeiteten Bestand nicht überliefert. - Dep.: Erw. im Revers des Sigmund Piers von 1478 März 30 im HHStA Wien (Sign. AUR 1478 III 30), Perg., 2 grüne SS des 
Ausst. und Niklas Raubers, Hauptmann von Triest und Mitterburg, in wachsf. Schüsseln an Ps, mit Rta-Vermerk auf der Rückseite. ${ }^{3}$

Reg.: Chmel, Mon. Habsb. I/2 S. 894 n. 1189.

Lit:: WoLSEGGer, Urbarium Gottschee S. 179 (zu Anm. 8).

1 Datierung und Ausstellungsort nach Reg. bei CHMEL.

2 Das Amt genannt ebd., nicht im Revers.

3 Reg. (Revers): CHMEL, Mon. Habsb. I/2 S. 893 n. 1185.

\section{März 30, Graz}

K.F. verleiht Clemens Parilo, Bürger von Krainburg, und dessen Erben von sundern gnaden wissenntlich mit dem brief eine in der Krainburger Pfarre gelegene und in das Amt Naklas zinspflichtige Hube im Dörflein Mlaka, auf der früher der von den Türken verschleppte Miclaw gesessen ist, zu rechtem Kaufrecht. Er verfügt, dass sie die Hube samt allem Zubehör stifftlich und pewlich innehaben und nutzen sollen, und verpflichtet sie, seinem Amtmann zu Naklas die gewöhnlichen Zinse und Dienste nach Inhalt seines Urbars jährlich zu entrichten, treu und gehorsam zu sein und alles gemäß Kaufrecht im Ftm. Krain zu tun, wozu auch die anderen in das genannte Amt zinsbaren Leute verpflichtet sind, andernfalls droht der Verlust des Kaufrechts. Er gebietet seinem Amtmann zu Naklas, dem Knaur, sowie allen künftigen Amtmännern, Parilo und dessen Erben bei dieser Gnade und dem Kaufrecht zu belassen und sie nicht zu behindern oder dies zu gestatten, vorbehaltlich seiner und seiner Erben genannten Zinse, Dienste und annder oberkait auf der Hube. An montag nach dem suntag Quasimodogeniti.

KVr: C.d.i.p. $-\mathrm{KVv}$ : Rta.

Org. im HHStA Wien (Sign. AUR 1478 III 30), Perg., rotes S 21 (zur Hälfte abgefallen) in wachsf. Schüssel mit wachsf. S 16 rücks. eingedr. an Ps.

Reg.: ChMel, Mon. Habsb. I/2 S. 894 n. 1187.

\section{[1478 April 1, Graz $]^{1}$}

K.F. überträgt Jörg von Obritschan ${ }^{2}$ bis auf Widerruf die Pflege von Schloss Schärffenberg, wie dessen verstorbener Vater Peter dieses innehatte, und überlässt ihm die zugehörigen Nutzen und Renten bestandweise gegen Zahlung von jährlich $200 \mathrm{fl}$. ung.

Org. oder Kop. im bearbeiteten Bestand nicht überliefert. - Dep.: Erw. im Revers Jörgs von Obritschan von 1478 April 1 im HHStA Wien (Sign. AUR 1478 IV 1), Perg., 2 SS des Ausst. 
(grün) und Jörgs von Tschernembl (rot), Hauptmann zu Adelsberg und auf dem Karst, ${ }^{3}$ in wachsf. Schüsseln an Ps, mit Rta-Vermerk auf der Rückseite.

Reg.: ChMel, Mon. Habsb. I/2 S. 733 n. $713 .{ }^{4}$

Lit:: IGÁLFFY-IGÁLY, Geschlecht der Obritschan S. 104.

1 Datierung und Ausstellungsort nach Reg. bei CHMEL.

2 Auch Obratschan, im Revers Obertschan.

3 Ksl. Rat, s. zu ihm Henng, Friedreich III./1 S. 233f.

4 CHMrL nennt auch den Revers sowie einen ksl. Befehl an die zum Schloss gehörigen Leute.

\section{April 4, Graz}

K.F. bevollmächtigt Kardinal Georg (Heßler) und seinen Sohn Ehz. Maximilian von Österreich und Burgund ${ }^{1}$ aus ksl. miltikeit und angeborner gute und tugent, auch auf Bitte Maximilians und aufgrund der treuen und gehorsamen dinstperkeit, die Ldgf. Hermann (IV.) von Hessen, Gubernator des Erzbistums Köln, ${ }^{2}$ ihm, dem Reich und seinem Sohn zu erweisen willig ist, aus ksl. Machtvollkommenheit wissentlich in craft diss unnsers keiserlichen brieves, sich an seiner statt mit Ldgf. Hermann hinsichtlich seiner (K.F.) finanziellen und sonstigen Forderungen sowie der Regalien gütlich zu einigen und diesem zuzusagen, ihn als Kölner Erzbischof mit dem Kurfürstentum und den Regalien zu bequemlicher und geburlicher zeit zu belehnen. ${ }^{3}$ Er verspricht bei seinen keiserlichen worten, sich an ihre Verhandlungsergebnisse und Zusagen zu halten, den Ldgf. mit den Regalien zu belehnen und ihn beim Papst hinsichtlich der Kölner Kirche zu fördern. Am vierden tag des monets aprilis. ${ }^{4}$

KVr: A.m.d.i.i.c. - KVv: Rta (Blattmitte). - procuratorium ad lanntgravium Hermannum (rechter Blattrand).

Org. im HHStA Wien (Sign. AUR 1478 IV 4), Perg., anh. S (wohl S 18 wie in n. 140) samt Ps ab und verloren. ${ }^{5}$

Druck: ChMel, Mon. Habsb. I/2 S. 348-350 n. 43.

Lit.: Bachmann, Reichsgeschichte 2 S. 662; HollweG, Georg Heßler S. 81; FuHs, Hermann IV. von Hessen S. 87; zu Hermann von Hessen s. auch GATZ, Bischöfe 2 S. 287f., zu Georg Heßler HeINIG, Friedrich III./1 S. 709-720.

Siehe n. 190.

1 Zur Titulatur Maximilians s. die Einleitung S. 22-24. 
2 Zur sog. „Kölner Stiftsfehde" und Ernennung Hermanns von Hessen zum Gubernator (1475 September 12) s. Regg.F.III. H. $27 \mathrm{nn} .220,270$.

3 Der noch amtierende, vom Papst gebannte Eb. Ruprecht (von der Pfalz) war im März 1478 von Ldgf. Heinrich III. von Hessen, dem Bruder Hermanns, gefangen genommen worden; zu dessen Resignation (1478 Juli 6) s. n. 190.

$4 \mathrm{Zu}$ der von Ehz. Maximilian und Georg Heßler vermittelten Einigung von 1478 August 18 s. Furs, Hermann IV. von Hessen S. 88.

5 Rücks. zeitnaher Vermerk: Gewaltsbrief von kay(ser) Fridrichen umb ettlich geltschuld und ander spruch und vordrung, so sein $k$ (ayserliche) m(ajesta) t zu landgraf Herman zu Hessen als gubernator des stiffts zu Coln, auch der regalien desselben stiffts etc. zu haben vermaint, zu verainen und zuvertragen (Blattmitte rechts).

\section{April 4, Graz}

K.F. bevollmächtigt Kardinal Georg (Heßler) und seinen Sohn Ehz. Maximilian von Österreich und Burgund ${ }^{1}$ aus ksl. miltikeit und angeborner gute und tugent, auch auf Bitte Maximilians und aufgrund der treuen und gehorsamen dinstperkeit, die Pfgf. Philipp bei Rhein ihm, dem Reich und seinem Sohn zu erweisen willig ist, aus ksl. Machtvollkommenheit wissenntlich in krafft diss brieves, sich an seiner statt mit Pfgf. Philipp hinsichtlich seiner (K.F.) Klagen und Forderungen, die noch von dem verstorbenen Friedrich, der sich Pfgf. bei Rhein und Hz. von Bayern genennet hat ${ }^{2}$ herrühren, samt Strafen und Bußen und allem bis heute daraus Folgenden gütlich zu einigen und diesem die Belehnung mit dem Kurfürstentum der Pfalz samt dem Erztruchsessenamt des Reichs und allen zur Pfalz gehörigen Fürstentümern und Ländern in einer bequemlichen zeit zuzusagen. Er verspricht bei seinen keiserlichen wortten, sich an ihre Verhandlungsergebnisse und Zusagen zu halten. Am vierden tag des monets aprilis.

KVr: A.m.d.i.i.c. - KVv: Rta (Blattmitte). - procuratorium ad d(ominum) palatinum (rechter Blattrand).

Org. im HHStA Wien (Sign. AUR 1478 IV 4), Perg., rotes S 18 in wachsf. Schüssel mit rücks. eingedr. S (Siegelbild nicht erkennbar, wohl wachsf. S 16) an Ps. - Kop.: Abschrift des 18. Jh. ebd. (Sign. Urkundenabschriften Österr. Urkunden, Kt. 46 sub dat.), Pap.

Druck: ChMEL, Mon. Habsb. I/2 S. 350f. n. 44.

Reg.: ChMel n. 7194; LichNowsky(-BIRK) 8 n. 37.

Lit:: BAchmANN, Reichsgeschichte 2 S. 662; HollweG, Georg Heßler S. 80; zu Georg Heßler S. auch HeINIG, Friedrich III./1 S. 709-720.

1 Zur Titulatur Maximilians s. die Einleitung S. 22-24.

2 Pfgf. Friedrich (der Siegreiche) († 1476) hatte nach dem Tod Kf. Ludwigs IV. zunächst die Vormund- 
schaft über dessen Sohn Philipp, seinen Neffen, übernommen und sich 1451/52 über dessen Adoption (in der röm.-rechtlichen Form der Arrogation) die Kurwürde angeeignet, wurde aber niemals von K.F. bestätigt und daher von ihm nur mit dem Vornamen oder als $\mathrm{Hz}$. Friedrich bezeichnet; zum Prozess gegen Pfgf. Friedrich s. ausführlich KrIeger, Prozeß, und DERs., Quelle, zu dessen Ächtung mit Urteil von 1474 Mai 27 s. Regg.F.II. H. 27 n. 232. - Nach dem Tod Pfgf. Friedrichs 1476 verweigerte K.F. auch dessen Arrogationssohn Philipp die Regalienleihe mit der Begründung, dieser hätte nicht im Jahr seiner Volljährigkeit 1470 die Herrschaft im Kurfürstentum angetreten und sich zum Empfang der Reichslehen innerhalb der vorgeschriebenen Frist erboten, weshalb seine Rechte auf die Pfalz als Erbe seines Vaters verjährt seien; zu den Verhandlungen im Jahr 1477 s. CHMEL, Mon, Habsb. I/3 S. 603-607 nn. 120, 122, S. 610f. n. 127, und Krieger, Prozeß S. 284.

\section{[Vor 1478 April 6, -]}

K.F. belehnt Margarethe, Witwe Augustin Erdingers, mit einem zu Thalheim bei der Kirche gelegenen Hof, der als Lehen ihres Mannes mit dessen Tod heimgefallen ist.

Org. oder Kop. im bearbeiteten Bestand nicht überliefert. - Dep.: Erw. im Revers Margarethe Erdingers von 1478 April 6 im HHStA Wien (Sign. AUR 1478 IV 6), Perg., 2 grüne SS Erhard Lembachers und Kaspar Harders in wachsf. Schüsseln an Ps, mit Rta-Vermerk auf der Rückseite. $^{1}$

1 Laut Revers war Margarethe Erdinger verpflichtet, dem Kaiser die Hälfte des Verkaufswerts oder bei Nichtverkauf des Schätzwerts innerhalb eines Jahres zu entrichten und den Hof als Fürpfand einzusetzen. Der rücks. Vermerk des 15. Jh. auf der Urkunde entspricht daher nicht ihrem Inhalt: Revers Margarethen Augustins Erdinger wittib umb einen hof der herrschafft Osterreich lehenschafft den nicht zuverkauffen und zuversetzen, ir lebtag lanng. - Reg.: CHMEL n. 7195; DERS., Mon. Habsb. I/2 S. 654 n. 511.

\section{April 15, Graz}

K.F. belehnt die Brüder Balthasar, Andreas und Kaspar Lueger aufgrund ihres Erbrechts nach dem Tod ihres Vetters Florian von Altenburg von sonndern gnaden wissenntlich mit dem brieve, waz wir in von rechten und gnaden wegen daran verleihen sullen oder mugen, mit dem Schloss Rudenegg (Rudegk) samt Zubehör, und verfügt, dass sie und ihre Erben das Schloss innehaben und nutzen sollen, ihm damit treu und gehorsam zu Diensten sein und es ihm offen halten sollen gemäß Lehens- und Landrecht. An mittichen nach dem suntag Jubilate.

KVr: C.d.i.i.c. $-\mathrm{KVv}$ : Rta

Org. im HHStA Wien (Sign. AUR 1478 IV 15), Perg., rotes S 21 in wachsf. Schüssel (beschädigt) mit wachsf. S 16 rücks. eingedr. an Ps. 
Reg.: ChMel, Mon. Habsb. I/2 S. 760 n. 807; GöTH, Urkunden-Regesten 9 n. 663.

Lit.: Zu Rudenegg s. PIRCHEGGER, Untersteiermark S. 198f.

\section{[Vor 1478 April 16, -]}

K.F. überlässt Richter, Rat und allen Bürgern der Stadt Völkermarkt bis auf Widerruf die Nutzen und Renten von Jahrmarkt ${ }^{1}$ und Maut zu Völkermarkt bestandweise ab Weihnachten 1478 (1477 Dezember 25) gegen Zahlung von jährlich 100 Pfd. Pf.

Org. oder Kop. im bearbeiteten Bestand nicht überliefert. - Dep.: Erw. im Revers von Richter, Rat und den Bürgern von Völkermarkt von 1478 April 16 im HHStA Wien (Sign. AUR 1478 IV 16), Perg., grünes S der Stadt Völkermarkt in wachsf. Schüssel an Ps, mit Rta-Vermerk auf der Rückseite. ${ }^{2}$

Reg.: ChMel, Mon. Habsb. I/2 S. 855 n. 1078 (zu April).

1 Am 20. April 1478 verlieh K.F. Völkermarkt zum Jahrmarkt im Herbst einen zweiten Jahrmarkt im Juni, S. Chmel, Mon. Habsb. I/2 S. 853 n. 1073.

2 Reg.: TOMASCHEK, Regesten Kärnten 3 n. 500; MC 11 n. 490.

\section{April 16, Graz}

K.F. ermahnt Kg. Ludwig (XI.) von Frankreich unter Androhung des gewaltsamen Vorgehens, die von diesem eroberte Stadt Cambrai und andere Güter des Reichs zurückzustellen, den gefangen genommenen $\mathrm{Bf}$. von Verdun ${ }^{1}$ freizulassen, die besetzten Schlösser und Städte des Bistums wieder herauszugeben und die von Hz. Karl von Burgund hinterlassenen Herrschaften nicht mehr zum Schaden seines Sohnes Ehz. Maximilian von Österreich und Burgund ${ }^{2}$ und dessen Gemahlin Hzn. Maria (von Burgund) zu bedrängen. Die sedecima mensis aprilis (nach Kop.).

KVr: A.m.p.d.i. (nach Kop.).

Org. im bearbeiteten Bestand nicht überliefert, der Kop. zufolge mit aufgedr. S. - Kop.: Zeitgleiche Abschrift (lat.) im HHStA Wien (Sign. AUR 1478 IV 16), Pap. (fol. 1rv); ${ }^{3}$ davon Abschrift des 18. Jh. ebd. (Sign. Urkundenabschriften Österr. Urkunden, Kt. 46 sub dat.), Pap.

Druck: ChMEL, Mon. Habsb. I/2 S. 317f. n. 8a. 
Reg.: Ein ausfiuhrliches Regest bieten die Regg.F.III. H. 4 n. 792 (nach Kop., mit Lit.), weiters Regg.F.III. H. 10 n. 440 (nach dt. Fassung); s. auch ChMEL n. 7196 und LichNowsky(-BIRK) 8 n. 42 .

Lit.: HollweG, Georg Heßler S. 78; WIESFLECKER, Kaiser Maximilian I./1 S. 141f.

1 Guillaume de Haraucourt (Bf. 1457-1500), der sich seit 1463 in Gefangenschaft des französischen Königs befand, s. zu ihm GaTz, Bischöfe 2 S. 257.

2 Zur Titulatur Maximilians s. die Einleitung S. 22-24.

3 Im Anschluss an das vorliegende Stück folgt von derselben Hand die Abschrift eines Entwurfs (lat., fol. 2r) für die Absage der Reichsfürsten und anderer Reichsuntertanen; Druck: CHMEL, Mon. Habsb. I/2 S. 318f. n. 8b. - Siehe in der Sache auch die zeitgleichen Abschriften von zehn zwischen 1478 Mai 24 und Juni 15 ausgestellten Absagebriefen deutscher Fürsten im HHStA Wien (Sign. AUR sub dat. 1478 V24), bis auf ein Stück (Absage Kf. Ernsts von Sachsen von 1478 Juni 12) alle gedruckt bei CHMEL, ebd. S. 321-328 nn. 10-14 und 16-19; s. auch Regg.F.III. H. 2 n. 165, H. 4 n. 790, H. 10 nn. 438, 441.

\section{[1478 April 18, Graz $]^{1}$}

K.F. überträgt Hans Gall von Buchenstein bis auf Widerruf die Pflege von Schloss Hohenmauthen und überlässt ihm die Maut sowie die Nutzen und Renten, wie sie der verstorbene Anton Hollenegger innehatte, bestandweise ab St. Georgstag 1478 (April 24) ${ }^{2}$ gegen Zahlung von jährlich $120 \mathrm{Pfd}$. Pf.

KVr: C.d.i. per manum propriam (nach CHMEL).

Org. oder Kop. im bearbeiteten Bestand nicht überliefert. - Dep.: Erw. im Revers Hans Galls von Buchenstein von 1478 April 18, Graz, im HHStA Wien (Sign. AUR sub dat. 1470 IV 18), Perg., 2 grüne SS des Ausst. und Erhard Lembachers in wachsf. Schüsseln an Ps, mit Rta-Vermerk auf der Rückseite. ${ }^{3}$

Reg.: ChMEL, Mon. Habsb. I/2 S. 743 n. 748.

Lit.: MUCHAR, Geschichte 8 S. 103; PIRCHEGGER, Untersteiermark S. 166.

1 Datierung und Ausstellungsort nach Reg. bei CHMEL.

2 Zur Datierung des St. Georgstags auf April 24 s. Regg.F.III. H. 12 n. 6, Anm. 2.

3 Reg.: Muchar, Urkunden-Regesten S. 473 n. 262; der Revers erw. bei ChMEL zu n. 748; s. dazu auch die ksl. Befehle an Hertnid Hollenegger, Sohn des früheren Pflegers Anton Hollenegger, das Schloss am St. Georgstag abzutreten, sowie an die zum Schloss gehörigen Leute bei CHMEL, ebd. S. 724 n. 674 und S. 745 n. 757. 
K.F. ${ }^{1}$ belehnt seinen Sohn Ehz. Maximilian von Österreich und Burgund ${ }^{2}$ und dessen Gemahlin Maria, geborene Hzn. von Burgund, sowie ihre gemeinsamen ehelichen Leibeserben aus ksl. Machtvollkommenheit und rechter wissen in crafft diss briefs, was wir ine von rechts und gnaden wegen daran zuverleihen haben, mit dem Hzm. Geldern samt der Gft. Zutphen und allen Schlössern, Städten, Märkten, Dörfern, Gebieten, Herrschaftsrechten, Lehenschaften, hohen und niederen Gerichten, zwingen, pennen, Mauten, Zöllen, Geleiten, Jagden, Wildbannen, Erzen, Bergwerken, Weidneien, Ehren, Rechten, Würden, Zierden, gerichtzwenngen, Nutzen, Gülten, Gefällen sowie allen anderen zugehörigen Rechten, die der verstorbene Hz. Karl von Burgund von ihm und dem Reich zu Lehen ${ }^{3}$ hatte und die sie gemäß Lehensrecht, Gewohneit und Herkommen innehaben, halten, besitzen, nutzen und gebrauchen sollen. Er verfügt, dass Maximilian bis sannt Michels tag schiristkunfftig (September 29) ${ }^{4}$ für sich und seine Gemahlin Kardinal Georg (Heßler) an seiner (K.F.) statt den gewöhnlichen Lehenseid leisten soll, und gebietet allen Untertanen des Hzm. Geldern und der Gft. Zutphen, ${ }^{5}$ nämlich den Prälaten, Gff., Freiherren, Rittern, Knechten, Adeligen, Städten und Landschaften, Mannen, Amtleuten, Bürgermeistern, regierenden Räten, Richtern, Vögten, Bürgern, Gemeinden, Hintersassen und allen anderen Personen, bei seiner und des Reichs schweren Ungnade und Strafe Treue und Gehorsam gegenüber Ehz. Maximilian, ihrem rechten naturlichen Herrn, Hzn. Maria und deren beider Erben sowie die Beachtung dieses Privilegs. Am newntzehenden tag des monets aprilis. ${ }^{6}$

KVr: A.m.d.i.p. referente d(omino) Sigismundo Pruschenck camer(ario). - KVv: Rta (Blattmitte). - B (rechter Blattrand). - Regalia domini archiducis Maximiliani super principatu Gelern (rechter Blattrand).

Org. im HHStA Wien (Sign. AUR 1478 IV 19), Perg., S samt Ss ab und verloren, der Siegelankündigung zufolge mit dem keiserlichen maiestat anhangendem insigel (wohl S 15). - Kop.: Vidimus $^{7}$ Abt Leonhards von Wilten von 1505 April 5, Wilten, ebd. (Sign. AUR 1478 IV 19), ${ }^{8}$ Perg., rotes (spitzovales) S des Klosters Wilten in wachsf. Schüssel an roter Ss; davon Abschrift des 18. Th. ebd. (Sign. Urkundenabschriften Österr. Urkunden, Kt. 46 sub dat.), Pap. - Abschrift (Org.) des 19. Jh. ebd. (Sign. Urkundenabschriften Österr. Urkunden, Kt. 46 sub dat.), Pap.

Druck: LÜNIG, RA 7 S. 116f. n. 19; CHMEL, Mon. Habsb. I/2 S. 396-398 n. 88/1 (nach Kop. [Vidimus von 1505]).

Reg.: ChMel n. 7198; Gross, Urkunden 1 S. 5 n. 7; Regg.F.III. H. 7 n. 624 (nach unzulänglicher Überlieferung).

Lit.: WIESFleCKeR, Kaiser Maximilian I./1 S. 141; HeINIG, Kaiser, Reich und Burgund S. 69; RoTHOFF-KraUs, Geldern und Habsburg S. 140f.; Böck, Herzöge und Konflikt S. 562. 
1 Im Vidimus von 1505, das alle drei Burgund betreffenden Lehensurkunden für Maximilian vom selben Tag beinhaltet (nn. 146-148), führt K.F. in der vorliegenden Urkunde für Geldern und Zutphen in der Intitulatio den Titel erczherczog zu Osterreich (so auch im Druck bei CHMEL), während im Original der Titel „Herzog zu Österreich" lautet.

2 Titulatur Maximilians: Ehz. zu Österreich, Burgund, Brabant, Lothringen, Steier, Kärnten, Krain, Limburg, Luxemburg und Geldern, Gf. zu Flandern, Habsburg, Tirol, Pfirt, Kyburg, Burgund und Artois, Pfgf. in Hennegau, Holland, Seeland, Namur und Zutphen, Mgf. des Heiligen Röm. Reiches und zu Burgau, Ldgf. im Elsass und Herr zu Friesland, auf der Windischen Mark, zu Portenau, Salins und Mecheln. In den nn. 147, 148 weicht die Titulatur Maximilians in der Reihenfolge der Herrschaften ab (s. n. 147, Anm. 1); zur Titulatur s. ausführlich die Einleitung S. 22-24.

3 Belehnung Hz. Karls von Burgund (als Hz. von Brabant) mit Geldern und Zutphen von 1473 November 6 auf dem Tag zu Trier, Druck: Gross, Urkunden 1 S. 2-4 n. 5; s. auch Regg.F.III. H. 27 n. 217. - Das von Karl dem Kühnen annektierte Hzm. Geldern war das einzige der zum Reich gehörigen burgundischen Territorien, mit dem dieser belehnt worden war; s. dazu Енм, Burgund und das Reich S. 134, 173; MüLLER, Herzöge von Burgund S. 448f:; Böck, Herzöge und Konflikt S. $544 f$.

4 Zur Leistung des Lehenseids s. n. 149.

5 Siehe Regg.F.III. H. 7 n. 625: Mandat an die Untertanen von Geldern und Zutphen von 1478 April 22.

6 Zum Revers Maximilians aus dem Jahr 1478 (Datum nicht bekannt) über die erfolgte Belehnung s. Regg.F.III. Supplemente 1 n. 28; s. dazu auch die Versicherung Maximilians von 1479 März 1, Geldern nicht ohne Zustimmung des $\mathrm{K}$. und des Reichs zu veräußern: Org. und Kop. (18. Jh.) im HHStA Wien (Sign. AUR 1479 III 1; Urkundenabschriften Österr. Urkunden, Kt. 46 sub dat.); Druck: CHMEL, Mon. Habsb. I/3 S. 165 n. 64; Reg.: ChMel n. 7266; LichNowsKy-(BIRK) 8 n. 148.

7 Das Vidimus von 1505 beinhaltet die nn. 146-148 und wurde ausgestellt auf Bitte des oberösterreichischen Regiments zu Innsbruck (Landhofmeister, Marschall, Kanzler, Statthalter und Regenten).

8 Unter dieser Sign. gemeinsam verwahrt mit den beiden Vidimus Kardinal Bernhards von 1538 (s. nn. 147, 148).

\section{April 19, Graz}

K.F. entspricht der Bitte seines Sohnes Ehz. Maximilian von Österreich und Burgund ${ }^{1}$ um schriftliche Belehnung, da ihm dieser vorgebracht hat, wegen des Krieges gegen den Kg. (Ludwig XI.) von Frankreich nicht abkömmlich zu sein, und belehnt mit wolbedachtem mute, guter zeittiger vorbetrachtung und rate und ausz rechter wissen ihn und dessen Gemahlin Maria, geborene Hzn. von Burgund, sowie ihre gemeinsamen ehelichen Leibeserben aus ksl. Machtvollkommenheit und rechter wissen mit und in crafft disz unsers keyserlichn briefs, was wir als Romischer keyser von recht billicheit und ausz sondern gnaden inen daran zuverleihen haben sollen oder mugen, mit den Herzogtümern Burgund, Brabant, Lothringen, Limburg, Luxemburg und Geldern, den Grafschaften Flandern, Burgund, Holland, Seeland, Artois, Namur und Zutphen, der Pfgft. zu Hennegau, der Mgft. des Reichs (Antwerpen) und den Herrschaften Friesland, Salins und Mecheln samt allen anderen Grafschaften und Herrschaften, zugehörigen Regalien, Lehen, weltlichen Rechten, Mannen, Mannschaften, Lehenschaften, geistlichen und weltlichen Herrschaftsrechten, Obrigkeiten, Erzen, Bergwerken, Wild- 
bannen, Weidneien, Ehren, Rechten, Würden, Zierden, hohen und niederen Gerichten, gerichtszwenngen, Nutzen, Gülten und Gefällen sowie allen anderen Rechten, die der verstorbene $\mathrm{Hz}$. Karl von Burgund innehatte und hinterlassen hat und die sie von ihm und dem Reich gemäß Lehensrecht, Gewohneit und Herkommen innehaben, halten, besitzen, nutzen und gebrauchen sollen. Er verfügt, dass Maximilian bis sanndt Michels tag schirstkunfftig (September 29) für sich und seine Gemahlin Kardinal Georg (Heßler) an seiner (K.F.) statt den gewöhnlichen Lehenseid ${ }^{2}$ leisten soll, jedoch soll bei Gelegenheit der persönliche Lehensempfang von ihm (K.F.) oder seinen Nachfolgern im Reich und die persönliche Eidesleistung erfolgen. K.F. gebietet allen Untertanen der genannten Herzogtümer, Grafschaften, Pfalzgrafschaften, Herrschaften und Länder, nämlich den Prälaten, Gff., Freiherren, Rittern, Knechten, Adeligen, Städten und Landschaften, Mannen, Amtleuten, Bürgermeistern, regierenden Räten, Richtern, Vögten, Bürgern, Gemeinden, Hintersassen und allen anderen Personen, bei seiner und des Reichs schweren Ungnade und Strafe Treue und Gehorsam gegenüber Ehz. Maximilian, ihrem rechten naturlichen Herrn, Hzn. Maria und deren beider Erben sowie die Beachtung dieses Privilegs. Am newnczehenden tag des monads aprilis.

KVr: A.m.d.i.p. - KVv: Rta Lucas Snitzer.

Org. im HHStA Wien (Sign. AUR 1478 IV 19), Perg., S samt Ss ab und verloren, der Siegelankündigung zufolge mit dem keyserlichen maiestat anhangendem insigel (wohl S 15). ${ }^{3}$ - Kop.: Vidimus ${ }^{4}$ Abt Leonhards von Wilten von 1505 April 5, Wilten, ebd. (Sign. AUR sub dat. 1478 IV 19), ${ }^{5}$ Perg., rotes (spitzovales) $\mathrm{S}$ des Klosters Wilten in wachsf. Schüssel an roter Ss; davon Abschrift des 18. Jh. ebd. (Sign. Urkundenabschriften Österr. Urkunden, Kt. 46 sub dat.), Pap. Vidimus ${ }^{6}$ Kardinal Bernhards (von Cles), Bf. von Trient, Präsident des Geheimen Rats, Oberster Kanzler und Statthalter der oberösterreichischen Länder, von 1538 Oktober 14, Linz, ebd. (Sign. AUR sub dat. 1478 IV 19), Perg., rotes S des Ausst. an rot-weißer Ss.; davon Abschrift des 18. Jh. ebd. (Sign. Urkundenabschriften Österr. Urkunden, Kt. 46 sub dat.), Pap. - Abschrift (Org.) des 19. Jh. ebd. (Sign. Urkundenabschriften Österr. Urkunden, Kt. 46 sub dat.), Pap.

Druck: ChMel, Mon. Habsb. I/2 S. 398-400 n. 88/2 (nach Kop. [Vidimus von 1505]).

Reg.: LichNowsKY(-BIRK) 8 n. $44 ;^{7}$ Gross, Urkunden 1 S. 4 f. n. $6 .^{8}$

Lit.: WIESFleCker, Kaiser Maximilian I./1 S. 141; HeINIG, Kaiser, Reich und Burgund S. 69; RothoFF-KRAUS, Einleitung S. 15f.; s. auch NiEDERSTäTTER, Jahrhundert der Mitte S. 170.

Die Belehnung mit den burgundischen Ländern wurde in Form von zwei Urkunden mit in der Dispositio grundlegenden Unterschieden ausgefertigt. Mit dem vorliegenden Stück wurden Maximilian und Maria bemerkenswerterweise mit allen von Karl dem Kühnen hinterlassenen Herzogtümern und Herrschaften belehnt, also auch mit den von Frankreich lehenrührigen Teilen des burgundischen Reichs, wie dem Hzm. Burgund, der Gft. Artois und großen Teilen Flanderns, 
während mit der zweiten Urkunde (n. 148) die Belehnung nur mit den burgundischen Reichslehen erfolgte.

Die französischen Lehen der burgundischen Länder waren nach dem Tod Karls des Kühnen 1477 sofort von Kg. Ludwig XI. als heimgefallene Lehen durch Erlöschen im Mannesstamm eingezogen worden. Demgegenüber gründete Maximilian sein Recht als legitimer Nachfolger auf Marias Geburtsrecht als geborene Herzogin von Burgund. Die von Karl dem Kühnen demonstrativ behauptete Souveränität gegenüber der französischen Krone war von Ludwig XI. nie akzeptiert worden. ${ }^{9}$ Unabhängig von den strittigen und im Erbfolgekrieg ausgetragenen Fragen des Erbfolgerechts Marias bzw. Nachfolgerechts Maximilians sowie der Souveränität Burgunds gegenüber Frankreich entbehrte die mit der vorliegenden Belehnung beanspruchte Eingliederung aller burgundischen Länder in den Reichslehensverband jeglicher rechtlichen Grundlage. Sie kann jedoch als politische Strategie gedeutet werden, zunächst „Wahrheiten zu schaffen“ ${ }^{10}$ die im Falle eines für Maximilian siegreichen Kriegsverlaufs etabliert und vielleicht hätten durchgesetzt werden können.

Die bis auf Teile der Dispositio nahezu wortgleichen Urkunden wurden bislang hinsichtlich ihres inhaltlichen Unterschieds und politischer Brisanz nicht wahrgenommen.

1 Titulatur Maximilians: Ehz. zu Österreich, Burgund, Steier, Kärnten, Krain, Brabant, Lothringen, Limburg, Luxemburg und Geldern, Gf. zu Habsburg, Tirol, Flandern, Burgund, Pfirt, Kyburg, Holland, Seeland, Artois, Namur und Zutphen, Pfgf. zu Hennegau, Mgf. des Heiligen Reichs und zu Burgau, Ldgf. im Elsass, Herr auf der Windischen Mark, zu Portenau, Friesland, Salins und Mecheln. Diese Titulatur Maximilians auch in n. 148, hingegen in n. 146 in der Reihenfolge der Herrschaften abweichend (s. ebd., Anm. 2); zur Titulatur s. ausführlich die Einleitung S. 22-24.

2 Zur Leistung des Lehenseids s. n. 149.

3 Rücks. Vermerk (16. Jh.): Dese brieven zijn geampetiret bij (gestrichen: keyser) hertoge Maximilian zue beginsel dat hij prince gewoorden es bij arrive, nyet weetende de previlegien ende nature vanden landen, ende zijn getemmiert (?) om dat de landen daer dore nyet en souden woorden vercort in huer vrijeheyden ende huer geleit veur vorsaatecheyt (?) (Blattmitte, Lesung unsicher).

4 Das Vidimus von 1505 beinhaltet die nn. 146-148 und wurde ausgestellt auf Bitte des oberösterreichischen Regiments zu Innsbruck (Landhofmeister, Marschall, Kanzler, Statthalter und Regenten).

5 Unter dieser Sign. gemeinsam verwahrt mit den beiden Vidimus Kardinal Bernhards von 1538 (zum 2. Vidimus s. n. 148)

6 Ausgestellt auf Bitte Kg. Ferdinands I.

7 Mit irrtümlichem Verweis auf LüNIG, RA 7 S. 116f. n. 19 (= Druck von n. 146).

8 Nach Gross unrichtig Belehnung mit den von Karl dem Kühnen hinterlassenen Reichslehen.

9 So führte Karl der Kühne den Titel eines souverain seigneur. Im Mai 1478 wurde in Paris posthum ein Prozess gegen ihn wegen Vergehens gegen die Souveränitat Frankreichs eröffnet, s. dazu WIESFLECKER, Kaiser Maximilian I./1 S. 143; PARAVICIN, Souverain seigneur, bes. S. 39f., 45-47; nach Bock, Maximilian S. 41, sei die rechtliche Stellung Marias als Erbin der burgundischen Länder unangreifbar gewesen.

10 Formulierung nach Herold, Ringen um den Text S. 325, hier bezogen auf die Belehnung Hz. Albrechts VI. von Österreich mit Brabant, Holland, Seeland und Hennegau im Jahr 1446. 
K.F. entspricht wie in n. 147 der Bitte seines Sohnes Ehz. Maximilian von Österreich und Burgund ${ }^{1}$ um schriftliche Belehnung und belehnt mit wolbedachtem mut, guter zeittiger vorbetrachtung und rate und aus rechter wissen ihn und dessen Gemahlin Maria, Hzn. von Burgund, sowie ihre gemeinsamen ehelichen Leibeserben aus ksl. Machtvollkommenheit und rechter wissen mit und in crafft diss unnsers keiserlichen briefs, was wir als Romischer keiser von recht billicheit und aus sonndern gnaden inen daran zu raichen und zuleihen haben sollen oder mogen, mit den Herzogtümern Brabant, Lothringen, Limburg, Luxemburg und Geldern, der Gft. Flandern, sovil der von dem heiligen reich zulehen rüret, den Grafschaften Burgund, Holland, Seeland, Namur und Zutphen, der Pfgft. zu Hennegau, der Mgft. des Reichs (Antwerpen), den Herrschaften Friesland, Salins und Mecheln samt allen anderen Grafschaften und Herrschaften, zugehörigen Regalien, Lehen, weltlichen Rechten, Mannen, Mannschaften, Lehenschaften, geistlichen und weltlichen Herrschaftsrechten, Obrigkeiten, Erzen, Bergwerken, Wildbannen, Weidneien, Ehren, Rechten, Würden, Zierden, hohen und niederen Gerichten, gerichtzzwenngen, Nutzen, Gülten, Gefällen sowie allen anderen Rechten, die der verstorbene $\mathrm{Hz}$. Karl von Burgund innehatte und hinterlassen hat und die sie von ihm (K.F.) und dem Reich gemäß Lehensrecht, Gewohneit und Herkommen innehaben, halten, besitzen, nutzen und gebrauchen sollen. K.F. verfügt, dass Maximilian bis sannt Michelstag schiristkünfftig (September 29) für sich und seine Gemahlin Kardinal Georg (Heßler) an seiner (K.F.) statt den gewöhnlichen Lehenseid ${ }^{2}$ leisten soll, jedoch soll bei Gelegenheit der persönliche Lehensempfang von ihm (K.F.) oder seinen Nachfolgern im Reich und die persönliche Eidesleistung erfolgen. K.F. gebietet allen Untertanen der genannten Herzogtümern, Grafschaften, Pfalzgrafschaften, Herrschaften und Länder, nämlich den Prälaten, Gff., Freiherren, Rittern, Knechten, Adeligen, Städten und Landschaften, Mannen, Amtleuten, Bürgermeistern, regierenden Räten, Richtern, Vögten, Bürgern, Gemeinden, Hintersassen und allen anderen Personen, bei seiner und des Reichs schweren Ungnade und Strafe Treue und Gehorsam gegenüber Ehz. Maximilian, ihrem rechten naturlichen Herrn, Hzn. Maria und deren beider Erben sowie die Beachtung dieses Privilegs. Am newnczehennden tag des monets aprilis.

KVr: A.m.p.d.i. - KVv: Rta Lucas Snitzer.

Org. im HHStA Wien (Sign. AUR 1478 IV 19), Perg., S samt Ss ab und verloren, der Siegelankündigung zufolge mit dem keiserlichen maiestat anhangendem insigel (wohl S 15). ${ }^{3}$ Kop.: Vidimus ${ }^{4}$ Abt Leonhards von Wilten von 1505 April 5, Wilten, ebd. (Sign. AUR sub dat. 1478 IV 19), ${ }^{5}$ Perg., rotes (spitzovales) S des Klosters Wilten in wachsf. Schüssel an roter Ss; davon Abschrift des 18. Jh. ebd. (Sign. Urkundenabschriften Österr. Urkunden, Kt. 46 sub dat.), Pap. - Vidimus ${ }^{6}$ Kardinal Bernhards (von Cles), Bf. von Trient, Präsident des Geheimen Rats, Oberster Kanzler und Statthalter der oberösterreichischen Länder, von 1538 Oktober 14, Linz, 
ebd. (Sign. AUR sub dat. 1478 IV 19), Perg., rotes S des Ausst. an rot-weißer Ss; davon Abschrift des 18. Jh. ebd. (Sign. Urkundenabschriften Österr. Urkunden, Kt. 46 sub dat.), Pap. - Abschrift (Org.) des 19. Jh. ebd. (Sign. Urkundenabschriften Österr. Urkunden, Kt. 46 sub dat.), Pap.

Reg.: Erw. bei ChMel, Mon. Habsb. I/2 S. 400 n. 88/3 (nach Kop. [Vidimus von 1505]) und bei Gross, Urkunden $1 \mathrm{~S} .4 \mathrm{f}$. zu n. $6 .^{7}$

Lit. siehe bei den nn. 146, 147; weiters Bock, Maximilian S. 40, der nur das vorliegende Stïck erwähnt.

Mit dieser zweiten, bis auf Teile der Dispositio mit n. 147 nahezu wortgleichen Urkunde erfolgte die Belehnung mit den burgundischen Reichslehen, also im Unterschied zu n. 147 ohne dem Hzm. Burgund und der Gft. Artois sowie beschränkt auf die zum Reich gehörigen Teile Flanderns (,Reichsflandern“). Auffallenderweise wird hier Maria auch nicht, wie in n. 147, als "geborene" Herzogin von Burgund bezeichnet.

Wohl aufgrund der unzulänglichen Angaben bei CHMEL und Gross (s. Anm. 7) galt die vorliegende Belehnungsurkunde als gleichlautende Ausfertigung von n. 147 mit geringfügigen Abweichungen und nicht als inhaltlich eigenständiges Stïck.

1 Titulatur Maximilians wie in n. 147 (Anm. 1), s. dazu ausführlich die Einleitung S. 22-24.

2 Zur Leistung des Lehenseids s. n. 149.

3 Rücks. Vermerke (16. Jh.): Dese brifven zijn geampetiret per hertoge Maximilian in beginsel dat hij prince gewoorden es bij arrive, ende nyet weetende de previlegien ende naturen vaanden landen ende zijn naemaels getaemiert (?) uuyt zijn handen ten regarde dat de landen daer bij nyet en souden woorden vercort in heuren vryheyden ende huer geleit om vorsaatecheyt (?) (Blattmitte links, Lesung unsicher). Darunter (in anderer Tinte): J. J.

4 Das Vidimus von 1505 beinhaltet die nn. 146-148 und wurde ausgestellt auf Bitte des oberösterreichischen Regiments zu Innsbruck (Landhofmeister, Marschall, Kanzler, Statthalter und Regenten).

5 Unter dieser Sign. gemeinsam verwahrt mit den beiden Vidimus Kardinal Bernhards von 1538 (zum 2. Vidimus s. n. 147)

6 Ausgestellt auf Bitte Kg. Ferdinands I.

7 Chmel bietet nur den Beginn der Urkunde (Wr Friderich von Gots gnaden Römischer kayser etc.) und verweist dann auf n. 147, da es sich um ein gleichlautendes Stück handeln würde mit Ausnahme des von ihm zitierten Zusatzes zur Gft. Flandern (sovil der von dem hailigen reich zu lehen rüret); desgleichen GROss, der das Vorliegen einer zweiten gleichlautenden Ausfertigung mit Ausnahme des einschränkenden Zusatzes bei Flandern erwähnt.

\section{[1478 zw. April 19 und August 20, -]}

K.F. befiehlt Kardinal Georg (Heßler), an seiner statt von seinem Sohn Ehz. Maximilian von Österreich und Burgund ${ }^{1}$ und dessen Gemahlin Hzn. Maria den Lehenseid für die ihnen von ihm und dem Reich verliehenen Lehen ${ }^{2}$ gemäß der ihm schriftlich übermittelten Eidesformel entgegenzunehmen. 
Org. oder Kop. im bearbeiteten Bestand nicht überliefert. - Dep.: Erw. in der Bestätigung Kardinal Georgs (Heßler) über die Leistung des Lehenseids durch Ehz. Maximilian von 1478 August 20, Brügge, im HHStA Wien (Sign. AUR 1478 VIII 20), Perg., S samt Ps ab und verloren; ${ }^{3}$ davon Abschrift des 18. Jh. ebd. (Sign. Urkundenabschriften Österr. Urkunden, Kt. 46 sub dat.), Pap.

Offen muss bleiben, ob Maximilian den Lehenseid gemäß den nn. 146 und 148 für Geldern, Zutphen und die burgundischen Reichslehen oder gemäß n. 147 für alle burgundischen Länder inklusive der französischen Kronlehen - geleistet hat.

1 Zur Titulatur Maximilians s. die Einleitung S. 22-24.

2 Siehe nn. 146-148.

3 Druck: Chmel, Mon. Habsb. I/2 S. 421 n. 110; Reg.: Lichnowsky(-BIRK) 8 n. 101; s. dazu HollweG, Georg Heßler S. 81.

\section{[Vor 1478 April 20, -]}

K.F. überlässt Gilg Prantner, Bürger von Cilli, und dessen Erben die Maut zu Cilli und zu Freudenthal ${ }^{1}$ bestandweise für drei Jahre ab St. Georgstag 1478 (April 24) ${ }^{2}$ gegen Zahlung von jährlich 750 Mark Pf.

Org. oder Kop. im bearbeiteten Bestand nicht überliefert. - Dep.: Erw. im Revers des Cillier Bürgers Gilg Prantner von 1478 April 20 im HHStA Wien (Sign. AUR 1478 IV 20), Perg., 2 SS des Ausst. und des Ritters Andreas Hohenwarter zu Gerlachstein an Ps ab und verloren, mit Rta-Vermerk auf der Rückseite.

1 Im Revers Frencz.

2 Zur Datierung des St. Georgstags auf April 24 s. Regg.F.III. H. 12 n. 6, Anm. 2.

\section{[Vor 1478 April 21, -]}

K.F. überträgt Wolfgang Praun bis auf Widerruf die Pflege von Schloss Kammer im Attersee und überlässt ihm die zugehörigen Nutzen und Renten bestandweise gegen Zahlung von jährlich 600 Pfd. Pf. ${ }^{1}$

Org. oder Kop. im bearbeiteten Bestand nicht überliefert. - Dep.: Erw. im Revers Wolfgang Prauns von 1478 April 21, Graz, im HHStA Wien (Sign. AUR sub dat. 1478 I 29), ${ }^{2}$ Perg., 2 grüne SS des Ausst. und Erhard Lembachers in wachsf. Schüsseln an Ps, mit Rta-Vermerk auf der Rückseite. $^{3}$ 
1 Siehe dazu auch die Urkunde Wolfgang Prauns von 1478 April 27 im HHStA Wien (Sign. AUR 1478 IV 27), in der er die Einigung mit K.F. bzgl. aller Schloss und Herrschaft Kammer und das Amt Gmunden betreffenden Forderungen und die Übergabe der entsprechenden Briefe an den $\mathrm{K}$. bestätigt; Reg.: CHMEL n. 7202; DERS., Mon. Habsb. I/2 S. 656 nn. 517, 518. Das Reg. n. 517, dem zufolge Praun von K.F. 2.000 fl. ung. erhalten hat, entspricht allerdings nicht der überlieferten Urkunde, die nur die Einigung mit K.F. und die Erledigung der Forderungen bestätigt, aber keine Geldsumme nennt.

2 Unter dieser Sign. gemeinsam verwahrt mit den nn. 114, 119, 152.

3 Reg.: Chmel n. 7115 (zu 1477 April 21); Lichnowsky(-BIRK) 8 n. 46; ChMel, Mon. Habsb. I/2 S. $655 f$ f. n. 516.

\section{[1478 April 21, Graz $]^{1}$}

K.F. überlässt Stephan Widmer das Amt zu Hochenegg ${ }^{2}$ samt Landgericht, Vogteien, Anleiten, Leitgebgeld, ${ }^{3}$ Nutzen, Renten und allem Zubehör, auch mit dem durch Flucht oder Abgang der Holden ihm (K.F.) zustehendem Gut, bestandweise für drei Jahre ab St. Georgstag 1478 (April 24) ${ }^{4}$ gegen Zahlung von jährlich 452 Mark und 21 Pf.

Org. oder Kop. im bearbeiteten Bestand nicht überliefert. - Dep.: Erw. im Revers Stephan Widmers von 1478 April 23, Graz, im HHStA Wien (Sign. AUR sub dat. 1478 I 29), ${ }^{5}$ Perg., 2 grüne SS des Ausst. und Erhard Lembachers in wachsf. Schüsseln an Ps, mit Rta-Vermerk auf der Rückseite. ${ }^{6}$

Reg.: Chmel, Mon. Habsb. I/2 S. 745 n. 758 (mit Nennung des Reverses).

Lit.: PIRCHEGGER, Untersteiermark S. 212.

1 Datierung und Ausstellungsort nach Reg. bei CHMEL.

2 Hochenegg in der Untersteiermark und nicht, wie der Rückvermerk des 16. Jh. unnder der Enns auf dem Revers nahelegt, Hohenegg in Niederösterreich.

3 Abgabe des Leitgebs (Wirt) für das Schankrecht, auch Leitgebpfennig, s. DRW 8 Sp. 1215; auch GrmM, Deutsches Wörterbuch 12, Sp. 737.

4 Zur Datierung des St. Georgstags auf April 24 s. Regg.F.III. H. 12 n. 6, Anm. 2.

5 Unter dieser Sign. gemeinsam verwahrt mit den nn. 114, 119, 151.

6 Reg.: ChMEL n. 7200; DERS., Mon. Habsb. I/3 S. 649 n. 54; erw. bei Muchar, Geschichte 8 S. 103.

\section{April 23, Graz}

K.F. fordert seinen Sohn Ehz. Maximilian von Österreich und Burgund ${ }^{1}$ auf, sich nicht, wie geplant, zu Verhandlungen mit den pundtgenossen nach Metz zu begeben, sondern in dessen Ländern zu bleiben und die Verbündeten zu sich kommen zu lassen 
oder durch Bevollmächtigte zu verhandeln, da in dessen Abwesenheit unrat und irrung droht. An phintztag vor sand Jorgen tag.

KVr: C.d.i.i.c. - KVv: Dem hochgeboren Maximilian erczherczogen zu Osterreich, zu Burgundi, zu Brabandt etc., graven zu Flanndern etc., unserm lieben sun und fursten (Adresse, Blattmitte).

Org. im HHStA Wien (Sign. AUR 1478 IV 23), Pap., rotes S 21 rücks. aufgedr. (großteils abgefallen).

Abb.: Kaiserurkunden in Abb., Lfg. XI, Tf. 24c.

Druck: ChmeL, Mon. Habsb. I/2 S. 403 n. 93.

Reg.: Kaiserurkunden in Abb., Textbd. S. $512 f$.

1 Zur Titulatur Maximilians s. die Einleitung S. 22-24.

[Um 1478 April 24, - ] $]^{1}$

K.F. überlässt Simon Krell von Sachsenburg Fron und Wechsel des Bergbaus in der Herrschaft Ortenburg und in den Gerichten Vellach, Rottenstein, Greifenburg und Kirchheim samt dem gewonnenen Erz aus den Anteilen des Kainachers ${ }^{2}$ bestandweise für zwei Jahre ab St. Georgstag 1478 (April 24) ${ }^{3}$ bis Weihnachten 1480 (1479 Dezember 25) gegen Zahlung von 600 Mark Silber.

Org. oder Kop. im bearbeiteten Bestand nicht überliefert. - Dep.: Erw. im Revers Simon Krells von 1480 Januar 26 im HHStA Wien (Sign. AUR 1480 I 26), Perg., 2 grüne SS des Ausst. und des Wiener Neustädter Ratsherrn Prokop Zinner ${ }^{4}$ in wachsf. Schüsseln an Ps, mit Rta-Vermerk auf der Rückseite. ${ }^{5}$

Lit.: WIESSNER, Kärntner Bergbau 1 S. 41f.; Fräss-EHRFELD, Geschichte Kärntens 1 S. 566, 671.

Siehe nn. 20, 22.

1 Zur Datierung: Im Revers Simon Krells von 1480 Januar 26 wird ein ksl. Bestandbrief für den Zeitraum von 1478 April 24 bis 1479 Dezember 25 erwähnt. Die Bestandgabe kann sowohl lange vor dem 24. April als auch später, dann rückwirkend, erfolgt sein.

2 Hans von Kainach († 1472), s. zu ihm HärTEL, Kainacher S. 13; zu dessen Anteilen s. den Kommentar zu n. 20.

3 Zur Datierung des St. Georgstags auf April 24 s. Regg.F.III. H. 12 n. 6, Anm. 2. 
4 Siehe zu ihm MAYER, Wiener Neustadt I/2 S. $491 \mathrm{f}$.

5 Druck: Chmel, Mon. Habsb. I/3 S. 387f. n. 167; Reg.: Chmel n. 7355.

\section{[1478 April 27, Graz $]^{1}$}

K.F. überlässt Heinrich Gabelhuber, Bürger von Bruck a.d. Mur, das Ungeld außerhalb von Bruck auf dem Land im Mürztal bis zum Semmering samt den Nebentälern zu Neuberg, in der Stanz und in der Veitsch, im gesamten Aflenzertal mit (Maria-)Zell, im Tragösser- und im Utschtal mit den Nutzen, Renten und allem Zubehör, wie es Gabelhuber von Hans Einpacher ${ }^{2}$ innehatte, bestandweise für zwei Jahre ab St. Laurentiustag 1478 (August 10) gegen Zahlung von jährlich 500 Pfd. Pf.

Org. oder Kop. im bearbeiteten Bestand nicht überliefert. - Dep.: Erw. im Revers des Brucker Bürgers Heinrich Gabelhuber von 1478 April 27, Graz, im HHStA Wien (Sign. AUR 1478 IV 27), Perg., 2 SS des Ausst. (grün) und des Andreas am Stain (rot), Erzpriester der Untersteiermark, ${ }^{3}$ in wachsf. Schüsseln an Ps, mit Rta-Vermerk auf der Rückseite. ${ }^{4}$

Reg.: CHMEL, Mon. Habsb. I/2 S. 750 n. 773.

1 Datierung und Ausstellungsort nach Reg. bei CHMEL.

2 Siehe zu ihm DIENES, Bürger von Graz S. LXXIf.

3 Andreas am Stain (von Stein), Sekretär in der erbländischen Kanzlei, hatte seit 1473 die Pfarre Gratwein und das mit dieser verbundene Erzpriesteramt (Archidiakonat) der Untersteiermark inne, s. HEINIG, Friedrich III./1 S. $626 \mathrm{f}$.

4 Reg.: GöTH, Urkunden-Regesten 9 n. 666; erw. bei MuchaR, Geschichte 8 S. 103 (zu April 26). Der Revers sowie ksl. Befehle an Hans Einpacher, das Ungeld abzutreten, sowie an die zum Ungeldbezirk gehörigen Leute auch erw. bei CHMEL zu n. 773 .

\section{[1478 April 30, Graz $]^{1}$}

K.F. überträgt seinem Kämmerer Sigmund Prüschenk bis auf Widerruf die Pflege von Schloss Bernstein und überlässt ihm die zugehörigen Nutzen und Renten bestandweise gegen Zahlung von jährlich 200 Pfd. Pf.. Er untersagt ihm, die zugehörigen Leute und Holden über die gewöhnlichen Zinse, Dienste und Robotleistungen gegen altes Herkommen zu belasten, jedoch soll von den mit seiner Zustimmung und auf seinen Befehl eingehobenen Steuern jeweils die Hälfte an ihn und Prüschenk fallen.

Org. oder Kop. im bearbeiteten Bestand nicht überliefert. - Dep.: Erw. im Revers Sigmund Prüschenks von 1478 Mai 3, Graz, im HHStA Wien (Sign. AUR Großformate 1478 V 3), ${ }^{2}$ Perg., 
2 grüne SS des Ausst. und des Ritters Wilhelm von Auersperg ${ }^{3}$ in wachsf. Schüsseln an Ps, mit Rta-Vermerk auf der Rückseite. ${ }^{4}$

Reg.: ChMel, Mon. Habsb. I/2 S. 657 n. 520.

Lit.: Prickier, Geschichte Bernstein S. 22; zu Sigmund Prüschenk s. Probszt, Brüder Prueschenk; ZeRnATTO, Herrenstand S. 69-72; HeINIG, Friedrich III./1 S. 78-88, bes. 80, und 206; MARIAN/ZeHETMAYER, Hardegg S. 560f., 563, 568f.

1 Datierung und Ausstellungsort nach Reg. bei СнмеL

2 Unter dieser Sign. gemeinsam verwahrt mit den nn. 163, 191.

3 Ksl. Kämmerer und Rat, s. zu ihm HeINIG, Friedrich III./1 S. 231f.

4 Reg.: CHMEL n. 7203; DERS., Mon. Habsb. I/2 S. 659 f. zu n. 528 (ksl. Befehl an den Pfleger Konrad Zirkendorfer, das Schloss abzutreten); zur Abtretung s. auch ebd. S. 659 n. 526 und S. 663 n. 538.

\section{Mai 3, -}

K.F. quittiert eigenhändig dem (Juden) Isaak und dessen Enkel ${ }^{1}$, ihre Steuer in der Weise entrichtet zu haben, dass der Jude Jekel für eine Gült, die der K. von dem Kagerer gekauft hat, von ihnen bezahlt wurde. Exaudi.

Eigenhändige Unterfertigung von K.F.: $p$ (er) m(anum) p(ropriam).

Org. (ksl. Autograph) im HHStA Wien (Sign. AUR 1478 V 3), Pap., rotes S 16 vorders. aufgedr. - Kop.: Abschrift des 18. Jh. ebd. (Sign. Urkundenabschriften Österr. Urkunden, Kt. 46 sub. dat.), Pap.

Abb.: Kaiserurkunden in Abb., Lfg. XI, Tf. $6 b$.

Druck: ChMEL, Mon. Habsb. I/2 S. 563 n. 273.

Reg.: Chmel n. 7204; Kaiserurkunden in Abb., Textbd. S. 482; WIENER, Regesten 1 S. 96 n. 113 .

Lit.: KolLER, Bedeutung S. 124; WAGENDORFER, Eigenhändige Unterfertigungen S. 251, zu den eigenhändigen Schreiben ebd. S. 221-226.

Zum Formular: Die erste Zeile nimmt mittig das $F$ für Friedrich ein, danach folgt der Urkundentext (drei Zeilen), wobei K.F. für sich die 1. Person Singular verwendet. Die Datierung (4. Zeile) beschränkt sich auf den Tag und das Jahr (exaudi 1478). Die Eigenhändigkeit wird durch den rechts unten befindlichen Vermerk per manum propriam bestätigt.

Das Stïck war lange Zeit in der AUR nicht mehr vorhanden und wurde im Jahr $2015 \mathrm{im}$ Bestand „Fridericiana“ (Kt. 4, Konv. 3) wiedergefunden, wo es vermutlich in den 1930er-Jahren nach einer Ausstellung im HHStA Wien versehentlich eingereiht worden war. 
1 In der Urkunde korrigiert aus Eidam: aidem gestrichen und enenkel über die Zeile geschrieben.

\section{Mai 5, Graz}

K.F. bestätigt aus ksl. Machtvollkommenheit seinem Vetter Ehz. Sigmund von Österreich und dessen Erben auf dessen Bitte und in Ansehung der treuen Dienste, die dieser und dessen Vorfahren ihm und dem Reich geleistet haben, mit wolbedachtem mute, guttem rate und aus rechter wissen folgende vier von diesem vorgelegte und von wortte zu wortte inserierte Urkunden mit rechter wissen in crafft diss briefs, was wir als Romischer keyser von rechts oberkeit oder aus gnaden daran confirmiren und bestetten mugen, nämlich die transsumpt und vidimus eines Schiedsspruchs $\mathrm{Hz}$. Albrechts (V.) von Österreich, einer Urkunde Bf. Alexanders von Trient ${ }^{1}$ und eines Mandats $\mathrm{Kg}$. Albrechts (II.) sowie ein recht original einer besiegelten Pergamenturkunde Kg. Albrechts (II.): (1) Spruchbrief Hz. Albrechts (V.) von Österreich als Kommissar $\mathrm{Kg}$. Sigismunds von 1435 Mai 6 zur Beendigung der Streitigkeiten zwischen Hz. Friedrich (IV.) von Österreich und Bf. Alexander von Trient, dem zufolge sich Bf. Alexander zum Verbleib bei der Gft. Tirol und Hz. Friedrich als Landesfürst und Vogt der Trienter Kirche zu Hilfe und Beistand verschreiben sollen; (2) Bündnisbrief Bf. Alexanders von Trient von 1435 Mai 9, mit dem er sich auch namens seiner Nachfolger auf ewig an die Gft. Tirol bindet; ${ }^{2}$ (3) Mandat Kg. Albrechts (II.) ${ }^{3}$ von 1439 Mai 1 an die Amtleute Bf. Alexanders von Trient, seinem Spruch nachzukommen und bei Nichteinhaltung seitens des Bischofs Hz. Friedrich gehorsam zu sein; (4) Mandat Kg. Albrechts (II.) von 1439 Mai 1 an die Lehensleute der Kirche von Trient, Bf. Alexander zur Einhaltung seines Spruchs zu bewegen, andernfalls Hz. Friedrich als ihrem Vogt Hilfe zu leisten. K.F. verfügt aus ksl. Machtvollkommenheit, dass diese Urkunden in Kraft bleiben und Ehz. Sigmund und dessen Erben sie überall nach Bedarf und Willen gebrauchen können. Er gebietet allen Reichsuntertanen, insbesondere den Prälaten, Pflegern, Hauptleuten, Amtleuten, Richtern und Untertanen des Bistums Trient, bei seiner und des Reichs schweren Ungnade und einer Pön von 50 Mark Gold, zahlbar je zur Hälfte an die Reichskammer und Ehz. Sigmund, die Beachtung der vier Urkunden und dieser Bestätigung. Am funfften tag des monadts may.

KVr: A.m.d.i.p. Jo(hannes) Waldner prothonotarius etc. - Rta Lucas Snitzer (fol. 5v).

Org. im HHStA Wien (Sign. AUR 1478 V 5), Perg.-Libell ( 5 fol.), wachsf. S 15 mit wachsf. (wohl ehedem rotem) 4 S 16 vorders. eingedr. an purpurf. Ss. - Kop.: Zwei Abschriften des 18./19. Jh. ebd. (Sign. Urkundenabschriften Österr. Urkunden, Kt. 46 sub dat.), Pap.

Druck: ChMel, Mon. Habsb. I/2 S. 482-493 n. 124. 
Reg.: ChMel n. 7205.

Die Urkunde steht im Kontext ${ }^{5}$ des - seit 1365 bzw. 1368 durch die sog. Kompaktaten vertraglich geregelten - Abhängigkeitsverhältnisses des Hochstifts Trient von den Gff. von Tirol, wobei sich zunehmend die Frage der Zugehörigkeit zur Grafschaft stellte. Als die selbstständige Außenpolitik Bf. Alexanders zum Konflikt mit Hz. Friedrich IV. führte, wurde im Schiedsspruch Albrechts V. von 1435 ausdrücklich der Herzog als Landesfürst und Vogt des Bischofs bezeichnet. In der Folgezeit betonte insbesondere Ehz. Sigmund die landesfürstliche Hoheit über das Hochstift, das er als Bestandteil der Gft. Tirol betrachtete. Mit den mit Bf. Johann Hinderbach abgeschlossenen Kompaktaten ${ }^{6}$ von 1468 gelang ihm die weitgehende Sicherung des Einflusses auf die bischöfliche Außenpolitik und die Amtleute durch den vom Tiroler Landesfürsten eingesetzten Hauptmann von Trient; auch die vorliegende Urkunde dürfte diesem Ziel gedient haben. Sigmunds Mediatisierungspolitik, die Stellung der Bischöfe als unmittelbare Reichsfürsten zu untergraben, und dessen fortdauernden Übergriffe bewogen schließlich K.F., zum Schutz des Bischofs einzuschreiten. ${ }^{7}$

1 Alexander von Masowien (Bf. 1423-1444), s. zu ihm Wos, Alessandro di Masovia; Gatz, Bischöfe 1 S. $786 f$

$2 \mathrm{Zu}$ den Verschreibungen Hz. Friedrichs IV. vom selben Tag s. BraNDIs, Tirol S. 562-566 nn. 156, 157, und dazu GöBEL, Entstehung S. 153f.

3 Zu den beiden Mandaten Albrechts II. von 1439 Mai 1 s. RI XII nn. 900, 901. Hintergrund der kgl. Mandate war, wie im Mandat an die Amtleute dargelegt, das selbstständige Engagement Bf. Alexanders im Krieg zwischen Mailand und Venedig, wodurch der Krieg auf Trienter Gebiet übergriff; s. BRANDSTÄTTER, Beziehungen S. 24; BAuM, Sigmund der Münzreiche S. 58.

4 Rote Wachsreste erkennbar.

5 Siehe zum Folgenden bes. BRANDSTÄTter, Reichskirche S. 292-294, und DERS., Beziehungen, bes. 2327; weiters Bellabarba, Principato vescovile S. 404-410; ein Überblick bei DörRER, Limitierte Landeshoheit; s. auch JÄGER, Geschichte Tirols 2,2 S. 228f.; GöBEL, Entstehung S. 136-170.

6 Druck: RIEDMANN, Rapporti S. 136-139 n. 2.

7 Siehe Regg.FIII. H. 35 n. 189 (1482 April 18).

\section{Mai 14, Graz}

K.F. belehnt Jörg Glojacher als Älteren und Lehensträger seines Bruders Ulrich wissenntlich mit dem brief, was wir im zu recht daran verleihen sullen oder mugen, mit folgenden von ihnen ererbten Stücken und Gütern, ${ }^{1}$ die sie und ihre Erben gemäß Lehens- und Landrecht innehaben und nutzen sollen: eine Hube zu Hausmannstätten aus der Lehenschaft des Ftm. Steier, auf der der Pömerl gesessen ist, sowie aus der Lehenschaft der verstorbenen Pettauer ${ }^{2}$ ein halber Acker zu Afram (Aferham), eine Hube zu Stocking (Stokharn), die der Wiener innehat, und eine Hube zu Stiefing (Stifen), die der Haushan innehat. An pfintztag nach dem heiligen Pfingstag. 
KVr: C.d.i.i.c. - KVv: Rta. - Rückseite der Wachsschüssel: Vermerk nicht lesbar.

Org. im HHStA Wien (Sign. AUR 1478 V 14), Perg., rotes S 21 in wachsf. Schüssel mit wachsf. S 16 rücks. eingedr. an Ps.

Reg.: Muchar, Urkunden-Regesten n. 265; ChMEL, Mon. Habsb. I/2 S. 768 n. 837; GöTH, Urkunden-Regesten 9 n. 668; STARZER, Lehen S. 221 n. 103/3 (zu Hausmannstätten).

Lit:: MUCHAR, Geschichte 8 S. 100.

1 Zur Belehnung der Familie Glojacher im Jahr 1447 mit den genannten Gütern s. Regg.F.II. H. 13 nn. 24, 25.

2 Die Herren von Pettau starben mit Friedrich von Pettau ( $\dagger 1438$ Januar 6) aus, S. PIRCHEGGER, Herren von Pettau S. 28.

\section{[Vor 1478 Juni 3, -]}

K.F. überträgt Hans von Trautmannsdorf bis auf Widerruf die Pflege von Schloss Kammern samt dem Landgericht und überlässt ihm die zugehörigen Nutzen und Renten, wie sie Gallus Zech innehatte, ${ }^{1}$ bestandweise gegen Zahlung von jährlich $700 \mathrm{Pfd}$. Pf.

Org. oder Kop. im bearbeiteten Bestand nicht überliefert. - Dep.: Erw. im Revers des Hans von Trautmannsdorf von 1478 Juni 3 im HHStA Wien (Sign. AUR 1478 VI 3), Perg., 3 grüne SS des Ausst., seines Vetters und Bürgen Wolfgang von Trautmannsdorf sowie Friedrich Holleneggers in wachsf. Schüsseln an Ps, mit Rta-Vermerk auf der Rückseite. ${ }^{2}$

Lit:: Muchar, Geschichte 8 S. 103 (zu Mai 5); Baravalle, Burgen S. 381; EbNer, Burgen Steiermark 2 S. 70.

1 Gallus Zech war von 1474 bis 1478 April 24 (St. Georgstag) Pfleger von Kammern und hatte die Erträge gegen jährlich 600 Pfd. Pf. in Bestand, s. CHMEL, Mon. Habsb. I/2 S. 793 n. 919. In einem ksl. Schreiben von 1478 Mai 14 wird er noch als Pfleger genannt (ebd. S. $767 f$ n. 835 und S. 778 n. 872), die Übergabe an Hans von Trautmannsdorf dürfte erst im Juni erfolgt sein.

2 Reg.: Muchar, Urkunden-Regesten n. 266 (mit Bestandgeld von jährlich 300 Pfd. Pf.); Götr, Urkunden-Regesten 9 n. 669 (zu Juni 2).

\section{Juni 5, Graz}

K.F. bestätigt Wolfgang Pandorfer, Bürger von Steyr, und dessen Erben, ihnen 200 Pfd. Pf. gemäß einem schuldbrieff seines Bruders Ehz. Albrecht VI. von Österreich für den Kauf von Pandorfers Schleifmühle zu Steyr unter der Steyrbrücke zu schulden, verspricht, das Geld über zwei Jahre ab Datum dieses briefs zu bezahlen, 
und verfügt, dass Pandorfer oder dessen Erben nach der Begleichung der Schulden die eribbrief unnd sidel unnd ain verczeichnuss herauszugeben haben. Am freytag nach sannd Erasmtag (nach Kop.).

KVr: C.d.i.p. (nach Kop.).

Org. im bearbeiteten Bestand nicht überliefert, der Kop. zufolge Perg. mit aufgedr. S. - Kop.: Vidimus Abt Ulrichs von Garsten von 1497 November 13 im HHStA Wien (Sign. AUR sub dat. 1478 VI 5), Perg., rotes S des Ausst. in wachsf. Schüssel an Ps. ${ }^{1}$

Reg.: ChMel n. 7210; DeRs., Mon. Habs. I/2 S. 664f. n. 543.

1 Rücks. zeitnaher Vermerk (Blattmitte oben): Panndorffer vidimus. Disen schuldbrief hat Panndorffer herrn aptt Ulrichen gegen der versechung des gegenschreiberambts zu Lynntz innhallt seiner verschreibung auff di camer gegeben.

\section{[1478 Juni 9, Graz $]^{1}$}

K.F. überträgt Andreas von Teufenbach bis auf Widerruf die Pflege von Schloss Eppenstein und überlässt ihm die zugehörigen Nutzen und Renten samt dem von den Gff. von Montfort an ihn gekommenen halben Markt Obdach und den Gütern aus dem Besitz Andreas Greiseneggers ${ }^{2}$, die der Ritter Hans Ramung von ihm bisher innehatte, bestandweise gegen Zahlung von jährlich $160 \mathrm{Pfd}$. Pf.

KVr: Per d. Sig. de Nidertor (nach CHMEL).

Org. oder Kop. im bearbeiteten Bestand nicht überliefert. - Dep.: Erw. im Revers des Andreas von Teufenbach von 1478 Juni 18 im HHStA Wien (Sign. AUR 1478 VI 18), Perg., 2 grüne SS des Ausst. und des Ritters Hans von Saurau in wachsf. Schüsseln an Ps, mit Rta-Vermerk auf der Rückseite. $^{3}$

Reg.: ChMel, Mon. Habsb. I/2 S. 787f. n. 903; s. auch Mell, Regesten Teufenbach 1 n. 458.

Lit.: PIRChegGer, Landesfïrst und Adel 3 S. 114; BrunneR, Teufenbach S. 342; zu Burg Eppenstein und Obdach s. EBNER, Burgen Ennstal S. 31-33 und 89.

1 Datierung und Ausstellungsort nach Reg. bei CHMEL.

2 Hingerichtet gemeinsam mit Andreas Baumkircher am 23. April 1471, s. SCHÄFFER, Baumkircherfehde S. 166f.; zur Person s. HeinIG, Friedrich III./1 S. $217 \mathrm{f}$.

3 Reg.: Muchar, Urkunden-Regesten n. 268; ChMEL, Mon. Habsb. I/2 S. 935 n. 1321; Göth, UrkundenRegesten 9 n. 672; MrLL, Regesten Teufenbach 1 n. 455; erw. bei Muchar, Geschichte 8 S. 104 (zu Juni 16). 
K.F. überträgt Konrad Zirkendorfer bis auf Widerruf die Pflege von Schloss Weitra samt Fischweide, Wildbann, Robot und Meierhof, benennt ihm jährlich $200 \mathrm{Pfd}$. Pf. für die Burghut und überlässt ihm dazu bestandweise seinen Teich zu Weitra mit der Verpflichtung, diesen mit Fischen zu besetzen und ihm vom Abfischen die Hälfte abzugeben. ${ }^{1}$

Org. oder Kop. im bearbeiteten Bestand nicht überliefert. - Dep.: Erw. im Revers Konrad Zirkendorfers von 1478 Juni 10 im HHStA Wien (Sign. AUR Großformate sub dat. 1478 V 3), ${ }^{2}$ Perg., 2 grüne SS des Ausst. und Erhard Lembachers in wachsf. Schüsseln an Ps, mit Rta-Vermerk auf der Rückseite. ${ }^{3}$

Reg.: ChMel, Mon. Habsb. I/2 S. 573 n. 307 (zu Mai 22). ${ }^{4}$

1 Laut Revers war Zirkendorfer verpflichtet, den Zeitpunkt des Abfischens bekanntzugeben, damit K.F. seine Leute zur Übernahme der ihm zustehenden Hälfte des Fangs entsenden konnte.

2 Unter dieser Sign. gemeinsam verwahrt mit den nn. 156, 191.

3 Reg.: ChMel n. 7211; auch erw. bei Dems., Mon. Habsb. I/2 S. 573 zu n. 307.

4 ChMel zufolge beinhaltet die ksl. Urkunde von 1478 Mai 22 (nach Kanzleibucheintrag) nur die bestandweise Überlassung des Teichs zu Weitra an den Pfleger Konrad Zirkendorfer. Sie ist möglicherweise mit dem vorliegenden erschlossenen Stück ident, doch ist nicht auszuschließen, dass über die Übertragung der Pflege von Weitra eine eigene ksl. Urkunde ausgestellt wurde.

\section{[Vor 1478 Juni 11, -]}

K.F. überträgt Niklas Haller, Bürger von Villach, bis auf Widerruf die Pflege von Schloss Landskron samt dem Landgericht und überlässt ihm die zugehörigen Nutzen und Renten, wie sie Paul Krabath innehatte, ${ }^{1}$ bestandweise gegen Zahlung von jährlich 50 Pfd. Pf.

Org. oder Kop. im bearbeiteten Bestand nicht überliefert. - Dep.: Erw. im Revers Niklas Hallers von 1478 Juni 11, Graz, im HHStA Wien (Sign. AUR 1478 VI 11), Perg., 2 grüne SS des Ausst. und Erhard Lembachers in wachsf. Schüsseln an Ps, mit Rta-Vermerk auf der Rückseite. ${ }^{1}$

Lit.: Zu Landskron s. MeTNITZ, Kärntner Burgenkunde 2 S. 89; WIESSNER/VYORAL-TsCHAPKA, Burgen Kärnten 3 S. 190-194.

Siehe n. 281.

1 Zur Übertragung an Paul Krabath im Jahr 1468 s. Regg.F.III. H. 22 n. 222.

2 Reg.: ToMAscheK, Regesten Kärnten 3 n. 503. 
K.F. überlässt Albrecht Kamrer, Bürger von Judenburg, bis auf Widerruf das Ungeld zu Judenburg samt Zubehör sowie den Aufschlag von dem Wein, der durch Judenburg an Orte ohne Ungeldeinhebung geführt wird, bestandweise gegen $Z_{\text {Zahlung }}^{2}$ von jährlich 500 fl. ung. ${ }^{3}$

Org. oder Kop. im bearbeiteten Bestand nicht überliefert. - Dep.: Erw. im Revers Albrecht Kamrers von 1478 Januar 19 oder Juni $15^{4}$ im HHStA Wien (Sign. AUR 1478 VI 15), Perg., 2 grüne SS des Ausst. und des Grazer Stadtrichters Christoph Patriarch ${ }^{5}$ in wachsf. Schüsseln an Ps, mit Rta-Vermerk auf der Rückseite. ${ }^{6}$

Reg.: ChMEL, Conzeptensammlung 1 S. 131 n. 152 (zu Januar 18).

Lit.: LeItHNeR, Judenburg S. 10 (zu Juni 15); Muchar, Geschichte 8 S. 103 (zu Mai 12); zu Albrecht Kamrer s. FeLSER, Herkunft XXIIf. (Kammerer).

1 Zur Datierung s. Anm. 4.

2 Laut Revers war Kamrer verpflichtet, das Bestandgeld zu Jahresbeginn im Voraus zu zahlen, und nicht, wie sonst üblich, ratenweise zu den Quatembern.

3 Albrecht Kamrer hatte das Ungeld und den Weinaufschlag nur kurzeitig in Bestand, bereits am 24. Februar 1479 erfolgte die Übertragung an Balthasar Eggenberger (s. n. 241).

4 Der Revers wurde am Montag nach St. Antoniustag 1478 ausgestellt; nach dem Festtag am 17. Januar (Antonius der Große, der Einsiedler) war es der 19. Januar, nach dem Festtag am 13. Juni (Antonius von Padua) der 15. Juni. Die Datierung (und daher Sign.) im HHStA Wien erfolgte nach dem Antoniustag im Juni.

5 Siehe zu ihm Popelka, Geschichte Graz 1 S. 486; Dienes, Bürger von Graz S. IXf., diesem zufolge war er auch ksl. Diener.

6 Reg.: ChMrL, Conzeptensammlung 1 S. 131 n. 153 (zu Januar 19); GötH, Urkunden-Regesten n. 679 (zu September 7).

\section{Juni 18, Graz}

K.F. lädt Konrad Seus, Bürger von Nürnberg, oder dessen bevollmächtigten Anwalt aufgrund der ihm vorgebrachten schriftlichen Appellation des Ehepaars Hans und Barbara Holfelder gegen ein zu dessen (Seus) Gunsten ergangenes Urteil ${ }^{1}$ sowie beschwerungen des Stadtgerichts Nürnberg auf den 45. Tag nach Erhalt dieser Ladung bzw. den ersten darauf folgenden Gerichtstag peremptorisch zu rechtlicher Verantwortung vor sich oder seinem Bevollmächtigten und weist darauf hin, dass auch in dessen Abwesenheit auf Erfordern der Gegenpartei verhandelt wird. Er teilt ihm überdies mit, dass er auss redlichen ursachen dem Ehepaar Holfelder im Falle des Versäumnisses der ge- 
bührenden Appellationsfrist aus ksl. Machtvollkommenheit die fehlende Zeit erstattet. Am achzehenden tag des monats junii (nach Kop.).

KVr: A.m.d.i. (nach Kop.).

Org. im bearbeiteten Bestand nicht überliefert, der Kop. zufolge mit rotem S rücks. aufgedr. Kop.: Notariatsinstrument des öff. Notars Konrad Reinhart von Karlstadt, Bistum Würzburg, von 1478 Juli 3 im HHStA Wien (Sign. AUR sub dat. 1479 VI 18), Pap., Notarssignet. $^{2}$

Lit.: Zum Prozess s. Protokoll- und Urteilsbücher 3 S. 1273f. Verfahrensnr. 460.

Siehe nn. 167, 275, 276.

1 Urteil von 1477 November 19, betreffend den Verkauf einer jährlichen Gülte durch das Ehepaar Holfelder an Konrad Seus, im HHStA Wien (Sign. AUR sub dat. 1477 IX 19); s. dazu auch die Urkunde der Holfelder von 1476 August 2 über den Verkauf der Gülte von $10 \mathrm{fl}$. rh. auf ihr Nürnberger Haus an Konrad Seus ebd. (Sign. AUR sub dat. 1476 VIII 3) sowie die Appellation des Konrad Seus von 1478 Oktober 22 (Notariatsinstrument) ebd. (Sign. AUR 1478 X 22).

2 Das Notariatsinstrument wurde am 3. Juli 1478 anlässlich der Übergabe der ksl. Ladung an Konrad Seus in dessen Haus in Nürnberg in Gegenwart der Zeugen Hans und Erhard Rotenburger aus Nürnberg ausgestellt. - Rücks. zeitnaher Vermerk: Instrumentum execucionis citationis Holfelder contra Seuss. Producta ad iudicium camere imperialis nona mensis octobris 1479. G(eorg) Knöringer (Blattmitte, rechts); zu Georg Knöringer s. HeINIG, Friedrich III./1 S. 761-763.

\section{[1478 Juni 18, Graz]}

K.F. lädt desgleichen den Nürnberger Bürger Hans Holfelder und dessen Ehefrau Barbara vor sich.

Org. oder Kop. im bearbeiteten Bestand nicht überliefert. - Dep.: Erw. in n. 275; ergibt sich zudem aus n. 166.

\section{Juni 20, Graz}

K.F. belehnt seinen Diener Erasmus Lueger für die ihm vor etlichen Jahren geleisteten Dienste und von sonndern gnaden mit jenen von ihm lehenrührigen Stücken und Gütern samt Zubehör, was wir im von rechtens oder gnaden wegen daran verleihen sullen oder mugen, ${ }^{1}$ die dessen verstorbener Vetter Haug, Bggf. von Lienz, zu Lehen hatte, aber als freies Eigen verkauft hat, wodurch sie ledig wurden und angefallen sind, und die nun Lueger und dessen Erben gemäß Lehens- und Landrecht innehaben und nutzen sollen. An sambstag nach sannd Veytstag. ${ }^{2}$ 
KVr: C.d.i.i.c. - KVv: Rta. - Rückseite der Wachsschüssel: Luger.

Org. im HHStA Wien (Sign. AUR 1478 VI 20), Perg., rotes S 21 in wachsf. Schüssel mit wachsf. S 16 rücks. eingedr. an Ps.

Reg.: ChMel, Mon. Habsb. I/2 S. 936 n. 1324.

Lit.: TROTTER, Burggrafen von Lienz S. 61; zu Erasmus Lueger s. auch SCHÄFFER, Christoph Ungnad S. 132.

1 Bei der Bedingungsformel (,was wir verleihen sollen oder mögen") fehlt hier die bei Belehnungen übliche Kombination mit „wissentlich". Die Formel könnte daher nach HAGENEDER, Kanonisches Recht S. 432f., conditionaliter bzw. bedingungsweise (soweit ihm das zusteht) und nicht - wie in Verbindung mit „wissentlich" - causaliter bzw. begründend und bekräftigend (weil er es verleihen soll und mag) interpretiert werden, wodurch die Urkunde nur eine eingeschränkte Rechtskraft hätte; s. dazu auch DERS., Rechtskraft S. 410.

2 Siehe dazu CнмеL, Conzeptensammlung 1 S. 155 n. 211: Belehnung Niklas Luegers (Vater des Erasmus) von 1478 Januar 29 mit den von dessen Vetter Haug ererbten Stücken und Gütern; auch erw. bei DEMS., Mon. Habsb. I/3 S. 726 n. 272 (hier statt Vetter „Vater').

\section{[Vor 1478 Juni 25, -]}

K.F. verschreibt Achaz von Ernau die Pflege von Schloss Falkenstein samt dem Amt satz- und pflegweise gegen ein Darlehen von $1.000 \mathrm{fl}$. ung. für die Ablösung des Schlosses von Gebhard Peuscher ${ }^{1}$ und überlässt ihm die zugehörigen Nutzen und Renten bestandweise für vier Jahre ab Weihnachten 1479 (1478 Dezember 25) gegen Zahlung von jährlich 95 Pfd. Pf. ${ }^{2}$

Org. oder Kop. im bearbeiteten Bestand nicht überliefert. - Dep.: Erw. im Revers des Achaz von Ernau von 1478 Juni 25 im HHStA Wien (Sign. AUR 1478 VI 25), Perg., 2 grüne SS des Ausst. und seines Vaters und Bürgen Jakob von Ernau in wachsf. Schüsseln an Ps, mit Rta-Vermerk auf der Rückseite. ${ }^{3}$

Reg.: ChMel, Mon. Habsb. I/2 S. 883 n. 1157 (zu Juli 1478).

Lit.: METNITZ, Kärntner Burgenkunde 2 S. 29; WIESSNER/VYORAL-TsCHAPKA, Burgen Kärnten 3 S. 51; LeITNER, Herren von Ernau S. 195.

1 Zur Verpfändung an Gebhard Peuscher im Jahr 1477 s. n. 83. - Zur Ablösung Falkensteins durch Achaz von Ernau s. Muchar, Urkunden-Regesten n. 277; ChMEL, Mon. Habsb. I/2 S. 929 n. 1302.

2 Laut Revers sollte im Falle einer Einlösung durch den K. nach dem ersten Jahr in den drei weiteren Jahren 
von den Erträgen ebenso viel geleistet werden wie vormals von Wolfgang Leininger, früher Pfleger von

Falkenstein; dieser für 1474 als Pfleger genannt, s. Regg.F.III. H. 27 nn. 227, 247.

3 Reg.: GöTH, Urkunden-Regesten 9 n. 673; ToMAschrK, Regesten Kärnten 3 n. 504; MC 11 n. 504.

\section{[1478 Juni 27, Graz ${ }^{1}$}

K.F. überlässt Hans Stubich das Ungeld in der Stadt Leoben und auf dem Land im Landgericht St. Peter ob Leoben so wie bisher bestandweise für zwei Jahre ab St. Peterund Paulstag 1478 (Juni 29) gegen Zahlung von jährlich 550 Pfd. Pf. ${ }^{2}$

Org. oder Kop. im bearbeiteten Bestand nicht überliefert. - Dep.: Erw. im Revers Hans Stubichs von 1478 Juli 7 im HHStA Wien (Sign. AUR 1478 VII 7), Perg., 2 grüne SS des Ausst. und Erhard Lembachers in wachsf. Schüsseln an Ps, mit Rta-Vermerk auf der Rückseite. ${ }^{3}$

Reg.: ChMel, Mon. Habsb. I/2 S. 795 n. 927.

Lit.: Muchar, Geschichte 8 S. 104 (zu Juli 4).

Siehe n. 282.

1 Datierung und Ausstellungsort nach Reg. bei CHMEL

2 Laut Revers sollte die Zahlung des Bestandgelds an Hans Einpacher zuhanden des K. erfolgen; zu Einpacher s. DiENEs, Bürger von Graz S. LXXIf.

3 Reg.: GöтH, Urkunden-Regesten 9 n. 674; der Revers sowie ksl. Befehle an die zum Ungeldbezirk gehörigen Leute und an Hans Stubich erw. bei CHMEL zu n. 927.

\section{[Nach 1478 Juli $1,-]^{1}$}

K.F. übermittelt Propst, Dekan und Kapitel sowie allen Kanonikern und personis der Kathedralkirche von Utrecht, denen die Wahl des Kirchenvorstehers zukommt, aus der seiner ksl. Würde entspringenden Sorge um geeignete, nicht nur den Gläubigen und der Kirche dienende, sondern auch dem Papst, ihm und dem Reich ergebene Hirten die inserierte Bulle Papst Sixtus' (IV.) von 1478 Juli 1. Dieser zufolge sollen sie bei Sedisvakanz auf ksl. Erfordern keinesfalls einen Bischof wählen, andernfalls sei die Wahl ungültig, jedoch soll die Zeit bis zur Bestimmung eines geeigneten, dem Apostolischen Stuhl und dem K. genehmen Kandidaten nicht auf die im (Wiener) Konkordat ${ }^{2}$ für die Wahl festgesetzte Frist angerechnet werden. K.F. befiehlt ihnen unter Androhung des Verlustes ihrer von ihm, seinen Vorgängern, röm. Kaisern und Königen, und von anderen erhaltenen Privilegien sowie der Strafe sub rebellionis und anderer schwerster Strafen und strenger Bußen, im Falle der Vakanz der Utrechter Kirche auf seine schriftliche Forderung und kraft der ihnen in seinen Briefen übermittelten apostolischen Schreiben 
in keiner Weise ad electionem, postulacionem, nominacionem seu provisionem eines Vorstehers zu schreiten, bevor gemäß der Bulle über die für diese Kirche zu bestellende persona ydonea sedi apostolice ac nobis grata eine Übereinkunft erzielt ist. Bei Zuwiderhandeln droht er mit den genannten Strafen und seiner und des Reichs schwersten Ungnade.

Org. im bearbeiteten Bestand nicht überliefert. - Konz. (lat., undatiert) mit Verzeichnis der Empfänger im HHStA Wien (Sign. AUR sub dat. 1478 VII 1), Pap. ${ }^{3}$

Druck: ChMeL, Mon. Habsb. I/2 S. 386-388 n. 80.

Lit.: BachmanN, Reichsgeschichte 2 S. 670f. (zu 1479); HollweG, Georg Heßler S. 88; FeINE, Kirchliche Rechtsgeschichte 1 S. 410; KRIEGER, Rechtliche Grundlagen S. 470f.

1 Datierung nach der inserierten Bulle Papst Sixtus' IV. von 1478 Juli 1.

2 Sog. „Wiener Konkordat “ von 1448 Februar 17, s. Regg.F.III. H. 13 n. 60.

$3 \mathrm{Zu}$ den weiteren Empfängern laut Verzeichnis s. die nn. 172-187. - Die inklusive Utrecht insgesamt 17 Bistümer bzw. Erzbistümer als Empfänger dieses Schreibens (ursprünglich mit Speyer 18, das gestrichen wurde) werden in der Literatur unvollständig angegeben: bei BACHMANN (S. 671, Anm. 1) fehlt Augsburg und statt Besançon wird Lausanne angeführt; FEINE (S. 410) nennt 14 Reichsbistümer, KRIEGER (S. 471) nennt 19, bei der Aufzählung (ebd., Anm. 26) sind es hingegen 17 (inklusive Speyer), wobei Straßburg fehlt.

\section{[Nach 1478 Juli 1, -]}

K.F.befiehlt desgleichen

- dem Domkapitel zu Lüttich.

- dem Domkapitel zu Cambrai. 173

- dem Domkapitel zu Trier. $\quad 174$

- dem Domkapitel zu Köln.

- dem Domkapitel zu Mainz.

- dem Domkapitel zu Würzburg. $\quad 177$

- dem Domkapitel zu Bamberg. 178

- dem Domkapitel zu Eichstätt. ${ }^{1}$

- dem Domkapitel zu Salzburg. $\quad 180$ 
- dem Domkapitel zu Straßburg.

- dem Domkapitel zu Passau.

- dem Domkapitel zu Augsburg.

- dem Domkapitel zu Freising.

- dem Domkapitel zu Münster.

- dem Domkapitel zu Regensburg. 186

- dem Domkapitel zu Besançon. 187

Orgg. im bearbeiteten Bestand nicht überliefert. - Erw. in dem in n. 171 (Konz.) genannten Empfängerverzeichnis.

1 Nach Eichstätt folgt im Empfängerverzeichnis Speyer, das gestrichen wurde.

\section{[Vor 1478 Juli 4, -]}

K.F. überträgt Hans von Laak bis auf Widerruf die Pflege von Schloss Haasberg samt dem Amt Stegberg und überlässt ihm die zugehörigen Nutzen und Renten, wie sie Balthasar Wagen ${ }^{1}$ innehatte, bestandweise ab St. Jakobstag im Schnitt 1478 (Juli 25) gegen Zahlung von jährlich $200 \mathrm{fl}$. ung.

Org. oder Kop. im bearbeiteten Bestand nicht überliefert. - Dep.: Erw. im Revers des Hans von Laak ${ }^{2}$ von 1478 Juli 4 im HHStA Wien (Sign. AUR 1478 VII 4), Perg., 3 grüne SS des Ausst., seines Bürgen Kaspar Rauber und des Ritters Wilhelm von Auersperg ${ }^{3}$ in wachsf. Schüsseln an Ps, mit Rta-Vermerk auf der Rückseite.

1 Im Juni 1478 noch als Pfleger von Haasberg genannt, s. CHMEL, Mon. Habsb. I/2 S. 907f. n. 1238

2 Hans von Laak am Ende des Reverses irrtümlich Sigmund genannt.

3 Ksl. Kämmerer und Rat, s. zu ihm HeINIG, Friedrich III./1 S. $231 \mathrm{f}$.

\section{[Vor 1478 Juli 5, -]}

K.F. überlässt Sigmund Piers bis auf Widerruf das Amt in der Rieg bestandweise ab St. Jakobstag im Schnitt 1478 (Juli 25) gegen Zahlung von jährlich 100 Pfd. Pf.

Org. oder Kop. im bearbeiteten Bestand nicht überliefert. - Dep.: Erw. im Revers des Sigmund Piers von 1478 Juli 5 im HHStA Wien (Sign. AUR 1478 VII 5), Perg., 3 grüne SS des 
Ausst., seines Bürgen Niklas Rauber und des Haasberger Pflegers Hans von Laak ${ }^{1}$ in wachsf. Schüsseln an Ps, mit Rta-Vermerk auf der Rückseite.

1 Siehe zu ihm n. 188.

[Nach 1478 Juli 6, - ] $]^{1}$

K.F. schreibt Papst Sixtus IV, ihm sei berichtet worden, dass Eb. Ruprecht von Köln ${ }^{2}$ zugunsten des Ldgf. Hermann (IV.) von Hessen ${ }^{3}$, Kanoniker der Kölner Kirche, resigniert habe und von beiden Seiten Gesandte ${ }^{4} \mathrm{zu}$ ihm (dem Papst) geschickt worden seien, um den Ldgf. auf die Kölner Kirche providieren und bestätigen zu lassen. Wegen der großen Bedeutung der Angelegenheit, die gut überlegt werden müsse und für ihn und das Reich von größtem Interesse sei, ersucht er ihn, diesbezüglich nicht precipitanter zu entscheiden und die Providierung nicht ohne seine (K.F.) Zustimmung vorzunehmen, zumal er es sowohl für den Papst und den Apostolischen Stuhl als auch für ihn und das Reich als angemessen erachte, wenn dies in beiderseitiger Übereinstimmung erfolge. Er weist darauf hin, dass ihn eine ohne seine Zustimmung getroffene Entscheidung wie in Mainz ${ }^{5}$ sehr schmerzlich treffen würde, er aber darauf vertraue, dass der Papst dies nicht zulassen werde.

Org. im bearbeiteten Bestand nicht überliefert. - Konz. (lat., undatiert) mit Korrekturen im HHStA Wien (Sign. AUR sub dat. 1478), Pap. (fol. 2rv). ${ }^{6}$

Lit.: Bachmann, Reichsgeschichte 2 S. 663 (etwa April 1479); FuHs, Hermann IV. von Hessen S. 87-99; s. auch die in n. 139 genannte Lit.

Eb. Ruprecht (von der Pfalz) wurde im März 1478 von Ldgf. Heinrich von Hessen, dem Bruder Hermanns, gefangen genommen und resignierte am 6. Juli 1478. Trotz schärfster Proteste und Hilfegesuche des Papstes ${ }^{7}$ wurde er nicht freigelassen, sondern starb zwei Jahre später in Gefangenschaft. Eine päpstliche Bestätigung seines Amtsverzichts erfolgte allerdings nicht, sodass er bis zu seinem Tod am 16. Juli 1480 amtierender Kölner Erzbischof war. Am 11. August 1480 erfolgte die einstimmige Wahl Hermanns von Hessen durch das Kölner Domkapitel, am 15. November folgte die Bestätigung des Papstes. ${ }^{8}$

Siehe n. 139.

1 Datierung nach der im Schreiben erwähnten Resignation Eb. Ruprechts von Köln am 6. Juli 1478, zu vermuten ist eine Ausstellung im ersten Viertel des Jahres 1479 (s. Anm. 4).

2 Pfgf. Ruprecht bei Rhein (Eb. 1464-1480), s. zu ihm GATz, Bischöfe 2 S. 605-607.

3 Seit 1475 Gubernator des Erzstifts Köln, Eb. von Köln 1480-1508, s. zu ihm bes. FuHs, Hermann IV. von Hessen; weiters Gatz, ebd. S. $287 f$. 
4 Gesandte sind im März 1479 in Rom belegt (s. PrieBatsch, Correspondenz 2 S. 485f. n. 524), ihre Bestallungsurkunden wurden nach JANSSEN, Verzicht S. 660, um die Jahreswende 1478/79 ausgefertigt. Das ksl. Schreiben an den Papst wurde daher vermutlich in den ersten Monaten des Jahres 1479 nach deren Entsendung ausgestellt.

5 Päpstliche Bestätigung des Mainzer Eb. Diethers von Isenburg-Büdingen (1475-1482).

6 Gemeinsam überliefert mit zwei weiteren Konzepten, s. nn. 121, 212.

7 Das päpstliche Hilfegesuch an K.F. von 1478 April 17 überliefert im HHStA Wien (Sign. AUR 1478 IV 17); Druck: Chmel, Mon. Habsb. I/2 S. 319f. n. 9; weitere päpstliche Schreiben in der Sache an etliche Reichsfürsten ebd. I/3 S. 449-451 nn. 20-34, u.a. an Hermann von Hessen (n. 24).

8 Siehe dazu Furs, Hermann IV. von Hessen S. 90f. und 100; zur Resignation Eb. Ruprechts ausführlich JANSSEN, Verzicht.

\section{[Vor 1478 Juli 8, -]}

K.F. überträgt Wilhelm Lesch bis auf Widerruf die Pflege von Schloss Mödling und überlässt ihm das dortige Ungeld, das Bergrecht und den Zehent samt Nutzen und allem Zubehör bestandweise gegen Zahlung von jährlich 800 Pfd. Pf.

Org. oder Kop. im bearbeiteten Bestand nicht überliefert. - Dep.: Erw. im Revers Wilhelm Leschs von 1478 Juli 8 im HHStA Wien (Sign. AUR Großformate sub dat. 1478 V 3), ${ }^{1}$ Perg., 5 anh. SS des Ausst., seiner drei Bürgen Sigmund Stuchsenhauser, Jörg vom Stein und Adam vom Rein sowie Erhard Lembachers in wachsf. Schüsseln an Ps, mit Rta-Vermerk auf der Rückseite. - Ergibt sich auch aus dem ebd. beiliegenden, zweiten Revers Wilhelm Leschs von 1478 Juli 9 , Graz, Pap., grünes S (Petschaft, unter Papieroblate) des Ausst. rücks. aufgedr. ${ }^{2}$

Lit.: WALTER, Ungeld S. 127.

In dem zweiten, eigenhändig geschriebenen Revers von 1478 Juli 9 erklärt Lesch, das Amtsgeld für ein Jahr bereits bezahlt zu haben, und verspricht, danach jährlich 16 Pfd. Pf. Amtsgeld in die ksl. Kanzlei zu entrichten.

1 Unter dieser Sign. gemeinsam verwahrt mit den nn. 156, 163

2 Reg.: ChMel nn. 7215, 7216; Ders., Mon. Habsb. I/2 S. 613f. nn. 420, 422; zur Übergabe von Schloss Mödling s. ebd. S. 613 n. 418, S. 625 f. nn. 454, 455.

\section{[Vor 1478 August 13, -]}

K.F. überlässt Paul Ebenauer das von Jörg von Reichenburg gekaufte Hals- und Landgericht zu Gleichenberg ${ }^{1}$ samt der zugehörigen Vogtei am Straden und in anderen Orten bestandweise für zwei Jahre ab St. Bartholomäustag 1478 (August 24) gegen Zahlung von jährlich 60 Pfd. Pf. 
Org. oder Kop. im bearbeiteten Bestand nicht überliefert. - Dep.: Erw. im Revers Paul Ebenauers von 1478 August 13 im HHStA Wien (Sign. AUR 1478 VIII 13), Perg., 3 grüne SS des Ausst., seines Bürgen Ritter Jörg von Reichenburg und des Ritters Ulrich von Fladnitz in wachsf. Schüsseln an Ps, mit Rta-Vermerk auf der Rückseite. ${ }^{1}$

1 Die Verkaufsurkunde Jörgs von Reichenburg von 1478 August 3 im HHStA Wien (Sign. AUR 1478 VIII 3); Reg.: GöTH, Urkunden-Regesten 9 n. 676; s. SCHÄFFER, Reinprecht von Reichenburg S. 133.

2 Reg.: CHMEL n. 7219; DeRs., Mon. Habsb. I/2 S. 825 n. 1007.

[Vor 1478 August 18, - ] ${ }^{1}$

K.F. sendet Papst Sixtus IV. ein Empfehlungsschreiben für Eb. Johann von Gran.

Org. oder Kop. im bearbeiteten Bestand nicht überliefert. - Dep.: Erw. im Breve Papst Sixtus' IV. an Eb. Johann von Gran von 1478 Oktober 6, Rom, im HHStA Wien (Sign. AUR 1478 X 6), Perg., rotes $S$ (Fischerringsiegel) des Ausst. rücks. aufgedr. ${ }^{2}$

Lit:: Zu Johann Beckenschlager (Beckensloer), Eb. von Gran 1472-1487, s. ZAISBERGER, Rohr, bes. S. 58-62; GATZ, Bischöfe 2 S. 36f.; HeINIG, Friedrich III./1 S. 449-452 mit weiterführender Lit.

In den Auseinandersetzungen um das Graner Erzbistum, das Eb. Johann von Kg. Matthias von Ungarn zurückforderte, ersuchte der Erzbischof den Papst in einem Schreiben von $1478 \mathrm{Au}-$ gust 18 um Unterstützung. In seiner Antwort von 1478 Oktober 6 bestätigte Sixtus IV. den Empfang dieses Schreibens zusammen mit dem Schreiben des Kaisers in commendationem tuam, das daher vor 1478 August 18 ausgestellt worden sein musste, und informiert ihn, in der Sache $\mathrm{Kg}$. Matthias - bekannt ist ein Breve ebenfalls von 1478 Oktober 6 - und Kardinal Gabriel von Erlau geschrieben zu haben. ${ }^{3}$

Siehe n. 273.

1 Zur Datierung s. den Kommentar.

2 Reg.: LichNowsKY(-BIRK) 8 n. 119

3 Siehe dazu Bachmann, Reichsgeschichte 2 S. 633f.; ZaisBerger, Rohr S. 69f; NeHRING, Matthias Corvinus S. $112 f$.

\section{August 19, Graz}

K.F. bestätigt und erneuert aus ksl. Macht und als regierender Herr und Landesfürst von Österreich dem Richter, Rat und allen Bürgern von Eggenburg für ihre in den vergangenen Kriegen ${ }^{1}$ gegen seine Feinde geleisteten Dienste und ihm als ihrem naturlichen 
Herrn und Landesfürsten erwiesene Treue sowie zur Förderung der Stadt von sundern gnaden wissenlich mit dem brief alle von seinen Vorgängern erhaltenen Privilegien und Rechte und gebietet allen Hauptleuten, Landmarschällen, Gff., Freiherren etc., Verwesern, Vizedomen, Pflegern, Bggff., Landrichtern, Bürgermeistern, Richtern, Räten, Bürgern, Gemeinden und allen anderen Amtleuten und Untertanen die Beachtung dieser Bestätigung. Am mitichen nach unser lieben Frawntag assumptionis.

KVr: C.d.i.i.c. - KVv: Rta (Blattmitte). - der stat freihaiten (oberer Blattrand).

Org. im HHStA Wien (Sign. AUR 1478 VIII 19), Perg., rotes S 21 in wachsf. Schüssel (beschädigt) mit wachsf. S 16 rücks. eingedr. an Ps.

Lit.: BRUNNER, Eggenburg 1 S. 218.

1 Krieg gegen Kg. Matthias von Ungarn 1477, s. BRUNNER, Eggenburg 1 S. $217 \mathrm{f}$.

\section{August 26, Graz}

K.F. setzt die Ammänner, Räte und Gemeinden der lennder Churwalden, Lenz, Schanfigg (Schaffnegk) zu St. Peter und Langwies (an der langen wyse) von der Beschwerde seines Vetters Ehz. Sigmund von Österreich in Kenntnis, der zufolge sie diesem die Huldigung verweigern und sich als ungehorsam erweisen würden, obgleich Sigmund sie von Vogt Gaudenz von Matsch ${ }^{1}$ rückgekauft und gelöst hat und sie daher diesem und dem hauss Österreich on mittel zugehörig seien. Er gebietet ihnen daher aus ksl. Macht und unter Androhung des Verlustes ihrer Freiheiten und Rechte, seiner und des Reichs schweren Ungnade sowie einer Pön von 50 Mark Gold, zahlbar je zur Hälfte an die ksl. Kammer und Ehz. Sigmund, diesem auf Erfordern als ihrem rechten naturlichen Erbherrn und Landesfürsten die Erbhuldigung und Eide zu leisten und ihm den gebührenden Gehorsam zu erweisen. Am sechsundzwaintzigisten tag des monats augusti (nach Kop.). ${ }^{2}$

KVr: A.m.p.d.i. (nach Kop.).

Kop.: Zeitgleiche Abschrift im HHStA Wien (Sign. AUR 1478 VIII 26), Pap. (fol. 1rv). ${ }^{3}$

Druck: ChMEL, Mon. Habsb. I/2 S. 500f. n. 130.

Reg.: LichNowsky(-BIRK) 8 n. 106; JeCKLIN, Materialien 1 S. 23 n. 102.

Lit.: LADURNER, Vögte von Matsch 3 S. $52 \mathrm{f}$.

Siehe nn. 196-199. 
1 Belehnung Ulrichs von Matsch, auch in Person seines Sohnes Gaudenz, von 1471 Juli 29 mit den von Sigmund unter Vorbehalt des ewigen Rückkaufs erworbenen Gerichten im Prättigau (CHMEL n. 6361); zum Rückkauf s. CHMEL, Mon. Habsb. I/2 S. 217-220 nn. 56, auch LichNowsky(-BIRK) 8 n. 138; s. dazu LADURNER, Vögte von Matsch 3 S. 35; Baum, Sigmund der Münzreiche S. 269f.; zur Belehnung Sigmunds von 1478 Dezember 27 s. Regg.F.III. H. 6 n. 138.

2 Siehe dazu den Vertrag von 1479 Mai 3 im HHStA Wien (Sign. AUR 1479 V 3), dem zufolge die vier Gerichte Ehz. Sigmund zugehören und diesem huldigen sollen; Druck: CHMEL, Mon. Habsb. I/3 S. $172 \mathrm{f}$. n. 68; Reg.: LichNowsKY(-BIRK) 8 n. 166.

3 Gemeinsam überliefert mit $n$. 196.

\section{August 26, Graz}

K.F. befiehlt dem Ammann und Rat von Schwyz aus ksl. Macht, den Ammännern, Räten und Gemeinden von Churwalden, Lenz, Schanfigg (Schaffnigk) zu St. Peter und Langwies (an der langen wisen), die sich Berichten zufolge Schwyz zuwenden und daher die Huldigung seines Vetters Ehz. Sigmund von Österreich verweigern würden, keinerlei Hilfe, Rat, Förderung und Beistand gegen Sigmund zu leisten, sie an der Huldigung gemäß seinem unter Androhung schwerer Strafe ergangenen Befehl ${ }^{1}$ nicht zu behindern und Sigmund diesbezüglich nicht zu beeinträchtigen. Am sechsundzwaintzigisten tag des monats augusti (nach Kop.).

KVr: A.m.p.d.i. (nach Kop.).

Kop.: Zeitgleiche Abschrift im HHStA Wien mit Verzeichnis der Empfänger ${ }^{2}$ (Sign. AUR 1478 VIII 26), Pap. (fol. 2rv). ${ }^{3}$

Druck: ChMEL, Mon. Habsb. I/2 S. 501f. n. 131; JeCKLIN, Materialien 2 S. 59 n. 60 (nach Org. $)^{4}$.

Reg.: LICHNOwsKY(-BIRK) 8 n. 107; JECKIIN, Materialien 1 S. 23 n. 103.

Lit.: LADURNER, Vögte von Matsch 3 S. $52 \mathrm{f}$.

1 Siehe n. 195.

2 Siehe nn. 197-199.

3 Gemeinsam überliefert mit n. 195.

4 Nach JECKLIN vier gleichlautende Orgg. (das vorliegende Stück und die nn. 197-199), alle Pap., mit rücks. aufgedr. ksl. S. 


\section{[1478 August 26, Graz]}

K.F. befiehlt desgleichen

- dem Ammann und Rat von Unterwalden. 197

- dem Ammann und Rat von Uri. $\quad 198$

- der Stadt Zürich.

Orgg. oder Kopien im bearbeiteten Bestand nicht überliefert. - Dep.: Erw. in dem in n. 196 genannten Empfängerverzeichnis.

\section{[Vor 1478 August 28, - $]^{1}$}

K.F. verpfändet Jobst Hauser Schloss Asparn (a.d. Zaya) für 3.500 fl. ung.

Org. oder Kop. im bearbeiteten Bestand nicht überliefert. - Dep.: Erw. im Revers Walter Hausers, des Bruders des verstorbenen Jobst Hauser, von 1479 Mai 22, Graz, im HHStA Wien (Sign. AUR 1479 V 22), Pap., grünes S des Ausst. rücks. aufgedr., mit Rta-Vermerk auf der Vorderseite. $^{2}$

1 Datierung nach einer ksl. Urkunde von 1478 August 28, in der Jobst Hauser als verstorben erwähnt wird, S. CHMEL n. 7221; Ders., Mon. Habsb. I/3 S. 672 n. 132.

2 Zum Revers: Nach der Rücklösung Asparns durch K.F versprach Walter Hauser für sich und seinen Bruder Leopold den seinem verstorbenem Bruder Jobst ausgestellten ksl. Pfandbrief innerhalb von sechs Wochen zu übergeben, was laut zeitgleichem Vermerk auf dem Revers am 18. Juni 1479 (Freitag vor St. Achaztag) erfolgte. - Reg.: ChMel n. 7282; Ders., Mon. Habsb. I/3 S. 641 n. 21.

\section{September 14, Graz}

K.F. lädt seinen Vetter Ehz. Sigmund von Österreich zur Beilegung der Streitigkeiten mit seiner swester, der verwitweten Ehzn. Mechthild von Österreich, geborenen Pfgfn. bei Rhein, und seinem swager Gf. Eberhard (V.) d.Ä. von Württemberg ${ }^{1}$ wegen der Auslösung von Hohenberg und Wehingen peremptorisch auf den nächsten Gerichtstag nach sannt Lucientag schiristkünfftig (Dezember 13) vor sich, nachdem Ehz. Sigmund durch Urteil ${ }^{2}$ seines Kammergerichts zwar Recht erhalten hatte, aber die jüngsten rechtsecz vor unser beschehen wegen seiner (K.F.) das Reich gescheffthalben betreffenden Belastungen unerledigt geblieben sind. Er versichert, sich um eine gütliche Einigung in der Sache bemühen zu wollen, andernfalls aber gemäß den jüngst beschehen rechtsetz zu verfahren, und weist darauf hin, dass bei Nichterscheinen einer Partei 
rechtlich verfahren wird, wie es sich nach seiner ordnung gebührt. Am vierzehennden tag des monets septembris. ${ }^{3}$

KVr: A.m.d.i. - KVv: Ladung herczog Sigmunden (oberer Blattrand).

Org. im HHStA Wien (Sign. AUR 1478 IX 14), Pap., rücks. aufgedr. rotes S 18 unter Papieroblate. - Kop.: Abschrift des 18. Jh. ebd. (Sign. Urkundenabschriften Österr. Urkunden, Kt. 46 sub dat.), Pap.

Druck: ChMEL, Mon. Habsb. I/2 S. 502f. n. 132.

Reg.: ChMel n. 7226; LichNowsKy(-BIRK) 8 n. 110.

Lit. siehe bei n. 25; zum Prozessverlauf s. Protokoll- und Urteilsbücher 3 S. 1329-1331 Verfahrensnr. 619 .

1 Eberhard (V.) d.A. ,im Bart" war Mechthilds zweitgeborener Sohn aus ihrer ersten Ehe mit Gf. Ludwig I. von Württemberg. Thre zweite Ehe mit Ehz. Albrecht VI. von Österreich begründete die Verschwägerung zwischen Württemberg und Habsburg.

2 Urteil von 1476 Juli 9, s. n. 25.

3 Zur Einigung zwischen den Streitparteien s. die Zustimmungsurkunde Ehzn. Mechthilds von 1481 Juni 5 im HHStA Wien (Sign. AUR 1481 VI 5); Reg.: ChMEL n. 7460.

\section{[1478 September 14, Graz]}

K.F. lädt die verwitwete Ehzn. Mechthild von Österreich, geborene Pfgfn. bei Rhein, zur Beilegung der Streitigkeiten mit Ehz. Sigmund von Österreich um Hohenberg und Wehingen vor sich.

Org. oder Kop. im bearbeiteten Bestand nicht überliefert. - Dep.: Erw. in n. 201.

Reg.: Regg.F.III. H. 23 n. 660 (nach unzulänglicher Überlieferung).

\section{[1478 September 14, Graz]}

K.F. lädt desgleichen Gf. Eberhard (V.) d.Ä. von Württemberg vor sich.

Org. oder Kop. im bearbeiteten Bestand nicht überliefert. - Dep.: Erw. in n. 201.

Reg.: Regg.F.III. H. 23 n. 659 (nach unzulänglicher Überlieferung). 
K.F. überträgt seinem Kämmerer Sigmund Prüschenk und dessen Erben wissenttlich mit dem brief Schloss und Herrschaft Weitenegg samt Nutzen, Renten, Zinse, Ungeld, Herrschaftsrechten und sonstigem Zubehör satz- und pflegweise für $5.000 \mathrm{fl}$. ung. und dazu die zum Schloss gehörigen Nutzen, Renten und Gülten im Yspertal für 1.500 fl. ung., wofür dieser das Schloss samt den Yspertaler Erträgen mit seiner (K.F.) Erlaubnis von seinem Kämmerer Kaspar von Rogendorf ${ }^{1}$ zuhanden des K. yecz ablösen wird. Er verpflichtet Prüschenk und dessen Erben, das Schloss auf eigene Kosten zu behüten, ihm und nach seinem Tod seinem Sohn Ehz. Maximilian von Österreich und Burgund ${ }^{2}$ und nach dessen Tod ihren Erben damit treu und gehorsam zu sein, es offen zu halten, ihn (K.F.) und seine Leute jederzeit aufzunehmen, jedoch auf Kosten des K. und unbeschadet des verschriebenen Pfandsatzes. Er untersagt ihnen, ohne seine Erlaubnis und sein Wissen vom Schloss aus Krieg zu führen, die zugehörigen Leute und Holden über die gewöhnlichen Renten, Zinse, Dienste und Robotleistungen ungebührlich gegen altes Herkommen zu belasten und den Entzug ksl. Rechte zuzulas-

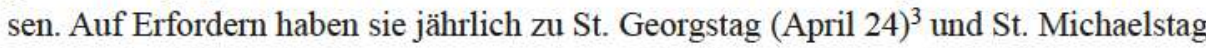
(September 29) die Ablösung zu gestatten und Schloss und Herrschaft mit den genannten Erträgen und samt zewg, Urbarregistern und sonstigem Zubehör gegen Bezahlung der $6.500 \mathrm{fl}$. ung. unverzüglich und ohne Widerspruch abzutreten. An phincztag sannd Ruprechts tag im herbst.

KVr: C.d.i.p. - KVv: Rta (Blattmitte). - Ist abgeschriben (oberer Blattrand).

Org. im HHStA Wien (Sign. AUR 1478 IX 24), ${ }^{4}$ Perg., rotes S 21 in wachsf. Schüssel mit wachsf. S 16 rücks. eingedr. an Ps.

Reg.: ChMet n. 7227; Lichnowsky(-BIRK) 8 n. 113; ChMel, Mon. Habsb. I/3 S. 640f. n. 18.

Lit.: Bergmann, Rogendorf S. 525 [S. 9]; zu Sigmund Prüschenk s. Probszt, Brüder Prueschenk; ZerNATTo, Herrenstand S. 69-72; HenNG, Friedrich III./1 S. 78-88, bes. 80, und 206; MARIAN/ZeHETMAYER, Hardegg S. 560f., 563, 568f.

1 Zur Verpfändung Weiteneggs an die Rogendorfer s. n. 205, Anm. 1.

2 Zur Titulatur Maximilians s. die Einleitung S. 22-24.

3 Zur Datierung des St. Georgstags auf April 24 s. Regg.FIII. H. 12 n. 6, Anm. 2.

4 Dem Privileg beiliegend $\mathrm{n} .205$. 


\section{September 24, Graz}

K.F. erklärt den Revers ${ }^{1}$ seines Kämmerers Kaspar von Rogendorf, Pfleger von Weitenegg, über die Verschreibung Schloss Weiteneggs samt den Nutzen und Renten im Yspertal, welcher diesem nach der Ablösung durch seinen Kämmerer Sigmund Prüschenk $^{2} \mathrm{zu}$ überantworten, aber nicht verfügbar ist, wissenntlich mit dem briefe für ganncz tod und krafftlos. Aus dessen Vorlage soll weder dem K. und seinen Erben ein Nutzen noch dem Rogendorfer und dessen Erben ein Schaden entstehen. K.F. verspricht, den Revers, sollte er zuhanden sein, gegen diesen tottbrief auszuhändigen. An phincztag sannd Ruprechts tag im herbst.

KVr: fehlt.

Org. im HHStA Wien (Sign. AUR 1478 IX 24), ${ }^{3}$ Perg., rotes S 21 rücks. aufgedr. (großteils abgefallen) und rotes $\mathrm{S} 16$ vorders. aufgedr.

Reg.: CHMEL n. 7228; DERS., Mon. Habsb. I/3 S. 641 zu n. 18.

Lit.: BeRgMANN, Rogendorf S. 524 [S. 8]; HeING, Friedrich III./1 S. 195; ZAIC, Kaspar von Roggendorf S. 14; NEIDHART, Geschichte Pöggstalls S. 42; zur Familie s. ZAJI, Rog[g]endorf S. 1207-1214; zu Weitenegg s. DAIM/KüHTREIBER, Burgen Waldviertel-Wachau S. 293-296.

1 Revers Sigmunds und Kaspars von Rogendorf von 1470 Mai 4, s. Regg.FIII. H. 27 n. 35; nach dem Tod Sigmunds im Jahr 1472 war Kaspar alleiniger Pfleger von Weitenegg.

2 Siehe n. 204.

3 Liegt n. 204 anbei.

\section{September 25, Graz ${ }^{1}$}

K.F. bestätigt allen seinen Leuten zu Tinjan (Tingnan) in der Gft. Mitterburg wissenntlich mit dem brief den Kauf des Berges Jesen von Hans Zechorner (Zekherner) und verfügt, dass der Kauf gemäß der darüber ausgestellten Urkunde in allen puncten und artikeln in Kraft bleiben soll und niemand dagegen handeln darf, jedoch unbeschadet allfälliger Rechte, die er daran haben sollte. Er gebietet allen Hauptleuten, Gff., Freiherren etc., Verwesern, Vizedomen, Pflegern, Bggff., Bürgermeistern, Richtern, Räten, Bürgern, Gemeinden und allen anderen Amtleuten und Untertanen die Beachtung dieser Bestätigung. An freytag nach sannd Ruprechts tag.

KVr: fehlt.

Org. im HHStA Wien (Sign. AUR sub dat. 1478 IV 3), Perg., wachsf. Schüssel an Ps, jedoch ohne Siegelabdruck, daher unbesiegelt. 
Reg.: ChMel n. 7193 (zu April 3).

Aufgrund des fehlenden Kanzleivermerks, der unvollständigen Besiegelung und der Überlieferung im Ausstellerarchiv ist anzunehmen, dass die Urkunde nicht ausgefertigt wurde.

1 Zur Datierung: Da bei den Herrscherjahren das 39. Jahr des Königtums gezählt wird (ab 1478 April 6), handelt es sich bei dem Ruprechtstag um den Festtag am 24. September (Translatio) und nicht um jenen am 27. März. Die Datierung (und daher Sign.) im HHStA Wien und bei CHMEL erfolgte nach dem Ruprechtstag im März.

\section{September 27, Graz}

K.F. belehnt seinen Vetter Ehz. Sigmund von Österreich mit wolbedachtem mutte, gutem rate und rechter wissen aus ksl. Macht wissentlich in krafft diss briefs, was wir im von rechts und gnaden wegen daran verleihen sullen und mugen, mit allen perckwerch und ertzt, es sei Gold, Silber, Kupfer, Blei oder anderes Metall, die in den zur Herrschaft Schelklingen gehörigen zirgklen und kreissen liegen und die dieser künftig durch seine Amtleute oder andere dazu befugte Personen suchen lassen und von ihm und dem Reich innehaben und nutzen kann. Er verfügt, dass Sigmund alle den anderen Bergbaustätten im Reich zustehenden Gnaden und Freiheiten haben und gebrauchen soll, jedoch unbeschadet seiner und des Reichs Rechte, und dass er bis zum suntag Letare schiristkunfftig (1479 März 21) Bf. Georg von Brixen an seiner (K.F.) statt den gewöhnlichen Lehenseid zu leisten hat. ${ }^{1}$ K.F. gebietet allen geistlichen und weltlichen Fürsten, Gff., Freiherren etc., Vizedomen, Vögten, Pflegern, Verwesern, Amtleuten, Schultheißen, Bürgermeistern, Richtern, Räten, Bürgern, Gemeinden und allen anderen Reichsuntertanen bei seiner und des Reichs schweren Ungnade und einer Pön von 50 Mark Gold, zahlbar je zur Hälfte an die ksl. Kammer und Ehz. Sigmund, diesen am Bergbau nicht zu behindern. Am sibenundzweintzigisten tag des monadts septembris.

KVr: A.m.d.i.p. Jo(hannes) Waldner prothonotarius etc. - KVv: Rta Lucas Sniczer (Blattmitte). - lehen h(erschafft) Sch(elklingen) (rechter Blattrand).

Org. im HHStA Wien (Sign. AUR 1478 IX 27), ${ }^{2}$ Perg., rotes S 18 in wachsf. Schüssel mit wachsf. S 16 rücks. eingedr. an Ps. - Kop.: Abschrift des 18. Jh. ebd. (Sign. Urkundenabschriften Österr. Urkunden, Kt. 46 sub dat.), Pap.

Druck: ChMEL, Mon. Habsb. I/2 S. 504f. n. 133.

Reg.: ChMel n. 7230; Lichnowsky(-BIRK) 8 n. 116.

1 Siehe n. 208.

2 Dem Privileg beiliegend n. 208. 


\section{September 27, Graz}

K.F. teilt Bf. Georg von Brixen ${ }^{1}$ mit, seinen Vetter Ehz. Sigmund mit den zur Herrschaft Schelklingen gehörenden Bergwerken und Erzen belehnt zu haben, wie der Bf. aus dem darüber ausgestellten lehenbrief ${ }^{2}$ entnehmen wird. Er gebietet ihm, in der darin festgesetzten Frist den Lehenseid an seiner statt von Sigmund entgegenzunehmen und ihn über die Eidesleistung under deinem insigel in Kenntnis zu setzen. Am sibemundzweinczigisten tag des monets septembris.

$\mathrm{KVr}$ : A.m.d.i.p. - KVv: Comiss(io) iuramenti (oberer Blattrand).

Org. im HHStA Wien (Sign. AUR 1478 IX 27), ${ }^{3}$ Pap., rotes S 18 rücks. aufgedr. (großteils abgefallen).

Reg.: ChMel n. 7231; Lichnowsky(-Birk) 8 n. 117; ChMEL, Mon. Habsb. I/2 S. 505f. n. 134.

1 Georg Golser (Elekt 1464, Bf. 1471-1488), s. zu ihm GaTz, Bischöfe 2 S. 232 f.

2 Siehe n. 207

3 Liegt n. 207 anbei.

\section{[Vor 1478 Oktober 5, - ]}

K.F. verkauft Hans Weinich ${ }^{1}$, Münzmeister zu Graz, und dessen Ehefrau Barbara für $400 \mathrm{fl}$. ung. sein Haus in Graz , in der Höll“" ${ }^{\text {, }}$, das mit dem vorderen Teil an den Friedhof der Minoriten grenzt und ehemals dem verstorbenen Hans Kamrer ${ }^{3}$ gehört hat.

Org. oder Kop. im bearbeiteten Bestand nicht überliefert. - Dep.: Erw. im Schuldbrief ${ }^{4}$ Hans und Barbara Weinichs von 1478 Oktober 5, Graz, im HHStA Wien (Sign. AUR 1478 X 5), Perg., 2 grüne SS Hans Weinichs und Erhard Lembachers in wachsf. Schüsseln an Ps. ${ }^{5}$

Lit.: PopelKA, Geschichte Graz 1 S. 526f.; Dienes, Bürger von Graz S. CCXV.

1 Auch Weinrich, im Revers Weinchen (Weinch).

2 Gegend zwischen Hauptplatz und Stadtmauer in der Umgebung des heutigen Franziskanerklosters, s. PoPELKA.

3 Grazer Bürger († vor 1467 Mai 1), s. zu ihm Dienes, Bürger von Graz S. XXXVII.

4 Schuldbrief über die auf die Kaufsumme noch ausständigen und binnen Jahresfrist zu zahlenden $300 \mathrm{fl}$. ung.

5 Reg.: ChMEL n. 7234; Ders., Mon. Habsb. I/2 S. 825 n. 1008. 
K.F. befiehlt Hans Vilshofer ${ }^{1}$ und Jörg Burckhart, von allen in die Ober- und Untersteiermark $^{2}$ hinein- und hinausgeführten Waren, ausgenommen von Vieh, das achtzigste Pfd. Pf. ${ }^{3}$ als Aufschlag zur Bezahlung des Kg. (Matthias) von Ungarn ${ }^{4}$ und anderer Gläubiger einzuheben, und bestellt sie zu Einnehmern des Aufschlags mit der Verpflichtung, das eingehobene Geld den von den ksl. Räten und Landleuten zu Österreich eingesetzten Einnehmern zu geben.

Org. oder Kop. im bearbeiteten Bestand nicht überliefert. - Dep.: Erw. im Revers Hans Vilshofers und Georg Burckharts von 1478 Oktober 11 im HHStA Wien (Sign. AUR 1478 X 11), Perg., 2 grüne SS der Grazer Bürger ${ }^{5}$ Stefan Judenhofer und Thomas Peheim, Ratsherr, in wachsf. Schüsseln an Ps, mit Rta-Vermerk auf der Rückseite. ${ }^{6}$

Lit.: MUCHAR, Geschichte 8 S. 105 (zu Oktober 12).

1 Siehe zu ihm Herng, Friedrich III./1 S. 807.

2 Im Revers in die Obern und Nidern Steyrmarch.

3 An anderer Stelle des Reverses heißt es: von allen Waren, die 80 Pfd. Pf. wert sind, jeweils ein Pfd. Pf. Aufschlag.

4 Gemäß dem Gmundner Vertrag von 1477 Dezember 2 (s. n. 106) schuldeten K.F. und das Hzm. Österreich Matthias Corvinus 100.000 fl.; zur Einhebung der dafür vorgesehenen Aufschläge und den von K.F. und den österreichischen Ständen gemeinsam eingesetzten Einnehmern s. CHмEL, Mon. Habsb. I/2 S. 549-555 n. 248, bes. S. 551f.; IsENMANN, Kaiserliche Obrigkeit S. 323.

5 Siehe zu ihnen DIENES, Bürger von Graz S. CXXXIII (Judenhofer) und XVIIIf. (Peheim).

6 Reg.: Muchar, Urkunden-Regesten n. 272; GöTH, Urkunden-Regesten 9 n. 682.

\section{Oktober 12, Graz}

K.F. bestätigt und erneuert als Kaiser und regierender Herr und Landesfürst von Steier dem Richter, Rat und allen Bürgern und Leuten der Stadt Friedberg sowie ihren Erben und Nachkommen zur Förderung der Stadt von sunndern gnaden wissenlich mit dem brief alle ihre von seinen Vorfahren, regierenden Herren und Landesfürsten von Steier, erhaltenen Privilegien und Rechte in allen puncten und artigkeln, als ob sie wortwörtlich inseriert wären, und gebietet allen Hauptleuten, Gff., Freiherren etc., Verwesern, Landschreibern, Vizedomen, Pflegern, Bggff., Bürgermeistern, Richtern, Räten, Bürgern, Gemeinden und allen anderen Amtleuten und Untertanen die Beachtung dieses Privilegs. Am montag vor sannd Kollmans tag.

KVr: C.d.i.i.c. - KVv: Rta. - Rückseite der Wachsschüssel: Fridb(erg) (Lesung unsicher). 
Org. im HHStA Wien (Sign. AUR 1478 X 12), Perg., rotes S 21 in wachsf. Schüssel (beschädigt) mit wachsf. S 16 rücks. eingedr. an Ps. - Kop.: Abschrift des 18. Jh. ebd. (Urkundenabschriften Österr. Urkunden, Kt. 46 sub dat.), Pap.

Druck: Chmel, Mon. Habsb. I/2 S. 826f. n. 1010.

Reg.: ChMel n. 7236.

\section{Oktober 13, Graz}

K.F. nimmt die Brüder Vitalis und Justus von Nikolitsch, Bürger von St. Veit am Pflaum (ad sanctum Vitum in terra nostra fluminis), denen er für ihre treuen Dienste eine gratiam specialem erweisen will, mit ihren beweglichen und unbeweglichen Gütern in seinen specialem protectionem, tuitionem ac salviguardiam und befreit sie von jeglicher Gerichtsbarkeit. Er verfügt, dass sie oder ihre Güter von niemandem gegen Recht und Billigkeit bedrängt oder verletzt werden dürfen und sie sich im Falle von Klagen nur vor ihm oder seinem dazu Bevollmächtigten rechtlich verantworten müssen. Er gebietet allen Hauptleuten, Gff., baronibus, militibus, militaribus, castellanis, Bggff., locum tenentibus, Vizedomen, Richtern, Räten, Bürgern, Gemeinden und allen anderen Amtleuten und Untertanen bei seiner schweren Ungnade die Beachtung dieses Privilegs. Die tredecima mensis octobris (nach Konz.).

Org. im bearbeiteten Bestand nicht überliefert, dem Konz. zufolge (Perg.) mit anh. S. - Konz. (lat.) mit Korrekturen, von anderer Hand datiert mit 1478 Oktober 13, im HHStA Wien (Sign. AUR sub dat. 1478), Pap. (fol. 1v-2r); ${ }^{1}$ davon Abschrift des 18. Jh. ebd. (Sign. AUR 1478 X 13), Pap.

Reg.: LichNowsky(-BIRK) 8 n. 123; ChMeL, Mon. Habsb. I/3 S. 726 n. 275.

1 Gemeinsam überliefert mit zwei weiteren Konzepten, s. nn. 121, 190.

\section{Oktober 16, Graz}

K.F. gewährt Hans Apfaltrer und dessen Erben wissenntlich mit dem brief die sonnder gnad des alleinigen Weinschankrechts in ihrer Taverne ${ }^{1}$ in dem zu St. Martin in der Littai (Luttey) unnder dem Roy gelegenen Dorf und gebietet allen Hauptleuten, Gff., Freiherren etc., Verwesern, Vizedomen, Pflegern, Bggff., Bürgermeistern, Richtern, Räten, Bürgern, Gemeinden und allen anderen Amtleuten und Untertanen die Beachtung dieses Privilegs. An freitag nach sannd Colmannstag (nach Kop.).

KVr: fehlt. - KVv: Rta (nach Kop.). 
[Org. im ARS Ljubljana, zuvor im HHStA Wien.] - Fotokop. vom Org. im HHStA Wien (Sign. AUR 1478 X 16).

Reg.: Regg.F.III. H. 29 n. 208 (nach Org.); s. auch CHMEL, Mon. Habsb. I/2 S. 827 n. 1011.

1 Zur Berechtigung des Tavernenbetriebs für Apfaltrer von 1470 Mai 15 s. Regg.F.III. H. 29 n. 156 (hier: im Dorf Littai zu St. Martin).

\section{Oktober 25, Graz}

K.F. gewährt Kaspar Harder wissenntlich mit dem brief die sonnder gnad, dass dessen Leute und Holden im Markt Gleisdorf das Marktrecht für Handel und Gewerbe mit Weinausschank und in annder wege wie die anderen Bürger und Einwohner haben sollen, und gebietet allen Hauptleuten, Gff., Herren etc., Verwesern, Landschreibern, Pflegern, Bggff., Bürgermeistern, Richtern, Räten, Bürgern, Gemeinden und allen anderen anderen Amtleuten und Untertanen, insbesondere aber Richter, Rat und den Bürgern von Gleisdorf, die Beachtung dieses Privilegs. An suntag nach sannd Ursulen tag.

KVr: C.d.i.i.c. - KVv.: Rta (Blattmitte). - Harder hat vor ain(en) solhen br(ief) gelost (Blattmitte unten). - Rückseite der Wachsschüssel: Vermerk nicht lesbar (wohl Name des Empfängers).

Org. im HHStA Wien (Sign. AUR Großformate 1478 X 25), Perg., rotes S 21 in wachsf. Schüssel mit wachsf. S 16 rücks. eingedr. an Ps.

Reg.: ChMel n. 7241; DeRs., Mon. Habsb. I/2 S. 827 n. 1012.

\section{[Vor 1478 Oktober 28, -]}

K.F. überträgt Andreas Hertenfelser bis auf Widerruf Schloss Neuburg in der Kanker und das Amt Primskau pfleg- und bestandweise gegen Zahlung von jährlich $174 \mathrm{fl}$. ung.

Org. oder Kop. im bearbeiteten Bestand nicht überliefert. - Dep.: Erw. im Revers Andreas Hertenfelsers von 1478 Oktober 28 im HHStA Wien (Sign. AUR 1478 X 28), Perg., 3 grüne SS des Ausst., seines Vaters und Bürgen Achaz Hertenfelser ${ }^{1}$ und Erhard Lembachers in wachsf. Schüsseln an Ps, mit Rta-Vermerk auf der Rückseite.

1 Zur zeitgleichen Übertragung des Krainer Vizedomamts an Achaz Hertenfelser s. dessen Revers von 1478 Oktober 27 im HHStA Wien (Sign. AUR sub dat. 1478 X 28). 
K.F. befiehlt Abt Konrad ${ }^{2}$ des Benediktinerklosters Allerheiligen zu Schaffhausen sowie dem dortigen Bürgermeister und Rat auf Beschwerde Gf. Sigmunds von Lupfen, den Wildbann und andere Hoheitsrechte in den Wäldern Randen, Gatterholz und Westerholz nicht zu gebrauchen, so lange die Streitsache vor dem Kammergericht anhängig ist.

Org. oder Kop. im bearbeiteten Bestand nicht überliefert. - Dep.: Erw. in einem Schreiben der Eidgenossen von 1479 Juni 2, Zürich, im HHStA Wien (Sign. AUR 1479 VI 2), Pap., S der Stadt Zürich als Verschluss rücks. aufgedr. (abgefallen). ${ }^{3}$

Hintergrund des Schreibens der Eidgenossen ist der jahrzehntelange Konflikt ${ }^{4}$ um die Hoheitsrechte im Randengebiet zwischen dem Kloster Allerheiligen und Schaffhausen einerseits, die sich zur gemeinsamen Verteidigung ihrer Rechte zusammengeschlossen hatten, ${ }^{5}$ und den Gff. von Lupfen andererseits. Die Eidgenossen ersuchten um Rücknahme des ksl. Verbots der Nutznießung unter Hinweis auf die jahrhundertealten Besitzrechte des Klosters auf die Wälder am Randen als Teil des Stiftungsguts des Gff. von Nellenburg im 11. Jh., deren Nichtgebrauch für die Dauer des Prozesses einen Schaden für das Kloster bedeuten würde. Die Gff. von Lupfen gründeten hingegen ihre Ansprüche auf eine Lehensurkunde ${ }^{6} \mathrm{Kg}$. Sigismunds von 1422 September 30, die aus Sicht des Klosters ein Entzug seines Besitzes bedeutete. Am 7. August 1479 belehnte K.F. ${ }^{7}$, der Urkunde Sigismunds folgend, Gf. Sigmund von Lupfen u.a. mit der Ldgft. Stühlingen, der Mundat (Immunitätsrechte) am Randen, dem Westerholz und dem Gatterholz. Der Konflikt um das Randengebiet wurde erst durch einen Schiedsspruch von 1491 August 27 beendet, dem zufolge Gf. Sigmund nur das Gatter- und Westerholz zugesprochen wurde, der restliche, größere Teil des Randen kam an das Kloster Allerheiligen bzw. Schaffhausen. ${ }^{8}$

1 Das Datum des ksl. Mandats wird im Schreiben der Eidgenossenschaft genannt: am funffen tag des manotz november nechstvergangen.

2 Zu Abt Konrad Dettikofer (1466-1488) s. Helvetia Sacra III/1,3 S. 1531-1533.

3 Druck: ChмеL, Mon. Habsb. I/3 S. 102f. n. 39; Reg.: Lichnowsky(-BIRK) 8 n. 174.

$4 \mathrm{Zu}$ den die Streitsache betreffenden Quellen s. HIIDBRAND, Herrschaft S. 328.

5 Nach ScHIB, Schaffhausen S. 166, handelte es sich bei den strittigen Rechten um den letzten Rest klösterlicher Hoheitsrechte, den sich das Kloster im Zuge der schrittweisen Eingliederung in die Stadt im Laufe des 15. Jh. zwar erhalten, aber mit Schaffhausen teilen musste, um gegen die Gff. von Lupfen zumindest ein theoretisches Besitzrecht wahren zu können.

6 RI XI 1 n. 5300.

7 UB Fürstenberg 7 S. 137f. n. 77.

8 Zum Schiedsspruch s. UB Fürstenberg 4 S. 126-129 n. 131; zur Streitsache s. Helvetia Sacra III/1,3 S. 1503f.; HIIDBRAND, Herrschaft S. 328-330. 
K.F. lädt Hans Oberhaimer, Pfleger von Starhemberg, auf Klage Hans Oberhaimers von Bernau zu rechtlicher Verantwortung vor sich.

Org. oder Kop. im bearbeiteten Bestand nicht überliefert. - Dep.: Erw. in n. 219.

\section{[Vor 1478 November 23, -]}

K.F. lädt Hans Oberhaimer, Pfleger von Starhemberg, auf Klage der Brüder Hans und Warmund Oberhaimer von Bernau zu rechtlicher Verantwortung vor sich.

Org. oder Kop. im bearbeiteten Bestand nicht überliefert. - Dep.: Erw. in n. 220.

\section{November 23, Graz}

K.F. befiehlt Hans Oberhaimer, Pfleger von Starhemberg, ${ }^{1}$ nachdem dieser auf seine Ladung $^{2}$ auf hewt nicht erschienen ist, im Streit mit Hans Oberhaimer von Bernau wegen einer von ihren verstorbenen Vätern herrührenden verbrieften Geldschuld dessen Forderungen zu befriedigen und ihn unclaghaft zu stellen. Dessen Klage zufolge hatte Kolman Oberhaimer (Vater des erstgenannten Hans) bei Matthias Oberhaimer von Bernau (Vater des zweitgenannten Hans) Schulden, die Hans Oberhaimer von Bernau nun von ihm als Erben Kolmans einfordere. Der K. lädt ihn im Falle eines Einspruchs von hewt über sechs wochen peremptorisch zu rechtlicher Verwantwortung vor sich oder seinem dazu Bevollmächtigten und weist darauf hin, dass bei Nichterscheinen er der anderen Partei recht ergeen lassen wird. An montag vor sannd Kathrein tag (nach Kop.).

Kop.: Zeitgleiche Abschrift im HHStA Wien (Sign. AUR 1478 XI 23), Pap. (fol. 1r), mit rücks. zeitgleichem Vermerk ultima citacio (Blattmitte). ${ }^{3}$

Reg.: ChMel n. 7246; DeRs., Mon. Habsb. I/2 S. 688 n. 568.

Lit.: Zur Familie Oberhaimer s. AsPERNIG, Beiträge S. 58.

1 Burg Starhemberg in Oberösterreich (Haag am Hausruck).

2 Siehe n. 217.

3 Gemeinsam überliefert mit n. 220. 
K.F. befiehlt Hans Oberhaimer, Pfleger zu Starhemberg, ${ }^{1}$ nachdem dieser auf seine Ladung $^{2}$ auf hewt nicht erschienen ist, im Streit mit den Brüdern Hans und Warmund Oberhaimer von Bernau wegen des halben Sitzes zu Affnang samt Höfen, Gütern und Lehen deren Forderungen zu befriedigen und sie unclaghaft zu stellen. Threr Klage zufolge haben sie ihm den Sitz für $150 \mathrm{fl}$. ung. verkauft, jedoch vorbehaltlich eines schriftlich vereinbarten Rückkaufrechts um dieselbe Summe innerhalb einer bestimmten Frist, und würden nun den Sitz trotz fristgerechter Forderung und Zahlungsangebot von ihm nicht zurückbekommen. Der K. lädt ihn im Falle eines Einspruchs von hewt über sechs wochen peremptorisch zu rechtlicher Verwantwortung vor sich oder seinem dazu Bevollmächtigten und weist darauf hin, dass bei Nichterscheinen er der anderen Partei recht ergeen lassen wird. An montag vor sannd Kathrein tag (nach Kop.).

Kop.: Zeitgleiche Abschrift im HHStA Wien (Sign. AUR 1478 XI 23), Pap. (fol. 2r), mit rücks. zeitgleichen Vermerk ultima citacio (Blattmitte). ${ }^{3}$

Reg.: ChMel n. 7247; DeRs., Mon. Habsb. I/2 S. 688f. zu n. 568.

1 Burg Starhemberg in Oberösterreich (Haag am Hausruck).

2 Siehe n. 218.

3 Gemeinsam überliefert mit n. 219.

\section{Dezember 5, Graz}

K.F. gewährt dem Heilig-Geist-Spital an der Marktstätte zu Konstanz aus ksl. Machtvollkommenheit und rechter wissen in crafft des brieves die sunnder gnad und fryhait, dass der Ammann und die zwölf Richter des mit Gericht, Zwing und Bann dem Spital zugehörigen Dorfes Sipplingen samt der ganzen Gemeinde mündiger mannspersonen sowie deren Erben und Nachkommen unverzüglich auf ewig den Pflegern und Spitalmeistern des Spitals jährlich den Eid zu leisten haben, dem Spital treu und gehorsam zu sein, es zu fördern und alles zun tun, was treue Hintersassen, Untertanen und zugewandte ihrer Herrschaft und oberkait aus rechter Gewohnheit oder altem Herkommen schuldig sind. Die Pfleger und Spitalmeister haben das Recht, jederzeit im Haus des Spitals zu Sipplingen, genannt Kelnhof, Amtleute ein- und abzusetzen, welche das Gericht und die anderen Spitalsangelegenheiten zu Sipplingen verwalten. K.F. verfügt, dass die Pfleger und Spitalmeister diese Gnaden und Freiheiten ungehindert gebrauchen sollen, jedoch unbeschadet seiner oberkait und seiner und des Reichs Rechte, und dass alle, die aus Sipplingen wegziehen, ihrer Eide ledig sind. Er gebietet aus ksl. 
Macht dem Ammann, den Richtern und der Gemeinde von Sipplingen, dieses Privileg zu beachten, sowie dem Landvogt in Schwaben und allen Reichsuntertanen, die mit diesem brief oder einer glaubwürdigen Abschrift darum ersucht werden, im Falle von Widersetzlichkeiten der Sipplinger den genannten Pflegern und Spitalmeistern Hilfe und Beistand zu leisten und die Ungehorsamen zu Huldigung, Eidesleistung und Einhaltung der Freiheiten zu bringen, denn andernfalls droht seine und des Reichs schwere Ungnade und eine Pön von 40 Mark Gold, zahlbar je zur Hälfte an die Reichskammer und an die Pfleger und Spitalmeister des Heilig-Geist-Spitals. Am funfften tag des monatts decembris (nach Kop.).

KVr: A.m.p.d.i. (nach Kop.).

Org. im bearbeiteten Bestand nicht überliefert, der Kop. zufoge (Perg.) mit anh. S. - Kop.: Zeitgleiche Abschrift im HHStA Wien (Sign. AUR 1478 XII 5), Pap., mit rücks. zeitgleichem Vermerk Nellemburg.

Druck: ChMEL, Mon. Habsb. I/2 S. 383-385 n. 78.

Reg.: LichNowsKY(-BIRK) 8 n. 129; KRAMML, Konstanz S. 452 n. 334 (nach anderer kopialer Überlieferung).

Lit:: KrammL, Konstanz S. 188-193, bes. 191.

Das Dorf Sipplingen verweigerte allerdings die Huldigung und Eidesleistung und appellierte an K.F., der in einem Schreiben ${ }^{1}$ an die Sipplinger von 1479 August 9 die Appellation zurückwies und ihnen befahl, binnen zehn Tagen nach Erhalt des Schreibens seinem Privileg Folge zu leisten. Da keine weiteren Reaktionen bekannt sind, wird angenommen, dass das Dorf dem ksl. Befehl nachkam (KRAMML S. 192).

1 Siehe KrammL, Konstanz S. 452 n. 342.

\section{Dezember 7, Graz}

K.F. belehnt seinen Kämmerer Kaspar von Rogendorf, Pfleger von Ybbs, ${ }^{1}{ }^{\text {wissentlich }}$ mit dem brief, was wir im zu recht daran verleihen sullen oder mugen, mit Schloss Pöggstall aus der Lehenschaft des Ftm. Österreich und zwar sowohl mit seinem Anteil, den der verstorbene Konrad Hölzler innehatte, auf den er (K.F.) aber wegen einer schuldigen, das Hubmeisteramt zu Österreich betreffenden raittung Hölzlers Ansprüche hat und den er dem Rogendorfer auf solh unser gerechtikait verkauft hat, als auch mit dem Anteil Matthias Hölzlers, den dieser ihm nach dem Verkauf an den Rogendorfer mit einem offenen besiegelten Brief mit der Bitte um Weiterverleihung aufgesandt hat. ${ }^{2} \mathrm{Er}$ 
verfügt, dass der Belehnte und dessen Erben das Schloss alles und ganntz samt Zubehör innehaben und nutzen und damit ihm als Lehensherrn gehorsam zu Diensten sein sollen gemäß Lehens- und Landrecht. An montag nach sannd Niclastag.

KVr: C.d.i.p. $-\mathrm{KVv}$ : Rta.

Org. im HHStA Wien (Sign. AUR 1478 XII 7), Perg., rotes S 21 in wachsf. Schüssel mit wachsf. S 16 rücks. eingedr. an Ps. - Kop.: Vidimus des Propstes Johann von Waldhausen von 1488 November 3 ebd. (Sign. AUR sub dat. 1478 XII 7), Perg., grünes (spitzovales) S des Ausst. in wachsf. Schüssel an Ps. ${ }^{3}$

Druck: ChMEL, Mon. Habsb. I/2 S. 631f. n. 162.

Reg.: ChMel nn. 7244 (zu November 12), 7249 (Kop.); Lichnowsky(-BIRK) 8 n. 130.

Lit.: Bergmann, Rogendorf S. 526 [S. 10]; NeUnLINGER, Pöggstall S. 65f.; ZAJIC, Kaspar von Roggendorf S. 17 und 25; NeIDHART, Geschichte Pöggstalls S. 42; DAIM/KüHTREIBER, Burgen Waldviertel-Wachau S. 361; Zалс, Herren und Aufsteiger S. 20; zur Person des Rogendorfers s. auch HeInIG, Friedrich III./1 S. 195f.; zur Familie s. ZAлI, Rog[g]endorf S. 1207-1214.

Kaspar von Rogendorf, 1480 in den Herrenstand erhoben, baute Pöggstall zur Familienresidenz und Zentrum eines weit ausgreifenden Herrschaftskomplexes in Niederösterreich aus. ${ }^{5}$

1 Zur Übertragung des Schlosses Ybbs s. den Pflegrevers Kaspar Rogendorfers von 1478 Oktober 19 im HHStA Wien (Sign. AUR sub dat. 1478 X 3); Reg.: CHMeL, Mon. Habsb. I/3 S. 640 n. 17.

2 Zur Wiener Ratsbürgerfamilie Hölzler als Besitzer von Pöggstall, das Konrad Hölzler (II./d.J., † 1478), Wiener Bürgermeister $(1450 / 51,1455)$ und langjähriger Hubmeister in Österreich $(1451-1453,1455-$ 1457, 1466-1468, 1472-1477), und dessen Brüder, darunter Matthias, im Jahr 1450 erwarben, s. PeRger, Wiener Ratsbürger S. 214; ZАлC, Herren und Aufsteiger S. 17-20; auch Regg.F.III. H. 13 n. 211.

3 Das Vidimus enthält auch n. 313 (Belehnung mit Ottenschlag).

4 Nach Chмег n. 7244 Belehnung Kaspar Rogendorfers von 1478 November 12 nur mit dem Anteil Matthias Hölzlers nach nicht genannter Überlieferung im HHStA Wien.

5 Siehe zur Familie Rogendorf und dem Residenzcharakter Pöggstalls die Beiträge in AICHINGER-RosenBERGER/ZAJC, Schloss Pöggstall.

\section{[Vor 1479 Januar 2, -]}

K.F. überlässt dem Bürgermeister, Richter und Rat der Stadt Graz für den Ausbau des Stadtgrabens ${ }^{1}$ das Ungeld und Landgericht zu Graz für drei Jahre ab dem Neujahrstag 1479 (Januar 1) mit der Verpflichtung, die Einkünfte daraus ausschließlich für die Bautätigkeit zu verwenden. ${ }^{2}$ 
Org. oder Kop. im bearbeiteten Bestand nicht überliefert. - Dep.: Erw. im Revers von Bürgermeister, Richter und Rat von Graz von 1479 Januar 2, Graz, im HHStA Wien (Sign. AUR 1479 I 2), Perg., S der Stadt Graz an Ps ab und verloren, mit Rta-Vermerk auf der Rückseite. ${ }^{3}$

Lit.: Muchar, Geschichte 8 S. 116; PoPelKa, Geschichte Graz 2 S. 20.

1 Laut Revers hatte die Stadt Graz auf Befehl des K. den Ausbau des Stadtgrabens übernommen.

2 Zur Übertragung des Ungelds s. auch n. 241.

3 Reg.: GöTH, Urkunden-Regesten 9 n. 685.

\section{[Vor 1479 Januar 9, -]}

K.F. belehnt Bernhard Stadler und Michael Reiffenberger mit Schloss Kirchstetten und etlichen Stücken und Gütern, die als verschwiegene Lehen des Ftm. Österreich heimgefallen sind. ${ }^{1}$

Org. oder Kop. im bearbeiteten Bestand nicht überliefert. - Dep.: Erw. im Revers Bernhard Stadlers und Michel Reiffenbergers von 1479 Januar 9 im HHStA Wien (Sign. AUR 1479 I 9), Perg., 4 grüne SS der Ausst., Kaspar Harders und Erhard Lembachers in wachsf. Schüsseln an Ps, mit Rta-Vermerk auf der Rückseite. ${ }^{2}$

Lit.: Zu Schloss Kirchstetten s. ReICHHALTER/KüHTREIBER, Burgen Weinviertel S. $292 \mathrm{f}$.

1 Laut Revers sollen Stadler und Reiffenberger dem K. 1.000 fl. ung. entrichten, sobald sie die Lehen in ihre Gewalt gebracht haben.

2 Reg.: CHMEL n. 7255; DERS., Mon. Habsb. I/3 S. 658 f. n. 95.

\section{[Vor 1479 Januar 10, - ] $]^{1}$}

K.F. ermahnt Eb. Bernhard (II.) von Salzburg, gemäß dessen in $\mathrm{Graz}^{2}$ gegebenen Zusage auf dem Salzburger Landtag zu resignieren.

Org. oder Kop. im bearbeiteten Bestand nicht überliefert. - Dep.: Ein ksl. Kredenzschreiben

erw. in einem Schreiben Eb. Bernhards von Salzburg an K.F. von 1479 Januar 17, Salzburg, im HHStA Wien (AUR sub dat. 1479 VI 13), Perg., rotes S des Ausst. als Verschluss rücks. aufgedr. (großteils abgefallen); ${ }^{3}$ ergibt sich auch aus n. 244.

Lit.: Zum Landtag s. BAChMAnN, Reichsgeschichte 2 S. 667; MAYER, Abdankung S. 188; WIDMANN, Geschichte Salzburgs 2 S. 316; ZAISBERGER, Rohr S. 65; DOPSCH, Geschichte Salz- 
burgs S. 547; KrammL, Salzburg und die Ungarn S. 116; zu Eb. Bernhard von Rohr s. auch GaTz, Bischöfe 2 S. 590-592.

Auf dem Salzburger Landtag am 10. Januar 1479 erfolgte nicht, wie mit K.F. in Graz vereinbart, der Amtsverzicht Eb. Bernhards (von Rohr). In seinem Schreiben an K.F. erklärte er, sich auf Wunsch des Domkapitels und der Stände entschlossen zu haben, im Amt zu bleiben, nachdem diese ihr Missfallen über die geheim gehaltenen Verhandlungen geäußert, ihn an seine eigene kanonische Wahl erinnert und die Befürchtung vorgebracht hätten, die Übergabe könnte die freie Wahl des Domkapitels verletzen.

Siehe nn. 244-265, 272, 273.

1 Die Datierung ergibt sich aus dem im Schreiben Eb. Bernhards genannten Datum des Salzburger Landtags, den 10. Januar 1479 (suntag nach sannd Erharts tag negstverschinen), auf dem die ksl. Räte das Kredenzschreiben vorlegten.

2 Eb. Bernhard hielt sich im November 1478 in Graz auf, wo er K.F. die Amtsübergabe an Eb. Johann von Gran schriftlich zusagte, s. MAYER, Abdankung S. 178 und 182; DoPsCH, Salzburg im 15. Jahrhundert S. 546.

3 Druck: CHмеL, Mon. Habsb. I/3 S. 3-6n. 1.

\section{[Vor 1479 Januar 11, -]}

K.F. überlässt Jörg Fleischhacker, Bürger von Bleiburg, das Amt Schwarzenbach bestandweise für zwei Jahre ab St. Georgstag 1479 (April 24) ${ }^{1}$ gegen Zahlung von jährlich 200 Pfd. Pf.

Org. oder Kop. im bearbeiteten Bestand nicht überliefert. - Dep.: Erw. im Revers Jörg Fleischhackers von 1479 Januar 11 im HHStA Wien (Sign. AUR 1479 I 11), Pap., 3 grüne SS des Ausst. (Petschaft), des Grazer Stadtrichters Tibold Brunner ${ }^{2}$ und des Grazer Ratsherm Hans Peer rücks. aufgedr., mit Rta-Vermerk auf der Vorderseite. ${ }^{3}$

1 Zur Datierung des St. Georgstags auf April 24 s. Regg.F.III. H. 12 n. 6, Anm. 2.

2 Stadtrichter 1478-1480, 1482-1484, s. zu ihm PopelkA, Geschichte Graz 1 S. 486f; DieNes, Bürger von Graz S. XXXIV.

3 Reg.: ToMAschEK, Regesten Kärnten 3 n. 506.

[Vor 1479 Januar 14, -]

K.F. überlässt Wolfgang Kransdorfer, Bürger von Wösendorf in der Wachau, das zum Schloss Dürnstein gehörige Ungeld bestandweise für drei Jahre ab Sonntag Reminiscere 1479 (März 7) gegen Zahlung von jährlich 232 Pfd. Pf. 
Org. oder Kop. im bearbeiteten Bestand nicht überliefert. - Dep.: Erw. im Revers Wolfgang Kransdorfers von 1479 Januar 14 im HHStA Wien (Sign. AUR 1479 I 14), ${ }^{1}$ Perg., 3 grüne SS des Ausst., des Grazer Stadtrichters Tibold Brunner ${ }^{2}$ und des Grazer Ratsherrn Hans Peer in wachsf. Schüsseln an Ps, mit Rta-Vermerk auf der Rückseite. ${ }^{3}$

Lit. WALTER, Ungeld S. 86.

1 Unter dieser Sign. gemeinsam verwahrt mit n. 229.

2 Stadtrichter 1478-1480, 1482-1484, s. zu ihm PoPelKA, Geschichte Graz 1 S. 486f.; DiENES, Bürger von Graz S. XXXIV.

3 Reg.: CHMEL n. 7256 (irrtümlich für Schloss Dürnstein); DERS., Mon. Habsb. I/3 S. 649 f. n. 55.

\section{[Vor 1479 Januar 15, -]}

K.F. überträgt Michael Reiffenberger bis auf Widerruf die Pflege von Schloss Hernstein mit der Verpflichtung, von den Nutzen und Renten das Schloss auf eigene Kosten zu behüten und ihm darüber hinaus jährlich $20 \mathrm{Pfd}$. Pf. zu entrichten.

Org. oder Kop. im bearbeiteten Bestand nicht überliefert. - Dep.: Erw. im Revers Michael Reiffenbergers von 1479 Januar 15 im HHStA Wien (Sign. AUR 1479 I 15), Perg., 3 grüne SS des Ausst., Erhard Lembachers und Andreas Prämers ${ }^{1}$ in wachsf. Schüsseln an Ps, mit Rta-Vermerk auf der Rückseite. ${ }^{2}$

Lit.: ZAHN, Geschichte von Hernstein S. 52.

1 Siehe zu ihm HeInIG, Friedrich III./1 S. 208f.

2 Reg.: ChMel n. 7257; Ders., Mon. Habsb. I/3 S. 641 n. 19.

\section{[Vor 1479 Januar 16, -]}

K.F. überlässt Peter Tenter, Bürger von Klosterneuburg, ${ }^{1}$ das Ungeld zu Klosterneuburg bestandweise für zwei Jahre ab Mariä Lichtmess 1479 (Februar 2) gegen Zahlung von jährlich 850 Pfd. Pf. ${ }^{2}$

Org. oder Kop. im bearbeiteten Bestand nicht überliefert. - Dep.: Erw. im Revers Peter Tenters von 1479 Januar 16, Wien, im HHStA Wien (Sign. AUR sub dat. 1479 I 14), ${ }^{3}$ Perg., 2 grüne SS des Ausst. und des Wiener Bürgers Jörg Reicholf $f^{4}$ in wachsf. Schüsseln an Ps, mit Rta-Vermerk auf der Rückseite. ${ }^{5}$

Lit.: WALTER, Ungeld S. 118. 
1 Wohl ident mit dem Klosterneuburger Ratsherrn Peter Rieder alias Tenter, S. PERGER, Klosterneuburg S. 158.

2 Siehe dazu den Revers Peter Tenters von 1479 Mai 16 über die Übertragung des Dorfes Kierling samt Amt im HHStA Wien (Sign. AUR sub dat. 1479 III 3); Reg.: ChMEL, Mon. Habsb. I/3 S. 650 n. 59.

3 Unter dieser Sign. gemeinsam verwahrt mit n. 227.

4 Siehe zu ihm PERger, Wiener Ratsbürger S. 233.

5 Reg.: ChMmL n. 7258 (Peter Teimter); Ders., Mon. Habsb. I/3 S. 650 n. 56.

\section{[Vor 1479 Januar $16-]^{1}$}

K.F. überlässt Bernhard Krannagel ${ }^{2}$, Richter von (Windisch-)Feistritz, das Amt Feistritz samt dem Gericht und der gewöhnlichen Stadtsteuer bestandweise für ein Jahr bis Weihnachten 1480 (1479 Dezember 25) gegen Zahlung von jährlich 300 fl. ung. und der Entrichtung der dem ksl. Pfleger für die Burghut jährlich zustehenden Menge an Wein und Getreide.

Org. oder Kop. im bearbeiteten Bestand nicht überliefert. - Dep.: Erw. im Revers Bernhard Krannagels von 1479 Januar 16 im HHStA Wien (Sign. AUR sub dat. 1479 VI 12), Pap., 3 grüne SS des Ausst. (Petschaft), des Grazer Stadtrichters Tibold Brunner und des Grazer Ratsherrn Thomas Peheim ${ }^{3}$ rücks. aufgedr., mit Rta-Vermerk auf der Vorderseite. ${ }^{4}$

Lit.: Schmutz, Lexikon Steiermark 1 S. 354 (Kramnagel, zu November 6); ${ }^{5}$ PIRCHEGGER, Untersteiermark S. 131 (Krumnagel).

1 Zur Datierung: Der Revers wurde am Samstag vor dem St. Antoniustag ausgestellt. Da der Bestand wohl mit Weihnachten 1478 begann, handelt es sich mit hoher Wahrscheinlichkeit um den Festtag am 17. Januar (Antonius der Große, der Einsiedler) und nicht um jenen am 13. Juni (Antonius von Padua). Die Datierung (und daher Sign.) im HHStA Wien erfolgte nach dem Antoniustag im Juni.

2 Im Revers Krannagl; in der Lit. verschiedene Schreibweisen des Namens: Kramnagel (ScHMUTz), Krannagel (GöTH), Grandnagel (MuCHAR), Krumnagel (PIRCHEGGER).

3 Zu Tibold Brunner (Grazer Stadtrichter 1478-1480, 1482-1484) und Thomas Peheim s. DiENEs, Bürger von Graz S. XVIIIf. (Peheim) und XXXIV (Brunner); zu Brunner auch PopelKA, Geschichte Graz 1 S. $486 f$.

4 Reg.: GötH, Urkunden-Regesten n. 698 (zu Juni 12); MuchaR, Urkunden-Regesten n. 291 (Grandnagel, zu Juni 12); erw. bei DEMS., Geschichte 8 S. 118.

5 Nicht auszuschließen ist, dass ScHMUTz mit der Urkunde von 1479 November 6 eine sonst nicht bekannte Verlängerung der Bestandgabe für 1480 nennt. 
K.F. überträgt Hans Raschberger, Mautner zu Rottenmann, bis auf Widerruf die Pflege von Schloss Wolkenstein samt dem zugehörigen Landgericht mit der Verpflichtung, von den Einkünften des Landgerichts das Schloss zu behüten sowie jährlich $200 \mathrm{fl}$. ung. und 100 Pfd. Pf. zu entrichten.

Org. oder Kop. im bearbeiteten Bestand nicht überliefert. - Dep.: Erw. im Revers Hans Raschbergers von 1479 Januar 18 im HHStA Wien (Sign. AUR sub dat. 1479 VI 14), Perg., 3 grüne SS des Ausst., seines Bürgen Kaspar Gräfenberger, Marchfutterer zu Graz, und Erhard Lembachers in wachsf. Schüsseln an Ps, mit Rta-Vermerk auf der Rückseite. ${ }^{2}$

1 Zur Datierung: Der Revers wurde am Montag nach dem St. Antoniustag ausgestellt; mit hoher Wahrscheinlichkeit handelt es sich - wie in den nn. 227 (Donnerstag vor St. Antonius), 228 (Freitag vor St. Antonius), 229 und 230 (beide Samstag vor St. Antonius) sowie 232 (Dienstag nach St. Antonius) - um den Festtag am 17. Januar (Antonius der Große, der Einsiedler) und nicht um jenen am 13. Juni (Antonius von Padua); so auch die Datierung bei GöTH (s. Anm. 2). Die Datierung (und daher Sign.) im HHStA Wien erfolgte nach dem Antoniustag im Juni.

2 Reg.: GöTH, Urkunden-Regesten 9 n. 686; erw. bei MuchaR, Geschichte 8 S. 117.

\section{Januar 19, Graz ${ }^{1}$}

K.F. belehnt Jörg Hauser als Ältesten und Lehensträger seiner Brüder Balthasar, Kaspar, Hans und Paul wissenlich mit dem brief ${ }^{2}$ mit folgenden von ihrem Vater Liebhard Hauser ererbten Gütern aus der Lehenschaft der Gft. Ortenburg, die sie und ihre Erben gemäß Lehens- und Landrecht innehaben und nutzen sollen: ein Hof zu Gröfelhof (Grefling), ein Gut an der Stainprugk, ein Gut an der Prelischk und ein Gut, genannt die Stöckhleinshueben. Weiters belehnt er Jörg Hauser allein auf Bitte Jakobs von Ernau, Pfleger von Karlsberg und Vizedom in Kärnten, ${ }^{3}$ mit zwei Höfen zu Rittersdorf (Rüettestorff) bei Oberdrauburg (Traburg) sowie auf Bitte der Brüder Sigmund und Philipp Leobenegger mit einem Hof am Rosenberg oberhalb von Oberdrauburg, Lehen des Ftm. Kärnten, die sie Jörg Hauser verkauft und mit ihren offenen besiegelten Briefen aufgesandt haben. Am eritag nach sannd Anthonientag.

KVr: C.d.i.p. - KVv: Rta. - Rückseite der Wachsschüssel: Vermerk nicht lesbar (Hauser?).

Org. im HHStA Wien (Sign. AUR sub dat. 1479 VI 15), Perg., rotes S 21 in wachsf. Schüssel mit wachsf. S 16 rücks. eingedr. an Ps.

Reg.: GöTH, Urkunden-Regesten 9 n. 687; TOMASCHEK, Regesten Kärnten 3 n. 515 (zu Juni 15). 
1 Zur Datierung: Die Urkunde wurde am Dienstag nach dem St. Antoniustag ausgestellt; aufgrund der Angabe der Herrscherjahre - 27. Jahr des Kaisertums (bis 19. März 1479) und 39. Jahr des Königtums (bis 6. April 1479) - handelt es sich um den Festtag am 17. Januar (Antonius der Große, der Einsiedler) und nicht um jenen am 13. Juni (Antonius von Padua). Die Datierung (und daher Sign.) im HHStA Wien erfolgte nach dem Antoniustag im Juni.

2 Hier fehlt die sonst bei Belehnungen üblichlicherweise mit „wissentlich" verbundene Bedingungsformel „was wir verleihen sollen oder mögen", die nach HAGENEDER, Kanonisches Recht S. 432f., causaliter bzw. bekräftigend (weil er es verleihen soll und mag) zu interpretieren ist.

3 1452-1479 Pfleger von Karlsberg und 1462-1479 Vizedom in Kärnten, s. zu ihm LerTNER, Herren von Ernau S. 193-195.

\section{[Vor 1479 Januar 21, - ]}

K.F. tauscht mit Andreas Rindscheit etliche Stücke und Güter zu Leutschach gegen dessen Eigengüter und Gülten ${ }^{1}$ in Radiga und im Dorf St. Johann im Saggautal.

Org. oder Kop. im bearbeiteten Bestand nicht überliefert. - Dep.: Erw. im Revers des Ritters Andreas Rindscheit von 1479 Januar 21 im HHStA Wien (Sign. AUR 1479 I 21), Perg., 2 grüne SS des Ausst. und Wulfings von Fladnitz d.Ä. in wachsf. Schüsseln an Ps, mit Rta-Vermerk auf der Rückseite. ${ }^{2}$

1 Im Revers werden die dem K. übergebenen Gülten Rindscheits aufgezählt.

2 Reg.: Muchar, Urkunden-Regesten n. 278; Göth, Urkunden-Regesten 9 n. 693; erw. bei Muchar, Geschichte 8 S. $116 f$.

\section{[1479 Januar 27, - $]^{1}$}

K.F. verkauft dem Wiener Neustädter Bürgermeister Wolfgang Schandel ${ }^{2}$ sein Haus in Wiener Neustadt am Fischmarkt, das zwischen den Häusern des Wiener Neustädter Bürgers Dr. med. Heinrich Hopf ${ }^{9}$ und des verstorbenen Senftenbergers liegt und vormals im Besitz des Laibacher Dompropsts Lienhard Jamnitzer ${ }^{4}$ war, um 360 fl.

Org. oder Kop. im bearbeiteten Bestand nicht überliefert. - Dep.: Erw. im Schuldbrief ${ }^{5}$ des Wiener Neustädter Bürgermeisters Wolfgang Schandel von 1479 Februar 1, Wiener Neustadt, im HHStA Wien (Sign. AUR 1479 II 1), Perg., 3 grüne SS des Ausst., des Wiener Neustädter Ratsherrn Augustin Pottensteiner, Kirchenmeister der Liebfrauen-Pfarrkirche, ${ }^{6}$ und des Wiener Neustädter Bürgers und Stadtschreibers Wolfgang Spitzweg ${ }^{7}$ in wachsf. Schüsseln an Ps, mit Rta-Vermerk auf der Rückseite. ${ }^{8}$

Lit.: MAYER, Wiener Neustadt I/2 S. 94 und 503. 
1 Datierung nach MAYER, Wiener Neustadt I/2 S. 94 (nach Org. im StadtA Wiener Neustadt).

2 Bürgermeister 1479-1482, s. ebd. S. 133.

3 Siehe zu ihm ebd. S. 282.

4 Zu Lienhard Jamnitzer (Jemnitzer, Jempnitzer) s. PERGER, Simon Pötel S. 38-42; DERS., Wiener Ratsbürger S. 216f.; HeING, Friedrich III./1 S. $603 \mathrm{f}$.

5 Schandel verpflichtete sich, die Kaufsumme binnen zwei Jahren zu bezahlen und verschrieb dem $\mathrm{K}$. bis zur Bezahlung das Haus als Fürpfand.

6 Kirchenmeister 1467-1487, s. MAYER, Wiener Neustadt I/2 S. 341.

7 Zu Wolfgang Sptzweg (Spitzwegk, Spitzweck), ksl. Diener, Sekretär und Notar in der röm. Kanzlei und ab 1473 Stadtschreiber von Wiener Neustadt, s. ebd. S. 106 und 135; HeINIG, Friedrich III./1 S. 749f.

8 Reg.: ChMel n. 7260; Ders., Mon. Habsb. I/3 S. 673 n. 138.

\section{[Vor 1479 Januar 30, -]}

K.F. überlässt Richter, Rat und allen Bürgern der Stadt Leoben den Aufschlag von Vieh und Fellen sowie die zur Instandhaltung von Brücken und Wegen eingehobene Maut zu Leoben $^{1}$ samt Zwiespil bestandweise für zwei Jahre ab St. Georgstag 1479 (April 24) ${ }^{2}$ gegen Zahlung von jährlich 1.300 Pfd. Pf.

Org. oder Kop. im bearbeiteten Bestand nicht überliefert. - Dep.: Erw. im Revers von Richter, Rat und den Bürgern von Leoben von 1479 Januar 30 im HHStA Wien (Sign. AUR 1479 I 30), Perg., grünes S der Stadt Leoben in wachsf. Schüssel an Ps, mit Rta-Vermerk auf der Rückseite. ${ }^{3}$ - Ebenfalls erw. im Bürgschaftsbrief der Leobener Bürger Gregor Leubner, Jörg Kätzler und Wolfgang Steyrer von 1479 Januar 30 ebd. (Sign. AUR 1479 I 30), Perg., 5 grüne SS der Ausst. und der Leobener Ratsherren Hans Reicher und Hans Pringsauf in wachsf. Schüsseln an Ps, mit Rta-Vermerk auf der Rückseite. ${ }^{4}$

1 Zur Leobener Maut s. SchILLINGER-PRASSL, Rechtsquellen Leoben S. 71f.

2 Zur Datierung des St. Georgstags auf April 24 s. Regg.F.III. H. 12 n. 6, Anm. 2.

3 Reg.: GöтH, Urkunden-Regesten 9 n. 690.

4 Die Aussteller verbürgten sich, im Falle von Zahlungsrückständen seitens der Stadt die Bezahlung des Bestandgeldes zu übernehmen (wie auch in n. 16). - Reg.: MUcHAR, Urkunden-Regesten n. 279 (irrtümlich Bestandgabe an die drei Leobener Bürger; so auch bei DEMS., Geschichte $8 \mathrm{~S}$. 117f.); GöTH, Urkunden-Regesten 9 n. 689. Von den als Bürgen oder Siegler beteiligten Leobener Bürgern hatten Leubner, Steyrer und Pringsauf in den 1470er- und 1480er-Jahren auch das Stadtrichteramt inne, S. ScHILLINGERPRAssL, Rechtsquellen Leoben, Anh. S. 314. 
K.F. überträgt Jörg Metnitzer ${ }^{1}$ bis auf Widerruf die Pflege von Schloss Arnfels und überlässt ihm die Nutzen, Renten, Herrschaftsrechte und das sonstige Zubehör, wie sie Jörg Hollenegger innehatte, bestandweise gegen Zahlung von jährlich $240 \mathrm{Pfd}$. Pf.

Org. oder Kop. im bearbeiteten Bestand nicht überliefert. - Dep.: Erw. im Revers Jörg Metnitzers von 1479 Februar 2 im HHStA Wien (Sign. AUR 1479 II 2), Perg., 2 grüne SS des Ausst. und Erhard Lembachers in wachsf. Schüsseln an Ps, mit Rta-Vermerk auf der Rückseite. ${ }^{2}$

Lit.: Baravalle, Burgen S. 312; zur Familie s. Metnitz, Kärntner Burgenkunde 2 S. IXf..

1 Im Revers Mottniczer.

2 Reg.: Muchar, Urkunden-Regesten n. 280; GötH, Urkunden-Regesten 9 n. 691; erw. bei Muchar, Geschichte 8 S. 117 (Jörg Minndorfer).

[Vor 1479 Februar 2, - $]^{1}$

K.F. überlässt Bernhard Murstetter, Bürger von Klosterneuburg, das Ungeld zu Herzogenburg für eine Quatember ab Dienstag Mariä Lichtmess (Februar 2) bis Dienstag nach dem Kreuztag Inventionis (Mai 4) 1479 zu treuen Händen. ${ }^{2}$

Org. oder Kop. im bearbeiteten Bestand nicht überliefert. - Dep.: Erw. im Revers des Klosterneuburger Bürgers Bernhard Murstetter von 1479 (ohne Tagesdatum), Graz, im HHStA Wien (Sign. AUR sub dat. 1479 XI 12), ${ }^{3}$ Pap., 2 grüne SS des Grazer Stadtrichters Tibold Brunner ${ }^{4}$ und des Grazer Ratsherrn Hans Peer rücks. aufgedr., mit Rta-Vermerk auf der Vorderseite. ${ }^{5}$

Lit.: WALTER, Ungeld S. 114.

1 Die Datierung ergibt sich aus dem im Revers als ,kommend“ bezeichneten Beginn der treuhändischen Verwaltung mit 1479 Februar 2 (Mariä Lichtmess).

2 Siehe dazu auch den Revers Murstetters von 1480 April 15 über die Übertragung des Herzogenburger Ungelds auf ein Jahr im HHStA Wien (Sign. AUR 1480 IV 15); Reg.: ChMEL n. 7368; Ders., Mon. Habsb. I/3 S. 653 n. 72.

3 Unter dieser Sign. gemeinsam verwahrt mit n. 325.

4 Stadtrichter 1478-1480, 1482-1484, s. zu ihm PopelKA, Geschichte Graz 1 S. 486f; Dienes, Bürger von Graz S. XXXIV.

5 Reg.: ChMEL n. 7329; DERS., Mon. Habsb. I/3 S. 652 n. 69; LiCHNOwSKY(-BrRK) 8 n. 221. 
K.F. belehnt Ruprecht Pretzner, Bürger von Klagenfurt, und dessen Ehefrau Elisabeth wissenntlich mit dem brieve, was wir in zu recht daran verleihen sullen oder mugen, mit vier ererbten mader Wiesmahd, gelegen bei Klagenfurt zwischen der Wiesmahd der Kirche St. Egid (Giligen) und der Gurnitzer Wiesmahd und an die Lankwart grenzend, aus der von den verstorbenen Schenken von Osterwitz ${ }^{1}$ an ihn gekommenen Lehenschaft, die sie und ihre Erben gemäß Lehens- und Landrecht innehaben und nutzen sollen. An mittichen nach sannd Appolonien tag.

KVr: C.d.i.p. - KVv: Rta. - Rückseite der Wachsschüssel: Vermerk nicht lesbar.

Org. im HHStA Wien (Sign. AUR 1479 II 10), Perg., rotes S 21 in wachsf. Schüssel mit wachsf. S 16 rücks. eingedr. an Ps.

Reg.: Muchar, Urkunden-Regesten n. 281; TOMASCHEK, Regesten Kärnten 3 n. 507.

1 Wilhelm Schenk von Osterwitz ( $\dagger$ 1472) hatte für den Fall des Todes ohne Nachkommen seinen Besitz letztwillig K.F. hinterlassen; dessen Bruder Jörg (Georg) starb in türkischer Gefangenschaft (vor 1478 Februar 10, s. MC 11 n. 481). Zudem waren die Schenken seit Mitte des 15. Th. dem Lehensempfang nicht mehr nachgekommen, ihre Güter wurden daher von K.F. eingezogen. Siehe dazu die Urkunde des Hans Schenk von Osterwitz, Sohn Jörgs und Neffe Wilhelms, von 1478 Mai 30 im HHStA Wien (Sign. AUR 1478 V 30); Druck: CHMet, Mon. Habsb. I/2 S. 863f. n. 1105; WIESSNER, Schenken von Osterwitz S. 128 f. n. 518, auch nn. 516, 517; s. auch CHMEL, Conzeptensammlung 1 S. 145 n. 186 und Conzeptensammlung 2 S. 188 n. 413; MC 11 nn. 481, 487, 495; HenNG, Friedrich III/1 S. 226.

\section{[Vor 1479 Februar 15, -]}

K.F. überträgt Wilhelm Kundorfer bis auf Widerruf die Pflege von Schloss Waxenegg 1 und benennt ihm für die Burghut 50 Pfd. Pf., ein Zehntel vom Getreide und von der beim Schloss gelegenen Wiesmahd, die Vogtrechte auf Hühner und Eier, die Fischweide, den Wildbann und die gewöhnliche Robot.

Org. oder Kop. im bearbeiteten Bestand nicht überliefert. - Dep.: Erw. im Revers Wilhelm Kundorfers von 1479 Februar 15 im HHStA Wien (AUR 1479 II 15), Perg., 2 grüne SS des Ausst. und Erhard Lembachers in wachsf. Schüsseln an Ps, mit Rta-Vermerk auf der Rückseite. ${ }^{2}$-Ebenfalls erw. im Revers Wilhelm Kundorfers von 1479 Februar 27 (s. n. 242).

Lit.: Baravalle, Burgen S. 610.

1 Zur Übertragung von Schloss Waxenegg s. auch n. 242.

2 Reg.: Muchar, Urkunden-Regesten n. 282. 
K.F. überträgt Gilg Slaher wissenntlich mit dem brief bis auf Widerruf die Pflege von Schloss Laxenburg samt Nutzen, Renten, Zinse und allem Zubehör sowie das Ungeld zu Himberg (Hynndperg) und in den anderen zum Ungeldbezirk gehörenden Dörfern. Er verpflichtet ihn, das Schloss von den Nutzen, Renten und dem Ungeld zu behüten, ihm und nach seinem Tod seinem Sohn Ehz. Maximilian von Österreich und Burgund ${ }^{1}$ und nach dessen Tod ihren Erben damit treu und gehorsam zu sein, es offen zu halten, ihn (K.F.) und seine Leute jederzeit aufzunehmen, die zum Schloss und zum Ungeldbezirk gehörigen Leute und Holden nicht über die gewöhnlichen Nutzen, Renten, Zinse, Dienste und Robotleistungen ungebührlich gegen altes Herkommen zu belasten, sondern sie vor Gewalt und Unrecht zu schützen, den Entzug ksl. Rechte nicht zuzulassen und auf Erfordern das Schloss samt Ungeld und Zubehör ohne Widerspruch abzutreten gemäß dessen Verschreibung ${ }^{2}$. An montag vor sannd Mathias tag des heiligen zwelifpoten.

KVr: C.d.i.p. $-\mathrm{KVv}:$ Rta.

Org. im HHStA Wien (Sign. FU n. 740), Perg., rotes S 21 in wachsf. Schüssel (beschädigt) mit wachsf. S 16 rücks. eingedr. an Ps.

Reg.: LichNowsKy(-BIRK) 8 n. 147 (zu März 1).

Lit.: WALTER, Ungeld S. 134; SPRINGER u.a., Laxenburg S. 47.

1 Zur Titulatur Maximilians s. die Einleitung S. 22-24.

2 Revers Gilg Slahers von 1479 Februar 22 im HHStA Wien (Sign. FU n. 741); Reg.: CHMEL n. 7264; DERS., Mon. Habsb. I/3 S. 461 n. 20.

\section{Februar 24, Graz}

K.F. überträgt Balthasar Eggenberger, Bürger von Graz, wissenntlich mit dem brief das Ungeld in der Stadt Judenburg samt Zubehör sowie den Aufschlag von dem Wein, der durch Judenburg an die ennde, da der ungelt sein ganng nit hat, gefiuhrt wird, als Ersatz für das Ungeld zu Graz, das er Eggenberger zusammen mit dem Aufschlag von dem zur Drau gefuihrten Wein zur Bezahlung des ihm vom lannd schuldigen Geldes übertragen hatte, ${ }^{1}$ er ihm aber befohlen hat, das Ungeld an die Grazer Bürger ${ }^{2}$ für den Bau der Stadt abzutreten. Er verfügt, dass Eggenberger das Ungeld und den Aufschlag zu Judenburg zusammen mit dem Aufschlag an der Drau zur Bezahlung der Schulden einnehmen, nach deren Begleichung Ungeld und Aufschläge samt Zubehör abtreten und über die Einnahmen Rechnung legen soll. An mittichen dem aschtag. 
KVr: C.d.i.p. - KVv: Rta. - Rückseite der Wachsschüssel: Egkenperger.

Org. im HHStA Wien (Sign. AUR 1479 II 24), Perg., rotes S 21 in wachsf. Schüssel (beschädigt) mit wachsf. S 16 rücks. eingedr. an Ps.

Lit.: LeITHNER, Judenburg S. 10 (zu 1479); zu Balthasar Eggenberger, der zu den Großfinanziers des K. gehörte, s. ausführlich DIENES, Bürger von Graz S. LXVII-LXX, bes. LXIX; weiters PopelkA, Geschichte Graz 1, S. 64, 66f., 72, 211, 236, und 2, S. 270 und 361 (Familie); HeINIG, Friedrich III./1 S. 79, 236, 807.

1 Zur Übertragung des Weinaufschlags und Grazer Ungelds s. den Revers Eggenbergers von 1478 Januar 3 im HHStA Wien (Sign. AUR 1478 I 3), nach DiENEs, Bürger von Graz S. LXVIII, zur Bezahlung der 12.000 fl., die Eggenberger den steirischen und kärntnerischen Ständen zur Beschaffung von Söldnern geliehen hatte. Der Revers auch erw. bei MuchaR, Geschichte 8 S. 102 (zu Januar 4); s. in der Sache auch die ksl. Urkunden von Januar 1478 bei CHMrL, Conzeptensammlung 1 S. 118 n. 116, S. 124f. n. 133 und Conzeptensammlung 2 S. 47 n. 240.

2 Zur Übertragung des Ungelds an die Grazer Bürger s. n. 223.

\section{[Vor 1479 Februar 27, -]}

K.F. überlässt Wilhelm Kundorfer bis auf Widerruf Amt und Landgericht zu Waxenegg zusätzlich zur Pflege des Schlosses ${ }^{1}$ bestandweise gegen Zahlung von jährlich 410 Pfd. Pf.

Org. oder Kop. im bearbeiteten Bestand nicht überliefert. - Dep.: Erw. im Revers Wilhelm Kundorfers von 1479 Februar 27 im HHStA Wien (AUR 1479 II 27), Perg., 2 grüne SS des Ausst. und Erhard Lembachers in wachsf. Schüsseln an Ps, mit Rta-Vermerk auf der Rückseite. ${ }^{2}$

Lit.: Baravalle, Burgen S. 610.

1 Siehe n. 239.

2 Reg.: Muchar, Urkunden-Regesten n. 283.

\section{[Vor 1479 März 6, -]}

K.F. überlässt Jörg Metnitzer ${ }^{1}$ bis auf Widerruf die Ämter zum Entrich (Mantrach) und zu Thunau bestandweise gegen Entrichtung von jährlich 40 Pfd. Pf. und drei Fässern Wein. 
Org. oder Kop. im bearbeiteten Bestand nicht überliefert. - Dep.: Erw. im Revers Jörg Metnitzers von 1479 März 6 im HHStA Wien (Sign. AUR 1479 III 6), Perg., 2 grüne SS des Ausst. und Erhard Lembachers in wachsf. Schüsseln an Ps, mit Rta-Vermerk auf der Rückseite.

Lit.: Schmutz, Lexikon Steiermark 4 S. 186 (pflegweise Übertragung); BaRAVALle, Burgen S. 339; zur Familie s. METNITZ, Kärntner Burgenkunde 2 S. IXf.

1 Im Revers Mottniczer.

\section{März 9, Graz}

K.F. befiehlt Bf. Christoph (I.) von Seckau ${ }^{1}$ unter Darlegung der von Eb. Bernhard von Salzburg $^{2}$ gebrochenen Vereinbarungen, diesen zur unverzüglichen Übergabe des Erzbistums gemäß dessen mündlicher und schriftlicher Zusage anzuhalten, um sich selbst und das Erzstift vor unrat und verderben zu bewahren und, sollte dieser in solichem seinem verirrten gemut beharren, ihm keinerlei Hilfe zu leisten, sondern auf ihn als Kaiser und obersten Vogt und Schirmherr der Kirche aufsehen zu haben. Er verspricht, ihn vor allfälligen Schäden zu bewahren, gemeinsam mit dem Eb. (Johann) von Gran ${ }^{3}$ Christophs Freiheiten und Privilegien sowie Hab und Gut zu schützen und sich mitsambt der billikeit mit Gnaden erkenntlich zeigen zu wollen.

Einleitend legt K.F. ausführlich dar, dass ihm als Erbvogt das zum Schaden des Erzstifts Salzburg gereichende Regiment des Eb. schon länger missfallen habe, er dies aber aus neigung zu geistlichen Personen, insbesondere zu Eb. Bernhard, und in der Hoffnung auf Besserung so lange geduldet habe, bis der Eb. sich selbst als untuglich erkannt und den Rücktritt angeboten habe. Auf seine (K.F.) Botschaft, dies nicht ohne sein Einverständnis zu tun und dessen eigenhändige schriftliche Bitte habe er (K.F.) ihn zu persönlichen Verhandlungen geladen. Bei der Zusammenkunft ${ }^{4}$ habe $\mathrm{Eb}$. Bernhard aus freiem Willen die Übergabe des Stifts nach Rat des K. zugesagt, woraufhin auf sein (K.F.) Ersuchen Eb. Johann von Gran der Übernahme zum Besten des Stifts und zur Betrachtung des eigenen Seelenheils, nicht aber aus mangel seiner zeittlichen narung zugestimmt habe. Es sei vereinbart worden, dass Eb. Bernhard mit Privilegien und sundern artickeln vorab persönlich ${ }^{5}$ und dann, um die Wahl nach altem Herkommen nicht zu verletzen, für das Stift seitens Papst (Sixtus IV.) und K. versorgt werden soll. Eb. Bernhard habe dies mit ganntz begirlichen willen angenommen und mündlich wie auch schriftlich mit eigener Hand ${ }^{6}$ der Resignation und entsprechenden Bemühungen des $\mathrm{K}$. beim Papst zugestimmt, selbst wenn das Domkapitel und die Stände nicht einwilligen sollten. Auf dessen gleuplich Zusage habe er (K.F.) sogleich dem Papst wegen solicher ubergab und freyheit, die wir der walehalben des stiffts erwerben sullen, ${ }^{7}$ berichtet und bei ihm arbeitten lassen, der sich, wie er überzeugt sei, diesbezüglich gutwillig zeigen 
werde. Eb. Bernhard habe dem Kapitel und den Ständen einen Tag nach Salzburg gesetzt und ihn gebeten, eine Gesandtschaft dorthin zu schicken. K.F. versichert, dass er nie gedacht hätte, der Eb. würde nach Rat jener, die aus Eigennutz das Wohl des Stifts kaum erwägen, seine Zusage nicht einhalten ${ }^{8}$ wie auch den ihm und dem $\mathrm{Eb}$. von Gran daraus entstandenen und keineswegs zu duldenden Spott nicht bedenken. Es sei daher erforderlich, über den Papst und auf anderen Wegen Eb. Bernhard zur Übergabe zu bewegen, woraus zu seinem Bedauern dem Erzstift Zerrüttung und Schaden erwachsen könne, was er lieber vermieden hätte. Am eritag nach sonntag Reminiscere (nach Konz.).

Org. im bearbeiteten Bestand nicht überliefert. - Konz., datiert mit 1479 März 9, im HHStA Wien (Sign. AUR 1479 III 9), Pap. (4 fol.), mit Verzeichnis der Empfänger (fol. 3v); davon Abschrift des 18. Jh. ebd. (Sign. Urkundenabschriften Österr. Urkunden, Kt. 46 sub dat.), Pap.

Druck: ChMEL, Mon. Habsb. I/3 S. 7-10 n. 3; MC 11 n. 519.

Reg.: LichNowsky(-BIRK) 8 n. 150; Regg.F.III. H. 4 n. 806 (nach unzulänglicher Überlieferung).

Lit.: $\mathrm{Zu}$ den Auseinandersetzungen um den Verzicht Eb. Bernhards auf den Salzburger Stuhl BACHMANN, Reichsgeschichte 2 S. 666-668; MAYER, Abdankung, bes. S. 188f.; WIDMANN, Geschichte Salzburgs 2 S. 316f.; ZAISBERGER, Rohr S. 64-66, 71f.; DOPSCH, Geschichte Salzburgs S. 546-549; NeHring, Matthias Corvinus S. 112f.; KRAMML, Salzburg und die Ungarn S. 117; Überblick bei NIEDERSTÄTTER, Jahrhundert der Mitte S. $185 f$.

Das vorliegende Konzept beginnt ohne Angabe eines Empfängers mit Ersamer lieber andechtiger (fol. 1v) und listet am Schluss die Empfänger auf (fol. $3 \mathrm{v}$ ), beginnend mit Bf. Christoph von Seckau. Auf fol. $4 \mathrm{r}$ ist vermerkt: Cedula inclusa. Und begern des ewer schrifflich furderlich annttwurt (Blattmitte).

Siehe nn. 225, 245-265, 272, 273.

1 Christoph von Trautmannsdorf (Bf. 1477-1480), s. zu ihm Amon, Bischöfe S. 149-155; Gatz, Bischöfe 2 S. 698. - Siehe in der Sache auch den Schutzbrief Kg. Matthias' von Ungarn für Bf. Christoph von 1479 November 17 im HHStA Wien (Sign. AUR 1479 XI 17).

2 Bernhard von Rohr (Eb. 1466-1481), s. zu ihm ZAISBERGER, Rohr; GATz, ebd. S. 590-592.

3 Johann Beckenschlager (Beckensloer) (Eb. von Gran 1472-1487), s. zu ihm ZAISBERGER, ebd., bes. S. 58-62; GATZ, ebd. S. 36f.; HeINIG, Friedrich III/1 S. 449-452 mit weiterführender Lit.

4 Im November 1478 in Graz, S. MAYER, Abdankung S. 178, 182; Dopsch, Salzburg im 15. Jahrhundert S. 546.

5 Eb. Bernhard sollte die Wiener Dompropstei mit jährlichen Einkünften von $2.000 \mathrm{fl}$. und von Johann von Gran eine Barzahlung von $6.000 \mathrm{fl}$. ung. sowie jährlich $4.000 \mathrm{fl}$. aus den Einkünften des Erzstifts Salzburg erhalten, s. MAYER, Abdankung S. 221 n. 7. 
6 Bekannt ist ein - heute nicht mehr vorhandener - eigenhändiger undatierter Zettel Bernhards von Rohr mit der Übergabezusage, allerdings mit Zustimmung von Kapitel und Landschaft, der auf 1478 datiert wird; der Zettel gedruckt bei ZAISBERGER, Rohr S. 165f. n. 39, demnach ehemals beiliegend einer Urkunde von 1481 November 29 im HHStA Wien (zu Urkunden unter diesem Datum s. Regg.F.III. H. 35 nn. 156-158); s. auch WIDMANN, Geschichte Salzburgs 2 S. 313.

7 Möglicherweise handelt es sich bei der erwähnten Freiheit um die Bulle von 1478 Juli 1 (s. n. 171), wonach bei Sedisvakanz das Domkapitel nur einen für Papst und K. genehmen Kandidaten wählen darf.

8 Zum Salzburger Landtag im Januar 1479 und dem Schreiben Eb. Bernhards von 1479 Januar 17, in dem er dem K. mitteilt, nicht auf sein Amt zu verzichten, s. n. 225.

\section{März 9, Graz}

K.F. befiehlt desgleichen

- Bf. Georg von Chiemsee.

- Bf. Johann von Lavant.

- Propst, Dekan und Kapitel des Augustiner-Chorherrenstifts Berchtesgaden. 247

- Propst, Dekan und Kapitel des Augustiner-Chorherrenstifts Baumburg. 248

- Propst, Dekan und Kapitel des Augustiner-Chorherrenstifts Gars.

- Propst, Dekan und Kapitel des Augustiner-Chorherrenstifts (Herren-) Chiemsee.

- Propst, Dekan und Kapitel des Augustiner-Chorherrenstifts Au am Inn. $\quad 251$

- Abt und Konvent des Benediktinerklosters St. Peter zu Salzburg. ${ }^{1}$

- Abt und Konvent des Benediktinerklosters Seeon.

- der Stadt Salzburg. $\quad \mathbf{2 5 4}$

- der Stadt Radstadt.

- der Stadt Hallein. $\quad \mathbf{2 5 6}$

- der Stadt Laufen. ${ }^{2} \quad \mathbf{2 5 7}$

- der Stadt Tittmoning. ${ }^{3} \quad \mathbf{2 5 8}$

- der Stadt Friesach.

- der Stadt Gmünd (in Kärnten). $\quad \mathbf{2 6 0}$

- der Stadt Pettau. $\quad 261$ 
- der Stadt Rann (Rayn).

- der Stadt St. Andrä im Lavanttal.

- allen Prälaten, Rittern, Städten und der Landschaft des Erzstifts Salzburg (Generalmandat).

Orgg. im bearbeiteten Bestand nicht überliefert. - Erw. in dem in n. 244 (Konz.) genannten Empfängerverzeichnis.

1 Org. im Archiv der Erzabtei St. Peter, Salzburg (Sign. Urk. Nr. 1449-1479 III 9), s. monasterium.net (ATStiASP, sub dat.); s. dazu ZaISBERGER, Rohr S. 166 n. 40 und S. 232-235 n. 68 (Druck).

2 Org. im HStA München (Sign. Salzburger Landschaft Urkunden n. 4), s. monasterium.net (DE-BayHStA, Bestand Salzburger Landschaft Urkunden, sub. dat.)

3 Nach Tittmoning folgt im Empfängerverzeichnis die Stadt Mühldorf, s. n. 265.

\section{März 9, Graz}

K.F. befiehlt desgleichen Richter, Bürgermeister, Rat und den Bürgern der Stadt Mühldorf (am Inn) (nach Kop.).

KVr: A.m.d.i.i.c. (nach Kop.).

Kop.: Abschrift in einer Hs. im HHStA Wien (Sign. AUR 1479-1482), Perg. (fol. 2r-3v), 15. Jh. $;^{1}$ die Urkunde auch erw. in dem in n. 244 genannten Empfängerverzeichnis.

Druck: ZAISBERGER, Rohr S. 232-235 n. 68.

Lit. und Kommentar s. n. 244.

1 Die Hs. beinhaltet die Verhandlungen mit Mühldorf bzgl. der Resignation Eb. Bernhards von Rohr (bei ZAISBERGER „HS. B 750“).

\section{[Vor 1479 März 10, -]}

K.F. erlässt für das Ftm. Kärnten eine Ordnung zur Einhebung des Wochenpfennigs.

Org. oder Kop. im bearbeiteten Bestand nicht überliefert. - Dep.: Erw. in n. 267. 
K.F. setzt alle Prälaten, Adeligen, Erzpriester, Pfarrer, Altaristen, Gesellpriester, Kapläne und Zechmeister der Pfarrkirchen in den Städten und Märkten sowie allen anderen geistlichen und weltlichen Personen im Ftm. Kärnten davon in Kenntnis, dass auf dem Landtag am sand Blasien tag nagstverganngen (Februar 3) wegen der schweren Verwüstungen durch die unglaubigen beschlossen wurde, zur Stärkung des Widerstands und umb gemains mitleiden willen den Wochenpfennig ${ }^{1}$ für jede Person auf ein weiteres Jahr $\mathrm{zu}$ veranschlagen, beginnend mit dem newen jarstag nagstvergangen (Januar 1), und befiehlt ihnen, die Einhebung für den Zeitraum der vergangenen, mit suntag Reminiscere (März 7) endenden Quatember wie folgt vorzunehmen: In jeder Pfarre erfolgt die Einhebung durch den Pfarrer und die Zechleute der Pfarrkirche gemeinsam mit einem in der Pfarre ansässigen Adeligen, die von den für das Wochengeld eingesetzten Einnehmern in den Landesvierteln gemäß seiner an sie ergangenen und öffentlich verkündeten Ordnung $^{2}$ ernannt wurden. K.F. weist insbesondere die Prälaten und Adeligen an, ihre Leute anzuhalten, den Wochenpfennig auch von den Ehefrauen und Dienstboten zu entrichten, da im Falle von Ungehorsam die Einnehmer das Hab und Gut der Leute gemäß der genannten Ordnung phenndten und aufhalten werden, und gemahnt sie an ihre Schuldigkeit gegenüber ihrem Glauben, ihm selbst sowie Land und Leuten. Er befiehlt den Pfarrern, Zechmeistern und Adeligen, den Einnehmern gemäß der Ordnung gehorsam zu sein, den Wochenpfennig mit fleiss einzubringen, das Geld nur den Einnehmern auf deren Erfordern gegen Quittung zu übergeben, diese für die künftige Abrechnung aufzubewahren und ihrerseits den Einnehmern eine schriftliche Bestätigung für die überantwortete Summe auszuhändigen, um eine ordnungsgemäße Abrechnung des Wochenpfennigs zu gewährleisten. An mittichen vor sand Gregorien tag. ${ }^{3}$

KVr: C.d.i.i.c. $-\mathrm{KVv}$ - - - Kay. Gescheff des wochen d. halben zugeben wider die Turcken (zeitnaher Vermerk, linke Blattseite).

Org. im HHStA Wien (Sign. AUR 1479 III 10), Pap., rotes S 21 rücks. aufgedr. (tlw. abgefallen).

1 Zur Einhebung des Wochenpfennigs (1 Pfennig wöchentlich für jeden Erwachsenen) für die Türkenabwehr s. Fräss-EHRFELD, Geschichte Kärntens I/2 S. 597, zu den Türkeneinfällen ebd. S. 598-602; zur Einhebung des Wochenpfennigs im Jahr 1478 s. CHMEL, Mon. Habsb. I/2 S. 866 n. 1114 (ksl. Mandat von 1478 Juni 8).

2 Siehe $n$. 266.

3 Die Angabe des Herrscherjahrs unsers kaysertumbs im achtundzwainzigisten jare widerspricht der Datumsangabe nach dem St. Gregorstag (12. März), da das 28. Jahr des Kaisertums erst mit dem 19. März 1479 begann, weshalb ein Schreiberfehler anzunehmen ist. 
K.F. verpfändet Eb. Johann von Gran Schloss und Herrschaft Kreuzenstein sowie 600 fl. ung. von seinen jährlichen Einkünften zu Klosterneuburg und 300 Pfd. Pf. von jenen zu Korneuburg für 11.000 fl. ung., die dieser Sigmund Schlick für die Ablösung bezahlt hat.

Org. oder Kop. im bearbeiteten Bestand nicht überliefert. - Dep.: Erw. im Revers Eb. Johanns von Gran von 1479 März 11, Graz, im HHStA Wien (Sign. AUR 1479 III 11), Perg., rotes S des Ausst. in wachsf. Schüssel an Ps, mit Rta-Vermerk auf der Rückseite. ${ }^{1}$

Lit.: STARZER, Korneuburg S. 128; Dopsch, Geschichte Salzburgs S. 546; zu Eb. Johann Beckenschlager (Beckensloer) s. ZAISBERGER, Rohr, bes. S. 58-62; GATZ, Bischöfe 2 S. 36f.; HEINIG, Friedrich III./1 S. 449-452 mit weiterführender Lit.

Siehe n. 93.

1 Reg.: ChMmL n. 7268; DeRs., Mon. Habsb. I/3 S. 656 n. 83; LichnowsKy(-BIRK) 8 n. 151.

\section{[Vor 1479 März 24, -]}

K.F. belehnt Wilhelm Kadauer mit dem durch den Tod des Heidenreich Truchsess (von Grub) ${ }^{1}$ ledig gewordenen halben Teil an dem (festen) Haus Buchberg, an der öden Feste Plank sowie an dem Hof und den Holden zu Neudorf. Weiters belehnt er ihn mit einem Hof zu Hagendorf samt 60 Joch Acker, Baumgärten, Wiesen, Baumstätten, einem halben Pfd. Geld auf zwei zugehörigen Hofstätten und einem Baumgarten in der Pewndt, mit dem Zehent auf neun ganze Lehen zu Hörersdorf in der Mistelbacher Pfarre sowie mit dem halben Teil der von Kg. Albrecht II. den Töchtern des Stephan Doss verliehenen und ledig gewordenen Lehen zu Hohenau, namentlich dem Hochgericht, zwei Teilen des Zehents, 2 Pfd. Geld sowie Maut und Zoll.

Org. oder Kop. im bearbeiteten Bestand nicht überliefert. - Dep.: Erw. im Revers Wilhelm Kadauers von 1479 März 24 im HHStA Wien (Sign. AUR 1479 III 24), Perg., 2 grüne SS des Ausst. und Erhard Lembachers in wachsf. Schüsseln an Ps, mit Rta-Vermerk auf der Rückseite. ${ }^{2}$

Lit.: MüLLNER, Herren von Kattau S. 98f.

1 Ksl. Rat, verstorben vor 1468, s. zu ihm HerNIG, Friedrich III/ 1 S. $285 \mathrm{f}$.

2 Druck: ChMEL, Mon. Habsb. I/3 S. 308f. n. 132; Reg.: ChMEL n. 7269. 
K.F. weist seinen Rat Andreas von Kraig, Pfleger von Gurkfeld, an, von seinen dortigen Nutzen und Renten der Agnes, Witwe des Eusebius Dietprant, $100 \mathrm{fl}$., die er ihr für die Ablösung verschiedener Güter schuldet, gegen Quittung zu geben, und versichert, die Summe gegen Vorlage der Quittung bei Rechnungslegung in Abzug zu bringen. An eritag nach dem suntag Judica in der vasten (nach Kop.).

KVr: C.d.i.p. - Rta (unterer Blattrand rechts) (nach Kop.).

[Org. im ARS Ljubljana, zuvor im HHStA Wien.] - Fotokop. vom Org. im HHStA Wien (Sign. AUR 1479 III 30).

Reg.: Regg.F.III. H. 29 n. 212 (nach Org., mit irrtümlicher Angabe einer rücks. statt vorders. Besiegelung); weiters CHMEL, Mon. Habsb. I/3 S. 727 n. 279.

Lit.: Zu Andreas Kraig, ksl. Rat und seit 1471 Erbkämmerer in Kärnten, s. HEINIG, Friedrich III./1 S. $212 \mathrm{f}$.

\section{[Vor 1479 April 7, -]}

K.F. verlängert Andreas von Spangstein den seit drei Jahren bestehenden und mit Sonntag Judica (1479 März 28) endenden Bestand von Schloss, Amt und Landgericht Eibiswald gegen Zahlung von jährlich $120 \mathrm{Pfd}$. Pf.

Org. oder Kop. im bearbeiteten Bestand nicht überliefert. - Dep.: Erw. im Revers des Andreas von Spangstein von 1479 April 7 im HHStA Wien (Sign. AUR 1479 IV 7), Perg., 2 grüne SS des Ausst. und Friedrich Holleneggers in wachsf. Schüsseln an Ps, mit Rta-Vermerk auf der Rückseite. $^{1}$

Lit.: Zu Schloss Eibiswald s. EBNER, Burgen Steiermark 3 S. 26.

1 Reg.: Muchar, Urkunden-Regesten n. 284; GöTH, Urkunden-Regesten 9 n. 697; erw. bei Muchar, Geschichte $8 \mathrm{~S} .117$.

\section{[Vor 1479 April 7, -]}

K.F. ersucht Papst Sixtus IV., Eb. Bernhard von Salzburg, ${ }^{1}$ der entgegen seinem Versprechen nicht zugunsten des Eb. (Johann) von $\mathrm{Gran}^{2}$ resignieren will, mit geeigneten Mitteln dazu zu veranlasssen. 
Org. oder Kop. im bearbeiteten Bestand nicht überliefert. - Dep.: Erw. in einer zeitgleichen, von der pästlichen Kanzlei ausgefertigten Abschrift eines Breves ${ }^{3}$ Papst Sixtus' IV. von 1479 April 7, Rom, im HHStA Wien (Sign. AUR 1479 IV 7), Pap.

Das Breve ist an Bf. Alexander von Forli, den Notar Ludwig de Agnellis und den Auditor Anton de Grassis adressiert, die der Papst beauftragte, sich über das Versprechen Eb. Bernhards zu informieren, ihn zur Einhaltung zu ermahnen und dessen Verwaltung zu untersuchen, um einen Weg zu finden, dem Ersuchen des Kaisers zu entsprechen, da er hinsichtlich der Resignation selbst, die frei und im vorgegebenen rechtlichen Rahmen erfolge, nichts tun könne. ${ }^{4}$

Siehe nn. 225, 244-265, 273.

1 Bernhard von Rohr (Eb. 1466-1481), s. zu ihm ZaIsBerger, Rohr, GATZ, Bischöfe 2 S. 590-592.

2 Johann Beckenschlager (Beckensloer) (Eb. von Gran 1472-1487), s. zu ihm die in n. 273 genannte Lit.

3 Org. des Breves im WStLA (Sign. HAUrk 4742), s. monasterium net (AT-WStLA, Bestand HauptarchivUrkunden, sub dat.); Druck: ChMEL, Mon. Habsb. I/3 S. 457f. n. 55 (nach Org.); Reg.: QGStW II/3 n. 4742 .

4 Siehe zum Breve BACHMANN, Reichsgeschichte 2 S. 668; ZAISBERgER, Rohr S. $71 \mathrm{f}$.

\section{April 13, Graz}

K.F. übersendet Eb. Johann von Gran hieinne beslossen das von seinem aus Rom zurückgekehrten Diener Hans Steinberg, Lehrer beider Rechte, ${ }^{1}$ mitgebrachte Breve des Papstes (Sixtus IV.), das Erzbistum Salzburg betreffend. Er teilt ihm überdies mit, dass ihn Steinberg auch hinsichtlich der anderen Angelegenheiten ${ }^{2}$ des Eb., worüber Steinberg und der Eb. (Andreas) von Krajina ${ }^{3}$ auf seinen (K.F.) Befehl mit dem Papst verhandelt hatten, unterrichtet habe und den Eb. sicher auch darüber unterrichten werde, und er (K.F.) dies nur so verstehen könne, dass sich der Papst gutwillig zeige, was er $\mathrm{ihm}$ (dem Eb.) nicht unverkundet lassen wolle. An eritag in den heiligen Osterfeirtagen.

KVr: C.d.i.i.c. - KVv: Dem erwirdigen Johannsen erczbischoven zu Gran, unserm fursten und lieben andechtigen (Adresse, Blattmitte).

Org. im HHStA Wien (Sign. AUR 1479 IV 13), Pap., rotes S 21 (unter Papieroblate) als Verschluss rücks. aufgedr.

Lit.: Zu Johann Beckenschlager (Beckensloer) (Eb. von Gran 1472-1487) s. ZAISBERGER, Rohr, bes. S. 58-62; GaTZ, Bischöfe 2 S. 36f.; HeInIG, Friedrich III./1 S. 449-452 mit weiterführender Lit.; zu den Konflikten um das Erzbistum Gran s. BACHMANN, Reichsgeschichte 2 S. 633f.; NeHRING, Matthias Corvinus S. 112f.

Siehe nn. 193, 225, 244-265, 272. 
1 Zu Hans (Johann) Steinberg s. HeINIG, Friedrich III/1 S. 521-523.

2 Wohl die Auseinandersetzungen mit $\mathrm{Kg}$. Matthias von Ungarn um das Erzbistum Gran.

3 Andreas Jamometić (Eb. 1476-1482), s. zu ihm PeTERsoHn, Personalakt; DERS., Gesandter, bes. S. 20-32.

\section{April 15, Graz}

K.F. beurkundet den von seinen Räten erzielten Vergleich zwischen Martin Scharffwindt, Pfarrer von Windischgraz, und Hans Gall von Buchenstein, Pfleger von Hohenmauthen (zu der Mawtt), ${ }^{1}$ in deren Streit um die Holznutzung des Pfarrers in dem Wald, genannt die Gemain am Perg und gelegen bei Windischgraz gegenüber dem Schloss schattnhalben oberhalb der Dörfer Rottenbach zu der Feichten bei St. Gertrud und Buchdorf. Dem Vergleich zufolge sollen Scharffwindt und die ihm nachfolgenden Pfarrer in dem Wald Brennholz für ihren Bedarf nehmen können und ihren freien gesuech darin haben, unbehindert von Gall, dessen Erben und Nachkommen. An phintztag vor dem suntag Quasimodogeniti (nach Kop.).

KVr: C.d.i.i.c. $-\mathrm{KVv}$ Rta (nach Kop.).

[Org. im ARS Ljubljana, zuvor im HHStA Wien.] - Fotokop. vom Org. im HHStA Wien (Sign. AUR 1479 IV 15).

Druck: ChMel, Mon. Habsb. I/3 S. 310f. n. 134.

Reg.: Regg.F.III. H. 29 n. 213 (nach Org.); s. weiters LichNowsKY(-BIRK) 8 n. 159.

1 Zur Übertragung der Pflege s. n. 145.

\section{April 20, Graz}

K.F. lädt Hans Holfelder, Bürger von Nürnberg, und dessen Ehefrau Barbara oder ihren bevollmächtigten Anwalt erneut auf den 45. Tag bzw. den ersten darauf folgenden Gerichtstag nach Erhalt dieser Ladung peremptorisch zu rechtlicher Verantwortung vor sich oder seinen Bevollmächtigten. Er teilt ihnen mit, dass nach der an sie ergangenen vormaligen Ladung ${ }^{1}$ aufgrund ihrer Appellation gegen ein zugunsten des Konrad Seus ergangenes Urteil des Nürnberger Stadtgerichts nun dieser an ihn appelliert habe, ${ }^{2}$ sein Kammergericht aber nicht in gemainer übung gewesen sei. Er versichert, die Sache gemäß der vormaligen Ladung im rechten anzuhören oder, sollte das Kammergericht nicht zusammentreten, für eine Anhörung am ksl. Hof zu sorgen und nach ordnunge des rechtens zu handeln, und weist darauf hin, im Falle des Nichterscheinens auf Erfor- 
dern der Gegenpartei rechtlich zu verfahren, wie es sich gebührt. Am zwaintzigsten tag des monads aprilis (nach Kop.).

KVr: A.m.d.i. (nach Kop.).

Org. im bearbeiteten Bestand nicht überliefert, der Kop. zufolge mit rotem S rücks. aufgedr. Kop.: Notariatsinstrument des öff. Notars Konrad Schilling aus Kronach, Bistum Bamberg, von 1479 Mai 21 im HHStA Wien (Sign. AUR sub dat. 1479 IV 20), Pap., Notarssignet. ${ }^{3}$

Lit.: Zum Prozess s. Protokoll- und Urteilsbücher 3 S. 1273f. Verfahrensnr. 460.

Siehe nn. 166, 167, 276.

1 Ladung der Holfelder von 1478 Juni 18, s. n. 167.

2 Appellation des Konrad Seus von 1478 Oktober 22 (Notariatsinstrument) im HHStA Wien (Sign. AUR $1478 \times 22)$.

3 Das Notariatsinstrument wurde anlässlich der Übergabe der ksl. Ladung an das Ehepaar Holfelder am 21. Mai 1479 in ihrem Haus in Nürnberg in Gegenwart der Zeugen Hans Kanig und Heinz Beck, Einwohner von Nürnberg, ausgestellt. - Rücks. zeitgleicher Vermerk: Sewss contra Holfelder producta xxvii $i^{\circ}$ septembris 1479. G(eorg) Knöringer (Blattmitte); zu Georg Knöringer s. HenNIG, Friedrich III./1 S. 761763. - Die Ladung des Ehepaars Holfelder auch erw. im zweiten Notariatsinstrument Konrad Schillings vom selben Tag (s. n. 276) sowie in der Bestellung der Kammergerichtsprokuratoren Georg Schrättl, Jakob Helmreich und Peter Staud (Stude) sowie des Ulrich Kurz als Bevollmächtigte Hans Holfelders von 1479 Juni 28 im HHStA Wien (Sign. AUR 1479 VI 28); zu den drei Kammergerichtsprokuratoren s. HeINIG, ebd. S. 139 und 771-774.

\section{[1479 April 20, Graz]}

K.F. lädt desgleichen den Nürnberger Bürger Konrad Seus vor sich.

Org. oder Kop. im bearbeiteten Bestand nicht überliefert. - Dep.: Erw. im Notariatsinstrument des öff. Notars Konrad Schilling aus Kronach, Bistum Bamberg, über die Prokuratorenbestellung des Dr. iur. Johann Seus, Sohn des Konrad Seus, von 1479 Mai 21 im HHStA Wien (Sign. AUR 1479 V 21), Pap., Notarssignet. ${ }^{1}$

Lit.: Zum Prozess s. Protokoll- und Urteilsbücher 3 S. 1273f. Verfahrensnr. 460.

Siehe nn. 166, 167, 275.

1 Rücks. zeitnahe Vermerke (Blattmitte): mandatum procur(ationis) doctoris Seus. - Sewss contra Holfelder producta ad iudicium camere imperialis xxvii ${ }^{\circ}$ mensis septembris 1479. G(eorg) Knöringer (Blattmitte); zu Georg Knöringer s. HeInG, Friedrich III./1 S. 761-763. 
K.F. belehnt Thomas Praitenwiser als nächsten Erben mit einem Hof zu Press samt einer Hofstatt, zwei Eimern Most Bergrecht am Presserberg und zwei, vormals von einem öden Weingarten zu dienenden Wassereimern Most sowie mit dem Hofanteil Matthias Katzianers ${ }^{1}$ samt vier Wassereimern Most Bergrecht am Presserberg aus der Lehenschaft des Ftm. Steier.

Org. oder Kop. im bearbeiteten Bestand nicht überliefert. - Dep.: Erw. im Aufsandbrief Thomas Praitenwisers mit Bitte um Weiterverleihung an Erasmus Königsfelder ${ }^{2}$ von 1479 April 24 im HHStA Wien (Sign. AUR 1479 IV 24), Pap., 2 grüne SS des Ausst. und seines Bruders Christoph Praitenwiser rücks. aufgedr., mit rücks. Vermerk: Sof(er) er die lehenbri(ef) pringt sol man im leihen. ${ }^{3}$

1 Als früherer Lehensinhaber wird außer Mathias Katzianer auch der verstorbene Kaspar Kapfensteiner genannt.

2 Nach Dienes, Bürger von Graz S. CCXXXVII, wohl nicht, wie von Muchar (s. Anm. 3) angegeben, Grazer Bürger, sondern vermutlich Bürger von Radkersburg.

3 Anlass der Aufsendung war die Übertragung der Lehen an Erasmus Königsfelder gegen lebenslange Versorgung Paitenwisers und eines Knechts; Reg.: Muchar, Urkunden-Regesten n. 287; GöTH, UrkundenRegesten 9 n. 700 (zu April 23).

\section{[1479 Mai 1, Graz $]^{1}$}

K.F. überlässt dem Abt und Konvent des Benediktinerklosters Ossiach bis auf Widerruf seine halbe Fischreuse in der Feistritz, die vormals Jakob von Ernau ${ }^{2}$ von ihm innehatte, bestandweise gegen Zahlung von jährlich $10 \mathrm{Pfd}$. Pf.

[Org. im KLA.] ${ }^{3}$ - Erw. im Revers Abt Leonhards ${ }^{4}$ und des Konvents des Klosters Ossiach von 1479 Mai 5 im HHStA Wien (Sign. AUR 1479 V 5), Pap., 2 SS des Abts (spitzoval, rot) und des Konvents (grün) rücks. aufgedr. (beschädigt), mit Rta-Vermerk auf der Vorderseite.

Lit.: BoDo, Ossiach S. 44 und 123.

1 Datierung und Ausstellungsort nach Org.: An sambstag sannd Philipps und sannd Jacobs tag.

2 1462-1479 Vizedom in Kärnten, s. zu ihm LerTNER, Herren von Ernau S. 193-195.

$3 \mathrm{KLA}$ (Sign. AT-KLA 418-B-A 1323 St), s. monasterium.net (AT-KLA, Bestand Allgemeine Urkundenreihe, sub.dat.).

4 Leonhard Zorn (Abt 1473-1484), s. zu ihm Bodo, Ossiach S. 206. 
K.F. gestattet den Landleuten der vier Stände zu Österreich ob und unter der Enns wissenlich mit dem brief, zur Bezahlung der 100.000 fl. an Kg. Matthias von Ungarn und Böhmen, seinen Sohn und Kurfürsten, wozu sie sich gemeinsam mit ihm (K.F.) verschrieben haben, ${ }^{1}$ eine Anleihe in Höhe von $100.000 \mathrm{fl}$. auf jene Aufschläge zu nehmen, die auf dem Landtag yetz zu Wien ${ }^{2}$ laut eines von ihnen dem Eb. Johann von Gran ${ }^{3}$ übergebenen Registers veranschlagt wurden, jedoch mit Wissen der von ihm eingesetzten Einnehmer. Er sichert ihnen zu, sie als ihr gnediger herr und vorstand zu schützen und für die Bezahlung der Anleihe aus den Aufschlägen zu sorgen. Am sunntag nach sannd Philips und sannd Jacobstag (nach Kop.).

KVr: C.d.i.i.c. $-\mathrm{KVv}$ : Rta (nach Kop.).

Kop.: Abschrift des 18. Jh. im HHStA Wien (Sign. AUR 1479 V 2), Pap.

Druck: ChMEL, Mon. Habsb. I/3 S. 328f. n. 142; SCHOBER, Eroberung S. 128f., Beil. n. 2 (jeweils nach Kop.).

Reg.: CHMEL n. 7275; LichNowsKy(-BIRK) 8 n. 165.

Lit.: VANCSA, Geschichte 2 S. 505; SCHOBER, Eroberung S. 20f.; IsenManN, Kaiserliche Obrigkeit S. 324.

1 Der gemeinsame Schuldbrief gedruckt bei CHмғL, Mon. Habsb. I/2 S. 314f. n. 4 (nach Konz.); zur Zahlungsverpflichtung gegenüber Matthias Corvinus gemäß dem Friedensvertrag von Gmunden s. n. 106, Artikel 7.

2 Zum Landtag vom April 1479 s. CHMeL, Mon. Habsb. I/3 S. 311-318 nn. 135, 136; ebd. S. 315 zu n. 135 eine ähnlich lautende lautende ksl. Urkunde wie das vorliegende Stück, vermutlich die von den Ständen formulierte Vorlage.

3 Johann Beckenschlager (Beckensloer) (Eb. von Gran 1472-1487), s. zu ihm ZAISBERGER, Rohr, bes. S. 58-62; Gatz, Bischöfe 2 S. 36f.; HEING, Friedrich III./1 S. 449-452 mit weiterführender Lit.

\section{Mai 4, Graz}

K.F. gewährt dem unmündigen Gf. Johann Ludwig (I.) von Nassau-Saarbrücken ${ }^{1}$ aus ksl. Machtvollkommenheit wissenntlich in crafft diss briefs eine Frist bis zum Alter von 16 Jahren für den Empfang der von dessen verstorbenem Vater Gf. Johann (II.) ererbten Reichslehen, die er bis dahin urlaubsweise innehaben und nutzen soll, jedoch unbeschadet seiner und des Reichs oberkeit, gewaltsam und gerechtigkeit sowie der Rechte anderer. Am vierdten tag des monets maii. ${ }^{2}$ 
KVr: fehlt. - KVv: Urlaub Sarbrugken (rechter Blattrand).

Org. im HHStA Wien (Sign. AUR 1479 V 4), Perg., S an Ps ab und verloren.

1 Der zum damaligen Zeitpunkt noch sechsjährige Gf. Johann Ludwig wurde nach dem Tod seines Vaters († 1472 Juli 25) am 20. Oktober 1472 geboren, s. Regg.F.III. H. 5 n. 246.

2 Siehe dazu ebd. n. 274 (ksl. Urkunde gleichen Inhalts von 1479 Mai 15) und n. 335 (Belehnung von 1490 September 2).

\section{[Vor 1479 Mai 5, - ]}

K.F. überträgt Hieronymus Leininger bis auf Widerruf die Pflege von Schloss Landskron samt dem Landgericht und überlässt ihm die zugehörigen Nutzen und Renten bestandweise ab St. Veitstag 1479 (Juni 15) gegen Zahlung von jährlich 60 Pfd. Pf.

Org. oder Kop. im bearbeiteten Bestand nicht überliefert. - Dep.: Erw. im Revers Hieronymus Leiningers von 1479 Mai 5 im HHStA Wien (Sign. AUR 1479 V 5), Perg., 2 grüne SS des Ausst. und Erhard Lembachers in wachsf. Schüsseln an Ps, mit Rta-Vermerk auf der Rückseite. ${ }^{1}$

Lit.: Zu Landskron s. METNITZ, Kärntner Burgenkunde 2 S. 89; WIESSNER/VYORAL-TsCHAPKA, Burgen Kärnten 3 S. 190-194.

Siehe n. 164.

1 Reg.: ToMAscheK, Regesten Kärnten 3 n. 512; MC 11 n. 521.

[Vor 1479 Mai 19, - ] ${ }^{1}$

K.F. überlässt Richter, Rat und allen Bürgern der Stadt Leoben bis auf Widerruf das Ungeld zu Leoben und auf dem Land im Landgericht St. Peter ob Leoben bestandweise ab St. Peters- und Paulstag 1480 (Juni 29) ${ }^{2}$ gegen Zahlung von jährlich 600 Pfd. Pf. ${ }^{3}$

Org. oder Kop. im bearbeiteten Bestand nicht überliefert. - Dep.: Erw. im Revers von Richter, Rat und den Bürgern von Leoben von 1479 Mai 19 im HHStA Wien (Sign. AUR 1479 V 19), Perg., grünes S der Stadt Leoben in wachsf. Schüssel an Ps, mit Rta-Vermerk auf der Rückseite. ${ }^{4}$

Lit.: MUCHAR, Geschichte 8 S. 118.

1 Datierung nach dem Revers von 1479 Mai 19 (am mittichenn nach sand Panngratienn tag nach Cristi gepurd viertzehenhundert und im newnundsibenntzigistenn jare), jedoch ist darauf hinzuweisen, dass für das Jahr 1479 die Datumsangabe nach dem St. Pankratiustag (12. Mai) nicht naheliegend erscheint, 
da dieser Festtag acht Tage davor war (Mittwoch der Vorwoche), hingegen Christi Himmelfahrt auf den folgenden Tag fiel (Donnerstag, 20. Mai). Es ist daher nicht auszuschließen, dass hier ein Schreiberfehler vorliegt, möglicherweise bei der Jahresangabe.

2 Bestandinhaber bis 1480 Juni 29 war Hans Stubich, s. n. 170.

3 Laut Revers sollte die Zahlung des Bestandgelds an Hans Einpacher zuhanden des K. erfolgen; zu Einpacher s. DIENES, Bürger von Graz S. LXXIf.

4 Reg.: GötH, Urkunden-Regesten 10 n. 701 (zu Mai 19 und Juli 14).

\section{[Vor 1479 Mai 20, -]}

K.F. überträgt Jörg Dürrer bis auf Widerruf die Pflege von Schloss Pemont und überlässt ihm die zugehörigen Nutzen und Renten samt dem Gut, den Häusern, den Weingärten und der Mühle, die von Peter Fintz ${ }^{1}$ an ihn (K.F.) gekommen sind, bestandweise gegen Zahlung von jährlich 200 Pfd. Pf.

Org. oder Kop. im bearbeiteten Bestand nicht überliefert. - Dep.: Erw. im Revers Jörg Dürrers von 1479 Mai 20 im HHStA Wien (Sign. AUR 1479 V 20), Perg., 2 grüne SS des Ausst. und des Sigmund Piers, Pfleger von Friedrichstein, in wachsf. Schüsseln an Ps, mit Rta-Vermerk auf der Rückseite.

1 Früher ebenfalls Pfleger von Pemont, s. BIRK, Urkunden-Auszüge (AÖG 10) n. 505 (1461), CHMEL nn. 3933 (1462), 5993 (1470).

\section{[Vor 1479 Mai 21, -]}

K.F. verschreibt Nikolesch Prothowicz Schloss Asparn a.d. Zaya satz- und pflegweise gegen ein Darlehen von 1.900 Pfd. Pf. zur Ablösung des Schlosses mit der Verpflichtung, von den Nutzen und Renten jährlich 200 Pfd. Pf. zu entrichten.

Org. oder Kop. im bearbeiteten Bestand nicht überliefert. - Dep.: Erw. im Revers des Nikolesch Prothowicz von 1479 Mai 21 im HHStA Wien (Sign. AUR 1479 V 21), Perg., 2 grüne SS des Ausst. und Ulrichs von Fladnitz in wachsf. Schüsseln an Ps, mit Rta-Vermerk auf der Rückseite. ${ }^{1}$

Lit.: TRESP, Söldner S. 117; zu Schloss Asparn a.d. Zaya s. REICHHALTER/KüHTREIBER, Burgen Weinviertel S. 52-56 (Asparn II).

1 Reg.: ChMel n. 7281; Ders., Mon. Habsb. I/3 S. 656 n. 84. 
K.F. belehnt Kaspar Zebinger zu Reitenau auf Bitte von dessen Ehefrau Anna wissenntlich mit dem brief, was wir im zurecht daran verleihen sullen oder mugen, mit nachstehenden, von dem verstorbenen Pettauer ${ }^{1}$ an ihn (K.F.) gekommenen Stücken und Gütern samt Zubehör, die Anna ihrem Mann ledigklich übergeben und mit einem offenen besiegelten Brief aufgesandt hat: vier Huben und acht Hofstätten in Rohrbach bei Pettau; acht Hofstätten am Rann (Rain) bei Pettau; vier Huben und 54 Eimer Bergrecht bei Unterzellnitz; acht ganze Huben und 60 Eimer Bergrecht bei Oberzellnitz; sieben Huben und 62 Eimer Bergrecht am kleinen Wischberg; vier Huben zu Polanzen (Polanitz). Er verfügt, dass Zebinger und dessen Erben die Lehen gemäß Lehens- und Landrecht innehaben und nutzen sollen. An freytag nach dem heiligen Auffarttag. ${ }^{2}$

$\mathrm{KVr}$ : C.d.i.i.c. $-\mathrm{KVv}$ : Rta.

Org. im HHStA Wien (Sign. AUR 1479 V 21), Perg., rotes S 21 in wachsf. Schüssel mit wachsf. S 16 rücks. eingedr. an Ps.

Reg.: GöтH, Urkunden-Regesten 10 n. 702.

Lit:: PIRCHEGGER, Untersteiermark S. 65.

1 Friedrich von Pettau ( $\dagger 1438$ Januar 6), s. PIRCHEGGER, Herren von Pettau S. 28.

2 Siehe dazu Regg.F.III. H. 12 n. 310: Belehnung Sigmund Raumschüssels von 1446 Februar 14; nach PIRCHEGGER waren die Lehen die Mitgift Anna Zebingers, der Tochter Konrad Raumschüssels.

\section{Mai 24, Graz}

K.F. überträgt Andreas am Greith (Gherewtt), gesessen im Amt (Ober-)Fladnitz, und dessen Erben wissentlich mit dem brief den einst öden Grund im Amt Gschaid, den dessen Vater gerewtt und ingefanngen hat, zinsweise gegen Entrichtung von jährlich 3 Sch. Pf. in das Amt (Ober-)Fladnitz. Er gebietet dem dortigen Amtmann Veit Merchl und dessen Nachfolgern sowie den zum Amt gehörigen Leuten und Holden, Andreas und dessen Erben bei der Innehabung und Nutzung des Grundes, vorbehaltlich des darauf liegenden Zinses, nicht zu behindern. An montag vor sannd Urbans tag.

KVr: C.d.i.p. - KVv: Rta (Blattmitte). - Hinlassbrief ains grunds in dem ambt Flednitz gelegen umb drey schilling d. jerlich davon zu geben (zeitnaher Vermerk, oberer Blattrand). - Rückseite der Wachsschüssel: Vermerk nicht lesbar (wohl Name des Empfängers).

Org. im HHStA Wien (Sign. AUR 1479 V 24), Perg., rotes S 21 in wachsf. Schüssel mit wachsf. S 16 rücks. eingedr. an Ps. 
Reg.: Göтн, Urkunden-Regesten 10 n. 703.

1479 Mai 24, Graz

K.F. befiehlt seinem Rat Andreas von Kraig, Pfleger von Gurkfeld, von den dortigen Nutzen und Renten Siguna von Kraig, Dienerin seiner Tochter Hzn. Kunigunde von Österreich, $160 \mathrm{Pfd}$. Pf. als Heiratssteuer für ihre Dienste im Frauenzimmer Kunigundes gegen Quittung ${ }^{1}$ zu geben, und verspricht, die Summe gegen Vorlage der Quittung bei Rechnungslegung in Abzug zu bringen. An montag nach dem heiligen auffarttag (nach Kop.).

KVr: C.d.i. per dominum Wilhelmum Aursperger camerarium. - Rta (unterer Blattrand rechts) (nach Kop.).

[Org. im ARS Ljubljana, zuvor im HHStA Wien.] - Fotokop. vom Org. im HHStA Wien (Sign. AUR 1479 V 24).

Reg.: Regg.F.III. H. 29 n. 214 (nach Org.); s. weiters ChMEL n. 7284 (100 fl. statt 160 fl.); DERS., Mon. Habsb. I/3 S. 727 n. 280.

Lit.: GRAF, Kunigunde S. 22; zu Andreas von Kraig, ksl. Rat und seit 1471 Erbkämmerer in Kärnten, s. HeINIG, Friedrich III./1 S. 212f., bes. 213.

1 Quittung Sigunas von Kraig von 1479 Mai 27 (Org. ehemals im HHStA Wien, Sign. AUR sub dat. 1479 V 24, nicht mehr vorhanden); s. CHMEL 7288 und DERS., Mon. Habsb. I/3 S. 727 zu n. 280 (nach Org. mit dem Hinweis, dass die Quittung von Wilhelm von Auersperg, dem Vetter Sigunas von Kraig, besiegelt wurde).

\section{[Vor 1479 Mai 25, -]}

K.F. überträgt Sigmund Tellitzer bis auf Widerruf die Pflege von Schloss Wald ${ }^{1}$ und überlässt ihm die zugehörigen Nutzen und Renten bestandweise gegen Zahlung von jährlich 200 Pfd. Pf.

Org. oder Kop. im bearbeiteten Bestand nicht überliefert. - Dep.: Erw. im Revers Sigmund Tellitzers von 1479 Mai 25 im HHStA Wien (Sign. AUR sub dat. 1479 III 3), ${ }^{2}$ Perg., 2 grüne SS des Ausst. und Erhard Lembachers in wachsf. Schüsseln an Ps, mit Rta-Vermerk auf der Rückseite. ${ }^{3}$

1 Zur Abtretung von Schloss Wald durch Ulrich von Grafenegg gemäß Vertrag von 1477 März 2 und Verpfändung an Sigmund Schlick s. n. 55, Anm. 10. - Zur Ablösung des Schlosses von Sigmund Schlick, wofür sich K.F. von Kaspar von Rogendorf 1.270 fl. ung. lieh, s. CHMEL n. 7283; Regg.F.III. H. 26 n. 730.

2 Unter dieser Sign. gemeinsam verwahrt mit den nn. 289, 291.

3 Reg.: ChMEL n. 7285; DERS., Mon. Habsb. I/3 S. 641f. n. 22. 
K.F. überlässt Sigmund Tellitzer bis auf Widerruf das Ungeld zu St. Pölten bestandweise gegen Zahlung von jährlich $650 \mathrm{Pfd}$. Pf.

Org. oder Kop. im bearbeiteten Bestand nicht überliefert. - Dep.: Erw. im Revers Sigmund Tellitzers von 1479 Mai 25 im HHStA Wien (Sign. AUR sub dat. 1479 III 3), ${ }^{1}$ Perg., 2 grüne SS des Ausst. und Erhard Lembachers in wachsf. Schüsseln an Ps, mit Rta-Vermerk auf der Rückseite. $^{2}$

Lit.: WALTER, Ungeld S. 113.

1 Unter dieser Sign. gemeinsam verwahrt mit den nn. 288, 291.

2 Reg.: CHMel n. 7279 (zu Mai 16) und DERS., Mon. Habsb. I/3 S. 650 n. 60.; LichNowsKY(-BIRK) 8 n. 172.

[Vor 1479 Mai 28, -]

K.F. überträgt Wolfgang Lembacher bis auf Widerruf die Pflege von Schloss Lankowitz und überlässt ihm die zugehörigen Nutzen und Renten bestandweise ab St. Georgstag 1479 (April 24) ${ }^{1}$ gegen Zahlung von jährlich 400 fl. ung.

Org. oder Kop. im bearbeiteten Bestand nicht überliefert. - Dep.: Erw. im Revers Wolfgang Lembachers von 1479 Mai 28 im HHStA Wien (Sign. AUR sub dat. 1479 V 18), Perg., 2 grüne SS des Ausst. und seines Bruders und Bürgen Erhard Lembacher in wachsf. Schüsseln an Ps, S Andreas Prämers ${ }^{2}$ samt Ps ab und verloren, mit Rta-Vermerk auf der Rückseite. ${ }^{3}$

Lit.: Baravalle, Burgen S. $555 ;{ }^{4}$ zu Schloss Lankowitz s. auch EBNER, Burgen Steiermark 3 S. 106.

1 Zur Datierung des St. Georgstags auf April 24 s. Regg.F.III. H. 12 n. 6, Anm. 2.

2 Siehe zu ihm HeInI, Friedrich III./1 S. $208 \mathrm{f}$.

3 Reg.: MuchaR, Urkunden-Regesten n. 288; GöTH, Urkunden-Regesten 10 n. 704.

4 Bei Baravalle irrtümlich eine Verleihung gegen ein Darlehen von $1.400 \mathrm{fl}$.

[Vor 1479 Mai 28, -]

K.F. überlässt Konrad Auer bis auf Widerruf Amt und Herrschaft Hornstein bestandweise ab Pfingsten (Mai 30) 1479 gegen Entrichtung von jährlich 300 Pfd. Pf. und 20 Fuder Heu. 
Org. oder Kop. im bearbeiteten Bestand nicht überliefert. - Dep.: Erw. im Revers Konrad Auers von 1479 Mai 28 im HHStA Wien (Sign. AUR sub dat. 1479 III 3), ${ }^{1}$ Perg., 3 grüne SS des Ausst., seines Bruders und Bürgen Willbold Auer und des Ritters Wilhelm von Auersperg ${ }^{2}$ in wachsf. Schüsseln an Ps, mit Rta-Vermerk auf der Rückseite. ${ }^{3}$

1 Unter dieser Sign. gemeinsam verwahrt mit den nn. 288, 289

2 Ksl. Kämmerer und Rat, s. zu ihm HeINIG, Friedrich III./1 S. $231 \mathrm{f}$.

3 Reg.: ChMel n. 7289; Ders., Mon. Habsb. I/3 S. 642 n. 23.

\section{[Vor 1479 Mai 30, - ] ${ }^{1}$}

K.F. teilt seinem Sohn Ehz. Maximilian von Österreich und Burgund ${ }^{2}$ mit, nicht per-

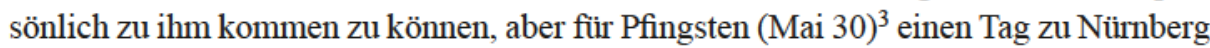
anberaumt zu haben, auf dem sich seine Gesandten für Hilfe aus dem Reich für ihn und dessen Länder einsetzen werden, und fordert ihn auf, ebenfalls Gesandte zu schicken. ${ }^{4}$

Org. oder Kop. im bearbeiteten Bestand nicht überliefert. - Dep.: Erw. im Antwortschreiben Ehz. Maximilians von 1479 Juni 8, Lille (Rijsel), im HHStA Wien (Sign. AUR 1479 VI 8), Pap., rotes S des Ausst. als Verschluss rücks. aufgedr. (abgefallen); ${ }^{5}$ davon Abschrift des 18. Jh. ebd. (Sign. Urkundenabschriften Österr. Urkunden, Kt. 46 sub. dat.), Pap.

1 Datierung nach dem im Schreiben Maximilians genannten Termin für den Nürnberger Tag.

2 Zur Titulatur Maximilians s. die Einleitung S. 22-24.

3 Gemäß den Ladungen von 1479 März 10 wurde der Nürnberger Tag dann auf den 7. Juni anberaumt, s. Regg.F.II. H. 4 nn. 808, 809; H. 7 n. 626 , H. 8 n. 406, H. 9 n. 344 , H. 10 nn. 444-449, H. 11 n. 509, H. 15 n. 359 , H. 16 n. 151.

4 Siehe dazu die ksl. Instruktion für die Gesandten auf dem Nürnberger Tag bei ChMEL, Mon. Habsb. I/3 S. 114-116 zu n. 44.

5 Inhalt des Schreibens Maximilians ist eine ausführliche Beschreibung der aktuellen kriegerischen Auseinandersetzungen mit Kg. Ludwig XI. von Frankreich und die dringliche Bitte um Reichshilfe; Druck: Chmel, Mon. Habsb. I/3 S. 176-178 n. 71; Reg.: ChMel n. 7291; Lichnowsky(-BIRK) 8 n. 176

\section{Juni 5, Graz}

K.F. erklärt aus ksl. Machtvollkommenheit wissentlich in crafft diss briefs auf Bitte und Beschwerde seines Vetters Ehz. Sigmund von Österreich, der zufolge die Appenzeller aufgrund etlicher von ihm (K.F.) erlangten Freiheiten ${ }^{1}$ dem Landgericht Rankweil gegen altes Herkommen keinen Gehorsam leisten und damit ihn (Sigmund) und das Haus Österreich schädigen würden, dass diese Freiheiten keinerlei Schmälerung, Schaden oder Verletzung des Landgerichts Rankweil bedeuten, sondern die Appenzeller dessen Gerichtszwang sowie Geboten und Verboten wie von alters her vor Gewährung der 
Freiheiten unterworfen sind und gehorsam zu sein haben. Am funfften tag des monadts junii.

KVr: A.m.p.d.i. - KVv: Rta (Blattmitte). - Declaracion Erczherczog Sigmund (rechter Blattrand).

Org. im HHStA Wien (Sign. AUR 1479 VI 5), Perg., wachsf. S 15 mit wachsf. S 16 vorders. eingedr. an purpurf. Ss. - Kop.: Vidimus des Bürgermeisters und Rats von Innsbruck von 1479 August 20, Innsbruck, ebd., dem Org. beiliegend, Perg., grünes S der Stadt Innsbruck in wachsf. Schüssel an Ps. ${ }^{2}-$ Abschrift des 18. Jh. ebd. (Sign. Urkundenabschriften Österr. Urkunden, Kt. 46 sub dat.), Pap.

Druck: ChMEL, Mon. Habsb. I/3 S. 175 n. 70.

Reg.: ChMel n. 7290; LichNowsKy(-BIRK) 8 n. 175.

1 Siehe dazu den fremde Gerichte betreffenden Freiheitsbrief von 1466 Juli 3 bei CHMEL n. 4548.

2 Das Vidimus wurde ausgestellt auf Bitte Ehz. Sigmunds.

\section{[Vor 1479 Juni 11, -]}

K.F. überträgt Hans Stubich bis auf Widerruf die Pflege von Schloss St. Peter ob Leoben samt dem Landgericht und überlässt ihm die zugehörigen Nutzen und Renten bestandweise ab dem St. Johannestag zur Sonnwende 1479 (Juni 24) gegen Zahlung von jährlich $120 \mathrm{Pfd}$. Pf.

Org. oder Kop. im bearbeiteten Bestand nicht überliefert. - Dep.: Erw. im Revers Hans Stubichs von 1479 Juni 11 im HHStA Wien (Sign. AUR 1479 VI 11), Perg., 4 grüne SS des Ausst., seiner beiden Bürgen Andreas Jostel und Gregor Leubner ${ }^{1}$ sowie Erhard Lembachers in wachsf. Schüsseln an Ps, mit Rta-Vermerk auf der Rückseite. ${ }^{2}$

Lit.: Baravalle, Burgen S. 374; zu Schloss St. Peter (St. Peter-Freienstein) s. auch EbNeR, Burgen Steiermark 2 S. 124.

Siehe nn. 120, 170.

1 Leobener Stadtrichter 1476 und 1483, s. SchIILINGER-PrassL, Rechtsquellen Leoben, Anh. S. 314.

2 Reg.: Muchar, Urkunden-Regesten n. 290; Göth, Urkunden-Regesten 10 n. 707; erw. bei Muchar, Geschichte 8 S. 117. 
K.F. nimmt Diebold Heller am Puhl und dessen Ehefrau Margarete mit ihren Kindern und ihrem Hab und Gut in seinen besonderen Schutz mit der Verpflichtung, jährlich zu St. Georgstag (April 24) 1 Pfd. Pf. in die ksl. Kammer zu zahlen.

Org. oder Kop. im bearbeiteten Bestand nicht überliefert. - Dep.: Erw. im Revers Diebold und Margarete Hellers von 1479 Juni 18 im HHStA Wien (Sign. AUR 1479 VI 18), Pap., 2 grüne SS (Petschaft) Diepold Hellers und des Klagenfurter Bürgers Stefan Krösel rücks. aufgedr. unter Papieroblate, mit Rta-Vermerk auf der Vorderseite. ${ }^{2}$

1 Zur Datierung des St. Georgstags auf April 24 s. Regg.F.III. H. 12 n. 6, Anm. 2.

2 Reg.: Chmel n. 7293 (zu Juni 10); Ders., Mon. Habsb. I/3 S. 721 n. 265.

\section{Juni 19, Graz}

K.F. belehnt Hans Dolacher auf Bitte Andreas Kolbrers wissenntlich mit dem brieve, was wir im zurecht daran verleihen sullen oder mugen, mit zwei zu Treßdorf im Goldensteiner Gericht gelegenen Huben aus der Lehenschaft des Ftm. Kärnten, die Kolbrer diesem verkauft und mit einem offenen besiegelten Brief aufgesandt hat, und verfügt, dass Dolacher und dessen Erben die Huben, von denen die eine von dem Nikl und die andere von Mert David als zulehen bebaut werden, gemäß Lehens- und Landrecht innehaben und nutzen sollen. An sambstag nach sannd Veitstag.

KVr: C.d.i.p. $-\mathrm{KVv}$ : Rta.

Org. im HHStA Wien (Sign. AUR sub dat. 1479 VI 12), Perg., rotes S 21 in wachsf. Schüssel mit wachsf. S 16 rücks. eingedr. an Ps.

Reg.: MuchaR, Urkunden-Regesten n. 294; ${ }^{1}$ Tomaschek, Regesten Kärnten 3 n. 514 (zu Juni 12).

1 Bei Muchar irtümlich eine Urkunde für Andreas Kolbrer.

\section{[Vor 1479 Juni 25, -]}

K.F. überträgt Jörg von Lamberg bis auf Widerruf die Pflege von Schloss Adelsberg und überlässt ihm die zugehörigen Nutzen und Renten samt der dortigen Maut bestandweise gegen Zahlung von jährlich $1.000 \mathrm{fl}$. ung. 
Org. oder Kop. im bearbeiteten Bestand nicht überliefert. - Dep.: Erw. im Revers Jörgs von Lamberg von 1479 Juni 25 im HHStA Wien (Sign. AUR 1479 VI 25), Perg., 3 grüne SS des Ausst., Georg Scheyrers und Sigmund Piers' in wachsf. Schüsseln an Ps.

\section{[Vor 1479 Juli 1, -]}

K.F. überlässt Kaspar Wenger, Pfleger von Litschau, bis auf Widerruf seinen Teich zu Waidhofen a.d. Thaya für die Burghut des Schlosses Litschau mit der Verpflichtung, ihm vom Abfischen Fische abzugeben.

Org. oder Kop. im bearbeiteten Bestand nicht überliefert. - Dep.: Erw. im Revers Kaspar Wengers von 1479 Juli $1 \mathrm{im}$ HHStA Wien (Sign. AUR sub dat. 1479 VI 11), ${ }^{1}$ Perg., S des Ausst. an Ps ab und verloren, grünes S Erhard Lembachers in wachsf. Schüssel an Ps, mit Rta-Vermerk auf der Rückseite. ${ }^{2}$

1 Unter dieser Sign. gemeinsam verwahrt mit n. 302 und (neben weiteren Reversen) einem Revers Leonhard Helds von 1479 Juni 11, s. dazu n. 39.

2 Reg.: ChMel n. 7299; Ders., Mon. Habsb. I/3 S. 651 n. 61.

\section{Juli 5, Graz}

K.F. belehnt Leopold von Wehingen als Ältesten und Lehensträger seiner Brüder Bernhard, Wolfgang und Achaz wissenntlich mit dem brief, was wir im zurecht daran verleihen sullen oder mugen, mit folgenden von ihren verstorbenen Brüdern Christoph und Wilhelm ererbten Stücken und Gütern aus der Lehenschaft des Ftm. Österreich: die Feste Sitzenberg; das Türhüteramt in Österreich; je ein Zehent zu Gutenbrunn, Hautzenfeld und Pottschall; 4 Pfd. 4 Sch. Pf. Geld sowie ein Weinzehent und eine Wiese zu Wilfersdorf (Wilberstorf); je ein Zehent am Bisamberg (Pusenperg) und zu Leobendorf. Er verfügt, dass die vier Brüder und ihre Erben die Lehen innehaben und nutzen, das Türhüteramt verwalten und mit der Feste ihm als Lehensherrn treu zu Diensten sein sollen gemäß altem Herkommen und Lehens- und Landrecht. An montag nach sannd Ulreichs tag.

KVr: C.d.i.p. $-\mathrm{KVv}$ : Rta.

Org. im HHStA Wien (Sign. AUR 1479 VII 5), Perg., rotes S 21 in wachsf. Schüssel mit wachsf. S 16 rücks. eingedr. an Ps.

Druck: ChMeL, Mon. Habsb. I/3 S. 332f. n. 145.

Reg.: CHMEL n. 7303. 
Lit.: StIERLE, Herren von Wehingen S. 71; HeINIG, Türhüter S. 362; s. auch Mrtis, Herren von Wehingen.

\section{Juli 13, Graz}

K.F. nimmt Jörg Wartenauer wegen dessen erberkait und gut sitten sowie künftiger treuer Dienste von sundern gnaden wissenlich mit dem brief als seinen Diener auf und samt Hab und Gut in seinen besonderen Schutz. Er verfügt, dass dieser alle den anderen unter seinem Schutz stehenden ksl. Dienern zustehenden Gnaden, Freiheiten und Rechte ungehindert haben und gebrauchen soll, und gebietet allen Hauptleuten, Gff., Freiherren etc., Verwesern, Vizedomen, Pflegern, Bggff., Bürgermeistern, Richtern, Räten, Bürgern, Gemeinden und allen anderen Amtleuten und Untertanen die Beachtung dieses Privilegs. An eritag nach sannd Margreten tag.

KVr: fehlt. - Rückseite der Wachsschüssel: Vermerk nicht lesbar (Warttenawer?).

Org. im HHStA Wien (Sign. AUR 1479 VII 13), Perg., rotes S 21 in wachsf. Schüssel mit wachsf. S 16 rücks. eingedr. an Ps.

Reg.: CHMel n. 7306.

\section{[Vor 1479 Juli 16, -]}

K.F. überträgt dem Ritter Heinrich Vogt von Summerau bis auf Widerruf die Pflege von Schloss Fürstenfeld sowie das Amt Feldbach und überlässt ihm die zugehörigen Nutzen und Renten bestandweise gegen Zahlung von jährlich 200 Pfd. Pf.

Org. oder Kop. im bearbeiteten Bestand nicht überliefert. - Dep.: Erw. im Revers Heinrich Vogts von Summerau von 1479 Juli 16 im HHStA Wien (Sign. AUR 1479 VII 16), Perg., 4 grüne SS des Ausst., seiner beiden Brüder und Bürgen Hans Heinrich, Lehrer beider Rechte, und Eitelhans, Vögte von Summerau, sowie des Ritters Andreas Prämer ${ }^{1}$ in wachsf. Schüsseln an Ps, mit Rta-Vermerk auf der Rückseite. ${ }^{2}$

Lit.: Baravalle, Burgen S. 136; zu Heinrich Vogt von Summerau s. HeInIG, Friedrich III./1 S. 74-78.

1 Siehe zu ihm HeInIG, Friedrich III./1 S. $208 \mathrm{f}$.

2 Reg.: Muchar, Urkunden-Regesten n. 295; Götr, Urkunden-Regesten 10 n. 708; erw. bei Muchar, Geschichte 8 S. 117. 
K.F. überträgt Hans Aichelperger bis auf Widerruf die Pflege von Schloss Klamm und überlässt ihm die zugehörigen Nutzen und Renten bestandweise ab St. Margaretentag 1479 (Juli 12) gegen Zahlung von jährlich $160 \mathrm{Pfd}^{\text {Pf. }}{ }^{1}$

Org. oder Kop. im bearbeiteten Bestand nicht überliefert. - Dep.: Erw. im Revers Hans Aichelpergers von 1479 Juli $18 \mathrm{im}$ HHStA Wien (Sign. AUR sub dat. 1479 VI 11), ${ }^{2}$ Perg., 2 grüne SS des Ausst. und Erhard Lembachers in wachsf. Schüsseln an Ps, mit Rta-Vermerk auf der Rückseite. $^{3}$

Lit.: Zu Klamm s. KÜHTREIBER u.a., Wehrbauten $1 \mathrm{~S} .124-136$, bes. 134.

1 Siehe dazu auch n. 48.

2 Unter dieser Sign. gemeinsam verwahrt mit n. 298 und (neben weiteren Reversen) einem Revers Leonhard Helds von 1479 Juni 11, s. dazu n. 39.

3 Im Revers verpflichtet sich Aichelperger, sobald K.F. hinaus zelannd kommt, innerhalb von vier Wochen die Bürgschaft mit vom K. akzeptierten Leuten zu leisten. - Reg.: CHMEL n. 7308; DERs., Mon. Habsb. I/3 S. 642 n. 26 (mit Druck des Passus bzgl. der Bürgschaftsleistung).

\section{Juli 18, Graz}

K.F. belehnt Andreas Stermoler (Stermeler) als Ältesten für sich und dessen Geschwister Heinrich, Margarete und Magdalena wissenntlich mit dem brief, was wir im zurecht daran verleiehen sullen oder mugen, mit nachfolgend genannten, von ihrem verstorbenen Vater Heinrich ererbten Stücken und Gütern aus der Lehenschaft der Gft. Cilli und der Herrschaft Rohitsch und verfügt, dass sie und ihre Erben die Güter innehaben und nutzen und mit dem Turm ihm als Lehensherrn treu zu Diensten sein sollen gemäß Lehens- und Landrecht.

Cillier Lehen: ein Hof am Gabernik mit Hofstatt und Bergrecht; drei Huben, davon eine zu Pulsgau, zwei zu Unterpulsgau (Nider Pultzkaw), und ein vor dem Gabernik gelegener Forst samt Zubehör; ein Hof und viereinhalb Huben zu Chorben; eine halbe supphuben zu Pogled; zwei Teile des Getreide- und Weinzehents zu Corben in dem Perg, zu Jeschowetz (Zelsowetz), Dragemeil (Daigameil), Tall und Hof; ein Drittel des Getreidezehents zu Wretzlowo (Bretzla) und Pregnitz (Pregaritz); acht Huben und elf Eimer Bergrecht am Sannd; eine Hube zu Werbno (Berben), fünf Huben zu Oberwodresch (Ober Beydros) und eine Hube zu Tall; ein Drittel des Getreidezehents zu Pöltschach, je zwei Teile des Getreidezehents zu Jörgendorf und Stokh; zwei Hofstätten zu Lemberg. 
Rohitscher Lehen: der Turm Rohitsch mit 15 Hofstätten, Fischweide, Gehölz, Weide und anderem Zubehör; vier Huben zu Marschetschendorf (Morschetzendorf) unter dem Rohitscherberg, fünf Huben am Schabnig (Sabiach), eine Hube zu Zerowetz, elf Huben zu Wrestovetz (Prestowetz), zwei Huben zu Swetscha (Swetzo); eine Mühle an der Sotla (Zatel); 65 zum Turm gehörige Eimer Bergrecht; zwei Huben zu Gerlitsche, ehedem Cillier Lehen, eine von Andreas Winter herrührende Hube zu Brosco (?) ${ }^{1}$ und eine von Andreas Losnitzer herrührende Hube zu Crestenitz. An suntag nach sannd Margrethen tag.

KVr: fehlt. - KVv: Rta. - Rückseite der Wachsschüssel: nur Stermeller lehen (?) lesbar.

Org. im HHStA Wien (Sign. AUR sub dat. 1479 VII 25), Perg., S (wohl rotes S 21, Siegelbild entfernt) $)^{2}$ in wachsf. Schüssel mit wachsf. S 16 rücks. eingedr. an Ps.

Reg.: Muchar, Urkunden-Regesten n. 296 (zu Juli 17); GöTH, Urkunden-Regesten 10 n. 709.

Lit.: Muchar, Geschichte 8 S. 115; Orožen, Lavant 7 S. 198f. (zu Juli 12); PIRCHEGGER, Untersteiermark S. 234f.

1 Lesung unsicher, auch Brosto möglich.

2 An der Stelle des Siegelbildes sind Kratzspuren erkennbar.

\section{Juli 20, Graz}

K.F. gestattet seinem Vetter Ehz. Sigmund von Österreich aus ksl. Macht, die an Johann (d.Ä.) Truchsess von Waldburg ${ }^{1}$ verpfändete Landvogtei Schwaben gegen Bezahlung der Pfandsumme ${ }^{2}$ von diesem einzulösen und um dieselbe Summe und nit hoher an andere geeignete Personen zu verpfänden, jedoch mit deren Verpflichtung, ihm und dem Reich die gewöhnlichen Eide zu leisten sowie treu und gehorsam zu sein gemäß den Pflichten jedes Landvogts gegenüber K. und Reich. Am zweinczigisten tag des monadts julii.

KVr: fehlt.

Org. im HHStA Wien (Sign. AUR 1479 VII 20), Perg, der Siegelankündigung zufolge mit keyserlichen anhungundem insigel, aber unbesiegelt. - Kop.: Abschrift des 18. Jh. ebd. (Sign. Urkundenabschriften Österr. Urkunden, Kt. 46 sub dat.), Pap.

Druck: Chmel, Mon. Habsb. I/3 S. 180f. n. 74.

Reg.: ChMel n. 7310; LichNowsKy(-BIRK) 8 n. 188. 
Lit.: VocheZER, Waldburg 2 S. 73; FeINE, Territorialbildung S. 282f.; GismanN, Beziehungen S. 480f;; BAuM, Habsburger S. 719; s. dazu auch HoFACKER, Reichslandvogteien S. 301-309.

Aufgrund des Fehlens von Kanzleivermerk und Besiegelung sowie der Überlieferung im Ausstellerarchiv ist anzunehmen, dass die Urkunde nicht ausgefertigt wurde.

1 Die Truchsessen von Waldburg waren seit 1415 im Pfandbesitz der Landvogtei Schwaben; s. zu ihnen HEINIG, Friedrich III/1 S. 367-370 und 2 S. 929-933 mit weiterführender Lit.

2 Gemäß dem Pfandbrief Kg. Sigismunds von 1422 November 17 (RI XI n. 5399) betrug die Pfandsumme $13.200 \mathrm{fl}$. rh. die - trotz zwischenzeitlicher Schwankungen - in Bezug auf die Einlösung unverändert blieb; s. zu den Bemühungen um Einlösung der Landvogtei Schwaben Regg.F.III. H. 13 nn. 1 und 246, H. 22 n. 28, H. 27 n. 212 . Die endgültige Pfandlösung für 13.200 fl. rh. erfolgte 1486 , s. die Abtretungsurkunde Johanns Truchsess von Waldburg von 1486 April 30 bei WeGELIN, Landvogtey Schwaben 2 S. $69 f$. n. 73 .

\section{Juli 21, Graz}

K.F. belehnt die Brüder Hans und Leonhard Rosegger (Rasegker), seine Diener, für ihre vor etlichen Jahren am Hof geleisteten Dienste von sonndern gnaden wissentlich mit dem brief, was wir in von rechttens und gnaden wegen daran verleihen sullen und mugen, mit einer in der Falkensteiner Herrschaft gelegenen Alm in der Redlein gegenüber der Hohenburg, auf die sie erbliche Rechte besitzen, aus der in den vergangenen Kriegen von den Gff. von Görz an den K. gekommenen Lehenschaft. Er verfügt, dass sie und ihre Erben die Alm gemäß Lehens- und Landrecht innehaben und nutzen sollen, jedoch vorbehaltlich einer Zugehörigkeit zu einem seiner Ämter. An mittichen vor sannd Maria Magdalenen tag.

KVr: fehlt. - KVv: Rta.

Org. im HHStA Wien (Sign. AUR 1479 VII 21), Perg., rotes S 21 in wachsf. Schüssel (beschädigt) mit wachsf. S 16 rücks. eingedr. an Ps.

Reg.: MUCHAR, Urkunden-Regesten n. 298.

\section{[1479 August 6, Graz ${ }^{1}$}

K.F. verkauft Barbara, Witwe des Wiener Bürgers Hans Neupaur ${ }^{2}$, sein zwischen den Häusern Hans Stegers ${ }^{3}$ und des Haringseers ${ }^{4}$ gelegenes Haus zu Wien, das einst Peter Strasser ${ }^{5}$ gehört hat, jedoch vorbehaltlich der ihm und seinen Erben auf ewig zustehenden Lehenschaft über die Kapelle und die Altar- und Messstiftungen samt Ornaten, Kelchen und anderem Zubehör (nach Druck). 
Kvr: C.d.i.p. (nach Druck).

Org. oder Kop. im bearbeiteten Bestand nicht überliefert. - Dep.: Genannt bei CHMEL nach einer Überlieferung im HHStA Wien.

Druck: CHMEL, Mon. Habsb. I/3 S. 674 n. 142 (Teildruck nach Org.).

Reg.: CHMel n. 7313.

Das von K.F. verkaufte Haus lag entweder zwischen dem Margaretenhof (Haus Hans Stegers) und dem Smerbeckenhof (Haringseer-Haus) in der Münzerstraße, oder es handelte sich um einen Teil des Haringseer-Hauses, das über eine Hauskapelle (Thomaskapelle) verfügte und ein großer, ursprünglich aus mehreren Häusern bestehender Gebäudekomplex (zwischen Bauernmarkt, Brandstätte und Rotenturmstraße) war.

1 Datierung und Ausstellungsort nach CHMEL (,Freitag nach St. Oswaldstag ${ }^{c c}$ ).

2 Zum Ratsbürger Hans Neupaur ( $\dagger$ 1473) s. PERGER, Wiener Ratsbürger S. 229.

3 Wohl der Wiener Stadtrichter und Bürgermeister Hans Steger ( $\dagger$ um 1459/61), der den Margaretenhof in der Münzerstraße (Teilstück des heutigen Bauernmarkts zwischen Freisinger- und Landskrongasse, s. Perger, Straßen S. 21f.) erworben hatte; s. zu ihm HüLbler, Hans Steger; auch Perger, Wiener Ratsbürger S. 250 .

4 Wohl der Wiener Bürgermeister Hans Haringseer ( $\dagger$ um 1457/59), der den Smerbeckenhof in der Münzerstraße erworben hatte; s. zu ihm CzEIKE, Hanns Haringseer, bes. S. 81f.; DERS., Bürgermeister S. 109; auch PERGER, Wiener Ratsbürger S. 208.

5 Wohl der Ratsbürger Peter Strasser ( $\dagger$ 1459/61), s. zu ihm PeRgER, Wiener Ratsbürger S. 251.

\section{August 11, Graz}

K.F. bestätigt und erneuert als röm. K. und Herr und Landesfürst von Österreich den Leinwatern der Leinwaterzeche zu Wien sowie ihren Erben und Nachkommen für ihre ihm und seinen Vorfahren geleisteten Dienste und zur Förderung der Stadt Wien von sundern gnaden wissentlich in krafft dits briefs alle ihnen von Bürgermeister und Rat von Wien gegebenen und in das Wiener Stadtbuch eingetragenen Artikel ihrer ordmung und hanndls. ${ }^{1}$ Er verfigt, dass diese Ordnung in allen puncten und articeln, als ob sie wortwörtlich inseriert wäre, in Kraft bleibt, von ihnen ungehindert genutzt werden soll und niemand dagegen handeln darf, und setzt fest, dass sie mit allerlei aus Flachs gesponnenem Leinentuch, genannt herbein, zwilich oder rupfen, gebleicht oder gefärbt, ${ }^{2}$ handeln dürfen. Auch erweist er ihnen in sunderhait die Gnade, im Falle der Verarmung oder sunst ihr Recht am Leinwandhandel verkaufen zu können, jedoch unter der Bedingung, dass der Käufer bei der Aufnahme in die Zeche zu den üblichen 1 Pfd. Pf. zusätzlich $5 \mathrm{fl}$. ung. in die Zechbüchse zahlt, die von alters her dazugehörigen Zinsen und Rechte des Spitals ${ }^{3}$ nicht beeinträchtigt und das Leinwaterrecht samt den Rechten 
des Spitals von ihm (K.F.) und seinen Erben nach Aufsendung durch den Verkäufer zu Lehen empfängt, andernfalls es an ihn und seine Erben verfällt und von ihm weiterverliehen werden kann. Er gebietet allen Hauptleuten, Landmarschällen, Gff., Freiherren etc., Pflegern, Bggff., Bürgermeistern, Richtern, Landrichtern, Räten, Mautnern, Bürgern, Gemeinden und allen anderen Amtleuten und Untertanen bei seiner schweren Ungnade und einer Pön von 10 Mark Gold, zahlbar je zur Hälfte an die ksl. Kammer und die Leinwater, die Beachtung dieses Privilegs. An mittichen nach sannd Laurentien tag.

KVr: C.d.i.p. $-\mathrm{KVv}$ : Rta.

Org. im HHStA Wien (Sign. AUR 1479 VIII 11), Perg., rotes S 21 in wachsf. Schüssel mit wachsf. S 16 rïcks. eingedr. an purpurf. Ss.

Druck: ChMEL, Mon. Habsb. I/3 S. 333-335 n. 146.

Reg.: ChMEL n. 7315.

Lit.: UhirIRZ, Gewerbe S. 735; PeRGER, Hoher Markt S. 53.

1 Ordnungen von 1453 August 4, 1455 Juli 26 und 1479 Juli 20, gedruckt bei GNEIss, Wiener Handwerksordnungsbuch nn. 274, 275, 277; s. dazu UhIRZ, Gewerbe S. 733, 735; PERGER, Hoher Markt S. 53.

2 Laut Schiedsspruch des Wiener Rats zwischen Krämern und Leinwatern von 1473 August 7 (s. GNerss, ebd. n. 294) war es nur den Krämern gestattet, mit gebleichter und gefärbter Leinwand zu handeln, den Leinwatern hingegen nur mit roher, ungebleichter Leinwand.

3 Das Leinwandhaus am Hohen Markt mit den Verkaufsständen der Leinwater war im Besitz des Wiener Bürgerspitals, s. PERGER, Hoher Markt S. 53, auch GNerss, ebd. S. 145.

\section{August 15, Graz}

K.F. ermahnt Jörg von Silberberg, seinen Diener Peter Knauer, Lehrer geistlicher Rechte und Propst zu Gurnitz, und Jörg Skodel, Pfleger von Weißenfels, dass er im Streit zwischen Bf. Philipp von Bamberg ${ }^{1}$ und dem ksl. Rat Christoph Ungnad ${ }^{2}$ um Gründe und Gemarkungen oberhalb von Villach gegenüber dem Bleiberg, weswegen sie vor ihm in verhör und recht steen, ${ }^{3}$ auf Bitte der Streitparteien, eine beschaw zu halten und kuntschafft nemen zelassen, dieweil die weter teg sein, dies mehrmals angeordnet hat, aber nichts geschehen sei. Er befiehlt ihnen daher, den Parteien einen Tag zu setzen und sie oder ihre Anwälte, auch die lewt, brief, kuntschafft und was sonst in der Sache beigebracht wird, hinsichtlich der von den Parteien vorgebrachten artigkl und frag anzuhören sowie, falls notwendig und gefordert, eine Begehung vor Ort vorzunehmen und ihm (K.F.) darüber schriftlich unter ihren Siegeln zu berichten. An suntag unserr lieben Frawen tag assumpcionis. 
KVr: C.d.i.p.

Org. im HHStA Wien (Sign. AUR 1479 VIII 15), Pap., rotes S. 21 (tlw. abgefallen) rücks. aufgedr. unter (abgelöster) Papieroblate. ${ }^{4}$

1 Philipp von Henneberg (Bf. 1475-1487), s. zu ihm Gatz, Bischöfe 2 S. $282 f$.

2 Siehe zu ihm HeINIG, Friedrich III./1 S. $179 \mathrm{f}$.

3 Strittig war das Gebiet zwischen dem Ursprung des Nötschbachs und dem Lerchbach auf der Villacher Alpe. Gegen die bischöflichen Ansprüche argumentierte Christoph Ungnad in seiner Stellungnahme (s. Anm. 3) mit der Zugehörigkeit des Gebiets zur Herrschaft (Wasser-)Leonburg und führte als Beweisstück u.a. eine ksl. Lehensurkunde für seinen Bruder Georg ( $\dagger$ 1468) an.

4 Beiliegend zahlreiche Schriftstücke zur Streitsache (foliiert, beginnend mit der ksl. Urkunde, insgesamt 18 fol.), darunter Christoph Ungnads Bevollmächtigung seiner Anwälte von 1479 September 7 (fol. 2rv), die Stellungnahmen (Artikel) beider Parteien (jene Ungnads mit umfangreicher Zeugenliste, fol. 9-12), die Fragen beider Parteien zu den Artikeln der Gegenpartei und für die Zeugenbefragung sowie der Bericht der Kommissare über die Begehung am 13. September 1479. Weiters ist im HHStA Wien ein Notariatsinstrument von 1479 September 13 über die Aussage des Anwalts Christoph Ungnads am Tag der Anhörung überliefert (Sign. AUR 1479 IX 13).

[Vor 1479 August 19, -]

309

K.F. überträgt Bajezid Ottman ${ }^{1}$ Schloss Rauhenstein satz- und pflegweise für 200 Pfd. Pf., um die dieser das Schloss mit seiner Erlaubnis von Ruprecht Kreuzer abgelöst hat.

Org. oder Kop. im bearbeiteten Bestand nicht überliefert. - Dep.: Erw. im Revers Bajezid Ottmans von 1479 August 19 im HHStA Wien (Sign. AUR 1479 VIII 19), Perg., 2 rote SS des Ausst. und Gf. Schaffrieds von Leiningen ${ }^{2}$ in wachsf. Schüsseln an Ps, mit Rta-Vermerk auf der Rückseite. $^{3}$

Lit.: BABINGER, Bajezid Osman S. 378; MAURER, Burg Baden S. $37{ }^{4}$

1 Zur Lebensgeschichte des unter päpstlicher Obhut aufgewachsenen, angeblichen Osmanenprinzen - er soll ein Halbbruder Sultan Mehmeds II. gewesen sein - s. ausführlich BABINGER, Bajezid Osman. Sein Taufname war Calixtus Ottoman(n)us (nach seinem Taufpaten Papst Calixt III.); der spätere, erstmals 1464 belegte Name Bajezid (Bayezid, Bajazit) nach dem Namen des erstgeborenen Sohnes Mehmeds II., des späteren Sultans Bayezid II., sollte wohl Ansprüche auf den osmanischen Thron verstärken (ebd. S. 366), was auch in der im Revers gebrauchten Selbstbezeichnung Bajazit Ottman, ain furst der Türkhen, im Gebrauch des Majestätsplurals in seinen Urkunden und in der zeitgenössischen Bezeichnung Turkisch kaiser (z.B. CHMEL, Mon. Habsb. I/2 S. 626 n. 456) zum Ausdruck kommt.

2 Ksl. Rat und Diener, s. zu ihm HerNg, Friedrich III./1 S. 417-423.

3 Reg.: ChMEL n. 7316, DERS., Mon. Habsb. I/3 S. 643 n 28. 
4 Nach BABINGer, dem MAURER folgt, datiert der Revers irrtümlich von 1479 Juli 19. - Siehe zu Bajezid Ottman auch dessen im bearbeiteten Bestand überlieferten Reverse von 1476 November 16 über die Übertragung der Burg Perchtoldsdorf (Sign. AUR 1476 XI 16), von 1477 Januar 20 über die Übertragung von Burg und Ungeld zu Baden (Sign. AUR sub dat. 1477 II 3) und von 1478 September 29 über die Übertragung eines Teichs bei Baden für den Wiederaufbau der durch die Ungarn zerstörten Burg (Sign. AUR sub dat. 1478 VIII 13); Druck: ChMel, Mon. Habsb. I/2 S. 259 f. n. 87 (Perchtoldsdorf), S. 626 n. 456 (Teich zu Baden).

[Vor 1479 August 26, -]

K.F. überlässt Hans Heidenreich bis auf Widerruf das Forstamt im Eisenerz ${ }^{1}$ bei Leoben und dazu die Fischweide im (Leopoldsteiner-)See und in den Zu- und Abflüssen ${ }^{2}$ im Innerberg bestandweise gegen Zahlung von jährlich 34 Pfd. Pf.

Org. oder Kop. im bearbeiteten Bestand nicht überliefert. - Dep.: Erw. im Revers Hans Heidenreichs von 1479 August 26 im HHStA Wien (Sign. AUR 1479 VIII 26), Perg., 2 grüne SS des Ausst. und Erhard Lembachers in wachsf. Schüsseln an Ps., mit Rta-Vermerk auf der Rückseite. ${ }^{3}$

Lit.: MUCHAR, Geschichte 8 S. 117.

1 Erzberg.

2 Im Revers zwyrichpechen.

3 Reg.: GöTH, Urkunden-Regesten 10 n. 711.

\section{September 4, Graz}

K.F. belehnt seinen Vetter Ehz. Sigmund von Österreich für die ihm und dem Reich mit darstreckhung seins leibs und guts geleisteten Dienste aus ksl. Machtvollkommenheit wissentlich in crafft diss briefs, was wir im von recht, pillicheit und gnaden wegen daran zuverleihen haben, mit allen ihm und dem Reich heimgefallenen Lehen des verstorbenen Petermann von Raron $\left(\right.$ Roraw) ${ }^{1}$ samt Rechten, Nutzen und Zubehör und verfügt, dass Sigmund die Lehen von ihm und dem Reich in lehennsweise innehaben und nutzen soll, jedoch unbeschadet seiner und des Reichs Rechte sowie der Rechte anderer. Am vierdten tag des monadts september.

KVr: A.m.p.d.i. - KVv: Rta (Blattmitte). - lehenbrief archiducis Sig(mundi) Austrie (oberer Blattrand).

Org. im HHStA Wien (Sign. AUR 1479 IX 4), ${ }^{2}$ Perg., rotes S 18 in wachsf. Schüssel mit wachsf. S 16 rücks. eingedr. an Ps. - Kop.: Abschrift des 18. Jh. ebd. (Sign. Urkundenabschriften Österr. Urkunden, Kt. 46 sub dat.), Pap. 
Druck: Chmel, Mon. Habsb. I/3 S. 184f. n. 77.

Reg.: ChMel n. 7319; LichNowsKy(-BIRK) 8 n. 204.

Siehe n. 312 .

1 Petermann von Raron verstarb als Letzter seines Geschlechts am 31. Juli 1479; s. zu ihm HAusER, Freiherren von Raron S. 528-530 [166-168].

2 Dem Privileg beiliegend n. 312.

\section{September 5, Graz}

K.F. gebietet allen Reichsuntertanen, so disz nachgeschriben sachen beruret und sich der annemen, aus ksl. Macht und unter Androhung seiner und des Reichs Strafe, seinen Vetter Ehz. Sigmund von Österreich, den er mit den heimgefallenen Lehen des verstorbenen Petermann von Raron (Roraw) laut seines lehennbriefs ${ }^{1}$ belehnt hat, bei diesen Lehen ungehindert zu lassen, um nicht für die hannthabung seines und des Reichs eigenthumb und der Lehenschaft Sigmunds zum Handeln gegen sie gezwungen zu werden. Am funfften tag des monadts september.

KVr: A.m.p.d.i. - KVv: Mandatum super littera feudorum domini archiducis Sig(mun)di Austrie (oberer Blattrand).

Org. im HHStA Wien (Sign. AUR sub dat. 1479 IX 4)², Perg., rotes S 18 rücks. aufgedr. (großteils abgefallen) unter (abgelöster) Papieroblate. - Kop.: Abschrift des 18. Jh. ebd. (Sign. Urkundenabschriften Österr. Urkunden, Kt. 46 sub dat.), Pap.

Reg.: ChMel n. 7320; LichNowsky(-BIRK) 8 n. 205.

1 Siehe n. 311.

2 Liegt $n .311$ anbei.

\section{September 6, Graz}

K.F. belehnt seinen Kämmerer Kaspar von Rogendorf, Pfleger von Ybbs, ${ }^{1}$ auf Bitte Wolfgang Seiseneggers ${ }^{2}$ mit Schloss Ottenschlag. An montag nach sannd Egidien tag (nach Kop.).

KVr: C.d.i.p. (nach Kop.). 
Kop.: Vidimus des Propstes Johann von Waldhausen von 1488 November 3 im HHStA Wien (Sign. AUR sub dat. 1478 XII 7), Perg., grünes (spitzovales) S des Ausst. in wachsf. Schüssel an Ps. ${ }^{3}$

Reg.: Ein ausführliches Regest auf der Grundlage des Org. bieten die Regg.F.III. H. 26 n. 732.

Lit. siehe Regg.F.III. H. 26 n. 732; weiters NeIDHART, Geschichte Pöggstalls S. 42; ZAJIC, Kaspar von Roggendorf S. 17; DERS., Herren und Aufsteiger S. 22; zur Person des Rogendorfers S. auch HeINIG, Friedrich III./1 S. 195f., zur Familie ZAлC, Rog[g]endorf S. 1207-1214; die Urkunde erw. bei STIERLE, Herren von Wehingen S. 66.

1 Zur Übertragung von Ybbs s. den Pflegrevers Kaspar Rogendorfers von 1478 Oktober 19 im HHStA Wien (Sign. AUR sub dat. $1478 \mathrm{X}$ 3).

2 Siehe zu ihm Turba, Ritterstand S. 119f;; HenNG, Friedrich III./1 S. $274 f$.

3 Das Vidimus enthält auch n. 222 (Belehnung mit Pöggstall).

\section{[Vor 1479 September 7, -]}

K.F. überlässt Jörg Kätzler, Bürger von Leoben, bis auf Widerruf den zum ksl. Amt gehörigen Hof im Winkl ${ }^{1}$ zu Leoben bestandweise gegen die Verpflichtung, Haus und Feld zu erhalten, und einem jährlichen Dienst von 13 Pfd. Pf., die zusammen mit den 11 Pfd. Pf. von dem anderen Hof im Winkl, den der K. von Hans Stubich ${ }^{2}$ gekauft und auf den Kätzler ein Kaufrecht hat, zu dienen sind.

Org. oder Kop. im bearbeiteten Bestand nicht überliefert. - Dep.: Erw. im Revers des Leobener Bürgers Jörg Kätzler von 1479 September 7 im HHStA Wien (Sign. AUR 1479 IX 7), Perg., 2 grüne SS des Ausst. und des Leobener Stadtrichters Hans Pringsauf in wachsf. Schüsseln an Ps, mit Rta-Vermerk auf der Rückseite. ${ }^{3}$

1 Siehe dazu Regg.F.III. H. 13 n. 299.

2 Hans Stubich, Pfleger von St. Peter ob Leoben (s. n. 294), erwarb den Hof 1478 von Kaspar von Rogendorf, der ihn von seinem verstorbenen Vater Sigmund geerbt hatte; s. die Verkaufsurkunde des Rogendorfers von 1478 August 31 im HHStA Wien (Sign. AUR 1478 VIII 31), in der Jörg Kätzler bereits als Inhaber mit Kaufrecht genannt wird. Im Juni 1479 erfolgte der Weiterverkauf des Hofs an K.F., s. die Urkunde von 1479 Juni 8 ebd. (Sign. AUR 1479 VI 8); Reg.: GöTH, Urkunden-Regesten 10 n. 706; erw. bei MUCHAR, Geschichte 8 S. 117.

3 Reg.: Muchar, Urkunden-Regesten n. 299; Göth, ebd. n. 712; SchILLINGER-PRAssL, Rechtsquellen Leoben S. 225 n. 195. 
K.F. belehnt als Herr und Landesfürst des Ftm. Österreich Michael Fritz, Bürger von Gmunden, mit einem Drittel des Zehents in den Pfarren Vöcklamarkt, Pöndorf und Gampern.

Org. oder Kop. im bearbeiteten Bestand nicht überliefert. - Dep.: Erw. im Aufsandbrief des Gmundner Bürgers Michael Fritz von 1479 September 11 im HHStA Wien (Sign. AUR 1479 IX 11), Pap., 2 SS der Gmundner Bürger Wolfgang Teurbanger und Peter Schymel rücks. aufgedr. unter Papieroblate. ${ }^{1}$

1 Michael Fritz sandte dem $\mathrm{K}$. die Lehen auf mit der Bitte um Weiterverleihung an den Gmundner Bürger Jörg Vaschang und dessen Gemahlin Sibylle, seine Enkelin. - Reg.: CHMEL n. 7323; DERs., Mon. Habsb. I/3 S. 710 n. 228.

\section{[Vor 1479 September 16, -]}

K.F. überlässt Richter, Rat und allen Bürgern des Marktes Aussee bis auf Widerruf das Ungeld zu Aussee bestandweise gegen Zahlung von jährlich 60 Pfd. Pf.

Org. oder Kop. im bearbeiteten Bestand nicht überliefert. - Dep.: Erw. im Revers von Richter, Rat und den Bürgern von Aussee von 1479 September 16, Aussee, im HHStA Wien (Sign. AUR 1479 IX 16), Perg., 2 grüne SS des Bürgen Rudolf Kirchpuchler, Bürger von Aussee, und Martin Hawnolds in wachsf. Schüsseln an Ps, mit Rta-Vermerk auf der Rückseite. ${ }^{1}$

Lit.: HollwöGER, Ausseer Land S. 62.

1 Reg.: GöTH, Urkunden-Regesten 10 n. 713

\section{[Vor 1479 September 18, -]}

K.F. überlässt Paul Engelhart bis auf Widerruf das Gericht zu Radmannsdorf bestandweise gegen Zahlung von jährlich 40 Mark Sch. Venediger Münze.

Org. oder Kop. im bearbeiteten Bestand nicht überliefert. - Dep.: Erw. im Revers Paul Engelharts von 1479 September 18 im HHStA Wien (Sign. AUR 1479 IX 18), Perg., 2 grüne SS des Ausst. und Erhard Lembachers in wachsf. Schüsseln an Ps, mit Rta-Vermerk auf der Rückseite. 
K.F. überlässt Hans Lengheimer ${ }^{1}$ bis auf Widerruf die Land- und Marktmaut zu Weixelburg bestandweise ab St. Michaelstag 1479 (September 29) gegen Zahlung von jährlich 150 Pfd. Pf.

Org. oder Kop. im bearbeiteten Bestand nicht überliefert. - Dep.: Erw. im Revers Hans Lengheimers von 1479 September 22 im HHStA Wien (Sign. AUR 1479 IX 22), Perg., 2 grüne SS des Ausst. und des Ritters Wilhelm von Auersperg ${ }^{2}$ in wachsf. Schüsseln an Ps, mit Rta-Vermerk auf der Rückseite.

1 Pfleger von Weixelburg, s. Regg.F.III. H. 29 n. 207 (1478 Juli 9); auch ChмEL, Mon. Habsb. I/2 S. 915f. nn. 1262-1265.

2 Ksl. Kämmerer und Rat, s. zu ihm HeINIG, Friedrich III./1 S. 231 f.

\section{[Vor 1479 Oktober 2, - ]}

K.F. überträgt Jörg Kranperger, Bürger von Wien, das Ungeld zu Nussdorf und Purkersdorf gegen ein Darlehen ${ }^{1}$ von 1.028 Pfd. 5 Sch. 24 Pf. und 157 fl. ung. bis zur Tilgung der Schuld.

Org. oder Kop. im bearbeiteten Bestand nicht überliefert. - Dep.: Erw. im Revers des Wiener Bürgers Jörg Kranperger von 1479 Oktober 2 im HHStA Wien (Sign. AUR sub dat. 1479 IX 7), ${ }^{2}$ Perg., 2 grüne SS des Ausst. und des Grazer Stadtrichters Tibold Brunner ${ }^{3}$ in wachsf. Schüsseln an Ps, mit Rta-Vermerk auf der Rückseite. ${ }^{4}$

Lit.: WALTER, Ungeld S. 120 (Krenberger); zu Jörg Kranperger S. PeRGER, Wiener Ratsbürger S. 181.

1 Laut Revers ein Darlehen zur Bezahlung ksl. Dienstleute und sonstigen Bedarfs.

$2 \mathrm{Zu}$ den ebenfalls unter dieser Sign. verwahrten Reversen Kaspar Rogendorfers und Heinrich Prüschenks S. n. 320 , Anm. 1.

3 Stadtrichter 1478-1480, 1482-1484, s. zu ihm PopelKA, Geschichte Graz 1 S. 486f:; DiENES, Bürger von Graz S. XXXIV.

4 Reg.: ChMel n. 7326; Ders., Mon. Habsb. I/3 S. 651 n. 63. 
K.F. überträgt Heinrich Prüschenk wissenlich mit dem brief die Pflege von Schloss Sarmingstein und überlässt ihm die dortige Maut bestandweise auf ein ganzes Jahr ab Datum dieses briefs gegen Zahlung von 1.500 Pfd. Pf. in guter landläufiger Münze. Er verpflichtet ihn, das Schloss trewlich innezuhaben und zu verwalten, von der Maut die 1.500 Pfd. Pf. nach Gewohnheit zu den Quatembern zu entrichten und vom Überschuss auf eigene Kosten das Schloss zu behüten und zu bewahren, den Entzug ksl. Rechte auf Schloss und Maut nicht zu gestatten und auf Erfordern das Schloss samt Zubehör und zewg ohne Widerspruch abzutreten gemäß dessen Verschreibung ${ }^{1}$. Am phintztag nach sannd Kollmanstag.

KVr: C.d.i.p. $-\mathrm{KVv}$ : Rta.

Org. im HHStA Wien (Sign. AUR 1479 X 14), Perg., rotes S 21 in wachsf. Schüssel mit wachsf. S 16 rücks. eingedr. an Ps. - Kop.: Abschrift des 18. Jh. ebd. (Sign. Urkundenabschriften Österr. Urkunden, Kt. 46 sub dat.), Pap.

Abb.: Kaiserurkunden in Abb., Lfg. XI, Tf. 22a.

Druck: Chmel, Mon. Habsb. I/3 S. 364f. n. 160.

Reg.: ChMEL n. 7328; Kaiserurkunden in Abb., Textbd. S. 506.

Lit.: Zu Heinrich und Sigmund Prüschenk s. Probszt, Brüder Prueschenk; Zernatto, Herrenstand S. 69-73; HeINIG, Friedrich III./1 S. 78-88, 206-208; MARIAN/ZeHETMAYER, Hardegg S. 560f., 563, 568f.; zur Sarmingsteiner Maut s. ausführlich HOFFMANN, Weinfuhren, und DERS., Salzmaut.

Gemeinsam mit dem vorliegenden Stück werden im HHStA Wien zwei eigenhändige ksl. Schreiben von 1480 und 1481 November 30 - beide unterfertigt mit per manum propriam und besiegelt mit vorders. aufgedr. S 16 - über die Verlängerung der Bestandgabe auf zunächst ein Jahr und dann auf zwei Jahre verwahrt. ${ }^{2}$

1 Revers Heinrich Prüschenks von 1479 Oktober 14 im HHStA Wien (Sign. AUR sub dat. 1479 IX 7); Reg.: ChMEL n. 7327; DERs., Mon. Habsb. S. 699 n. 196. - Unter dieser Sign. wird auch n. 319 sowie der Pflegrevers Kaspar Rogendorfers über die Übertragung von Werfenstein samt Maut von 1479 September 7 verwahrt (s. CHMEL n. 7322; DERS., Mon. Habsb. I/3 S. 698 n. 195); zu der im Revers des Rogendorfers erwähnten ksl. Urkunde vom selben Tag s. Regg.F.III. H. 26 n. 733.

2 Siehe Regg.F.III. H. 35 nn. 1 und 159; zu den ksl. Autographen s. Koller, Bedeutung; WAGENDorfER, Eigenhändige Unterfertigungen S. 226, 252. 
K.F. überlässt Wilhelm von Auersperg bis auf Widerruf seine Fischweide im Mürztal auf der Mürz ober- und unterhalb von Kindberg bestandweise ab St. Martinstag 1479 (November 11) gegen Zahlung von jährlich $50 \mathrm{fl}$. ung. und der Verpflichtung, bei Aufenthalten des K. in Kindberg oder bei der Durchreise Fische für den ksl. Hof zu liefern. ${ }^{1}$

Org. oder Kop. im bearbeiteten Bestand nicht überliefert. - Dep.: Erw. im Revers Wilhelms von Auersperg von 1479 Oktober 17 im HHStA Wien (Sign. AUR sub dat. 1479 X 23), Perg. (fleckig), 2 grüne SS des Ausst. und Erhard Lembachers (beschädigt) in wachsf. Schüsseln an Ps, mit Rta-Vermerk auf der Rückseite. ${ }^{2}$

Lit.: Muchar, Geschichte 8 S. 117; zu Wilhelm von Auersperg s. HeINIG, Friedrich III./1 S. $231 \mathrm{f}$.

1 Siehe dazu auch $\mathrm{n} .122$.

2 Reg.: GöTH, Urkunden-Regesten 10 n. 714.

[Vor 1479 November 1, -]

K.F. überlässt Jörg Dachauer die Kaltmaut ${ }^{1}$ zu Korneuburg und Wien bestandweise für ein Jahr bis Dienstag vor St. Gallentag 1480 (Oktober 10) gegen Zahlung von 100 Pfd. Pf.

Org. oder Kop. im bearbeiteten Bestand nicht überliefert. - Dep.: Erw. im Revers Jörg Dachauers von 1479 November 1, Korneuburg, im HHStA Wien (Sign. AUR sub dat. 1479 X 25), Perg., 2 grüne SS des Ausst. und des Korneuburger Bürgers Hans Trost ${ }^{2}$ (beschädigt) in wachsf. Schüsseln an Ps, mit Rta-Vermerk auf der Rückseite. ${ }^{3}$

1 Die Kaltmaut oder Kalte Maut wurde zwischen Kolomani (Oktober 13) und dem Dreikönigstag (Januar 6) eingehoben, s. CzeIKE, Lexikon Wien 3 S. 438; SABLIK, Korneuburg S. 140.

2 Langjähriger Korneuburger Stadtrichter (1453, 1462-1480), s. StARZER, Korneuburg S. $273 f$.

3 Reg.: Chmel n. 7334; Ders., Mon. Habsb. I/3 S. 652 n. 66; erw. bei Starzer, ebd. S. 128.

\section{[Vor 1479 November 8, -]}

K.F. überlässt Seifreid Kötzler, Bürger von Graz, das Ungeld zu Leibnitz samt Zubehör, wie dieser es von (Hans) Einpacher ${ }^{1}$ innehatte, bestandweise für ein Jahr ab St. Katharinentag 1479 (November 25) gegen Zahlung von 320 Pfd. Pf. 
Org. oder Kop. im bearbeiteten Bestand nicht überliefert. - Dep.: Erw. im Revers des Grazer Bürgers Seifried Kötzler von 1479 November 8 im HHStA Wien (Sign. AUR 1479 XI 8), Perg., 2 grüne SS des Ausst. und Erhard Lembachers in wachsf. Schüsseln an Ps, mit Rta-Vermerk auf der Rückseite. ${ }^{2}$

Lit.: MUChaR, Geschichte 8 S. 118; DiENES, Bürger von Graz S. XIIX (Közler).

1 Siehe zu ihm Dienes, Bürger von Graz S. LXXIf.

2 Reg:: Muchar, Urkunden-Regesten n. 302; Göth, Urkunden-Regesten 10 n. 717.

\section{November 10, Graz}

K.F. überlässt seinem Kämmerer Sigmund Prüschenk wissentlich mit dem brief die in seinen Herrschaften Gutenstein, Windischgraz und Holenstein gelegenen Ämter, die er (K.F.) von ihm und dessen Bruder (Heinrich) gekauft hat, ${ }^{1}$ samt Nutzen, Renten, Zinse und Zubehör bestandweise für ein Jahr bis zum St. Nikolaustag 1480 (Dezember 6) und danach bis auf Widerruf gegen Zahlung von jährlich $100 \mathrm{Pfd}$. Pf. guter landläufiger Münze. Er verfügt, dass Sigmund die Ämter innehaben und nutzen soll, und verpflichtet ihn, das Bestandgeld nach Gewohnheit zu den Quatembern zu entrichten, die zu den Ämtern gehörigen Leute und Holden nicht über die gewöhnlichen Nutzen, Renten, Zinse, Dienste und Robotleistungen ungebührlich gegen altes Herkommen zu belasten und auf Erfordern die Ämter abzutreten gemäß dessen Verschreibung. An mittichen vor sannd Mertten tag.

Eigenhändiger Vermerk von K.F. auf der Plica: und darczue czbainczig t(alenta).

KVr: -. - KVv: Rta.

Org. im HHStA Wien (Sign. AUR 1479 XI 10), Perg., rotes S 21 in wachsf. Schüssel (beschädigt) mit wachsf. S 16 rücks. eingedr. an Ps.

Reg.: ChMfl n. 7336; Ders., Mon. Habsb. I/3 S. 721 n. 266.

Lit.: WAGENDORFER, Eigenhändige Unterfertigungen S. 234, 247f., 263; zu Sigmund Prüschenk s. Probszt, Brüder Prueschenk; Zernatto, Herrenstand S. 69-72; HeINIG, Friedrich III./1 S. 78-88, bes. 80, und 206; MARIAN/ZeHETMAYER, Hardegg S. 560f., 563, $568 \mathrm{f}$.

Sigmund Prüschenk als einer der Hauptfinanziers Friedrichs III. - er gehörte zu dessen finanziellem „Grundgerüst“ (HeINIG S. 79) - erhielt die meisten Urkunden mit autographen Vermerken des $\mathrm{K}$.

1 Siehe dazu die Verkaufsurkunde der Brüder Prüschenk von 1479 Dezember 6 über zahlreiche Güter in den genannten Herrschaften im HHStA Wien (Sign. AUR 1479 XII 6); Druck: CHMEL, Mon. Habsb. I/3 S. $372-378$ n. 163. 
K.F. überlässt Hans Gressl bis auf Widerruf das Hansgrafenamt im Ftm. Österreich bestandweise gegen Zahlung von jährlich $60 \mathrm{Pfd}$. Pf.

Org. oder Kop. im bearbeiteten Bestand nicht überliefert. - Dep.: Erw. im Revers Hans Gressls von 1479 November 12, Wien, im HHStA Wien (Sign. AUR 1479 XI 12), ${ }^{1}$ Perg., 2 grüne SS des Ausst. und des Wiener Ratsherrn und Stadtkämmerers Thomas Tenk ${ }^{2}$ in wachsf. Schüsseln an Ps, mit Rta-Vermerk auf der Rückseite. ${ }^{3}$ - Dem Revers beiliegend ein Zettel (Pap.) mit der Mitteilung: Lieber maister Hanns, unser her der kaiser will ein penüegen an det (?) hansgraffen zu Wienn verschreiben. Sigmund von Nidertor. ${ }^{4}$

Lit:: PERGER, Wiener Ratsbürger S. 203.

1 Unter dieser Sign. gemeinsam verwahrt mit n. 237.

2 Siehe zu ihm PERGER, Wiener Ratsbürger S. 185.

3 Reg.: CHMEL n. 7337; Ders., Mon. Habsb. I/3 S. 652 n. 67.

4 Zum ksl. Rat und Kämmerer Sigmund von Niedertor s. HenNIG, Friedrich III./1 S. 301-304.

\section{November 24, Graz}

K.F. bestätigt durch Besiegelung die von ihm, eciam vigore literarum apostolicarum, ${ }^{1}$ mit Rat des Kardinalpriesters Georg (Heßler) von S. Lucia in Silice, des päpstlichen Legaten Bf. Alexander von Forli ${ }^{2}$ und Eb. Johanns von $\mathrm{Gran}^{3}$ finaliter beschlossenen Artikel über die Einigung im Konstanzer Bistumsstreit zwischen dem Elekten Otto von Sonnenberg ${ }^{4}$ und dem Providierten Ludwig von Freiberg ${ }^{5}$, um die hoch verschuldete Konstanzer Kirche in sui preeminencia wiederherzustellen, den Frieden zu sichern und die beiden genannten Kontrahenten von ihren großen Mühen und Aufwendungen zu entlasten.

(1) ${ }^{6}$ Es wird erneut und nun endgültig festgesetzt, dass alle zwischen K.F. und dem Bf. von Caithness ${ }^{7}$ früher beschlossenen Artikel $^{8}$ in Kraft bleiben, insbesondere aber, dass der Elekt Otto der wahre und außer Zweifel stehende pastor et rector der Konstanzer Kirche ist, er die volle Gewalt über Spiritualien und Temporalien wie ein wahrer Bischof pleno iure ausüben soll und ihm darüber vom Apostolischen Stuhl Provision, Bestätigung und Weihe mit entsprechenden Briefen gewährt werden muss. Ludwig von Freiberg wird untersagt, unter dem Vorwand der Provision oder eines anderen vermeintlichen Rechts auf die Konstanzer Kirche den Elekten Otto oder jemand anderen in dessen Namen wegen des Bistums, dessen Nutzungsrechte oder sonstiger Einkünfte direkt oder indirekt zu behelligen. Der Apostolische Stuhl soll ihm darüber auf ewig 
Schweigen auferlegen, den Streit für nichtig erklären und entsprechende Briefe zugunsten Ottos in meliori forma ausstellen. Hinsichtlich der von Ludwig an die Apostolische Kammer bereits bezahlten Annaten und Servitia Minuta sowie dessen sonstigen hohen Auslagen wird als Gegenleistung beschlossen, dass Otto beim Bf. von Augsburg literas plene quietacionis seu liberacionis hinterlegt, mit denen er für sich und seine Brüder, den Gff. von Sonnenberg, ${ }^{9}$ quittiert, von Ehz. Sigmund von Österreich $18.000 \mathrm{fl}$. rh. erhalten zu haben, die dieser Gf. Eberhard (I.) von Sonnenberg und dessen Erben schul$\operatorname{det}^{10}$ und die von Sigmunds anderen Schulden bei Gf. Eberhard abgezogen werden. Desgleichen soll Ludwig Quittanzbriefe beim Bf. von Augsburg hinterlegen, mit denen er für sich und seine Erben bestätigt, in der Person Ehz. Sigmunds von Otto gänzlich bezahlt worden zu sein und die genannten $18.000 \mathrm{fl}$. rh. erhalten zu haben, die dieser ihm für die Annaten, die Servitia Minuta und sonstige Auslagen schuldet. Zudem hat Ludwig dafür zu sorgen, dass Otto von den für Wahl und Provision zu zahlenden Annaten und Servitia Minuta befreit wird und diesem über deren Bezahlung Quittanzbriefe der Apostolischen Kammer ohne Unkosten ausgestellt werden, die ebenfalls beim Bf. von Augsburg zu hinterlegen sind. Erst nach Hinterlegung der genannten Quittanzbriefe, der vom Apostolischen Stuhl zu erwirkenden Briefe über die Provision Ottos, das Schweigegebot und die Nichtigkeit des Streits sowie der Gratialbriefe über den von Otto als subsidium caritativum einzufordernden Zehent ${ }^{11}$ beim Bf. vom Augsburg kann dieser die Briefe den Parteien übergeben.

(2) Ludwig soll so bald wie möglich auf alle kirchlichen Benefizien und Pfründen, die der Elekt Otto vor seiner Wahl innehatte, in recompensam gemäß den Statuten der Konstanzer Kirche canonice providiert werden, jedoch ohne dem Domkapitel die Zahlung der primariorum fructuum zu schulden und mit dem Nutznießungsrecht auch in Abwesenheit, so wie andere Kanoniker bei Abwesenheit zum Studium generale.

(3) Das zum Konstanzer Bistum gehörige Schloss Küssaberg mit allem Zubehör wird Ludwig zur Nutzung übertragen mit der Verpflichtung, das Schloss nach dem Willen des Bf. und der Konstanzer Kirche offen zu halten, von den Erträgen zu behüten und nichts zu verkaufen, zu verpfänden oder zu entfremden. Im Falle seiner Beförderung an eine andere Kathedralkirche oder seines Todes ist das Schloss samt Zubehör dem jeweiligen Bf. und der Konstanzer Kirche wieder abzutreten. Ludwig hat sich dazu mit ebenfalls beim Bf. von Augsburg zu hinterlegenden offenen Briefen für sich und seine Erben zu verpflichten.

(4) Zur Wahrung der Freiheit, des Friedens und des Schutzes der Konstanzer Kirche soll zwischen dem Haus Österreich (domum Austrie) und dem Elekten Otto gutes Einvernehmen bestehen, jedoch ohne Nachteil für den Apostolischen Stuhl und das Reich. Alle Kanoniker der Konstanzer Kirche, die Parteigänger Ludwigs waren und sind, sollen sicher und ungehindert zu ihren Benefizien zurückkehren und deren Einkünfte beziehen. Über jene während des Streits von den Domherren nicht bezogenen Erträge sollen je zwei vom Domkapitel und von den Kanonikern gewählte Männer 
einen Vergleich erzielen; gelingt dieser nicht, ist der Spruch eines von K.F. gewählten Schiedsrichters von den Parteien einzuhalten.

(5) Der Elekt Otto kann nach Gutdünken die beiden früher in Konstanz tätigen Notare Konrad Armbruster und Michael von Meersburg wieder zum Notariatsamt zulassen, ${ }^{12}$ andernfalls hat er jedem von ihnen $100 \mathrm{fl}$. rh von dem als subsidium caritativum einzuhebenden Zehent zu zahlen, wobei sie sich bei der Einhebung im Auftrag Ottos als fideles zu erweisen haben. ${ }^{13}$

(6) K.F. und Ehz. Sigmund werden sich beim Apostolischen Stuhl und bei ihren und des Reichs Untertanen mit geeigneten Mitteln dafür einsetzen, dass zur Wiederherstellung der Konstanzer Kirche pro caritativo subsidio der Zehent aller Einkünfte und Erträge vom nicht exemten Klerus der Klöster und Kirchen der Stadt und der Diöz. Konstanz, Regulierte wie Säkulare beiderlei Geschlechts, einmal in doppelter Höhe oder zweimal und von den exemten Prälaten und Klerikern einmal eingehoben wird sowie ein vollkommenen Jubel-Ablass auf fünf Jahre für die Konstanzer Kirche vom Apostolischen Stuhl erwirkt wird. ${ }^{14}$ Alle Einnahmen sollen zum Nutzen der Konstanzer Kirche und des Elekten Otto verwendet werden.

(7) Ludwig soll zusammen mit dem Klerus seiner Pfarrkirche Ehingen ${ }^{15}$ in der Diöz. Konstanz auf Lebenszeit von der bischöflichen Jurisdiktion exemt sein.

(8) Abschließend wird festgesetzt, dass alles Unrecht und alle Zwietracht zwischen Otto und Ludwig sowie ihren Parteigängern, Untertanen und Familiaren gänzlich aufgehoben sind und keine Partei die andere diesbezüglich via iuris aut facti belangen soll. Bei Zuwiderhandeln oder Nichteinhaltung der Beschlüsse durch eine Partei wird der Verlust aller ihr zustehenden Rechte hinsichtlich der Konstanzer Kirche und eine Pön von 100 Pfd. Gold angedroht, zahlbar je zur Hälfte an die Apostolische Kammer und die ksl. Kammer. Alle päpstlichen, kaiserlichen oder von einer anderen Autorität im Zuge des Streits verhängten Strafen über Universitäten, Gemeinschaften, Domkapitel und Konvente der Kirchen, Städte und Dörfer oder über geistliche und weltliche Personen wie Exkommunikation, Interdikt, Suspension, Bann etc. werden aufgehoben; für die Absolution und die Amnestie sollen der Apostolische Stuhl und K.F. an ihrer statt Personen mit voller Gewalt ausstatten. Vicesimaquarta die mensis novembris.

KVv: Abrede des bistumbs halben zu Constenntz (Blattmitte).

Von K.F. besiegelte Notel (lat.) mit Korrekturen im HHStA Wien (Sign. AUR 1479 XI 24), Pap. (1 fol., Hochformat), rotes S 21 vorders. unter dem Text auf fol. 1v aufgedr. (tlw. abgefallen).

Druck: CHMEL, Mon. Habsb. I/3 S. 22-27 n. 9 (korrigierte Textfassung ohne Tilgungen).

Reg.: REC 5 n. 15268. 
Lit.: VocheZER, Waldburg 1 S. 862f.; HollweG, Georg Heßler S. 87f.; GöLLER, Konstanzer Bistumsstreit S. 42; DANN, Besetzung S. 87; KRAMML, Konstanz S. 225f.; BaUM, Habsburger S. 656; Helvetia Sacra I/2 S. 364f;; zahlreiche Fridericiana zum Konstanzer Bistumsstreit bieten die Regg.F.III. H. 23.

Der als abrede bezeichnete und korrigierte Textentwurf beginnt mit Infrascripti sunt articuli concepti et finaliter conclusi per serenissimum [...] Fridericum und nennt in den Artikeln K.F. wie auch alle Vertragsparteien in objektiver Form in der 3. Person; hingegen erfolgt die Siegelankündigung durch K.F. in der 1. Person. Durch die Besiegelung erhielt der vorliegende Vertragsentwurf rechtliche Verbindlichkeit. Da die Korrekturen des Textes von derselben Hand stammen, kann mit hoher Wahrscheinlichkeit angenommen werden, dass es sich bei dem von K.F. bestätigten Textentwurf um die korrigierte Fassung handelte. Nicht ganz auszuschließen ist allerdings, dass die Korrekturen ein Ergebnis weiterer Verhandlungen waren, weshalb die gestrichenen Textteile in den Anmerkungen angegeben wurden.

Ludwig von Freiberg lehnte auch diese Vereinbarung ab und begab sich nach Rom, um seine Ansprüche geltend zu machen, verstarb aber dort im Herbst 1480. Sein Tod beendete den jahrelangen Bistumsstreit; Otto von Sonnenberg wurde am 10. November 1480 vom Papst als Konstanzer Bischof bestätigt.

1 Papst Sixtus IV. hatte dem K. am 12. März 1479 die Schlichtung des Konstanzer Bistumsstreits übertragen, s. REC 5 n. 15151; DANN, Besetzung S. 84.

2 Alexander Numai (Bf. 1470-1483), s. zu ihm ERfle, Alexander Numai.

3 Johann Beckenschlager (Beckensloer) (Eb. von Gran 1472-1487), s. zu ihm ZAISBERGER, Rohr, bes. S. 58-62; GATZ, Bischöfe 2 S. 36f.; HeING, Friedrich III./1 S. 449-452 mit weiterführender Lit.

4 Gf. Otto von Sonnenberg aus der gräflichen Linie der Truchsessen von Waldburg, seit 1474 September 30 (vom Domkapitel) erwählter Bf. von Konstanz; s. zu ihm Helvetia Sacra I/2,1, S. 366-371; GAtz, ebd. S. 669f:; auch HenNG, ebd. S. $487 f$.

5 Ludwig von Freiberg, Dr. iur. utr., wurde am 9. September 1474 von Sixtus IV. zum Koadjutor des Konstanzer Bischofs mit Nachfolgerecht ernannt; s. zu ihm und dem Bistumsstreit Helvetia Sacra I/2,1, S. 361-366; GaTz, ebd. S. $197 f$.

6 Die Nummerierung der Artikel folgt der Gliederung des Vertragstextes, in dem jeder Artikel mit conclusum est eingeleitet wird.

7 Prosper Camogli de Medici (Bf. 1478-1484).

8 Vereinbarung von 1479 April 15 (REC 5 n. 15162), die von Ludwig von Freiberg beim Papst beeinsprucht wurde.

9 Eberhard (II.), Andreas und Johannes, Söhne Eberhards I. von Sonnenberg.

$10 \mathrm{Ehz}$. Sigmund schuldete Gf. Eberhard I. von Sonnenberg, Truchsess von Waldburg, für den Verkauf der Gft. Sonnenberg $34.000 \mathrm{fl}$. gemäß Vertrag von 1474 August 31, s. VocHEZER, Waldburg 1 S. $587 f$.

11 Am linken Rand (fol. 1r) super decima pro caritativo subsidio [...] korrigiert aus super caritativo subsidio per eundem Ottonem electum (gestrichen).

12 Konrad Armbruster und Michael Scriptoris von Meersburg, beide Kler. des Bistums Konstanz, standen als bischöfliche Notare im Dienst Ludwigs von Freiberg und waren vom Amt suspendiert worden; s. zu ihnen SCHULER, Notare Südwestdeutschlands S. 14-16 n. 26 u. S. 426f. n. 1257. 
13 Am linken Rand (fol. 1r) decima pro caritativo subsidio tradere debet eingefugt als Korrektur aus caritativo subsidio tradere teneatur (im Text gestrichen) sowie Erweiterung des Artikels um den Treueerweis der Notare bei der Einhebung des Zehents.

14 Der Passus über das subsidium caritativum wurde inhaltlich sehr weitreichend korrigiert (Streichung und Korrektur unterhalb des Textes auf fol. 1r): Dem getilgten Text zufolge sollte das subsidium caritativum ursprünglich von allen Prälaten und dem gesamten Klerus der Stadt und der Diöz. Konstanz, exemt wie nicht exemt, einmal in doppelter Höhe oder zweimal eingehoben werden (gestrichen: caritativum subsidium duplicatum una vice seu successive duabus vicibus ab omnibus prelatis et toto clero civitatis et diocesis Constantiensis tam exemptis quam non exemptis exigatur et exactum ad usum et commoditatem dicti Ottonis electi et inprimis Constantiensis ecclesie impendatur).

15 Die Pfarre Ehingen hatte Ludwig von Freiberg seit 1459 inne, s. GATZ, Bischöfe 2 S. 197.

\section{[Vor 1479 November 26, -]}

K.F. belehnt Hans Halder und Jörg Stadler ${ }^{1}$ mit dem Sitz Tandlesbach samt dem Bauhof und etlichen Zehenten, Stücken und Gütern als verwirkte und heimgefallene Lehen des Ftm. Österreich ob der Enns und verpflichtet sie, nach der Inbesitznahme ihm oder seinen Erben die Hälfte herauszugeben oder sich diesbezüglich mit ihm zu einigen.

Org. oder Kop. im bearbeiteten Bestand nicht überliefert. - Dep.: Erw. im Revers Hans Halders und Jörg Stadlers von 1479 November 26 im HHStA Wien (Sign. AUR 1479 XI 26), Perg., 3 SS Hans Halders (grün), des Gratweiner Pfarrers Andreas am Stain (rot) und des Gurnitzer Propstes Lic. decr. Peter Knauer (rot) ${ }^{2}$ in wachsf. Schüsseln an Ps, mit Rta-Vermerk auf der Rückseite. $^{3}$ - Ebenfalls erw. in den nn. 328, 329.

Siehe nn. 328, 329.

1 Zum Kanzleischreiber Jörg (Georg) Stadler s. HEINIG, Friedrich III./1 S. 625.

$2 \mathrm{Zu}$ Andreas am Stain, Sekretär in der ksl. Kanzlei und Erzpriester der Untersteiermark (verbunden mit der Gratweiner Pfarre), und Peter Knauer s. HeING, ebd. S. 477 und $626 \mathrm{f}$.

3 Reg.: ChMEL n. 7341; DeRs., Mon. Habsb. I/3 S. 710 n. 230.

\section{November 28, Graz}

K.F. teilt seinem Rat Bernhard von Schärffenberg, Hauptmann ob der Enns, ${ }^{1}$ mit, dass er Hans Halder und seinen Kanzleischreiber Jörg Stadler ${ }^{2}$ mit dem Sitz Tandlesbach samt dem Bauhof und etlichen anderen Stücken, Zehenten und Gütern mit allem Zubehör als verwirkte und heimgefallene Lehen von gnaden laut seines briefs $^{3}$ belehnt hat, nachdem damit nicht gemäß Lehens- und Landrecht gehanndlt worden war. Er bevollmächtigt ihn, auf Erfordern der Belehnten oder ihres Anwalts mit etlichen seiner (K.F.) Lehensleute zu Österreich ein lehennsrecht zu besetzen, über Ladungen und sonst in der Sache 
Nötiges rechtlich zu entscheiden und Halder und Stadler gegen jene, die diese Lehen innehaben, Recht ergehen zu lassen gemäß Lehens- und Landrecht. Abschließend verweist er auf seinen brieff an die Lehensleute, Schärffenberg diesbezüglich Gehorsam zu leisten, den dieser wol zugebrauchen wisse. An suntag nach sannd Kathrein tag.

KVr: C.d.i.i.c.

Org. im HHStA Wien (Sign. AUR 1479 XI 28), ${ }^{5}$ Pap., rotes S 21 rücks. aufgedr. (tlw. abgefallen).

Reg.: ChMel n. 7342; DERS., Mon. Habsb. I/3 S. 710f. n. 231.

Lit.: HEINIG, Friedrich III./1 S. 626; zu Bernhard von Schärffenberg s. ebd. S. $291 f$.

1 Zur Übertragung der Hauptmannschaft an Bernhard von Schärffenberg s. dessen Revers von 1478 September 12 im HHStA Wien (Sign. AUR sub dat. 1478 VIII 13)

2 Siehe zu ihm HeInig, Friedrich III./1 S. 625.

3 Siehe n. 327.

4 Siehe n. 329.

5 Der Vollmacht beiliegend n. 329.

\section{November 28, Graz}

K.F. befiehlt seinen Lehensleuten im Ftm. Österreich mit Verweis auf seine in der Sache ausgegangenen brief $^{1}$ unter Androhung des Lehensverlustes, seinem Rat Bernhard von Schärffenberg, Hauptmann ob der Enns, gehorsam zu sein, wenn dieser sie zu dem lehensrechten erfordert, um zwischen Hans Halder und seinem Kanzleischreiber Jörg Stadler ${ }^{2}$ einerseits, die von ihm mit dem Sitz Tandlesbach samt dem Bauhof und etlichen anderen Stücken, Zehenten und Gütern als verwirkte und heimgefallene Lehen belehnt worden sind, und den Inhabern dieser Lehen andererseits rechtlich zu entscheiden. An suntag nach sannd Kathrein tag.

$\mathrm{KVr}$ : C.d.i.i.c.

Org. im HHStA Wien (Sign. AUR 1479 XI 28) ) $^{3}$ Pap., rotes S 21 rücks. aufgedr.

Reg.: ChMel n. 7343; DeRs., Mon. Habsb. I/3 S. 711 zu n. 231.

Lit. siehe bei $\mathrm{n} .328$.

1 Siehe nn. 327, 328.

2 Siehe zu ihm HeINIG, Friedrich III./1 S. 625.

3 Liegt $n .328$ anbei. 
K.F. überträgt Erasmus, Bggf. von Lienz und zum Lueg, die Pflege von Schloss (Ober-)Stein in Krain, wie sie der verstorbene Pfleger Valentin von Lamberg innehatte, ${ }^{1}$ und überlässt ihm Amt, Maut und Landgericht zu Stein bis auf Widerruf bestandweise ab dem Dreikönigstag 1480 (Januar 6) gegen Zahlung von jährlich 750 fl. ung.

Org. oder Kop. im bearbeiteten Bestand nicht überliefert. - Dep.: Erw. im Revers Erasmus Luegers von 1479 Dezember 16 im HHStA Wien (Sign. AUR 1479 XII 16), Perg., 3 grüne SS des Ausst. und seiner beiden Bürgen Leonhard von Herberstein und Niklas Lueger, Bruder des Erasmus, in wachsf. Schüsseln an Ps, mit Rta-Vermerk auf der Rückseite.

Lit.: TROTTER, Burggrafen von Lienz S. 61-63, bes. 61 (Revers zu 1479 August 12)2 ; zu Erasmus Lueger s. auch ScHÄFFER, Christoph Ungnad S. 132; HeINIG, Friedrich III./1 S. 227, 374.

1 Siehe n. 115, bes. Anm 3.

2 Nach einer Überlieferung im StLA Graz.

\section{Dezember 16, Graz}

K.F. belehnt Georg Scheyrer auf Bitte Lienhard Weichselbergers wissenntlich mit dem brieve, was wir im zurecht daran verleihen sullen oder mugen, mit dem Zehent und Bergrecht an den beiden (Wein-)Bergen Arenberg und Neuberg samt Zubehör aus der Lehenschaft des Ftm. Krain, die Weichselberger diesem verkauft und mit einem offenen besiegelten Brief aufgesandt hat, und verfügt, dass Scheyrer und dessen Erben die Lehen gemäß Lehens- und Landrecht innehaben und nutzen sollen. An phintztag nach sannd Lucein tag.

KVr: C.d.i.i.c. - KVv: Rta. - Rückseite der Wachsschüssel: Vermerk nicht lesbar.

Org. im HHStA Wien (Sign. AUR 1479 XII 16), Perg., rotes S 21 in wachsf. Schüssel mit wachsf. S 16 rücks. eingedr. an Ps.

\section{[Vor 1479 Dezember 29, -]}

K.F. überlässt Kaspar vom Stein bis auf Widerruf das Amt Primskau bestandweise ab St. Georgstag 1480 (April 24) ${ }^{1}$ gegen Zahlung von jährlich 150 fl. ung.

Org. oder Kop. im bearbeiteten Bestand nicht überliefert. - Dep.: Erw. im Revers Kaspars vom Stein von 1479 XII 29 im HHStA Wien (Sign. AUR sub dat. 1480 XII 21), Perg., 2 grüne SS 
des Ausst. und des Kaspar Melz, Verweser (der Hauptmannschaft) in Krain, in wachsf. Schüsseln an Ps, mit Rta-Vermerk auf der Rückseite.

1 Zur Datierung des St. Georgstags auf April 24 s. Regg.F.III. H. 12 n. 6, Anm. 2. 


\section{Quellen- und Literaturverzeichnis}

Aichinger-Rosenberger, P., ZAJic A., Schloss Pöggstall. Adelige Residenz zwischen Region und Kaiserhof, St. Pölten 2017 (= Menschen und Denkmale, Katalog des NÖ Landesmuseums NF 537).

Amon, K. (Hg.), Die Bischöfe von Graz-Seckau 1218-1968, GrazWien-Köln 1969.

AsPernig, W., Beiträge zur Besitzgeschichte des Schlosses Irnharting, in: Jahrbuch des Musealvereines Wels 17 (1970/71), S. 56-62.

- Der Wiener Bürgermeister Jakob Starch und die Storchen zu Klaus in Oberösterreich, in: AsPeRnIG, W. u.a., Wiener Bürgermeister im Spätmittelalter, Wien 1980 (= Forschungen und Beiträge zur Wiener Stadtgeschichte 7), S. 43-58.

BABINGer, F., „Bajezid Osman“ (Calixtus Ottomanus), ein Vorläufer und Gegenspieler Dschem-Sultans, in: La nouvelle Clio 3/9-10 (1951), S. 347-388.

Bachmann, A., Deutsche Reichsgeschichte im Zeitalter Friedrich III. und Max I. Mit besonderer Berücksichtigung der österreichischen Staatengeschichte, 2 Bde., Leipzig 1884/94.

- Urkundliche Nachträge zur österreichisch-deutschen Geschichte im Zeitalter Kaiser Friedrichs III., Wien 1892 (= Fontes Rerum Austriacarum, 2. Abt.: Diplomataria et Acta 46).

Baravalle, R., Burgen und Schlösser der Steiermark, Graz 1961 (ND 1995).

BARTLOVÁ S. ČORNEJ

Battenberg, F. s. Protokoll- und Urteilsbücher

Baum, W., Die Habsburger in den Vorlanden 1386-1486. Krise und Höhepunkt der habsburgischen Machtstellung in Schwaben am Ausgang des Mittelalters, Wien u.a. 1993.

- Sigmund der Münzreiche. Zur Geschichte Tirols und der habsburgischen Länder im Spätmittelalter, Bozen 1987 (= Schriftenreihe des Südtiroler Kulturinstitutes 14).

Baumert, E. H., Grüll, E., Burgen und Schlösser in Oberösterreich, Bd. 2: Innviertel und Alpenvorland, 2. erg. Aufl. Wien 1985, Bd. 3: Salzkammergut und A1penland, 2. Aufl. Wien 1983.

Begert, A., Böhmen, die böhmische Kur und das Reich vom Hochmittelalter bis zum Ende des Alten Reiches. Studien zur Kurwürde und zur staatsrechtlichen Stellung Böhmens, Husum 2003 (= Historische Studien 475).

Bellabarba, M., Il principato vescovile di Trento nel Quattrocento: 
poteri urbani e poteri signorili, in: Storia del Trentino, Bd. 3: L'età medievale, hg. v. A. $\mathrm{CA}_{\mathrm{A}}$ STAGNETTI U. G. M. VARANINI, Bologna 2004, S. 385-415.

Benna, A. H., Hut oder Krone? Ein Beitrag zur Ikonographie des Erzherzogshutes, in: MÖSTA 24 (1971), S. 87-139.

BergmanN, J., Über die Freiherren und Grafen zu Rogendorf, Freiherren auf Mollenburg, in: Sitzungsberichte der phil.hist. Klasse der Akademie der Wissenschaften Wien (1851), S. 519-626 (= Sonderdruck, 1851, S. 3-110).

BIRK, E., Urkunden-Auszüge zur Geschichte Kaiser Friedrich des III. in den Jahren 1452-1467 aus bisher unbenützten Quellen, in: AÖG 10 (1853), S. 175-240, 369-443; 11 (1853), S. 141176.

- $\quad$ s. LICHNOWSKY

BLENDINGER, F., Art. Meuting, in: NDB 17, Berlin 1994, S. 275-277.

Bock, D., Maximilian als Herzog der Niederlande (1477-1493), Diss., Graz 1970.

Bodo, I., Geschichte des Benediktinerstiftes Ossiach in Kärnten in der Zeit von 1028-1783, Diss., Wien 1966.

Böck, M., Herzöge und Konflikt. Das spätmittelalterliche Herzogtum Geldern im Spannungsfeld von Dynastie, ständischen Kräften und territorialer Konkurrenz (1339-1543), Geldern 2013 (=
Veröff. des Historischen Vereins für Geldern und Umgegend 10).

Bӧнм, C., Die Handschriften des kaiserlichen und königlichen Haus-, Hof- und Staatsarchivs, Wien 1873.

BRANDIS, C. W., Tirol unter Friedrich von Österreich, Wien 1823.

BrandL, M., Gmunden. Politischer Bezirk Gmunden, in: Die Städte Oberösterreichs, red. v. H. KNTTTLER, Wien 1968 (= Österreichisches Städtebuch 1: Oberösterreich), S. 153-167.

- Steyr. Stadt mit eigenem Statut, unter Mitarbeit v. J. OFNER, in: Die Städte Oberösterreichs, red. v. H. KNITTLER, Wien 1968 (= Österreichisches Städtebuch 1: Oberösterreich), S. 275-298.

BRANDSTÄTteR, K., Die Beziehungen zwischen Tirol und Trient im späten Mittelalter / Le relazione tra Trento e il Tirolo nel tardo medioevo, in: Studi Trentini di Scienze Storiche 1/75 (1996), S. 3-59.

- $\quad$ Reichskirche zwischen Nord und Süd: die habsburgische Politik gegenüber Brixen und Trient, in: Höfe und Residenzen geistlicher Fürsten. Strukturen, Regionen und Salzburgs Beispiel in Mittelalter und Neuzeit, hg. v. W. Wüst u.a., Ostfildern 2010 (= Residenzenforschung 24), S. 285-302 .

BRUnNer, L., Eggenburg. Geschichte einer niederösterreichischen 
Stadt, Bd. 1: Urzeit und Mittelalter, Eggenburg 1933.

Brunner, O., Die Finanzen der Stadt Wien von den Anfängen bis ins 16. Jahrhundert, Wien 1929 (= Studien aus dem Archiv der Stadt Wien $\mathrm{I} / 2$ ).

Brunner, W., Teufenbach. Ein Ort stellt sich vor, Teufenbach 1996.

Buzzi, Ch., Die Herren von Pottendorf, in: Jahrbuch der Heraldisch-Genealogischen Gesellschaft Adler 3/11 (1983), S. 75-145.

Chmel, J., Conzeptensammlung $1=$ Urkundliches zur Geschichte K. Friedrichs IV., Auszüge aus einem Kanzleibuche (ConceptenSammlung) K. Friedrichs's IV., in: AÖG 3 (1849), S. 77-157.

- Conzeptensammlung 2 = Auszüge aus einem Kanzleibuche (Concepten-Sammlung) K. Friedrichs IV. Vom Jahre 1478, in: Notizenblatt 2, Beilage zum AÖG (1852), S. 43-48, 58-64, 93-96, 110-112, 118-124, 138-144, 157-160, 172-176, 183-189, 317-320, 340-350, 361-368, 377-384.

- Materialien zur österreichischen Geschichte. Aus den Archiven und Bibliotheken gesammelt $u$. hg. v. J. Chmel, 2 Bde., LinzWien 1832-1838.

- Monumenta Habsburgica. I. Abt:: Aktenstïcke und Briefe zur Geschichte des Hauses Habsburg im Zeitalter Maximilians I., 3 Bde., Wien 1854-1858 (ND Hildesheim 1968).
- Regesta chronologico-diplomatica Friderici IV. Romanorum Regis (Imperatoris III.). Auszug aus den im k. k. geheimen Haus-, Hof- und Staats-Archive zu Wien sich befindenden Reichsregistraturbüchern vom Jahre 1440-1493. Nebst Auszügen aus Original-Urkunden, Manuskripten und Büchern (mit einem Anhang: Einige Urkunden Königs Friedrichs IV. in vollständigem Abdruck), Wien 1838 (ND Hildesheim 1962).

- Verzeichnis von 283 OriginalUrkunden im Franzens-Museum zu Brünn, die sich auf ,Oesterreich" (Erzherzogthum) beziehen, in: Notizenblatt. Beilage zum Archiv für Kunde österreichischer Geschichtsquellen 1 (1851), S. 106-111, 119-123.

CoRI, J. N., Die Grenzfehden zwischen Böhmen und Oberösterreich zur Zeit des Kaisers Friedrich III., in: Bericht über das Museum Francisco-Carolinum 44 (1886), S. 1-45.

ČorneJ, P., BARTLová M., Velké dějiny zemí koruny ceské [Große Geschichte der Länder der böhmischen Krone], Bd. 6: 14371526, Praha u.a. 2007.

Czerke, F., Hanns Haringseer. Ein Wiener Bürgermeister des 15. Jahrhunderts, in: Wiener Geschichtsblätter 11 (1956), S. 80-84.

- $\quad$ Historisches Lexikon Wien in 6 Bänden, Wien 1992-2004.

- Wien und seine Bürgermeister. 
Sieben Jahrhunderte Wiener Stadtgeschichte, Wien-München 1974.

Daim, F., KüHtreiber K. u. Th. (Hg.), Burgen Waldviertel, Wachau, Mährisches Thayatal, mit Beiträgen v. M. Jeitler u.a., 2. Aufl. Wien 2009.

DanN, W., Die Besetzung des Bistums Konstanz vom Wormser Konkordat bis zur Reformation, in: ZGO 100/NF 61 (1952), S. 3-96.

Deutsches Rechtswörterbuch (DRW), 13 Bde., Stuttgart 1914-2017.

Dienes, G. M., Die Bürger von Graz. Örtliche und soziale Herkunft (Von den Anfängen bis 1500), Graz 1979 (= Dissertationen der Universität Graz 46).

Diestelkamp, B. s. Protokoll- und Urteilsbücher

Dimitz, A., Geschichte Krains von der ältesten Zeit bis auf das Jahr 1813, T1. 1: Von der Urzeit bis zum Tode Kaiser Friedrichs III. (1493), Laibach 1874.

Doblinger, M., Die Herren von Walsee. Ein Beitrag zur österreichischen Adelsgeschichte, Wien 1906 (Separatdruck) (= AÖG 95 [1906], S. 235-578).

DöRRER, F., Die „limitierte Landeshoheit" der Bischöfe von Trient und Brixen in Beziehung zur gefürsteten Grafschaft Tirol, in: Landeshoheit. Beiträge zur Entstehung, Ausformung und Typologie eines Verfassungselements des römisch-deutschen Reiches, hg. v. E. RIEDENAUER, München 1994 (= Studien zur bayerischen Verfassungs- und Sozialgeschichte 16), S. 135-144.

Dopsch, H. (Hg.), Geschichte Salzburgs. Stadt und Land, Bd. I: Vorgeschichte, Altertum, Mittelalter, Tl. 1, 2. Aufl. Salzburg 1983.

EBNER, H., Die Bürgerfamilie Judel zu Voitsberg, in: Blätter für Heimatkunde 53 (1979), S. 9-14.

- $\quad$ (Burgen und Schlösser in der Steiermark 1:) Schlösser im Ennstal und Murboden, 2. erg. Aufl. Wien 1976.

- Burgen und Schlösser in der Steiermark 2: Mürztal und Leoben, 2. erg. Aufl. Wien 1979.

- Burgen und Schlösser in der Steiermark 3: Graz, Leibnitz, West-Steiermark, 2. erg. Aufl. Wien 1981.

- Die Herrschaft Katsch, in: PIRChegGer, H., Landesfürst und Adel in Steiermark während des Mittelalters, Bd. 2, Graz 1955 (= Forschungen zur Verfassungs- und Verwaltungsgeschichte der Steiermark 13), S. 114-129.

Енм, P., Burgund und das Reich. Spätmittelalterliche Außenpolitik am Beispiel der Regierung Karls des Kühnen (1465-1477), München 2002 (= Pariser Historische Studien 61).

Ehrenberg, R., Das Zeitalter der Fugger. Geldkapital und Kreditver- 
kehr im 16. Jahrhundert, 2 Bde., Jena 1906.

EIBL, E.-M., Zwischen Entwurf, Original und Kopie. Bemerkungen zu Formen von Urkunden und Briefen aus den Kanzleien Kaiser Friedrichs III., in: Archiv für Diplomatik 44 (1998), S. 19-41.

- $\quad$ s. Regesten Kaiser Friedrichs III.

Erfle, B., Alexander Numai, Bischof von Forlì, als Diplomat in Diensten von Papst und Kaiser (1470-1483), Diss., Marburg 2002.

ERNST, F., Eberhard im Bart. Die Politik eines deutschen Landesherrn am Ende des Mittelalters, Stuttgart 1933.

EtTElT-SchöNEwALD, B., Kanzlei, Rat und Regierung Herzog Ludwigs des Reichen von Bayern-Landshut (1450-1479), 2 Teilbde., München 1996/99 (= Schriftenreihe der Bayerischen Landesgeschichte 97/I, 97/II).

FALKe, Jacob, Geschichte des fürstlichen Hauses Liechtenstein, Bd. 1, Wien 1868.

FeIGL, H., Geschichte des Marktes und der Herrschaft Trautmannsdorf an der Leitha, Wien 1974 (= Forschungen zur Landeskunde von Niederösterreich 20).

Feine, H. E., Kirchliche Rechtsgeschichte, Bd. 1: Die katholische Kirche, 5. Aufl. Köln-Wien 1972.

- Die Territorialbildung der Habsburger im deutschen Südwesten vornehmlich im späten Mittel- alter, in: ZRG Germanistische Abt. 67 (1950), S. 176-308.

Felser, R., Herkunft und soziale Schichtung der Bürgerschaft obersteirischer Städte und Märkte während des Mittelalters. Unter besonderer Berücksichtigung der Bürger der Stadt Judenburg, Wien 1977 (= Dissertationen der Universität Graz 38).

FrÄss-EhrFeld, C., Geschichte Kärntens, Bd. 1: Das Mittelalter, Klagenfurt 1984.

Fraknó, W., Mathias Corvinus, König von Ungarn 1458-1490, Freiburg im Breisgau 1891.

Fuchs, A. (Bearb.), Urkunden und Regesten zur Geschichte des Benedictinerstiftes Göttweig, 3. T1.: 1468-1500, Wien 1902 (= Fontes Rerum Austriacarum, 2. Abt.: Diplomataria et Acta 55).

FÜRSTENBERGISCHES URKUNDENBUCH. Sammlung der Quellen zur Geschichte des Hauses Fürstenberg und seiner Lande in Schwaben, Bde. 4 u. 7, Tübingen 1879/91.

Furs, M., Hermann IV. von Hessen, Erzbischof von Köln (1480 1508), Köln u.a. 1995 (= Kölner Historische Abhandlungen 40).

GATz, E. (Hg.), Bischöfe 1 = Die Bischöfe des Heiligen Römischen Reiches 1198 bis 1448. Ein biographisches Lexikon, Berlin 2001.

- $\quad$ Bischöfe 2 = Die Bischöfe des Heiligen Römischen Reiches 
1448 bis 1648 . Ein biographisches Lexikon, Berlin 1996.

GefFCken, P., Schmölz-Häberlein

M., Art. Meuting (Meiting, Mütting), Kaufmanns-, Patrizierfamilie, in: Stadtlexikon Augsburg: https://www.wissner. com/stadtlexikon-augsburg/arti$\mathrm{kel} /$ stadtlexikon/meuting/4783 (abgerufen am 11.10.2019) (= Augsburger Stadtlexikon. Geschichte, Gesellschaft, Kultur, Recht, Wirtschaft, 2. Aufl. 1998).

Germania Benedictina II/3: Die Männer- und Frauenklöster der Benediktiner in Bayern, München 2014.

Gismann, R., Die Beziehungen zwischen Tirol und Bayern am Ausgang des Mittelalters. Herzog Sigmund der Münzreiche und die Wittelsbacher in Landshut und München von 1439 bis 1479, Diss., Innsbruck 1976.

GNeIss, Markus, Das Wiener Handwerksordnungsbuch (13641555). Edition und Kommentar, Wien 1917 (= QIÖG 16).

GöBEL, W., Entstehung, Entwicklung und Rechtsstellung geistlicher Territorien im deutsch-italienischen Grenzraum. Dargestellt am Beispiel Trients und Aquileias, Diss., Würzburg 1976.

Göller, E., Sixtus IV. und der Konstanzer Bistumsstreit (14741480), in: Freiburger Diözesanarchiv 52/NF 25 (1924), S. 1-60.
Göтн, G., Urkunden-Regesten für die Geschichte von Steiermark vom Jahre 1252 bis zum Jahre 1580, in: MHVSt 9 (1859), S. 283-304, MHVSt 10 (1861), S. 314-336.

Graf, K., Kunigunde, Erzherzogin von Österreich und Herzogin von Bayern-München (1465-1520) - Eine Biographie, Diss., Mannheim 2000.

Grimm, J. u. W., Deutsches Wörterbuch, 16 Bde. in 32 Teilbänden, Leipzig 1854-1961, Quellenverzeichnis 1971.

Gross, L. (Bearb.), Urkunden und Aktenstücke des Reichsarchivs Wien zur reichsrechtlichen Stellung des burgundischen Kreises, Bd. 1, unter Mitwirkung v. R. LACroIx, Wien 1944 (= Veröff. des Reichsarchivs Wien).

GRÜLL S. BAUMERT

Gubo, A., Geschichte der Stadt Cilli vom Ursprung bis auf die Gegenwart, Graz 1909.

HäRTEL, R., Die Kainacher. Genealogie, Besitz, Ämter und Urkunden des steirischen Geschlechtes, Diss., Graz 1969.

Hageneder, O., Kanonisches Recht, Papsturkunde und Herrscherurkunde. Überlegungen zu einer vergleichenden Diplomatik am Beispiel der Urkunden Friedrichs III., in: Archiv für Diplomatik 42 (1996), S. 419-443.

- Die Rechtskraft spätmittelalterlicher Papst- und Herrscherurkunden „ex certa scientia“, 
„,non obstantibus“ und ,propter importunitatem petentium“, in: Papsturkunde und europäisches Urkundenwesen. Studien zu ihrer formalen Kohärenz vom 11. bis 15. Jahrhundert, hg. v. P. HeRdE u. H. JAKOBS, Köln u.a. 1999, S. 401-429.

Haller-Reiffenstein, B., Kaiser Friedrich III. und Andreas Baumkircher, in: Andreas Baumkircher und seine Zeit. „Schlaininger Gespräche 1982“, red. v. R. Kropf u. W. Meyer, Eisenstadt 1983 (= Wissenschaftliche Arbeiten aus dem Burgenland 67), S. 63-104.

- Ulrich von Grafeneck und seine Nachkommen - ein Parallelfall?, in: Andreas Baumkircher Erben und Nachfolger. „Schlaininger Gespräche 1989“, bearb. v. U. Döcker u. R. KROPF, Eisenstadt 1992 (= Wissenschaftliche Arbeiten aus dem Burgenland 88), S. 117-154.

Hauser, E., Geschichte der Freiherren von Raron, in: Schweizer Studien zur Geschichtswissenschaft 8 (1915/16), S. 363-567 (= Schweizerische Studien 8/2 [1916], S. 1-205).

HeINIG, P.-J. Kaiser Friedrich III. (1440-1493). Hof, Regierung und Politik, 3 Bde., Köln u.a. 1997 (= Forschungen zur Kaiser- und Papstgeschichte des Mittelalters, Beihefte zu J. F. Böhmer, Regesta Imperii 17).

- Kaiser, Reich und Burgund.
Habsburgs ,neue Westpolitik“ im 15. Jahrhundert, in: Zeitschrift des Aachener Geschichtsvereins 106 (2004), S. 55-76.

- Zur Kanzleipraxis unter Kaiser Friedrich III. (1440-1493), in: Archiv für Diplomatik 31 (1985), S. 383-442.

- Die Türhüter und Herolde Kaiser Friedrichs III. Studien zum Personal deutscher Herrscher im 15. Jahrhundert, in: Kaiser Friedrich III. (1440-1493) in seiner Zeit, hg. v. P.-J. HeInIG, Köln u.a. 1993 (= Forschungen zur Kaiser- und Papstgeschichte des Mittelalters, Beihefte zu J. F. Böhmer, Regesta Imperii 12), S. 355-375.

- $\quad$ s. Regesten Kaiser Friedrichs III. Helvetia Sacra

- Abt. I, Bd. 1: Schweizerische Kardinäle. Das apostolische Gesandtschaftswesen in der Schweiz. Erzbistümer und Bistümer I (A-Ch), red. v. A. BRUCKNER, Bern 1972.

- $\quad$ Abt. I, Bd. 2: Erzbistümer und Bistümer II. Das Bistum Konstanz. Das Erzbistum Mainz. Das Bistum St. Gallen, T1. 1, red. v. B. Degler-Spengler, Basel-Frankfurt am Main 1993.

- $\quad$ Abt. III, Bd. 1: Frühe Klöster, die Benediktiner und Benediktinerinnen in der Schweiz, T1. 3, red. v. E. GiLOMEN-SCHENKEL, Bern 1986.

Herold, P., Das Ringen um den Text. Die Lehensurkunden von 
1446/47 für Herzog Philipp von Burgund als Beispiel für Genese, Wirkungsweise und Scheitern von Urkundentexten, in: Vom Nutzen des Schreibens. Soziales Gedächtnis, Herrschaft und Besitz im Mittelalter, hg. v. W. PoHL u. P. Herold, Wien 2002 (= Forschungen zur Geschichte des Mittelalters $5=$ Denkschriften der phil.-hist. Klasse der Österreichischen Akademie der Wissenschaften 306).

- $\quad$ s. Regesten Kaiser Friedrichs III. Hildbrand, Th., Herrschaft, Schrift und Gedächtnis. Das Kloster Allerheiligen und sein Umgang mit Wissen in Wirtschaft, Recht und Archiv (11.-16. Jahrhundert), Zürich 1996.

Hirsch, V., Der Hof des Basler Bischofs Johannes von Venningen (1458-1478). Verwaltung und Kommunikation, Wirtschaftsführung und Konsum, Ostfildern 2004 (= Residenzenforschung 16).

Hoensch, J. K., Matthias Corvinus. Diplomat, Feldherr und Mäzen, Graz u.a. 1998.

HofACKER, H.-G., Die schwäbischen Reichslandvogteien im späten Mittelalter, Stuttgart 1980 (= Spätmittelalter und Frühe Neuzeit, Tübinger Beiträge zur Geschichtsforschung 8).

Hoffmann, A., Die Salzmaut zu Sarmingstein in den Jahren 148087, in: MIÖG (1954), S. 447-
459 (ND in: DERs., Studien und Essays, Bd. 2: Österreich und das Land ob der Enns, Wien 1981, S. 188-204).

- Die Weinfuhren auf der österreichischen Donau in den Jahren 1480-87, in: Aus Verfassungs- und Landesgeschichte. Festschrift zum 70. Geburtstag von Theodor Mayer, Bd. 2, Sigmaringen 1955, S. 329-345 (ND in: DERS., Studien und Essays, Bd. 2: Österreich und das Land ob der Enns, Wien 1981, S. 216238).

Hollweg, W., Dr. Georg Heßler. Ein kaiserlicher Diplomat und römischer Kardinal des 15. Jahrhunderts. Versuch einer Biographie, Leipzig 1907.

Hollwöger, F., Das Ausseer Land. Geschichte der Gemeinden Bad Aussee, Altaussee, Grundlsee, Mitterndorf und Pichl, Bad Aussee 1956.

HoLtz, E., Kaiser Friedrich III. (14401493) und die Länder der böhmischen Krone (Böhmen, Mähren, Schlesien), in: Jahrbuch für die Geschichte Mittel- und Ostdeutschlands 59 (2013), S. 23-58.

- $\quad$ s. Regesten Kaiser Friedrichs III. HÜBLER, H., Ritter Hans Steger, Bürgermeister von Wien, in: ASPERNIG, W. u.a., Wiener Bürgermeister im Spätmittelalter, Wien 1980 (= Forschungen und Beiträge zur Wiener Stadtgeschichte 7), S. 27-42. 
Hurnagl, F., Die Maut zu Gmunden. Entwicklungsgeschichte des Salzkammergutes, Wien u.a. 2008.

HugGLE, F., Geschichte der Stadt Neuenburg am Rhein, 3 Tle., Freiburg im Breisgau 1876-1881.

IgÁLFFY-IGÁLY, L., Das slwowenische Geschlecht der Obritschan von Altenburg und seine europäischen Verflechtungen, in: Adler. Zeitschrift für Genealogie und Heraldik 20 (XXXIV) (19992000), S. 98-106.

IsENMANN, E., Kaiserliche Obrigkeit, Reichsgewalt und ständischer Untertanenverband. Untersuchungen zu Reichsdienst und Reichspolitik der Stände und Städte in der zweiten Hälfte des 15. Jahrhunderts, Habil.-Schr., Tübingen 1983.

JäGER, A., Geschichte der landständischen Verfassung Tirols, Bd. 2/2: Die Blütezeit der Landstände Tirols. Von dem Tode des Herzogs Friedrich mit der leeren Tasche 1439 bis zum Tode des Kaisers Maximilian I. 1519, Innsbruck 1885 (ND 1970).

JäGer-Sunstenau, H., Das Archiv der Stadt Klosterneuburg, Klosterneuburg 1962.

JANSSEN J., Frankfurts Reichscorrespondenz nebst anderen verwandten Aktenstücken von 1376-1519, Bd. 2, Abt. 1: Aus der Zeit Kaiser Friedrichs III. bis zur Wahl König Maximilians
1440-1486, Freiburg im Breisgau 1866.

JANSSEN, W., Der Verzicht des Erzbischofs Ruprecht von der Pfalz auf das Erzbistum Köln um die Jahreswende 1478/79, in: Köln. Stadt und Bistum in Kirche und Reich des Mittelalters. Festschrift für Odilo Engels zum 65. Geburtstag, hg. v. H. VolLRATH u. St. Weinfurter, Köln u.a. 1993 (= Kölner Historische Abhandlungen 39).

JECKLIN, F. (Hg.), Materialien zur Standes- und Landesgeschichte Gemeiner III Bünde (Graubünden) 1464-1803, Tl. 1: Regesten, Tl. 2: Texte, Basel 1907-1909.

KaISERURKunden IN ABbildungen, Text- u. Tafelbd., hg. v. Heinrich v. Sybel u. Th. Sickel, Berlin 1880-1891.

Kalous, A., Matyáš Korvín (14431490). Uherský a český král [Matthias Corvinus. Ungarischer und böhmischer König], Česke Budějovice 2009.

Keiblinger, I. F., Die Burg Aggstein in Österreich im Kreise ob dem Wienerwald, in: Berichte und Mittheilungen des AlterthumsVereines zu Wien 7 (1864), S. 1-98.

Klein, A. A., Zur Geschichte der Türkeneinfälle in Steiermark während der Regierung Friedrichs III., in: ZHVStmk 19 (1924), S. 103-125.

KNoLLE, U., Studien zum Ursprung und zur Geschichte des Reichsfiska- 
lats im 15. Jahrhundert, München 1965.

KoнLA, F. X., Kärntner Burgenkunde 1 = Kärntens Burgen, Schlösser, Ansitze und wehrhafte Stätten. Ein Beitrag zur Siedlungstopographie. Mit Ergänzungen, Exkursen und Nachträgen v. G. Moro, 2. erg. Aufl. Klagenfurt 1973 (= Aus Forschung und Kunst 17/1).

Kollar s. Ursinus Velius

Koller, H., Zur Bedeutung der eigenhändigen Briefe Kaiser Friedrichs III., in: Geschichte der Zentraljustiz in Mitteleuropa. Festschrift für Bernhard Distelkamp zum 65. Geburtstag, hg. v. F. BATTENBERG U. F. RANIERI, Weimar u.a. 1994, S. 119-129.

- Die Probleme der Regierung Kaiser Friedrichs III., in: Österreich in Geschichte und Literatur mit Geographie 34/3 (1990), S. 144-152.

- $\quad$ s. Regesten Kaiser Friedrichs III. KorAK, B., Burggrafen und Burgpfleger in Kärnten bis zum Jahr 1500, Diss., Wien 1984.

Kos, D., In Burg und Stadt. Spätmittelalterlicher Adel in Krain und Untersteiermark, Wien-München 2006 (=VIÖG 45).

KovÁcs, P. E., Ungarn im Spätmittelalter (1382-1526), in: Geschichte Ungarns, hg. v. I. G. TóTH, Budapest 2005, S. 143-223.

Krackowizer, F., Geschichte der Stadt Gmunden in Ober-Oesterreich, Bd. 2, Gmunden 1899.
KrammL, P. F., Kaiser Friedrich III. und die Reichsstadt Konstanz (1440-1493). Die Bodenseemetropole am Ausgang des Mittelalters, Sigmaringen 1985 (= Konstanzer Geschichts- und Rechtsquellen 29).

- Salzburg und die Ungarn. Fakten, Lügen, Propaganda, in: Stadt, Land und Kirche. Salzburg im Mittelalter und in der Neuzeit. Beiträge der Tagung zur Emeritierung von Heinz Dopsch in Salzburg vom 13. bis 24. September 2011, Salzburg 2012 (= Salzburg Studien 13), S. 113-136.

Krieger, K.-F., Der Prozeß gegen Pfalzgraf Friedrich den Siegreichen auf dem Augsburger Reichstag vom Jahre 1474, in: ZHF 12 (1985), S. 257-286.

- $\quad$ Eine bisher unbekannte Quelle zum Prozeß Kaiser Friedrichs III. gegen den Pfalzgrafen Friedrich den Siegreichen (1474), in: Palatinus semper illustrandus. Beiträge zur Geschichte, Literatur, Volkskunde und Geographie der Kurpfalz. Festschrift zum 65. Geburtstag von Hansjörg Probst, hg. v. H. WIEGAND, Sigmaringen 1997 (= Mannheimer Geschichtsblätter NF 4).

- Rechtliche Grundlagen und Möglichkeiten römisch-deutscher Königsherrschaft im 15. Jahrhundert, in: Das spätmittelalterliche Königtum im europäischen Vergleich, hg. v. R. 
SCHNEIDER, Sigmaringen 1987

(= Vorträge und Forschungen 32), S. 465-489.

- $\quad$ s. Regesten Kaiser Friedrichs III.

KüHtreiber K. u. Th., Mochty Ch., WeLtin, M., Wehrbauten und Adelssitze Niederösterreichs. Das Viertel unter dem Wienerwald: Bd. 1, St. Pölten 1998 (= Sonderreihe der STUF 1).

- $\quad$ s. Daim; Mochty-Weltin; REICHHALTER

KunNert, H., Beiträge zur Geschichte des Bergbaues im Berggerichtsbezirk Schladming in den Jahren 1304 bis 1616, Diss., Wien 1927.

- Zur Geschichte des Schladminger Bergbaues, in: Die Stadt Schladming. Festschrift zur 50. Wiederkehr der zweiten Stadterhebung, koordiniert v. W. STIPPERGER, Schladming 1975, S. $45-53$.

Kurz, F., Oesterreich unter Kaiser Friedrich dem Vierten, 2 Bde., Wien 1812.

LACKNER, Ch., Vom Herzogtum zum Erzherzogtum Österreich, in: MIÖG 112 (2004), S. 290-305.

- Rat, Kanzlei und Regierung der österreichischen Herzoge (1365-1406), Wien-München 2002 (= MIÖG Erg.-Bd. 41).

- $\quad$ s. Regesten Kaiser Friedrichs III.

LADURner, J., Die Vögte von Matsch, später auch Grafen von Kirchberg, 3. Abt., in: Zeitschrift des Ferdinandeums für Tirol und Vorarlberg, 3. Folge, H. 18 (1873), S. 5-158.

LeIthner, A. F., Versuch einer Monographie über die k.k. Kreisstadt Judenburg und ihren Pfarrbezirk nebst Schilderung einiger der nächsten Umgebungen. Ein Gedenkbuch nach bewährten Quellen, Wien 1840.

Leitner, F. W., Die Herren von Ernau. Eine Genealogie, in: Kärntner Landesgeschichte und Archivwissenschaft. Festschrift für A. Ogris zum 60. Geburtstag, hg. v. W. WADL, Klagenfurt 2001 (= Archiv für vaterländische $\mathrm{Ge}$ schichte und Topographie 84), S. 191-214.

Lichnowsky, E. M., Geschichte des Hauses Habsburg. Regesten v. E. Birk, 8 Bde., Wien 1836 1844.

LÜNIG, J. C., Das Teutsche Reichs-Archiv, Bd. 7, Leipzig 1711.

Luger, D., „Daz ... unser gedechtnuß dest lennger und seligclicher gehalten werde." Die Bestätigung des Privilegium maius durch Kaiser Friedrich III., in: Privilegium maius. Autopsie, Kontext und Karriere der Fälschungen Rudolfs IV. von Österreich, hg. v. Th. Just u.a., Wien u.a. 2018 (= VIÖG 69 = MÖSTA Sonderbd. 15).

- Humanismus und humanistische Schrift in der Kanzlei Kaiser Friedrichs III. (1440-1493), Wien 2016 (= MIÖG Erg.-Bd. 60). 
- $\quad$ s. Regesten Kaiser Friedrichs III.

Luschin-EBengreuth s. PopelKa

MARIAN, G., Studien zum mittelalterlichen Adel im Tullnerfeld, St. Pölten 2017 (= Forschungen zur Landeskunde von Niederösterreich 39).

Marian, G., Zehetmayer, R., Hardegg (A., B.), in: Höfe und Residenzen im spätmittelalterlichen Reich. Grafen und Herren, Teilbd. 1, hg. v. W. PARAVICINI, Ostfildern 2012 (= Residenzenforschung 15/IV), S. 559-573.

Maurer, R., Die Burg Baden. Ihre Herren - ihre Herrschaft, Baden 2006 (= Katalogblätter des Rollettmuseums Baden 61).

MAYER, F. M., Über die Abdankung des Erzbischofs Bernhard von Salzburg und den Ausbruch des dritten Krieges zwischen Kaiser Friedrich III. und König Mathias von Ungarn (1477-1481), in: AÖG 55 (1877), S. 169-246.

MAYER, J., Geschichte von Wiener Neustadt, Bd. I, Tl. 2: Eine Glanzperiode der Stadt (1440-1500), Wiener Neustadt 1926.

MeINDL, K., Geschichte der Stadt Wels in Oberösterreich, 2 Bde., Wels 1878.

MelL, A., Regesten zur Geschichte der Familien von Teufenbach in Steiermark, T1. 1: 1074-1547, Graz 1905 (= Veröff. der Historischen Landes-Kommission für Steiermark 20).

MERK, W. (Bearb.), Oberrheinische Stadtrechte 2. Abt.: Schwäbi- sche Rechte, 3. Heft: Neuenburg am Rhein, Heidelberg 1913.

Metnitz, G. A., Kärntner Burgenkunde 2 = Quellen- und Literaturhinweise zur geschichtlichen und rechtlichen Stellung der Burgen, Schlösser und Ansitze in Kärnten sowie ihrer Besitzer, Klagenfurt 1973 (= Aus Forschung und Kunst 17/2).

Milbradt, H., Die Parteien in ihren Prozessen vor König und königlichem Kammergericht in der 2. Hälfte des 15. Jahrhunderts, Diss., Mainz 1979.

Miris, O., Die schwäbischen Herren von Wehingen in Österreich ein Beispiel für Familienwanderung im Mittelalter, in: Jahrbuch für Landeskunde von Niederösterreich NF 23 (1930), S. 76-92. Mochty-Weltin, Ch., Kühtreiber K. u. Th., ZehetmaYer, A., Wehrbauten und Adelssitze Niederösterreichs. Das Viertel unter dem Wienerwald: Bd. 3, St. Pölten 2014 (= Sonderreihe der STUF 3).

- $\quad$ S. KÜHTREIBER

Monumenta historica ducatus CARINTHIAE (MC). Geschichtliche Denkmäler des Herzogtums Kärnten, Bd. 11: Die Kärntner Geschichtsquellen 1414-1500, hg. v. H. WIESSNER, Klagenfurt 1972.

Muchar, A., Geschichte des Herzogthums Steiermark, Bd. 8: Geschichte der Steiermark 
als Herzogthum in den Jahren 1458-1558, Graz 1867.

- $\quad$ Urkunden-Regesten für die Geschichte Innerösterreichs vom Jahre 1312 bis zum Jahre 1500 , in: AÖG 2 (1849), S. 429-510.

MÜLleR, H., Warum nicht einmal die Herzöge von Burgund das Königtum erlangen konnten, in: DERS., Frankreich, Burgund und das Reich im späten Mittelalter. Ausgewählte Aufsätze, hg. v. G. ANNAS u.a., Tübingen 2011 (= Spätmittelalter, Humanismus, Reformation 56), S. 421-461.

Müllner, J., Die Herren von Kattau, die ersten Besitzer der Herrschaft Kattau, in: Heimatkundliches Jahrbuch des Waldviertler Heimatbundes 2 (1978/79), S. $87-110$.

NeHring, K., Matthias Corvinus, Kaiser Friedrich IIII. und das Reich. Zum hunyadisch-habsburgischen Gegensatz im Donauraum, München 1975 (= Südosteuropäische Arbeiten 72).

- Quellen zur ungarischen Außenpolitik in der zweiten Hälfte des 15. Jahrhunderts, Tl. I: Briefe und Urkunden (Regesten), Tl. II: Verträge (Quellen- und Editionshinweise), in: Levéltári Közlemények [Archivalische Mitteilungen] 47 (1976), S. 87-120 (T1. I), 247-268 (T1. II).

Neidhart, H., Aus der Geschichte Pöggstalls. Von den Anfängen bis zur Gegenwart, Pöggstall 2007.
Neumann, D., Das Kärntner Lesachtal. Werden und Wandlungen einer bergbäuerlichen Kultur- und Wirtschaftslandschaft, 2 . verbesserte Aufl. Klagenfurt 1987 (= Das Kärntner Landesarchiv $6)$.

Neunlinger, L., Beiträge zur Geschichte der Herrschaft Pöggstall, Diss., Wien 1969.

NIEDERSTÄTTER, A., Das Jahrhundert der Mitte. An der Wende vom Mittelalter zur Neuzeit, Wien 1996 (= Österreichische Geschichte, hg. v. H. Wolfram).

- $\quad$ s. Regesten Kaiser Friedrichs III.

Orožen, I., Das Bisthum und die Diözese Lavant, Bd. 7: Das Dekanat Rohitsch, Marburg 1887.

Otorepec, B., Gradivo za zgodovino Ljubljane v srednjem veku, H. VI.: listine 1444-1499, Ljubljana 1961.

PALAckÝ, F., Geschichte von Böhmen, größtentheils nach Urkunden und Handschriften, Bd. 5/1: Das Zeitalter der Jagelloniden, 1. Abt.: K. Wladislaw II von 1471 bis 1500 , Prag 1865.

Paravicin, W., „Mon souverain seigneur", in: Power and Persuasion. Essays on the Art State Building in Honour of W. P. Blockmans, hg. v. P. HOPPENBRouWERs, A. JANSE u. R. STEIN, Turnhout 2010, S. 27-48.

Perger, R., Der Hohe Markt, WienHamburg 1970 (= Wiener Geschichtsbücher 3).

- $\quad$ Klosterneuburg im Mittelalter, 
in: Klosterneuburg. Geschichte und Kultur, Bd. 1: Die Stadt, hg. v. F. Röhrig, G. Otruba u. M. Duscher, Klosterneuburg-Wien 1992, 139-208.

- Simon Pötel und seine Handelsgesellschaft, in: Jahrbuch des Vereins für Geschichte der Stadt Wien 40 (1984), S. 7-88.

- Straßen, Türme und Basteien. Das Straßennetz der Wiener City in seiner Entwicklung und seinen Namen. Ein Handbuch, Wien 1991.

- Die Wiener Ratsbürger 1396 bis 1526. Ein Handbuch, Wien 1988 (= Forschungen und Beiträge zur Wiener Stadtgeschichte 18).

Petersohn, J., Kaiserlicher Gesandter und Kurienbischof. Andreas Jamometić am Hof Papst Sixtus' IV. (1478-1481). Aufschlüsse aus neuen Quellen, Hannover 2004 (= MGH Studien und Texte 35).

- Zum Personalakt eines Kirchenrebellen. Name, Herkunft und Amtssprengel des Basler Konzilsinitiator Andreas Jamometić († 1484), in: ZHF 13 (1986), S. 1-14.

Pirchegger, H., Die Herren von Pettau, in: ZHVStmk 42 (1951), S. 3-36.

- Landesfürst und Adel in Steiermark während des Mittelalters, Bd. 2, 3, Graz 1955/58 (= Forschungen zur Verfassungs- und Verwaltungsgeschichte der Steiermark 13, 16).
- Die Untersteiermark in der Geschichte ihrer Herrschaften und Gülten, Städte und Märkte, München 1962 (= Buchreihe der Südostdeutschen Historischen Kommission 10).

\section{- $\quad$ S. PopelKa}

Popelka, F., Geschichte der Stadt Graz, Bd. 1: mit dem Häuserund Gassenbuch der inneren Stadt Graz v. A. Luschin-EBENGREUTH, Bd. 2: mit dem Häuserund Gassenbuch der Vorstädte am rechten Murufer v. H. PIRCHEGGER, 2. Aufl. Graz 1959/60.

PraY, G., Annales regum Hungariae ab anno Christi 997 ad annum 1564, Bd. 4: Complectens res gestas ab coronatione Mathiae Corvini ad Ludovicum II., Wien 1762.

Press, V., Eberhard im Bart von Württemberg als Graf und Fürst des Reiches, in: Eberhard und Mechthild. Untersuchungen zur Politik und Kultur im ausgehenden Mittelalter, hg. v. H.-M. MAURER, Stuttgart 1994 (= Lebendige Vergangenheit, Schriftenreihe des Württembergischen Geschichts- und Altertumsvereins 17), S. 9-34.

Prickler, H., Geschichte der Herrschaft Bernstein, Eisenstadt 1960 (= Burgenländische Forschungen 41).

Priebatsch, F. (Hg.), Politische Correspondenz des Kurfürsten Albrecht Achilles, Bd. 2: 1475-1480, Leipzig 1897 (= 
Publicationen aus den k. Preußischen Staatsarchiven 67).

Pritz, F. X., Beschreibung und Geschichte der Stadt Steyr und ihrer nächsten Umgebung, nebst mehreren Beilagen betreffend die Geschichte der Eisengewerkschaft und der Klöster Garsten und Gleink, Linz 1837.

- Geschichte des einstigen Collegiatstiftes weltlicher Chorherren zu Spital am Pyrn im Lande ob der Enns, in: AÖG 10 (1853), S. 241-328.

- Geschichte des Landes ob der Enns von der ältesten bis zur neuesten Zeit, 2 Bde., Linz 1846/47.

Probszt, G., Die Brüder Prueschenk, in: Oberösterreichische Heimatblätter 14, H. 2 (1960), S. 115127.

Die Protokoll- und URTEILSBÜChER des Königlichen Kammergerichts aus den Jahren 1465 bis 1480. Mit Vaganten und Ergänzungen, 3 Bde., hg. v. F. BATTENBERG u. B. DiestelKamp, bearb. v. C. Helm, Ch. Magin, J. MAurer u. Ch. WAGNer, Köln u.a. 2004 (= Quellen und Forschungen zur höchsten Gerichtsbarkeit im alten Reich 44).

QUELlen ZUR Geschichte DER STADT WIEN (QGStW), hg. v. Alterthumsvereine zu Wien (später: Verein f. Geschichte der Stadt Wien):

- $\quad$ I/5 = Abt. 1: Regesten aus inund ausländischen Archiven mit Ausnahme des Archives der Stadt Wien, Bd. 5, bearb. v. J. LAMPEL u. K. UhLIRZ, Wien 1906.

- $\quad \quad I I / 3=$ Abt. 2 : Regesten aus dem Archiv der Stadt Wien, Bd. 3: Verzeichnis der Originalurkunden des städtischen Archives 1458-1493, bearb. v. K. UHLIRZ, Wien 1904.

RaUSCH, K., Die burgundische Heirat Maximilians I., quellenmäßig dargestellt, Wien 1880.

RÁzsó, G., Die Feldzüge des Königs Matthias Corvinus in Niederösterreich 1477-1490, 3. Aufl. Wien 1982 (= Militärhistorische Schriftenreihe 24).

REGESTA CHRONOLOGICO-DIPLOMATICA Friderici IV. S. CHMEL

Regesta ePiscoporum ConstantienSIUM (REC), Regesten zur Geschichte der Bischöfe von Konstanz, von Bubulcus bis Thomas Berlower (517-1496), Bd. 5: 1474-1480, bearb. v. K. J. RIEDER, Innsbruck 1931.

Regesta ImperiI XI. Die Urkunden Kaiser Sigmunds (1410-1437), 2 Bde., verzeichnet v. W. ALtMANN, Innsbruck 1896-1900 (ND Hildesheim 1968).

REGESTA IMPERII XII. Albrecht II. 14381439, bearb. v. G. HöDL, Wien u.a. 1975.

REgesta IMPERII XIV. Ausgewählte Regesten des Kaiserreiches unter Maximilian I. 1493-1519, Bd. 4/1: Maximilian I. 1502-1504, bearb. v. H. WIESFleckeR, I. 
WIESFLECKER-FRIEDHUBER U. M.

HollegGeR, unter Mitwirkung

v. Ch. BeER, Wien u.a. 2002.

Regesten Kaiser Friedrichs III. (1440-1493), nach Archiven und Bibliotheken geordnet, hg. v. (ab H. 28 begründet v.) $\mathrm{H}$. Koller, P.-J. Heinig (ab H. 9), A. NiederstätTer (ab H. 13) u. Ch. Lackner (ab H. 28):

- $\quad$ H. 2: Urkunden und Briefe aus Klosterarchiven im Bayerischen Hauptstaatsarchiv (München), bearb. v. C. E. JANOTTA, Wien u.a. 1983.

- $\quad$ H. 4: Die Urkunden und Briefe aus dem Stadtarchiv Frankfurt am Main, bearb. v. P.-J. HeINIG, Wien u.a. 1986.

- $\quad$ H. 5: Die Urkunden und Briefe aus dem Hessischen Hauptstaatsarchiv Wiesbaden, bearb. v. R. NeumanN, Wien u.a. 1988.

- $\quad$ H. 6: Die Urkunden und Briefe des Kantons Zürich (vornehmlich aus dem Staatsarchiv Zürich), bearb. v. A. NIEDERSTäTTER, Wien u.a. 1989.

- $\quad$ H. 7: Die Urkunden und Briefe aus den Archiven und Bibliotheken des Regierungsbezirks Köln, bearb. v. Th. R. KRAUS, Wien u.a. 1990.

- $\quad$ H. 8: Die Urkunden und Briefe aus den Archiven der Regierungsbezirke Darmstadt und Gießen, bearb. v. D. RüBSAMEN, Wien u.a. 1993.

- $\quad$ H. 9: Die Urkunden und Briefe aus den Archiven und Biblio- theken der Regierungsbezirke Koblenz und Trier, bearb. v. R. Neumann, Wien u.a. 1996.

- $\quad$ H. 10: Die Urkunden und Briefe aus den Archiven und Bibliotheken des Landes Thüringen, bearb. v. E. HoLtz, Wien u.a. 1996.

- $\quad$ H. 11: Die Urkunden und Briefe aus den Archiven und Bibliotheken des Freistaates Sachsen, bearb. v. E.-M. EIBL, Wien u.a. 1998.

- $\quad$ H. 12: Die Urkunden und Briefe des Österreichischen Staatsarchivs in Wien, Abt. Haus-, Hofund Staatsarchiv: Allgemeine Urkundenreihe, Familienurkunden und Abschriftensammlungen (1440-1446), bearb. v. Th. WiLLICH, Wien u.a. 1999.

- $\quad$ H. 13: Die Urkunden und Briefe des Österreichischen Staatsarchivs in Wien, Abt. Haus-, Hofund Staatsarchiv: Allgemeine Urkundenreihe, Familienurkunden und Abschriftensammlungen (1447-1457), bearb. v. P. Herold u. K. HolzNer-ToBISCH, Wien u.a. 2001.

- $\quad$ H. 15: Die Urkunden und Briefe aus den Beständen „Reichsstadt" und „Hochstift" Regensburg des Bayerischen Hauptstaatsarchivs in München sowie aus den Regensburger Archiven und Bibliotheken, bearb. v. F. Fuchs u. K.-F. KRIEGER, Wien u.a. 2002.

- $\quad$ H. 16: Die Urkunden und Briefe 
aus den Archiven und Bibliotheken des Bundeslandes Sachsen-Anhalt, bearb. v. E. HoLtz, Wien u.a. 2002.

- $\quad$ H. 17: Die Urkunden und Briefe aus den Archiven und Bibliotheken der Stadt Speyer, bearb. v. J. KEMPER, Wien u.a. 2002.

- $\quad$ H. 18: Die Urkunden und Briefe des Österreichischen Staatsarchivs in Wien, Abt. Haus-, Hofund Staatsarchiv: Allgemeine Urkundenreihe, Familienurkunden und Abschriftensammlungen (1458-1463), bearb. v. S. DÜNNEBEIL u. P. HeRold, Wien u.a. 2004.

- $\quad$ H. 20: Die Urkunden und Briefe aus den Archiven und Bibliotheken der Bundesländer Berlin, Brandenburg und MecklenburgVorpommern sowie des Archiwum Państwowe w Szczecinie/ Staatsarchivs Stettin für die historische Provinz Pommern, bearb. v. E.-M. EIBL, Wien u.a. 2004.

- $\quad$ H. 22: Die Urkunden und Briefe des Österreichischen Staatsarchivs in Wien, Abt. Haus-, Hofund Staatsarchiv: Allgemeine Urkundenreihe, Familienurkunden und Abschriftensammlungen (1464-1469), bearb. v. Ch. OTTNER, Wien u.a. 2007.

- $\quad$ H. 23: Die Urkunden und Briefe aus dem Landesarchiv BadenWürttemberg, Abt. Hauptstaatsarchiv Stuttgart, Bestand A 602: Württembergische Regesten, bearb. v. P.-J. HeINIG, Wien u.a. 2007.

- $\quad$ H. 26: Die Urkunden und Briefe aus den Archiven und Bibliotheken der Tschechischen Republik, bearb. v. E. HoLtz, Wien u.a. 2012.

- $\quad$ H. 27: Die Urkunden und Briefe des Österreichischen Staatsarchivs in Wien, Abt. Haus-, Hofund Staatsarchiv: Allgemeine Urkundenreihe, Familienurkunden und Abschriftensammlungen (1470-1475), bearb. v. S. DüNNEBEIL u. D. Luger, Wien u.a. 2012.

- $\quad$ H. 29: Die Urkunden und Briefe aus den Archiven und Bibliotheken der Republik Slowenien, Tl. 1: Die staatlichen, kommunalen und kirchlichen Archive in der Stadt Laibach/Ljubljana, bearb. v. J. KeMPeR, J. VolČJAK u. M. ARMGART, Wien u.a. 2014.

- $\quad$ H. 30: Die Urkunden und Briefe des Österreichischen Staatsarchivs in Wien, Abt. Haus-, Hofund Staatsarchiv: Allgemeine Urkundenreihe, Familienurkunden und Abschriftensammlungen (1483-1488), bearb. v. P. GRETZEL, Wien u.a. 2014.

- $\quad$ H. 35: Die Urkunden und Briefe des Österreichischen Staatsarchivs in Wien, Abt. Haus-, Hofund Staatsarchiv: Allgemeine Urkundenreihe, Familienurkunden und Abschriftensammlungen (1480-1482), bearb. v. 
P. HeINICKER U. A.-K. Kunde, Wien u.a. 2019.

- $\quad$ Supplemente Kaiser und Reich in der Regierungszeit Friedrichs III. 1: Regesten zur burgundisch-niederländischen $\mathrm{Ge}-$ schichte unter Maximilian I. bis zum Tode Friedrichs III. (14771493) aus den Archives générales du Royaume/dem Algemeen Rijksarchief in Bruxelles/Brüssel, Bestand: Manuscrits divers, bearb. v. C. RotThoff-Kraus, Wien u.a. 2008.

ReichHalter, G., Kühtreiber K. u. Th., Burgen Weinviertel, mit Beiträgen von G. MARIAN u. R. ZeHETMAYER, hg. v. F. DAim, Wien 2005.

Reinle, Ch., Die Peuscher. Zum sozialen Aufstieg eines bayerischen Niederadelsgeschlechts im späten Mittelalter, in: Zeitschrift für bayerische Landesgeschichte 58 (1995), S. 901-957.

RIEBARTSCH, J., Augsburger Handelsgesellschaften des 15 . und 16 . Jahrhunderts. Eine vergleichende Darstellung ihres Eigenkapitals und ihrer Verfassung, Bergisch-Gladbach 1987.

RIEDMANN, J., Rapporti del principato vescovile di Trento con il conte del Tirolo: le cosiddette „,compattate" del 1468, in: Il princicipe vescovo Johannes Hinderbach (1465-1486) fra tardo Medioevo e Umanesimo, hg. v. I. RogGer u. M. Bellabarba, Bologna-Trient 1992 (= Pubbli- cazioni dell'Istituto di Scienze Religiose in Trento, Series maior 3), S. 119-146.

Roscher, M., Geschichte der Cistercienserabtei Viktring, Diss., Wien 1953.

Rotthoff-Kraus, C., Geldern und Habsburg zur Zeit Maximilians I. als Herzog von Burgund (1477-1492), in: Gelre - Geldern - Gelderland. Geschichte und Kultur des Herzogtums Geldern, hg. v. J. STINNER u. K.-H. Tekath, Geldern 2001, 139-144.

- $\quad$ s. Regesten Kaiser Friedrichs III. Supplemente

SABLIK, K., Korneuburg. Politischer Bezirk Korneuburg, in: Die Städte Niederösterreichs, Tl. 2: H-P, red. v. F. Goldmann, E. Oberhammer u. J. Pradel, Wien 1976 (= Österreichisches Städtebuch 4/2), S. 131-143.

SCHÄFFER, R., Die Baumkircherfehde (1469-1471), in: Andreas Baumkircher und seine Zeit. „Schlaininger Gespräche 1982“, red. v. R. KRoPf u. W. MEYER, Eisenstadt 1983 (= Wissenschaftliche Arbeiten aus dem Burgenland 67), S. 151-182.

- $\quad$ Reinprecht von Reichenburg (1434-1505). Feldhauptmann und Landeshauptmann in Steier. Die steirische Landesverwaltung um 1500, Graz 2016 (= Forschungen zur geschichtlichen Landeskunde der Steiermark 75): http://www. 
hlk.steiermark.at/cms/dokumente/12687503_97168013/ fa $872 \mathrm{~d} 0 \mathrm{a} /$ Online_Schaeffer_Reichenburg_Version_1WZ_\%282016-05-02\%29.pdf (abgerufen am 11.10.2019).

- Das Todesdatum des Christoph Ungnad. Inschrift und Relief der Tumba in Eberndorf, in: Carinthia I 168 (1978), S. 127-146.

- $\quad$ Untreue und Verrat im Urteil ihrer Zeit am Beispiel der Hinrichtung Baumkirchers und Greiseneggers (1471), in: ZHVStmk 69 (1978), S. 87-98.

SchIв, K., Geschichte der Stadt und Landschaft Schaffhausen, Schaffhausen 1972.

Schillinger-Prassl, Ch. (Bearb.), Die Rechtsquellen der Stadt Leoben Wien-Köln-Weimar 1997 (= Fontes Rerum Austriacarum, 3. Abt.: Fontes iuris 14).

SCHMÖLZ-HäBERLEIN S. GEFFCKEN

SchmuTz, C., Historisch-topographisches Lexicon von Steyermark, 4 Bde., Graz 1822/23.

Schober, K., Die Eroberung Niederösterreichs durch Mathias Corvinus in den Jahren 1482-1490, Wien 1879 (= Sonderdruck aus den Blättern des Vereines für Landeskunde von Niederösterreich, Jg. 1879).

Schuler, P.-J., Notare Südwestdeutschlands. Ein prosopographisches Verzeichnis für die Zeit von 1300 bis ca. 1520, 2 Bde., Stuttgart 1987 (= Veröff. der Kommission für geschichtliche
Landeskunde in Baden-Württemberg, Reihe B/90, 99).

Schwarz, J., Von der Mitte an den Rand: Johann Waldner (ca. 1430-1502) in den Netzwerken der Höfe Kaiser Friedrichs III. und Maximilians I., in: Grenzen des Netzwerks, hg. v. K. HrtzBLECK U. K. HÜBNER, Ostfildern 2014, S. 113-136.

- $\quad$ Ein Salzburger Kürschnersohn am Wiener Kaiserhof. Biographische Skizzen zu Johann Waldner (ca. 1430-1502), in: Salzburg Archiv 31 (2006), S. 45-64.

SCRIPTORES Rerum SILESIACARUm, Bd.

13: Politische Correspondenz Breslaus im Zeitalter des Königs Matthias Corvinus, 1. Abt.: $1469-1479$, hg. v. B. KRONTHAL u. H. WeNDT, Breslau 1893.

SeEbach S. Wiessner

SIEVERT, A., Archivalien aus Orten des Amtsbezirks Müllheim (1), in: ZGO 40/NF 1 (1886), S. m7$\mathrm{m} 31$.

Speck, D., Treffeisen J., Neuenburg am Rhein. Stadt und Landstände im vorderösterreichischen Breisgau, Neuenburg am Rhein 2000.

SPRINGER, E. u.a., M., Laxenburg - Juwel vor den Toren Wiens. Eine Ortschronik der Marktgemeinde Laxenburg, Laxenburg 2013.

Starzer, A., Geschichte der landesfürstlichen Stadt Klosterneuburg, Klosterneuburg 1900.

- Geschichte der landesfürstlichen 
Stadt Korneuburg, Korneuburg 1899.

- Die landesfürstlichen Lehen in Steiermark von 1421-1548, Graz 1902 (= Veröff. der Historischen Landes-Commission für Steiermark 17).

SteIner, R., Die Meuting in Augsburg, München 1978 (= Genealogia Boica 3/I).

Stierle, L., Die Herren von Wehingen. Ein schwäbisches Rittergeschlecht im Dienste der Grafen von Hohenberg, der Babenberger, König Ottokars II. von Böhmen und der Habsburger. Seine verschiedenen Zweige in Niederösterreich und Mähren, in Tirol und in der angestammten Heimat, Sigmaringen 1989.

TeleKI, J., Hunyadiak kora Magyarországon [Das Zeitalter der Hunyaden in Ungarn], Bd. 12, Pest 1857.

Tepperberg, Ch., Die Herren von Puchheim im Mittelalter. Beiträge zur Geschichte des landsässigen Adels von Niederösterreich, Diss., Wien 1978.

Tomascher, I., Regesten zur Geschichte Kärnten's 3, in: Archiv für vaterländische Geschichte und Topographie 8 (1863), S. 111-133.

- Urkunden-Regesten zur Geschichte Krain's 2, in: Mitteilungen des historischen Vereins für Krain 18 (1863), S. 88-90.

TREFFEISEN S. SPECK

Trotter, K., Die Burggrafen von
Lienz und zum Lueg, Innsbruck 1954 (= Schlern-Schriften 105).

Turba, G., Der Ritterstand in Österreich um die Mitte des 15. Jahrhunderts, Diss., Wien 1970.

UB FüRSTENBERG S. Fürstenbergisches Urkundenbuch

Uhlirz, K., Das Gewerbe, in: Geschichte der Stadt Wien II/2: Von der Zeit der Landesfürsten aus habsburgischem Hause bis zum Ausgange des Mittelalters, Wien 1905, S. 592-740.

- $\quad$ s. Quellen zur Geschichte der Stadt Wien

Ursinus Velius, C., De bello Pannonico libri X, hg. v. A. F. KollaR, Wien 1762.

VANCSA, M., Geschichte Nieder- und Oberösterreichs, Bd. 2: 1283 bis 1522, Stuttgart-Gotha 1927 (= Deutsche Landesgeschichten $6 / 2$ ).

VILfAN, S., Andreas Baumkircher in Krain, in: Andreas Baumkircher und seine Zeit. „Schlaininger Gespräche 1982“, red. v. R. Kropf u. W. MEYER, Eisenstadt 1983 (= Wissenschaftliche Arbeiten aus dem Burgenland 67), S. 263-289.

- Der echte Baumkircherturm und das Schicksal seines Besitzes, in: Andreas Baumkircher. Erben und Nachfolger. ,Schlaininger Gespräche 1989“, red. v. U. Döcker u. R. Kropf, Eisenstadt 1992 (= Wissenschaftliche Arbeiten aus dem Burgenland 88), S. 301-311. 
VOCHEZER, J., Geschichte des fuerstlichen Hauses Waldburg in Schwaben, 3 Bde., Kempten 1888-1907.

VYORAL-TSCHAPKA S. WIESSNER

WADL, W., Geschichte der Juden in Kärnten im Mittelalter. Mit einem Ausblick bis zum Jahr 1867, Klagenfurt 1981 (= Das Kärntner Landesarchiv 9).

WAGENDORFER, M., Eigenhändige Unterfertigungen Kaiser Friedrichs III. auf seinen Urkunden und Briefen, in: König und Kanzlist, Kaiser und Papst. Friedrich III. und Enea Silvio Piccolomini in Wiener Neustadt, hg. v. F. Fuchs, P.-J. Heinig u. M. WAGENDORFER, Wien u.a. 2013 (= Forschungen zur Kaiser- und Papstgeschichte des Mittelalters, Beihefte zu J. F. Böhmer, Regesta Imperii 32).

WaLTER, J., Das Ungeld im Herzogtume Österreich im 15. und beginnenden 16. Jahrhundert. Ein Beitrag zur Geschichte der indirekten Steuern, Diss., Wien 1941.

WeGELIN, R. J., Gründlich-Historischer Bericht von der Kayserlichen und Reichs Landvogtey in Schwaben wie auch dem Frey Kayserlichen Landtgericht auf Leutkircher Haid und in der Pirß, 2 Bde., o.O. 1755.

WeISE, E. (Hg.), Die Staatsverträge des Deutschen Ordens in Preußen im 15. Jahrhundert, Bd. 3, Marburg 1969.

Wellner, M., Klosterneuburg. Politi- scher Bezirk Wien-Umgebung, in: Die Städte Niederösterreichs, Tl. 2: H-P, red. v. F. GoLDMANN, E. Oberhammer u. J. Pradel, Wien 1976 (= Österreichisches Städtebuch 4/2), S. 113-130.

\section{WELTIN S. KÜHTREIBER}

WidmanN, H., Geschichte Salzburgs, Bd. 2: Von 1270 bis 1519 , Gotha 1909 (= Allgemeine Staatengeschichte, Abt. 3: Deutsche Landesgeschichten 9).

WIENER, M. (Bearb.), Regesten zur Geschichte der Juden in Deutschland während des Mittelalters, T1. 1, Hannover 1862.

WIESFLECKER, H., Kaiser Maximilian I. Das Reich, Österreich und Europa an der Wende zur Neuzeit, Bd. 1: Jugend, burgundisches Erbe und Römisches Königtum bis zur Alleinherrschaft 14591493, Wien 1971.

- $\quad$ s. Regesta Imperii XIV.

WIESSNER, H., Geschichte des Kärntner Bergbaus, Bd. 1: Geschichte des Kärntner Edelmetallbergbaues, Klagenfurt 1950 (= Archiv für vaterländische Geschichte und Topographie 32).

- Die Kärntner Geschichtsquellen 1414-1500, s. Monumenta historica ducatus Carinthiae.

- Die Schenken von Osterwitz. Geschichte eines durch fünf Jahrhunderte führenden Kärntner Ministerialiengeschlechtes, Klagenfurt 1977.

Wiessner, H., Seebach, G., Burgen und Schlösser in Kärnten, Bd. 2: 
Klagenfurt, Feldkirchen, Völkermarkt, 2. erg. Aufl. Wien 1980.

WiesSNER, H., VyoRAL-TSChAPKA, M., Burgen und Schlösser in Kärnten, Bd. 3: Hermagor, Spittal/ Drau, Villach, 2. erg. Aufl. Wien 1986.

WolsegGer, P., Das Urbarium der Herrschaft Gottschee vom Jahre 1574. Ein Beitrag zur Kenntnis der volkswirtschaftlichen, nationalen und politischen Verhältnisse von Gottschee bis zur definitiven Erwerbung des Ländchens durch die Auersperge, in: Mittheilungen des Musealvereines für Krain 3 (1890), S. 140-183.

Woś, J. W., Alessandro di Masovia vescove di Trento (1423-1444). Un profilo introduttivo, Trento 1990 (= Civis. Studi e testi, Supplemento 6).

WuRM, H., Die Jörger von Tollet, Linz u.a. 1955 (= Forschungen zur Geschichte Oberösterreichs 4).

ZAHN, J., Geschichte von Hernstein in Niederösterreich und den damit vereinigten Gütern Starhemberg und Emmerberg, Wien 1889 (= Hernstein in Niederösterreich, sein Gutsgebiet und das Land im weiteren Umkreis II/2).

ZAISBERGER, F., Bernhard von Rohr und Johann Beckenschlager, Erzbischof von Gran, zwei Salzburger Kirchenfürsten in der 2. Hälfte des 15. Jahrhunderts, Diss., Wien 1963.
- Briefe des Breslauers Johann Beckenschlager, Erzbischof von Gran und Administrator von Salzburg, aus den Jahren 14821484, in: Archiv für schlesische Kirchengeschichte 28 (1970) S. 153-175.

ZAJIC, A., Große Herren und Aufsteiger, Fürstendiener und Hochverräter - Bausteine zu einer Nutzungsgeschichte von Schloss und Herrschaft Pöggstall, in: Schloss Pöggstall. Adelige Residenz zwischen Region und Kaiserhof, hg. v. P. AICHINGER-ROSENBERGER U. A. ZAJIC, St. Pölten 2017 (= Menschen und Denkmale, Katalog des NÖ Landesmuseums NF 537), S. 13-51.

- $\quad$ Kaspar von Roggendorf (gest. 1506), Karrierist und Kunstliebhaber, in: Waldviertler Biographien, Bd. 2, hg. v. H. HItz u.a., Horn-Waidhofen/Thaya 2004, S. 9-32.

- $\quad$ Rog[g]endorf A., B., in: Höfe und Residenzen im spätmittelalterlichen Reich. Grafen und Herren, Teilbd. 2, hg. v. W. PARAVICINI, Ostfildern 2012 (= Residenzenforschung 15/IV), S. 1207-1219.

- $\quad$ S. AICHINGER-ROSENBERGER

ZehetMayer s. Marian; MochtyWeLtiN

ZeIBIG, H. J., Urkunden-Buch der Stadt Klosterneuburg (1298-1565) = Monumenta Claustroneoburgensia 3, in: AÖG 7 (1851), S. 309-346. 
Zernatto, E., Die Zusammensetzung des Herrenstandes in Öster- reich ob und unter der Enns von 1406-1519, Diss., Wien 1967. 


\section{Register}

Ach (vmtl. Seeache, Fluss zwischen

Mondsee [Abfluss] und Attersee

[Zufluss] bei Unterach am Attersee,

Oberösterreich) 103

Achau (ö. Mödling, Niederösterreich)

-Fischweide 6

Adelsberg (Postojna, sw. Laibach/

Ljubljana, Slowenien), Schloss

138,297

- Hauptmann zu $\sim$ und auf dem Karst s.

Jörg von Tschernembl

- Maut 297

- Pfleger s. Jörg von Lamberg

Aferham s. Afram

Affnang (Ober- und Unteraffnang, sw.

Grieskirchen, Oberösterreich), Sitz

220

Aflenzertal (Tal bei Aflenz, n. Bruck

a.d. Mur, Steiermark)

- Ungeld im 155

Afram (bei Wildon, n. Leibnitz, Steiermark) 159

Aggstein (nö. Melk, Niederösterreich), Schloss 38

Agleier (Münze) s. Aquileia

Agnellis, Ludwig de , päpstl. Notar

272

Ahnherrnschloss s. Spangstein

Aich (Dob, bei Domschale/Domžale, nö. Laibach/Ljubljana, Slowenien) 24

Aichberg, Hans von , Ritter, Hofmeister Eb. Bernhards II. von Salzburg 83

Aichelperger Hans, Pfleger von Klamm

302
Aichperger, Wolfgang, Bürgermeister von Krems und Stein 98

Albrecht II., röm.-dt. Kg. (1438-1439), $\mathrm{Kg}$. von Böhmen und Ungarn, $\mathrm{Hz}$. von Österreich (Albrecht V.) 158, 269

Albrecht VI. s. Österreich, Hz. von

Allerheiligen, Benediktinerkloster s. Schaffhausen

Almegg (sw. Steinerkirchen a.d. Traun, Oberösterreich) s. Hans Sachs zu

Altdorfer, Georg s. Chiemsee, Bf. von $\sim$

Altenburg (Vrbovec in Nazarje, nw. Cilli/Celje, Slowenien), Schloss 94

- Herren (rittermäßig) von (Altenburger)

- - Florian, Vetter der Brüder Lueger 142

- Pfleger s. Wenko von Lusthal

Altenhauser, Peter, Stallmeister Maximilians I. 55

Altfinkenstein s. Finkenstein

Altmannsdorf(Wien XII) 6

Altspaur s. Spaur

Antignana s. Tinjan

Antwerpen (Belgien), Markgrafschaft (des Reichs) 146-148

- Markgrafen von s. Karl von Burgund, Maria von Burgund, Maximilian I.

- Untertanen 147, 148

Apfaltrer, (rittermäßige Krainer) Familie

- Hans 213

Apostolischer Stuhl s. Papst 
Appenzell (Schweiz), die von (Appenzeller) 293

Aquileia (sö. Udine, Italien), Patriarchat

- Patriarch Marcus (Barbo von Venedig) von $\sim$, Kardinallegat (14701491) 55

- Diözese 73

- Münze (Agleier) 96

Aragón s. Neapel

Arenberg (bei Lipnik, ö. Treffen/Trebnje, Slowenien), (Wein-)Berg 331

Armbruster, Konrad, öff. Notar, Kler. des Bistums Konstanz 326

Arnfels (sw. Leibnitz, Steiermark), Schloss 236

- Pfleger s. Jörg Hollenegger, Jörg Metnitzer

Artois (Dép. Nord und Dép. Pas-deCalais, Frankreich), Grafschaft 146-148

- Grafen von s. Karl von Burgund, Maria von Burgund, Maximilian I.

- Untertanen 147

Asparn a.d. Zaya (nw. Mistelbach, Niederösterreich), Schloss 200, 284

- Pfleger s. Nikolesch Prothowicz

Aspersdorf s. Grafenegg

Attersee (See sw. Vöcklabruck, Oberösterreich) 103, 151

- Schloss im s. Kammer

Atzgersdorf (Wien XXIII)

- in der Eben $\mathrm{zu} \sim 6$

$\mathrm{Au}$ (bei Trieben, sö. Liezen, Steiermark)

- Gut bei der Gaysgassen 6

Au am Inn (sw. Mühldorf am Inn, Bayern), Augustiner-Chorherrenstift

- Propst, Dekan und Kapitel 251
Auer, Konrad, Ritter, Pfleger von Hornstein 291

- Willbold, Bruder Konrads 291

Auersperg (Turjak, sö. Laibach/

Ljubljana, Slowenien), Herren (rittermäßig) von (Auersperger)

- Jörg, Bruder Wilhelms 111

- Osterman 49

- Wilhelm, Ritter, Kämmerer und Rat Friedr. III. 45, 54, 55, 111, 112 156, 188, 287, 291, 318, 321

- Verwandte s. Siguna von Kraig

Augsburg (Bayern), Stadt 97

- Bürger s. Meuting

- Bürgermeister und Rat 97

- Bistum 171

- - Bischof 326

- - Domkapitel 183

Aursperger s. Auersperg

Aussee (Bad Aussee, n. Schladming, Steiermark), Markt 3, 316

- Bürger s. Kirchpuchler

- Richter, Rat und Bürger 316

- Pfarre

--Gut in der , gen. die Gnoppen 6

- Saline 3, 127

--Verweser s. Andreas Fuchsberger, Andreas Wagen

- See bei $\sim$ s. Grundlsee

- Ungeld 316

-DO 316

Austria s. Österreich

Awen (Flur) s. Ferndorf

Aychaw s. Achau

Bad Aussee s. Aussee

Bad Bellingen s. Bellingen

Bad Fischau s. Fischau

Bad Radkersburg s. Radkersburg 
Baden (Baden-Württemberg), Markgraf Georg von s. Metz, Bf. von

Baden (sw. Wien, Niederösterreich), Burg 309

- Pfleger s. Bajezid Ottman

- Teich 309

- Ungeld 309

Bakócz, Thomas s. Gran, Eb. von Bamberg (Bayern), Bistum 275, 276

- Bischof Philipp (von Henneberg) (1475-1487) 308

- Domkapitel 178

Bánfi, Nikolaus, Gespan von Pressburg 106

Barbo, Marcus s. Aquileia, Patriarch von

Basel (Schweiz), Bistum

-Bischof 64, 65

- - Johann (von Venningen) (14581478) 66

- Domherr s. Antonius von Regisheim

- Erzpriesterhof 66

-- Prokurator s. Anton Retzinger

- Hochstift 64, 66

--Dörfer s. Schliengen, Steinenstadt Baumburg (nw. Traunreut, Bayern), Augustiner-Chorherrenstift

- Propst, Dekan und Kapitel 248

Baumkircher, (Krainer) Familie

- Andreas, Freiherr von Schlaining (seit 1463) († 1471) 123, 130, 162

- Baumkircherturm s. Wippach

Bayern, Herzogtum

- Herzöge von Bayern(-Landshut) 244

- - Ludwig IX. (der Reiche) (14501479) 50,53

- - - Hofgericht (zu Landshut) 52

- - - sein Rat s. Reichart Kärgl

- Herzöge von Bayern(-München)
--Albrecht IV. (der Weise) (14651508) 50

- s. Pfalzgrafen bei Rhein

Bayezid II. s. Osmanisches Reich, Sultan von

Beck, Heinz, Einwohner von Nürnberg 275

Beckenschlager (Beckensloer), Johann s. Gran, Eb. von

Beckensloer s. Beckenschlager

Bela Peč s. Weißenfels

Bellingen (Bad Bellingen, sü. Neuenburg am Rhein, Baden Württemberg) 65

Berben s. Werbno

Berchtesgaden (sö. Bad Reichenhall, Bayern), Augustiner-Chorherrenstift

- Propst, Dekan und Kapitel 247

Bergbau s. Greifenburg, Ortenburg, Rottenstein, Schelklingen, Vellach

Berlower s. Prelokar

Bernau (Pernau) (in Fischlham, sw. Wels, Oberösterreich), Schloss

217-220

- Inhaber s. Oberhaimer

Bernstein (nö. Pinkafeld, Burgenland), Schloss 156

- Pfleger s. Sigmund Prüschenk, Konrad Zirkendorfer

Besançon (Dép. Doubs, Frankreich), Bistum 66, 171

- Domkapitel 187

Biedermannsdorf (sü. Wien, Niederösterreich)

- Teich 2

Bisamberg (Erhebung n. Wien, Niederösterreich) 299

Bišečki Vrh s. Wischberg

Bistra s. Freudenthal 
Blay, Hans, Pfleger von Grafenwarth 45

Bleiberg (Berg ö. Villach, Kärnten) 308

Bleiburg (Pliberk, sö. Völkermarkt, Kärnten), Stadt 226

- Bürger s. Fleischhacker

Bodrež (Wodresch) s. Oberwodresch Böhmen (Tschechien), Königreich (Krone) und Kurfürstentum (Erzschenkenamt) $42,69,82,87,106$, 107

- Könige von $\sim 107,108$

- - Wladislaw II., Kg. von und Ungarn $(\dagger 1516) 28,38, \mathbf{4 2}, \mathbf{8 2}, \mathbf{8 7}$, 106, 107

- - - sein Hauptmann 42

--- seine Erblande 42

- - s. auch Albrecht II., Ferdinand I., Matthias Corvinus von Ungarn,

Sigismund

- Adelige 107

- Einwohner 107

- Erzschenkenamt 82

- Kanzlei 42

- Kronvasallen 107

- Oberster Hauptmann s. Bohuslav von Schwanberg

- Oberster Münzmeister s. Benesch von Weitenmühl

- Obersthofmeister s. Bohuslav von Schwanberg

- Stände 82

- Städte 107

- Untertanen 42, 87, 107

Bösing (Pezinok, nö. Pressburg/Bratislava, Slowakei), Grafen von St. Georgen und

- Sigmund ( $\dagger$ 1492) 121

Borgia, Alfonso s. Calixt III., Papst

Boršt s. Worst
Brabandt s. Brabant

Brabant (Belgien), Herzogtum 146148

- Herzöge von s. Karl von Burgund,

Maria von Burgund, Maximilian I.

- Untertanen 147, 148

Branković, Katharina s. Cilli

Bratislava s. Pressburg

Brecljevo s. Wretzlowo

Breg s. Rann

Breslau (Wrocław, Polen), Stadt

- Frieden von $\sim(1474)$ 28, 38, 42

Bressanone s. Brixen

Brestanica s. Reichenburg

Brestovec s. Wrestovetz

Bretzla s. Wretzlowo

Brežice s. Rann

Brixen (Bressanone, Südtirol, Italien), Bistum

- Bischof Georg (Golser) von (Elekt 1464, Bf. 1471-1488, † 1489) 207, 208

Brosco (Brosto?) (vmtl. bei Rohitsch/

Rogatec, ö. Cilli/Celje, Slowenien) 303

Bruck a.d. Leitha (sö. Wien, Niederösterreich), Stadt und Schloss (Prugg) 55

Bruck a.d. Mur (Steiermark), Stadt 155

- Bürger s. Gabelhuber

Brügge (Bruck in Flandern) (Belgien), Stadt 75, 80

-DO 149

Brunn (Brunn a.d. Schneebergbahn, w. Wiener Neustadt, Niederösterreich), Burg 6

Brunner, Tibold, Bürger, Ratsherr und Stadtrichter von Graz 118, 226, 227, 230, 237, 319

Brunpeck, Bartholomäus 132 
Buchberg am Kamp (sü. Horn, Niederösterreich), Burg (festes Haus) 269

Buchdorf (Bukovska Vas, n. Windischgraz/Slovenj Gradec, Slowenien), Dorf 274

Buchenstein (Pukštajn, n. Windischgraz/Slovenj Gradec, Slowenien) s. Hans Gall von

Buda s. Ofen

Büchsenmeister s. Hans Krafft

Büderich (linksrheinischer Stadtteil von Wesel, Nordrhein-Westfalen) s. Wilhelm ten Putte von

Bukovska Vas s. Buchdorf Burckhart, Jörg 210

Burgau (sö. Günzburg, Bayern), Markgrafschaft 146, 147

Burgund (Burgundische Länder) 72, $75,76,80,139,140,144,146-148$

- Burgundische Länder (Herzogtümer, Grafschaften, Herrschaften) s. Antwerpen, Artois, Brabant, Flandern, Friesland, Geldern, Hennegau, Holland, Limburg, Lothringen, Luxemburg, Mecheln, Namur, Salins, Seeland, Zutphen

- (Frei-)Grafschaft (Franche Comté) 146-148

- Herzogtum 146-148

- Herzog/Herzogin von 105

- Karl (der Kühne) (1467-1477) 50, 78-80, 144, 146-148

- - - seine Ehefrau s. Margarete von York

- Maria, Tochter Karls, Ehefrau Maximilians I. (1477-1482) 51, 75, 77, 80, 144, 146-148, 149

-- s. Maximilian I.

-- Gesandte 105

- Reichslehen 147, 148
- Untertanen 79, 80, 147, 148

Burgundi s. Burgund

Caithness (Schottland), Bistum

- Bischof Prosper (Camogli de Medici, auch Prospero Cannilio de Jauna) von $\sim(1478-1484) 326$

Calixt III. (Alfonso Borgia), Papst (1455-1458) 309

Cambrai (Dép. Nord, Frankreich), Stadt 144

- Bistum

--Domkapitel 173

Camogli de Medici, Prosper (auch Prospero Cannilio de Jauna) s. Caithness, Bf. von

Cappel (sü. Marburg, Hessen), Hartung d.J. von (Molitoris von Kappel), Dr. iur. utr., Rat Friedr. III. und Beisitzer des KG 25

Celje s. Cilli

Cerovec s. Zerowetz

Český Šternberk s. Sternberg

Chechelwanng s. Kalwang

Chiemsee (sö. Wasserburg, Bayern), Bistum

- Bischof Georg (Altdorfer) (14771495) 245

- s. Herrenchiemsee

Chorben s. Corben

Christenheit 42

Churwalden (sö. Chur, Kanton Graubünden, Schweiz), Gericht

- Ammann, Rat und Gemeinde $\mathbf{1 9 5}$, 196

Cilli (Celje, nö. Laibach/Ljubljana, Slowenien), Stadt 150

- Bürger s. Prantner

- Maut 150

- Grafschaft 303 
- - Grafen von $\sim$ und zu Ortenburg

--- Friedrich $(\dagger$ 1454) 117

- - - Ulrich (II.), Sohn Friedrichs

(† 1456) 117

- - - - seine Witwe Katharina

(Branković) 117

- s. Prelokar (Prelager, Berlower) von $\sim$, Thomas

Cimburk s. Zinnburg

Cles, Bernhard von s. Trient, Bf. von

Corben, Chorben (bei Pöltschach/

Poljčane, sü. Windisch-Feistritz/

Slovenska Bistrica, Slowenien)

- Hof 303

- Perg (Flur), im 303

Costentzer, Jörg 132

Crestenitz (vmtl. bei Rohitsch/Rogatec,

ö. Cilli/Celje, Slowenien) 303

Črna na Koroškem s. Schwarzenbach

Črnomelj s. Tschernembl

Čušperk s. Zobelsberg

Dachauer, (rittermäßige) Familie

- Heinrich 38

- Jörg 322

Dachaw s. Dachauer

Daigameil s. Dragemeil

David, Mert 296

Derr von Hundsheim, Simon 58

Dettikofer, Konrad s. Schaffhausen,

Abt von Allerheiligen

Dietprant, Eusebius 270

- Agnes, Ehefrau (Witwe) des Eusebius 270

Dob s. Aich

Döbriach (ö. Spittal a.d. Drau, Kärnten)

- Fischweide 124

Dol s. Lusthal

Dolacher, Hans 296
Dolenje Medvedje selo s. Unterbärenthal

Donau (Fluss) 6, 55, 106

Donaudorf (nw. Ybbs a.d. Donau, Niederösterreich) 100

Donauwörth (Bayern) s. Leonhard Vetter von

Dorenji/Dolenji Podšumberk s. Schönberg

Dorffer s. Dorfner

Dorfner, Andreas, Bürger von Steyr 101

- Dorothea, Ehefrau des Andreas 101

Dornhaws s. Voitsberg

Dosse, Stephan 269

- seine Töchter 269

Dragemeil (Dragomila) (Dragomilo, bei St. Marein bei Erlachstein/ Šmarje pri Jelšah, ö. Cilli/Celje, Slowenien) 303

Dragomila, Dragomilo s. Dragemeil

Drau (Nebenfluss der Donau) 10, 241

Drauburg s. Oberdrauburg

Dürnstein (w. Krems, Niederösterreich), Schloss 227

- Ungeld 227

Dürrer (von der Dürr, Durrer), (rittermäßige Krainer) Familie

- Friedrich, Hauptmann von Portenau 113

- Jörg (Georg), Pfleger von Pemont 283

- Niklas 49

Durrer s. Dürrer

Ebelsberg (Stadtteil von Linz, Oberösterreich)

- Maut und Zoll 36

Eben (Flur) s. Atzgersdorf, Simmering 
Ebenauer, Paul, Landrichter zu Gleichenberg 192

Ebenthal (nö. Gänserndorf, Niederösterreich) 55

Ebersdorf (nw. Melk, Niederösterreich)

- Tabore 55

Ebersdorf(Kaiserebersdorf, Wien XI), Herren von

- Veit 38

Ebersperg s. Ebelsberg

Eberstorffs. Ebersdorf

Eger s. Erlau

Eggenberger, Balthasar, Bürger von Graz 165, 241

Eggenburg (ö. Horn, Niederösterreich), Stadt 68

- Richter, Rat und Bürger 194

- Pfarrer s. Johannes Rehwein

Eggersdorf bei Graz (nö. Graz, Steiermark), Pfarre 135

Egkenperger s. Eggenberger

Ehingen (sw. Ulm, Baden-Württemberg), Pfarre 326

-Klerus 326

- Pfarrer s. Ludwig von Freiberg

Ehr(e)nau s. Ernau

Eibiswald (sü. Deutschlandsberg, Steiermark), Schloss 271

-Amt 271

- Landgericht 271

- Pfleger s. Andreas von Spangstein

Eichstätt (nw. Ingolstadt, Bayern), Bistum 53

- Domkapitel 179

- Kleriker s. Ulrich Mair, Wolfgang Staud

Eidgenossen 216

- s. Schwyz, Unterwalden, Uri, Zürich

Einpacher, Hans, Bürger von Graz 155, $170,282,323$
Eisenerz (Erzberg, nw. Leoben, Steiermark)

-Forstamt im $\sim 310$

- Amtmann s. Hans Heidenreich

- s. auch Innerberg

Eitzing (nw. Ried im Innkreis, Oberösterreich), Herren von $\sim$ (Eitzinger)

- Sigmund $\sim$ von Loosdorf, Rat und Gesandter Friedr. III. 28

-Stephan 68

Elacher, Thomas, Hauptmann zu Mitterburg 111

Elsass (Dép. Bas-Rhin und Dép. HautRhin, Frankreich), Landgrafschaft 146, 147

Elssenberger, Gilg, Pfleger von Greifenburg 41

Engelhart, Paul 317

Enns (Nebenfluss der Donau)

- Ennstal 6

- unnder der Enns s. Niederösterreich, Österreich

Ennser, Ruprecht, Brückenmeister der Wiener Donaubrücken 1

Entrich, zum (Mantrach, sw. Leibnitz, Steiermark), Amt 243

- Amtmann s. Jörg Metnitzer

Epishauser, Christoph, Bürger und Bürgermeister (1456) von Graz 86

- seine Ehefrau (Witwe) s. Ursula Lembacher

- Verwandte s. Huber

Eppenstein (sö. Judenburg, Steiermark), Schloss 162

- Pfleger s. Andreas von Teufenbach

Erdberg (Wien III)

-Zehent, gen. im Gussen 6

Erdinger, Augustin 141

- Margarethe, Ehefrau (Witwe) Augustins 141 
Erlau (Eger, nö. Budapest, Ungarn), Bistum

- Bischof Gabriel (Rangoni von Verona) (1474-1486), päpstl. Legat 106, 193

Ernau (Ehrnau bei Mautern, w. Leoben, Steiermark), von $\sim$, (rittermäßige Kärntner) Familie

- Achaz, Sohn Jakobs, Ritter 169

- Jakob, Pfleger von Karlsberg und Vizedom in Kärnten (1462-1479) († 1479) 133, 169, 232, 278

Erzberg s. Eisenerz

Eschelweck, Jörg 7

- seine Ehefrau (Witwe) s. Afra von Rottau

Esztergom s. Gran

Etzkestorffs. Atzgersdorf

Falkenstein (Oberfalkenstein, bei Obervellach, nw. Spittal a.d. Drau, Kärnten), Schloss 83, 169

-Amt 169

- Herrschaft 305

- Pfleger s. Achaz von Ernau, Wolfgang Leininger, Gebhard Peuscher

Feicht (bei Lendorf, nw. Spittal a.d. Drau, Kärnten)

- Gut 44

Feistritz (Nebenfluss der Glan, Kärnten)

- Fischweide 278

Feistritz s. Windisch-Feistritz

Feistritzer, Peter, Amtmann zu Stockenboi 19

Feldbach (sö. Graz, Steiermark), Amt 301

- Pfleger s. Heinrich Vogt von Summerau

Feldkirchen (Kärnten) 29
- s. Gradisch

Ferdinand I., röm.-dt. Kg. (15311564), K. (1556), Kg. von Ungarn (1526) und Böhmen (1527) 82, 147,148

Ferndorf (nö. Villach, Kärnten)

-Familie von

--Barbara, Tochter Walters 10

- - Walter 10

-Amt 10

- Fluren (in der Awen, die Lakhen) 10

- Mühle an der Drau 10

- Haus 10

- Taverne 10

Fewcht s. Feicht

Filipec, Johann s. Großwardein, Bf. von

Findorfer s. Vindorfer

Finkenstein (Altfinkenstein, sö. Villach, Kärnten), Schloss 47

- Pfleger s. Sigmund Skodel

Fintz, Peter, Pfleger von Pemont 283 Fischau (Bad Fischau, w. Wiener Neustadt, Niederösterreich), Pfarre 6

Fischmeister s. Vischmeister

Fiume s. St. Veit am Pflaum

Fladnitz (Fladnitz a.d. Teichalm, nw. Weiz, Steiermark), Herren (rittermäßig) von $\sim$ (Fladnitzer)

- Ulrich, Ritter 192, 284

- Wulfing (ident mit Wulfing d.Ä.?) 122

- Wulfing d.Ä. 131, 233

Fladnitz s. Oberfladnitz

Flandern (Belgien), Grafschaft 146, 147

- Grafen von $\sim$ s. Karl von Burgund, Maria von Burgund, Maximilian I. - Untertanen 147, 148 
zum Reich gehörig (,Reichsflandern") 148

Flanndern s. Flandern

Flednitz s. Fladnitz

Fleischhacker, Jörg, Bürger von Bleiburg und Amtmann zu Schwarzenbach 226

Forlì (sö. Bologna, Italien), Bistum

- Bischof Alexander (Numai) (1470 1485), päpstl. Legat 272, 326

Formbach s. Vornbach

Frangepan zu Veglia (Krk, sö. Rijeka, Kroatien), Grafen von - Ivan, Gf. von Veglia 11

Frankfurt (Hessen), Stadt 80, 53

Frankreich, Königreich 147

- König Ludwig XI. (1461-1483) 144,

147, 148, 292

Frawnfeld s. Schwechat

Freiberg (Freyberg, ö. Biberach, Baden-Württemberg), Ludwig von s. Konstanz, Bf. von

Freinperger, Thomas, Rektor der Pfarrkirche in Reiffnitz und Hofkaplan Friedr. III. 73

Freising (nö. München, Bayern), Bistum 66

-Domkapitel 184

- Kleriker s. Johannes Lingk

Frencz s. Freudenthal

Freudenthal (Bistra, sw. Laibach/

Ljubljana, Slowenien)

- Maut 150

Freyberg s. Freiberg

Fridrihštajn s. Friedrichstein

Friedberg (Steiermark), Stadt 211

- Richter, Rat, Bürger und Leute 211

Friedrich III., röm.-dt. Kg. (1440-

1493), K. (1452), Kg. von Ungarn
(1459) (s. auch Kärnten, Krain, Österreich, Steiermark)

-Familie

-- Bruder s. Albrecht VI. von Österreich

- - Schwägerin s. Mechthild, Pfalzgräfin bei Rhein, Ehefrau Albrechts VI.

--- ihr Sohn s. Eberhard von Württemberg

-- Sohn s. Maximilian I.

-- (adoptierter) Sohn s. Matthias Corvinus von Ungarn

-- Tochter s. Kunigunde von Österreich

-- Vetter s. Sigmund von Österreich

- Diener s. Leonhard Harracher, Reichart Kärgl, Peter Knauer, Schaffried Leiningen, Erasmus Lueger, Ludwig Meuting, Christoph Patriarch, Christoph von Rappach, Hans Rosegger, Leopold Rosegger, Jörg Schrott, Wolfgang Spitzweg, Hans Steinberg, Jörg Wartenauer

-Dienstleute 56

- Erblande 42, 105, 106, 110

- Gesandte, Bevollmächtigte 53, 93, $105,106,133,166,212,219,220$, 275, 282, 292; s. Sigmund von Eitzing, Georg Heßler, Eb. Andreas von Krajina, Raphael Lischinsky, Bf. Georg von Metz, Wilhelm von Puchheim, Virgil Schrutauer, Rüdiger von Starhemberg, Hans Steinberg, Leopold von Wulzendorf - Hauptmann 42; s. Friedrich Dürrer, Thomas Elacher, Berthold Mager (Verweser), Kaspar Melz (Verweser), Niklas Rauber, Jakob Raunacher, Wilhelm von Saurau (Verweser), Bernhard von Schärf- 
fenberg, Jörg von Tschernembl, Haug von Werdenberg

- Hof 119, 122, 201, 305, 321

- Hofkaplan s. Thomas Freinperger,

Hans Winkler

- Hofmarschall s. Georg Fuchs von

Fuchsberg

- Kammer 12, 195, 207, 295, 326

- Kämmerer s. Wilhelm von Auersperg, Sigmund von Niedertor,

Sigmund Prüschenk, Pankraz Rindscheit, Kaspar von Rogendorf

- - Silberkämmerer s. Sigmund Skodel

- Kammergericht 25-27, 85, 201, 216, 275

- - Beisitzer s. Hartung von Cappel, Thomas von Cilli, Georg Fuchs von Fuchsberg, Bf. Sigmund von Laibach, Bartholomäus von Lichtenstein, Johannes Rehwein, Abt Johann von St. Lambrecht, Wilhelm von Thierstein, Bf. Antonius von Triest, Haug von Werdenberg

--Fiskalprokurator 25, 53, 85; s. Johann Keller

- - Prokuratoren s. Jakob Helmreich, Georg Schrättl, Peter Staud (Stude)

- Kanzlei

- - römische $\sim 13,21,107,234,327$

- - - Leiter s. Johann Waldner

- - - Protonotare s. Georg Heßler, Johann Waldner

- - - Registrator s. Lukas Snitzer

- - - Schreiber (Notare, Sekretäre) s. Georg Knöringer, Johann Laventaler, Wolfgang Spitzweg, Jörg Stadler

- österreichische $\sim 25,68,86,125$, $155,191,327$

- - - Leiter s. Johannes Rehwein
- - - Protonotar s. Thomas Prelokar von Cilli, Johannes Rehwein

- - - Schreiber (Notare, Sekretäre) s. Jörg Stadler, Andreas am Stain,

- (seine) Länder/Herrschaften, Land und Leute 38, 42, 106, 132

- Landleute 38, 42

-Räte 86, 210, 225, 274; s. Wilhelm von Auersperg, Hartung von Cappel, Thomas von Cilli, Sigmund von Eitzing, Georg Fuchs von Fuchsberg, Georg Heßler, Johann Keller, Andreas von Kraig, Schaffried von Leiningen, Bf. Georg von Metz, Christoph Mindorfer, Christoph von Mörsberg, Sigmund von Niedertor, Georg von Pottendorf, Heinrich von Puchheim, Wilhelm von Puchheim, Jakob von Raunach, Johannes Rehwein, Pankraz Rindscheit, Bernhard von Schärffenberg, Hans von Spaur, Rüdiger von Starhemberg, Wilhelm von Thierstein, Bf. Antonius von Triest, Heidenreich Truchsess von Grub, Christoph Ungnad, Reinprecht von Wallsee, Balthasar von Weißpriach, Haug von Werdenberg, Stephan Zmollner

- Söldner 56, 57, 106

- (sein) Stadtanwalt im Wiener Rat s.

Virgil Schrutauer

- Truchsess s. Kaspar von Rogendorf

- Türhüter s. Andreas Wagen

- Untertanen 42, 55, 106

--Amtleute und Untertanen 5, 12, 15, $30,110,117,133,134,194,206$, 211-214, 300, 307

$--\mathrm{s}$. auch Burgund (Burgundische 
Länder), Kärnten, Österreich,

Reich, Trient

Friedrich IV. (von Tirol) s. Österreich,

Hz. von

Friedrichstein (Fridrihštajn, sü. Gott-

schee/Kočevje, Slowenien),

Schloss 136, 283

- Pfleger s. Sigmund Piers

Friesach (nö. St. Veit a.d. Glan, Kärnten), Stadt 259

Friesland (Landschaft in Niedersachsen und in den Niederlanden), Herrschaft 146-148

- Herren von s. Karl von Burgund,

Maria von Burgund, Maximilian I.

- Untertanen 147, 148

Fritz, Michael, Bürger von Gmunden

315

- seine Enkelin s. Sibylle Vaschang

Frostl, Vitus, Rektor der Pfarrkirche in Grafenwarth 73

Fuchs von Fuchsberg, Jörg (Georg),

Ritter, Hofmarschall und Rat

Friedr. III., Beisitzer des KG, Pfleger von Starhemberg 4, 18, 19, 25, $29,32,33,41,42,47$

Fuchsberger, Andreas, Verweser der

Saline Aussee 127

Fuchsperger s. Fuchsberger

Fürstenfeld (Steiermark), Schloss 301

- Pfleger s. Heinrich Vogt von Summerau

Gabelhuber, Heinrich, Bürger von Bruck a.d. Mur 155

Gabernik (bei Oberpulsgau/Zgornja

Polskava, nö. Windisch-Feistritz/

Slovenska Bistrica, Slowenien)

- Hof am $~ 303$

-Forst vor dem $\sim 303$
Gaimersheim (nw. Ingolstadt, Bayern)

s. Ulrich Mair von

Gall, (rittermäßige Krainer) Familie

- Hans von Buchenstein (Pukštajn), Pfleger von Hohenmauthen 123,

145, 274

Galler, (rittermäßige steirische) Familie

- Wolfgang 91

Gampern (sw. Vöcklabruck, Oberösterreich), Pfarre 315

Gars am Inn (nö. Wasserburg am Inn, Bayern), Augustiner-Chorherrenstift

- Propst, Dekan und Kapitel 249

Gars am Kamp (sü. Horn, Niederösterreich), Pfarre 68

- Pfarrer s. Johannes Rehwein

Garsten (sw. Steyr, Oberösterreich), Benediktinerkloster

- Abt Ulrich (Pranauer) (1495-1524) 161

Gatterholz (bei Schleitheim, Kanton

Schaffhausen, Schweiz), Wald 216

Gaysgassen s. Au

Geldern (Niederlande), Herzogtum 146-148

- Herzog von s. Karl von Burgund, Maria von Burgund, Maximilian I.

- Untertanen 146-148

Geler s. Galler

Gelern s. Geldern

Gerlachstein (Kolovec, sö. Stein in Krain/Kamnik, Slowenien) s. Hohenwart zu

Gerlice s. Gerlitsche

Gerlitsche (Gerlice, bei St. Marein bei

Erlachstein/Šmarje pri Jelšah, ö.

Cilli/Celje, Slowenien) 303

Gherewtt s. Greith 
Giebinger, Andreas, Pfleger von Katsch 4

Glanfurt (Fluss) s. Lankwart

Glanhofer, Hans, Bürger von Laibach 116

Gleichenberg (bei Bad Gleichenberg, sü. Feldbach, Steiermark), Burg 192

- Hals- und Landgericht 192

- - Landrichter s. Paul Ebenauer

Gleisdorf (ö. Graz, Steiermark), Markt 214

- Bürger und Einwohner 214

- Leute und Holden (des Kaspar Harder) 214

- Richter und Rat 214

Gletarn s. Kledering

Gloggnitz (sw. Neunkirchen, Niederösterreich), Propstei (des Klosters Vornbach) 12

Glojacher (von Glojach, nö. Leibnitz, Steiermark), (rittermäßige) Familie 159

- Jörg 159

- Ulrich, (jüngerer) Bruder Jörgs 159

Gmünd in Kärnten (nö. Spittal a.d. Drau, Kärnten), Stadt 260

Gmunden (Oberösterreich), Stadt 32, 105-108, 110, 114, 126, 127, 210, 279,315

- Bürger 114; s. Fritz, Pinter, Schymel, Studnitzer, Teurbanger, Tainsteter, Traint

- Richter und Rat 114, 127

- - Stadtrichter s. Hans Pinter

- - Ratsherren s. Konrad Studnitzer, Kaspar Tainsteter, Wolfgang Traint -Amt (Salzamt) 32, 114, 126, 127, 151

--Amtleute, Salzfertiger 32, 114

- - Pfleger s. Wilhelm Lesch
- Maut und Zoll 36

- Ungeld 32, 33, 114

- AO 104-109

- s. auch Hallstatt

Gnoppen (Gut) s. Aussee

Görz, Grafschaft 9

- Grafen von $\sim$ (Görzer) 8, 305

--Leonhard († 1500) 9

- - - sein Pfleger von Reifenberg 9

--- sein Verweser 9

Gösting (nw. Graz, Steiermark), Schloss 120

- Pfleger s. Andreas Prämer

Götzendorf a.d. Leitha (w. Bruck a.d. Leitha, Niederösterreich) 55

Goldenstein (ö. Kötschach-Mauthen, Kärnten), Gericht 296

Golser, Georg s. Brixen, Bf. von Goppo, Anton de $\sim$ s. Triest, Bf. von Gorizia, (Nova) Gorica s. Görz Gottschee (Kočevje, sö. Laibach/ Ljubljana, Slowenien), Landgericht in $\operatorname{der} \sim 136$

Graben (zu Kornberg, n. Feldbach, Steiermark), Ulrich von $\sim 55$

Gradisch (sü. Feldkirchen, Kärnten), Burgstall am 29

Gräfenberger, Kaspar, Marchfutterer zu Graz 231

Grafenegg (ö. Krems, Niederösterreich, benannt nach Ulrich von Grafenegg, zuvor Aspersdorf, Tahenstein, [Neu-]Wolfenreut), (seit 1465) Herren von $\sim$ (Grafenegger)

- Ulrich, Schwager Jörgs von Potten$\operatorname{dorf}(\dagger$ 1487) 38, 55, 93, 288

- - seine (2.) Ehefrau s. Katharina von Pottendorf

- Wolf, Sohn Ulrichs 55 
Grafenwarth (Kostel, sö. Gottschee/

Kočevje, Slowenien)

- Pfarrkirche 73

- - Rektor s. Vitus Frostl, Johannes Grumel

- Schloss 45

- - Pfleger s. Hans Blay

Gran (Esztergom, nw. Budapest, Ungarn), Erzbistum 193, 273

-Erzbischof

-- Johann (Beckenschlager, Beckensloer) (1472-1487) 55, 93, 193, 225, 244, 268, 272, 273, 279, 326

- - - seine Amtleute und Diener 93

- - Thomas (Tamás) (Bakócz), Kardinal (1497-1521) 106

Grandnagel s. Krannagel

Grasser, Matthias, Pfleger am Kahlenberg 59,62

Grassis, Anton de , päpstl. Auditor 272

Gratwein (nw. Graz, Steiermark), Pfarre 155, 327

- Pfarrer s. Andreas am Stain

Gratzen (Nové Hrady, sö. Budweis/

České Budějovice, Tschechien),

Schloss und Stadt 55

Gravenegkh s. Grafenegg

Graz (Steiermark), Stadt 80, 86, 118, 123, 165, 209, 210, 223, 225-227, 230, 231, 237, 241, 244, 319, 323

- Bürger und Einwohner 241; s. Brunner, Eggenberger, Einpacher, Epishauser, Judenhofer, Kamrer, Kötzler, Krafft, Payer, Peer, Peheim

- - Büchsenmeister s. Hans Krafft

- Bürgermeister, Richter und Rat 223

--Bürgermeister s. Christoph Epishauser, Hans Payer
- - Ratsherren s. Hans Payer, Hans Peer, Thomas Peheim

- - Stadtrichter s. Tibold Brunner, Christoph Patriarch, Hans Payer

- Burg 35

--Burggraf s. Christoph von Mörsberg

- Haus 209; Besitzer s. Albrecht Kamrer, Hans Weinich

- Kirchen und Klöster

- - (Dom-)Pfarrkirche St. Ägidius (Gilgen) 30 (Margaretenaltar)

--Franziskanerkloster 209

--Minoritenkloster 209

- Landgericht 74, 223

--Landrichter s. Hans Weidinger

- Marchfutterer s. Kaspar Gräfenberger

- Münzmeister s. Hans Weinich

- Stadtgraben 223

- Stadtmauer 209

- Straßen und Plätze

- - Hauptplatz 209

--, ,in der Höll“ (Gegend zw. Hauptplatz und Stadtmauer in der Umgebung des heutigen Franziskanerklosters) 209

- Ungeld 223, 241

-AO 110-114, 116-119, 122, 124, $127,129,130,133-140,142,144-$ $148,152,153,155,156,158,159$, 161, 162, 166-168, 170, 194-199, 201-208, 211-214, 219-222, 232, 238, 240, 241, 244-265, 267, 270, 273-276, 278-280, 285-287, 293, 296, 299, 300, 303-308, 311-313, $320,324,326,328,329,331$

-DO 112, 119, 145, 151, 152, 155 , 156, 164, 191, 200, 209, 223, 237. 268

Grefling s. Gröfelhof 
Greifenburg (Greifenberg) (sw. Spittal a.d. Drau, Kärnten), Burg 41

-Amt 41

- Gericht 20, 22, 154

- Pfleger s. Gilg Elssenberger (von Greifenburg)

Greilhueb s. Kematen

Greisenegg (untere Burg Voitsberg, Steiermark), Herren (rittermäßig) von $\sim$ (Greisenegger)

- Andreas († 1471) 130, 162

- - seine Güter 162

Greith (Weizer Greith, nw. Weiz, Steiermark), Andreas am 286

Gressl (Gresl), (rittermäßige steirische) Familie

- Hans, Hansgraf in Österreich 325 Grißheim (n. Neuenburg am Rhein, Baden-Württemberg) 65

Gröfelhof (bei Irschen, sw. Spittal a.d. Drau, Kärnten)

- Hof 232

Groß, Niklas d.Ä., Bürger von Nürnberg 56

Großkirchheim (nw. Spittal a.d. Drau, Kärnten)

-Amt 23

-Amtleute s. Jörg Peurbeck, Kaspar Pischofer

- Landgericht 23, 81

Großsachsenheim s. Sachsenheim

Großwardein (Oradea, Rumänien), Bistum

- Bischof (Elekt) Johann (Filipec) (1477-1490) 106

Großweikersdorf s. Weikersdorf

Grub s. Truchsess von Grub

Gruber, Simon, Bürger und Ratsherr von Wiener Neustadt 43, 67

Grünberg s. Strakonitz
Grumel, Johannes, Kler. der Diöz. Speyer, Rektor der Pfarrkirche in Grafenwarth 73

Grundlsee (See nö. Bad Aussee, Steiermark) 3

Gschaid bei Birkfeld (nö. Weiz, Steiermark), Amt 286

Gspan s. Span

Gurkfeld (Krško, sö. Cilli/Celje, Slowenien), Stadt 117

- Friedhof (Hofstätte neben dem ) 117

- Pfarre

- - Pfarrer Martin 117

-Schloss 117, 270, 287

- - Pfleger s. Andreas von Kraig

- Spital (Heilig-Geist-Spital) 117

- Urbar 117

- - Dorf am Rain im 117

Gurnitz (sö. Klagenfurt, Kärnten), Kollegiatstift 308, 327

- Propst s. Peter Knauer

- Wiese 238

Gussen (Flur) s. Erdberg

Guštanj s. Gutenstein

Gutenbrunn (nö. Herzogenburg, Niederösterreich) 299

Gutenstein (Guštanj, heute Ravne na Koroškem, Slowenien), Herrschaft 324

-Amt 324

- Leute und Holden 324

Gwaltshofer, Sigmund, Wiener Bürger und Münzmeister in Österreich 74, 90

Haag am Hausruck (sö. Ried im Innkreis, Oberösterreich) 219, 220 Haasberg (Hašperk, bei Planina, sw. Laibach/Ljubljana, Slowenien), Schloss 188, 189 
- Pfleger s. Hans von Laak, Balthasar Wagen

Habsburg (sw. Brugg, Kanton Aargau, Schweiz), Grafschaft 146, 147

Hack, Georg s. Trient, Bf. von

Hafner (Hoffner), Hermann 135

- Elisabeth, Ehefrau Hermanns 135

- Kristina, Tochter Elisabeths und Hermanns, Ehefrau des Andreas von Spangstein 135

Hagenau (Haguenau, Dép. Bas-Rhin, Frankreich) 14

Hagendorf (sö. Laa a.d. Thaya, Niederösterreich)

- Hof (mit Äckern, Baumgärten, Wiesen, Baumstätten, Hofstätten) 269

- Pewndt (Flur), Baumgarten in der 269

Haguenau s. Hagenau

Haiden

- Laurenz, Ritter, Bürger von Wien 94

- Martin, Dr. iur. utr. 48

Hainburg (Niederösterreich), Stadt 106

Haindorfer, Hans, Bürger und Ratsherr von Leoben 16

Halder, Hans 327, 328, 329

Hallein (Land Salzburg), Stadt 256

Haller, Niklas, Bürger von Villach und Pfleger von Landskron 164

Hallstatt (sü. Bad Ischl, Oberösterreich), Markt 127

-Burgleute 126, 127

- Hofschreiber s. Bartholomäus Rietmair

- Saline (Salzsieden) 29, 32, 114, 126, 127

-- Pfannstätten 127

Haraucourt, Guillaume (Wilhelm) de s. Verdun, Bf. von
Hardegg (nö. Horn, Niederösterreich), Grafschaft

- Grafen von $\sim$ s. Maidburg (Magdeburg), Bggff. von

- Burg 121

Harder, Kaspar 39, 141, 214, 224

- Leute und Holden s. Gleisdorf

Haringseer, Hans (d.Ä.), Bürgermeister von Wien († um 1457/59) 306

- Hans, Sohn des Hans d.Ä. († 1464) 306

Harnstein, Ulrich, Pfleger von Schloss Lavamünd 99

Harracher, (rittermäßige österr.) Familie

- Leonhard (Lienhard), Diener Friedr. III., Pfleger von Weitersfeld 125

Haselbach (Landkreis Landshut, Bayern) s. Pirckheimer zu

Hašperk s. Haasberg

Haus im Ennstal (nö. Schladming, Steiermark), Pfarre 6

Hauser, Familie

- Balthasar, Sohn Liebhards 232

- Hans, Sohn Liebhards 232

- Jörg, (ältester) Sohn Liebhards 232

- Kaspar, Sohn Liebhards 232

- Liebhard 232

- Paul, Sohn Liebharts 232

Hauser, Familie

- Jobst 56, 200

- Leopold, Bruder Jobsts 200

- Walter, Bruder Jobsts 200

Haushan, der $\sim 159$

Hausmannstätten (sö. Graz, Steiermark)

- Hof 129

- Hube 159

Hautzenfeld (abgeg. bei Langmanners- 
dorf, sö. Herzogenburg, Niederösterreich) 299

Hawnold, Martin 316

Hawser s. Hauser

Heberstorf s. Ebersdorf

Heidenreich, Hans, Amtmann im

Eisenerz 310

Held, Familie

- Jörg, Bruder Leonhards 39

- Leonhard, Pfleger von Schönau 39,

298, 302

Heller, Diebold am Puhl 295

- Margarete, Ehefrau Diepolds 295

- ihre Kinder 295

Helmreich, Jakob, Prokurator am KG 275

Henneberg, Philipp von s. Bamberg, Bf. von

Hennegau (Belgien), (Pfalz-)Grafschaft 146-148

- (Pfalz-)Grafen von s. Karl von

Burgund, Maria von Burgund, Maximilian I.

- Untertanen 147, 148

Herberstein (sw. Hartberg, Steiermark),

Herren (rittermäßig) von (Herbersteiner)

- Leonhard (Lienhard), Pfleger von

Wippach († 1511) 9, 11, 111, 123, 330

Herbersteiner s. Herberstein

hergruben s. St. Christoph

Herly s. Hörlin

Hernstein (nw. Wiener Neustadt, Niederösterreich), Schloss 228

- Pfleger s. Michael Reiffenberger

Herrenchiemsee (ö. Rosenheim, Bayern), Augustiner-Chorherrenstift

- Propst, Dekan und Kapitel 250

Hertenfelder s. Hertenfelser
Hertenfelser (Hertenfelder), Familie

- Achaz, Vizedom in Krain 215

- Andreas, Sohn des Achaz, Pfleger von Neuburg in der Kanker und dem Amt Primskau 215

Herzogenburg (nö. St. Pölten, Niederösterreich)

- Ungeld 237

Hessen, Landgrafschaft

- Landgrafen von

-- Heinrich III. (1458-1483) 139, 190

- - Hermann, Bruder Heinrichs s.

$\mathrm{Köln}, \mathrm{Eb}$. von

Heßler, Georg, Dr., Kardinalpriester

von S. Lucia in Silice (Rom), Rat

Friedr. III., Protonotar in der röm.

Kanzlei († 1482) 50, 51, 139, 140,

146-148, 149, 326

Heustadl, Balthasar 132

Himberg (sö. Wien, Niederösterreich)

- Ungeld 240

Hinderbach, Johann s. Trient, Bf. von Hochenegg (Vojnik, n. Cilli/Celje, Slowenien)

-Amt 152

- - Amtmann s. Stephan Widmer

- Holden 152

- Landgericht 152

Hochosterwitz (ö. St. Veit a.d. Glan, Kärnten) s. Schenken von Osterwitz

Hochwart s. Hohenwart

Hölzler, (Wiener) Ratsbürgerfamilie

- Konrad (II./d.J.), Bürgermeister von

Wien, Hubmeister in Österreich

(† 1478) 222

- Matthias, Bruder Konrads 222

Hörersdorf (nw. Mistelbach, Niederösterreich)

- Lehen 269 
Hörlin, (Augsburger) Familie

- Lukas, Bruder des Matthias 57

- Matthias 57

Hof (vmtl. in der Gegend ö. Cilli/Celje, Slowenien) 303

Hoffner s. Hafner

Hohenau an der March (nö. Zistersdorf, Niederösterreich)

- Gericht 269

- Maut 269

Hohenberg (bei Schörzingen, ö.

Rottweil, Baden-Württemberg),

Burgstall 25-27, 201

Hohenburg (bei Pusarnitz, w. Spittal a.d. Drau, Kärnten), Burg 305

Hohenegg (nw. St. Pölten, Niederösterreich) 152

Hohenmauthen (zu der Maut) (Muta, bei Vuzenica, nö. Windischgraz/ Slovenj Gradec, Slowenien), Schloss 145, 274

- Leute 145

- Maut 145

- Pfleger s. Hans Gall, Anton Hollenegger

Hohenwart (Hochwart, bei Köstenberg, nö. Villach, Kärnten), Herren (rittermäßig) von $\sim$ (Hohenwarter)

- Andreas zu Gerlachstein, Ritter 150

Holenstein (Jamnik, bei Gonobitz/

Slovenske Konjice, nö. Cilli/Celje), Herrschaft 324

-Amt 324

- Leute und Holden 324

Holfelder, Hans, Bürger von Nürnberg 166, 167, 275

- Barabara, Ehefrau des Hans 166, 167, 275

- ihr Haus in Nürnberg 166, 275

Holič s. Weißkirchen
Holland (Niederlande), Grafschaft 146-148

- Grafen von s. Karl von Burgund, Maria von Burgund, Maximilian I.

- Untertanen 147, 148

Hollenburg (sw. Klagenfurt, Kärnten), Burg 134

- Pfleger s. Konrad Lochner

Hollenegg (sö. Deutschlandsberg, Steiermark), Herren (rittermäßig) von $\sim$ (Hollenegger)

- Anton, Pfleger von Hohenmauthen 145

-Friedrich 125, 160, 271

- Hertnid, Sohn Antons 145

- Jörg, Pfleger von Arnfels 236

Holobersy Hans, vmtl. ident mit Jan Holub von Štokov, Ritter 1, 2

Holub s. Holobersy

Hopf, Heinrich, Dr. med., Bürger von Wiener Neustadt 234

Horensperg s. Hornsburg Hornsburg (bei Kreuttal, sw. Mistelbach, Niederösterreich), Burg 62 Hornstein (nw. Eisenstadt, Burgenland), Herrschaft 291

- Amt 291

- Pfleger s. Konrad Auer

Huber, Familie

- Elisabeth, Schwester Ulrichs und des Pankraz, Ehefrau Jakob Rehweins 86

- Pankraz, Bruder Elisabeths, Bürger von Radkersburg 86

- Ulrich, Lic. iur. can., Bruder Elisabeths, Chorherr zu Laibach 86

- ihr Vetter s. Christoph Epishauser

Hundsheim (sü. Hainburg, Niederösterreich)

- Hof 58 
- Weingarten, gen. Rainfal 58

- s. Derr von Hundsheim

Hynndperg s. Himberg

Ingolstadt (Bayern), Stadt

- DO 53

Innerberg (Eisenerz, nw. Leoben,

Steiermark; auch Bezeichnung für die nw. Seite des Erzbergs) 310

Innsbruck (Tirol), Stadt 293

- Bürgermeister und Rat 293

- Regiment (oberösterr.) zu 146-148

-DO 72, 132, 293

Isaak, (wohl) Jude 157

- sein Enkel 157

Isenburg-Büdingen, Diether von $\sim \mathrm{s}$.

Mainz, Eb. von

Jamnik s. Holenstein

Jamnitzer (Jemnitzer, Jempnitzer),

Lienhard, Dompropst von Laibach 234

Jamometić, Andreas s. Krajina, Eb. von

Jekel, der Jude 157

Jemnitzer, Jempnitzer s. Jamnitzer

Jeschowetz (Ješovec pri Šmarju, ö.

Cilli/Celje, Slowenien) 303

Jesen (Ježenjski brig, Ježenjski vrh),

Berg s. Tinjan

Ješovec pri Šmarju s. Jeschowetz

Ježenjski brig, Ježenjski vrh (Jesen) s.

Tinjan

Jörgendorf(St. Georgen bei Cilli/

Šentjur pri Celju oder St. Georgen

bei Rohitsch/Rogatec, ö. Cilli/

Celje, Slowenien) 303

Jörger (zu Tollet, nw. Grieskirchen,
Oberösterreich), (rittermäßige oberösterr.) Familie 92

- Bernhard 92, 102

- Christoph, Pfleger von Roith ( $†$ 1518)

92, 102

- - sein Vetter s. Hans von Sinzendorf

Jostel, Andreas 294

Jude s. Isaak, Jekel, Kifel

- Judensteuer 157

Judel, Hans, Bürger von Voitsberg 90

Judenburg (Steiermark), Stadt 21, 90, 165,241

- Bürger s. Kamrer, Rösler, Scheller

- Stadtrichter s. Stephan Scheller

- Ungeld und Weinaufschlag 165, 241

- -Amtleute s. Balthasar Eggenberger,

Albrecht Kamrer

-Wiese bei $\sim$, gelegen an der zu den

Kellchen führenden Straße 21

Judenhofer, Stefan, Bürger von Graz 210

Kadauer (zu Kattau, n. Eggenburg, Niederösterreich), (rittermäßige) Familie

- Wilhelm 269

Kärgl zu Wolfersdorf, Reichart, Ritter, Diener Friedr. III., Rat Ludwigs IX. von Bayern(-Landshut) 17, 29, 52, 53

Kärnten, Herzogtum 29, 34, 81, 83, $117,133,146,147,169,232,266$, $267,278,296$

- Ämter

--Erbkämmerer s. Andreas von Kraig

- - Verweser der Landeshauptmannschaft s. Berthold Mager

--(Viertel-)Einnehmer des Wochenpfennigs 267

- - Vizedom s. Jakob von Ernau 
- Ämter (landesfürstliche) 305

- Landesviertel 267

- Landstände 241

- Landtag 267

- Untertanen

- - geistliche und weltliche Personen (bes. Prälaten, Adelige, Erzpriester, Pfarrer, Altaristen, Gesellpriester, Kapläne, Zechmeister) 267

Kätzler (Ketzler), Jörg (Georg), Bürger von Leoben 16, 235, 314

-s. auch Kötzler

Kagerer, der $\sim 157$

Kahlenberg (heute Leopoldsberg, Erhebung im Norden Wiens, Wien XIX), Burg am 59, 62

- Pfleger am s. Matthias Grasser

Kainach (Kleinkainach, nw. Voitsberg, Steiermark), Herren (rittermäßig) von $\sim$ (Kainacher)

- Hans, Pfleger von Ortenburg 20, 154

- Heinrich 88, 89

Kaiserebersdorf s. Ebersdorf

Kalwang (w. Leoben, Steiermark) 6

Kammer (sw. Vöcklabruck, Oberösterreich), Schloss (im Attersee) und Herrschaft 151

- Pfleger s. Wolfgang Praun

Kammerer s. Kamrer

Kammern (Kammerstein) (bei Kammern im Liesingtal, nw. Leoben, Steiermark), Schloss 160

- Landgericht 160

- Pfleger s. Hans von Trautmannsdorf, Gallus Zech

Kamnik s. Stein in Krain

Kamrer (Kammerer)

- Albrecht, Bürger, Mautner und Ungelter von Judenburg 165

- Hans, Bürger von Graz 209
Kanig, Hans, Einwohner von Nürnberg 275

Kapfensteiner, Kaspar $(\dot{\dagger}) 277$

Kapler (Kapliř), Peter s. Sulewitz

Kappel, Hartung s. Cappel

Karl V., röm.-dt. Kg. (1519-1556), K. $(1520 / 30)(\dagger 1558) 82$

Karlinger, Bernhard, Mautner zu Stein 99

Karlsberg (sw. St. Veit a.d. Glan, Kärnten), Burg 133, 232

- Pfleger s. Jakob von Ernau

Karlstadt (nw. Würzburg, Bayern) s.

Konrad Reinhart von

Karlstein (Karlštejn, sw. Prag/Praha, Tschechien), Burggraf von $\sim$ s. Benesch von Weitenmühl

Karlštejn s. Karlstein

Karst (Kras, Landschaft in Italien, Slowenien und Kroatien) 138 Hauptmann zu Adelsberg und auf dem s. Jörg von Tschernembl

Kastav (w. Rijeka, Kroatien), Kastell und Amt 112

-Amtleute s. Niklas Rauber, Jakob Raunacher

Kastilien (Spanien), Königreich

- König von 105

- Gesandte 105

Katsch (nö. Murau, Steiermark), Schloss 4

- Pfleger s. Andreas Giebinger

Kattau s. Kadauer

Katzendorfer, Jörg, Pfleger von Montpreis 131

Katzianer, Matthias 277

Kellchen s. Judenburg

Keller, Johann (Hans) von Nürnberg, Lic. oder Dr. iur. utr., Fiskalproku- 
rator und Rat Friedr. III., Anwalt

Sigmunds von Österreich 25, 53, 85

- s. auch Kellner

Kellerberger, (rittermäßige Kärntner)

Familie

- Meinhard 29

Kellner (Keller), Martin, Dr., Anwalt

Mechthilds von Österreich und

Eberhards von Württemberg 26, 27

Kelnhof s. Sipplingen

Kematen a.d. Krems (sö. Wels, Oberösterreich), Pfarre 101

- Hube, gen. die Greilhueb 101

Ketzler s. Kätzler

Kierling (Stadtteil von Klosterneuburg,

Niederösterreich), Dorf 229

-Amt 229

Kifel, der Jude 133

Kindberg (nö. Bruck a.d. Mur, Steiermark)

- Fischweide bei 122, 321

Kirchpuchler, Rudolf, Bürger von Aussee 316

Kirchstetten (ö. Laa a.d. Thaya, Niederösterreich), Schloss 224

Klagenfurt (Kärnten), Stadt 238, 295

- Bürger s. Krösel, Pretzner

- Kirche St. Egid

- - Wiesen 238; s. auch Gurnitzer Wiese

Klamm (sw. Neunkirchen, Niederösterreich), Schloss 48, 302

- Pfleger s. Hans Aichelperger, Jörg

Schrott

- Pfarre 6

Klaus (Klaus a.d. Pyhrnbahn, sü.

Kirchdorf a.d. Krems, Oberösterreich), Schloss 88, 89

- Maut 36

- Ungeld in der $\sim 36$
Kledering (Stadtteil von Schwechat, Niederösterreich) 6

Kleinkainach s. Kainach

Kleinmannsburg s. Pfaffendorf

Kleinwilfersdorf s. Wilfersdorf

Klingler, Leonhard s. Wilten, Abt von

Klosterneuburg (n. Wien, Niederösterreich), Stadt 15, 55, 63, 105, 229, 237,268

- Bürger 15; s. Murstetter, Tenter

- Richter und Rat 15, 63

- - Ratsherr s. Peter Tenter (Rieder)

- Gericht 63

- Jahrmarkt 15

- Maut 63

- Ungeld 229

- s. Kierling

Knauer, Peter, Lic. decr., Propst von Gurnitz, Diener Friedr. III. 308, 327

Knaur, der , Amtmann zu Naklas 137

Knöringen (Unterknöringen, sö. Günzburg, Bayern), Burkhard von , Ritter 132

Knöringer, Georg, Sekretär in der röm. Kanzlei Friedr. III. 166, 275, 276

Kobinger, Wolfhard 132

Kočevje s. Gottschee

Kočevska Reka s. Rieg

Köln (Nordrhein-Westfalen), Stadt 51, 80

- Bürgermeister und Rat 51

-Zoll 51

- Erzbistum 85, 139, 190

--Erzbischöfe 139

- - - Hermann IV., Ldgf. von Hessen

(Gubernator 1475-1480, Eb. 14801508) 139,190

- - - - seine Gesandten 190

- - Ruprecht, Pfgf. bei Rhein (14631480) 139,190 
- - - - seine Gesandten 190

--Domkapitel 175, 190

-- Gubernator s. Eb. Hermann IV.

- Kleriker s. Wilhelm ten Putte

- Kurfürstentum 139

Königsfelder, Erasmus, vmtl. Bürger von Radkersdorf 277

Köppl, Ernreich, Ratsherr von Wien 95

Kötzler (Közler), Seifried, Bürger von Graz 323

- s. auch Kätzler

Kogl s. Neu-Attersee

Kolbrer (Kolber, Kolberer), Andreas 296

Kolman, Primos, Bürger von Laibach 24

Kolovec s. Gerlachstein

Konstanz (Baden-Württemberg), Stadt 221,326

- Heilig-Geist-Spital 221

- - Pfleger und Spitalmeister 221

- - Spitalsbesitz s. Sipplingen

- Marktstätte 221

- Bistum 326

- - Bischof 326

- - Ludwig (von Freiberg), Dr. iur. utr. (Koadjutor 1474, providiert 1474-1480) 326

- - Otto (von Sonnenberg) (Elekt 1475, Bf. 1480-1491) 326

--Domkapitel 326

- - - Domherren 326

- - - Dompropst s. Thomas Prelokar

-- Klerus 326; s. Konrad Armbruster, Michael Scriptoris

- - - Prälaten 326

Koppel, Konrad, wohl Kler. des Bistums Würzburg 53

Korneuburg (n. Wien, Niederösterreich), Stadt 55, 93, 106, 268, 322
- Bürger 93

- Stadtrichter (und Bürger) s. Hans Trost

- Ämter 93

-- Einnehmer 93

- Kaltmaut 322

-DO 105-108, 322

Kostel s. Grafenwarth

Krabath

- Andreas von Lappitz 13

- Paul, Pfleger von Landskron 164

Krafft, Hans, Büchsenmeister, Bürger und Ratsherr von Graz 118

Kraig (n. St. Veit a.d. Glan, Kärnten), Herren von

- Andreas, Rat Friedr. III., Erbkämmerer in Kärnten, Pfleger von Gurkfeld 117, 270, 287

- Siguna, Dienerin Kunigundes von Österreich 287

- - ihr Vetter s. Wilhelm von Auersperg Krain (Slowenien), Herzogtum 49, 73, $116,146,147,215,331,332$

- Verweser der Hauptmannschaft s. Kaspar Melz

- Vizedomamt (zu Laibach) 46

- - Vizedom s. Achaz Hertenfelser Krainburg (Kranj, Slowenien), Stadt 137

- Bürger s. Parilo

- Pfarre 137

Krajina (Grenzlandschaft im Norden Albaniens und Süden Montenegros), Erzbistum

- Erzbischof Andreas (Jamometić) (1476-1482, † 1484) 273

Kramnagel s. Krannagel

Kranj s. Krainburg

Krannagel (Kramnagel, Grandnagel, 
Krumnagel), Bernhard, Richter von Windisch-Feistritz 230

Kranperger (Krenberger), Jörg, Bürger von Wien 319

Kransdorfer, Wolfgang, Bürger von

Wösendorf 227

Kras s. Karst

Krautgeb (vmtl. Flur bei Wien), Amt 6

Krell von Sachsenburg (nw. Spittal a.d.

Drau, Kärnten), Simon 20, 22, 154

Krems (ehemals Doppelstadt Krems-

Stein, Niederösterreich), Stadt 98

- Bürgermeister (zu und Stein) s.

Wolfgang Aichperger

- Stadtschreiber s. Hans Schurger

- Tag zu (Landtag) 106

-AO 96, 97, 100

-DO 98

- s. Stein

Kremser, Peter, Bürger von Stein in Krain 24

Kremsmünster (sö. Wels, Oberösterreich)

- Ungeld 102

Krenberger s. Kranperger

Kreuzenstein (n. Korneuburg, Niederösterreich), Schloss 93, 268

- Herrschaft 268

Kreuzer, (rittermäßige Kärntner) Familie

- Ruprecht, Pfleger von Rauhenstein 309

Krk s. Frangepan

Krösel, Stefan, Bürger von Klagenfurt 295

Kronach (ö. Coburg, Bayern) s. Konrad Schilling aus

Krško s. Gurkfeld

Krumelsee s. Grundlsee

Krumnagel s. Krannagel
Küssaberg (Küssaburg, Küssenberg) (sö. Waldshut-Tiengen, Baden-

Württemberg), Schloss 326

Kundorfer, Wilhelm, Pfleger von

Schloss Waxenegg 239, 242

Kunigunde, Tochter Friedr. III. s. Österreich, Hzn. von

Kurz, Ulrich 275

Kyburg (sü. Winterthur, Kanton Zürich, Schweiz), Grafschaft 146, 147

Laa a.d. Thaya (Niederösterreich),

Stadt 71

- Richter und Rat 71

- Gericht 71

- Maut 71

- Ungeld 71

-DO 71

Laak bei Mannsburg (Loka pri

Mengšu, nö. Laibach/Ljubljana,

Slowenien), Hans von , Pfleger

von Haasberg und Amtmann zu

Stegberg 188, 189

Lafnitz (Gewässer bei Vorau, Steiermark)

- Fischweide 119

Laibach (Ljubljana, Slowenien), Stadt 24, 46, 116

- Bürger s. Glanhofer, Kolman, Melz

- Richter, Rat und Bürger 46

- Fleischhacker 46; s. Michse Melz

- Obere Brücke (Schusterbrücke/

Čevljarski most)

- - Fleischbänke auf der $\sim 46$

- Pfarre 24

- Vizedomamt 46

- Bistum

- - Bischof Sigmund (von Lamberg),

Beisitzer des KG (1463-1488) 25

-- Chorherr s. Ulrich Huber 
--Dompropst s. Lienhard Jamnitzer

Lakhen (Flur) s. Ferndorf

Lamberg (zu Ortenegg/Ortnek, bei Soderschitz/Sodražica, nw. Gottschee/ Kočevje, Slowenien), Herren (rittermäßig) von (Lamberger)

- Jörg (Georg), Pfleger von Adelsberg 297

- Kaspar, Vetter Valentins 115

- Sigmund s. Laibach, Bf. von

- Valentin, Pfleger von Stein in Krain 115,330

Landsee (nw. Oberpullendorf, Burgenland), Schloss 55

Landshut (Bayern), Stadt

- Hofgericht 52

Landskron (nö. Villach, Kärnten), Schloss 164, 281

- Landgericht 164, 281

- Pfleger s. Niklas Haller, Paul Krabath, Hieronymus Leininger

Langauer, Peter $\mathbf{8 1}$

- seine Ehefrau s. Barbara Waching

langen, an der $\sim$ wisen/wyse s. Langwies

Langwies (sö. Chur, Kanton Graubünden, Schweiz) Gericht

- Ammann, Rat und Gemeinde 195, 196

Lankowitz (nw. Voitsberg, Steiermark), Schloss 290

- Pfleger s. Wolfgang Lembacher

Lankwart (später Glanfurt, Fluss sü.

Klagenfurt, Kärnten) 238

Lanngenawer s. Langauer

Lanzendorf(sü. Wien, Niederösterreich) 6

Lappitz s. Krabath

Laufen (nw. Freilassing, Bayern), Stadt 257
Lausanne (Schweiz), Bistum 171

Lavamünd (ö. Völkermarkt, Kärnten), Markt 98

- Bürger s. Waitzinger

-Amt 98

- Fischweide (oberhalb der Mühle) 98

- Mühle 98

- Schloss 99

-- Pfleger s. Ulrich Harnstein

Lavant (Nebenfluss der Drau, Kärnten), Bistum (Sitz in St. Ändrä im Lavanttal)

- Bischof Johann (Rot[h] von Wemding) (1468-1482) 246

Laventaler, Johann, Notar in der röm. Kanzlei Friedr. III. 85

Laxenburg (sü. Wien, Niederösterreich), Schloss 240

- Leute und Holden 240

- Pfleger s. Gilg Slaher

Lehner, Nikolaus, ksl. Notar 53

Leibnitz (sü. Graz, Steiermark)

- Ungeld 323

Leiningen (Altleiningen, sw. Grünstadt, Rheinland-Pfalz), Grafen von (Leiningen-Dagsburg)

- Schaffried, Rat und Diener Friedr. III. 309

Leininger

- Hieronymus, Pfleger von Landskron 281

- Wolfgang, Pfleger von Falkenstein 169

Lembacher, (rittermäßige steirische)

Familie

- Erhard (Erhart) 86, 113, 141, 145, $151,152,163,164,170,191,209$, $215,224,228,231,236,239,242$, 243, 269, 281, 288-290, 294, 298, $302,310,317,321,323$ 
-- Ursula, Ehefrau Erhards, Witwe Christoph Epishausers 86

- Wolfgang, Bruder Erhards, Pfleger von Lankowitz 39, 290

Lemberg (Lemberg pri Šmarju, nö. Cilli/Celje, Slowenien)

- Hofstätten 303

Lengbach s. Neulengbach

Lengheimer, Hans, Pfleger von Weixelburg $\mathbf{3 1 8}$

Lenz (sü. Chur, Kanton Graubünden, Schweiz), Gericht

- Ammann, Rat und Gemeinde 195, 196

Leoben (Steiermark), Stadt 16, 235, $282,310,314$

- Bürger 235, 282; s. Haindorfer, Kätzler, Leubner, Pringsauf, Reicher, Steyrer

- Richter und Rat 16, 235, 282

- - Ratsherren s. Hans Haindorfer, Hans Pringsauf, Hans Reicher

- - Stadtrichter s. Gregor Leubner, Hans Pringsauf, Wolfgang Steyrer

-Amt 314

- Brücken und Wege 16, 235

- Hof 294

- Maut 16, 235

- Ungeld 170, 282

- Winkl (im Stadtteil Göss), Höfe im 314

Leobendorf (n. Korneuburg, Niederösterreich) 299

Leobenegg (nö. Gmünd in Kärnten, Kärnten), Herren (rittermäßig) von $\sim$ (Leobenegger)

- Philipp 232

- Sigmund, Bruder Philipps 232

Leonburg s. Wasserleonburg

Leopoldsberg s. Kahlenberg
Leopoldsteinersee (Bergsee, nw. Leoben, Steiermark)

- Fischweide 310

Lerchbach (Gewässer am Hermsberg, w. Villach, Kärnten) 308

Lesach (Lesachtal, nw. Hermagor, Kärnten)

-Amt 34

--Amtmann s. Ruprecht Steiner

- Hube (auf dem perg ob der wisen, genant am Ort), vmtl. Orthner/OrtherGut in Xaveriberg 8

Lesch, Wilhelm, Pfleger von Wildenstein, Mödling und dem Amt Gmunden 32, 33, 114, 127, 191

Leschenprant, Hans, Bürger von Wien 101

Leubner, Gregor, Bürger und Stadtrichter von Leoben 16, 235, 294

Leutschach (sw. Leibnitz, Steiermark) 233

Lichtenperger, Hans, Bürger von Wien 84

Lichtenstein (bei Leifers, sü. Bozen, Südtirol, Italien), Bartholomäus von $\sim$, Hofmeister Maximilians I., Beisitzer des KG 25

Liechtenstain $\mathrm{s}$. Liechtenstein

Liechtenstein (w. Mödling, Niederösterreich), Herren von

- Heinrich von $\sim$ zu Nikolsburg († 1483) 38, 55

Liechtmoss (vmtl. Flur im Enns- oder

Paltental, Steiermark), im $\sim 6$

Lienz (Tirol), Burggrafen von $\sim$ s. Lueger

Lieserhofen (n. Spittal a.d. Drau, Kärnten)

- Maut 17

- Taverne 17 
Liezen (Steiermark) 6

Lille (Rijsel) (Dép. Nord, Frankreich), Stadt

- AO 292

Limburg (Limbourg, Gebiet in Belgien und den Niederlanden), Herzogtum 146-148

- Herzöge von $\sim$ s. Karl von Burgund, Maria von Burgund, Maximilian I.

- Untertanen 147, 148

Lingk, Johannes von München, öff.

Notar, Kler. des Bistums Freising 66

Linz (Oberösterreich), Stadt

- Gegenschreiberamt 161

-DO 147, 148

Lischinsky, Raphael, Gesandter

Friedr. III. 28

Litija s. Littai

Litschau (Niederösterreich), Stadt 55

- Schloss 55, 298

- - Pfleger s. Kaspar Wenger

Littai (Litija, ö. Laibach/Ljubljana,

Slowenien), Dorf 213

Lobkovice s. Lobkowitz

Lobkowitz (Lobkovice, n. Prag/Praha, Tschechien), Herren von

- Diepold 69

Lochner, Konrad, Pfleger von Hollenburg 134

Loka pri Mengšu s. Laak bei Mannsburg

Losnitzer, Andreas 303

Lothringen, Herzogtum 146-148

- Herzöge von s. Karl von Burgund,

Maria von Burgund, Maximilian I.

- Untertanen 147, 148

Ludmansdorfer, Wolfgang 60

Lueg (Predjamski Grad, nw. Adelsberg/
Postojna, Slowenien), Burggrafen von Lienz und zum $\sim \mathrm{s}$. Lueger

Lueger

- Andreas, Bruder Balthasars und Kaspars 142

- Balthasar, Bruder des Andreas und Kaspars 142

- Erasmus, Sohn des Niklas, Bggf. von Lienz und zum Lueg, Diener Friedr. III., Pfleger von Stein in Krain $(\dagger$ 1484) 168, 330

- Haug (Hugo), Bggf. von Lienz 168

- Kaspar, Bruder des Andreas und Balthasars 142

- Niklas, Vetter Haugs 168

- Niklas, Sohn des Niklas, Bruder des Erasmus 330

- s. Florian von Altenburg, Vetter der Brüder Andreas, Balthasar und Kaspar

Lüttich (Belgien), Bistum

- Domkapitel 172

Luger s. Lueger

Lupfen (nw. Tuttlingen, Baden-Württemberg), Grafen von

- Sigmund, Ldgf. von Stühlingen 216

Lusthal (Dol, ö. Laibach/Ljubljana,

Slowenien), Wenko von $\sim 94$

Luttey s. Littai

Lutzen s. Liezen

Luxemburg, Herzogtum 146-148

- Herzöge von s. Karl von Burgund, Maria von Burgund, Maximilian I.

- Untertanen 147, 148

Madau (sü. Passau, Bayern) 7

Mähren (Tschechien), Markgrafschaft $28,42,82,107$

- Markgrafen von $\sim 108$

$--\mathrm{s}$. Matthias Corvinus von Ungarn 
$--\mathrm{s}$. Wladislaw II. von Böhmen

- Hauptmann von $\sim$ s. Ctibor von Zinnburg und Tobitschau

Magdeburg s. Maidburg

Mager, Berthold, Verweser der Hauptmannschaft von Kärnten (14761493) 34

Maidburg (Magdeburg, Sachsen-Anhalt), Burggrafen von

- Michael, Gf. von Hardegg 121

Maierhof (Pristava, Teil von Mannsdorf/Mengeš, nö. Laibach/ Ljubljana, Slowenien) 24

Mailand (Milano, Italien), Herzogtum $72,105,158$

- Herzog/Herzöge von 105

- - Gian Galeazzo Maria Sforza (1476-1494) 105

- - - seine Mutter s. Bona von Savoyen

- (Reichs-)Vikariat 105

Mainz (Rheinland-Pfalz), Erzbistum 190

- Erzbischof Dieter (von IsenburgBüdingen) (1475-1482) 190

- Domkapitel 85, 176

Mair, Ulrich von Gaimersheim, Kler. des Bistums Eichstätt 53

Mali Mengeš s. Kleinmannsburg

Mannigspurg s. Mannsburg

Mannsburg (Mengeš, nö. Laibach/ Ljubljana, Slowenien) 24

- Herren (rittermäßig) von (Mannsburger) 24

Mantrach s. Entrich

Maria Lankowitz s. Lankowitz

Mariazell (Zell) (nö. Bruck a.d. Mur, Steiermark) 155

Markgrafschaft des Reichs s. Antwerpen

Marschetschendorf(Marsecka ves, bei
Rohitsch/Rogatec, ö. Cilli/Celje, Slowenien) 303

Marsecka ves s. Marschetschendorf

Martin, Pfarrer s. Gurkfeld

Masowien, Alexander von $\sim$ s. Trient, Bf. von

Matsch (nö. Schluderns, Südtirol, Italien), Vögte von

- Gaudenz, Sohn Ulrichs 195

- Ulrich (IX.) 195

Matthias Corvinus s. Ungarn, Kg. von

Maurer, Simon, Bürger von Vilshofen 7

Mawtt, zu der $\sim \mathrm{s}$. Hohenmauthen

Maximilian I., röm.-dt. Kg. (14861519), K. (1508), Sohn Friedr. III., $\mathrm{Hz}$ /Ehz. von Österreich, Hz. von Burgund 13, 35, 50, 55, 72, 75, 80, $93,139,140,144,146,147,148$, 149, 153, 204, 240, 292

-Familie

--Ehefrau s. Maria von Burgund

-- Schwester s. Kunigunde von Österreich

-- Vater s. Friedrich III.

-- Vetter s. Sigmund von Österreich

- Hof

- - Hofmeister s. Bartholomäus von Lichtenstein

- - Stallmeister s. Peter Altenhauser

- Kammer (niederösterr.) 161

- Kammergericht 55

- Kanzlei 14

-- Kanzler s. Johann Waldner

- (seine) Länder 153

- Regiment (oberösterr.) zu Innsbruck (Landhofmeister, Marschall, Kanzler, Statthalter und Regenten) 146-148

- Verbündete 153 
Mecheln (n. Brüssel, Belgien), Herrschaft 146-148

- Herren von s. Karl von Burgund, Maria von Burgund, Maximilian I.

- Untertanen 147, 148

- DO 51

Meersburg (nw. Friedrichshafen, Baden-Württemberg) s. Michael Scriptoris von

Mehmed II. s. Osmanisches Reich, Sultan von $\sim$,

Melz, (Laibacher) Familie

- Kaspar, Bürger von Laibach, Verweser der Hauptmannschaft in Krain $24,116,332$

- Michse, Fleischhacker, Bürger von Laibach 46

Mengeš s. Mannsburg

Merchl, Veit, Amtmann zu Oberfladnitz 286

Metlika s. Möttling

Metnitzer (von Metnitz, nw. St. Veit a.d. Glan, Kärnten), Jörg, Pfleger von Arnfels und der Ämter zum Entrich und zu Thunau 236, 243

Metz (Dép. Moselle, Frankreich), Stadt 153

- Bistum

-- Bischof Georg (Mgf. von Baden), Rat und Gesandter Friedr. III. (1459-1484) 50, 51

Meuting, (Augsburger) Kaufmannsund Patrizierfamilie

- Ludwig d.Ä., Bürger von Augsburg, Diener Friedr. III. 97

- - seine Familie 97

Miclaw, Einwohner von Mlaka 137

Mikulov (Nikolsburg) s. Liechtenstein

Milano s. Mailand

Millstätter See (Kärnten) 124
Mindorfer (von Mindorf), Christoph, Rat Friedr. III. 111

Mistelbach (Niederösterreich), Pfarre 269

Mittelhausen (bei Brumath, sw. Hagenau/Haguenau, Dép. Bas-Rhin, Frankreich), Hans von $\sim 14$

Mitterburg (Pazin, sw. Rijeka, Kroatien), Schloss und Kastell 111

- Grafschaft 206

- Hauptmannschaft 111, 112, 116, 136

--Hauptleute s. Thomas Elacher, Niklas Rauber, Jakob Raunacher

-Leute s. Tinjan

Mlada Gora s. Neuberg

Mlaka pri Kranju (n. Krainburg/Kranj, Slowenien), Dorf 137

- Einwohner s. Miclaw

Mödling (sw. Wien, Niederösterreich), Schloss 191

- Pfleger s. Wilhelm Lesch

- Ungeld 191

Mörsberg (Morimont, sw. Pfirt, Dép. Haut-Rhin, Frankreich), Herren von

- Christoph, Rat Friedr. III., Bggf. zu Graz 35

Möttling (Metlika, Slowenien), Stadt 128

- Richter, Rat und Bürger 128

- Gericht 128

- Maut 128

Molln (sö. Kirchdorf a.d. Krems, Oberösterreich), Amt 88, 89

Montfort (n. Feldkirch, Vorarlberg), Grafen von $\sim 162$

Montpreis (Planina pri Sevnici, sö. Cilli/Celje, Slowenien), Schloss 96, 131 
- Pfleger s. Jörg Katzendorfer, Reinprecht von Reichenburg

Mordax (rittermäßige Kärntner Familie), $\operatorname{der} \sim 123$

Mores, Klaus 43

Morimont s. Mörsberg

Morschetzendorfs. Marschetschendorf

Mošćenice s. Moschenitz

Moschenitz (Mošćenice, sw. Rijeka, Kroatien), Kastell und Amt 112

-Amtleute s. Niklas Rauber, Jakob

Raunacher

Moser, Matthias, Bergrichter zu

Schladming 118

Mottniczer s. Metnitzer

Moyker, Hans 58

- Dorothea, Ehefrau des Hans 58

Moykerin s. Moyker Dorothea

Mühldorf am Inn (Bayern), Stadt 258

- Richter, Bürgermeister, Rat und Bürger 265

München (Bayern) s. Johannes Lingk von

Münster (Nordrhein-Westfalen), Bistum

- Domkapitel 185

Mürz (Fluss, Steiermark)

- Fischweide 122, 321

- Mürztal

- - Ungeld im 122, 321

Murstetter

- Bernhard, Bürger von Klosterneuburg 237

- Hans, Bürger und Stadtrichter von Wien 84

Muta s. Hohenmauthen

Naklas (Naklo, nw. Krainburg/Kranj,

Slowenien), Amt 137

-Amtmann s. Knaur
Naklo s. Naklas

Namur (Belgien), Grafschaft 146-148

- Grafen von $\sim$ s. Karl von Burgund,

Maria von Burgund, Maximilian I.

- Untertanen 147, 148

Nassau, Grafen von $\sim$ s. Saarbrücken

Neapel (Italien), Königreich

- Könige von

- - Ferdinand (Ferrante) I. (von Aragón), Titularkönig von Sizilien (1458-1494) 105

- - - seine Gesandten 105, 106

- - Beatrix, Prinzessin von $\sim$, Toch-

ter Kg. Ferdinands I., Ehefrau

Kg. Matthias' von Ungarn (in 2.

Ehe Wladislaws II. von Böhmen)

(† 1508) 105

- - Friedrich, Prinz von $\sim$, Sohn Kg.

Ferdinands I., (später Kg. von Aragón) (1496-1501/04) 105

Nellenburg (Baden-Württemberg),

Grafen von $\sim 216$

Neu-Attersee (später Kogl, bei

St. Georgen im Attergau, sw.

Vöcklabruck, Oberösterreich),

Schloss 103

$-\mathrm{s}$. Vischmeister

Neuberg (Mlada Gora, ö. Treffen/Trebnje, Slowenien), (Wein-)Berg 331

Neuberg a.d. Mürz (nw. Mürzzuschlag, Steiermark)

- Ungeld im Tal zu 155

Neuburg in der Kanker (Turn unter

Neuburg/Turn pod Novim gradom, nö. Krainburg/Kranj, Slowenien),

Schloss 215

- Pfleger s. Andreas Hertenfelser

Neudorf(vmtl. Neudorf bei Staatz, ö.

Laa a.d. Thaya, Niederösterreich)

- Hof und Holden 269 
Neudorf s. Wiener Neudorf

Neuenburg am Rhein (Baden-Württemberg), Stadt 64, 65, 66

Neuhaus s. Weißkirchen

Neulengbach (Lengbach) (ö. St. Pölten, Niederösterreich)

- Ungeld 60

Neumarkt in Steiermark (sö. Murau, Steiermark)

- Maut 43

Neunkirchen (sw. Wiener Neustadt, Niederösterreich), Markt 40

- Bürger s. Schottl

- Gericht 40

- Maut 40

- Ungeld 40

Neupaur, Hans, Bürger von Wien 306

- Barbara, Ehefrau (Witwe) des Hans 306

Neuschloss s. Weißkirchen

Neuss (Nordrhein-Westfalen), Stadt 85

Neuwolfenreut s. Gafenegg

Newnburg s. Klosterneuburg

Nicolspurg s. Liechtenstein

Nider Pultzkaw s. Unterpulsgau

Nidern Urfar/Ursa/Ursar s. Unterurscha

Nidersperntal s. Unterbärenthal

Niederösterreich 18, 152; s. auch Österreich (unter der Enns)

Niedertor (in Bozen, Südtirol, Italien),

Sigmund von , Kämmerer und Rat

Friedr. III. 162, 325

Nikl, der $\sim 296$

Nikolitsch, Justus von , Bürger von

St. Veit am Pflaum 212

- Vitalis, Bruder des Justus, Bürger von

St. Veit am Pflaum 212

Nikolsburg (Mikulov, Tschechien) s.

Liechtenstein
Nötschbach (Zufluss der Gail, w. Villach, Kärnten) 308

Notare, öff. s. Armbruster, Lehner, Lingk, Putte, Reinhart, Schilling, Scriptoris, Staud

Nové Hrady s. Gratzen

Nürnberg (Bayern), Stadt 56, 166, 167, 275,276

- Bürger und Einwohner s. Beck, Groß, Holfelder, Kanig, Rotenburger, Seus

- Haus 166, 275; Besitzer s. Holfelder, Konrad Seus

- Stadtgericht 166, 275

- Tag zu 292 (1479)

Numai, Alexander s. Forli, Bf. von

Nußdorf (im Chiemgau, nw. Bad Reichenhall, Bayern), Ulrich von $\sim \mathrm{s}$.

Passau, Bf. von

Nussdorf(Wien XIX)

- Ungeld 319

Obdach (sü. Judenburg, Steiermark), Markt 90, 162

Ober Beydros s. Oberwodresch

Oberdrauburg (Drauburg) (sw. Spittal

a.d. Drau, Kärnten) 232

Oberfalkenstein s. Falkenstein

Oberfellabrunn (w. Hollabrunn, Niederösterreich) 59

Oberfladnitz (Fladnitz) (n. Weiz, Steiermark), Amt 286

- Amtmann s. Veit Merchl

Oberg s. Obritschan

Oberhaimer, (rittermäßige oberösterr.)

Familie

- Hans, Sohn Kolmans, Pfleger von

Starhemberg 217-220

- Hans von Bernau, Sohn des Matthias, 
Bruder Warmunds (von Bernau)

217-220

- Kolman 219

- Matthias von Bernau 219

- Warmund von Bernau, Bruder des

Hans (von Bernau) 218, 220

Obernburger, Niklas, Pfleger von Prem

111

Oberösterreich 18, 219, 220; s. auch

Österreich (ob der Enns)

Oberpulsgau s. Pulsgau

Oberstein s. Stein in Krain

Obertschan s. Obritschan

Obervellach s. Vellach

Oberwaltersdorf s. Waltersdorf

Oberwodresch (Wodresch) (Bodrež, bei St. Marein bei Erlachstein/ Šmarje pri Jelšah, ö. Cilli/Celje, Slowenien) 303

Oberzellnitz (Zgornja Selnica, bei Selnica ob Dravi, w. Marburg/Maribor, Slowenien) 285

Obretz (bei Rann/Brežice, sö. Gurkfeld/Krško, Slowenien), Dorf zu 117

Obritschan (Obratschan) (Oberg/Obrh [Obrh pri Dragatušu], sw. Tschernembl/Černomelj, Slowenien), (rittermäßige Krainer) Familie

- Jörg (Georg), Pfleger von Schärffenberg 138

- Peter, Vater Jörgs, (früherer) Pfleger von Schärffenberg 138

Obrh, Obrh pri Dragatušu s. Obritschan Österreich (ob und unter der Enns),

Herzogtum 6, 7, 12, 15, 28, 42, 55 , $59,62,82,90,93,100,101$ (ob der Enns), 104, 105, 141, 146, 147, 152 (unter der Enns), 194, 210, 222,
224, 299, 307, 315, 325, 327 (ob

der Enns)

- Haus 109, 195, 293, 326

- (Erz-)Herzöge von (Habsburger)

106

- -Albrecht V. s. Albrecht II.

--Albrecht VI. (1416-1463), Bruder

Friedr. III. 25, 89, 147, 161

- - - seine Ehefrau s. Mechthild, Pfalzgräfin bei Rhein

- - Friedrich IV. (von Tirol) (14061439) 158

- Kunigunde, Tochter Friedr. III. (1465-1520) 105, 287

- - Dienerin s. Siguna von Kraig

- - - ihr Frauenzimmer 287

-- Maximilian, Sohn Friedr. III. s. Maximilian I.

- - - seine Ehefrau s. Maria von Burgund

- - Sigmund (von Tirol), Vetter

Friedr. III. (1446-1490, † 1496) 25, 26, 27, 72, 76, 80, 109, 132, 158, 195, 196, 201, 202, 203, 207, 208, 293, 304, 311, 312, 326

- - -Anwälte 158; s. Johann Keller

--- Diener s. Jörg Schetzer

- - - Vorfahren 158

- Adelige 106, 110

- Ämter

- - Einnehmer 210

- - Erbschenk s. Jörg von Pottendorf

- Erbtruchsess s. Heinrich von Puchheim

-- Gegenschreiberamt (zu Linz) 161

- - Hansgrafenamt (Sitz in Wien) 325;

Hansgraf s. Hans Gressl

- - Hauptmann ob der Enns s. Bern-

hard von Schärffenberg 
-- Hubmeisteramt (Sitz in Wien) 222; Hubmeister s. Konrad Hölzler

--lanndesman (Landrecht) 38

--Münzmeister (Sitz in Wien) s. Sigmund Gwaltshofer

-- (erbliches) Türhüteramt 299; Türhüter s. Wehingen

- Landstände (Landleute) 38, 42, 106, 210,279

-- Prälaten 105

- Landtag s. Krems, Wien

- Lehensleute 328, 329

- Münze 38

- Untertanen 28, 42, 104

Ofen (Buda, Stadtteil von Budapest, Ungarn), Stadt 121

- Tag zu $\sim 121$

Oradea s. Großwardein

Ortenburg (w. Spittal a.d. Drau, Kärnten), Grafschaft/Herrschaft 10, 19, $20,22,44,70,124,154,232$

- Grafen zu $\sim$ s. Cilli

- Bergbau 20, 22, 154

- Vizedom s. Leonhard Saldorfer

Orth a.d. Donau (ö. Wien, Niederösterreich), Herrschaft 58

Orthner/Orther-Gut s. Xaveriberg

Osmanisches Reich

- Sultan

- - Mehmed II. (1444-1446, 14511481) 309

- - - sein angeblicher Halbbruder s. Bajezid Ottman

- - Bayezid II., Sohn Mehmeds II. (1481-1512) 309

- Osman s. Ottman

- Osmanen s. Türken

Ossiach (nö. Villach, Kärnten), Benediktinerkloster
- Abt Leonhard (Zorn) (1473-1484) und Konvent 278

Osterreich s. Österreich

Osterwitz (Hochosterwitz) s. Schenken von

Ottakring (Wien XVI), Amt 6

Ottenschlag (sü. Zwettl, Niederösterreich), Schloss 222, 313

Ottman (Osman), Bajezid (Bayezid, Bajazit) (Calixtus Ottomanus), türkischer Exulant (angeblicher Osmanenprinz), Pfleger von Perchtoldsdorf, Baden und Rauhenstein 309

Pandorfer, Wolfgang, Bürger von Steyr 161

Papst, Papsttum 106, 139, 171, 190, 244, 273; s. Calixt III., Sixtus IV.

- Apostolischer Stuhl (Heiliger Stuhl)

$171,190,326$

- Auditor s. Anton de Grassis

- Kammer 326

- Kanzlei 272

- Legaten und Oratoren s. Ludwig de Agnellis, Patriarch Marcus von Aquileia, Bf. Gabriel von Erlau, Bf. Alexander von Forli, Anton de Grassis

- Notare s. Ludwig de Agnellis, Georg Schönberg

- s. auch Wiener Konkordat

Parilo, Clemens, Bürger von Krainburg 137

Passau (Bayern), Bistum

- Bischof Ulrich (von Nußdorf)

(1451/54-1479) 55

- Domkapitel 182

Patriarch, Christoph, Diener Friedr. III., Stadtrichter von Graz († 1478) 118, 165 
Paulseer, Wilhelm, Amtmann zu Stockenboi 70

Payer mit dem Bären, Hans, Ratsherr, Bürgermeister und Stadtrichter von Graz 118

Payerbach (sw. Neunkirchen, Niederösterreich), Pfarre 6

Pazin s. Mitterburg

Peddersdorfer, Pankraz 86

Peer, Hans, Ratsherr von Graz 226, 227, 237

Peheim, Thomas, Bürger und Ratsherr von Graz 123, 210, 230

Peltel Georg s. Schönberg

Pemont (bei Motovun, nw. Mitterburg/ Pazin, Kroatien), Schloss 283

- Gut, Häuser, Weingärten und Mühle 283

- Pfleger s. Jörg Dürrer, Peter Fintz

Perchtoldsdorf (sw. Wien, Niederösterreich), Burg 309

- Pfleger s. Bajezid Ottman perg, auf dem $\sim$ s. Sigmund

Perl, Veit 116

Perler, Wolfgang, gesessen auf der Sperlmul (?, mglw. bei Obdach, sü. Judenburg, Steiermark) 90

Pettau (Ptuj, sö. Marburg/Maribor, Slowenien), Stadt 261, 285

- Herren von $\sim 159$

- - Friedrich († 1438) 159, 285

Peurbeck, Jörg, Amtmann zu Großkirchheim 20, 22, 23, 154

Peuscher, Gebhard, Pfleger von Falkenstein 55, 83, 169

Pewndt (Flur) s. Hagendorf

Pezinok s. Bösing

Pfaffendorf (Kleinmannsburg/

Mali Mengeš, Ortsteil von
Mannsburg/Mengeš, nö. Laibach/

Ljubljana, Slowenien) 24

Pfalzgrafschaft bei Rhein, Kurfürstentum (Erztruchsessenamt) 140

- Pfalzgrafen bei Rhein, Herzöge von Bayern

-- Friedrich I., Bruder Ludwigs IV. und Ruprechts, Onkel, Vormund und Adoptivvater Philipps (14511476) 140

- - Mechthild, Tochter Kf. Ludwigs III., Ehefrau (1) Ludwigs I. von Württemberg und (2) Albrechts VI. von Österreich, Schwägerin Friedr. III. († 1482) 25, 26 , 201, 202, 203

- - ihr Sohn s. Eberhard V. von Württemberg

- - - ihre Anwälte s. Martin Kellner, Hermann von Sachsenheim, Bernhard Schöferlin

-- Ludwig IV., Kf. (1436-1449) 140

- - Philipp, Sohn Ludwigs IV., Neffe und Adoptivsohn Friedrichs I., Kf. (1476-1508) 140

--Ruprecht, Bruder Ludwigs IV. und Friedrichs I. s. Köln, Eb. von Pfirt (sö. Mühlhausen, Dép. Haut-Rhin, Frankreich), Grafschaft 146, 147

Pickl, Hans, Amtmann zu Voitsberg 130

Pielachperg (?, mglw. Pielachberg, nö. Melk, Niederösterreich) 6

Pierbach s. Payerbach

Piers, Sigmund, Pfleger von Friedrichstein und Amtmann in der Rieg 136, 189, 283, 297

Pinter, Hans, Stadtrichter von Gmunden 114

Pirchach s. Pyhra 
Pirckheimer (Pirkheimer) (zu Wolfersdorf, Haselbach und Pöttmes), Familie

- Sigmund 52, 53

Pischofer, Kaspar, Amtmann zu Großkirchheim 23

Pittersberg (bei Kötschach-Mauthen, nw. Hermagor, Kärnten), Schloss 34

- Pfleger s. Ruprecht Steiner

Planck, Stephan, Kastner zu Vilshofen 7

Planina pri Sevnici s. Montpreis

Plank am Kamp (sü. Horn, Niederösterreich), Burg (öde) 269

Plesser, Jörg 2

Pliberk s. Bleiburg

Pöggstall (nw. Melk, Niederösterreich), Schloss 222, 313

- Inhaber s. Hölzler, Kaspar von Rogendorf

Pöltschach (Poljčane, nö. Cilli/Celje, Slowenien) 303

Pömerl, der $~ 159$

Pöndorf (w. Vöcklabruck, Oberösterreich), Pfarre 315

Pöttmes (nö. Augsburg, Bayern) s. Pirckheimer zu

Pogled (Pogleth) (bei Gonobitz/Slovenske Konjice, nö. Cilli/Celje, Slowenien) 303

Polanitz s. Polanzen

Polanzen (Polenci, nö. Pettau/Ptuj, Slowenien) 285

Polen, Königreich

- König Kasimir II. (1447-1492) 28, 38,106

- s. Wladislaw II. von Böhmen, Sohn Kasimirs II.

Polenci s. Polanzen
Poljčane s. Pöltschach

Polskava s. Pulsgau

Ponhaimer, Hans, Bürger von Wien 1

Pordenone s. Portenau

Porrentruy s. Pruntrut

Portenau (Pordenone, sw. Udine, Italien), Schloss und Stadt 113

- Hauptmann s. Friedrich Dürrer

- Herrschaft 146, 147

Posch, Melchior, Einwohner von Spittal a.d. Drau 124

Postojna s. Adelsberg

Potendorffs. Pottendorf

Pottendorf(sö. Baden, Niederösterreich), Herren von

-Friedrich (Linie Kirchschlag) 38, 55

- Jörg (Georg) (Linie Ebenfurt), Erbschenk in Österreich 38, 55

- Katharina, Schwester Jörgs, Ehefrau Ulrichs von Grafenegg 55

Pottensteiner, Augustin, Ratsherr von Wiener Neustadt und Kirchenmeister der Liebfrauenkirche 234

Pottschach (sw. Neunkirchen, Niederösterreich), Schloss 55

Pottschall (ö. Herzogenburg, Niederösterreich) 299

Prämer, Andreas, Ritter, Pfleger von Gösting 120, 228, 290, 301

Prättigau (Tal der Landquart, Kanton Graubünden, Schweiz) 195

Prag (Praha, Tschechien), Stadt

- Landtag zu (1476) 42

- Erzbistum 82

--Administrator s. Ernst von Schleinitz

Praha s. Prag

Praitenwiser, Thomas 277

- Christoph, Bruder des Thomas 277

Pranauer, Ulrich s. Garsten, Abt von 
Prantner, Gilg, Bürger von Cilli 150

Praun (rittermäßige oberösterr. und steirische), Familie

- Wolfgang 3, 114, 151

Predjamski Grad s. Lueg

Pregaritz s. Pregnitz

Pregnitz (bei Rohitsch/Rogatec, ö.

Cilli/Celje, Slowenien) 303

Prein (Gegend bei Prein a.d. Rax, sw. Neunkirchen, Niederösterreich) 6

Prelager s. Prelokar

Prelischk(?, Kärnten), Gut an der 232

Prelokar (Prelager, Berlower), Thomas von Cilli, Dr. iur. utr., Dompropst zu Konstanz, Rat Friedr. III., Protonotar der österr. Kanzlei, Beisitzer des KG (ca. 1421-1496) 25

Prem (sü. Adelsberg/Postojna, Slowenien), Schloss 96, 111

-Familie von

- - Achaz 96

- - Wolfgang, Sohn des Achaz 96

- Herrschaft 96

- Hof, gen. Striell 96

- Leute und Holden 96

- Maut bei 116

- Pfleger s. Niklas Obernburger

- Urbaramt 96

Press (bei Oberradkersburg/Gornja

Radgona, Slowenien)

- Hof 277

- Presserberg (Flur) 277

Pressburg (Bratislava, Slowakei), Stadt 106

- Gespan s. Nikolaus Bánfi

- Propst s. Georg Schönberg

Prestowetz s. Wrestovetz

Pretzner, Ruprecht, Bürger von Klagenfurt 238
- Elisabeth, Ehefrau Ruprechts 238

Primskau (Primskovo, Stadtteil von

Krainburg/Kranj, Slowenien), Amt

215,332

- Amtleute s. Andreas Hertenfelser,

Kaspar von Stein

Primskovo s. Primskau

Pringsauf, Hans, Bürger, Ratsherr und

Stadtrichter von Leoben 16, 235, 314

Pristava s. Maierhof

Prothowicz, Nikolesch, Pfleger von

Asparn a.d. Zaya 284

Prüschenk, (ab 1480) Herren zu Stettenberg (Stattenberg/Štatenberg, sö. Windisch-Feistritz/Slovenska Bistrica, Slowenien)

- Heinrich, Bruder Sigmunds, Pfleger von Sarmingstein 319, 320, 324

- Sigmund, Kämmerer Friedr. III., Pfleger von Bernstein und Weitenegg 13, 124, 130, 146, 156, 204, 205,324

Prueschinckh, Prueschinkh, Pruschenck, Pruschinckh s. Prüschenk Prugg (Schloss) s. Bruck a.d. Leitha Prunn (bei Mannsburg/Mengeš, nö.

Laibach/Ljubljana, Slowenien)

- Hof 24

Pruntrut (Porrentruy, Kanton Jura, Schweiz), Schloss 66

Ptuj s. Pettau

Puchaim s. Puchheim

Pucher, Jakob, Einwohner von Tilliach 8

Puchheim (Schloss heute in AttnangPuchheim, ö. Vöcklabruck, Oberösterreich), Herren von

- Georg (Jörg), Sohn Heinrichs 55 
- Heinrich, Erbtruchsess in Österreich, Rat Friedr. III. († 1478) 38, 55

- Wilhelm, Rat und Gesandter

Friedr. III. († 1483) 28

Puhl s. Heller

Pukštajn s. Buchenstein

Pulsgau (Oberpulsgau/Zgornja Pols-

kava, nö. Windisch-Feistritz/Slo-

venska Bistrica, Slowenien) 303

-Forst s. Gabernik

- s. Unterpulsgau

Purkersdorf (w. Wien, Niederösterreich)

- Ungeld 319

Pusenperg s. Bisamberg

Putte, Wilhelm ten $\sim$ von Büderich, öff.

Notar 85

Pyhra (bei Gnadendorf, nw. Mistelbach, Niederösterreich), Dorf 62

Rackenhof (abgeg., heute Flur Ragenhof bei Zwerndorf, ö. Gänserndorf, Niederösterreich) 55

Radiga (sw. Leibnitz, Steiermark) 233

Radkersburg (Bad Radkersburg, Steiermark), Stadt 86, 277

- Bürger s. Huber, Königsfelder

Radmannsdorf (Radovljica, nw. Laibach/Ljubljana, Slowenien)

- Gericht 317

Radovljica s. Radmannsdorf

Radstadt (Land Salzburg), Stadt 255

Ragenhof (Flur) s. Rackenhof

Rain, am s. Gurkfeld

Rainfal (Weingarten) s. Hundsheim

Rajhenburg s. Reichenburg

Ramung, Hans, Ritter 162

Randen (Höhenzug im Kanton Schaffhausen, Schweiz, und in BadenWürttemberg) 216
- Wald 216; s. Gatterholz, Westerholz Rangoni, Gabriel von Verona s. Erlau, Bf. von

Rankweil (nö. Feldkirch, Vorarlberg), Landgericht 293

Rann (Brežice, sö. Gurkfeld/Krško, Slowenien), Stadt 262

- Urbar 117

--Dorf im s. Obretz

Rann (Breg, sw. Pettau/Ptuj, Slowenien) 285

Ranten (nw. Murau, Steiermark) 90

Rappach (bei Rottenmann, sö. Liezen, Steiermark), Herren von

- Christoph, Diener Friedr. III. († 1485) 6, 18

Raron (w. Brig, Kanton Wallis, Schweiz), Herren von

- Petermann (Peter) $(\dagger$ 1479) 311, 312

Raschberger, Hans, Mautner zu Rottenmann und Pfleger von Wolkenstein 231

Rasegker $\mathrm{s}$. Rosegger

Rauber, (rittermäßige Krainer) Familie

- Hermann, Pfleger von Zobelsberg 111

- Kaspar 188

- Niklas, Hauptmann zu Triest und Mitterburg, Amtmann zu Kastav, Veprinitz und Moschenitz 111, 112, 116, 136, 189

Rauhenstein (w. Baden, Niederösterreich), Schloss 309

- Pfleger s. Ruprecht Kreuzer, Bajezid Ottman

Raumschüssl, Familie

- Anna, Tochter Konrads, Ehefrau Kaspar Zebingers 285

- Konrad 285

- Sigmund 285 
Raunacher, (rittermäßige Krainer) Familie

- Jakob, Rat Friedr. III., Hauptmann zu

Mitterburg 111, 112

Ravne na Koroškem s. Gutenstein

Rayn s. Rann

Rechwein s. Rehwein

Redlein (Flur bei Hohenburg, w. Spittal a.d. Drau, Kärnten), Alm (Alpe) in der $\sim 305$

Regensburg (Bayern), Bistum

- Domkapitel 186

Regisheim (Réguisheim, n. Ensisheim, Dép. Haut-Rhin, Elsass, Frankreich), Antonius von , Domherr zu Basel 66

Regkenhof s. Rackenhof

Réguisheim s. Regisheim

Rehwein (Rechwein, Reben), Familie

- Johannes (Hans), Lic. iur. utr., Pfarrer von Gars und Eggenburg, Rat Friedr. III., Protonotar und Leiter der österr. Kanzlei, Beisitzer des KG (ca. 1430-1481) 25, 68, 86

- Jakob (d.J.), Bruder des Johannes, Bürger von Wien 68, 86

- - seine Ehefrau s. Elisabeth Huber

- - seine Schwäger s. Ulrich und Pankraz Huber

Reich, Heiliges (römisches) Reich 12, $14,38,50,51,53,65,66,75,80$, $82,87,97,105-108,139,140,144$, 146-149, 158, 171, 190, 195, 201, $207,221,280,292,304,311,312$, 326

- Erzschenkenamt 82, 87, 107

- Erztruchsessenamt 140

- Könige und Kaiser 171; s. Albrecht II., Ferdinand I., Fried- rich III., Karl V., Maximilian I., Sigismund

- Kurfürsten 75, 80

- Markgrafschaft des s. Antwerpen

- Reichsbistïmer 171; s. Augsburg,

Bamberg, Basel, Besançon, Cambrai, Eichstätt, Freising, Köln,

Konstanz, Laibach, Lausanne, Lüttich, Mainz, Metz, Münster, Passau, Prag, Regensburg, Salzburg, Speyer, Straßburg, Trient, Trier, Utrecht, Verdun, Würzburg

- Reichsfürsten 75, 80, 107, 144, 158, 191

- Reichshilfe 292

-Reichskammer 158, 221

- Reichslandvogtei s. Schwaben

- Reichsuntertanen 12, 65, 75, 80, 87, 97, 109 (Pfandnehmer), 144, 158, 207, 221, 312, 326

- Reichsvikariat s. Mailand

Reichenauer, Sebald 59, 62

Reichenburg (Rajhenburg [Brestanica], sö. Cilli/Celje, Slowenien), Herren (rittermäßig) von $\sim$ (Reichenburger)

- Jörg (Georg), Ritter, Bruder Reinprechts 192

- Reinprecht, Pfleger von Montpreis 96

Reicher, Hans, Bürger und Ratsherr von Leoben 235

Reicholf, Jörg, Bürger von Wien 229

Reifenberg (Rihemberk, n. Triest, Slowenien), Burg

- Pfleger 9

Reiffenberger, Michael, Pfleger von Schloss Hernstein 224, 228

Reiffnitz (Ribnica, nw. Gottschee/ Kočevje, Slowenien)

- Pfarrkirche 73

-- Rektor s. Thomas Freinperger 
Reihenawer s. Reichenauer

Rein, Adam vom 191

Reinberg (nö. Vorau, Steiermark)

-Fischweide 119

Reinhart, Konrad von Karlstadt, öff.

Notar 166

Reisacher, (rittermäßige steirische)

Familie

-Hans 122

Reisenberg (ö. Baden, Niederösterreich), Dorf 55

Reitenau (n. Hartberg, Steiermark) s. Kaspar Zebinger zu

Reitering (Stadtteil von Ybbs a.d. Donau, Niederösterreich) 100

Retzinger, Antonius, Prokurator des Erzpriesterhofs zu Basel 66

Rhein (Fluss) 64, 66, 85

-Rheinauen 65; s. Bellingen, Grißheim

-s. Pfalzgrafschaft bei

Ribnica s. Reiffnitz

Rieder, Peter s. Tenter

Rieg (Kočevska Reka, sö. Gottschee/

Kočevje, Slowenien), Amt in der 189

- Amtmann s. Sigmund Piers

Rietmair, Bartholomäus, Hofschreiber zu Hallstatt 29

Riettarn s. Reitering

Rihemberk s. Reifenberg

Rijeka s. St. Veit am Pflaum

Rijsel s. Lille

Rindscheit, (rittermäßige steirische)

Familie

- Andreas, Ritter, Sohn des Pankraz 30, 233

- Pankraz, Kämmerer und Rat

Friedr. III., Landschreiber in der

Steiermark, Beisitzer des KG 30
Ringkl, Kaspar, Pfleger von Schauenstein 55

Rittersdorf (ö. Oberdrauburg, Kärnten) 232

Rösler, Wolfgang, Bürger von Judenburg 21

- Katharina, Ehefrau (Witwe) Wolfgangs 21

Rogatec s. Rohitsch

Rog[g]endorf (zu Pöggstall, nw. Melk, Niederösterreich), Herren (ab 1480, zuvor rittermäßig) von $\sim($ Rogendorfer) 31

- Kaspar, Sohn Sigmunds, Truchsess und Kämmerer Friedr. III., Pfleger von Weitenegg, Ybbs und Werfenstein († 1506) 13, 31, 204, 205, 222, 288, 313, 314, 319, 320

- Sigmund, Vater Kaspars 205, 314

Roggendorf bei Röschitz s. Ruckendorfer

Rogoznica s. Rohrbach

Rohitsch (Rogatec, ö. Cilli/Celje, Slowenien), Herrschaft 303

- Turm 303

- Rohitscher Berg 303

Rohr, Bernhard von $\sim$ s. Salzburg, Eb. von

Rohrbach (Rogoznica, nw. Pettau/Ptuj, Slowenien) 285

Roith (in Taufkirchen a.d. Trattnach, nw. Grieskirchen, Oberösterreich), Schloss 92

- Pfleger s. Christoph Jörger

Rojach (bei Lendorf, nw. Spittal a.d. Drau, Kärnten), Familie von

- Afra, Tochter des Niklas, Schwester Christians 44

-Barbara, Tochter des Niklas, Schwester Christians 44 
- Christian, Sohn des Niklas 44

- Clemens, Sohn des Niklas, Bruder Christians 44

- Niklas 44

Rom (Roma, Italien), Stadt 273

- Kirche S. Lucia in Silice (Selci, auch

S. Lucia in Orphea)

- - Kardinalpriester s. Georg Heßler

-DO 193, 272

Roma s. Rom

Roraw s. Raron

Rorbeck

- Thomas, Schuster, Bürger von Wien 95

- - Anna, Ehefrau des Thomas 95

- Wolfgang, Bürger und Ratsherr von Wiener Neustadt 40

Rosegger, Hans, Diener Friedr. III. 305

- Leonhard (Lienhard), Bruder des

Hans, Diener Friedr. III. 305

Rosenberg (Rožmberk, sw. Kaplitz/

Kaplice, Böhmen, Tschechien),

Herren von

- Wok $(\dagger 1505) 69$

Rosenberg (n. Oberdrauburg, Kärnten),

Hof am $\sim 232$

Rot(h) von Wemding, Johann s. Lavant, Bf. von

Rotenburger, Erhard, Einwohner von

Nürnberg 166

- Hans, Einwohner von Nürnberg 166

Rottau (nö. Pocking, Bayern) zu Ma-

dau, Wilhelm von $\sim$, Pfleger von

Vilshofen 7

- Afra, Ehefrau Wilhelms, Witwe Jörg

Eschelwecks 7

Rottenbach (Troblje, bei Windischgraz/

Slovenj Gradec, w. Marburg/Mari-

bor, Slowenien), Dorf 274
Rottenburg am Neckar (Baden-Württemberg), Stadt

- DO 26

Rottenmann (sö. Liezen, Steiermark)

231

- Mautner s. Hans Raschberger

Rottenstein (sw. Spittal a.d. Drau,

Kärnten), Gericht 20, 22, 154

Roy, unnder dem $\sim \mathrm{s}$. Littai

Rožmberk s. Rosenberg

Ruckendorfer (von Roggendorf bei

Röschitz, nö. Eggenburg, Niederös-

terreich), (rittermäßige) Familie

- Wolfgang 55

Rudegk s. Rudenegg

Rudenegg (bei Rietz/Rečica ob Savinji, sw. Prassberg/Mozirje, Slowenien),

Schloss 142

Rüettestorffs. Rittersdorf

Saarbrücken (Saarland), Grafschaft

- Grafen von Nassau-Saarbrücken

- - Johann II. (1442-1472) 280

- - Johann Ludwig I., Sohn Johanns II.

(1472/90-1545) 280

Sabiach s. Schabnig

Sachs, Familie

- Hans zu Almegg 100

- Jesse, Bruder Wolfgangs 100

- - seine Kinder Jörg, Jesse, Christoph, Leonhard und Hans 100

- Pilgrim, Bruder Wolfgangs 100

- Wolfgang, Bruder Jesses und Pilgrims 100

Sachsen, Herzogtum und Kurfürstentum

- Herzöge von

--Ernst, Kf. (1464-1486) 144

Sachsenburg s. Krell

Sachsenheim (Großsachsenheim, nw. 
Ludwigsburg, Baden-Württemberg), Hermann von , Ritter, Anwalt Mechthilds von Österreich und Eberhards von Württemberg 25-27

Sachsenlander Christoph, Pfleger von

Starhemberg 18

Saggautal s. St. Johann

Sailer, Philipp, Bürger von Wien 37

- Barbara, Ehefrau (Witwe) Philipps 37

Sailerin s. Sailer

Saldorfer, Leonhard (Lienhard), Vizedom zu Ortenburg 124

Salins (Salins-les-Bains, Dép. Jura,

Frankreich), Herrschaft 146-148

- Herren von s. Karl von Burgund,

Maria von Burgund, Maximilian I.

- Untertanen 147, 148

Salzburg (Österreich), Stadt 80, 254

- Benediktinerkloster St. Peter

- - Abt und Konvent 252

- Erzbistum 171, 225, 244, 273

- - Erzbischof Bernhard II. (von Rohr)

(1466-1481), Administrator zu

St. Stephan in Wien $(1482 / 84$

1487) $(\dagger$ 1487) 83, 225, 244, 265 , 272

--- sein Hofmeister s. Hans von

Aichberg

--Domkapitel 180, 225, 244

-- Landstände 225, 244, 264 (Prälaten, Ritterschaft, Städte)

- Landtag zu 225, 244

-DO 225

Sanctus Vitus in terra nostra fluminis s.

St. Veit am Pflaum

St. Ägidius s. Graz, Klagenfurt

(St. Egid)

St. Andrä im Lavanttal (sü. Wolfsberg,

Kärnten), Stadt 263
St. Christoph (im Bereich des Navje in Laibach/Ljubljana, Slowenien)

- Äcker hinter der hergruben bei $\sim 24$

St. Egid s. Klagenfurt

St. Georgen s. Jörgendorf

St. Georgen (Svätý Jur, nö. Pressburg/ Bratislava, Slowakei) s. Bösing, Grafen von

St. Gertrud (bei Windischgraz/Slovenj Gradec, w. Maribor, Slowenien) 274

St. Johann im Saggautal (sw. Leibnitz, Steiermark), Dorf 233

St. Lambrecht (sö. Murau, Steiermark), Benediktinerkloster

- Abt Johann (Schachner), Rat Friedr. III., Beisitzer des KG (1455-1478) 25

St. Lorenzen im Lesachtal (nw. Hermagor, Kärnten) 8

St. Martin bei Littai (Šmartno pri Litiji, ö. Laibach/Ljubljana, Slowenien)

213

- Dorf zu s. Littai

St. Peter s. Salzburg, Schanfigg

St. Peter ob Leoben (St. Peter am Freienstein, nw. Leoben, Steiermark), Schloss 120, 170, 294

- Landgericht 120, 170, 282, 294

- Pfleger s. Stephan Zmollner

- Ungeld 170, 282

St. Pölten (Niederösterreich), Stadt

- Ungeld 289

St. Ulrich (Sveti Urh in Vihre, sö. Gurkfeld/Krško, Slowenien), Dorf (öde) zu 117

St. Veit a.d. Glan (Kärnten), Stadt 133

- Einwohner s. Kifel

St. Veit am Pflaum (Fiume, heute Rijeka, Kroatien), Stadt 212 
- Bürger s. Nikolitsch

Sannd (Gegend bei Lemberg/Lemberg pri Šmarju, ö. Cilli/Celje, Slowenien), am $\sim 303$

Sannd Gilgen, Giligen s. St. Ägidius (Egid)

Sannd Vyor (bei Prem, sü. Adelsberg/ Postojna, Slowenien)

- Maut 116

Sarbrugken s. Saarbrücken

Sarmingstein (bei St. Nikola a.d. Donau, ö. Grein, Oberösterreich), Schloss 55, 320

- Maut 320

- Pfleger s. Heinrich Prüschenk

Saurau (nö. Murau, Steiermark), von , (rittermäßige steirische) Familie

- Hans, Ritter 162

- Wilhelm, Verweser der Hauptmannschaft von Steier 130

Savoyen (Frankreich), Herzogtum

- Bona von , Tochter Ludwigs I. von Savoyen, Ehefrau Galeazzo Maria Sforzas († 1503) 105

- - ihr Sohn Gian Galeazzo s. Mailand, Hz. von

Schabnig (Zabnik, Gegend bei Rohitsch/Rogatec, ö. Cilli/Celje, Slowenien), am $~ 303$

Schachner, Johann s. St. Lambrecht, Abt von

Schärffenberg (Scharf[f]enberg) (Svibno, sw. Cilli/Celje, Slowenien), Herren (rittermäßig) von

- Bernhard, Rat Friedr. III., Hauptmann ob der Enns 328, 329

- Schloss 138

- - Leute 138

- - Pfleger s. Obritschan

Schaffhausen (Schweiz), Stadt 216
- Bürgermeister und Rat 216

- Benediktinerkloster Allerheiligen 216

- Abt Konrad (Dettikofer) (14661488) 216

Schaffnegk, Schaffnigk s. Schanfigg

Schandacher, Jörg 100

Schandel, Wolfgang, Bürgermeister von Wiener Neustadt 234

Schanfigg zu St. Peter (ö. Chur, Kanton Graubünden, Schweiz), Gericht

-Amman, Rat und Gemeinde 195, 196

Scharf(f)enberg s. Schärffenberg

Scharffwindt, Martin, Pfarrer von Windischgraz 274

Schauenstein (bei Krug, sw. Horn, Niederösterreich), Schloss 55

- Pfleger s. Kaspar Ringkl

Schaunberg (nw. Eferding, Oberösterreich), Grafen von $\sim$ (Schaunberger)

- Wolfgang $\sim 55$

Scheck von (vom) Wald, (rittermäßige) Familie 55

- Georg 55

Schelklingen (n. Ehingen, BadenWürttemberg), Herrschaft 207, 208

- Bergbau 207, 208

Scheller, Stephan, Bürger und Stadtrichter von Judenburg 21

Schenken von Osterwitz (Hochosterwitz, ö. St. Veit a.d. Glan, Kärnten), (Kärntner) Familie (Herren) 238

- Hans, Sohn Jörgs 238

- Jörg (Georg) 238

- Wilhelm, Bruder Jörgs 238

Schetzer, Jörg (Georg), Diener Hz. Sigmunds von Österreich 25

Scheyrer, Georg (Jörg) 297, 331

Schilling, Konrad aus Kronach, öff. Notar 275, 276 
Schködl s. Skodel

Schladming (Steiermark), Stadt

- Bergbau 118

- Bergrichter s. Matthias Moser

- Pfarre 118

Schlaining (Stadtschlaining, nö. Oberwart, Burgenland) s. Baumkircher

Schleinitz (w. Meißen, Sachsen), Ernst von , Administrator des Erzbistums Prag (1525-1544) 82

Schlick, (Egerer) Familie

- Sigmund, Herr zu Weißkirchen, Pfleger von Schloss Wald 55, 268, 288

Schliengen (sw. Mühlheim, BadenWürttemberg), Dorf 64, 66

Schnitzer s. Snitzer

Schöder (nw. Murau, Steiermark) 90

Schödl s. Skodel

Schöferlin, (württembergische) Familie

- Bernhard, Dr., Anwalt Mechthilds von Österreich und Eberhards von Württemberg 26, 27

Schönau (Schönau a.d. Triesting, sü. Baden, Niederösterreich), Schloss 39

- Pfleger s. Leonhard Held

Schönberg (Dorenji/Dolenji

Podšumberk, sö. Treffen/Trebnje, Slowenien), Dorf 11

Schönberg, Georg (von ), Propst zu Pressburg, päpstl. Protonotar 105, 106

Schottl, Franz, Bürger von Neunkirchen $\mathbf{4 0}$

Schottwien (sw. Neunkirchen, Niederösterreich), Markt 6

Schrättl, Georg, Lic. decr., Prokurator am KG 275

Schrott, (steirische) Familie
- Jörg (Georg), Diener Friedr. III., Pfleger von Klamm 24, 48, 115

Schrutauer, Virgil, Stadtanwalt Friedr. III. in Wien und ksl. Gesandter 28, 90

Schurger, Hans, Stadtschreiber von Krems und Stein 98

Schwaben, Landvogtei 304

- Landvogt 221; s. Truchsess von Waldburg

Schwanberg (Schwamberg) (Švamberk bei Krasikov, nw. Pilsen/Plzeň, Tschechien), Herren von

- Bohuslav, Oberster Hauptmann und Oberster Hofmeister des Kgr. Böhmen 69

- Jan (Johann), Großprior des Johanniterordens zu Strakonitz 69

Schwarzenbach (Črna na Koroškem, sw. Unterdrauburg/Dravograd, Slowenien), Amt 226

- Amtmann s. Jörg Fleischhacker

Schwechat (sö. Wien, Niederösterreich)

- Feld (Unser Frawnveld) zu $\sim 6$

Schweiz(er) s. Eidgenossen

Schwyz (Schweiz), Gemeinde

- Ammann und Rat 196

Schymel, Peter, Bürger von Gmunden 315

Scriptoris, Michael von Meersburg, öff. Notar, Kler. des Bistums Konstanz 326

Seckau (n. Knittelfeld, Steiermark), Bistum

- Bischof Christoph (I.) (von Trautmannsdorf) (1477-1480) 244

Seeache (Fluss) s. Ach

Seefelder, Kaspar, Bürgermeister von

Wiener Neustadt 20-23 
Seeland (Niederlande), Grafschaft 146-148

- Grafen von s. Karl von Burgund, Maria von Burgund, Maximilian I.

- Untertanen 147, 148

Seeon (nö. Rosenheim, Bayern), Benediktinerkloster

- Abt und Konvent 253

Seisenegg (nö. Amstetten, Niederösterreich), Herren (rittermäßig) von (Seisenegger)

-Wolfgang 313

Semmering (Gebirgspass sw. Neunkirchen zw. Niederösterreich und Steiermark) 155

Senftenberger, der $\sim$, Bürger von Wiener Neustadt 234

Seus, Konrad, Bürger von Nürnberg 166, 167, 275, 276

- Johann, Dr. iur., Sohn Konrads 276

Sforza s. Mailand, Hz. von

Sigismund, röm.-dt. Kg. (1410-1437), K. (1433), Kg. von Ungarn und Böhmen 158, 216, 304

Sigmund (von Tirol) s. Österreich, Hz. von

Sigmund auf dem perg (vmtl. in/bei Xaveriberg bei St. Lorenzen im Lesachtal, nw. Hermagor, Kärnten) 8

Silberberg (nö. Friesach, Kärnten), Jörg (Georg) von , Ritter 308

Simmering (Wien XI)

- in der Eben zu $\sim 6$

Sinzendorf (bei Nußbach, nö. Kirchdorf a.d. Krems, Oberösterreich), Herren (rittermäßig) von (Sinzendorfer)

- Hans 92, 102

- - sein Vetter s. Christoph Jörger
Sipplingen (nw. Überlingen, BadenWürttemberg), Dorf 221

- Ammann und Richter 221

- Gemeinde 221

- Kelnhof (Sitz der Amtleute des Konstanzer Heilig-Geist-Spitals) 221

Sitzenberg (w. Tulln, Niederösterreich), Burg 299

Sixtus IV. (Francesco della Rovere), Papst (1471-1484) 55, 82, 171, 190, 193, 244, 272, 273, 326

- Legaten s. Patriarch Marcus von Aquileia, Bf. Gabriel von Erlau

- Protonotar s. Georg Schönberg Sizilien (Insel im Mittelmeer, Italien),

Könige von $\sim$ s. Neapel

Skodel (Schködl, Schödl, Skodl, Sködl), (rittermäßige Kärntner)

Familie

- Jörg (Georg), Pfleger von Weißenfels 308

- Sigmund, Silberkämmerer Friedr. III., Pfleger von Finkenstein 47

Skodl, Sködl s. Skodel

Slaher Gilg, Pfleger von Laxenburg 240

Slovenj Gradec s. Windischgraz

Slovenska Bistrica s. WindischFeistritz

Šmartno pri Litiji s. St. Martin bei Littai

Snitzer (Schnitzer), Lukas, Registrator in der röm. Kanzlei Friedr. III. 82, 147, 148, 158, 207

Sollenau (n. Wiener Neustadt, Niederösterreich)

- Maut 39

Sonnegg s. Ungnad

Sonnenberg (Burg bei Nüziders, nw. 
Bludenz, Vorarlberg), Grafen von

s. Truchsessen von Waldburg

Sotla (Fluss in Slowenien)

- Mühle 303

Span, (Marburger) Familie

- Andreas, Vetter Barbaras 129

- Barbara 129

- Leonhard (Lienhard), Vater Barbaras 129

Spangstein (auch Ahnherrnschloss)

(Burg bei Schwanberg, sw.

Deutschlandsberg, Steiermark),

Herren (rittermäßig) von (Spangsteiner)

- Andreas 135, 271

- seine Ehefrau s. Kristina Hafner

Spaur (Altspaur/Spormaggiore, nw.

Trient/Trento, Italien), Hans (Jo-

hann) von , Rat Friedr. III., Pfleger

von Starhemberg 18

Sperlmul, gesessen auf der $\sim$ s. Perler

Speyer (Rheinland-Pfalz), Bistum 73,

171,179

- Kleriker s. Johannes Grumel

Spiš s. Zips

Spital am Pyhrn (sü. Windischgarsten,

Oberösterreich), Kollegiatstift

- Dekan und Kapitel 88, 89, 104

- - Dekan Urban (von Weix) († 1496)

88,89

Spittal a.d. Drau (Kärnten), Markt 124

- Einwohner s. Melchior Posch

Spitzweg (Spitzwegk, Spitzweck),

Wolfgang, Diener Friedr. III., Se-

kretär und Notar in der röm. Kanz-

lei, Bürger und Stadtschreiber von

Wiener Neustadt 21, 234

Spodnja Polskava s. Unterpulskau

Spodnja Selnica s. Unterzellnitz

Spormaggiore s. Spaur
Stadler

- Bernhard 224

- Jörg (Georg), Kanzleischreiber

Friedr. III. 327, 328, 329

Stain (Stein), Andreas am (1420/30 1489), Sekretär Friedr. III., Pfarrer von Gratwein und Erzpriester der

Untersteiermark 155, 327

Stainer s. Steiner

Stainprugk (?, mglw. sw. Spittal a.d.

Drau, Kärnten), Gut an der $\sim 232$

Stanz (bei Kindberg, nö. Bruck a.d.

Mur, Steiermark), in der $\sim, \mathrm{Tal}$

- Ungeld 155

Starhemberg (in Haag am Hausruck, sö. Ried im Innkreis, Oberösterreich), Schloss 18, 217-220

- Herren von

- - Johann (Jan) 55

--Rüdiger ( $†$ 1480), Rat und Gesandter Friedr. III. 28

- Pfleger s. Hans Oberhaimer

Starhemberg (nw. Wiener Neustadt, Niederösterreich), Schloss 18

- Pfleger s. Jörg Fuchs von Fuchsberg, Christoph Sachsenlander, Hans von Spaur

Stari grad (Oberstein) s. Stein in Krain Stattenberg (Štatenberg) s. Prüschenk

Staud (Stude), Peter, Prokurator am KG 275

Staud, Wolfgang, Meister, öff. Notar, Kler. des Bistums Eichstätt 53

Šteberk s. Stegberg

Stecher, Christoph 60

Stegberg (Šteberk, bei Zirknitz/Cerknica, sü. Laibach/Ljubljana, Slowenien), Amt 188

- Amtleute s. Hans von Laak, Balthasar Wagen 
Steger, Hans, Bürgermeister und Stadtrichter von Wien († um 1459/61) 306

Steier/Steiermark, Herzogtum 6, 30, $74,129,130,135,146,147,159$, 211, 277

-Amt/Ämter

--Einnehmer (Aufschlag) s. Jörg Burckhart, Hans Vilshofer

--Erzpriester (Archidiakonat) der Untersteiermark s. Andreas am Stain

-- Landschreiber s. Pankraz Rindscheit

--Marchfutterer s. Kaspar Gräfenberger

--Münzmeister s. Hans Weinich

- - Verweser der Hauptmannschaft s. Wilhelm von Saurau

- - Weisbote s. Hans Weidinger

- Landstände 241

- Ober- und Untersteiermark (Obern und Nidern Steyrmarch) 210

--Untersteiermark 152, 155, 327

Stein (ehemals Doppelstadt KremsStein, heute Stadtteil von Krems, Niederösterreich), Stadt 98, 99

- Bürgermeister zu Krems und $\sim$ s. Wolfgang Aichperger

- Mautner s. Bernhard Karlinger

- Stadtschreiber s. Hans Schurger

$-\mathrm{s}$. Krems

Stein in Krain (Kamnik, nö. Laibach/ Ljubljana, Slowenien), Stadt 24, 114

- Bürger 24; s. Kremser

-Amt 115, 330

- Landgericht 115, 330

- Maut 115, 330

- Schloss (Oberstein/Stari grad, oberhalb von Stein in Krain) 330
-- Pfleger s. Valentin von Lamberg, Erasmus Lueger

Stein, vom

- Jörg 191

- Kaspar, Amtmann zu Primskau 332

Stein s. Stain

Steinberg, Hans (Johann), Dr. iur. utr., Diener und Gesandter Friedr. III. $(\dagger 1500) 273$

Steinenstadt (Stadtteil von Neuenburg am Rhein, w. Mühlheim, BadenWürttemberg), Dorf 64, 66

Steiner, Ruprecht, Pfleger von Pittersberg und Amtmann zu Lesach 34

Steirer s. Steyrer

Stermeler s. Stermol

Stermol (Strmol in Rogatec/Rohitsch, ö. Cilli/Celje, Slowenien), von , (rittermäßige Krainer) Familie (Stermoler, Stermeler)

- Andreas, Sohn Heinrichs, Bruder Heinrichs (d.J.), Margaretes und Magdalenas 303

- Heinrich 303

- Heinrich (d. J.), Sohn Heinrichs, (jüngerer) Bruder des Andreas 303

- Magdalena, Tochter Heinrichs, (jüngere) Schwester des Andreas 303

- Margarete, Tochter Heinrichs, (jüngere) Schwester des Andreas 303

Sternberg (Český Šternberk, ö. Beneschau/Benešov, Tschechien), Zdenko von $\sim 55$

Stettenberg s. Prüschenk zu

Stettner, Berthold 17

Steyr (Oberösterreich), Stadt 55, 93, 101,161

- Bürger 93; s. Dorfner, Pandorfer

- Haus 55; Inhaber s. Ulrich von Grafenegg 
- Herrschaft 89

- Scheckenamt 55

- Schleifmühle 161

- Schloss 55, 93

- Steyrbrücke 161

- Ungeld 93

- AO 101, 103

Steyrer (Steirer), Wolfgang, Bürger und Stadtrichter von Leoben 16, 235

Stiefing (bei Wildon, n. Leibnitz, Steiermark) 159

Stifen s. Stiefing

Stockenboi (sü. Spittal a.d. Drau, Kärnten)

-Amt 19, 70

--Amtleute s. Peter Feistritzer, Wilhelm Paulseer

- Brücke 19, 70

Stockern (ö. Horn, Niederösterreich), Schloss 68

- Familie s. Stockhorner

- Teich 68

Stockhorner (Stok[h]arner, Stockerner), (rittermäßige) Familie 68 - der $\sim 68$

Stocking (bei Wildon, n. Leibnitz, Steiermark) 159

Stöckhleinshueben (?, Kärnten), Gut, gen. $\sim 232$

Stokarner, Stokharner s. Stockhorner Stokh (bei St. Marein bei Erlachstein/ Šmarje pri Jelšah, ö. Cilli/Celje, Slowenien) 303

Stokharn s. Stocking

Štokov s. Holobersy

Stoll, Alexius s. Wilten, Abt von

Straden (sü. Feldbach, Steiermark)

- Vogtei am 192

Strakonitz (Strakonice/Grünberg, Tschechien) s. Jan von Schwanberg
Straßburg (Strasbourg, Elsass, Dép.

Bas-Rhin, Frankreich), Bistum 171

- Domkapitel 181

Strasser

- Leonhard s. Vornbach, Abt von

- Peter, Bürger von Wien († 1459/61) 306

Striell (Hof) s. Prem

Strmol s. Stermol

Stubich (Stübich), Hans, Pfleger von

St. Peter ob Leoben 170, 282, 294 , 314

Stuchsenhauser, Sigmund 191

Stude s. Staud

Studnitzer, Konrad, Ratsherr von

Gmunden 114

Stübich s. Stubich

Stühlingen (nö. Waldshut-Tiengen, Baden-Württemberg), Landgrafschaft 216

- Landgrafen s. Lupfen, Gff. von Stupenský, Peter 69

Sulewitz (Sulevice/Sulejovice, sw.

Leitmeritz/Litoměřice, Tschechien),

Peter Kapler (Kapliř) von und

Winterberg (Vimperk) 69

Summerau s. Vogt von Summerau

Svätý Jur s. St. Georgen

Švamberk s. Schwanberg

Sveča s. Swetscha

Sveti Urh s. St. Ulrich

Svibno s. Schärffenberg

Swetscha (Sveča, sw. Pettau/Ptuj, Slowenien) 303

Swetzo s. Swetscha

Symaning s. Simmering

Tahenstein s. Grafenegg

Tainsteter, Kaspar, Ratsherr von

Gmunden 114 
Talhaimer, Jörg, Bürger von Wien 1

Tall (bei Lemberg pri Šmarju, nö. Cilli/ Celje, Slowenien) 303

Tandlesbach (bei Hötzendorf, sw. Rohrbach, Oberösterreich), Sitz 327-329

- Bauhof 327-329

Tellitzer, Sigmund, Pfleger von Schloss Wald $55, \mathbf{2 8 8}, \mathbf{2 8 9}$

Tenk, Thomas, Ratsherr, Stadtrichter und Stadtkämmerer von Wien 95, 325

Tenter, Peter, wohl ident mit Peter Rieder alias Tenter, Bürger und Ratsherr von Klosterneuburg 229

Teuf[f]enbacher (von Teufenbach, ö. Murau, Steiermark), (rittermäBige steirische) Familie

- Andreas 162

Teurbanger, Wolfgang, Bürger von Gmunden 315

Thalheim bei Wels (sü. Wels, Oberösterreich)

- Hof 141

- Kirche 141

Thierstein (sö. Laufen, Kanton Solothurn, Schweiz), Grafen von

- Wilhelm, Rat Friedr. III., Beisitzer des KG $(\dagger$ 1498) 25

Thunau (in Oberhaag, sw. Leibnitz, Steiermark), Amt 243

- Amtmann s. Jörg Metnitzer

Tieminger, Pankraz 55

Tilliach (Ober- und Untertilliach, sw.

Lienz, Tirol) 8

- Einwohner s. Pucher

Tingnan s. Tinjan

Tinjan (ital. Antignana, w. Mitterburg/ Pazin, Kroatien)
-Berg Jesen (Ježenjski brig, Ježenjski vrh) 206

- Leute 206

Tirol, Grafschaft 146-158

- Grafen von $~ 158$

-- s. Friedrich IV. von Österreich, Sigmund von Österreich

Tittmoning (sw. Burghausen, Bayern), Stadt 258

Tobitschau (Tovačov, sü. Olmütz/Olomouc, Tschechien), Herren von $\sim \mathrm{s}$. Zinnburg

Tollet s. Jörger

Tovačov s. Tobitschau

Traburg s. Oberdrauburg

Trafey $\mathrm{s}$. Trofaiach

Tragössertal (Tal bei Tragöß, nö. Bruck a.d. Mur, Steiermark)

- Ungeld 155

Traint, Wolfgang, Ratsherr von Gmunden 114

Trautmannsdorf a.d. Leitha (w. Bruck a.d. Leitha, Niederösterreich), Herrschaft 55

- DO 55

Trautmannsdorf in Oststeiermark

(Ortsteil von Bad Gleichenberg, sü. Feldbach, Steiermark), Herren (rittermäßig) von (Trautmannsdorfer)

- Christoph s. Seckau, Bf. von

-Hans 160

- Wolfgang, Vetter des Hans 160

Trebnje s. Treffen

Treffen (Trebnje, nw. Novo Mesto, Slowenien), Pfarre 49

Trento s. Trient

Treßdorf (bei Kirchbach, sö. Kötschach-Mauthen, Kärnten) 296

Trient (Trento, Italien), Bistum 
- Bischöfe 158

- - Alexander (Hz. von Masowien) (1423-1444) 158

-- Bernhard (von Cles), Kardinal, Präsident des Geheimen Rats Ferdinands I., Oberster Kanzler und Statthalter der oberösterr. Länder (1514-1539) 147, 148

-- Johann (Hinderbach), Dr. decr. (1465-1486) 158

- Amtleute, Lehensleute, Untertanen 158

- Hochstift 158

- Vogt s. Friedrich IV. von Österreich

Trier (Rheinland-Pfalz), Stadt

- Erzbistum

- - Domkapitel 174

- Tag zu (1473) 146

Triest (Trieste, Italien), Stadt 111, 112, 116,136

- Hauptmann s. Niklas Rauber

-Bistum

--Bischof Anton (de Goppo), Rat Friedr. III., Beisitzer des KG (1451-1485) 25

Troblje s. Rottenbach

Trofaiach (nw. Leoben, Steiermark), Pfarre 6

Trost, Hans, Bürger und Stadtrichter von Korneuburg 322

Truchsessen von Grub (nw. Horn, Niederösterreich), (österr. Freiherren-) Familie

- Heidenreich, Rat Friedr. III. († vor 1468) 269

Truchsessen von Waldburg (sö. Ravensburg, Baden-Württemberg), Familie

- Andreas, Sohn Eberhards d.Ä., Gf. von Sonnenberg $(\dagger 1511) 326$
- Eberhard (I.) d.Ä., Gf. von Sonnenberg $(\dagger 1479) 326$

- Eberhard (II.), Sohn Eberhards d.Ä. $(\dagger 1483) 326$

- Johann (Hans) d.Ä., Landvogt in Schwaben $(\dagger$ 1504) 304

- Johann, Sohn Eberhards d.Ä.., Gf. von Sonnenberg 326

- Otto, Sohn Eberhards d.Ä. s. Konstanz, Bf. von

Tschernembl (Črnomelj, sö. Gottschee/ Kocevje, Slowenien), Herren von

- Jörg (Georg), Hauptmann zu Adelsberg und auf dem Karst 138

Türken 42, 83, 105, 238 (türkische Gefangenschaft), 267 (Ungläubige), 309

- Sultan s. Osmanisches Reich

- s. auch Bajezid Ottman (,Fürst der Türken")

Turcken s. Türken

Turjak s. Auersperg

Turn unter Neuburg (Turn pod Novim gradom) s. Neuburg in der Kanker

Ungarn, Königreich 42, 106, 121, 309

- Einwohner 82

- Könige von 38, 106, 210

- - Matthias (I.) Corvinus (14581490), Kg. von Böhmen 28, 38, 42, 61, 69, 82, 105-108, 110, 121, 193, $210,244,273,279$

---Adoptivvater s. Friedrich III.

- - Ehefrau s. Beatrix von Neapel

---Gesandte, Bevollmächtigte 105, 106

- - - (seine) Länder und Herrschaften 106,110

--- Leute (Diener, Bürger, Leute, Dienstleute, Holden) 110 
---Söldner 106

--- Verbündete 42, 106

- s. auch Albrecht II., Sigismund,

Wladislaw II. von Böhmen

- Stände 121

- - Prälaten, Barone, Ritter und Städte 121

- Untertanen 106, 110

Ungnad, Herren zu Sonnegg (bei

Eberndorf, sü. Völkermarkt, Kärnten)

- Christoph, Rat Friedr. III. († 1481)

55, 308

- Georg (Jörg), Bruder Christophs

(† 1468) 308

Unserr Frawnveld s. Schwechat

Unterach am Attersee (nw. Bad Ischl, Oberösterreich) 103

Unterbärenthal (Dolenje Medvedje selo, Ortsteil von Treffen/Trebnje, Slowenien), Dorf 49

Unterknöringen s. Knöringen

Unterpulsgau (Spodnja Polskava, nö.

Windisch-Feistritz/Slovenska Bistrica, Slowenien) 303

- s. auch Pulsgau (Oberpulsgau)

Unter-Urscha (Urscha, Stadtteil von

Gleisdorf, ö. Graz, Steiermark),

Dorf 135

Unterwalden (Schweiz), Gemeinde

- Ammann und Rat 197

Unterzellnitz (Spodnja Selnica, bei Selnica ob Dravi, w. Marburg/Maribor, Slowenien) 285

Uri (Schweiz), Gemeinde

- Ammann und Rat 198

Urscha s. Unter-Urscha

Utrecht (Niederlande), Bistum

- Bischof (Kirchenvorsteher) 171

- Domkapitel (Propst, Dekan, Kapitel,
Kanoniker, sonstige Würdenträger) 171

Utschtal (Tal bei Oberaich, w. Bruck a.d. Mur, Steiermark)

- Ungeld 155

Uttweiler (n. Buchsweiler, Dép. BasRhin, Frankreich), Herren von

- Adam 14

- - Brüder und Vettern

-- -Adolf 14

- - - Eberhard 14

- - - Friedrich 14

-- - Georg 14

--- Heinrich 14

---Melchior 14

--- Michael 14

Varst, under dem $\sim \mathrm{s}$. Worst

Vaschang, Jörg, Bürger von Gmunden

315

- Sibylle, Ehefrau Jörgs, Enkelin des

Michael Fritz 315

Vedendorffs. Ferndorf

Veglia s. Frangepan zu

Veitmile s. Weitenmühl

Veitsch (w. Mürzzuschlag, Steiermark), in der $\sim$ Tal

- Ungeld 155

Vellach (Obervellach, nw. Spittal a.d.

Drau, Kärnten), Gericht 20, 22, 154

Venedig (Venezia, Italien) 158

- Marcus Barbo von s. Aquilea, Patriarch von

- Münze 116, 123, 317

Venezia s. Venedig

Venningen, Johann von $\sim$ s. Basel, Bf. von

Veprinac s. Veprinitz

Veprinitz (Veprinac, w. Rijeka, Kroatien), Kastell und Amt 112 
- Amtleute s. Niklas Rauber, Jakob Raunacher

Verdun (Dép. Meuse, Frankreich), Bistum 144

- Bischof Guillaume (Wilhelm) (de Haraucourt) (1457-1500) 144

Verona (Italien), Gabriel Rangoni von $\sim$ s. Erlau, Bf. von $\sim$

Vetter, Leonhard von Donauwörth 70

Viktring (sw. Klagenfurt, Kärnten), Zisterzienserkloster 134

-Abt 134

- Pfarrkirchen (inkorporiert) 134

- - Pfarrhöfe 134

- - Priester und Seelsorger (seeltrager) 134

Villach (Kärnten), Stadt 164, 308

- Bürger s. Haller

Villacher Alpe (Berg w. Villach, Kärnten) 308

-Bäche s. Lerchbach, Nötschbach

Vilshofen a.d. Donau (Bayern), Stadt 7

- Bürger s. Maurer

- Kastner s. Planck

- Pfleger s. Wilhelm von Rottau

Vilshofer, Hans 210

Vimperk s. Winterberg

Vindorfer Gotthard, Bürger von Wiener

Neustadt 40, 41, 67

Vipava s. Wippach

Vischmeister, Hieronymus zu Neu-

Attersee (Schloss Kogl) 103

Višnja Gora s. Weixelburg

Vöcklamarkt (w. Vöcklabruck, Oberösterreich), Pfarre 315

Völkermarkt (Kärnten), Stadt 143

- Richter, Rat und Bürger 143

- Jahrmarkt 143

- Landtag zu 267

- Maut 143
Vogel, der , Einwohner von Voitsberg 130

Vogt von Summerau (sw. Wangen, Baden-Württemberg), Familie

- Eitelhans, Bruder Heinrichs 301

- Hans Heinrich, Lehrer beider Rechte, Bruder Heinrichs 301

- Heinrich, Ritter, Pfleger von Schloss

Fürstenfeld und Amtmann zu Feldbach 301

Voit, Wolfgang 39

Voitsberg (Steiermark), Stadt 90, 130

- Amtmann s. Hans Pickl

- Bürger und Einwohner s. Judel, Vogel

- Häuser

--Dornhaws 130

- - Haus des Vogel 130

- Hauptplatz (am Platz) 130

- Judlamt 90

- Kirchengasse 130

Vojnik s. Hochenegg

Vorau (nw. Hartberg, Steiermark),

Augustiner-Chorherrenstift

- Propst Leonhard und Konvent 119

- Kellermeister 119

Voraubach (Gewässer bei Vorau, Steiermark)

-Fischweide 119

Vornbach (Formbach) (sü. Passau, Bayern), Benediktinerkloster

- Abt Leonhard (Strasser) (1474-1501) und Konvent 12

- Klosterleute 12

- Propstei s. Gloggnitz

Vrbovec in Nazarje s. Altenburg

Wachau (Donautal zw. Melk und Krems, Niederösterreich) 227

Waching, Familie 
- Barbara, Tochter Margaretes und Gabriels, Ehefrau Peter Langauers 81

- Gabriel 81

- Margarete, Ehefrau Gabriels 81

Währing (Wien XVIII) 55

Wagen, (Krainer) Familie

- Andreas, Türhüter Friedr. III., Verweser der Saline Aussee 3, 127

- Balthasar, Pfleger von Haasberg und Amtmann zu Stegberg 188

Wagram (vmtl. Wagram am Wagram, Ortsteil von Grafenwörth, nö. Krems, Niederösterreich), Dorf 55

Waidhofen a.d. Thaya (Niederösterreich), Stadt

- Teich 298

Waidhofen a.d. Ybbs (Niederösterreich), Stadt

- Ungeld 13, 31

Waitzinger, Jörg, Bürger von Lavamünd 98

Wald (bei Pyhra, sö. St. Pölten, Niederösterreich), Schloss 55, 288

- Pfleger s. Sigmund Schlick, Sigmund Tellitzer

Wald s. Scheck von

Waldburg (sö. Ravensburg, BadenWürttemberg) s. Truchsess von Waldburg

Waldhausen (im Strudengau, nö. Grein, Oberösterreich), Augustiner-Chorherrenstift

- Propst Johann 222, 313

Waldner, Johann, aus Salzburg, Protonotar und Leiter der röm. Kanzlei Friedr. III., österr. Kanzler Maximilians I. († 1502) 13, 82, 87, 158, 207

Wallsee (Niederwallsee: nw. Amstetten, Niederösterreich, Oberwallsee: bei Feldkirchen a.d. Donau, nw. Linz, Oberösterreich), Herren von 55

- Reinprecht (V.), Rat Friedr. III. († 1483) 55, 69, 95, 96

Waltersdorf (Oberwaltersdorf, sö. Baden, Niederösterreich), Schloss 55

Wartenauer, Jörg, Diener Friedr. III. 300

Warttenawer s. Wartenauer

Wasserleonburg (Schloss bei Nötsch im Gailtal, w. Villach, Kärnten), Herrschaft 308

Waxenegg (bei Anger, nö. Weiz, Steiermark), Schloss 239, 242

-Amt 239, 242

- Landgericht 239, 242

- Pfleger s. Wilhelm Kundorfer

Wehingen (sö. Rottweil, BadenWürttemberg), Burgstall 25-27, 201-203

- Herren (rittermäßig) von $\sim$ (österr. Zweig), Inhaber des (erblichen) Türhüteramts in Österreich (Wehinger)

--Achaz, (jüngerer) Bruder Leopolds 299

- Bernhard, (jüngerer) Bruder Leopolds 299

- Christoph, Bruder Leopolds († 1479) 299

-- Leopold 299

-- Wilhelm, Bruder Leopolds († 1479) 299

- -Wolfgang, (jüngerer) Bruder Leopolds 299

Weichselberger, Lienhard 331

Weidinger, Hans, Weisbote in Steier, Landrichter zu Graz 74 
Weikersdorf(Großweikersdorf, nw.

Tulln, Niederösterreich), Markt 55

- Landgericht 54

- Ungeld 54

Weinchen $\mathrm{s}$. Weinich

Weinich (Weinrich, Weinch), Hans,

Münzmeister zu Graz 209

- Barbara, Ehefrau des Hans 209

Weißenfels (Bela Peč, nw. Kronau/

Kranjska Gora, Slowenien) 308

- Pfleger s. Jörg Skodel

Weißkirchen (auch Neuhaus/Neu-

schloss) (Holič, sw. Skalica, Slowa-

kei), Herr zu s. Schlick

Weißpriach (nw. Tamsweg, Salzburg),

Herren von

- Balthasar, Rat Friedr. III. († 1484) 55

Weitenegg (w. Melk, Niederösterreich),

Schloss und Herrschaft 31, 204,

205

- Leute und Holden 204

- Pfleger s. Sigmund Prüschenk, Kaspar von Rogendorf

Weitenmühl (Veitmile, bei Neu Bydschow/Novy Bydžov, nö. Prag/

Praha, Tschechien), Herren von

- Benesch (Beneš), Bggf. von Karlstein und Oberster Münzmeister von

Böhmen 42

Weitersfeld (w. Mureck, Steiermark),

Schloss 125

- Pfleger s. Leonhard Harracher

Weitra (sw. Gmünd, Niederösterreich),

Schloss 163

- Fischweide 163

- Meierhof 163

- Pfleger s. Konrad Zirkendorfer

- Teich 163

Weix, Urban von $\sim$ s. Spital am Pyhrn

(Kollegiatstift)
Weixelburg (Višnja Gora, sö. Laibach/

Ljubljana, Slowenien), Markt

-Land- und Marktmaut 318

- Pfleger s. Hans Lengheimer

Weizer Greith s. Greith

Wels (Oberösterreich), Stadt

- Richter, Rat und Bürger 36

- Gericht 36

- Umland 36

- Ungeld 36

Welser, Hans, Ratsherr von Wien 95

Wemding (ö. Nördlingen, Bayern) s.

Johann Roth von

Wenger, Kaspar, Pfleger von Litschau 298

Werbno (Berben) (bei Reicheneck/Rifnik, sö. Cilli/Celje, Slowenien) 303

Werdenberg (nw. Buchs, Kanton

St. Gallen, Schweiz), Grafen von

- Haug (XI.), Gf. von Werdenberg-

Heiligenberg, Rat Friedr. III.,

Beisitzer des KG, Oberster (Feld-)

Hauptmann 25, 56, 57, 69

Werfenstein (ö. Grein, Oberösterreich),

Schloss 320

- Maut 320

- Pfleger s. Kaspar von Rogendorf

Westerholz (bei Schleiheim, Kanton

Schaffhausen, Schweiz), Wald 216

Widmer, Stephan, Amtmann zu

Hochenegg 152

Wien (Österreich), Stadt 1, 6, 61, 68,

$74,86,90,94,95,101,106,121$,

$222,229,288,306,307,319$

- Bürger und Einwohner s. Gwaltsho-

fer, Haiden, Haringseer, Hölzler,

Köppl, Kranperger, Leschenprant,

Lichtenperger, Murstetter, Neupaur,

Ponhaimer, Rehwein, Reicholf, 
Rorbeck, Sailer, Steger, Strasser, Talhaimer, Tenk, Welser

- Bürgermeister 61, 86, 307; s. Hans

Haringseer, Konrad Hölzler, Hans

Steger

- Bürgermeister und Rat 307

- Bürgermeister, Rat, Genannte, Gemein 61

- Bürgermeister, Richter, Rat 86

- Rat 28, 61, 86, 307

--Ratsherren s. Ernreich Köppl, Thomas Tenk, Hans Welser

- - s. Bürgermeister, Stadtanwalt, Stadtrichter

- Ämter s. Krautgeb, Ottakring

- Bürgerspital 307

- Burgfried 37

- Donaubrüicken 1, 61

- - Brückenmeister s. Ruprecht Ennser

- Häuser 55, 306

- - Häuser in der Münzerstraße

- - - Margaretenhof 306

- - - Smerbeckenhof (mit Hauskapelle) 306

- - Haus gegenüber dem Propsthof 86

- - Haus bei St. Michael am Eck 95

--Leinwandhaus 307

- Handwerk und Handel

- - Krämer 307

- - Leinwater/Leinwaterzeche 307

- - Schuster s. Thomas Rorbeck

- Kaltmaut 322

- Kirchen und Klöster

- - Dompropstei von St. Stephan 244

- - - Propsthof 86

-- Kapelle 37, 306

- - Minoritenkloster

- - - Guardian und Konvent 37

- - St. Michael 95
- - St. Thomaskapelle (Hauskapelle im Smerbeckenhof) 306

- Landesverwaltung (Sitz in Wien:

Hansgraf, Hubmeister, Münzmeister) s. Österreich

- Stadtanwalt Friedr. III. im Rat s. Virgil Schrutauer

- Stadtbuch 307

- Stadtgericht 84

- Stadtkämmerer s. Thomas Tenk

- Stadtrichter 86; s. Hans Murstetter, Hans Steger, Thomas Tenk

- Straßen und Plätze

- - Bauernmarkt 306

- - Brandstätte 306

- - Freisingergasse 306

- - Hoher Markt 307

- - Landskrongasse 306

- - Münzerstraße 306

- - Rotenturmstraße 306

- Tag zu 105, 106, 279

- Wiener Konkordat (1448) 171

- s. Altmannsdorf, Atzgersdorf, Erdberg, Krautgeb, Ottakring, Simmering

-AO 49, 51, 53, 55-59, 61, 62, 65, 66, $73,75,80-82,85-87,91$

-DO 2, 54, 61, 84, 93, 229, 325

Wiener, der $\sim 159$

Wiener Neudorf (Neudorf) (sü. Wien, Niederösterreich), Maut 39

Wiener Neustadt (Niederösterreich), Stadt 6, 18, 20-23, 40, 43, 67, 154, 234

- Bürger und Einwohner s. Gruber, Hölzler, Hopf, Rorbeck, Spitzweg, Vindorfer; s. auch Hausbesitzer - Rat

--Bürgermeister s. Wolfgang Schandel, Kaspar Seefelder 
- - Ratsherren s. Simon Gruber, Augustin Pottensteiner, Wolfgang Rorbeck, Prokop Zinner

-- Stadtschreiber s. Wolfgang Spitzweg

- Häuser 55, 234

- - Haus am Fischmarkt 234

- - Hausbesitzer s. Grafenegger, Hopf, Jamnitzer, Schandel, Senftenberger

- Kammergericht 55

- Pfarrkirche (Dom) Unserer Lieben

Frau (Liebfrauen-Pfarrkirche, Liebfrauendom) 234

- Kirchenmeister s. Augustin Pottensteiner

- Tag zu 105

- Ungeld 67

-AO 3, 5, 6, 8-12, 14, 15, 24-31, $35-38,42,44,46,47$

-DO 19-23, 42, 45, 234

Wienn s. Wien

Wieselburg (Niederösterreich), Pfarre 5

- Pfarrer s. Hans Winkler

Wilberstorf s. Wilfersdorf

Wildenstein (sw. Bad Ischl, Oberösterreich), Schloss und Herrschaft 32, 33,114

- Landgericht 32, 114

- Pfleger/Verweser s. Gmunden, Wilhelm Lesch

Wilfersdorf (Kleinwilfersdorf, nö. Stockerau, Niederösterreich)

- Wiese zu $~ 299$

Wilsberg (bei Zabern/Saverne, Dép.

Bas-Rhin, Frankreich), Konrad von $\sim 14$

Wilshausen (nw. Hochfelden, Dép.

Bas-Rhin, Frankreich) 14

Wilten (Stadtteil von Innsbruck, Tirol), Prämonstratenserkloster 146-148
- Äbte

--Alexius (Stoll) (1470-1492) 109

- Leonhard (Klingler) (1498-1530) 146-148

-DO 109, 146-148

Windische Mark (sö. Teil von Krain, Slowenien), Herrschaft auf der 146, 147

Windisch-Feistritz (Feistritz) (Slovenska Bistrica, sw. Marburg/Maribor, Slowneien), Stadt 230

-Amt 230

- Gericht 230

- Richter s. Bernhard Krannagl

- Schloss

-- Pfleger 230

- Stadtsteuer 230

Windischgraz (Slovenj Gradec, sw. Marburg/Maribor, Slowenien) 274

-Amt 324

- Herrschaft 324

- Leute und Holden 324

- Pfarrer s. Martin Scharffwindt

- Schloss 274

- Wald bei , gen. die Gemain am Perg 274

Winkl (sü. Kirchberg am Wagram, Niederösterreich), Schloss 55

- Herren von $\sim 55$

Winkl, im $\sim$ s. Leoben

Winkler, Hans, Pfarrer von Wieselburg, Hofkaplan Friedr. III. 5

Winklern (nw. Spittal a.d. Drau, Kärnten), Turm 81

Winter, Andreas 303

Winterberg (Vimperk, nw. Budweis/ České Budějovice, Tschechien) s. Sulewitz

Wippach (Vipava, nw. Postojna/Adelsberg, Slowenien), Herrschaft 9, 11 
- Leute und Holden 9

- Pfleger s. Leonhard von Herberstein

- Turm (Baumkircherturm) 123

- - Inhaber s. Andreas Baumkircher

-- Pfleger s. Leonhard von Herberstein

Wischberg (Bišečki Vrh, n. Pettau/Ptuj, Slowenien), am kleinen $\sim$, Erhebung 285

wisen/wyse, an der langen $\sim \mathrm{s}$. Langwies

Wladislaw II. s. Böhmen, Kg. von

Wodresch s. Oberwodresch

Wörth s. Donauwörth

Wösendorf in der Wachau (bei Weizenkirchen in der Wachau, sw. Krems, Niederösterreich), Markt 227

- Bürger s. Kransdorfer

Wolfenreut s. Grafenegg

Wolfersdorf (n. Freising, Bayern), Schloss 52, 53

-s. Reichart Kärgl zu , Pirckheimer $\mathrm{zu} \sim$

Wolkenstein (sw. Liezen, Steiermark), Schloss 231

- Landgericht 231

- Pfleger s. Hans Raschberger

Worst (Boršt, Stadtteil von Laibach/ Ljubljana, Slowenien) 24

Wrestovetz (Brestovec, bei Rohitsch/ Rogatec, ö. Cilli/Celje, Slowenien) 303

Wretzlowo (Brecljevo, bei St. Marein bei Erlachstein/Šmarje pri Jelšah, ö. Cilli/Celje, Slowenien) 303

Wrocław s. Breslau

Württemberg (Baden-Württemberg), Grafschaft

- Grafen von $\sim($ Linie zu Urach)

--Ludwig (I.) d.Ä. (1412-1450) 25
--- seine Ehefrau s. Mechthild, Pfalzgräfin bei Rhein

--Eberhard (V.) d.Ä. (,,im Bart"), Gf. von $\sim$ und Mömpelgard, Sohn Ludwigs I. und Mechthilds (1445/50 1496) 25, 26, 27, 201, 202, 203

- - - seine Anwälte s. Martin Kellner, Hermann von Sachsenheim, Bernhard Schöferlin

Würzburg (Bayern), Bistum 53, 166

- Domkapitel 177

- Kleriker s. Konrad Koppel

Wulzendorf (abgeg. bei Breitenlee, Wien XXII), Herren (rittermäßig) von $\sim$ (Wulzendorfer)

- Leopold, Ritter, Gesandter Friedr. III. 28, 55

Xaveriberg (bei St. Lorenzen im Lesachtal, nw. Hermagor, Kärnten)

- Orthner/Orther-Gut 8

Ybbs a.d. Donau (nö. Amstetten, Niederösterreich), Stadt

- Pfarre 100

- Schloss 222, 313

--Pfleger s. Kaspar von Rogendorf

- Ungeld 13, 31

York (Großbritannien), Herzogin Margarete von $\sim$, Ehefrau Karls (des Kühnen) von Burgund (1446-1503) 78, 80

Yspertal (Tal des Flusses Ysper [Isper], n. Ybbs a.d. Donau, Niederösterreich) 204, 205

Zabnik s. Schabnig

Zapolya, Emmerich, Graf in der Zips 106

Zatel s. Sotla 
Zebinger (von Zöbing zu Kranichberg, sw. Neunkirchen, Niederösterreich), Kaspar zu Reitenau 285

- seine Ehefrau s. Anna Raumschüssel

Zech, Gallus, Ritter, Pfleger von Kammern 160

Zechorner, Hans 206

Zekherner s. Zechorner

Zell s. Mariazell

Zelsowetz s. Jeschowetz

Zerowetz (Cerovec, ö. Cilli/Celje, Slowenien) 303

Zgornja Polskava (Oberpulskau) s. Pulskau

Zgornja Selnica s. Oberzellnitz

Zinnburg (Cimburk, vmtl. Nennung nach Cimburk bei Městečko

Trnàvka, sö. Zwittau/Svitavy, und nicht nach Cimburk bei Koritschan/ Koryčany, ö. Brünn/Brno, Tschechien), Herren von

- Ctibor (Stibor) von $\sim$ und Tobitschau,
Hauptmann von Mähren (14691494) 28

Zinner, Prokop, Ratsherr von Wiener

Neustadt 20, 22, 23, 154

Zips (Spiš, Gebiet in der nö. Slowakei), Graf von $\sim$ s. Emmerich Zapolya

Zirkendorfer, Konrad, Pfleger von Schloss Weitra 163

Zmollner, Stephan, Pfleger von St. Peter bei Leoben 120

Zobelsberg (Čušperk, sö. Laibach/

Ljubljana, Slowenien), Schloss 111

- Pfleger s. Hermann Rauber

Zorn, Leonhard s. Ossiach, Abt von

Zürich (Schweiz), Stadt 199, 216

-DO 216

Zutphen (sö. Apeldoorn, Niederlande), Grafschaft 146-148

- Grafen von s. Karl von Burgund,

Maria von Burgund, Maximilian I.

- Untertanen 146-148 


\title{
REGESTA IMPERII XIII: \\ REGESTEN KAISER FRIEDRICHS III. (1440-1493) NACH ARCHIVEN UND BIBLIOTHEKEN GEORDNET
}

\author{
Herausgegeben von Paul-Joachim Heinig | Christian Lackner | Alois Niederstätter
}

Band 29: Die Urkunden und Briefe aus den Archiven und Bibliotheken der Republik Slowenien

Teil 1: Die staatlichen, kommunalen und kirchlichen Archive in der Stadt Laibach/ Ljubljana

Bearb. von Jure Volcjak.

2013. 226 Seiten, Paperback

$€ 42,00 \mathrm{D} \mid € 44,00 \mathrm{~A}$

ISBN 978-3-205-79485-1

Band 28: Die Urkunden und Briefe aus den Archiven und Bibliotheken der Stadt Nürnberg

Teil 3: 1456-1463

Bearb. von Dieter Rübsamen.

2013. 263 Seiten, Paperback

$€ 49,90 \mathrm{D} \mid € 51,30 \mathrm{~A}$

ISBN 978-3-205-78877-5

Band 27: Die Urkunden und Briefe des Österreichischen Staatsarchives in Wien (1470-1475)

Abteilung Haus-, Hof- und Staatsarchiv: Allgemeine Urkundenreihe, Familienurkunden und Abschriftensammlungen (1470-1475)

Bearb. von Sonja Dünnebeil und Daniel Luger. 2012. 260 Seiten, Paperback $€ 44,90 \mathrm{D} \mid € 46,20 \mathrm{~A}$

ISBN 978-3-205-78707-5
Band 26: Die Urkunden und Briefe aus den Archiven und Bibliotheken der Tschechischen Republik

Bearb. von Eberhard Holtz.

2012. 461 Seiten, Paperback

$€ 79,00 \mathrm{D} \mid € 79,00 \mathrm{~A}$

ISBN 978-3-205-78852-2

Band 25: Die Urkunden und Briefe aus den Kurmainzer Beständen des Bayerischen Staatsarchivs in Würzburg sowie den Archiven und Bibliotheken der Stadt Mainz

Bearb. von Petra Heinicker. 2010. 217 Seiten, Paperback $€ 42,00 \mathrm{D} \mid € 44,00 \mathrm{~A}$ ISBN 978-3-205-78521-7

Band 24: Die Urkunden und Briefe aus dem historischen Staatsarchiv Königsberg im Geheimen Staatsarchiv Preußischer Kulturbesitz Berlin aus den Staatsarchiven Gdansk, Torun, Riga sowie aus dem Stadtarchiv Tallinn für die historischen Landschaften Preußen und Livland

Bearb. von Elfie-Marita Eibl 2010. 255 Seiten, Paperback $€ 53,00 \mathrm{D} \mid € 55,00 \mathrm{~A}$ ISBN 978-3-205-78509-5 
REGESTA IMPERII XIII:

REGESTEN KAISER FRIEDRICHS III. (1440-1493) NACH ARCHIVEN UND BIBLIOTHEKEN GEORDNET

\author{
Herausgegeben von Paul-Joachim Heinig | Christian Lackner |Alois Niederstätter
}

Band 35: Regesten Kaiser Friedrichs III. Die Urkunden und Briefe des Österreichischen Staatsarchivs in Wien, Abt. Haus-, Hof- und Staatsarchiv: Allgemeine Urkundenreihe, $\mathrm{Fa}$ milienurkunden und Abschriftensammlungen (1480-1482)

Bearb. von Petra Heinicker, Anne-Katrin Kunde. 2019. 201 Seiten, kartoniert

$€ 40,00 \mathrm{D} \mid € 42,00 \mathrm{~A}$

ISBN 978-3-205-23214-8

Band 34: Regesten Kaiser Friedrichs III. Die Urkunden und Briefe des Österreichischen Staatsarchivs in Wien, Abt. Haus-, Hofund Staatsarchiv:Allgemeine Urkundenreihe, Familienurkunden und Abschriftensammlungen (1476-1479)

Bearb. von Kornelia Holzner-Tobisch nach Vorarbeiten von Anne-Katrin Kunde. 2019. 272 Seiten, gebunden $€ 50,00 \mathrm{D} \mid € 52,00 \mathrm{~A}$

ISBN 978-3-205-23216-2

Band 33: Regesten Kaiser Friedrichs III. (1440-1493)

Die Urkunden und Briefe aus den Archiven und Bibliotheken des deutschen Bundeslandes Nieder sachsens (mit Ausnahme der HAB Wolfenbüttel)

Bearb. von Paul-Joachim Heinig.

2018. 375 Seiten, Paperback

$€ 60,00 \mathrm{D} \mid € 62,00 \mathrm{~A}$

ISBN 978-3-205-20072-7
Band 32: Regesten Kaiser Friedrichs III. (1440-1493)

Die Urkunden und Briefe aus dem Staatsarchiv Bamberg und den Archiven und Bibliotheken des Regierungsbezirks Oberfranken sowie aus dem Bestand Rep. 106a (Fehdeakten) des Staatsarchivs Nürnberg

Bearb. von Elfie-Marita Eibl.

2018. 561 Seiten, Paperback

$€ 95,00 \mathrm{D} \mid € 98,00 \mathrm{~A}$

ISBN 978-3-205-20071-0

Band 31: Regesten Kaiser Friedrichs III. (1440-1493)

Die Urkunden und Briefe aus den Archiven und Bibliotheken der deutschen Bundesländer Bremen, Hamburg und Schleswig-Holstein sowie der skandinavischen Länder

Bearb. von Eberhard Holtz.

2016. 310 Seiten, Paperback

$€ 40,00 \mathrm{D} \mid € 42,00 \mathrm{~A}$

ISBN 978-3-205-79419-6

Band 30: Die Urkunden und Briefe des Österreichischen Staatsarchivs in Wien Abt. Haus-, Hof- und Staatsarchiv: Allgemeine Urkundenreihe, Familienurkunden und Abschriftensammlungen (1483-1488)

Bearb. von Peter Gretzel. 2014. 280 Seiten, Paperback $€ 44,90 \mathrm{D} \mid € 46,20 \mathrm{~A}$ ISBN 978-3-205-79623-7 
www.vandenhoeck-ruprecht-verlage.com

ISBN 978-3-205-23216-2 